\title{
Effects of Various Shaped Roughness Elements in Two-Dimensional High Reynolds Number Turbulent Boundary Layers
}

\author{
Jeremy Lawrence Bennington
}

Thesis submitted to the faculty of Virginia Polytechnic Institute and State University in partial fulfillment of the requirements for the degree of

\author{
Master of Science \\ In \\ Aerospace Engineering \\ Dr. Roger L. Simpson (Chair) \\ Dr. William J. Devenport \\ Dr. Wayne Neu
}

August $24^{\text {th }}, 2004$

Blacksburg, VA

Keywords: Isolated roughness elements, Distributed roughness, Near-wall flow structure, Drag correlation, Circulation decay, Turbulent kinetic energy, Turbulent boundary layer, Subsonic, Laser-Doppler Velocimetry 


\title{
Effects of Various Shaped Roughness Elements in Two- Dimensional High Reynolds Number Turbulent Boundary \\ Layers
}

\section{Jeremy Lawrence Bennington}

\begin{abstract}
$\underline{\text { Abstract }}$
Modeling the effects of surface roughness is an area of concern in many practical engineering applications. Many current roughness models to this point have involved the use of empirical 'constants' and equivalent sand grain roughness. These underdeveloped concepts have little direct relationship to realistic roughness and cannot predict accurately and consistently the flow characteristics for different roughness shapes. In order to aid in the development of turbulence models, the present research is centered around the experimental investigation of seven various shaped single roughness elements and their effects on turbulence quantities in a two-dimensional turbulent boundary layer.
\end{abstract}

The elements under scrutiny are as follows: cone, cone with spatial variations equal to the smallest sublayer structure length scale, cone with spatial variations equal to 2.5 times the smallest sublayer structure length scale, Gaussian-shaped element, hemisphere, cube aligned perpendicular to the flow (cube at $90^{\circ}$ ), and a cube rotated $45^{\circ}$ relative to the flow. The roughness element heights, $\mathrm{k}^{+}$, non-dimensionalized by the friction velocity $\left(\mathrm{U}_{\tau}\right)$ of the approaching turbulent boundary layer, are $145,145,145,145,80,98$, and 98 respectively. Analysis of a three-dimensional fetch of the same Gaussian-shaped elements described previously was also undertaken. In order to analyze the complex flow fields, detailed measurements were obtained using a fine-measurement-volume (50 micron diameter) three-velocity component laser-Doppler velocimetry (LDV) system.

The data reveals the formation of a horseshoe vortex in front of the element, which induces the downwash of higher momentum fluid toward the wall. This 'sweep' motion 
not only creates high Reynolds stresses $\left(\overline{v^{2}}, \overline{w^{2}},-\overline{u v}\right)$ downstream of the element, but also leads to higher skin-friction drag. Triple products were also found to be very significant near the height of the element. These parameters are important in regards to the contribution of the production and diffusion of the turbulent kinetic energy in the flow. The 'peakiness' of the roughness element was found to have a direct correlation to the production of circulation, whereas the spatial smoothing does not have an immense effect on this parameter. The peaked elements were found to have a similar trend in the decay of circulation in the streamwise direction. These elements tend to show a decay proportional to $(\mathrm{x} / \mathrm{d})^{-1.12}$, whereas the cube elements and the hemisphere do not have a common trend.

A model equation is proposed for a drag correlation common to all roughness elements. This equation takes into account the viscous drag and pressure drag terms in the calculation of the actual drag due to the roughness elements presence in the boundary layer. The size, shape, frontal and wetted surface areas of the roughness elements are related to one another via this model equation. Flow drawings related to each element are presented which gives rise to a deeper understanding of the physics of the flow associated with each roughness element. 


\section{Acknowledgements}

First and foremost, I would like to thank my wonderful and lovely wife Nicole for her constant support and encouragement throughout my time in Graduate school. Many thanks also go to my family for always being there for me in my times of need.

I would like to thank the numerous people who made this research a success. Primarily my advisor and mentor Dr. Roger Simpson whose knowledge and guidance throughout this research was invaluable. The members of my committee: Dr. William Devenport who taught me how to think and study like a graduate student when I first came to Virginia Tech and Dr. Wayne Neu whose time and assistance has been much appreciated as I finished my Master's thesis.

Many thanks go to my friends and co-workers who answered my questions and were always there to lend a helping hand. Devin Stewart, Todd Lowe, Genglin Tang, and Gwibo Byun who helped me learn the basic operation of the LDV systems. Jacob George, Kevin Pisterman, Qing Tian, Shereef Sadek, and Edgar Orsi who helped me in the setup of the experiments. I would also like to thank the shop technicians, Bruce Stanger, Mike Vaught, James Lambert, and Steve Edwards who were always there to keep the project moving forward in a timely manner.

Much appreciation is also extended to all of the professors that I had the pleasure to learn from during my time at Virginia Tech. Thank you to the AOE office staff of Betty Williams, Gail Coe, and Wanda Foushee who were always able to lend a helping hand whenever needed.

Finally, I would like to thank the United States Office of Naval Research for sponsoring this research. Without the support of the Office of Naval Research grant, this research would not have been possible. 


\section{Table of Contents}

ABSTRACT .................................................................................................................................................... II

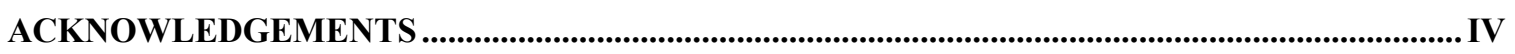

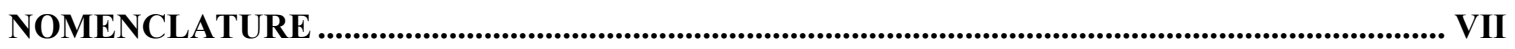

LIST OF FIGURES.............................................................................................................................. XII

LIST OF TABLES........................................................................................................................................ XIX

CHAPTER 1 INTRODUCTION _........................................................................................................... 1

LAMINAR AND TURBULENT Flow OVER ISOLATED PRotUBERANCES......................................... 2

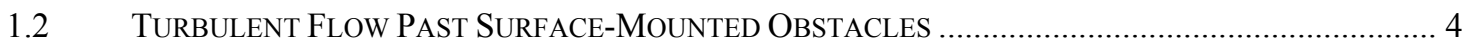

1.3 ROUGH-WALL TURBULENT BOUNDARY LAYERS................................................................. 7

1.4 RELATED MODELING AND COMPUTATIONAL EFFORTS …...................................................... 8

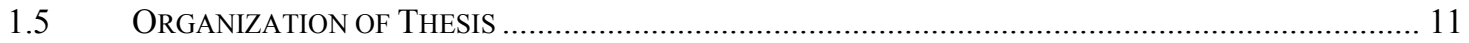

CHAPTER 2 APPARATUS AND INSTRUMENTATION.............................................................. 12

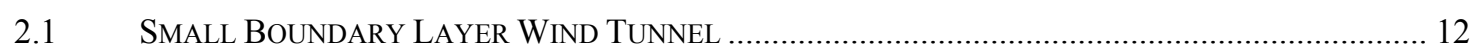

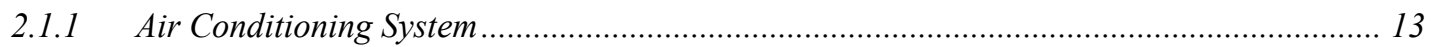

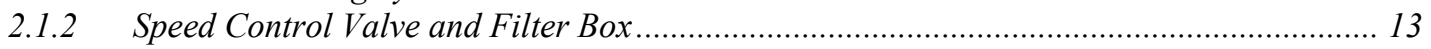

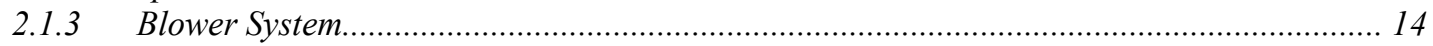

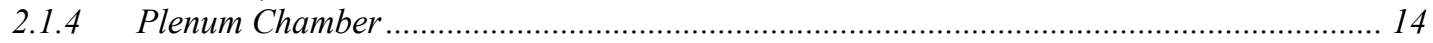

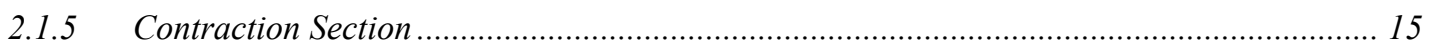

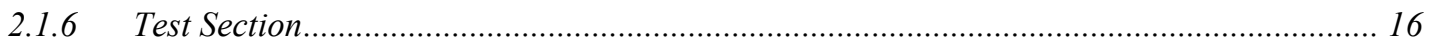

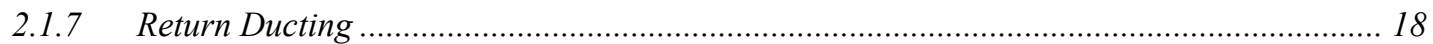

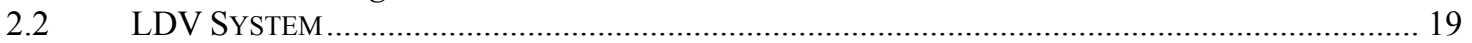

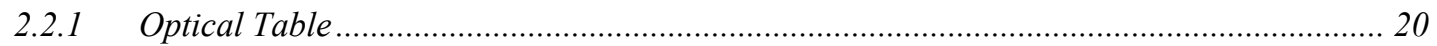

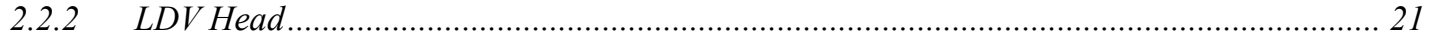

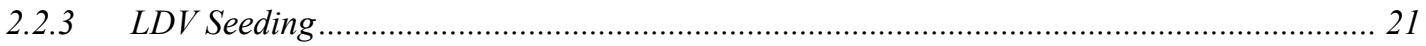

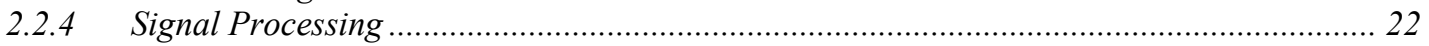

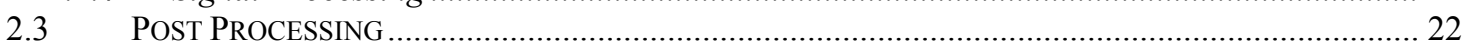

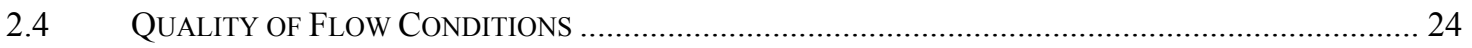

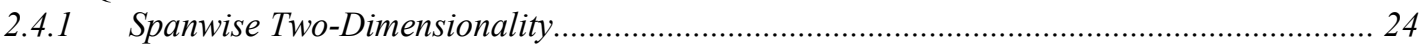

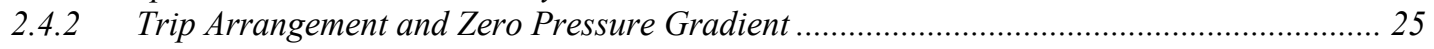

2.4.3 Profile Comparison and Boundary Layer Characteristics ............................................... 26

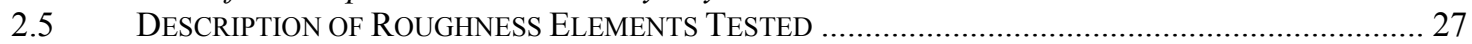

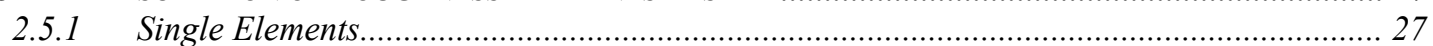

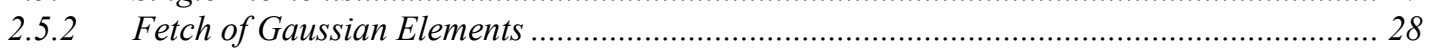

2.6 ALIGNMENT OF LDV HEAD WITH WIND TUNNEL AND ROUGHNESS ELEMENTS ............................ 30

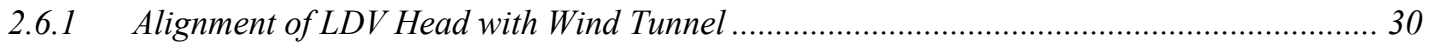

2.6.2 Alignment of LDV Head with Roughness Elements ....................................................... 30

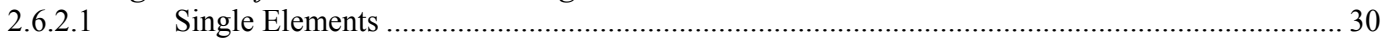

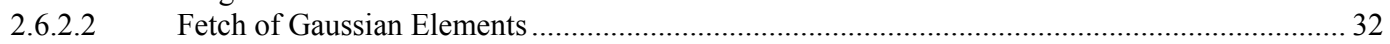

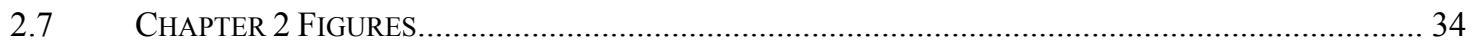

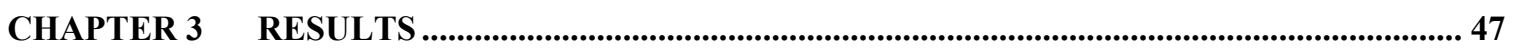

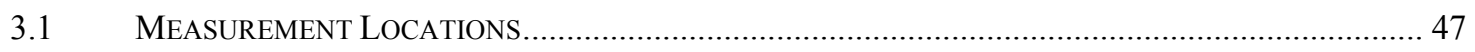

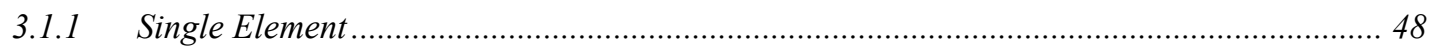

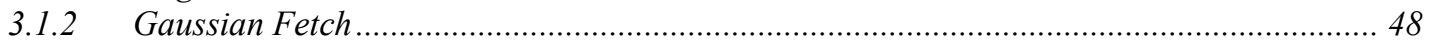

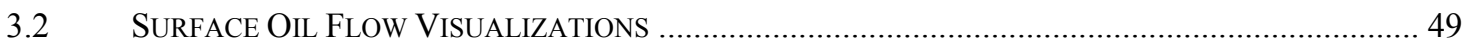

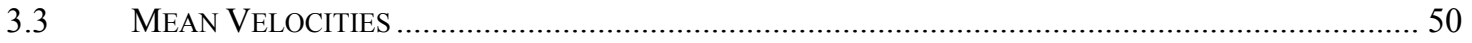

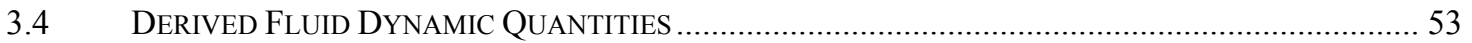

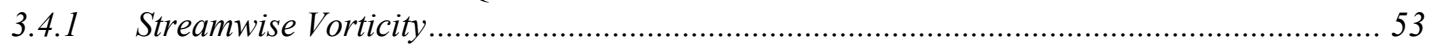

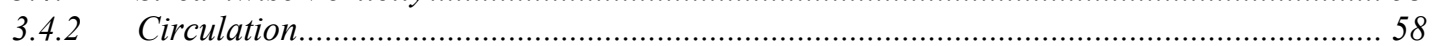




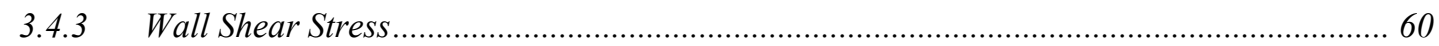

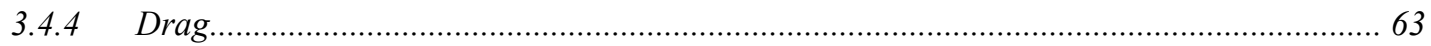

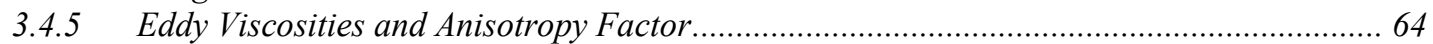

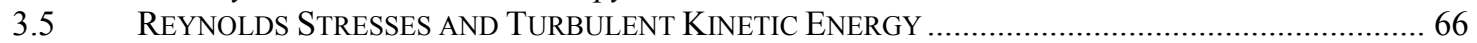

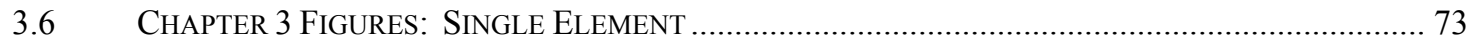

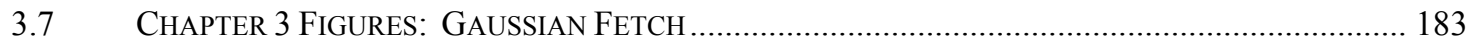

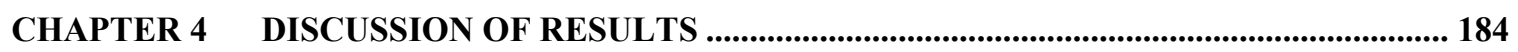

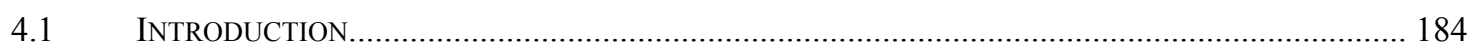

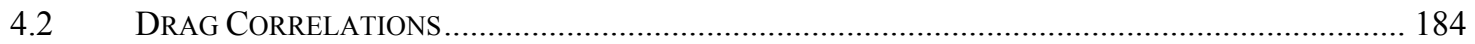

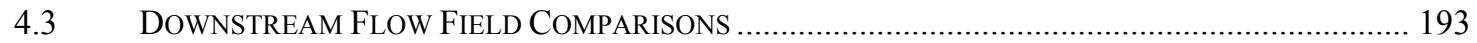

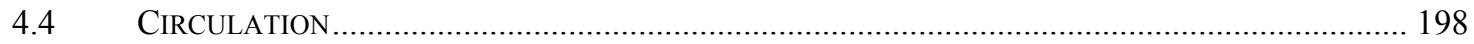

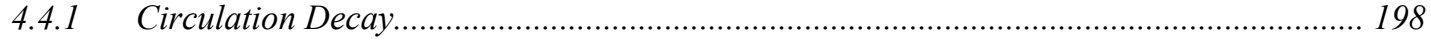

4.4.2 Streamwise and Spanwise Circulation Relationship ..................................................... 199

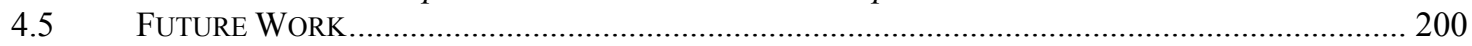

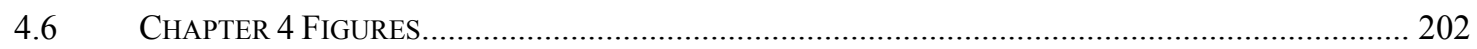

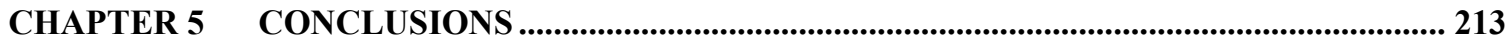

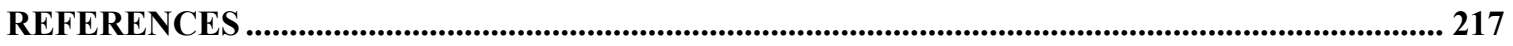

APPENDIX A TRIP ARRANGEMENTS.......................................................................................... 226

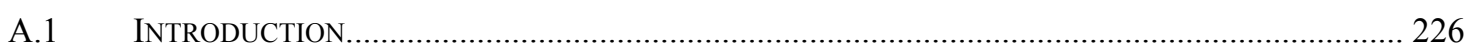

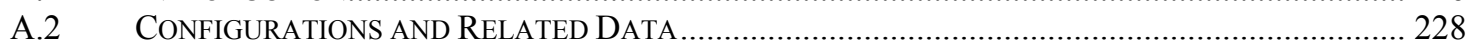

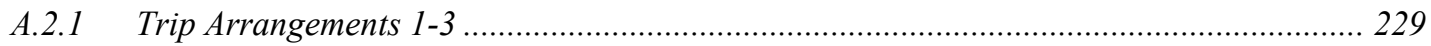

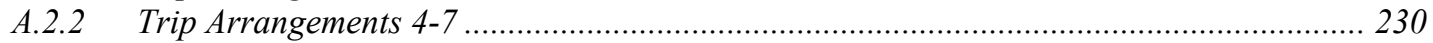

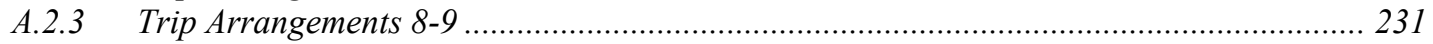

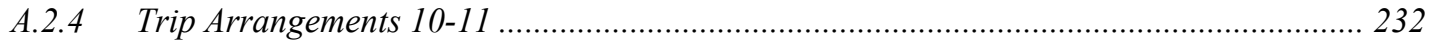

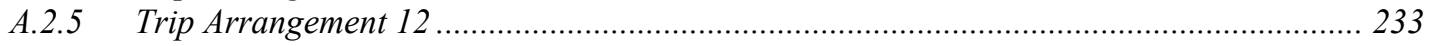

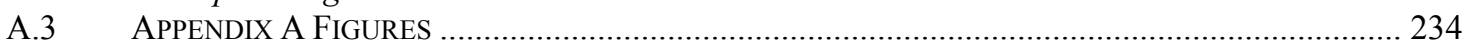

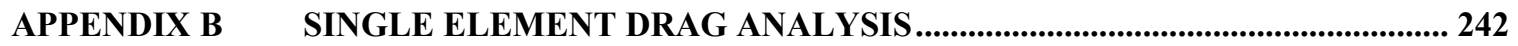

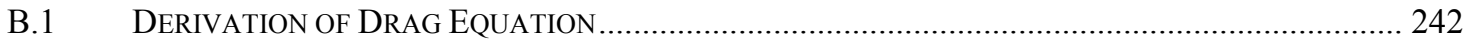

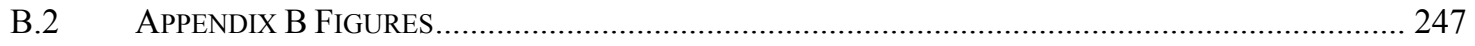

APPENDIX C UNCERTAINTY ANALYSIS ................................................................................... 248

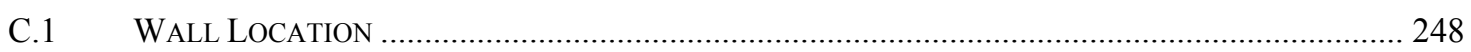

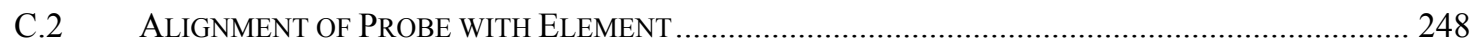

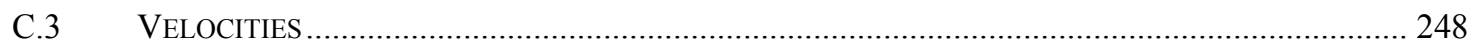

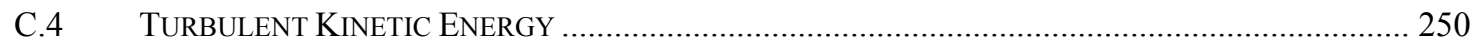

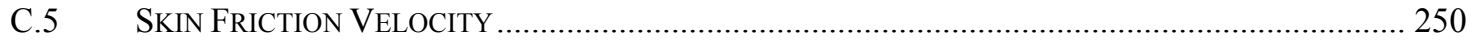

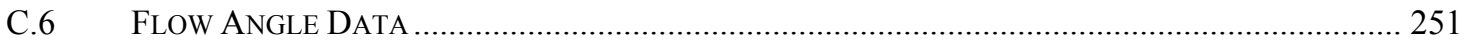

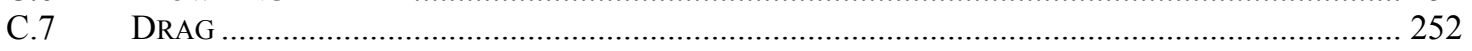

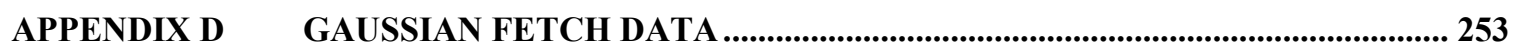

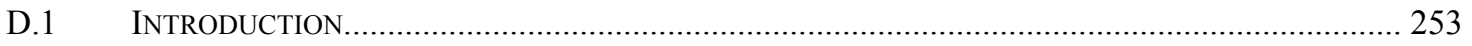

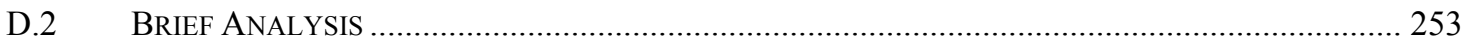

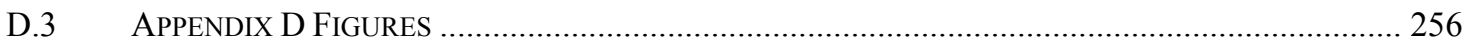

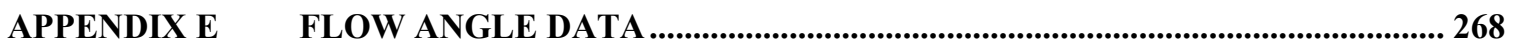

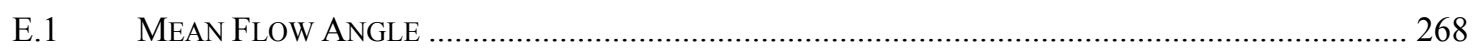

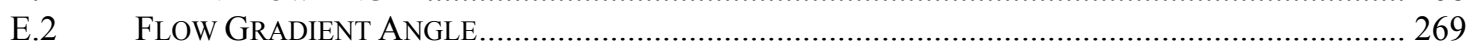

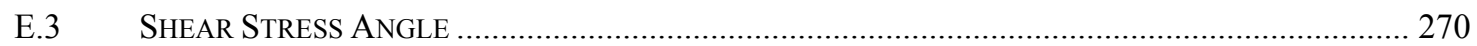

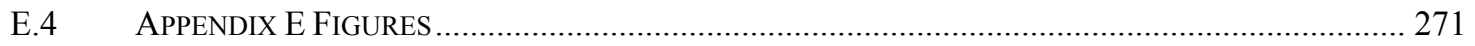

APPENDIX F ELEMENT PROFILE SHAPE DATA...................................................................... 285

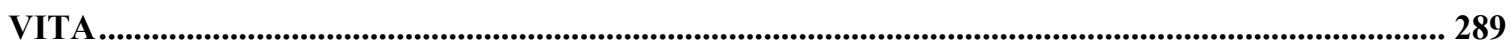




\section{Nomenclature}

\section{Roman}

2DTBL

3DTBL

$\mathrm{A}_{\mathrm{f}}$

A proj_outer, $_{\text {proj_inner }}$

$\mathrm{A}_{\mathrm{W}}$

$\mathrm{A}_{\mathrm{ww}}$

$\mathrm{b} / \mathrm{l}$

CFD

$\mathrm{C}_{1}, \mathrm{C}_{2}$

$C_{d(\text { frontal_or_wetted })}$

$C_{d_{-} \text {Wieghardt }}$

$\mathrm{C}_{\mathrm{dp}}, \mathrm{C}_{\mathrm{d} v}$

$\mathrm{C}_{\mathrm{f}}$

$\mathrm{C}_{\mathrm{f} \_ \text {outer }}, \mathrm{C}_{\mathrm{f} \text { inner }}$

$\mathrm{C}_{\mathrm{p}}$

CV

$\mathrm{C}_{\mathrm{WWA}}$

d

$\mathrm{d}^{+}$

$\mathrm{d}_{\max }$

D

DNS

DOP
Two-Dimensional Turbulent Boundary Layers

Three-Dimensional Turbulent Boundary Layers

Frontal projected area of roughness element

Projected floor surface areas of the respective wake regions within the control volume

Wetted area of roughness element

Windward wetted area of roughness element

Boundary layer

Computational fluid dynamics

Coefficients for curve fit of skin friction velocity

Drag coefficient defined as $\Delta D_{E_{-} \text {data }} /(1 / 2) \rho U_{e}^{2} A_{f_{-} \text {or }} w$

Drag coefficient defined as $\Delta D_{E_{-} \text {data }} / \bar{q} A_{\text {frontal }}$

Coefficients acquired via a least squares fit in Eqn. 4.3

Skin friction coefficient

Skin friction coefficients in the outer and inner wake regions within the control volume

Coefficient of pressure

Control volume

Coefficient that is associated with the fraction of total surface area that sees attached flow

Base diameter of axisymmetric elements, edge length of cube at $90^{\circ}$, diagonal length of cube at $45^{\circ}$

$\mathrm{d}^{+}=\mathrm{dU}_{\tau} / \mathrm{v}$

Average of the half of the differences between two data values

Diameter of the LDV measurement volume $\approx 50 \mu \mathrm{m}$

Direct Numerical Simulation

di-octyl phthalate (seeding material used for LDV system) 


\begin{tabular}{|c|c|}
\hline $\overrightarrow{d s}$ & Elemental length of circuit for integration around a closed loop \\
\hline$D_{x}$ & Component of drag acting in the $\mathrm{x}$-direction \\
\hline $\mathrm{E}(\mathrm{y})$ & $y$-variation of the width of the roughness element \\
\hline$e_{x}, e_{y}, e_{z}$ & Unit vectors in the $\mathrm{x}, \mathrm{y}, \mathrm{z}$ directions respectively \\
\hline FA & Flow angle: $\tan ^{-1}(\mathrm{~W} / \mathrm{U})$ \\
\hline FGA & Flow gradient angle: $\tan ^{-1}\left[\frac{\partial W / \partial y}{\partial U / \partial y}\right]$ \\
\hline$f$ & $\begin{array}{l}\text { Function particular to each roughness element's incremental } \\
\text { variation in the length of its surface as a function of } y\end{array}$ \\
\hline $\mathrm{H}$ & Shape factor \\
\hline$\dot{i}, \vec{j}, \vec{k}$ & Unit vectors in the $\mathrm{x}, \mathrm{y}, \mathrm{z}$ directions respectively \\
\hline $\mathrm{k}, \mathrm{h}$ & Roughness element height \\
\hline $\mathrm{k}^{+}$ & Non-dimensional roughness height: $\mathrm{k}^{+}=\mathrm{kU}_{\tau} / \mathrm{v}$ \\
\hline LDV & Laser Doppler Velocimeter \\
\hline $\mathrm{n}$ & Total number of valid samples when dealing with uncertainty \\
\hline $\mathrm{N}$ & Anisotropy factor: $N=\frac{\varepsilon_{z}}{\varepsilon_{x}}=\frac{-\overline{v w} /(\partial W / \partial y)}{-\overline{u v} /(\partial U / \partial y)}=\frac{\tan (S S A)}{\tan (F G A)}$ \\
\hline$p_{\text {ref, static }}$ & Static pressures of the undisturbed free stream \\
\hline$p_{\text {ref, total }}$ & Stagnation pressures of the undisturbed free stream \\
\hline QSV & Quasi-streamwise vortex \\
\hline $\bar{q}$ & $\begin{array}{l}\text { Stagnation pressure averaged over the frontal area of the } \\
\text { roughness element, as done by Wieghardt (1946) }\end{array}$ \\
\hline$R_{\alpha \beta}$ & $\begin{array}{l}\text { Reynolds stresses when dealing with Schumann's realizability } \\
\text { conditions: } R_{\alpha \beta}=\overline{u_{\alpha} u_{\beta}}\end{array}$ \\
\hline$R^{2}$ & Correlation coefficient of the curve fit \\
\hline $\operatorname{Re}_{\theta}$ & Momentum thickness Reynolds number: $\mathrm{U}_{\mathrm{e}} \theta / v$ \\
\hline $\operatorname{Re}_{x}$ & $\begin{array}{l}\text { Reynolds number based on distance from contraction exit: } \\
\mathrm{U}_{\mathrm{e}} \mathrm{x} / v\end{array}$ \\
\hline SSA & Shear stress angle: $\tan ^{-1}(-\overline{v w} /-\overline{u v})$ \\
\hline Spanwise & $\begin{array}{l}\text { Direction aligned with the z-axis in the wind tunnel coordinate } \\
\text { system }\end{array}$ \\
\hline Streamwise & $\begin{array}{l}\text { Direction aligned with the } \mathrm{x} \text {-axis in the wind tunnel coordinate } \\
\text { system }\end{array}$ \\
\hline
\end{tabular}




\section{TA}

TBL

TKE

$\mathscr{W}, \mathscr{V}, \mathscr{W}$

$u, v, w$

$\mathrm{U}, \mathrm{V}, \mathrm{W}$

$\mathrm{U}_{\mathrm{c}}^{+}$

$U_{e}$

$\mathrm{U}_{\text {top }}$

$\overline{u^{2}}, \overline{v^{2}}, \overline{w^{2}}$

$\overline{u v}, \overline{u w}, \overline{v w}$

$\overline{u u v}, \overline{u u w}, \overline{v v w}, \overline{u v v}, \overline{u w w}$,

$\overline{v w w}, \overline{u v w}, \overline{u u u}, \overline{v v v}$,

$\overline{w w w}$

$\mathrm{U}_{\tau}=\sqrt{\frac{\tau_{w}}{\rho}}$

$\mathrm{U}^{+}$

$\mathrm{V}^{+}$

$\vec{V}$

$V_{q} / U_{\tau}$

$W_{q} / U_{\tau}$

$\mathrm{W}^{+}$

$\mathrm{x}, \mathrm{y}, \mathrm{z}$

$\mathrm{x}_{\mathrm{o}}$

$\mathrm{x}_{1}$

$\mathrm{y}_{\text {shift }}$

$\mathrm{y}^{+}$
Trip arrangement

Turbulent Boundary Layer

Normalized turbulent kinetic energy, q, $\left\lfloor\left(\overline{q^{2}} / 2\right) / U_{\tau}^{2}\right\rfloor$ : where $\overline{q^{2}}=\left(\overline{u^{2}}+\overline{v^{2}}+\overline{w^{2}}\right)$

Instantaneous velocities ( $\mathrm{x}, \mathrm{y}$, and $\mathrm{z}$ axes)

Velocity fluctuations (x, y, and $\mathrm{z}$ axes)

Mean velocities

Streamwise velocity at vortex center location

Free-stream velocity $(27.5 \mathrm{~m} / \mathrm{s})$

Streamwise velocity at top of control volume

Reynolds normal stresses

Reynolds shear stresses

Triple products

Wall-shear-stress velocity

Non-dimensional streamwise velocity, $U^{+}=U / U_{\tau}$

Non-dimensional normal-to-wall velocity, $V^{+}=V / U_{\tau}$

Velocity vector

Non-dimensional diffusion velocity vector in normal-to-wall direction: where $V_{q} / U_{\tau}=\left(\overline{v q^{2}} / \overline{q^{2}}\right) / U_{\tau}$

Non-dimensional diffusion velocity vector in spanwise direction: where $W_{q} / U_{\tau}=\left(\overline{w q^{2}} / \overline{q^{2}}\right) / U_{\tau}$

Non-dimensional spanwise velocity, $W^{+}=W / U_{\tau}$

Coordinate system for wind tunnel

Location of downstream edge of sandpaper for trip arrangement

Location of $b / 1$ profile measurements, see Eqn. A.5

Wall location refinement

Non-dimensional distance from wall: $\mathrm{y}^{+}=\mathrm{yU}_{\tau} / \nu$ 


$\mathrm{y}_{\mathrm{c}}^{+}, \mathrm{z}_{\mathrm{c}}^{+}$
$\mathrm{y}_{\text {top }}$
$-\mathrm{z}_{2 \mathrm{D}}$

\section{Greek}

$\alpha$

$\Delta \mathrm{D}_{\mathrm{E}_{-} \text {incremental }}$
$\Delta \mathrm{D}_{\mathrm{E}_{-} \text {actual }}$
$\Delta \mathrm{D}_{\mathrm{E}_{-} \text {calculated }}$
$\Delta \mathrm{y}_{\mathrm{c}}^{+}, \Delta \mathrm{z}_{\mathrm{c}}^{+}$
$\delta$
$\delta^{*}$
$\delta()$
$\Delta y$
$\varepsilon_{x}$
$\varepsilon_{z}$
$\Gamma$
$\tau_{\mathrm{w}}$
$\Omega_{\mathrm{x}}$
$\Omega_{\mathrm{z}}$
$\sigma$
$\theta$
$\sigma$

\section{Subscripts}

$1,2,3,4$
Location of vortex center non-dimensionalized by $U_{\tau} / \nu$

Distance from wall at top of control volume

Distance at which the b/l sees no effect from the element's presence and resembles a smooth wall 2DTBL

\section{Vertex angle of cone}

Half of the incremental drag calculated on the roughness element

Half of the actual drag calculated on the roughness element Half of the actual drag calculated on the roughness element, from the correlation in Equation 4.3

Change in location of peak $\Omega_{\mathrm{x}}$

Boundary layer thickness defined as the distance from the wall where the local velocity is $99 \%$ of $U_{e}$

Displacement thickness

Uncertainty of quantity with 20:1 odds

$y$-shift value in the refinement of wall position

Eddy viscosity in streamwise, x-direction: $\varepsilon_{x}=-\overline{u v} /(\partial U / \partial y)$

Eddy viscosity in spanwise, z-direction $\varepsilon_{z}=-\overline{v w} /(\partial W / \partial y)$

Circulation in y-z plane: $\Gamma=\oint_{C} \vec{V} \cdot \overrightarrow{d s}$

Ratio of: (projected frontal area to flow)/(total surface area)

Kinematic viscosity

Momentum thickness

Density

Standard deviation

Wall shear stress of the undisturbed reference 2DTBL

Local wall shear stress

Streamwise vorticity

Spanwise vorticity

Quantity at Face 1, 2, 3, and 4 in the drag analysis 
avg

E

top

$\mathrm{w} / \mathrm{oE}$
Average of quantity

Quantity with the element present

Quantity at the top of the control volume

Quantity without the roughness element present 


\section{List of Figures}

Figure 2.1: Schematic of small boundary layer wind tunnel setup (Adapted from Smith, et al. 1990) ....... 34

Figure 2.2: Small boundary layer wind tunnel setup (view from front) ............................................ 35

Figure 2.3: Small boundary layer wind tunnel setup (view from back) ................................................ 35

Figure 2.4: View of baffle plate in plenum chamber (looking from blower) ........................................ 36

Figure 2.5: Seeding tube and drip pan on downstream side of baffle plate .......................................... 36

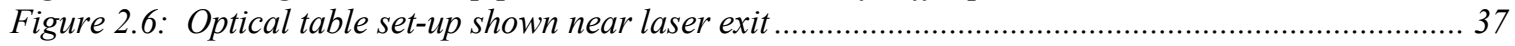

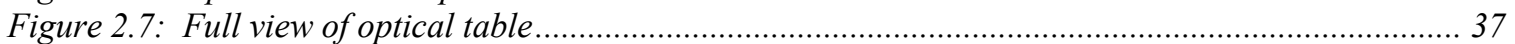

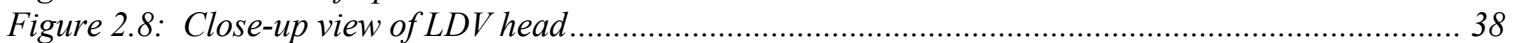

Figure 2.9: LDV head during data acquisition (view is above the tunnel ceiling looking upstream) ......... 38

Figure 2.10: Spanwise velocity profile at center of wind tunnel ....................................................... 39

Figure 2.11: Comparison between profiles and DNS data ( $x=$ distance from contraction exit, $z=$ spanwise

distance from tunnel centerline) ............................................................................ 40

Figure 2.12: Three-Dimensional drawings of single element cases.................................................... 41

Figure 2.13: Close-up views of single isolated roughness elements ....................................................43

Figure 2.14: Element profile plots showing element width varying with respect to element height .......... 44

Figure 2.15: Close-up view of Gaussian roughness fetch (center-to-center spacing $=0.216$ inches)......... 45

Figure 2.16: Top view of test section, looking upstream, with wind tunnel ceiling removed .................... 45

Figure 2.17: Data being acquired for the fetch of Gaussian roughness ............................................. 46

Figure 3.1: Measurement locations for single element cases (except fine grooved) ................................ 73

Figure 3.2: Measurement locations for fine grooved element............................................................... 73

Figure 3.3: Oil flows for the cube and hemisphere elements .............................................................. 74

Figure 3.4: Oil flows for the Gaussian, fine grooved, large grooved, and cone elements ......................... 75

Figure 3.5: Close-up views of oil flows for the cube and hemisphere elements .................................... 76

Figure 3.6: Close-up views of the oil flows for the Gaussian, large grooved, fine grooved, and cone

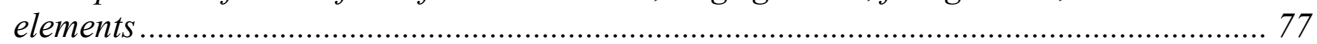

Figure 3.7: $U / U_{\tau}$ vs. $y U_{\tau} /{ }_{v}$, streamwise mean velocity profiles along the centerline ........................ 78

Figure 3.8: Contours of $U / U_{\tau}$ in the $y-z$ plane at a location of $x / d=2.75$ (Note: Fine Grooved $x / d=1.36$ )

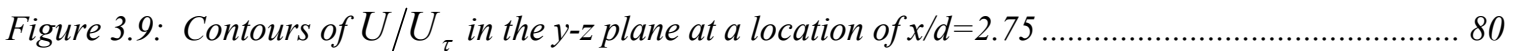

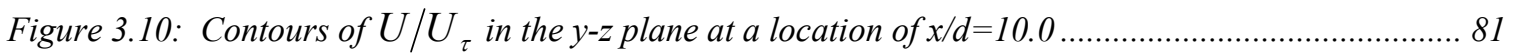

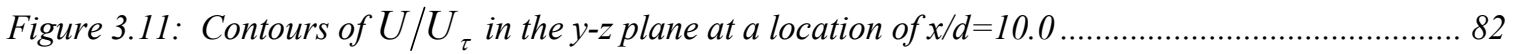

Figure 3.12: $V / U_{\tau}$ vs. $y U_{\tau} / v$, normal to wall mean velocity profiles along the centerline...................83

Figure 3.13: Contours of $V / U_{\tau}$ in the $y$-z plane at a location of $x / d=2.75$ (Note: Fine Grooved $\left.x / d=1.36\right)$

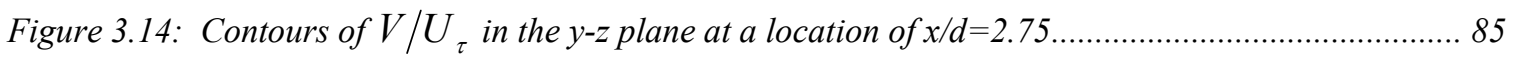

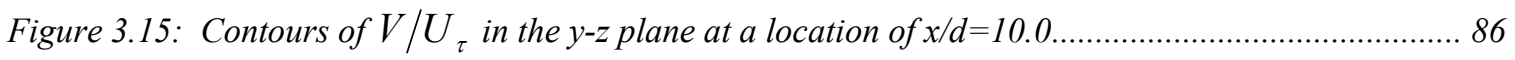

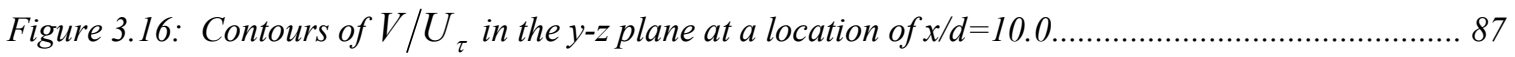

Figure 3.17: Contours of $W / U_{\tau}$ in the $y-z$ plane at a location of $x / d=2.75$ (Note: Fine Grooved $x / d=1.36$ )

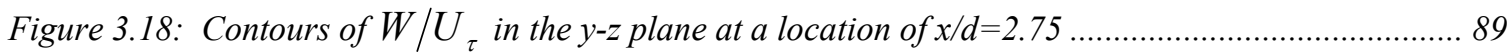

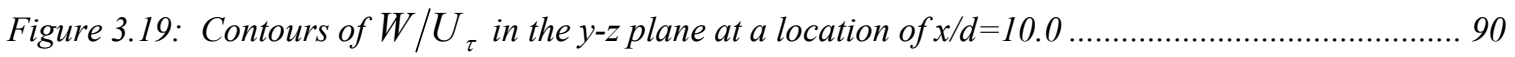


Figure 3.20: Contours of $W / U_{\tau}$ in the $y$-z plane at a location of $x / d=10.0$.

Figure 3.21: Contours of normalized streamwise vorticity, $\Omega_{x} \sqrt{A_{f}} / U_{\tau}$, with normalized secondary velocity vectors $V / U_{\tau}$ and $W / U_{\tau}$ in the $y$-z plane at a location of $x / d=2.75$ (Note: Fine Grooved $x / d=1.36)$

Figure 3.22: Contours of normalized streamwise vorticity, $\Omega_{x} \sqrt{A_{f}} / U_{\tau}$, with normalized secondary velocity vectors $V / U_{\tau}$ and $W / U_{\tau}$ in the $y$-z plane at a location of $x / d=2.75$.

Figure 3.23: Contours of normalized streamwise vorticity, $\Omega_{x} \sqrt{A_{f}} / U_{\tau}$, with normalized secondary velocity vectors $V / U_{\tau}$ and $W / U_{\tau}$ in the $y$-z plane at a location of $x / d=10.0$..... 94

Figure 3.24: Contours of normalized streamwise vorticity, $\Omega_{x} \sqrt{A_{f}} / U_{\tau}$, with normalized secondary velocity vectors $V / U_{\tau}$ and $W / U_{\tau}$ in the $y-z$ plane at a location of $x / d=10.0 \ldots \ldots \ldots \ldots . . .95$

Figure 3.25: Variation of circulation, $\Gamma /\left(U_{\tau} \sqrt{A_{f}}\right)$, with streamwise distance, $x / d$............................... 96

Figure 3.26: Variation of normalized wall shear $\left(\tau_{w} / \tau_{o}\right)$ along centerline ........................................... 97

Figure 3.27: Variation of normalized wall shear $\left(\tau_{w} / \tau_{0}\right)$ at $x / d=2.75$ plane ....................................... 97

Figure 3.28: Variation of normalized wall shear $\left(\tau_{w} / \tau_{o}\right)$ at $x / d=10.0$ plane ...................................... 98

Figure 3.29: $\overline{u^{2}} \int_{U_{\tau}^{2}} v s . \quad y U_{\tau} / v$, streamwise Reynolds normal stress profiles along the centerline....... 99

Figure 3.30: Contours of $\overline{u^{2}} / U_{\tau}^{2}$ in the $y$-z plane at a location of $x / d=2.75$ (Note: Fine Grooved $x / d=1.36)$

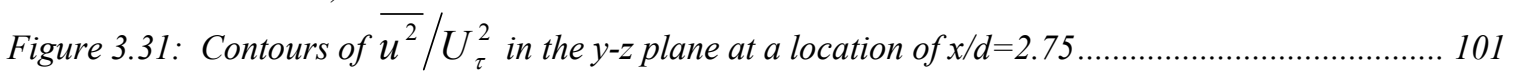

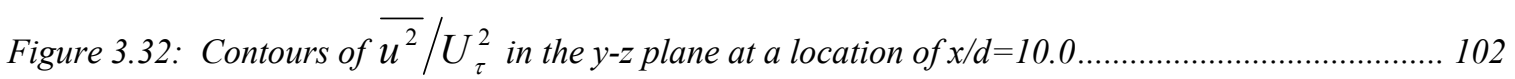

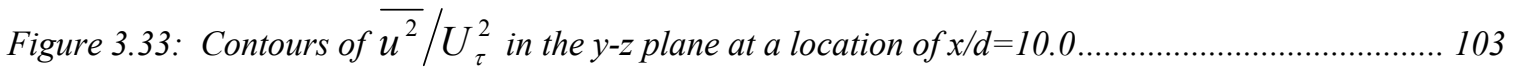

Figure 3.34: $\overline{v^{2}} \int_{U_{\tau}^{2}} v s .9 U_{\tau} / v$, normal to wall Reynolds normal stress profiles along the centerline 104

Figure 3.35: Contours of $\overline{v^{2}} / U_{\tau}^{2}$ in the $y$-z plane at a location of $x / d=2.75$ (Note: Fine Grooved $x / d=1.36)$

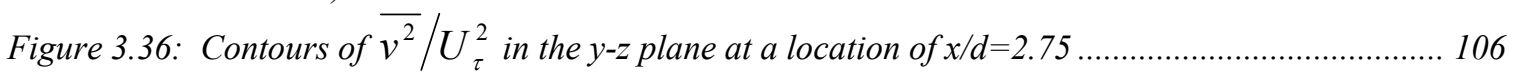

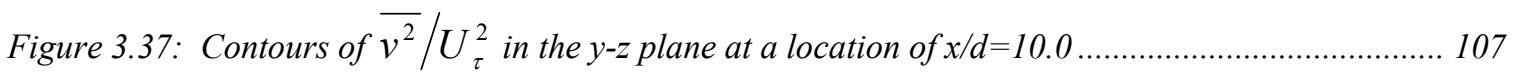

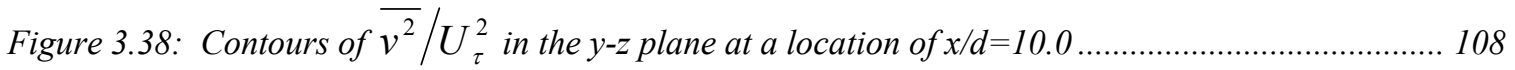

Figure 3.39: $\overline{w^{2}} / U_{\tau}^{2}$ vs. $y U_{\tau} / v$, spanwise Reynolds normal stress profiles along the centerline ....... 109

Figure 3.40: Contours of $\overline{w^{2}} / U_{\tau}^{2}$ in the $y$-z plane at a location of $x / d=2.75$ (Note: Fine Grooved $x / d=1.36)$

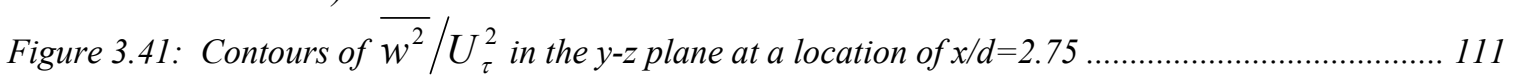

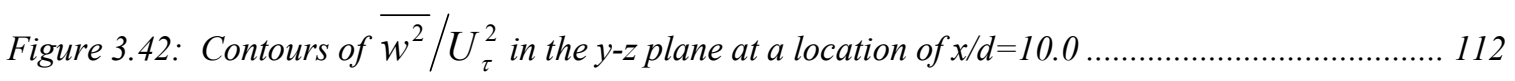

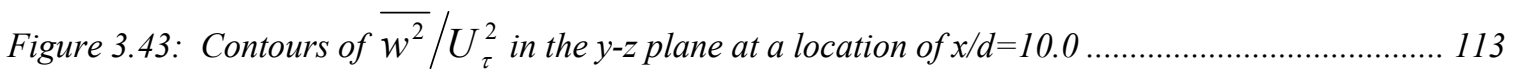


Figure 3.44: $\overline{u v} / U_{\tau}^{2}$ vs. $y U_{\tau} / v$, streamwise Reynolds shear stress profiles along the centerline ........ 114

Figure 3.45: Contours of $\overline{u v} / U_{\tau}^{2}$ in the $y-z$ plane at a location of $x / d=2.75$ (Note: Fine Grooved $x / d=1.36)$...

Figure 3.46: Contours of $\overline{u v} / U_{\tau}^{2}$ in the $y$-z plane at a location of $x / d=2.75 \ldots \ldots \ldots \ldots \ldots \ldots \ldots \ldots \ldots \ldots \ldots \ldots \ldots \ldots . . . .116$

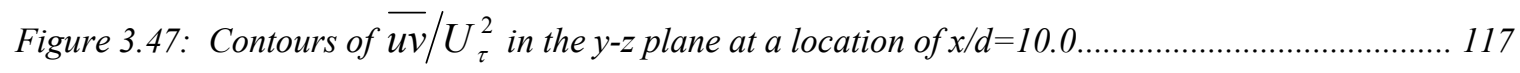

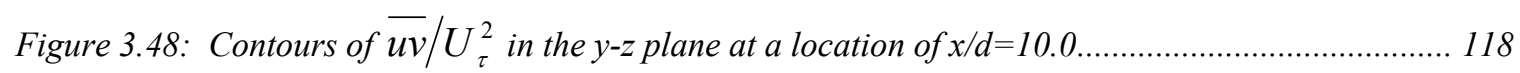

Figure 3.49: Contours of $\overline{u w} / U_{\tau}^{2}$ in the $y$-z plane at a location of $x / d=2.75$ (Note: Fine Grooved $x / d=1.36)$..

Figure 3.50: Contours of $\overline{u w} / U_{\tau}^{2}$ in the $y$-z plane at a location of $x / d=2.75 \ldots \ldots \ldots \ldots \ldots \ldots \ldots \ldots \ldots \ldots \ldots \ldots \ldots . . . . .120$

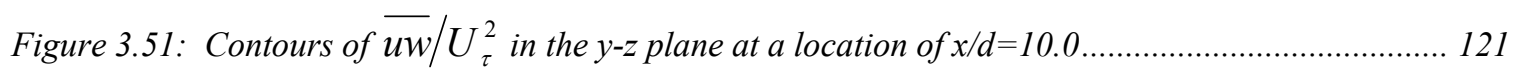

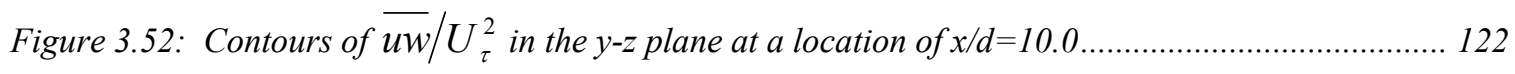

Figure 3.53: Contours of $\overline{v w} / U_{\tau}^{2}$ in the $y$-z plane at a location of $x / d=2.75$ (Note: Fine Grooved $x / d=1.36)$..

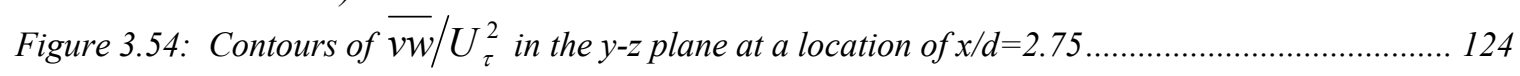

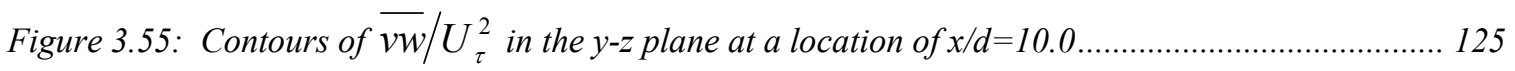

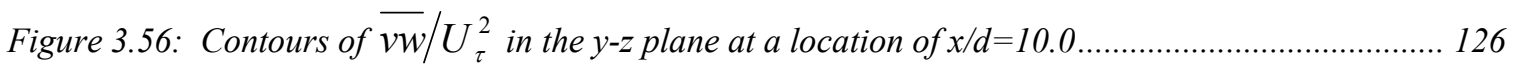

Figure 3.57: $\overline{u^{2} v} \int_{U_{\tau}^{3}}$ vs. $y U_{\tau} / v$ profiles along the centerline ........................................................ 127

Figure 3.58: Contours of $\overline{u^{2} v} / U_{\tau}^{3}$ in the $y$-z plane at a location of $x / d=2.75$ (Note: Fine Grooved

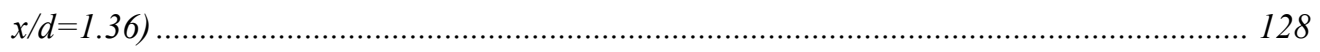

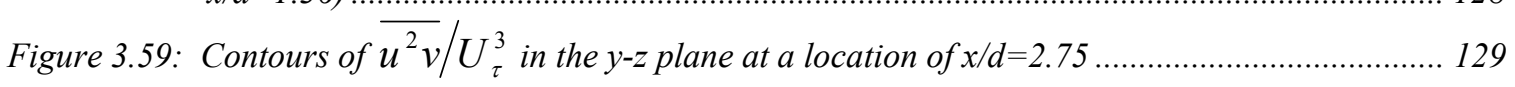

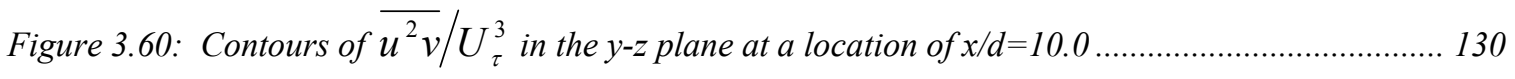

Figure 3.61: Contours of $\overline{u^{2} v} / U_{\tau}^{3}$ in the $y$-z plane at a location of $x / d=10.0$...................................... 131

Figure 3.62: Contours of $\overline{u^{2} w} / U_{\tau}^{3}$ in the $y$-z plane at a location of $x / d=2.75$ (Note: Fine Grooved $x / d=1.36)$...

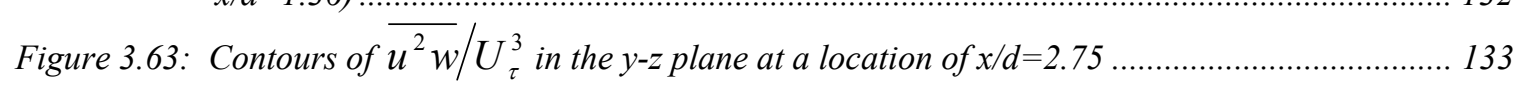

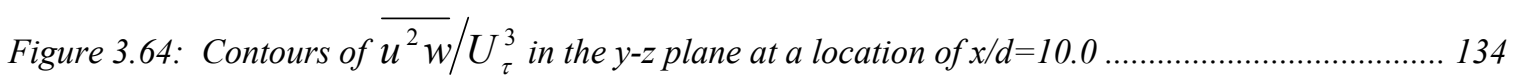

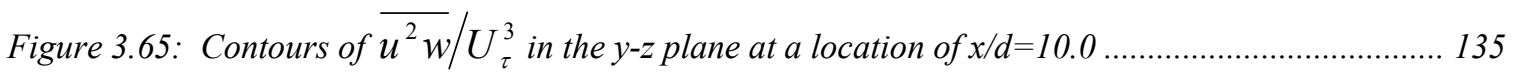

Figure 3.66: Contours of $\overline{v^{2} w} / U_{\tau}^{3}$ in the $y$-z plane at a location of $x / d=2.75$ (Note: Fine Grooved $x / d=1.36)$.. 136

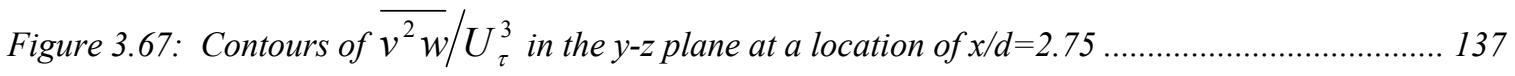

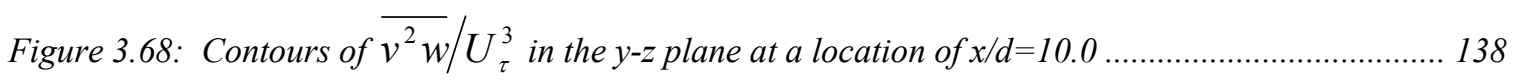

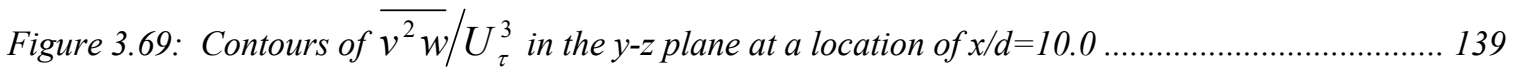


Figure 3.70: $\overline{u v^{2}} /_{U_{\tau}^{3}} v s$.

Figure 3.71: Contours of $\overline{u v^{2}} / U_{\tau}^{3}$ in the $y$-z plane at a location of $x / d=2.75$ (Note: Fine Grooved $x / d=1.36$ )

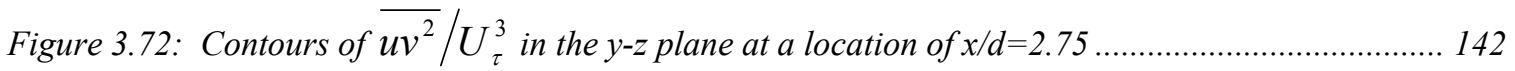

Figure 3.73: Contours of $\overline{u v^{2}} / U_{\tau}^{3}$ in the $y$-z plane at a location of $x / d=10.0$....................................... 143

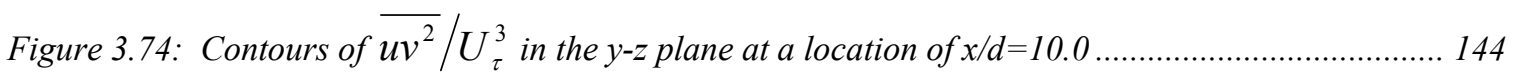

Figure 3.75: $\overline{u w^{2}} \int_{U^{3}} v s . U_{\tau} / v$ profiles along the centerline ....................................................... 145

Figure 3.76: Contours of $\overline{u w^{2}} / U_{\tau}^{3}$ in the $y$-z plane at a location of $x / d=2.75$ (Note: Fine Grooved $x / d=1.36$ )

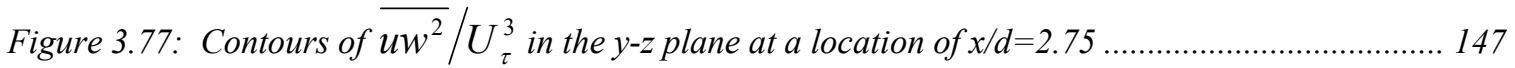

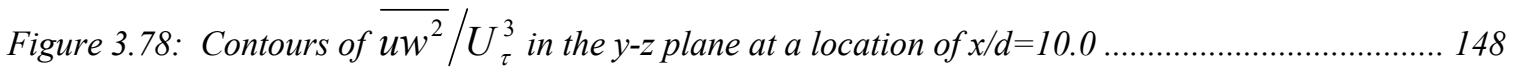

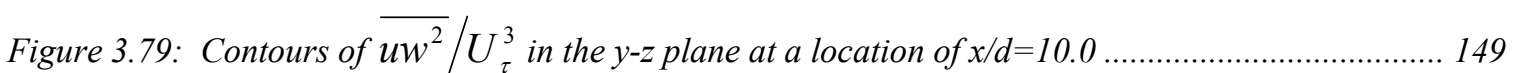

Figure 3.80: $\overline{v w^{2}} /_{U_{\tau}^{3}} v s . \quad y U_{\tau} / v$ profiles along the centerline

Figure 3.81: Contours of $\overline{v w^{2}} / U_{\tau}^{3}$ in the $y-z$ plane at a location of $x / d=2.75$ (Note: Fine Grooved $x / d=1.36$ )

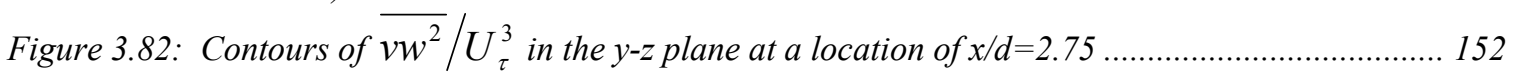

Figure 3.83: Contours of $\overline{v w^{2}} / U_{\tau}^{3}$ in the $y$-z plane at a location of $x / d=10.0 \ldots \ldots \ldots \ldots \ldots \ldots . . . . . . . . . . . . . . . . . . .153$

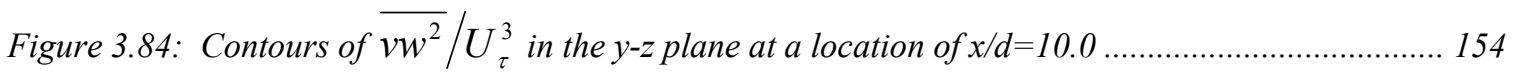

Figure 3.85: Contours of $\overline{u v w} / U_{\tau}^{3}$ in the $y$-z plane at a location of $x / d=2.75$ (Note: Fine Grooved $x / d=1.36)$..

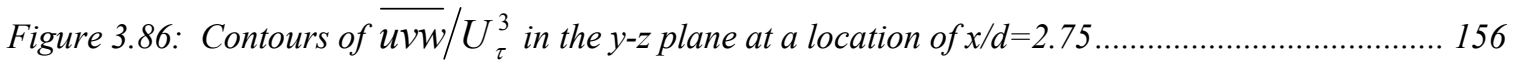

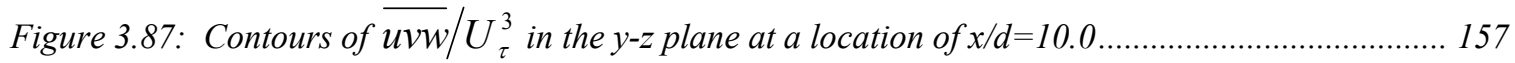

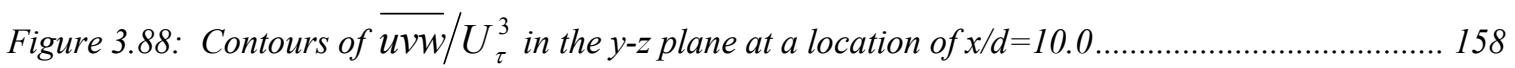

Figure 3.89: $\overline{u^{3}} / U_{\tau}^{3}$ vs. $y U_{\tau} / v$ profiles along the centerline ............................................................. 159

Figure 3.90: Contours of $\overline{u^{3}} / U_{\tau}^{3}$ in the $y$-z plane at a location of $x / d=2.75$ (Note: Fine Grooved $x / d=1.36$ ) 160

Figure 3.91: Contours of $\overline{u^{3}} / U_{\tau}^{3}$ in the $y$-z plane at a location of $x / d=2.75 \ldots \ldots \ldots \ldots \ldots \ldots \ldots \ldots \ldots \ldots \ldots \ldots \ldots \ldots . . .161$

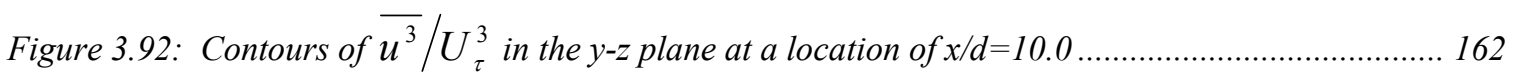

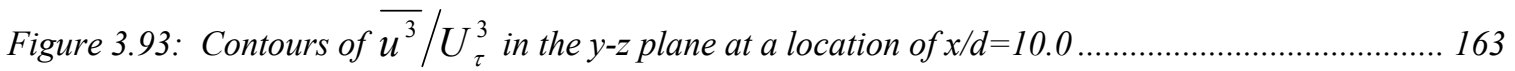


Figure 3.94: $\overline{v^{3}} / U_{\tau}^{3}$ vs. $y U_{\tau} / v$ profiles along the centerline. 164

Figure 3.95: Contours of $\overline{v^{3}} / U_{\tau}^{3}$ in the $y$-z plane at a location of $x / d=2.75$ (Note: Fine Grooved $x / d=1.36$ )

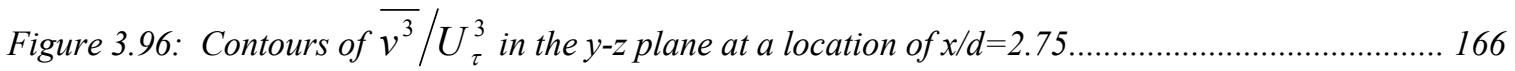

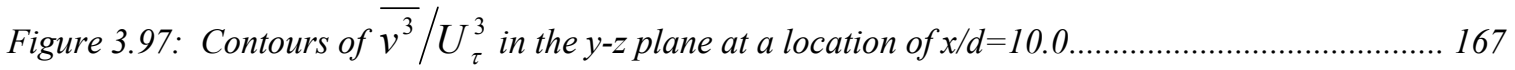

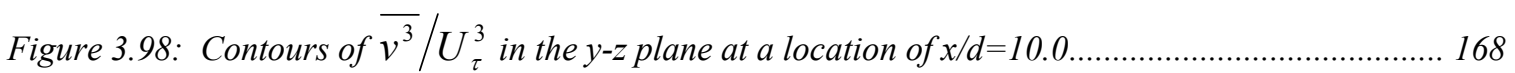

Figure 3.99: Contours of $\overline{w^{3}} / U_{\tau}^{3}$ in the $y$-z plane at a location of $x / d=2.75$ (Note: Fine Grooved $x / d=1.36$ )

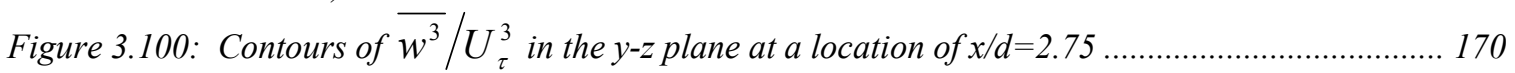

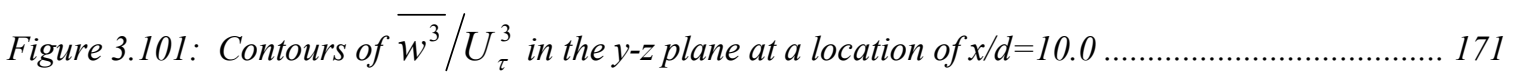

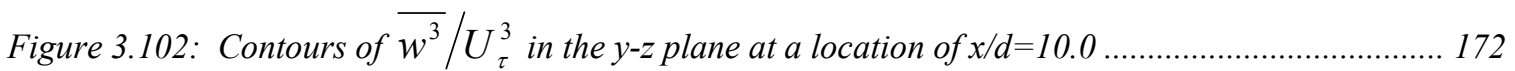

Figure 3.103: Turbulent Kinetic Energy (TKE), $\left(\left[\overline{q^{2}} / 2\right) / U_{\tau}^{2}\right\rfloor v s$.

Figure 3.104: Contours of TKE $\left\lfloor\left(\overline{q^{2}} / 2\right) / U_{\tau}^{2}\right\rfloor$ with diffusion velocity vectors, $V_{q} / U_{\tau}=\left(\overline{v q^{2}} / \overline{q^{2}}\right) / U_{\tau}$ and $W_{q} / U_{\tau}=\left(\overline{w q^{2}} / \overline{q^{2}}\right) / U_{\tau}$, in the $y$-z plane at a location of $x / d=2.75$ (Note: Fine Grooved $x / d=1.36$ ) 174

Figure 3.105: Contours of TKE $\left[\left(\overline{q^{2}} / 2\right) / U_{\tau}^{2}\right]$ with diffusion velocity vectors, $V_{q} / U_{\tau}=\left(\overline{v q^{2}} / \overline{q^{2}}\right) / U_{\tau}$ and $W_{q} / U_{\tau}=\left(\overline{w q^{2}} / \overline{q^{2}}\right) / U_{\tau}$, in the $y$-z plane at a location of $x / d=2.75$

Figure 3.106: Contours of TKE $\left[\left(\overline{q^{2}} / 2\right) / U_{\tau}^{2}\right]$ with diffusion velocity vectors,

$V_{q} / U_{\tau}=\left(\overline{v q^{2}} / \overline{q^{2}}\right) / U_{\tau}$ and $W_{q} / U_{\tau}=\left(\overline{w q^{2}} / \overline{q^{2}}\right) / U_{\tau}$, in the $y$-z plane at a location of $x / d=10.0$

Figure 3.107: Contours of TKE $\left[\left(\frac{1}{q^{2}} / 2\right) / U_{\tau}^{2}\right]$ with diffusion velocity vectors, $V_{q} / U_{\tau}=\left(\overline{v q^{2}} / \overline{q^{2}}\right) / U_{\tau}$ and $W_{q} / U_{\tau}=\left(\overline{w q^{2}} / \overline{q^{2}}\right) / U_{\tau}$, in the $y$-z plane at a location of $x / d=10.0$ 177

Figure 3.108: Centerline variation of normalized streamwise eddy viscosity, $\varepsilon_{x} / v$ vs. $y U_{\tau} / v \ldots \ldots .178$

Figure 3.109: Contours of eddy viscosity ratio, $N_{\varepsilon}=\varepsilon_{z} / \varepsilon_{x}$, in the $y$-z plane at $x / d=2.75$ (Fine Grooved $x / d=1.36)$ 179

Figure 3.110: Contours of eddy viscosity ratio, $N_{\varepsilon}=\varepsilon_{z} / \varepsilon_{x}$, in the $y$-z plane at $x / d=2.75 \ldots \ldots \ldots \ldots . . . .180$

Figure 3.111: Contours of eddy viscosity ratio, $N_{\varepsilon}=\varepsilon_{z} / \varepsilon_{x}$, in the $y$-z plane at $x / d=10.0 \ldots \ldots \ldots . . . . .181$

Figure 3.112: Contours of eddy viscosity ratio, $N_{\varepsilon}=\varepsilon_{z} / \varepsilon_{x}$, in the $y$-z plane at $x / d=10.0$................ 182

Figure 3.113: Measurement locations for the Gaussian fetch of roughness ............................................ 183

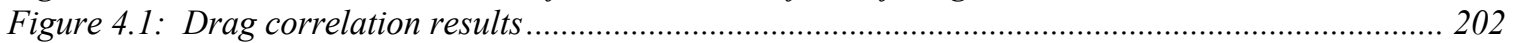

Figure 4.2: Variation of normalized drag coefficient based on frontal area $C_{d(f r o n t a l)} / C_{f} \ldots \ldots \ldots \ldots \ldots \ldots \ldots \ldots . . . . . . . . . . .203$

Figure 4.3: Variation of normalized drag coefficient based on wetted area $C_{d(\text { wetted })} / C_{f} \ldots \ldots . . . . . . . . . . . . . . . . . . . .2203$

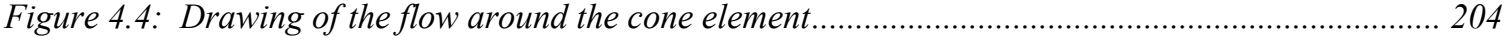

Figure 4.5: Drawing of the flow around the Gaussian element ............................................................ 205 
Figure 4.6: Drawing of the flow around the large grooved element ..................................................... 206

Figure 4.7: Drawing of the flow around the fine grooved element ...................................................... 207

Figure 4.8: Drawing of the flow around the cube at the $90^{\circ}$ orientation ..................................................208

Figure 4.9: Drawing of the flow around the cube at the $45^{\circ}$ orientation ............................................... 209

Figure 4.10: Drawing of the flow around the hemisphere ........................................................ 210

Figure 4.11: Log-log plot of the decay of circulation, $\Gamma /\left(U_{\tau} \sqrt{A_{f}}\right)$, with streamwise distance, x/d..... 211

Figure 4.12: Various roughness regimes that could be investigated in the future .................................. 212

Figure A.1: Boundary layer impact probe and traverse system .................................................... 234

Figure A.2: Tested trip arrangements: TA 1-TA 12 (Note that TA 2 and TA 3 are not shown) .............. 238

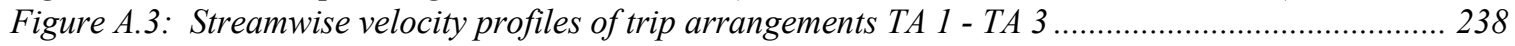

Figure A.4: Streamwise velocity profiles of trip arrangements TA 4 - TA 6 .................................... 239

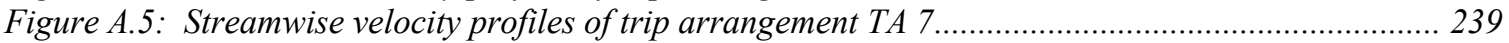

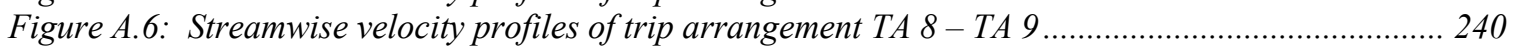

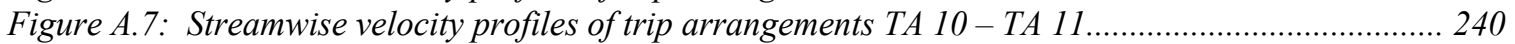

Figure A.8: Streamwise velocity profiles of trip arrangement TA 12 ......................................... 241

Figure B.1: Views of control volume used in drag analysis ......................................................... 247

Figure D.1: Centerline profiles of Gaussian fetch (mean velocities, Reynolds stresses, and triple products)

Figure D.2: Contours of $U / U_{\tau}$ in the $y$-z plane at a location of $x / d=1.08$ with normalized secondary

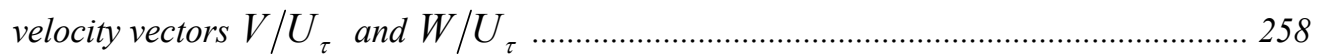

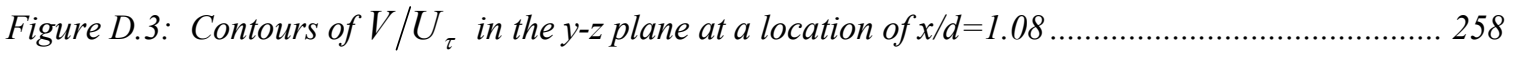

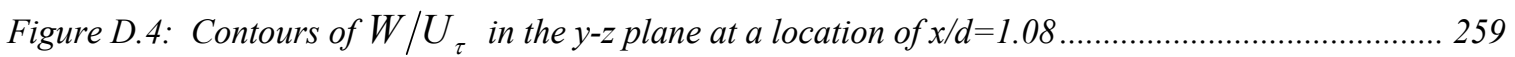

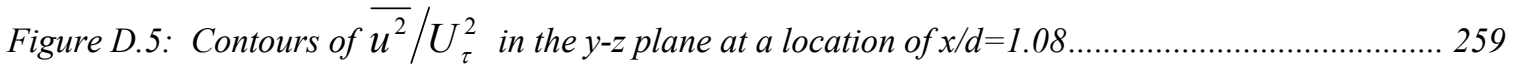

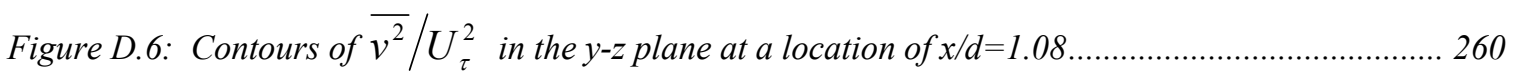

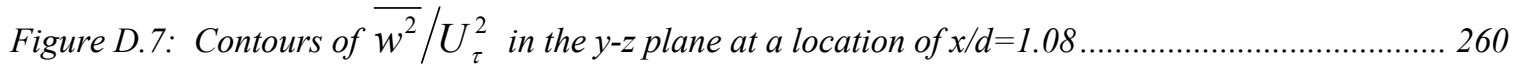

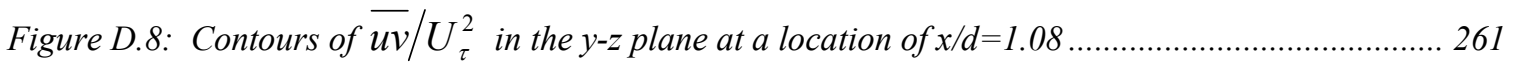

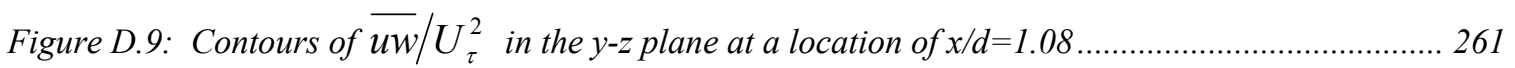

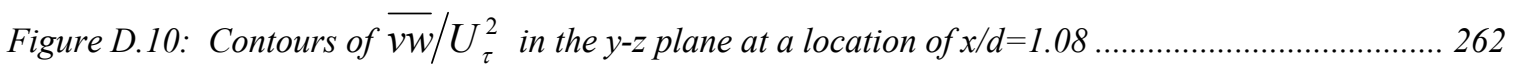

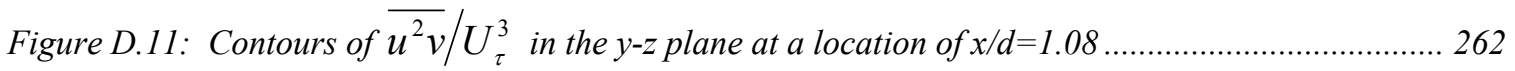

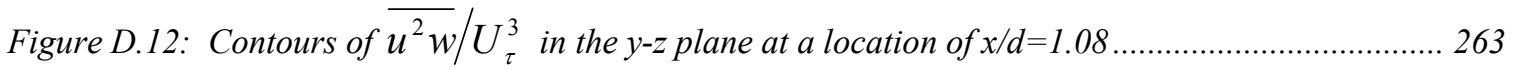

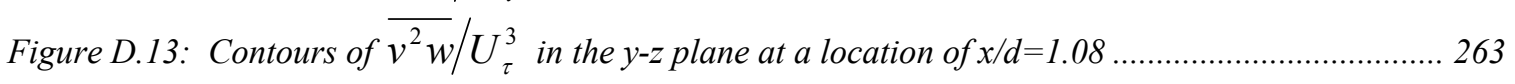

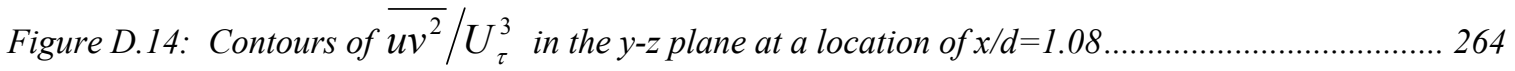

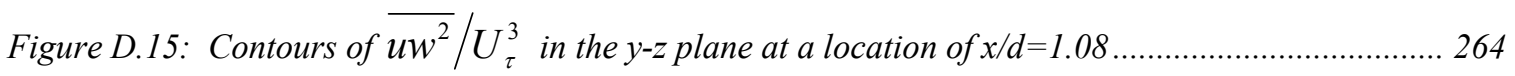

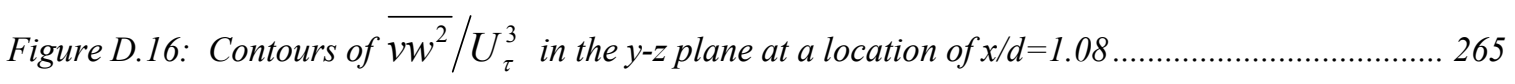

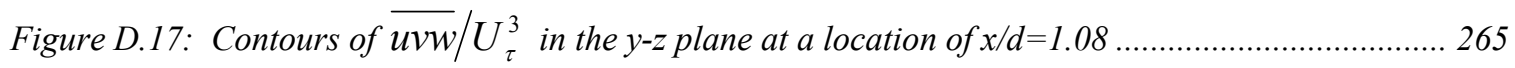

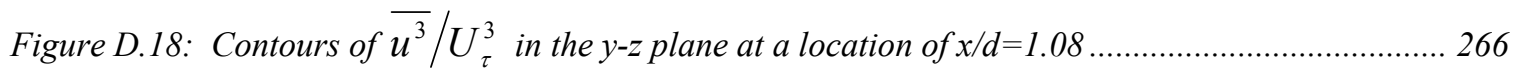

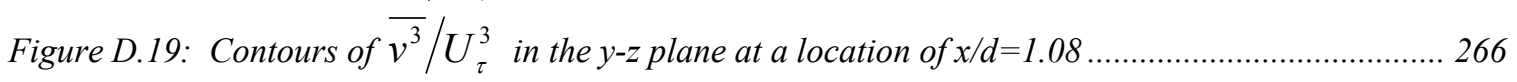

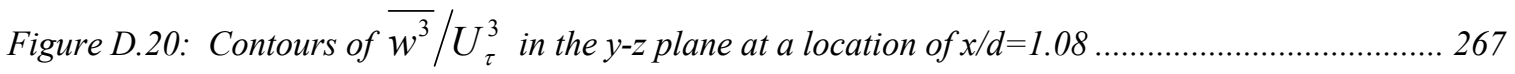


Figure D.21: Contours of TKE $\left\lfloor\left(\overline{q^{2}} / 2\right) / U_{\tau}^{2}\right\rfloor$ with diffusion velocity vectors,

$$
V_{q} / U_{\tau}=\left(\overline{v q^{2}} / \overline{q^{2}}\right) / U_{\tau} \text { and } W_{q} / U_{\tau}=\left(\overline{w q^{2}} / \overline{q^{2}}\right) / U_{\tau} \text {, in the } y \text {-z plane at } x / d=1.08267
$$

Figure E.1: Mean flow angle variation along the centerline, where $F A=\tan ^{-1}(W / U) \ldots \ldots \ldots \ldots \ldots . . . . .271$

Figure E.2: Contours of mean flow angle (FA) in the $y$-z plane at $x / d=2.75$ (Fine Grooved $x / d=1.36) \ldots 272$

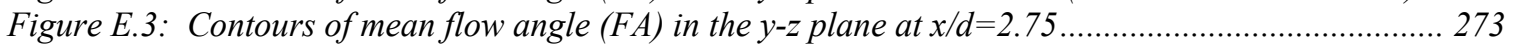

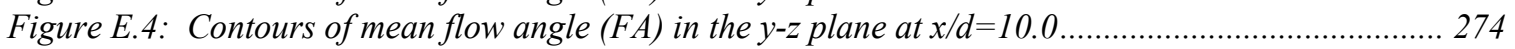

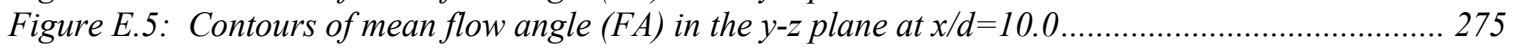

Figure E.6: Contours of flow gradient angle (FGA) in the $y$-z plane at $x / d=2.75$ (Fine Grooved $x / d=1.36$ )

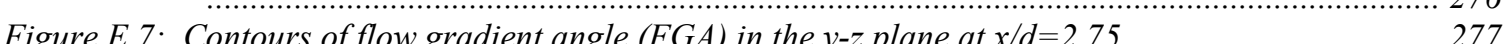

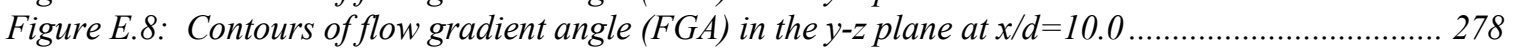

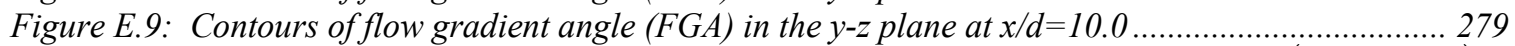

Figure E.10: Shear stress angle (SSA) variation along the centerline, where $S S A=\tan ^{-1}(-\overline{v w} /-\overline{u v})$

Figure E.11: Contours of shear stress angle (SSA) in the $y-z$ plane at $x / d=2.75($ Fine Grooved $x / d=1.36)$

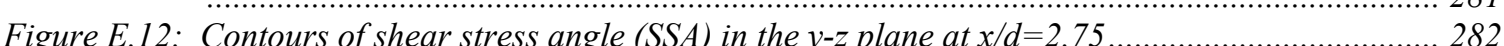

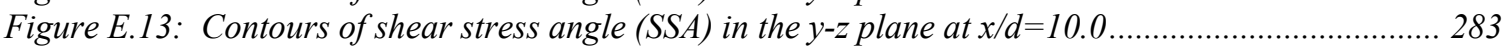

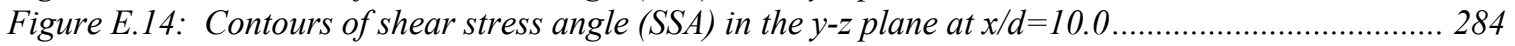




\section{List of Tables}

Table 2.1: Port \#'s and related distance from contraction exit............................................................... 17

Table 2.2: Ceiling height as a function of distance from contraction exit (for $d p / d x=0$ flow) ................... 26

Table 3.1: Locations of maximum streamwise vorticity ....................................................................... 56

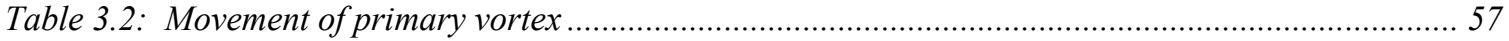

Table 3.3: Circulation, $\Gamma$, as a function of streamwise distance, $x / d$........................................................ 59

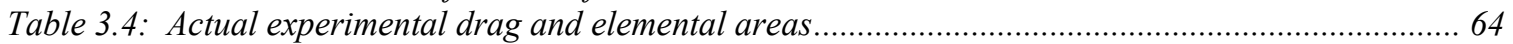

Table 4.1: Contributions of terms to related drag equations .............................................................. 187

Table 4.2: Comparison of actual and calculated drag values........................................................... 191

Table A.1: Trip arrangements and related abbreviations ................................................................ 228

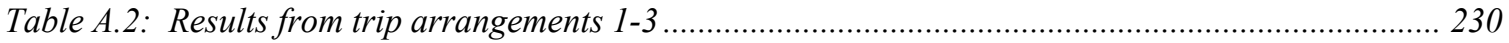

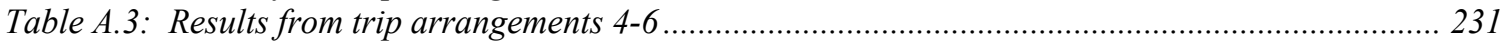

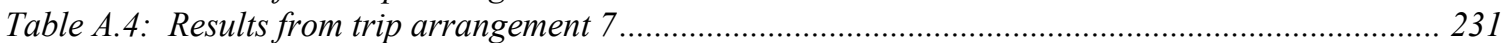

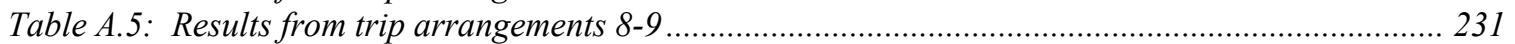

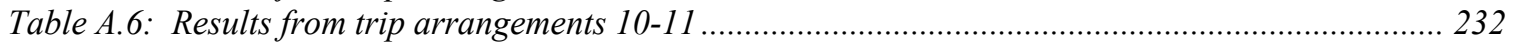

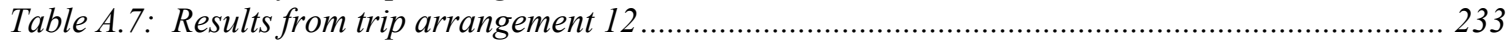

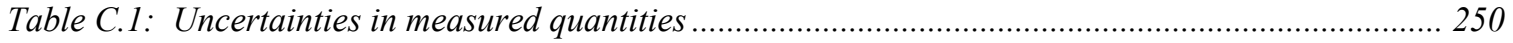

Table F.1: Coordinate locations for profile shapes of grooved elements............................................... 285 


\section{Chapter 1 Introduction}

The primary goal of this research is to fully measure and understand the effects of various shaped roughness elements in two-dimensional and three-dimensional high Reynolds number turbulent boundary layers. Roughness elements with heights less than $7 \%$ of the boundary layer thickness are analyzed in considerable detail. Mean flow and turbulence characteristics associated with the various protuberances are discussed extensively. Another significant attribute of this study is to determine the effects of spatial smoothing of roughness elements on the decay of turbulence quantities in a turbulent boundary layer. This is the first time that a detailed fundamental study has been done on roughness elements of these shapes and sizes. Moreover, it is essential to note that detailed analysis has not been done previously concerning the spatial smoothing of related roughness elements. The end result of this research, combined with the previous efforts of George and Simpson and continued future efforts, is to provide detailed measurements that will enable one to obtain a deterministic conceptual scheme in order to better define how roughness affects the physics of the flow in turbulent boundary layers. This study is also very significant in that it will provide detailed modeling test cases which will allow comparison with and adjustment to turbulence models that are used to calculate such flows.

The rest of this chapter is broken up into five different sections. Section 1.1 will discuss laminar and turbulent flows over isolated protuberances. Section 1.2 will go over previous efforts related to turbulent flow past surface mounted obstacles. This section will look at obstacles that have a height more comparable to that of the boundary layer thickness. Previous literature on the distribution of roughness elements and rough-wall turbulent boundary layers will be looked at in section 1.3. A brief look into some related modeling and computational efforts used to calculate roughness flows can be found in section 1.4. Finally, the organization of the research and thesis is presented in section 1.5 . 


\subsection{Laminar and Turbulent Flow Over Isolated Protuberances}

The earlier experiments involving flows over single protuberances were conducted in laminar boundary layers. The emphasis of these early laminar boundary layer studies was on defining a critical Reynolds number based on the element height which would cause transition to turbulent flow. Due to the fact that studies conducted in laminar boundary layers are not directly related to the current research, only a few examples will be discussed on this topic. Tani (1961) studied the effect of two-dimensional and isolated roughness in laminar boundary layers. Through this research it was discovered that if the fluid speed is low and the height of the roughness element is small compared to the boundary layer thickness, then transition will not occur and the effect of the roughness element will only propagate downstream a short distance. Flows involving laminar boundary layers can have relevance to the current research in regards to the formation and propagation of horseshoe vortices. These vortices will only be present in laminar boundary layers if there is the existence of a pressure gradient large enough to cause shear layer roll-up at the upstream junction of the roughness element base and the wall. Experiments that involve the development of the horseshoe vortex structure can be found in Sedney (1973) and Gregory and Walker (1955). Gregory and Walker performed a qualitative analysis on isolated protuberances using the china-clay and smoke visualization techniques at wind tunnel speeds of $37 \mathrm{~m} / \mathrm{s}$ and $5 \mathrm{~m} / \mathrm{s}$ respectively. Elements tested were on the order of the same size as the present research while having a roughness height to boundary layer thickness ratio $(\mathrm{k} / \delta)$ less than 1 in all cases. Varying heights, $5.1 \mathrm{~mm}$ and smaller, of a $60^{\circ}$ cone and cylinders were tested. The horseshoe vortex structure related to each element was observed. Sedney also investigated flow past cones, hemispheres and cylinders using a smoke visualization technique. Again, the presence of horseshoe vortices in these flows was detected and the qualitative flow features analyzed. Other experiments that have been performed on isolated roughness elements in laminar boundary layers with the intent to study the effects of flow transition or the development of vortex structures due to the presence of roughness element are as follows. Ichimaya (1999) performed experiments on a $2 \mathrm{~mm}$ high cylinder in a $2.2 \mathrm{~mm}$ thick boundary layer in order to determine the effects of a single roughness on boundary layer transition. Barrett et al. (1993) took measurements at various plane locations in the 
downstream wake region of a $0.8 \mathrm{~mm}$ diameter sphere in a $1.4 \mathrm{~mm}$ thick boundary layer and has shown the development of vortical structures. Finally, Klebanoff et al. (1992) investigated the development of a turbulent boundary layer induced by a hemisphere placed on a flat plate.

There is a very limited amount of literature related to isolated roughness elements in already developed turbulent boundary layers, hence the necessity for the present research. Previous studies have mainly been restricted to the evaluation of the drag induced by these small protuberances. Wieghardt (1946) conducted experiments in order to quantify the drag of various sizes of cylinders. These experiments were performed in a $67 \mathrm{~mm}$ thick boundary layer at a $\operatorname{Re}_{\mathrm{x}}=7.2\left(10^{6}\right)$, where $\mathrm{x}$ is the streamwise distance over which the flow develops. Hoerner (1958) presents results for common protrusions such as bolt and rivet heads, brackets and various other joints. Relations of protuberance sizes and shapes to their associated drag characteristics were investigated and presented as drag coefficients normalized by an 'effective' dynamic pressure. These results were garnered from Hoerner's work as well as various other people's work such as Nikuradse, Schlichting, and Moody. Hoerner also discusses the characterization of roughness by relating it to the concept of sand grain roughness. Young and Patterson (1981) reported on aircraft excrescence drag which also involved Wieghardt's data. These data were presented as a drag coefficient normalized by the undisturbed friction coefficient versus the undisturbed roughness Reynolds number with respect to the element height and undisturbed friction velocity, $\mathrm{U}_{\tau}$. Gaudet (1987) examined the drag on various forms of three-dimensional excrescences including cylinders and mushroom shaped rivet heads. These results as well as Wieghardt's data are presented as a functional form related to wall variables.

Previous work that does investigate the turbulence structure behind wall mounted protrusions is that of Fontaine and Deutsch (1996). The flow field behind a wall mounted Gaussian bump with a height of 16.4 wall units $\left(\mathrm{k}^{+}=16.4\right)$ and a base diameter of 13 wall units was analyzed in a turbulent boundary layer with a momentum thickness Reynolds number, $\operatorname{Re}_{\theta}$, equal to 730 . This $\operatorname{Re}_{\theta}$ is based on a skin friction velocity, $U_{\tau}$, 
equal to $0.4 \mathrm{~m} / \mathrm{s}$ and an equivalent momentum thickness based on centerline velocity. It was found that the protrusion produced a pair of counter-rotating vortices, each 15 wall units in diameter. Reynolds-stress-producing events such as sweeps and ejections were discovered to be retarded within the convergence region of the vortices. The work of George and Simpson (2001) is directly applicable due to the fact that the current research is an extension of these previous experiments. Previous work completed by George and Simpson was performed on three cylinders and one Gaussian shaped element in the same boundary layer conditions as are described in Chapter 2. The three cylinders tested had a base diameter of $1.98 \mathrm{~mm}$ and were $0.38 \mathrm{~mm}, 0.76 \mathrm{~mm}$, and $1.52 \mathrm{~mm}$ tall. The Gaussian element also had a height equal to $1.52 \mathrm{~mm}$. These experiments will be discussed in more detail in the following chapters. It is important to note that higher Reynolds shear stresses were found downstream of the elements due to the horseshoe vortices. An increase in skin friction drag was also found behind the element. This is due to the significant amount of fluid being drawn toward the wall.

\subsection{Turbulent Flow Past Surface-Mounted Obstacles}

Studies discussed in this section are ones related to obstacles having dimensions more comparable to the boundary layer thickness, or larger, than those of the protuberances discussed previously. As compared to section 1.1, flows in this regime have flow features that are more prevalent, easier to distinguish, and easier to make qualitative conclusions about. There have been many two-dimensional shapes examined including square bars, triangular cylinders and forward and backward facing steps to name a few. There are a few important differences between two-dimensional and three-dimensional flows as is described by Hunt et al. (1977) and Martinuzzi and Tropea (1993). These key differences, respectively, are that two-dimensional flows have a closed mean flow separation bubble behind the element whereas three-dimensional flows do not, and flows over two-dimensional obstacles take longer to reattach as compared to flows over threedimensional obstacles. To this end, only three-dimensional obstacles will be discussed in the rest of the section. 
Wind effects over three-dimensional bluff bodies has been the center of many studies concerned with determining drag and pressure distributions on man-made obstacles as well as natural obstacles. The study of Okamoto et al. (1977) was motivated by the flow around cones and how they could be related to the flow around a mountain. Cones with vertex angles equal to $60^{\circ}, 90^{\circ}, 120^{\circ}$, and $150^{\circ}$ were used. All cones had base diameters equal to $100 \mathrm{~mm}$ and heights equal to $86 \mathrm{~mm}, 50 \mathrm{~mm}, 29 \mathrm{~mm}$, and $13 \mathrm{~mm}$ respectively. Experiments were conducted in a turbulent boundary layer with a thickness of $7.6 \mathrm{~mm}$ and a free-stream velocity of $10 \mathrm{~m} / \mathrm{s}$. Surface pressure measurements on the cone and flat plate, and wake velocity measurements were taken to analyze the flow field. Drag and lift coefficients calculated from the surface-pressure distributions were presented as well. Surface oil flows were also performed to visualize the formation of horseshoe vortices as well as to define the separation points on the cone surface. Another study by Savory et al. (1988) focused on flow over a hemisphere and its relation to flow over a domed building. This particular investigation involved a hemisphere with a base diameter equal to $190 \mathrm{~mm}$ in three separate turbulent boundary layers with thicknesses equal to 367,258 , and $86 \mathrm{~mm}$. All flows had a free-stream speed of $10.7 \mathrm{~m} / \mathrm{s}$. Drag measurements, pressure measurements and wake velocity profiles were taken in order to quantify the hemisphere's presence in the boundary layer. Cases in which the obstacle was more or less contained within the boundary layer are Arie et al. (1975a and b), Taniguchi et al. (1981), Sakamato et al. (1982), and Sakamato (1985). Different aspects of circular cylinders, rectangular cylinders, and cube-shaped obstacles were examined. Obstacle characteristics such as aspect ratio, ratio of obstacle height to boundary layer thickness, and the ratio of friction velocity to free stream velocity were related to the form drag of each obstacle. Conclusions stemming from this work are that the obstacle shape and size and the thickness of the boundary layer are key factors in describing the flow characteristics.

A recent study was performed involving the flow around surface mounted pyramids in thick and thin turbulent boundary layers, Martinuzzi and AbuOmar (2003). The effects of apex angle and angle of attack were related to wake periodicity and vortex shedding based on surface pressure measurements taken on the wall and pyramid sides, velocity 
measurements, and mean flow surface patterns. For broad pyramids it was found that periodicity in the wake does exist but cannot be related directly to vortex shedding. Periodic fluctuations are seen in the surface pressure measurements on the sides of the pyramids as well as in the velocity field in the wake. Also, for pyramids placed in thick boundary layers no wake periodicity is observed and the wake characteristics are different as compared to those of the pyramid in a thin boundary layer.

The flow around a surface-mounted cube has been the focus of numerous studies; a few of these are Castro and Robins (1977), Hunt et al. (1978), Scholfield and Logan (1990), Martinuzzi and Tropea (1993), and Sousa (2002). The investigation by Castro and Robins is one of the few previous studies that has analyzed the effect that orientation has on the flow field. Mean velocity and turbulence intensities were measured in the wake of each case. These measurements show the effect that the vortices have on the near wake flow regions. The velocity deficit in each case was shown to have fully decayed at a location equal to six lengths downstream. It was also discovered that the cube oriented $45^{\circ}$ relative to the flow produced a stronger downstream vortex pair than the cube oriented $90^{\circ}$ relative to the flow. The same result has also been found to be the case in the present research. Schofield and Logan's analysis concentrates on how the major flow features are influenced by the model geometry as well as the incident shear flow. Sousa utilized digital particle image velocimetry to identify the location of the large-scale vortical structures present within the flow field surrounding the cube.

Another study by Logan and Lin (1982) evaluates the wall shear recovery behind prisms of different aspect ratios at downstream centerline locations. This study concluded that a quicker recovery was seen by three-dimensional obstacles as opposed to two-dimensional obstacles due to the presence of the streamwise vortex structure. One of the most complete flow field pictures of the flow around a cube is given by Martinuzzi and Tropea (1993). Two-component laser-Doppler anemometry measurements along with surface pressure measurements and surface oil flow visualizations were utilized to describe the flow field. The aim of this study was to determine the separation and reattachment 
patterns on the prism as well as to obtain quantitative data that describes the mean and fluctuating velocity field.

\subsection{Rough-Wall Turbulent Boundary Layers}

Due to the limited amount of directly applicable data obtained in the present research, this section will be a very restricted look into the previous research done on rough-wall turbulent boundary layers. For a more in-depth look at previous studies done to analyze the flow structure in sparsely distributed rough-wall turbulent boundary layers please refer to George (2004). The current subset of data will be compared with the data and analysis done by George and Simpson (2000-04) in later chapters.

Past studies of rough-wall turbulent boundary layers have mainly involved the characterization of roughness elements as equivalent sand grain roughness. This idea was stemmed from Nikuradse (1933) and has been in wide use ever since. A significant amount of attention has also been devoted to the effect of two-dimensional roughness elements (ribs, grooves, etc.). Perry et al. (1969), Perry et al. (1987), and Krogstad and Antonia (1994) are a few examples found in previous literature related to twodimensional roughness effects. George and Simpson have taken a more systematic approach to describe rough-wall turbulent boundary layers. They have performed experiments on only the isolated roughness element and then used that very same element to construct an array of roughness elements. Information gathered from these efforts has been extensive in terms of the mean velocities, turbulence quantities, and skin friction, as well as the related fluid dynamic quantities that can be derived from the above parameters. The current research is directly related to that of George and Simpson in that seven isolated roughness elements are tested as well as a fetch of one of those isolated elements (Gaussian element). Consequently, with the addition of the current research, the combined data base will encompass a wide range of the effects that each roughness element has on the flow field. This knowledge in turn can be assembled into a deeper understanding of how the physics of the different flows are connected. 


\subsection{Related Modeling and Computational Efforts}

Modeling the effects of surface roughness is an area of concern in many practical engineering applications. Consequently, there has been a lot of effort in recent years to calculate flows over different rough surfaces using various computational fluid dynamic (CFD) models. Many current roughness models to this point have involved the use of empirical 'constants' and equivalent sand grain roughness. These underdeveloped concepts have little direct relationship to realistic roughness and cannot predict accurately and consistently the flow characteristics for different roughness shapes. The calculations of various roughness flows are greatly influence by the choice of turbulence model. Most models have a difficult time with the calculation of mean flow characteristics. This difficulty only becomes more pronounced when turbulence quantities are calculated. The shortcomings of these models are due to effects not taken into account in the calculation methods. Patel (1998) also discusses the downfalls of current CFD models and comments that high Reynolds number flows over rough surfaces is the 'Achilles Heel' of CFD. Patel suggests the need for further experimental research concerning the effects of roughness in high Reynolds number turbulent boundary layers. This section gives some general insight as to what the objectives of some models are, how they have been used to calculate different roughness flows, and the results of such efforts.

Patel (1998) shows the experimental data of Parthasarathy and Muste (1994) compared to the calculations of Yoon et al. (1997) using the standard k- $\omega$ model in an asymmetric channel flow. A $2 \mathrm{~mm}$ thick wire mesh and a series of rib-roughness, $8.9 \mathrm{~mm}$ wide x 13 $\mathrm{mm}$ tall (50.2 $\mathrm{mm}$ spacing), were tested using an equivalent sand grain roughness determined from empirical correlations. Mean flow characteristics were calculated to within no less than a $7 \%$ difference. In contrast, the Reynolds shear stress was not calculated very close at all. Conclusions from this experiment were that the standard k- $\omega$ model does not capture the physics of the flow and deteriorates for increasing roughness size.

A force field approach using large eddy simulation (LES) is discussed in Cui et al. (2003). A body-fitted grid is avoided by applying an orthogonal Cartesian grid to 
complex geometry and additional stability limits are not introduced by the computational scheme. The body force magnitude is determined internally during numerical solution, thus making it a more general model than those requiring empirical inputs. This force field LES approach was compared to a two-dimensional wavy rough boundary with a mean pressure gradient and separation (data of Buckles et al.). In this case, the model calculates premature separation, overestimates reverse flow, and calculates late reattachment. Although this model does have its downfalls, it does show improvements from previous models.

A modified van Driest damping function model was introduced by Krogstad (1991). This model simulates the viscous stresses near the wall by manipulating the amount of viscous damping applied. Through this 'manipulation' process various parameters are adjusted to match experimental results. This model was compared with data from a three-dimensional wing body junction flow using 24-grit sandpaper to roughen the surface. The model was tested on two different streamlines within the flow: line 1 is a line of symmetry that leads up to an ordinary separation point, and line 3 passes outside the separated region. Line 2 leads up to the horseshoe vortex where three-dimensional separation exists and was not calculated. The model predicts early separation on line 1 due to the fact that no viscous-inviscid interaction is accounted for. The model does calculate line 3 better due to the smaller pressure gradients and less interaction seen along this line.

Shim et al. (2000 and 2001) compare four different turbulent models used to calculate the flow field, namely the lift and drag coefficients, involving iced airfoils at various angles of attack (data from Addy et al.). Two different elemental flow fields are examined. A somewhat smooth Gaussian-shaped element (rime ice) and a similarly shaped element with prominent horns near the peak of the element (glaze ice). In these flow regimes two 1-equation models are utilized and two 2-equation models are used. The 1-eqn. models are the Baldwin-Barth (BB) model and the Spalart and Allmaras (SA) model. The BB model is derived from the $\mathrm{k}-\varepsilon$ model and its objective is to accurately calculate the turbulence phenomena in separated regions and shear layers which cannot be handled by 
algebraic models. The SA model has the same objective as the BB model but is not derived from a 2-eqn. model. The SA model includes a destruction term that depends on the distance to the wall and is forgiving in terms of near-wall resolution and stiffness. This model also provides a smooth laminar to turbulent transition but only at points specified by the user. The SA model is less grid sensitive than the BB model and more accurate in calculating boundary layer profiles, skin friction and other wake properties.

The 2-eqn. models are the Chien $\mathrm{k}-\varepsilon(\mathrm{k}-\varepsilon)$ model and the shear stress transport (SST) model. The $\mathrm{k}-\varepsilon$ model includes terms describing the behavior of the turbulent shear stress, turbulent kinetic energy (TKE), and rate of dissipation near a solid wall using a Taylor Series expansion. This model retains a proper physical behavior of the balance between dissipation and molecular diffusion of the TKE. The SST model is developed from the original $k-\omega$ model of Wilcox. The SST is identical to the $k-\omega$ model in the inner region of flow and changes gradually to the standard k- $\varepsilon$ model toward the outer edge of the boundary layer. As compared with the original k- $\omega$ model, the SST model has the ability to account for the transport of the turbulent shear stress in boundary layers with adverse pressure gradients.

All models were found to have good results at low angles of attack but the $\mathrm{k}-\varepsilon$ model did not converge at high angles of attack. The SST model was determined to give the best calculation for the rime ice case. The maximum percent difference for the lift coefficient was $\approx 13 \%$ whereas the maximum percent difference for the drag coefficient was $\approx 9 \%$. However, the SA model gave the best calculations for the glaze ice flow regime. The maximum percent difference for the lift coefficient was $\approx 7 \%$ whereas the maximum percent difference for the drag coefficient was $\approx 8 \%$. The deviations of the models at higher angles of attack are attributed to the formation and shedding of large separation bubbles and the resultant unsteadiness within the flow. 


\subsection{Organization of Thesis}

This thesis is comprised of five main chapters and six appendices. Each chapter discusses the most significant aspects of the research whereas the appendices contain supporting material related to the main discussion. Chapter 2 discusses the apparatus and instrumentation employed to conduct the research. Information contained in this chapter is related to the wind tunnel setup, LDV measurement system, data post-processing, quality of flow conditions, description of the tested roughness elements, and LDV probe alignment techniques. Chapter 3 contains the results of the research conducted. All mean velocities, turbulence quantities, skin friction, and other quantities that are derived from the previous parameters can be found in this chapter. Oil flow visualizations are also described within Chapter 3. Chapter 4 provides the related discussion to the experimental results as well as data correlations and flow field schematics. These schematics give a description of the flow field using the data gathered from Chapter 3. Chapter 5 provides the conclusions and results gathered from the previous four chapters. The references used in this study can be found immediately following Chapter 5.

Appendices A through $\mathrm{F}$ can be found at the end of the thesis. Appendix A contains information related to various trip arrangements and the generation of the desired boundary layer characteristics. Appendix B is the derivation of the drag equation used in the single roughness element analysis. Appendix $\mathrm{C}$ contains the uncertainties that are pertinent to the conducted research. Appendix D includes a brief analysis of the data related to the Gaussian fetch of roughness. A discussion related to the flow angle data can be found in Appendix E. Finally, Appendix F gives the $y-z$ coordinates that define the shape of both the large and fine grooved elements. 


\section{Chapter 2 Apparatus and Instrumentation}

The purpose of this chapter is to familiarize the reader with the experimental apparatus and techniques used in conducting the present research in the Department of Aerospace and Ocean Engineering at Virginia Polytechnic Institute and State University. Virginia Tech's small boundary layer wind tunnel was resurrected and many modifications were performed in order to conduct the present research. Pitot tube and three-velocity component laser-Doppler velocimetry (LDV) measurements were taken and used to determine the flow characteristics of the wind tunnel following setup. All LDV data obtained for the various roughness cases was obtained in the same fashion and are discussed below. Other details that will be discussed include the following: wind tunnel, LDV system, flow conditions, tested roughness elements and their respective manufacturing techniques, and data acquisition and post processing.

\subsection{Small Boundary Layer Wind Tunnel}

The function of the wind tunnel is to deliver low turbulence air at constant temperature and velocity to the test section. The wind tunnel is a closed-circuit design and can accommodate the necessary seeding of the flow for LDV measurements. The small boundary layer wind tunnel previously located in Lab \#7 in the basement of Randolph Hall is now located in Lab \#6. In order to accommodate the ever increasing need for space, many modifications were made to the previous wind tunnel setup. For information concerning the previous setup see E. J. Smith et al. (1990), as well as K. R. Saripalli and R. L. Simpson (1980). The result is the most space efficient and versatile design for the small boundary layer wind tunnel. The dimensions of the wind tunnel setup are approximately; 6.25 meters long, 1.73 meters wide and 2.20 meters high. There are seven main parts to the wind tunnel system: air conditioning system, speed control valve and filter box, blower system, plenum chamber, contraction section, test section, and the return ducting. Figures of the following descriptions can be found at the end of the chapter. Figure 2.1 gives a schematic of the wind tunnel arrangement while Figures 2.2 and 2.3 show different views of the actual setup of the wind tunnel. The modifications 
will be discussed below along with a description of the wind tunnel setup in the order the flow travels through the system.

\subsubsection{Air Conditioning System}

The first component that the flow encounters in the experimental setup is the air conditioner (AC). The flow travels through $30.48 \mathrm{~cm}$ diameter ducting that is attached to the AC. In order to accommodate the duct work, the AC rests on top of a $7.62 \mathrm{~cm}$ by $3.81 \mathrm{~cm}$ aluminum $\mathrm{C}$-channel support bracket bolted to the top of the plenum chamber, see Section 2.2.4. The function of the $\mathrm{AC}$ was to maintain a constant temperature in the wind tunnel test section. The AC utilized is a model ACM244XL0 manufactured by Whirlpool with a 1 phase $60 \mathrm{~Hz}$ motor and has a capacity of 25.3 MJ (24,000 BTU's). By controlling only the fan and cooling adjustments on the AC, it was possible to obtain an equilibrium temperature condition in the test section of $\pm 0.56^{\circ} \mathrm{C}$ at $25^{\circ} \mathrm{C}\left( \pm 1^{\circ} \mathrm{F}\right.$ at $77^{\circ} \mathrm{F}$ ). It is important to note that the $\mathrm{AC}$ condenser never ceased running in order to maintain a constant temperature. If such a condition did happen, and only the fan was left running for a period of time, there would be an unwanted temperature fluctuation within the system.

A series of ductwork was constructed and added to the AC in order to expel unwanted hot air out of the laboratory. Sheet metal was cut and subsequently fitted and sealed to the $\mathrm{AC}$ exit and then connected to a rectangular duct system that measured $41.91 \mathrm{~cm}$ by $21.59 \mathrm{~cm}$ and was $185.42 \mathrm{~cm}$ tall. This ducting then connected to the wall which led to an outlet for the unwanted hot air. The normal room AC and heating system was utilized in order to keep the temperature of the laboratory between $22.22^{\circ} \mathrm{C}$ and $24.44^{\circ} \mathrm{C}\left(72^{\circ} \mathrm{F}\right.$ to $\left.76^{\circ} \mathrm{F}\right)$. With this accomplished it was possible to maintain the $\pm 0.56^{\circ} \mathrm{C}$ at $25^{\circ} \mathrm{C}\left( \pm 1^{\circ} \mathrm{F}\right.$ at $77^{\circ} \mathrm{F}$ ) condition within the test section.

\subsubsection{Speed Control Valve and Filter Box}

The next component in the flow circuit is the speed control valve. This valve is a butterfly valve contained inside the $30.48 \mathrm{~cm}$ diameter ducting. It is possible to limit the amount of air ingested by the blower by rotating the valve in order to obtain a constant speed in the test section. With the valve completely open, the wind tunnel can achieve a 
test section velocity of approximately $44 \mathrm{~m} / \mathrm{s}$. Following the butterfly valve the flow passes into the filter box. The filter box is constructed out of $1.27 \mathrm{~cm}$ thick plywood on all sides except for the side that it connects to the blower intake. This side is constructed of $0.64 \mathrm{~cm}$ thick aluminum in order to bolt the filter box to the blower intake. A $0.32 \mathrm{~cm}$ thick rubber gasket lies between the filter box and the intake flange of the blower in order to reduce the transmission of vibration throughout the system. The dimensions of the box are as follows; $68.90 \mathrm{~cm}$ tall, $64.77 \mathrm{~cm}$ wide, and $43.82 \mathrm{~cm}$ deep. The ducting connects to the filter box via a sheet metal connection. A fine wire mesh screen is fastened to the inside top of the filter box in order to ensure that there will be no debris taken into the blower.

\subsubsection{Blower System}

Once the air passes through the filter box it enters the suction side of the blower. A new blower was selected in order to provide the most versatility to the small wind tunnel setup. The final blower selection made was a New York Blower Size 194 GI-DH Series 20 Fan, Arrangement 10 with a DH wheel and clockwise rotation. This general industrial, centrifugal blower is driven by a constant speed v-belt drive, $5.59 \mathrm{~kW}(7.5$ horsepower), $3500 \mathrm{rpm}, 230$ Volt motor which is covered for safety precautions. This blower is capable of delivering $62.30 \mathrm{~m}^{3} / \min (2200 \mathrm{cfm})$ of air at $30.48 \mathrm{~cm} \mathrm{H}_{2} 0$ static pressure at $2529 \mathrm{rpm}, 5.16 \mathrm{~kW}(6.92 \mathrm{Bhp})$, and $21.11^{\circ} \mathrm{C}\left(70^{\circ} \mathrm{F}\right)$.

\subsubsection{Plenum Chamber}

As the air exits the blower it enters the plenum chamber. The purpose of the plenum is twofold. First, the air 'settles' and large scale fluctuations produced by the blower are eliminated. Second, the acoustic noise is absorbed and the flow is quieted. The plenum is a rectangular box that is constructed out of $1.91 \mathrm{~cm}$ thick plywood with inside dimensions of $116.91 \times 116.91 \times 157.48 \mathrm{~cm}$. The inlet and outlet dimensions of the plenum are $50.80 \times 50.80 \mathrm{~cm}$ and $58.42 \times 58.42 \mathrm{~cm}$ respectively. The plenum is symmetric about the centerline that extends from the inlet to the exit of the plenum. The plywood is supported via $0.318 \mathrm{~cm}$ thick $3.81 \times 3.81 \mathrm{~cm}$ steel angle which is bolted to the plywood sides. The plenum is connected to the blower outlet flange and the contraction via 0.318 
$\mathrm{cm}$ thick rubber in order to eliminate any mechanical vibrations to the test section. The entire plenum chamber is sealed with silicone rubber in order to prevent any leaks.

To facilitate the 'mixing' of the flow, there is a baffle plate placed inside the plenum chamber, see Figure 2.4. The baffle plate is $63.50 \times 63.50 \mathrm{~cm}$ constructed from $1.91 \mathrm{~cm}$ thick plywood and secured to the top and bottom walls of the plenum by means of the previously discussed steel angle. It is positioned $63.50 \mathrm{~cm}$ aft of the plenum exit and centered between the plenum side walls. Directly behind the downstream side of the baffle plate, a $1.27 \mathrm{~cm}$ diameter copper tube extends to the center of the baffle plate, see Figure 2.5. This tube introduces the 'seed' particles to the flow to ensure the air will get uniformly mixed with 'seed' before the flow reaches the contraction and test section. The seeding particles used in the current research are di-octyl phthalate (DOP). The liquid DOP is converted to nearly constant diameter particles, having a Gaussian distribution centered around $1 \mu \mathrm{m}$ in size, via an aerosol generator system. Once the DOP is in its aerosol form it is introduced into the plenum chamber. In order to catch excess liquid DOP, a $20.32 \times 20.32 \times 6.35 \mathrm{~cm}$ stainless steel drip pan is located directly under the copper tubing on the bottom wall of the plenum. A ball valve is attached to the drip pan on the outside of the plenum that enables one to release the contents of the pan.

A $7.62 \mathrm{~cm}$ thick layer of polyester urethane foam with a standard anechoic wedge surface geometry (Smith, et al. 1990) covers the inlet, top, bottom, and side walls of the plenum as well as the upstream side of the baffle plate. This foam helps to absorb the acoustic noise generated by the blower. For more information on the foam selection see Smith et al. 1990. The final effective inside dimensions of the plenum chamber are $101.60 \times 101.60 \times 149.86 \mathrm{~cm}$.

\subsubsection{Contraction Section}

Following the exit of the plenum chamber, the flow enters the contraction section. The contraction section consists of two different contractions as well as a rectangular section that houses three screens and a honeycomb (Saripalli and Simpson, 1980). The purpose of the contraction is to connect the plenum to the test section as well as increase the flow velocity and reduce the turbulence levels of the flow. The contraction section is 
constructed from $0.71 \mathrm{~mm}$ thick sheet metal. The first contraction that leads to the rectangular section is $45.72 \mathrm{~cm}$ in length and has inlet and outlet dimensions as follows, $53.34 \times 53.34 \mathrm{~cm}$ and $38.10 \times 22.86 \mathrm{~cm}$. Once the flow has entered the rectangular section it encounters the honeycomb which serves the purpose of eliminating large scale turbulence within the flow. After passing through the honeycomb, the flow reaches the three screens. These screens are located $13.97 \mathrm{~cm}$ apart with the purpose of letting the wakes from each of the previous screens die out before reaching the following screen. The main function of the screens is to reduce the turbulence level of the flow. The second and final contraction has an inlet dimension of $40.64 \times 24.13 \mathrm{~cm}$ and an outlet dimension of $10.16 \times 24.13 \mathrm{~cm}$.

\subsubsection{Test Section}

Once the air exits the final contraction, it enters the test section. The test section and contraction are lined up to facilitate a smooth flow transition. Diagrams of the test section within the wind tunnel setup can be seen in Figures 2.1 and 2.2. The test section has an overall length of $199.39 \mathrm{~cm}$ and is $24.13 \mathrm{~cm}$ wide. The test section floor is constructed of hard 'fin-form' plywood with a polished surface. There are two openings in the test section floor, in which inserts can be placed, that make it possible for data acquisition. The opening nearest the contraction measures $40.64 \mathrm{~cm}$ by $23.50 \mathrm{~cm}$ and has a $0.48 \mathrm{~cm}$ thick smooth aluminum plate inserted into the opening. The aluminum plate is supported via three evenly spaced sections of wood laid perpendicular to the tunnel floor. Each piece of wood is routered on their respective ends to provide the necessary support and a smooth continuation of the test section floor. The second opening in the floor measures $71.12 \mathrm{~cm}$ by $23.50 \mathrm{~cm}$ and has $0.79 \mathrm{~cm}$ thick Plexiglas inserted into the opening. This Plexiglas insert is supported in a similar fashion as discussed previously. The only difference being that four wooden sections were utilized for support, one on each edge, in order to allow the necessary LDV access needed for data acquisition. There is a $20.32 \mathrm{~cm}$ diameter hole cut into the Plexiglas in which a $20.32 \mathrm{~cm}$ diameter, $0.64 \mathrm{~cm}$ thick piece of float glass can be placed. It is through this piece of float glass where the LDV measurements are taken. Actual locations of measurements will be discussed in further detail in the following chapters. It is important to note however, that the center of 
the glass disc is located $116.72 \mathrm{~cm}$ from the contraction exit. The side walls are $20.96 \mathrm{~cm}$ high and are constructed out of $0.64 \mathrm{~cm}$ thick glass.

The test section ceiling has three main sections. There are two removable/adjustable sections and a permanent section, all of which are constructed from $0.95 \mathrm{~cm}$ thick Plexiglas. The permanent section is located the last $13.97 \mathrm{~cm}$ of the test section and has a height of $10.48 \mathrm{~cm}$. The rest of the ceiling is split into two parts in order to facilitate the removal and replacement of the trip arrangement discussed previously. It is important to note that the two removable ceiling sections are reinforced with $2.54 \mathrm{~cm}$ by $1.27 \mathrm{~cm}$ aluminum c-channel and are secured together in a manner which permits a smooth transition from one section to the other. The forward most section has a length of 51.44 $\mathrm{cm}$ and is the section that can be removed for the replacement of the sandpaper, etc. The rest of the removable ceiling has a length of $133.98 \mathrm{~cm}$ and transitions smoothly to the permanent ceiling. There are twenty-four $0.95 \mathrm{~cm}$ holes drilled along the centerline of the removable ceiling in order to provide the necessary pitot probe access to obtain a zero-pressure gradient flow. The first hole is $22.86 \mathrm{~cm}$ from the contraction exit. All subsequent holes are spaced $6.35 \mathrm{~cm}$ apart, except between holes ten and eleven. The spacing between these two holes is $12.70 \mathrm{~cm}$. This is due to the fact that a support beam interferes with the regular hole placement. At hole number twenty, there are two more holes drilled $3.81 \mathrm{~cm}$ on either side of the centerline hole. These holes give access to the tunnel in order to probe the boundary layer and determine the two-dimensionality characteristics of the flow. See Table 2.1 below for port \#'s and their related locations relative to the contraction exit.

Table 2.1: Port \#'s and related distance from contraction exit

\begin{tabular}{|c|c|c|}
\cline { 2 - 3 } \multicolumn{1}{c|}{} & \multicolumn{2}{c|}{$\begin{array}{c}\text { Dist. from Contraction } \\
\text { Exit }\end{array}$} \\
\hline Port \# & Inches & Centimeters \\
\hline 1 & 9 & 22.86 \\
\hline 2 & 11.5 & 29.21 \\
\hline 3 & 14.0 & 35.56 \\
\hline 4 & 16.5 & 41.91 \\
\hline 5 & 19.0 & 48.26 \\
\hline 6 & 21.5 & 54.61 \\
\hline 7 & 24.0 & 60.96 \\
\hline 8 & 26.5 & 67.31 \\
\hline
\end{tabular}




\begin{tabular}{|c|c|c|}
\hline 9 & 29.0 & 73.66 \\
\hline 10 & 31.5 & 80.01 \\
\hline 11 & 36.5 & 92.71 \\
\hline 12 & 39.0 & 99.06 \\
\hline 13 & 41.5 & 105.41 \\
\hline 14 & 44.0 & 111.76 \\
\hline $\begin{array}{c}\text { Center of } \\
\text { Glass Disc }\end{array}$ & 45.95 & 116.72 \\
\hline 15 & 46.5 & 118.11 \\
\hline 16 & 49.0 & 124.46 \\
\hline 17 & 51.5 & 130.81 \\
\hline 18 & 54.0 & 137.16 \\
\hline 19 & 56.5 & 143.51 \\
\hline 20 & 59.0 & 149.86 \\
\hline 21 & 61.5 & 156.21 \\
\hline 22 & 64.0 & 162.56 \\
\hline 23 & 66.5 & 168.91 \\
\hline 24 & 69.0 & 175.26 \\
\hline
\end{tabular}

There is a single static pressure port $148.59 \mathrm{~cm}$ from the contraction exit. This port has a diameter of $0.05 \mathrm{~cm}$ and was utilized in probing the boundary layer. The ceiling has five aluminum crossbars that allow it to be bolted to an aluminum c-channel, attached to the glass side walls, to alleviate unwanted wandering of the setup. Two bolts located at each ceiling crossbar position enable one to adjust the ceiling height. The ceiling height was adjusted in order to produce a zero pressure gradient flow. Actual ceiling height measurements are discussed for the final configurations (single element and Gaussian fetch) in Section 2.4.2, Table 2.2.

It is important to note that the blower, contraction section, and test section are all bolted to the floor to alleviate any wandering of the wind tunnel system.

\subsubsection{Return Ducting}

Once the flow exits the test section it enters a rectangular to circular expansion having inlet dimension of $24.13 \mathrm{~cm}$ by $10.48 \mathrm{~cm}$ and outlet dimensions of $30.48 \mathrm{~cm}$ in diameter. The expansion takes place over a length of $26.67 \mathrm{~cm}$. This expansion then connects with the rest of the circular ducting which is also $30.48 \mathrm{~cm}$ in diameter. The ducting utilized is a general commercial type of ducting. In order to assist the space efficiency of the tunnel setup, the ducting was fashioned in a vertical return loop. For safety precautions, as well 
as ease of use, the bottom of the ducting is $182.88 \mathrm{~cm}$ off of the floor and is located directly above the test section. A $0.32 \mathrm{~cm}$ thick section of rubber acts as a joint between two pieces of ducting within the return loop help to alleviate any mechanical vibrations to the test section. The return system is supported via three $1.91 \mathrm{~cm}$ by $1.91 \mathrm{~cm}$ steel angle brackets. Two brackets are directly on either side of the rubber connection and the other bracket can be found attached to a steel angle located on the front end of the plenum chamber. The return ducting is attached to the $\mathrm{AC}$ and then continues into the filter box. All connections between ductwork and other various parts of the wind tunnel system are sealed with silicon rubber in order to prevent leaks.

\subsection{LDV System}

The LDV system utilized consists of a miniature LDV head, optical table, a signal conditioning arrangement, 3 digital signal processors, traverse system, and a particle seeding system. This setup allows the coincident and instantaneous measurement of the $\mathrm{U}, \mathrm{V}$, and $\mathrm{W}$ components of velocity. Consequently, all mean velocities, Reynolds stresses, and triple products can be gathered from this measurement scheme. This measurement system is also described by Chesnakas and Simpson (1994) as well as Tian (2003) and Tang (2004). The LDV system has a nearly spherical measurement volume of $\approx 50 \mu \mathrm{m}$ in diameter and fringe spacing equal to $\approx 5 \mu \mathrm{m}$. These parameters were calculated using equations from Durst et. al (1995) and Durst et. al (1981) respectively. Very near-wall measurements can be obtained with this LDV system. Measurements with this system closely agree with direct numerical simulation (DNS) data for a twodimensional turbulent boundary layer which gives added confidence in the obtained data. An uncertainty analysis for the LDV system and results can be found in Appendix C. Kuhl and Simpson (2001) have investigated the velocity bias effects and transient time broadening effects of the measurement system and determined them to be negligible. Likewise, Olcmen et. al (1998) investigated the instrument broadening effects on the measured frequency by the digital signal processors (Macrodyne FDP3100) and they too were determined to be negligible. 


\subsubsection{Optical Table}

A Coherent argon-ion laser powers the LDV measurement system. The laser is encased in a Plexiglas box that has a small air conditioning unit placed inside. Both the box and the air conditioner serve to keep the laser in a clean dust-free environment. A view of the optical table setup near the laser exit can be seen in Figure 2.6, whereas a full view of the optical table is shown in Figure 2.7. Following the exit of the main beam out of the laser, the beam is directed by a series of mirrors into a prism. The main beam is a conglomeration of various wavelengths. Thus, the prism separates the colors and associated wavelengths in order for the desired green $(514.5 \mathrm{~nm})$ and blue $(488 \mathrm{~nm})$ beams to be utilized. Once the colors are separated the beams are allowed to travel a sufficient distance in order for them to spread out from one another. The green beam is then directed by a mirror into a polarization rotator. This rotator enables the manual shifting of beam power (vertically or horizontally polarized laser light) in order for optimum signal quality. From the polarization rotator the green beam passes through a beam splitting cube. This cube splits the vertically and horizontally polarized beams and directs them $90^{\circ}$ away from one another. After the green beams are split they travel into their respective Bragg Cells which shift the beams by a known frequency amount, which in this case is $-27 \mathrm{MHz}, 0 \mathrm{MHz}$, and $50 \mathrm{MHz}$. The purpose of the Bragg Cell is to enable one know the direction in which the fringes are moving. This in turn will eliminate any directional ambiguity that may arise within the measured Doppler signal. It is worthwhile to note that, for this system, the maximum data rate was obtained when more power was in the unshifted $0 \mathrm{MHz}$ green beam as opposed to an equal distribution of power. The blue beam requires no beam splitting cube and is split into two equal intensity beams via the $40 \mathrm{MHz}$ Bragg Cell driver. An unshifted beam of $0 \mathrm{MHz}$ and a shifted beam of $40 \mathrm{MHz}$ will come from this Bragg Cell. In order to have a clear signal from each of the 3 beam pairs, it is necessary to shift the beams by a frequency amount large enough that will eliminate cross-talk between the signals. All five beams, $-27 \mathrm{MHz}$, $0 \mathrm{MHz}, 50 \mathrm{MHz}$ (green) and $0 \mathrm{MHz}, 40 \mathrm{MHz}$ (blue), are then directed into their respective beam launchers where a microscope objective focuses the beam into the fiber optics. From there the beams travel through polarization preserving optical fibers and into the LDV head itself. 


\subsubsection{LDV Head}

The LDV head is comprised of two separate aluminum beam assemblies that house the necessary optics to transmit the 3 green beams $(514.5 \mathrm{~nm})$ and 2 blue beams $(488 \mathrm{~nm})$. The beam assemblies are at a right angle with respect to one another. Within these assemblies are beam collimating lenses as well as focusing lenses which make it possible to obtain a constant diameter laser beam that can be aligned at the focal point of the lens. Figure 2.8 shows a close-up view of the head where Figure 2.9 shows the LDV head while acquiring data. The off-axis backscatter signal is received through an array of receiving optics that is also located in the center of the LDV head. Both the green beam assembly as well as the receiving optics assembly are adjustable which aids in the beam alignment process.

The LDV head is attached to a base which in turn is attached to a three-dimensional traverse system. The current system is on the same traverse base as a comprehensive LDV system being developed by Lowe (2004). This enables the full utilization of both systems without constant realignment issues. This traverse system allows full mobility of the LDV head in the flow field. The vertical (y) traversing is accomplished via a National Aperture Inc. MC-4B Series (Servo 3000) controller, where the spanwise (z) and streamwise (x) directions are controlled with a Velmex VP9000 Series controller. For additional information on the $\mathrm{x}-\mathrm{z}$ traverse system see Lowe (2004). The entire traverse system and the LDV head were situated underneath the tunnel floor so as to not interfere with the flow field in any way. Measurements could be made through a 20.32 $\mathrm{cm}$ diameter, $0.64 \mathrm{~cm}$ thick piece of float glass inserted into the wind tunnel floor. The center of this glass disc is $116.72 \mathrm{~cm}$ from the contraction exit.

\subsubsection{LDV Seeding}

An aerosol generator designed by Echols and Young (1963) was used to seed the wind tunnel. This generator used dioctal phthalate as the seeding material. The mean particle size is $\approx 1 \mu \mathrm{m}$ in diameter. The air pressure going into the generator was kept near $11 \mathrm{psi}$ in order to maintain optimum signal quality. The seeding material was introduced into the wind tunnel via a 0.5 inch $(1.27 \mathrm{~cm})$ diameter copper pipe. The air/seed mixture 
exhausted from the air conditioner was sent through an Aprilaire Electronic Air Cleaner before being exhausted outside.

\subsubsection{Signal Processing}

With all five laser beams crossing at the receiving lens focal point, instantaneous coincident three-velocity component data is sent to the signal setup. The signal receiving setup, stationed on the optical table, gathered the Doppler bursts from the multimode receiving optical fiber. Two photo-multiplier (PM) tubes from Electron Tubes Limited, models $9125 \mathrm{~B}$ and 9124B, received the separated green and blue light respectively. The signal from the photo-multiplier tubes was then transmitted into an electrical voltage signal and sent to the signal conditioning electronics.

All of the electronics are located on a single circuit board. The green signal coming out of its respective PM tube is divided into two signals related to the $50 \mathrm{MHZ}$ and $-27 \mathrm{MHz}$ shifted beams. These two signals are then taken along with the blue $40 \mathrm{MHz}$ signal and amplified as well as mixed with their respective RF signals. These RF signals are produced by three variable RF generators that allow the extraction of the meaningful Doppler signals. These mixed signals are then transferred to their respective Macrodyne FDP 3100 frequency processors. The three Macrodynes were set with a coincidence window equal to $10 \mu \mathrm{s}$ in order to obtain coincident data from the three signals. A validation ratio from each of the three Macrodynes was always within a range of $97 \%$ to $99 \%$. The acquired number of samples per point varied between 15,000 near the wall to 30,000 away from the wall. The Macrodyne signals were sent to an IBM 386/33C PC with a Dostek 1400A Laser Velocimeter Interface, and a TCEM daughter board option, via three 25-pin parallel cables.

\subsection{Post Processing}

Due to the significant amount of electronics used to measure the LDV signals there is an inherent amount of noise that must be taken into account when processing the LDV data. The method utilized to remove the irrelevant noise from the data was that same as that used by Olcmen and Simpson (1995). A parabola was fit to each side of the logarithm of 
the velocity component histogram ordinate over a range of $1 \%$ to $80 \%$ of the peak histogram value. Histogram values above and on the outskirts of the parabolic fit to the histogram were considered noise and discarded. If one velocity component was deemed unacceptable then all three velocity components were discarded. Following the initial noise reduction the data were rotated to match the tunnel coordinate system. The same process was again followed and the rotated histograms were fit with parabolas and the noise discarded.

Determining the position of the wall relative to the measurement volume is critical to obtaining meaningful results. When the measurement volume is positioned on the wall a strong signal is sent from the spectrum analyzer to the oscilloscope. This signal can then be utilized to find the position of the wall. Although this method is extremely useful, it is difficult to consistently determine the wall position to less than $\pm 30 \mu \mathrm{m}$ uncertainty. Factors that lead to this discrepancy are associated with the traverse movements as well as the size of the measurement volume, $50 \mu \mathrm{m}$. Consequently, it is necessary to have a more refined approach to finding the actual wall position. This is done by applying a least square fit to the theoretical mean velocity sublayer equation, as used by Olcmen and Simpson (1995) and Kuhl (2001),

$Q=C_{1} y+C_{2} y^{4}$

where $Q=\sqrt{U^{2}+W^{2}}$, and $\mathrm{C}_{1}$ and $\mathrm{C}_{2}$ are coefficients to be solved for. A curve is fit through $\mathrm{Q}=0$ at $\mathrm{y}=0$ using at least 4 points in the viscous sublayer $\mathrm{y}^{+}<10$. This fit was done in an iterative fashion by changing the y-shift value in order to maximize the correlation coefficient. All y-shifts that were obtained were on the order of the measurement volume diameter, $\pm 50 \mu \mathrm{m}$, which is the uncertainty in finding the wall. Nearly all y-shift values were in the range of 20-30 $\mu \mathrm{m}$. Using the curve fit equation, it is possible to calculate the friction velocity via the following relationship with the wall shearing stress, $\tau_{w} / \rho=v[\partial Q / \partial y]_{\text {wall }}=U_{\tau}^{2}$, where $C_{1} v=U_{\tau}^{2}$. When the skin friction velocity becomes zero, or even negative, the previous method of finding a y-shift value does not work. Consequently, in the near element measurement regions where there is separated flow and backflow an average y-shift value was used. As was discussed 
previously, all y-shift values obtained for the unseparated profiles were between 20 and 30 microns. Thus, an average value of 25 microns was used as the y-shift value for the near element profiles.

With all LDV systems it is impossible to achieve perfect alignment of the LDV coordinate system with the wind tunnel coordinate system. Consequently, it is important to perform a series of final rotations on the data sets. A slight rotation about the tunnel coordinate system $\mathrm{x}, \mathrm{y}$, and $\mathrm{z}$ axes (roll, yaw, and pitch angles) was performed on the two-dimensional mean flow data in order for it to reach minimum values within a set of tolerances. Final rotations of the data were always on the order of $1^{\circ}$. For more information on these rotations, see Kuhl (2001).

\subsection{Quality of Flow Conditions}

\subsubsection{Spanwise Two-Dimensionality}

After the initial setup of the small boundary layer wind tunnel it was necessary to determine the two-dimensionality of the flow field. An initial series of pitot tube measurements were taken without any trip arrangement inserted into the tunnel. The sole purpose of these measurements was simply to indicate that there was a constant velocity core within the test section. Thus, the free-stream speed is not set exactly to the desired $27.5 \mathrm{~m} / \mathrm{s}$ during these tests. Pressure readings were taken with a calibrated Series 475 Mark III Digital Manometer by Dwyer with an uncertainty of \pm 0.01 inches of water. Figure 2.10 shows spanwise velocity profiles at a constant height of 2.23 inches (5.66 $\mathrm{cm})$ from the tunnel floor and a distance of 50.38 inches $(127.95 \mathrm{~cm})$ from the contraction exit. This height is the vertical center of the wind tunnel test section. Measurements, for this data set only, were taken starting at the starboard side wall $(\mathrm{z}=$ $0)$. All other data sets presented use the designated axis system with $z=0$ being the centerline of the wind tunnel. The profiles are very repeatable and show a constant velocity core of about $12 \mathrm{~cm}$. The profiles tend to follow a smooth curve except that some edge effects can be seen near both tunnel walls. LDV measurements are taken well inside these areas and therefore these regions will not affect the data in any way. 


\subsubsection{Trip Arrangement and Zero Pressure Gradient}

Previous research directly related to the present research has been conducted in Virginia Tech's Boundary Layer Wind Tunnel. Consequently, to obtain meaningful results that can be related to previous work it is imperative that the flow conditions be matched in Virginia Tech's Small Boundary Layer Wind Tunnel. In order to assist the production of the necessary turbulent boundary layer characteristics, the first $45.72 \mathrm{~cm}$ of the test section floor were modified. A $0.32 \mathrm{~cm}$ square rod was placed directly at the contraction exit followed by another $0.32 \mathrm{~cm}$ square rod $5.40 \mathrm{~cm}$ downstream of the contraction exit. Silicon carbide, 20 grit sandpaper with a width of $20.32 \mathrm{~cm}$, manufactured by Norton, was inserted everywhere on the first $45.72 \mathrm{~cm}$ of the test section floor not occupied by a square rod. The placement of the second $0.32 \mathrm{~cm}$ square rod was determined by giving the flow sufficient distance to reattach before applying another step. Thus, with the discussed arrangement, a thicker boundary layer with a greater momentum deficit was created in order to obtain the necessary turbulent boundary layer characteristics for the particular tests. For more information on various trip arrangements and relevant data, please refer to Appendix A: Trip Arrangements.

Following the selection and insertion of the trip arrangement, a zero pressure gradient flow was obtained. The wind tunnel ceiling height was adjusted the entire length of the test section to ensure a constant velocity flow $\left[d U_{e} / d x=0 \therefore d p / d x=0\right]$. Velocity measurements were made at each of the port \#'s, see section 2.1.6, along the length of the test section. The ceiling height was adjusted until the velocity differences between each port was \pm 0.01 inches of water. The height of the ceiling was adjusted to obtain a zero pressure gradient flow for all single element tests as well as the fetch of Gaussian roughness. Ceiling height measurements as a function of streamwise, $\mathrm{x}$, distance from the contraction exit can be found in Table 2.2 below. 
Table 2.2: Ceiling height as a function of distance from contraction exit (for $\mathbf{d p} / \mathbf{d x}=\mathbf{0}$ flow)

\begin{tabular}{|c|c|c|c|}
\hline \multicolumn{2}{|c|}{ Single Elements } & \multicolumn{2}{|c|}{ Gaussian Fetch } \\
\hline $\begin{array}{c}\text { Ceiling } \\
\text { Height }(\mathrm{cm})\end{array}$ & $\begin{array}{c}\text { Distance from } \\
\text { Contraction Exit }(\mathrm{cm})\end{array}$ & $\begin{array}{c}\text { Ceiling } \\
\text { Height }(\mathrm{cm})\end{array}$ & $\begin{array}{c}\text { Distance from } \\
\text { Contraction Exit }(\mathrm{cm})\end{array}$ \\
\hline 10.87 & 49.53 & 10.72 & 49.53 \\
\hline 10.91 & 58.42 & 10.87 & 58.42 \\
\hline 10.95 & 73.66 & 11.11 & 73.66 \\
\hline 11.03 & 86.36 & 11.31 & 86.36 \\
\hline 11.15 & 96.52 & 11.35 & 96.52 \\
\hline 11.27 & 109.22 & 11.39 & 109.22 \\
\hline 11.35 & 124.46 & 11.47 & 124.46 \\
\hline 11.43 & 137.16 & 11.55 & 137.16 \\
\hline 11.55 & 165.1 & 11.67 & 165.1 \\
\hline 11.67 & 182.88 & 11.91 & 182.88 \\
\hline
\end{tabular}

\subsubsection{Profile Comparison and Boundary Layer Characteristics}

LDV boundary layer profiles were taken at various locations within the flow field to determine two-dimensionality and agreement with direct numerical simulation (DNS) data. Figure 2.11 shows profiles located at the center of the glass disc $(\mathrm{x}=116.72 \mathrm{~cm}, \mathrm{z}$ $=0 \mathrm{~cm}), 2.26 \mathrm{~cm}$ on either side of center, and $5.08 \mathrm{~cm}$ upstream of center. The different streamwise locations were taken because all axisymmetric elements were placed on the glass disc at the upstream location whereas the cube element was placed at the center of the glass disc. This different element location was to ensure that all of the desired LDV profiles could be taken for the elements within the glass disc. The spanwise, z-direction, locations for the boundary layer profiles were chosen to be outside of the bounds that LDV measurements would be taken on the roughness elements. Meaning that all LDV profiles associated with the roughness elements are taken within this spanwise range of $\pm 2.26 \mathrm{~cm}$. If two-dimensionality was satisfied at these locations as is shown in Figure 2.11 , then it is assumed to be satisfied within the bounds as well. The two sets of DNS data are for a turbulent boundary layer over a flat plate and are from Spalart (1988). Differences between all LDV profiles are small (less than 5\%) and are within the experimental uncertainties, see Appendix C. Therefore, the flow conditions are very near two-dimensional. 
The boundary layer characteristics for the single elements tested are as follows: $\mathrm{U}_{\mathrm{e}}=27.5$ $\mathrm{m} / \mathrm{s}, \operatorname{Re}_{\theta} \approx 7500, \delta=39.3 \mathrm{~mm}, \mathrm{H}=\delta^{*} / \theta=1.33$, and $U_{\tau}=0.95 \mathrm{~m} / \mathrm{s}$. Here $U_{\tau}$ is determined from Equation 2.1, $\theta$ from Equation A.1, and $\delta^{*}$ from Equation A.2. The boundary layer thickness, $\delta$, is defined as the distance from the wall where $\mathrm{U}=0.99 \mathrm{U}_{\mathrm{e}}$. The boundary layer characteristics for the fetch of Gaussian roughness are $U_{e}=27.5 \mathrm{~m} / \mathrm{s}, \operatorname{Re}_{\theta} \approx 10500$, $\delta=52.2 \mathrm{~mm}$, and $\mathrm{U}_{\tau}=1.43 \mathrm{~m} / \mathrm{s}$. All parameters are determined in a similar fashion as above except for the value of $U_{\tau}$. In the case of the Gaussian fetch, $U_{\tau}$ is defined via Equation D.1. Due to the fact that there are not any other DNS data sets available that have a higher and more representative $\mathrm{Re}_{\theta}$ value with which to compare to the current two-dimensional boundary layer data, the two DNS data sets from Spalart (1988) are used. Thus, the difference between the DNS profiles and the experimental profiles is attributed to the large difference in the $\mathrm{Re}_{\theta}$ values. All profiles, of the mean streamwise velocity (U), Reynolds normal stresses $\left(\overline{u^{2}}, \overline{v^{2}}, \overline{w^{2}}\right)$, and Reynolds shear stress $(\overline{u v})$, do show the same trends as the DNS data. It is known from AGARD (1996) that differences in the $\operatorname{Re}_{\theta}$ have an effect on the peak values of the Reynolds stresses. Thus it is not expected that the experimental profiles match exactly with the DNS profiles due to the large difference in the value of $\operatorname{Re}_{\theta}$. The very small differences between the LDV profiles themselves, along with the same trends as DNS data, give confidence in the quality of the flow within the wind tunnel.

\subsection{Description of Roughness Elements Tested}

\subsubsection{Single Elements}

All single elements tested were machined from aluminum and made in the Virginia Tech Aerospace and Ocean Engineering machine shop. All elements were machined so as to have a post slightly less than $1 / 16$ inches $(0.159 \mathrm{~cm})$ in diameter protruding from the bottom of the element. This post was $1 / 8$ inches $(0.318 \mathrm{~cm})$ long in order to secure it to the glass disc. The post at this diameter is necessary to leave adequate room for the glue used to secure it to the glass disc via a $1 / 16$ inch $(0.159 \mathrm{~cm})$ diameter hole. Seven single elements were tested in total and are listed here: cone, Gaussian bump, cone with spatial 
variations equal to the smallest sublayer structure length scale $\left(12 v / U_{\tau}\right)$ (hereafter fine grooved), cone with spatial variations equal to 2.5 times the smallest sublayer structure length scale (hereafter large grooved), hemisphere, cube aligned $90^{\circ}$ relative to the flow, and a cube aligned $45^{\circ}$ relative to the flow. The spatial variations of $\left(12 v / U_{\tau}\right)$ were chosen via an average value for the smallest turbulent length scale. This value has been shown by Jimenez and Moin (1991) to give sufficient resolution in order to resolve the turbulent scales. Jimenez and Moin showed a grid resolution in the spanwise and streamwise directions equal to $\Delta \mathrm{z}^{+}=5-10$ and $\Delta \mathrm{x}^{+}=8-16$. Figure 2.12 shows threedimensional drawings of each element and Figure 2.13 shows close-up views of the actual elements. Dimensions of these elements and other details are discussed below.

The cone has a height of 0.1 inches and a base diameter equal to 0.1 inches. The Gaussian bump has a height of 0.1 inches, a base diameter equal to 0.1 inches and has its profile defined by the following equation,

$y=0.1 \exp \left(-1843 z^{2}\right)$

where $\mathrm{y}$ is in the vertical direction and $\mathrm{z}$ is in the spanwise direction. The fine grooved element has the same frontal projected area as the cone, a height equal to 0.1 inches, and a base diameter equal to 0.106 inches. The large grooved element also has the same frontal projected area as the cone, a height of 0.1 inches, and a base diameter of 0.118 inches. The hemisphere is defined by a base diameter equal to 0.110 inches. Finally, the cube has an edge length of 0.068 inches. Element profile plots, showing the variation of element width with respect to element height, of the more complex elements (Gaussian, fine grooved, and large grooved) can be seen in Figure 2.14. Similarly, Table F.1 in Appendix $\mathrm{F}$ gives the $\mathrm{y}-\mathrm{z}$ coordinates that define the shape of both the large and fine grooved elements.

\subsubsection{Fetch of Gaussian Elements}

The fetch of Gaussian elements, shown in Figures 2.15 through 2.17, was constructed by means of a vacuum bagging technique. A specially made Gaussian shaped end-mill cutter with the same profile as described by Equation 2.2 was used to drill holes at a constant spacing of 0.216 inches into a 12 inch $\times 12$ inch x 1/8 inch Teflon mold. The 
spacing was determined to give a desired value of $\lambda=0.088$ where $[(\lambda=$ projected frontal area to flow)/(total surface area)]. Vent holes with a diameter equal to 0.005 inches were also drilled at the center of each Gaussian shaped cavity. These holes continue the rest of the way through the mold. The vent hole allows the escape of excess resin as well as air bubbles that can create voids.

A layer of vacuum bagging film was secured to a table top with duct tape. The mold was placed on this film and raised off of the vacuum bagging surface by metal shims that facilitate the escape of resin and air. A fiber compound was then placed over the mold (see note below). An epoxy mixture was used as the base resin for the elements. This mixture was poured over the mold and fiber compound. A layer of plastic film was then placed over the epoxy in order to create a protective interface between the vacuum bagging film and resin. Finally, the outermost layer of vacuum bagging film was secured to the table. All leaks and connections within the vacuum bagging setup were sealed with vacuum bagging sealant tape. The vacuum pump was left running until a sample of the epoxy resin was hard, a minimum of 5 hours. Once the resin hardened, the Gaussian fetch was removed from the mold. The 'spikes' created by the vent holes could easily be removed by brushing a ruler over the top of the fetch. The fetch was then cut into 8 inch $\mathrm{x} 12$ inch sections and placed in the wind tunnel via rubber cement. The fetch for the measurement region was cut into an 8 inch diameter circle. This circle was then aligned with the upstream and downstream fetches and secured to the glass disc using a thin layer of rubber cement. The fetch of roughness covered the tunnel floor from the end of the trip arrangement to $20.32 \mathrm{~cm}$ past the downstream edge of the glass disc.

The carbon-fiber substrate was used for the fetch everywhere on the tunnel floor except over the measurement volume. The measurement volume region utilized a thinner cheesecloth substrate. Various sections of this cheesecloth substrate needed to be removed in order for data acquisition to occur. A thin substrate was required in order for the 'lip' between the glass and the fetch to be minimized. 


\subsection{Alignment of LDV Head with Wind Tunnel and Roughness Elements}

Alignment of the LDV head with the roughness elements tested is absolutely essential in obtaining meaningful and repeatable results. Consequently, very vigilant care was taken with this aspect of the measurement process. First the LDV head and traverse system base had to be aligned with the tunnel axis. Following this procedure it was then possible to align the LDV head with the roughness elements. The following two procedures are discussed below.

\subsubsection{Alignment of LDV Head with Wind Tunnel}

The traverse system was designed and constructed to have three-orthogonal axes. Thus, it was necessary to align only one of these axes with the desired axis in the wind tunnel. The resolution of the traverse system in the horizontal ( $\mathrm{x}-\mathrm{z}$ plane) is $5 \mu \mathrm{m}$ whereas the resolution in the $\mathrm{y}$-direction is $\approx 2 \mu \mathrm{m}$. These fine resolutions give significant confidence in the final alignment and position of the traverse system. Refer to Figure 2.1 for a description of the wind tunnel axis system. The streamwise $\mathrm{x}$-axis was the axis chosen for the alignment of the traverse system and the wind tunnel. A 12 inch long 1/8 inch thick metal engineer's ruler was placed on the centerline of the wind tunnel over the glass disc measurement area. The measurement volume of the LDV head was then placed on the surface of the glass disc at the edge of the ruler. The LDV head was traversed in the $\mathrm{x}$-direction along the edge of the ruler over the entire measurement range. If the measurement volume strayed in any one direction over this range, the traverse base was moved and the procedure repeated. After a number of iterations it was possible to get the measurement volume to follow the centerline of the tunnel over the entire measurement range. A secondary check was made along the spanwise z-axis of the wind tunnel to ensure the orthogonality of the traverse system.

\subsubsection{Alignment of LDV Head with Roughness Elements}

\subsubsection{Single Elements}

All single elements tested were placed on a $20.32 \mathrm{~cm}$ diameter $0.64 \mathrm{~cm}$ thick piece of float glass through which LDV measurements could be taken. Each element was machined so as to have a post slightly less than $1 / 16$ inches $(0.159 \mathrm{~cm})$ in diameter 
protruding from the bottom of the element. This post was $1 / 8$ inches $(0.318 \mathrm{~cm})$ long in order to secure it to the glass. The float glass disc had a $1 / 16$ inch $(0.159 \mathrm{~cm})$ diameter hole drilled $1 / 8$ inches $(0.318 \mathrm{~cm})$ deep into it with a diamond coated glass drill bit from Precision Diamond Drills. The roughness element was then secured to the glass disc with super glue. All axisymmetric elements were placed 2 inches $(5.08 \mathrm{~cm})$ upstream of center on the glass disc. This extra downstream space was necessary in providing the LDV head access to all measurement locations. The cube element was placed at the center of the disc in order to facilitate the taking of measurements at the $90^{\circ}$ and $45^{\circ}$ orientations relative to the oncoming flow. As is discussed previously, boundary layer profiles were taken at both locations (center and $5.08 \mathrm{~cm}$ upstream) on the glass disc and there was no discernable difference between the boundary layer characteristics.

The alignment procedure for the cone, Gaussian, fine grooved, and large grooved element was identical. The measurement volume was placed at the surface of the glass and the LDV head was aligned along the centerline of the tunnel. With the LDV head aligned with the centerline of the tunnel, the probe was traversed in the vertical direction while moving the glass disc so the beams would be at the edges and center of the element. The LDV head was then traversed in the vertical direction until the measurement volume was at the height of the roughness element. Using laser goggles it was possible to follow the beams up the side of the element until it reached the peak. The glass disc was rotated in the tunnel in order to do the final alignment of the peak of the element with the measurement volume. By watching the beam profiles change along the sides of the element and the wall of the tunnel (when the measurement volume was higher than the element height) it was possible to have an uncertainty in positioning of \pm 60 microns in the $\mathrm{x}$ and $\mathrm{z}$ directions. After the element was aligned with the probe volume, the glass disc was taped to the wind tunnel floor ensuring that no positioning change occurred.

The hemisphere was aligned in a similar fashion as that described above. The only difference being that the probe volume could not be aligned with the element peak. The head was traversed back and forth along the base of the hemisphere and the glass disc rotated until the measurement volume was moved the same distance from the center of 
the tunnel to each spanwise edge of the element. A secondary check that ensured alignment was traversing the LDV head in the streamwise direction and checking that the distance from the spanwise located element center was also equidistant from the streamwise edges of the hemisphere.

The cube alignment method is as follows. With the glass disc outside of the tunnel, a fine scaled ruler (1/100 inch divisions) was used to find the center of one edge of the cube. A jeweler's eye loop was utilized for this procedure. Once the center of the cube was found, the ruler was taped to the glass disc. A line on the edge of the glass disc was then made with a permanent marker that signified the center of the cube's edge (hereafter the $90^{\circ}$ line). Using this $90^{\circ}$ line another line was drawn at an angle of $45^{\circ}$. The centerline of the tunnel was then marked and the glass disc inserted into the tunnel floor. After aligning the $90^{\circ}$ line on the disc with the tunnel centerline a series of secondary checks was performed. The measurement volume was traversed along the ruler's edge (note that the cube's edge is much too short to ensure proper alignment) ensuring that no deviation of the measurement volume could be detected from the edge of the ruler. Finally, the distance from the now known element center to each edge of the cube was checked to guarantee that the center of the element was known. If anything did not check out with the previous measurements and alignments, the entire process was repeated.

Alignment for the cube rotated $45^{\circ}$ relative to the flow consisted of rotating the glass disc until the $45^{\circ}$ line on the disc was aligned with the centerline of the wind tunnel. Secondary checks were again made to ensure that all corners were aligned with the tunnel coordinate system and equidistant from the center of the element.

Once the center of each roughness element was located, the traverse system zero location was determined. All measurement locations were relative to this center location.

\subsubsection{Fetch of Gaussian Elements}

The majority of the Gaussian roughness was made on a carbon-fiber substrate in sections that were 8 inches $(20.32 \mathrm{~cm})$ wide by 12 inches long. These sections were centered in the wind tunnel and rubber cemented to the floor. Measurements from each side of the 
roughness fetch to its respective tunnel wall were made to ensure that the fetch was centered in the test section. The roughness extended from the end of the trip arrangement to 8 inches $(20.32 \mathrm{~cm})$ past the end of the glass disc. Figure 2.16 shows a view with the wind tunnel ceiling removed looking upstream at the roughness fetch.

As is discussed previously, the fetch of Gaussian roughness that was made to cover the measurement region has a cheesecloth substrate. In order to obtain measurements from this setup, sections of cheesecloth had to be removed for the passage of the laser beams, see Figure 2.17. Please note that all sections that were removed upstream were replaced before taking measurements downstream. Very few Gaussian elements were missing from the fetch of roughness. The ones that were missing were far enough away from the measurement region in the upstream, downstream, and spanwise directions so as to not affect the obtained measurements. This conclusion was determined from previous results and experience done by George and Simpson (2002 and 2004). A He-Ne laser located on the optical table was used to align the probe with the desired Gaussian element. The red beam coming from the laser was launched into the receiving optical fiber and thus directed out of the receiving lens and into the wind tunnel. It was then a matter of iteration to center the LDV head on the Gaussian element. The probe was traversed vertically until the red beam could be seen through the element. The probe was then traversed in the streamwise and spanwise directions until the center of the beam extending from the receiving lens was at the center of the peak of the Gaussian element. The traverse system zero location was defined and measurement locations were relative to the center of the element. 


\subsection{Chapter 2 Figures}

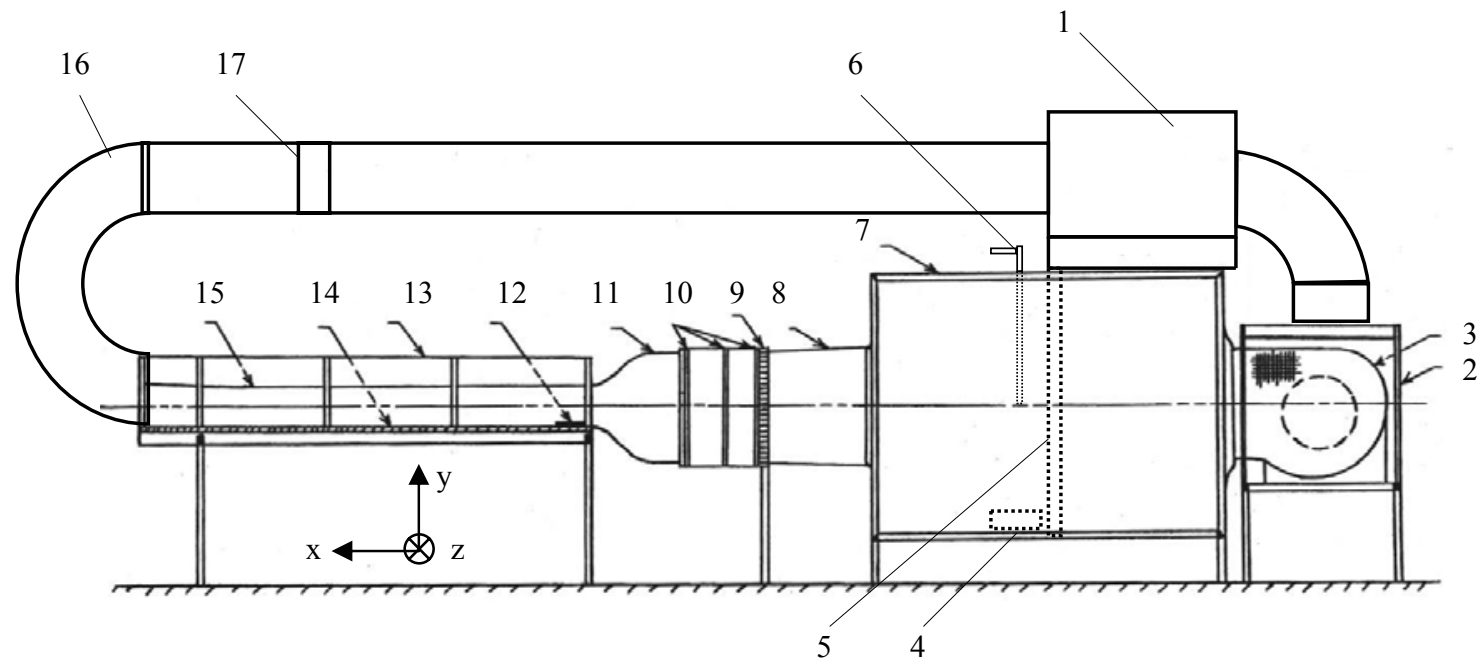

Figure 2.1: Schematic of small boundary layer wind tunnel setup (Adapted from

Smith, et al. 1990)

1. Air Conditioner

2. Filter Box

3. Blower

4. Drip Pan

5. Baffle Plate

6. Seeding Pipe

7. Plenum Chamber

8. Contraction

9. Honeycomb
10. Screens

11. Contraction

12. Trip Arrangement

13. Glass Side Walls

14. Bottom Wall

15. Top Wall

16. Return Ducting

17. Rubber Joint 


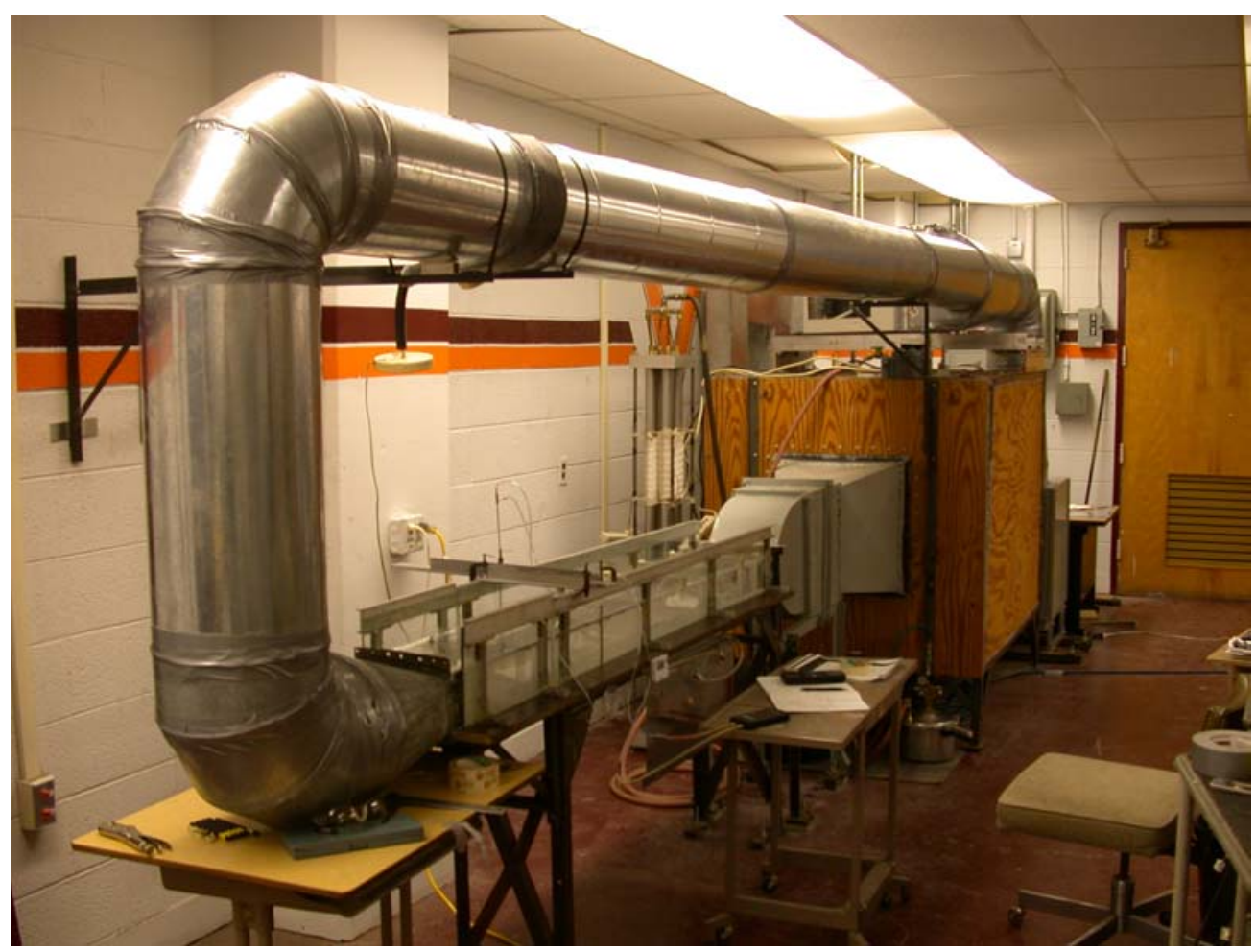

Figure 2.2: Small boundary layer wind tunnel setup (view from front)

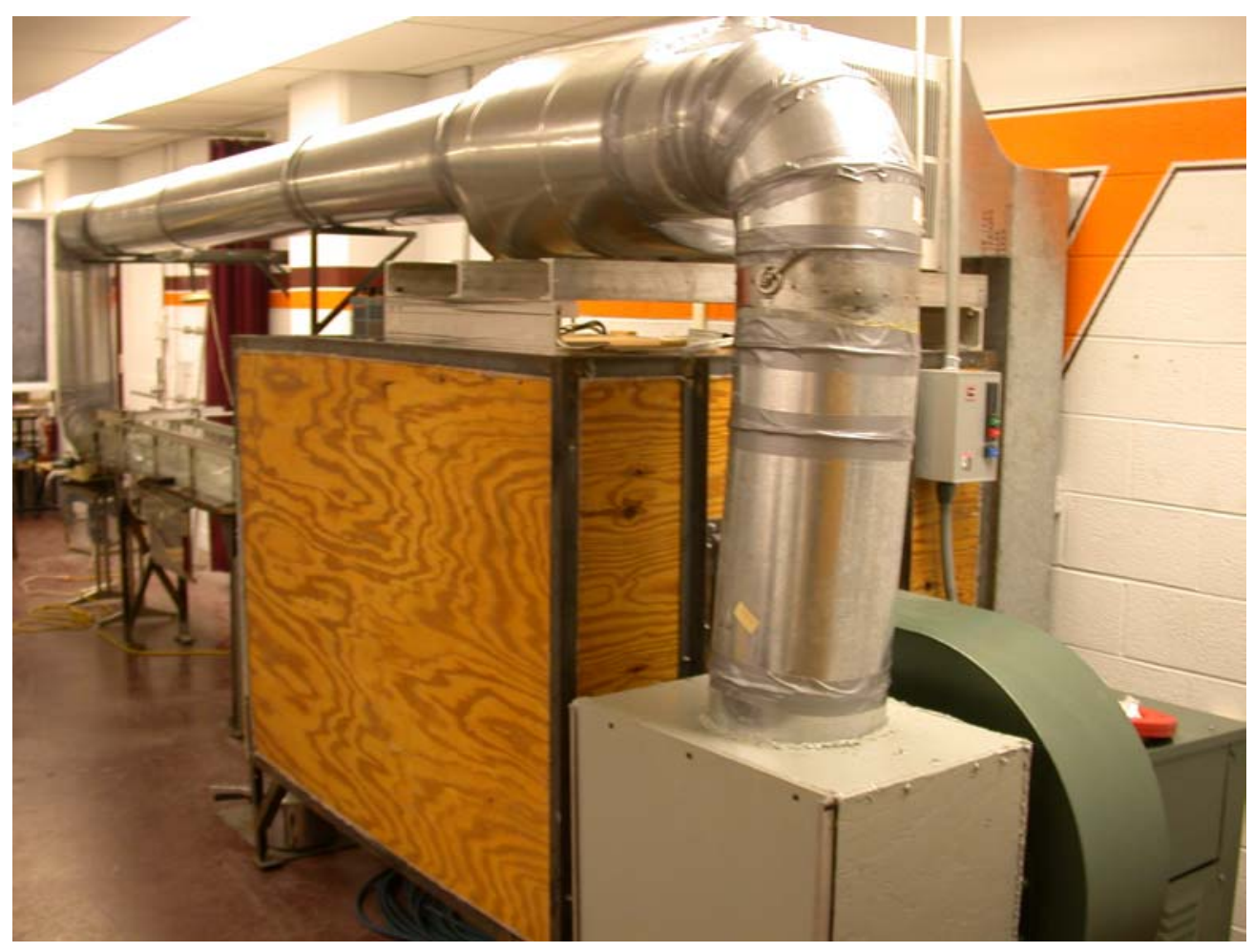

Figure 2.3: Small boundary layer wind tunnel setup (view from back) 


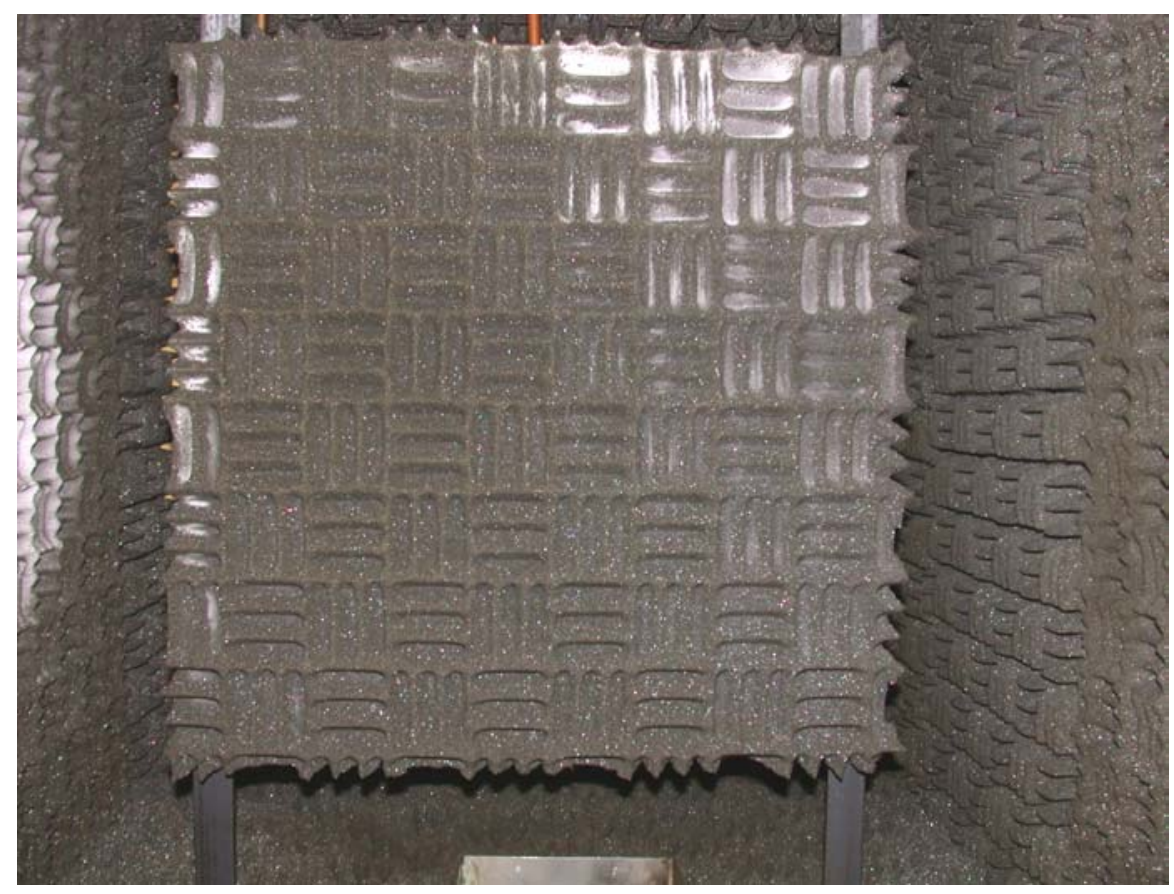

Figure 2.4: View of baffle plate in plenum chamber (looking from blower)

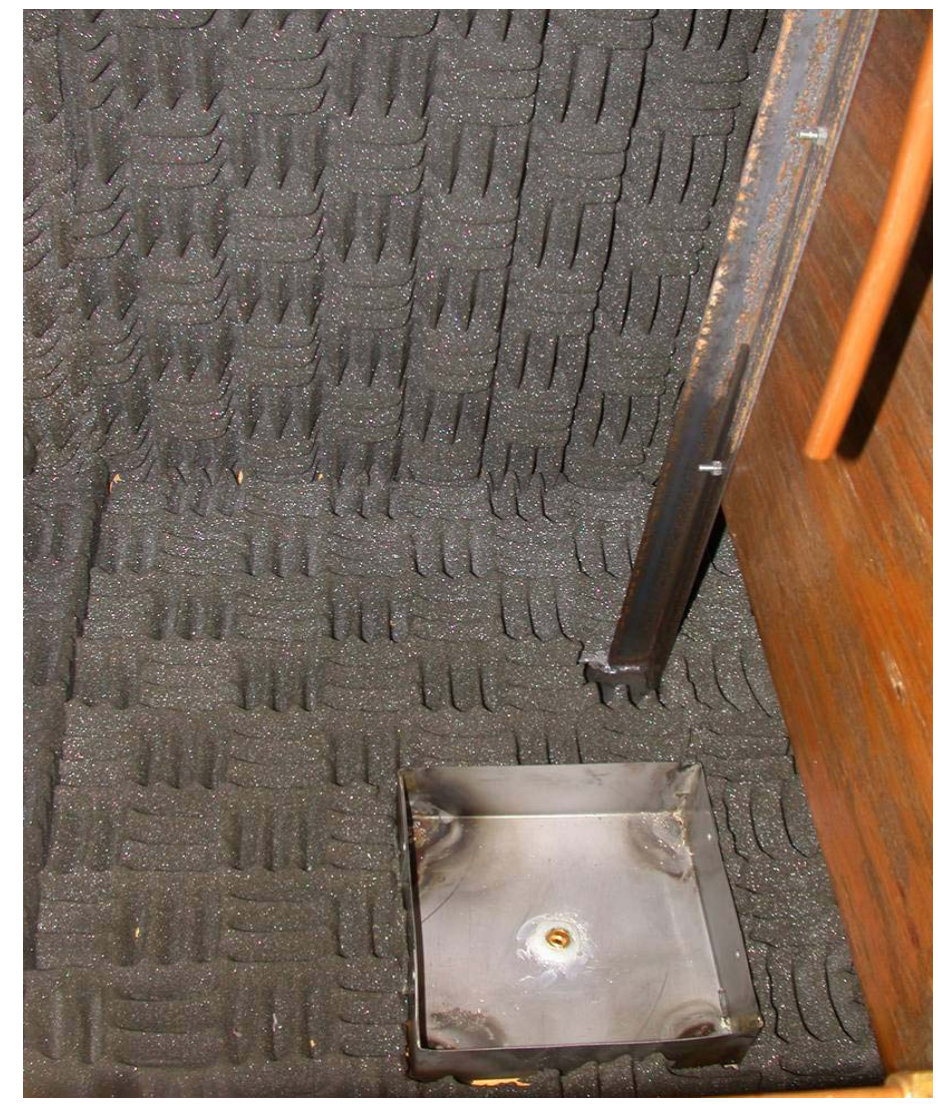

Figure 2.5: Seeding tube and drip pan on downstream side of baffle plate 


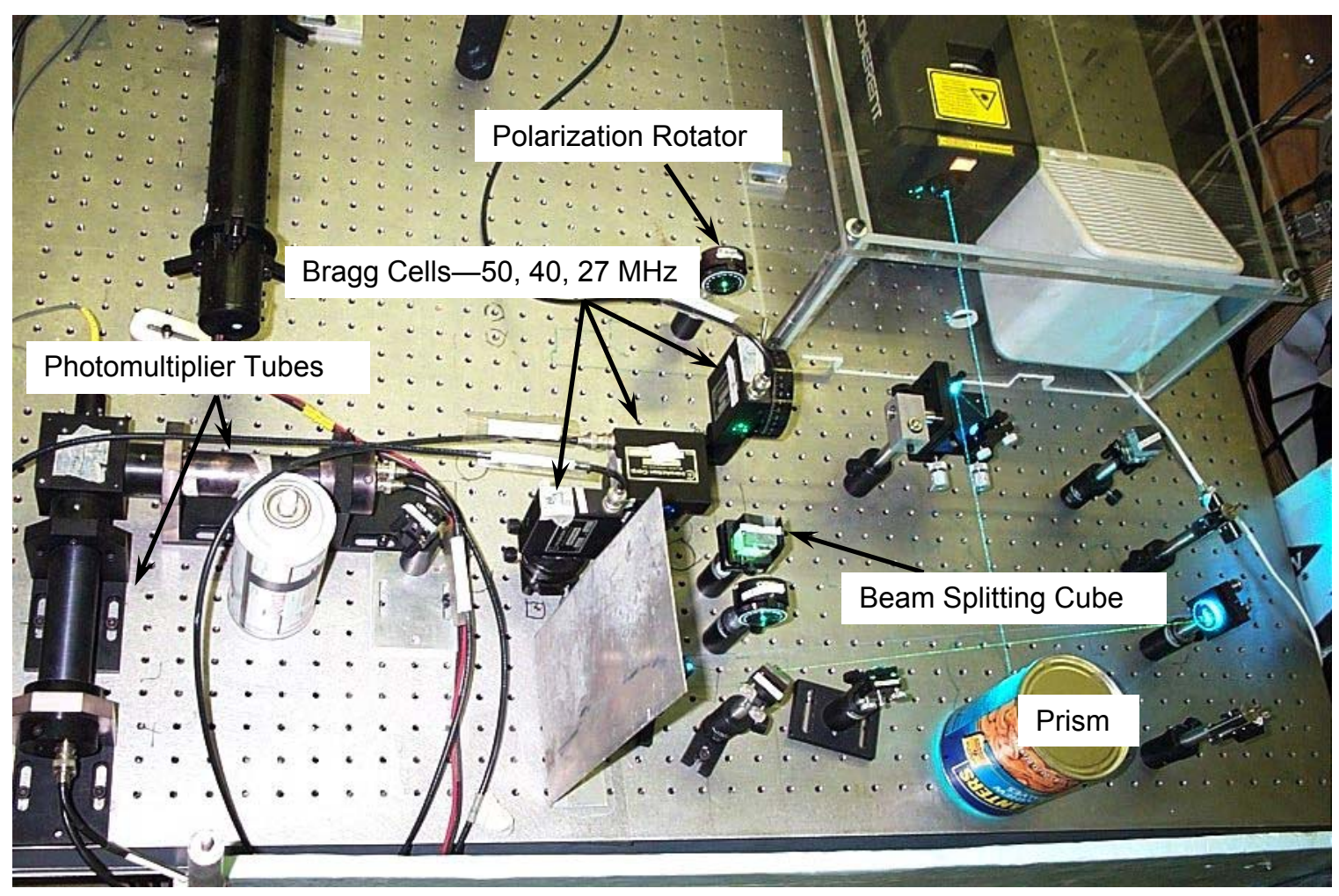

Figure 2.6: Optical table set-up shown near laser exit

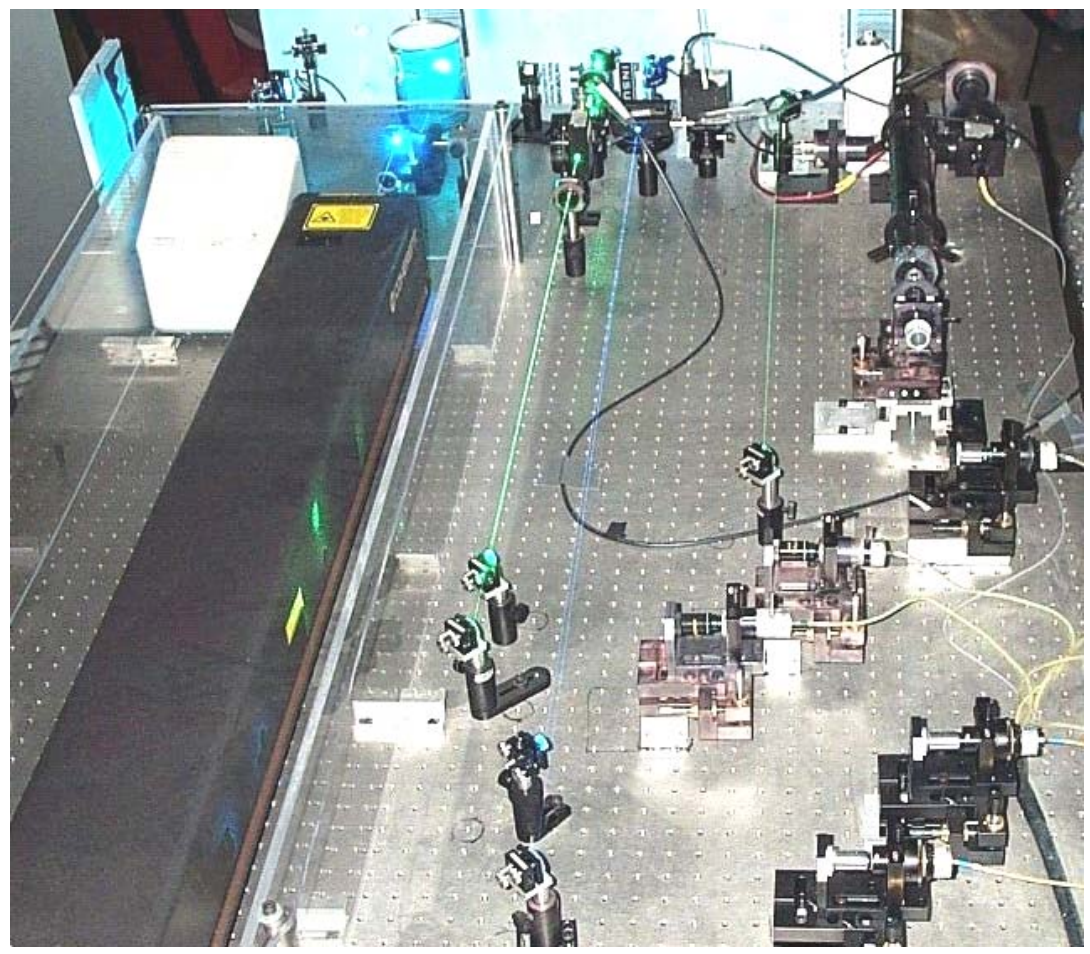

Figure 2.7: Full view of optical table 


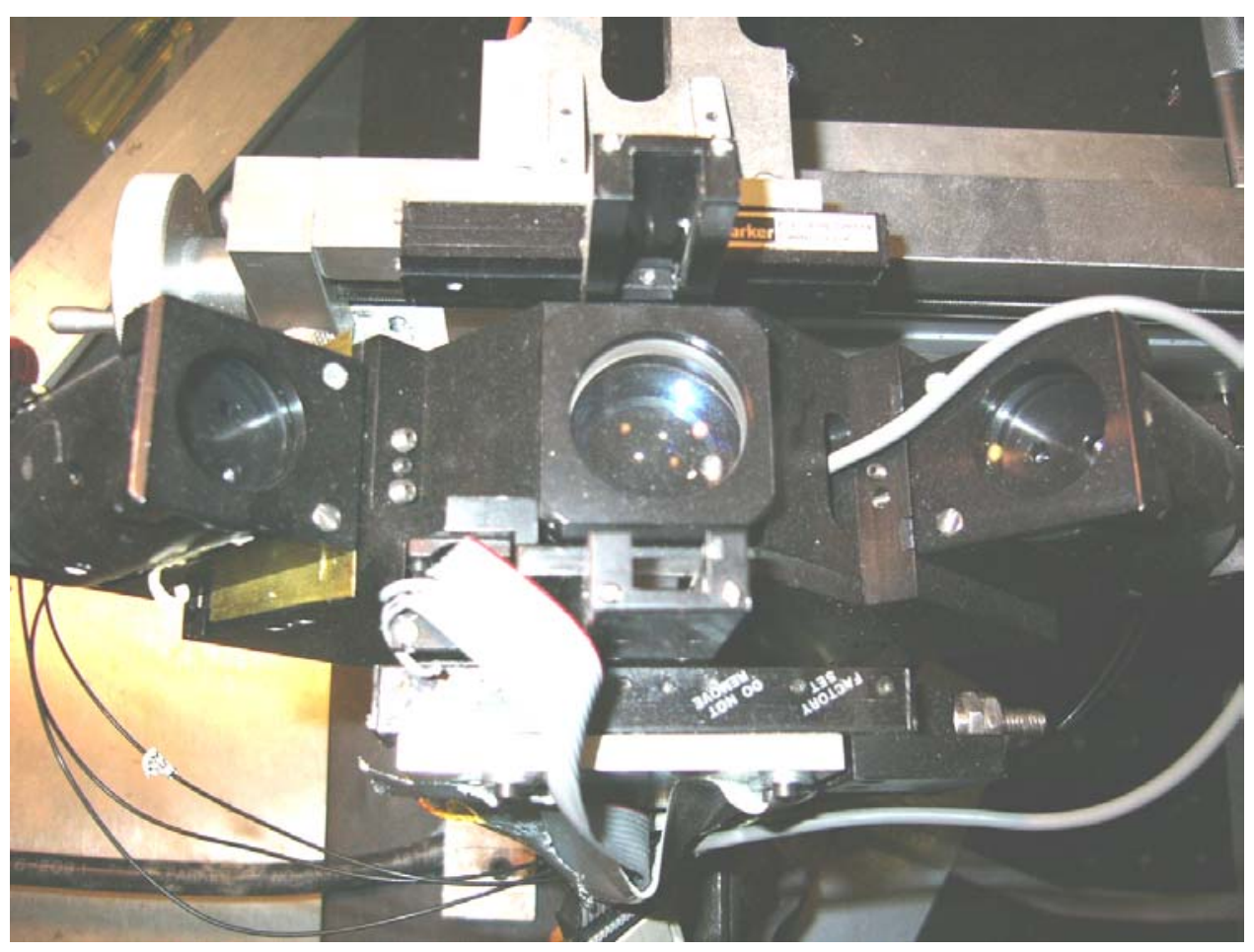

Figure 2.8: Close-up view of LDV head

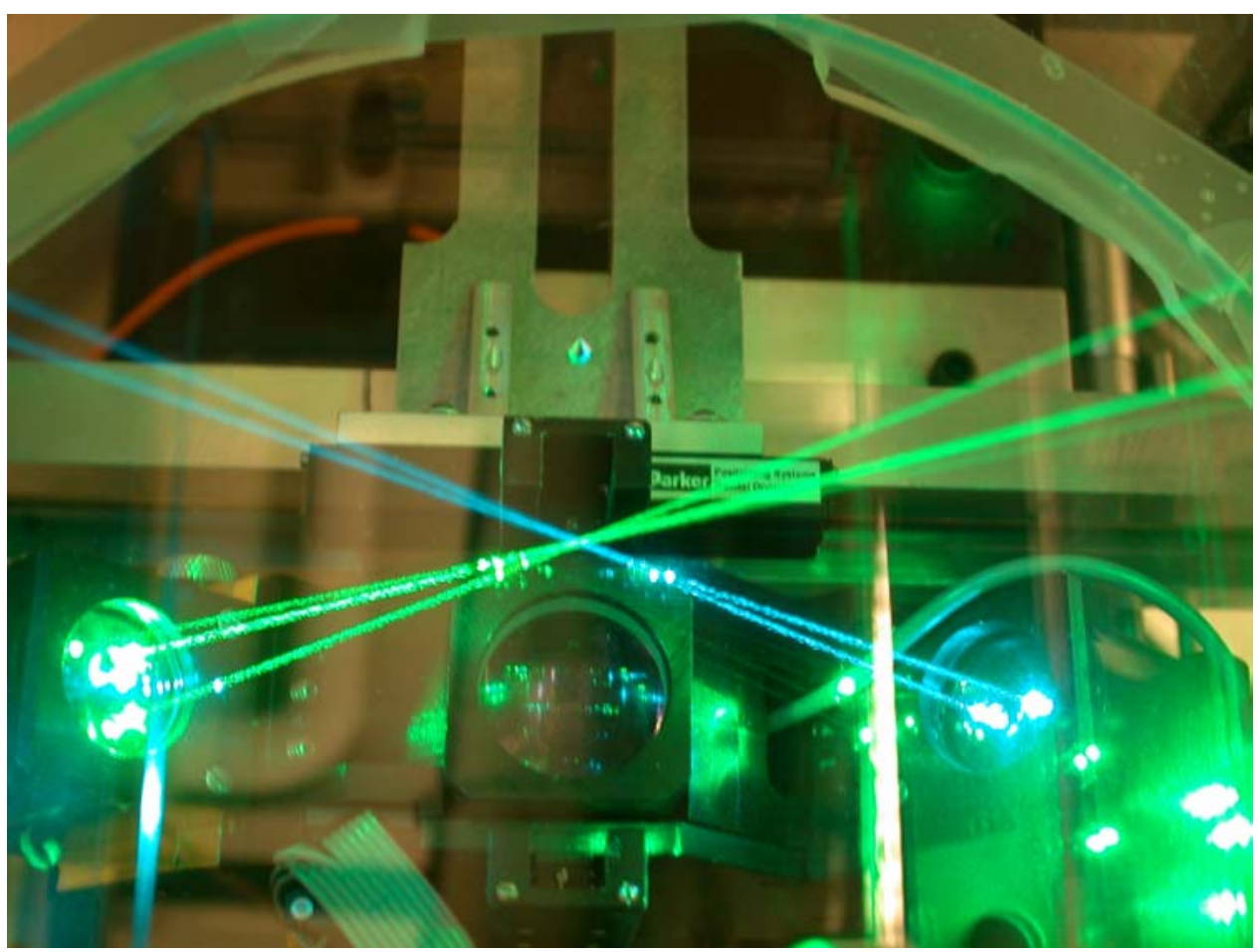

Figure 2.9: LDV head during data acquisition (view is above the tunnel ceiling looking upstream) 


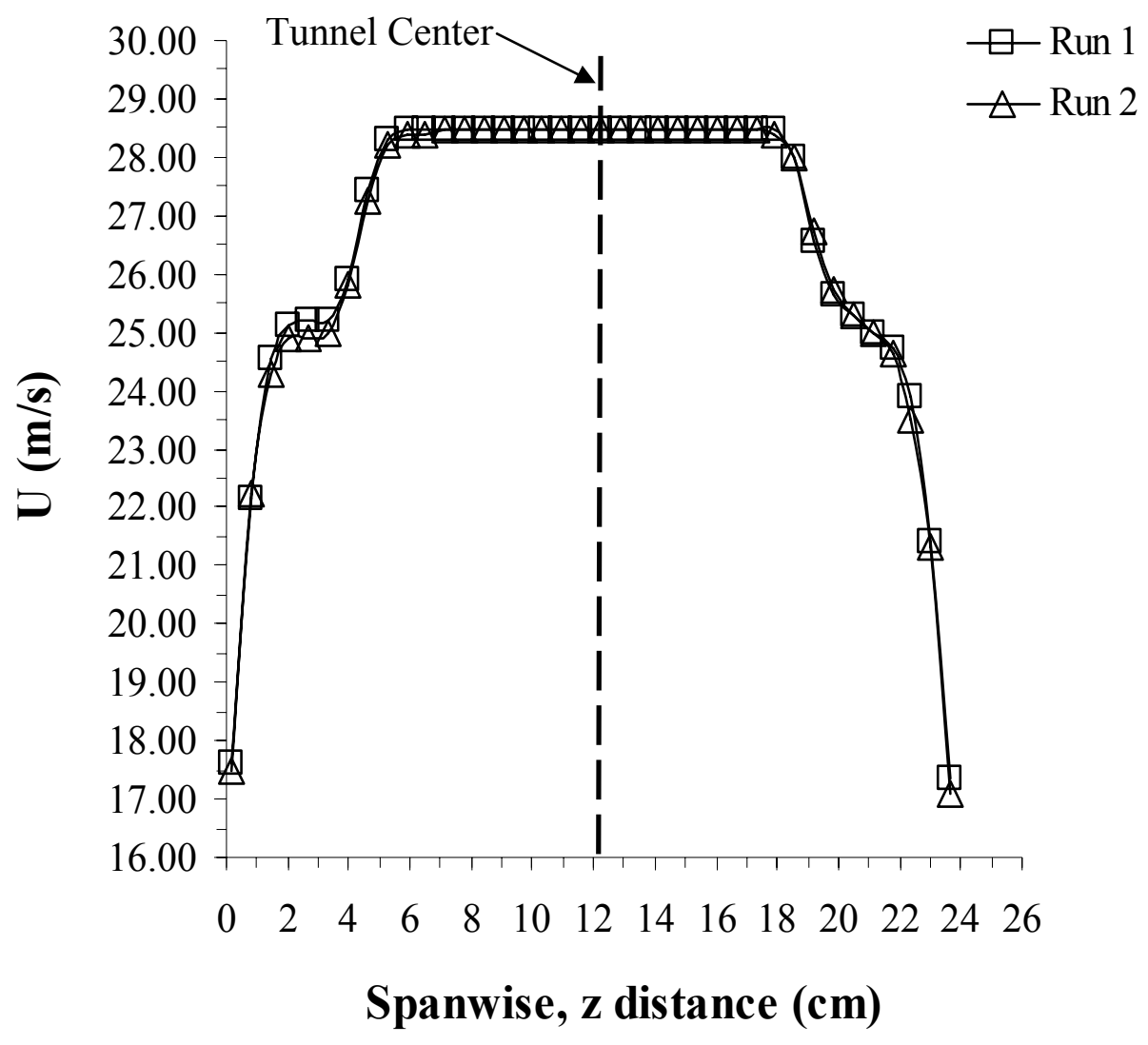

Figure 2.10: Spanwise velocity profile at center of wind tunnel 


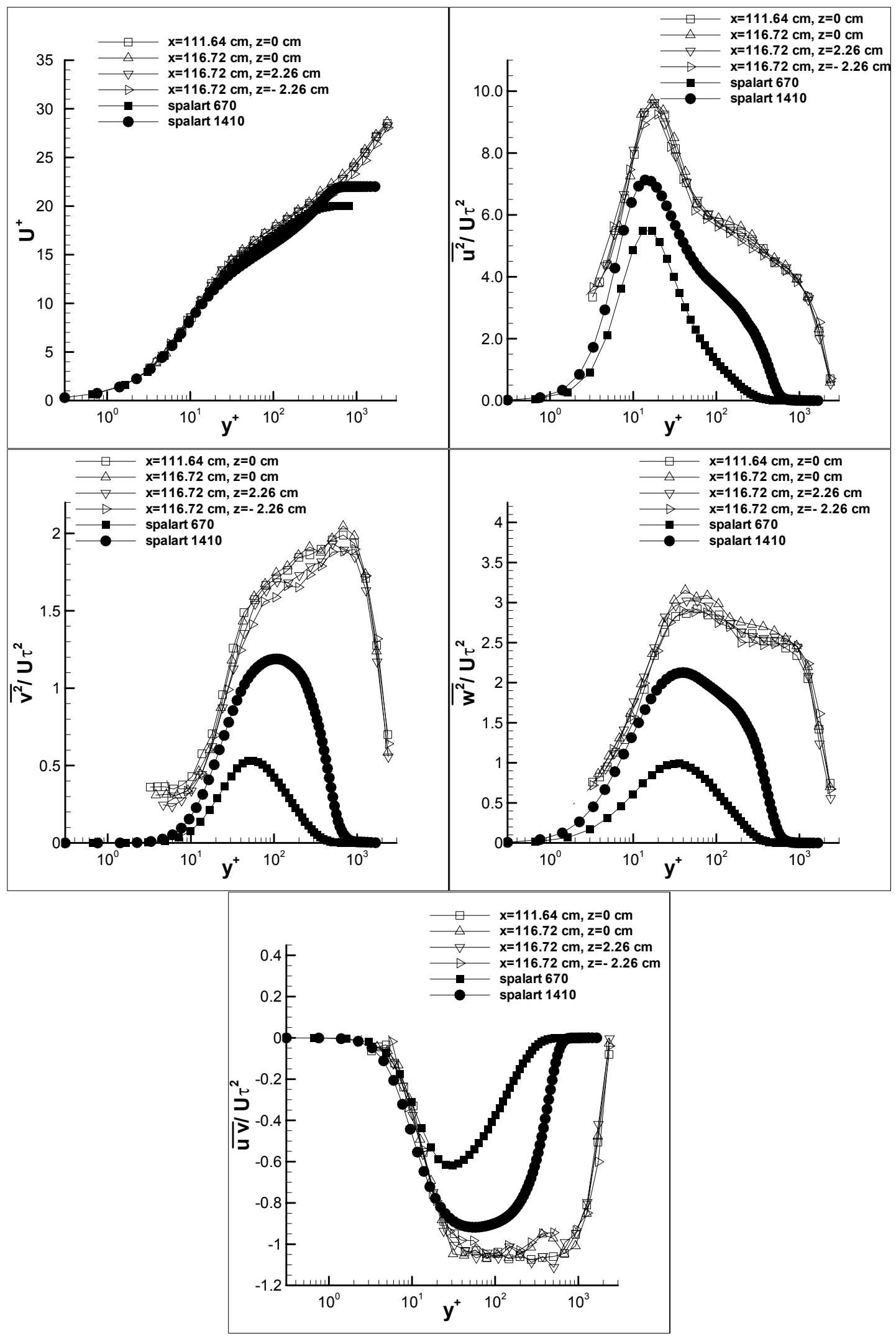

Figure 2.11: Comparison between profiles and DNS data $(x=$ distance from contraction exit, $z=$ spanwise distance from tunnel centerline) 


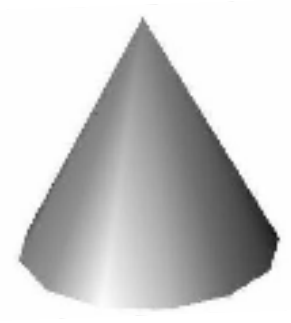

Cone

Height $=0.1 "$

Diameter $_{\text {Base }}=0.1 "$

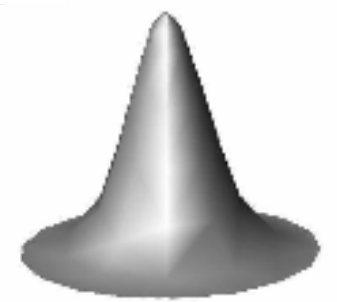

Gaussian Bump

Height $=0.1 "$

Diameter $_{\text {Base }}=0.1 "$

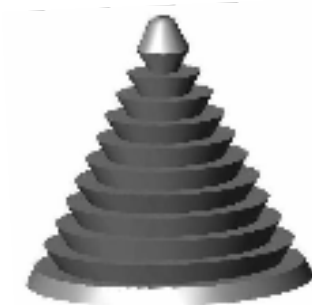

Groove Size $=1 \times$ Smallest

Sublayer Structure Length Scale

Same height and frontal area

as cone (fine grooved)

Groove $_{\text {height }}=0.0080 "$

Groove $_{\text {width }}=0.0086 "$

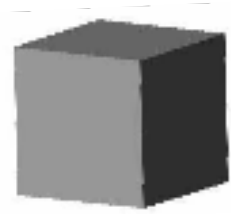

Cube

Length $_{\text {Edge }}=0.068 "$

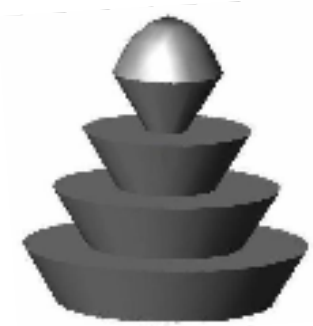

Groove Size $=2.5 \times$ Smallest

Sublayer Structure Length Scale

Same height and frontal area as cone (large grooved)

Groove $_{\text {height }}=0.020$ "

Groove $_{\text {width }}=0.022 "$

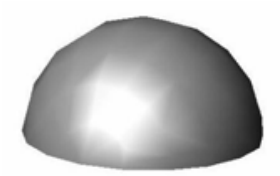

Hemisphere

Height $=0.055^{\prime \prime}$

Diameter $_{\text {Base }}=0.110$ "

Figure 2.12: Three-Dimensional drawings of single element cases 

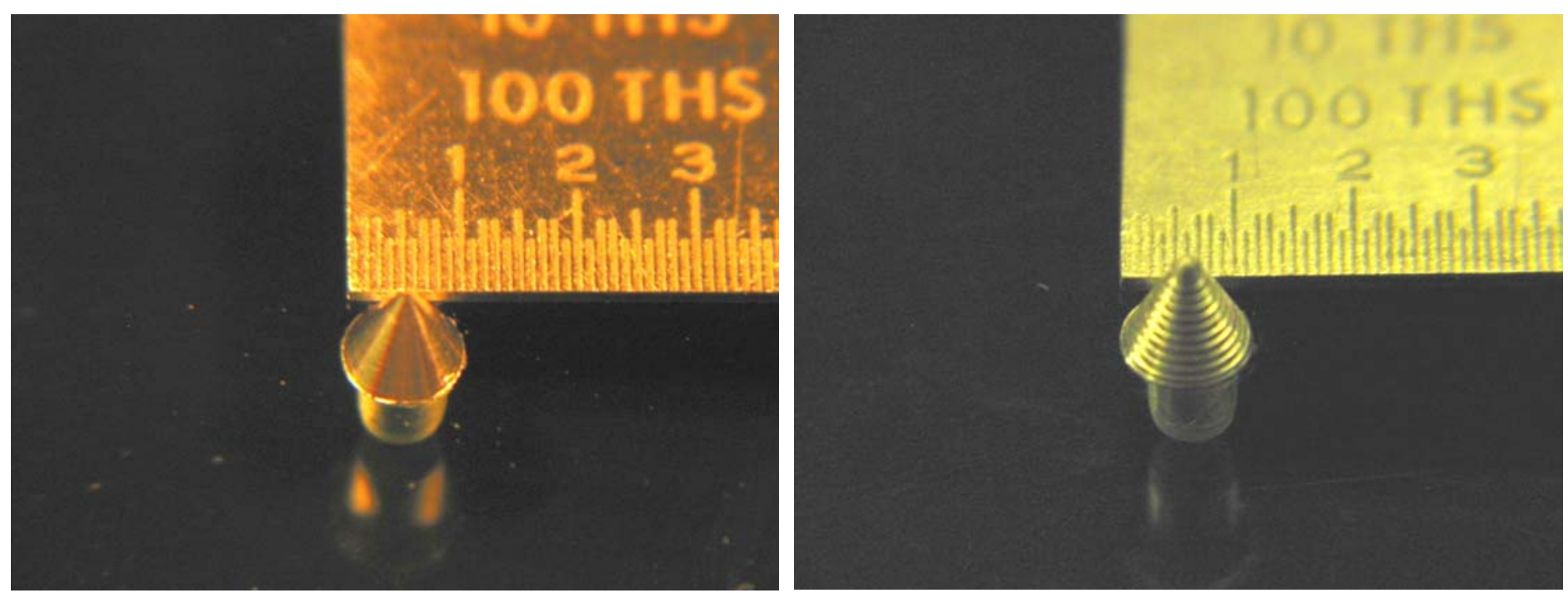

Cone Element

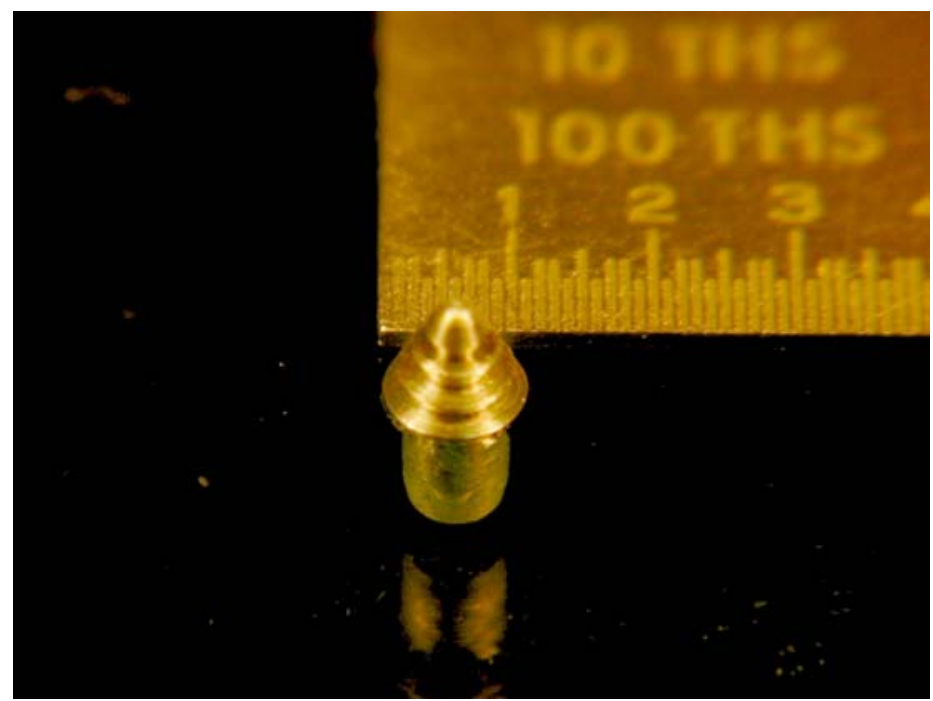

Large Grooved Element
Fine Grooved Element

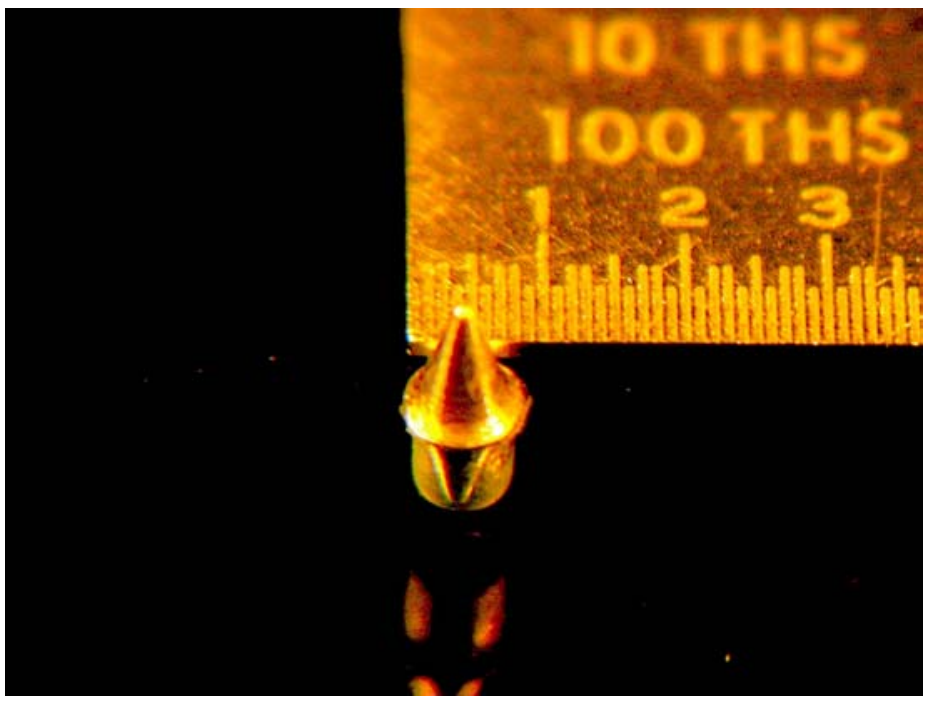

Gaussian Element

(cont.) 


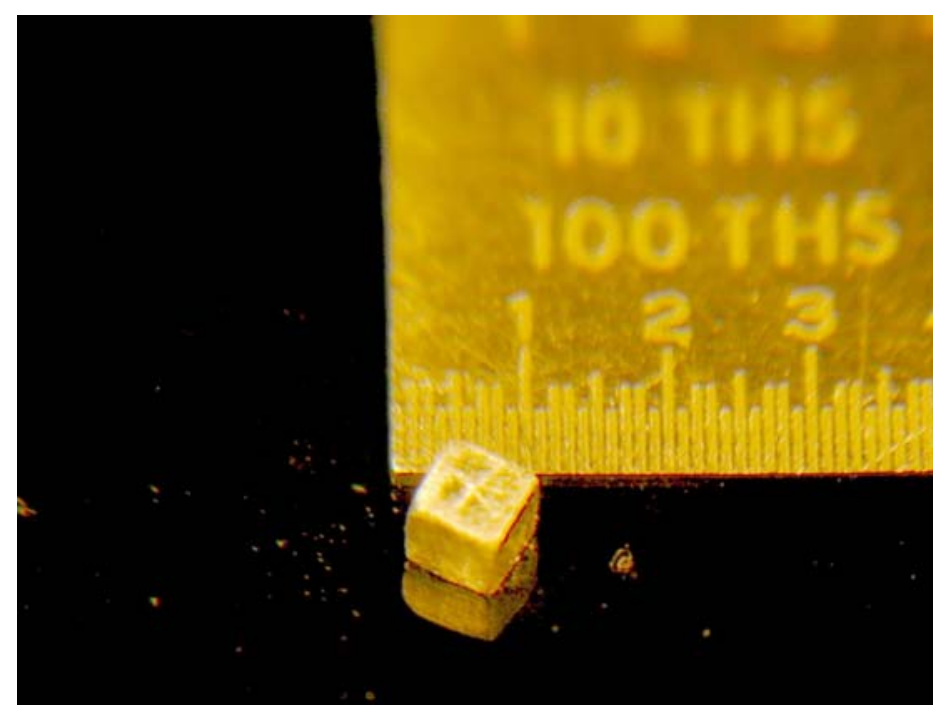

Cube Element

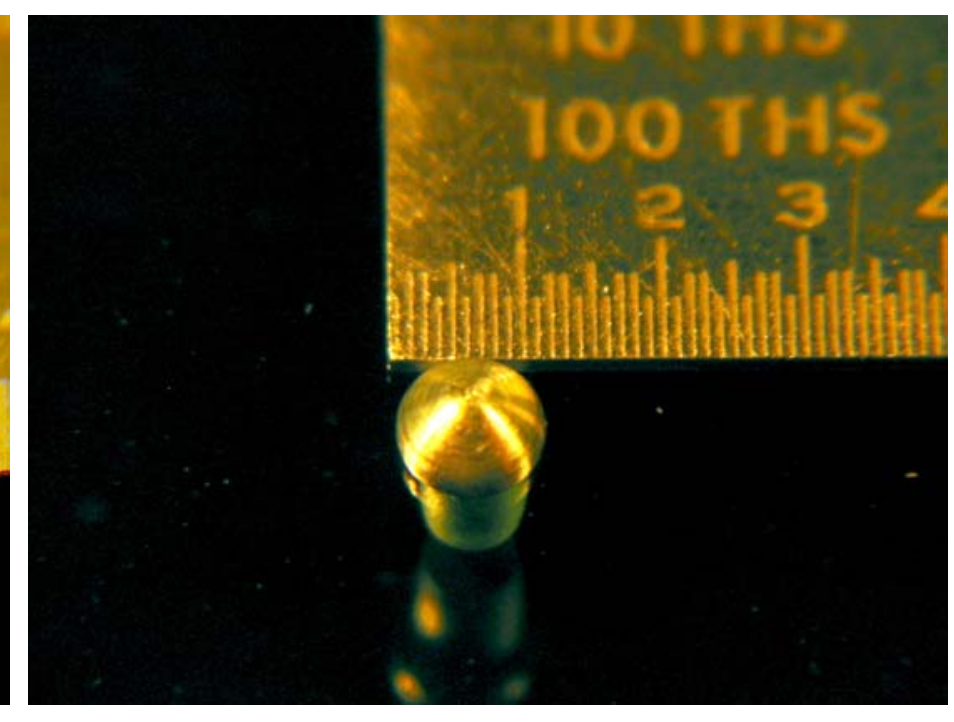

Hemisphere Element

Figure 2.13: Close-up views of single isolated roughness elements 

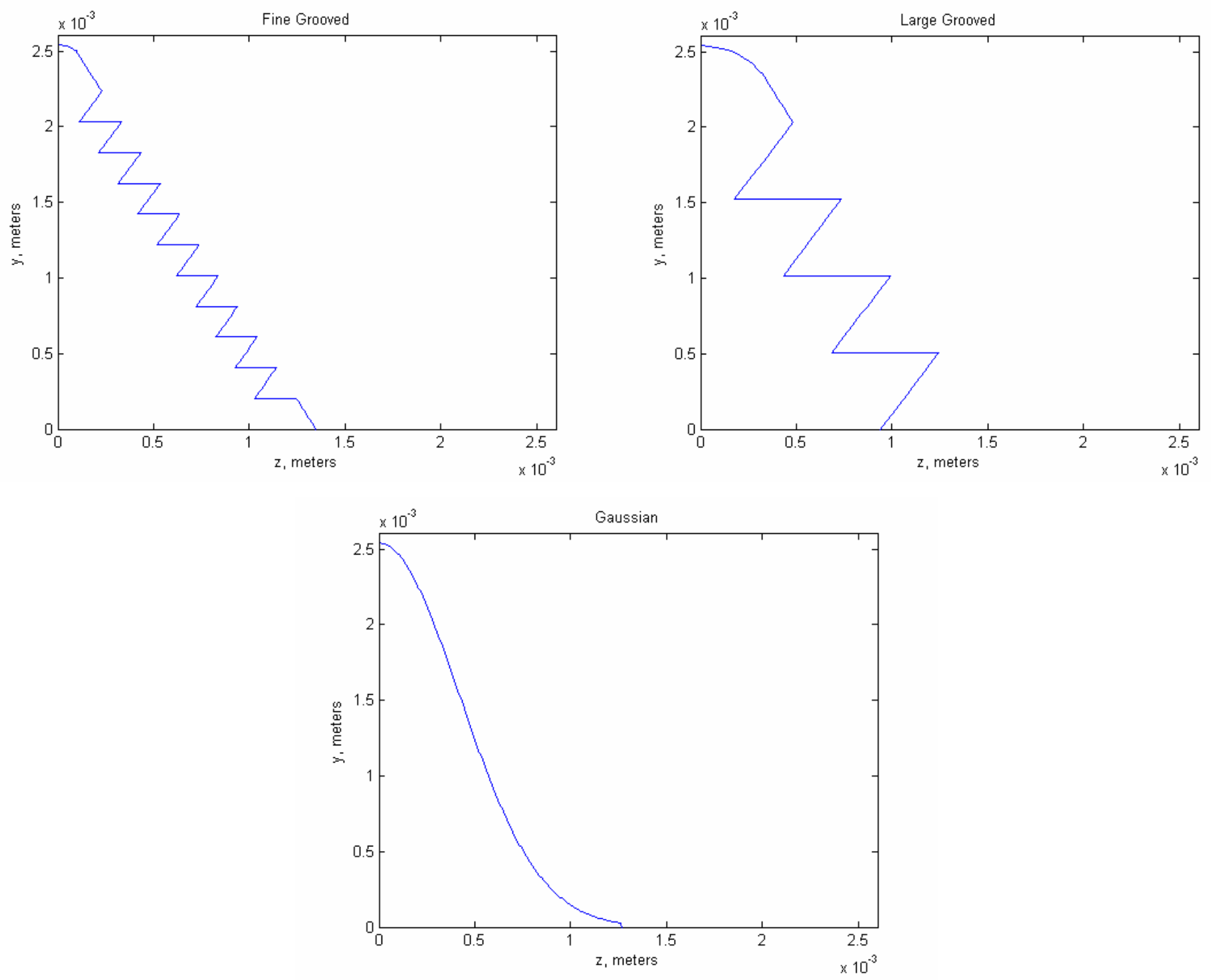

Figure 2.14: Element profile plots showing element width varying with respect to element height 


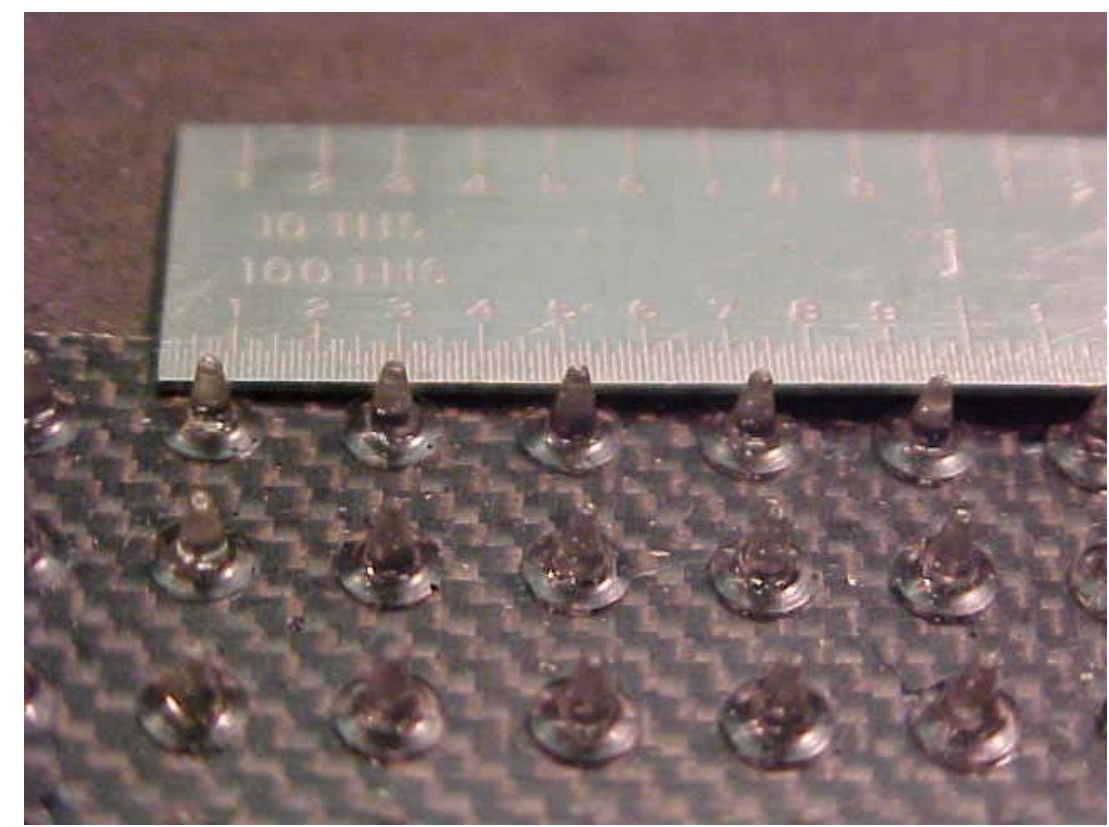

Figure 2.15: Close-up view of Gaussian roughness fetch (center-to-center spacing $=0.216$ inches)

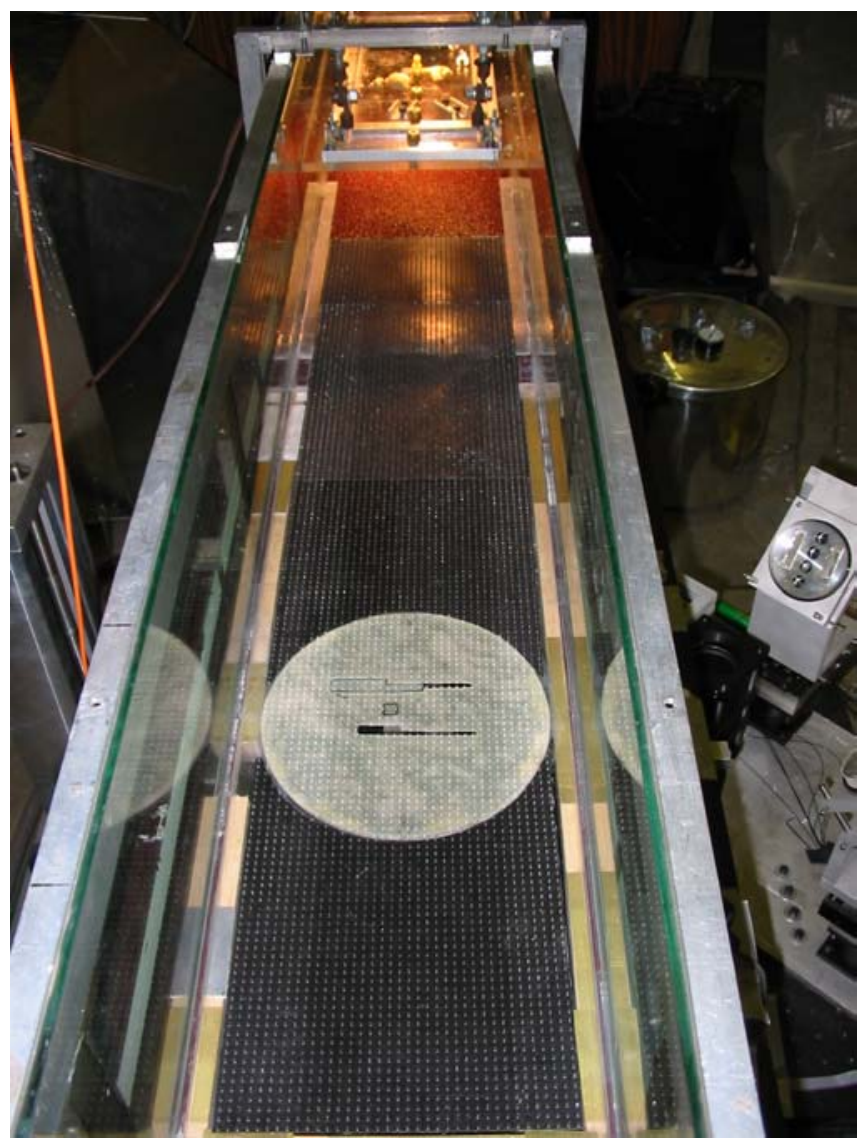

Figure 2.16: Top view of test section, looking upstream, with wind tunnel ceiling removed 


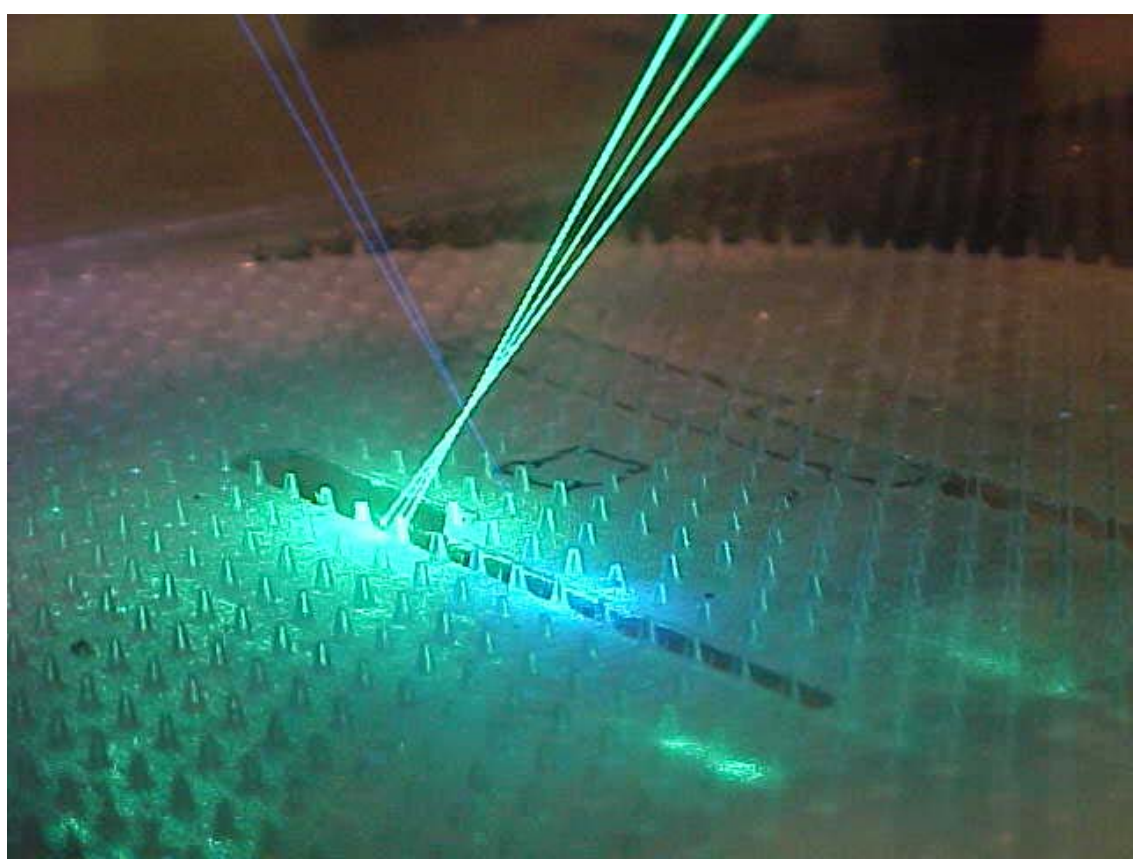

Figure 2.17: Data being acquired for the fetch of Gaussian roughness 


\section{Chapter 3 Results}

This chapter discusses the results of the data obtained within the analyzed flow fields of the single elements and Gaussian fetch of roughness. Comparisons between elements are made for all mean velocities, turbulence quantities, and skin friction. Roughness element comparisons can also be found for other fluid dynamic quantities derived from the previous parameters. Oil flow visualizations are also presented and described within this chapter. Please note that the spanwise normalization scheme uses the parameter (d). This value for all axisymmetric elements is the base diameter of the element. For brevity, this same parameter is the edge length of the cube (used to normalize the cube at $90^{\circ}$ ) and the diagonal of the cube (used to normalize the cube at $45^{\circ}$ ).

\subsection{Measurement Locations}

The twenty-six boundary layer profile measurement locations for the single elements can be seen in Figures 3.1 and 3.2. In an effort to keep the data and figures of the isolated roughness elements separate from the Gaussian fetch of roughness, the figures associated with the Gaussian fetch can be found following the figures related to the isolated roughness elements. As a result, the thirteen measurement locations for the fetch of Gaussian shaped roughness can be seen in Figure 3.113. Single point LDV measurements, 18 to 24 in number, were taken in logarithmically spaced increments at each shown profile location. All measurements were taken in the downstream flow field of the elements. The coordinate systems used are also defined in the figures listed above. The origin of the coordinate systems is located at the center of each roughness element under investigation. The $\mathrm{x}$-direction is taken to be along the centerline of the wind tunnel with $(+) \mathrm{x}$ being downstream of the element. The y-direction is normal to the wind tunnel floor (wall) where $(+) y$ is up from the floor. The coordinate system is completed following the right hand rule. Please note that all axisymmetric single elements were placed at a distance of $111.64 \mathrm{~cm}$ from the contraction exit and the cube elements were placed $116.72 \mathrm{~cm}$ from the contraction exit. There was no discernable difference in the boundary layer characteristics at these two locations (see section 2.4.3). 


\subsubsection{Single Element}

Centerline measurements were taken at six downstream $\mathrm{x} / \mathrm{d}$ locations in order to determine the distance the boundary layer needed to recover from the presence of the roughness element. These centerline locations are $\mathrm{x} / \mathrm{d}=1.36,2.75,5.0,10.0,20.0$, and 40.0. Please note that the last downstream measurement location for the cube at $45^{\circ}$ is located at $\mathrm{x} / \mathrm{d}=31.0$. This is due to insufficient room to traverse the LDV probe downstream and still take measurements through the glass disc. Two different planes of data were taken at $\mathrm{x} / \mathrm{d}$ locations of 2.75 and 10.0 for all elements except the fine grooved element. Data for the fine grooved element were taken at an $\mathrm{x} / \mathrm{d}=1.36$ plane. The $\mathrm{x} / \mathrm{d}=1.36$ plane was initially thought to provide further insight into the vortical structures emanating from the peak of the roughness element. All planes of data make it possible to determine contours of both measure and derived quantities. Hundreds of data points were used to formulate each grid used to make the contour plots. Measurements were mainly taken in the $-\mathrm{z}$ direction for symmetry reasons. One profile was taken in the $+\mathrm{z}$ direction at the centerline locations in order to assure that the flow was symmetric. Results for the single elements are shown with the reference smooth wall results. The smooth wall profile was taken at the location of the element without the element being present.

\subsubsection{Gaussian Fetch}

Boundary layer profiles for the Gaussian fetch of roughness are similar to those for the single element cases described previously. Three centerline locations, $\mathrm{x} / \mathrm{d}=0.79,1.08$, and 1.37, were taken downstream of the chosen roughness element. A plane of data at the $\mathrm{x} / \mathrm{d}=1.08$ location (halfway between elements) was again taken in order to develop contours of the measured and derived quantities. Two profiles were taken in the $+z$ direction at this plane location in order to assure flow symmetry. A profile directly between roughness elements was also taken at $\mathrm{x} / \mathrm{d}=2.16$ and $\mathrm{z} / \mathrm{d}=-1.08$. Finally a centerline profile at $\mathrm{x} / \mathrm{d}=1.08$ was taken at a location 8 cells downstream of the shown configuration. For data and a brief analysis related to the Gaussian fetch of roughness, see Appendix D. A more thorough analysis of this data set will be done by Stewart (2004). 


\subsection{Surface Oil Flow Visualizations}

The oil flow visualization technique helps to give a qualitative idea of what the global flow field looks like on the wall. The surface oil flow visualizations were done with a mixture of $15 \mathrm{ml}$ titanium dioxide, $40 \mathrm{ml}$ kerosene, and $1 \mathrm{ml}$ oleic acid. This mixture was brushed onto a piece of black contact paper that was attached to each element's respective glass disc. The tunnel was then turned on and left running until the mixture was dry. Digital pictures of the oil flows done for each element can be seen in Figures 3.3 and 3.4. The vertical lines shown in Figure 3.3 and Figure 3.4 are the streamwise locations where planes of data were obtained.

In front of all elements it is possible to see the flow separation and related formation of a horseshoe vortex. For each cube element there is evidence of high scouring, denoted by the black regions, directly behind the element. Consequently, this suggests the formation of an arch vortex which promotes backflow in the downstream regions near the element. This vortex looks to be similar in both cases of when the cube is rotated $45^{\circ}$ relative to the flow and when the cube is oriented at $90^{\circ}$ relative to the flow. It can also be seen that there is a significant region directly behind the cube elements where the wake region experiences a 'necking' down effect. In the close-up views of the elements, Figures 3.5 and 3.6, flow separation is easily noticed at the sharp edges of the cubes. There is a larger region of flow separation in the cube at $45^{\circ}$ as would be expected at this particular

orientation. Further downstream of the cube elements, lines of particle deposits can be seen. These deposits give rise to the conclusion that there is a significantly lower amount of wall shear in these areas as compared to the areas along the centerline of the element. Flow reattaches behind the arch vortex region as the accelerating fluid over the top and around the sides of each element converges toward one another near the centerline. A significant amount of scouring is also seen directly behind the hemisphere element. Fluid rushing over the top of the element impinges on the wall and causes the noticeable black region. Flow separation can also be seen on the back half of the hemisphere, indicated by the line of tracer particles extending downstream from the edges of the element. Again shear layer roll-up is found in front of the element which leads to the formation of a 
horseshoe vortex structure. The wake behind the hemisphere looks to be spreading in the spanwise direction fairly rapidly.

All peaked elements in Figure 3.4 seem to have a similar wake structure. The formation of a horseshoe vortex structure can be seen in front of the elements which in turn extends downstream of the elements. All elements have a region of flow separation and backflow directly behind the element followed by a region of particle deposits. Directly downstream of these deposits flow reattaches due to the fluid coming around the sides and over the peaks of the element to converge towards the floor. The wake behind the 'peaked' elements tends to be more of a wedge shape as compared to the cubes and hemisphere. Regions of tracer particles are also noticeable away from the centerline of the elements which again show a lower region of wall shear.

As discussed previously, away from the centerline in the spanwise z-direction there are noticeable deposits of tracer particles which indicate a region of lower shear as compared to the centerline. The regions of lower shear are attributed to less mixing due to the counter-rotating vortices creating a region of upwash. Whereas, in the high regions of shear there will be greater mixing due to the downwash of the counter-rotating vortex pair. There will also be some fluid acceleration along the centerline that will have a slight affect on the increase of wall shear. The presence of the wake can be seen in all elements to around ten diameters downstream. The axisymmetric elements' wakes tend to dissipate first followed by the cube at $90^{\circ}$ and then the cube at $45^{\circ}$. This phenomenon suggests that the strength of the vortex structure produced by the elements' presence in the turbulent boundary layer is significantly stronger in the cube at $45^{\circ}$.

\subsection{Mean Velocities}

Centerline profiles of the mean streamwise velocity are presented in non-dimensional form as $U / U_{\tau}$ vs. $y U_{\tau} / v$ in Figure 3.7. The value used for the skin friction velocity, $\mathrm{U}_{\tau}$, is from the approach boundary layer just upstream of a given element. Contours of the same mean velocity for $\mathrm{x} / \mathrm{d}$ locations of 2.75 (1.36 for the fine grooved cone) and 10.0 
can be seen in Figures 3.8 through 3.11. At the first measurement location of $\mathrm{x} / \mathrm{d}=1.36$, all elements have a backflow region present except for the Gaussian element. The largest magnitude of backflow can be seen in the cube elements. Here the cube at $45^{\circ}$ has a $\mathrm{U}^{+}$ value very near -7 . The fine grooved element and hemisphere are very comparable initially, but the hemisphere recovers to the reference smooth wall sooner due to its lower height. The cone and large grooved elements follow with still smaller values of backflow. It is reasonable to surmise that the large grooved element causes less blockage to the oncoming flow, due to its sizeable grooves, and in return has a smaller backflow region. All elements have a high velocity gradient near their respective heights as all profiles show a collapse to the reference 2D boundary layer above the element heights. Each element collapses in the order of its height except the cube at $45^{\circ}$. This element tends to a later collapse than the cube at $90^{\circ}$. A drastic change is seen in the profiles by $\mathrm{x} / \mathrm{d}=2.75$. At this location the separated flow downstream of all elements has reattached and the flow for most of the axisymmetric elements has collapsed to one another. The cube elements still have the most significant velocity deficit due to the amount of blockage and flow separation that they create. At $\mathrm{x} / \mathrm{d}=5.0$ the mean $U / U_{\tau}$ profiles for all elements have collapsed to one another except for the cube at $90^{\circ}$. Profiles for all elements collapse to the 2D reference boundary layer slightly above their height. The cube at $90^{\circ}$ is the only element that shows signs of a velocity defect at $\mathrm{x} / \mathrm{d}=10.0$, and by $\mathrm{x} / \mathrm{d}=20.0$ the profiles for all elements have collapsed to the reference boundary layer values. At $\mathrm{x} / \mathrm{d}=40.0$ there are no effects of the elements' presence in the boundary layer. The $\mathrm{U}^{+}$velocity contours in Figures 3.8 through 3.11 show the spanwise effect that the elements have on the oncoming flow. As is expected, the cubes show the most significant effect at the upstream location. By $\mathrm{x} / \mathrm{d}=10.0$ most discernable effects have dissipated for all elements. Please note that in all contour plots, crude outlines of the tested elements are shown. Due to the fact that the plots are in log scale, the outlines are not drawn to scale. These outlines are simply presented in order to give a general understanding of the height of each element with respect to the presented contours.

Centerline profiles of the mean normal-to-wall velocity are presented in non-dimensional form as $V / U_{\tau}$ vs. $y U_{\tau} / v$ in Figure 3.12. Contours of the same mean velocity for $\mathrm{x} / \mathrm{d}$ 
locations of 2.75 (1.36 for fine grooved) and 10.0 can be seen in Figures 3.13 through 3.16. At the first measurement location, $\mathrm{x} / \mathrm{d}=1.36$, there is an extremely significant amount of fluid being brought toward the wall in all cases. In all cases the peak value is reached at the roughness element height or slightly below. Most elements have a peak value at the forward most location of around $\mathrm{V}^{+} \approx-3.3$ with the cube at $90^{\circ}$ being significantly less at $\mathrm{V}^{+} \approx-1.9$. This large difference can be attributed to the increased blockage effects of the cube. There will be a region of separated and more stagnant flow directly behind the element. The profiles downstream of the elements do not collapse to the reference boundary layer until well above their heights. No effects can be seen above three element heights. At $\mathrm{x} / \mathrm{d}=2.75$ there is a very drastic difference in the behavior of the normal-to-wall mean velocity between elements. The cube at $45^{\circ}$ has a peak value of $\approx-3.4$ which is over twice the amount seen by any other element. This drastic difference is caused by the large downwash of fluid coming over the top of the element along with the strong horseshoe vortex structure bringing a large amount of fluid toward the wall. The cube at $90^{\circ}$ has the next largest magnitude followed by a close conglomeration of the peaked elements and finally the hemisphere follows with the least amount of normal-towall velocity. All elements present a noticeable peak that occurs below the height of the element.

The magnitudes of velocity continue to decrease at $\mathrm{x} / \mathrm{d}=5.0$ with the cube at $45^{\circ}$ still having the largest effect on the boundary layer. At $\mathrm{x} / \mathrm{d}=10.0$ the hemisphere has collapsed to the reference boundary layer and all elements, save the cube at $45^{\circ}$, have collapsed to each other. At $\mathrm{x} / \mathrm{d}=20.0$ and 40.0 the elements have collapsed back to the reference 2D boundary layer. As is evidenced by the contour plots in Figures 3.13 and 3.14 , the wall-ward rush of fluid is greatest near the centerline with a peak value slightly below the element height. The velocity magnitude decreases with spanwise distance from the centerline. The most extensive effects in the spanwise direction can be seen in the cubes followed by the cone, Gaussian, fine grooved (estimate due to measurement location), large grooved, and finally the hemisphere. A noticeable peak of normal-towall velocity can still be seen near the element height, albeit a small amount, in all elements except for the hemisphere. The streamwise decay of the $\mathrm{V}$ velocity is fairly 
drastic near the element. This decay smoothes out substantially as the streamwise distance is increased.

Contours of the mean normalized spanwise velocity component, $\mathrm{W}^{+}$, can be seen in Figures 3.17 through 3.20. These plots show a significant amount of velocity in the spanwise direction for the cube elements as compared to the peaked elements and the hemisphere. Flow symmetry can be confirmed by these plots, thus giving confidence to the quality of flow obtained for these extremely small roughness elements. More discussion concerning the spanwise velocity and related vorticity can be found in section 3.4.1.

\subsection{Derived Fluid Dynamic Quantities}

\subsubsection{Streamwise Vorticity}

In order to analyze the horseshoe vortex structure more thoroughly, contour plots of streamwise vorticity $\left(\Omega_{\mathrm{x}}\right)$ for all single elements are presented in Figures 3.21 through 3.24. The streamwise vorticity for all elements was calculated using Equation 3.1.

$\Omega=\operatorname{curl} \vec{V}=\left|\begin{array}{ccc}e_{x} & e_{y} & e_{z} \\ \partial / \partial x & \partial / \partial y & \partial / \partial z \\ U & V & W\end{array}\right| \therefore \Omega_{x}=\frac{\partial W}{\partial y}-\frac{\partial V}{\partial z}$

The boundary layer profiles used to calculate the derivatives involved with the streamwise vorticity were first interpolated to a fine spacing. This interpolation step is important in order to have sufficient resolution to perform numerical differentiation on the data. The contours of streamwise vorticity are in the $y-z$ plane at $x / d$ locations of 2.75 (1.36 for fine grooved) and 10.0. The vorticity is normalized by $\sqrt{A_{f}} / U_{\tau}$ where $\mathrm{A}_{\mathrm{f}}$ is the projected frontal area of each element. George and Simpson (2004) found that the length scale $\sqrt{A_{f}}$ was the most appropriate parameter to use to normalize the vorticity. The normalized secondary flow vectors $V / U_{\tau}$ and $W / U_{\tau}$ are also shown with the contours of streamwise vorticity. 
At the $\mathrm{x} / \mathrm{d}=2.75$ element locations, the secondary velocity vectors show a large amount of fluid being brought toward the wall by the counter-rotating horseshoe vortex structures. All elements at this location show a significant normal-to-wall (V) velocity at the centerline. These nearly perpendicular velocity vectors also show flow symmetry about the centerline plane. The velocity vectors curve sharply toward and away from the centerline near the height of the elements and at the wall, respectively. The flow shown near the wall is nearly parallel to the wall in the negative z-direction. The secondary velocity vectors for the fine grooved element at the $\mathrm{x} / \mathrm{d}=1.36$ plane show fluid being swept in around the sides of the element. At this location the horseshoe vortex structure has not yet taken over the secondary flow field. Therefore, the dominant secondary flow structure appears to be the flow coming around the sides of the element rather than the counter-rotating structure shown at the $\mathrm{x} / \mathrm{d}=2.75$ plane. The velocity vectors along the centerline directly behind the element are very small which also denotes that the horseshoe vortex structure has not yet started to bring large amounts of fluid toward the wall. The highest magnitude of secondary flow can be seen in the two cube elements, followed by the hemisphere, cone, Gaussian, and the large grooved element. As is evidenced by the $\mathrm{V}^{+}$plots discussed previously (Figure 3.12), the fine-grooved element's secondary flow at the $\mathrm{x} / \mathrm{d}=2.75$ plane is very near the magnitude of the Gaussian element. Further downstream at $\mathrm{x} / \mathrm{d}=10.0$, the secondary flow has lost a significant amount of strength as compared to the $\mathrm{x} / \mathrm{d}=2.75$ plane. The cube at $45^{\circ}$ shows the largest magnitude of secondary flow followed by the cube at $90^{\circ}$. All other elements' secondary flow vectors are very similar in magnitude. However, the fine-grooved element is slightly larger. Flow is still being directed toward and away from the centerline for all elements in a similar fashion as discussed previously. A noticeable difference in the secondary flow structure is the increase in distance away from the wall and centerline as compared to the upstream measurement plane. This incident is due to the induced velocity from the 'wall image vortex' on the actual horseshoe vortex structure.

Readily identifiable regions of positive streamwise vorticity can be seen at the upstream measurement plane for all elements. The cube elements have the highest pockets of vorticity as well as the most spanwise skewed contour lines. The presence of negative 
vorticity very close to the wall is also present at this measurement location. This nearwall opposite sign vorticity is due to the 'no slip' condition of the wall creating large velocity gradients in the spanwise, W, velocity. Furthermore, the presence of these negative regions originates from the convection of wall induced vorticity caused by the interaction of the horseshoe vortex at the wall. The negative regions of vorticity at the upstream measurement locations are smaller than and not as developed as the primary positive vortex structures. Although not as developed as the positive regions, these negative regions of vorticity impact the primary vortex by generating an exchange of vorticity that is created by near wall induced velocities. As the vortices propagate downstream to the $\mathrm{x} / \mathrm{d}=10.0$ plane, the negative regions of vorticity develop and become larger. As is shown in Figures 3.23 and 3.24, the size of the negative regions of vorticity are very close to the same size as their respective positive regions. At the downstream location, the negative vorticity is of greater magnitude than that of the positive vorticity. These negative vorticity values are comparable to the upstream positive vorticity values.

Following previous studies such as Wendt and Greber (1992), the location of the vortex center is taken to be the point of maximum streamwise vorticity, $\Omega_{\mathrm{x}_{-} \max }$. However, it is important to note that in general the $\mathrm{V}=\mathrm{W}=0$ location is not the same as the $\Omega_{\mathrm{x}_{-} \max }$ location. Locations of the vortex centers for each element can be found in Table 3.1 below. Also presented are the maximum vorticity, $\left(\Omega_{x} \sqrt{A_{f}} / U_{\tau}\right)_{\max }$, and streamwise velocity at the vortex center location. 
Table 3.1: Locations of maximum streamwise vorticity

\begin{tabular}{|c|c|c|c|c|c|c|c|c|c|c|}
\cline { 2 - 12 } \multicolumn{1}{c|}{} & \multicolumn{4}{c|}{$\mathbf{x} / \mathbf{d}=\mathbf{2 . 7 5}$ (fine grooved $\boldsymbol{x} / \mathbf{d}=1.36)$} & \multicolumn{5}{c|}{ x/d=10.0 for all elements } \\
\hline Element & $\mathrm{z}_{\mathrm{c}}^{+}$ & $\mathrm{y}_{\mathrm{c}}^{+}$ & $\mathrm{U}_{\mathrm{c}}^{+}$ & $\left(\frac{\Omega_{x} \sqrt{A_{f}}}{U_{\tau}}\right)$ & $\Omega_{\mathrm{x}}\left(\mathrm{s}^{-1}\right)$ & $\mathrm{z}_{\mathrm{c}}^{+}$ & $\mathrm{y}_{\mathrm{c}}^{+}$ & $\mathrm{U}_{\mathrm{c}}^{+}$ & $\left(\frac{\Omega_{x} \sqrt{A_{f}}}{U_{\tau}}\right)$ & $\Omega_{\mathrm{x}}\left(\mathrm{s}^{-1}\right)$ \\
\hline Cube at $45^{\circ}$ & -115.4 & 13.6 & 9.3 & 11.35 & 5263 & -154.4 & 44.0 & 14.2 & 2.46 & 1143 \\
\hline Cube at $90^{\circ}$ & -81.6 & 8.8 & 7.0 & 11.59 & 6394 & -136.7 & 32.9 & 12.9 & 1.63 & 899 \\
\hline Cone & -120.0 & 12.0 & 9.3 & 6.80 & 3607 & -200.7 & 45.7 & 14.8 & 0.68 & 361 \\
\hline Gaussian & -79.9 & 13.0 & 9.1 & 5.03 & 2940 & -160.7 & 44.5 & 14.4 & 0.68 & 398 \\
\hline Large Grooved & -142.6 & 13.9 & 9.5 & 4.44 & 2353 & -159.1 & 43.9 & 14.1 & 0.68 & 362 \\
\hline Hemisphere & -87.8 & 14.5 & 10.3 & 7.64 & 4159 & -221.5 & 57.9 & 16.0 & 0.73 & 395 \\
\hline Fine Grooved & -84.3 & 5.6 & 5.3 & 23.63 & 12533 & -170.4 & 43.7 & 14.2 & 0.99 & 527 \\
\hline
\end{tabular}

At the $\mathrm{x} / \mathrm{d}=2.75$ location, the horseshoe vortex structures are located $\approx 9-15$ wall units off of the wall and $\approx 80-143$ wall units from the centerline. In order to relate the location of maximum streamwise vorticity to the location of the sides of each element, the normalized half diameter values $\left(\mathrm{d}^{+} / 2\right)$ for each element are listed below. Each value is in the same order as its respective element shown in Table 3.1. The values of $\mathrm{d}^{+} / 2$ for each element are 69.6, 49.2, 72.4, 72.4, 85.5, 79.7, and 79.8, respectively. As can be seen in Table 3.1, the hemisphere and Gaussian elements show a location of maximum streamwise vorticity that is located very close to the half diameter location of each element. On the other hand, all of the other elements tend to push the point of $\Omega_{\mathrm{x}_{-} \max }$ further away from the centerline. For the fine grooved element at $\mathrm{x} / \mathrm{d}=1.36$ the vortex structure is located $\approx 6$ units off of the wall and $\approx 84$ units away from the centerline. The vortex structure produced by the cube at $90^{\circ}$ is centered the closest to the wall whereas the structure produced by the hemisphere is the farthest from the wall. The maximum value of streamwise vorticity at $\mathrm{x} / \mathrm{d}=2.75$ is seen in the cube elements. The cube at $90^{\circ}$ shows the largest magnitude with the cube at $45^{\circ}$ slightly less. The rest of the elements tend to be fairly close to one another with the large grooved element having the smallest vorticity maximum.

At the $\mathrm{x} / \mathrm{d}=10.0$ location, the vortex structures produced by the cube elements still maintain a significant amount of strength, whereas all other elements' vortex structures 
have dissipated greatly. These axisymmetric elements experience streamwise vorticity nearly an order of magnitude less than the upstream location. This tells us that due to the sharp edges on the cube elements, there is a significantly stronger vortex structure produced that has the ability to maintain its strength greater streamwise distances within the turbulent boundary layer. Due to the fact that the vortices produced by the elements are located very close to the wall (Table 3.1), viscous forces will have a major contribution in the decay of the vortices as they propagate downstream. Another aspect that is related to the diffusion and transport of the vortices is the entrainment of fluid from the boundary layer. As is given by Cutler and Bradshaw (1993b) and stated in George (2004), the equation governing the transport of streamwise vorticity is, $U \frac{\partial \Omega_{x}}{\partial x}+V \frac{\partial \Omega_{x}}{\partial y}+W \frac{\partial \Omega_{x}}{\partial z}=\Omega_{x} \frac{\partial U}{\partial x}+\Omega_{y} \frac{\partial U}{\partial y}+\Omega_{z} \frac{\partial U}{\partial z}+\left(\frac{\partial^{2}}{\partial y^{2}}-\frac{\partial^{2}}{\partial z^{2}}\right)(-\overline{v w})+\frac{\partial^{2}}{\partial y \partial z}\left(\overline{v^{2}}-\overline{w^{2}}\right)+v \nabla^{2} \Omega_{x}$

Terms 4 and 5 on the right hand side of this equation, the gradients of the normal and shear stresses, are the main contributors to the diffusion of the vortices.

Table 3.2 presents the change in location of the primary vortex structure as it proceeds downstream. The $\Delta \mathrm{z}_{\mathrm{c}}{ }^{+}$value is the normalized change of the peak $\Omega_{\mathrm{x}}$ vorticity location in the spanwise direction whereas the $\Delta \mathrm{y}_{\mathrm{c}}^{+}$value is the normalized change in the normal-towall direction. All values are differences from the upstream measurement plane $\mathrm{x} / \mathrm{d}=2.75$ (1.36 fine grooved) and the downstream $\mathrm{x} / \mathrm{d}=10.0$ measurement plane.

Table 3.2: Movement of primary vortex

\begin{tabular}{|c|c|c|}
\hline Element & $\Delta \mathrm{z}_{\mathrm{c}}^{+}$ & $\Delta \mathrm{y}_{\mathrm{c}}^{+}$ \\
\hline Cube at $45^{\circ}$ & -39.1 & 30.4 \\
\hline Cube at $90^{\circ}$ & -55.1 & 24.1 \\
\hline Cone & -80.7 & 33.7 \\
\hline Gaussian & -80.8 & 31.6 \\
\hline Large Grooved & -16.5 & 30.1 \\
\hline Hemisphere & -133.7 & 43.4 \\
\hline Fine Grooved & -86.2 & 38.1 \\
\hline
\end{tabular}


As the vortex structures proceed downstream, they diffuse and are transported away from the wall and centerline. The movement and strength of the vortex structure will be affected a great deal by the generation of secondary vorticity. In this region the near wall viscous forces will cause the loss of strength in the primary vortex. All vortex structures move a very comparable distance, $\approx 24-43$ wall units, in the $\mathrm{y}$-direction away from the wall. The vortex transport in the spanwise z-direction for all elements is not nearly as comparable as it was in the y-direction. The peaked elements' vortices all move a similar distance except for the large grooved element whose initial vortex structure was already a significant distance from the centerline at the upstream measurement location $(\mathrm{x} / \mathrm{d}=2.75)$. The cube elements also have a $\Delta \mathrm{z}_{\mathrm{c}}{ }^{+}$value that is reasonably comparable with respect to one another. The peak $\Omega_{\mathrm{x}}$ location produced by the cube at $45^{\circ}$ does not move as much in the spanwise direction as does the vortex produced by the cube at $90^{\circ}$. The peak location of $\Omega_{\mathrm{x}}$ for the hemisphere moves the greatest distance in the spanwise direction, as it is at least $55 \%$ greater than all other elements.

\subsubsection{Circulation}

The circulation, $\Gamma$, was calculated by integrating the velocity vector along a closed rectangular circuit in the $\mathrm{y}-\mathrm{z}$ plane using the following equation,

$$
\Gamma=\oint_{C} \vec{V} \cdot \overrightarrow{d s}
$$

where $\vec{V}$ is the velocity vector and $\overrightarrow{d s}$ is an element of the circuit C. The centerline $(\mathrm{z}=0)$ and the wall $(\mathrm{y}=0)$ are two sides of the circuit. The other two sides are formed by $\mathrm{y}=$ constant and $\mathrm{z}=$-constant values where the boundary layer shows no effect of the presence of the roughness element. Due to the fact that $\mathrm{W}=0$ at all planes along the path of integration (flow symmetry at $\mathrm{z}=0$, no-slip condition at the wall, and two-dimensional flow at the two remaining planes), the circulation is only dependent on the $\mathrm{V}$ velocity profile at the centerline. Table 3.3 gives the circulation of the vortex produced by each element at successive streamwise locations. This table is presented for raw comparisons between the respective roughness elements only. 
Cutler and Bradshaw (1986) have defined a non-dimensional parameter involving the circulation of a vortex as $\Gamma /\left(U_{e} \delta\right)$, where $\delta$ is the boundary layer thickness and $\mathrm{U}_{\mathrm{e}}$ refers to the free-stream speed of the flow. According to this notation, vortices in the range of 0.08 to 2 are considered weak. For the vortices generated by the elements under current investigation, the largest value obtained using the Cutler and Bradshaw notation is 0.009. Consequently, the vortices under scrutiny are much weaker than those defined by Cutler and Bradshaw. Even though the present vortices are an order of magnitude weaker than those defined as 'weak' by Cutler and Bradshaw, they still have a very significant effect on altering the structure of the turbulent boundary layer (especially in the near wall region).

Table 3.3: Circulation, $\Gamma$, as a function of streamwise distance, $x / d$

\begin{tabular}{|c|c|c|c|c|c|c|c|}
\cline { 2 - 9 } \multicolumn{1}{c|}{} & \multicolumn{9}{c|}{$\Gamma\left(\mathbf{m}^{2} / \mathbf{s}\right)$} \\
\hline Element & $\mathrm{x} / \mathrm{d}=1.36$ & $\mathrm{x} / \mathrm{d}=2.75$ & $\mathrm{x} / \mathrm{d}=5.0$ & $\mathrm{x} / \mathrm{d}=10.0$ & $\mathrm{x} / \mathrm{d}=20.0$ & $\mathrm{x} / \mathrm{d}=31.0$ & $\mathrm{x} / \mathrm{d}=40.0$ \\
\hline Cube at $45^{\circ}$ & $5.43 \mathrm{E}-03$ & $6.19 \mathrm{E}-03$ & $3.27 \mathrm{E}-03$ & $1.22 \mathrm{E}-03$ & $3.28 \mathrm{E}-04$ & ------- & N/A \\
\hline Cube at $90^{\circ}$ & $2.39 \mathrm{E}-03$ & $2.64 \mathrm{E}-03$ & $1.41 \mathrm{E}-03$ & $2.18 \mathrm{E}-04$ & ------- & N/A & ------ \\
\hline Cone & $9.67 \mathrm{E}-03$ & $6.53 \mathrm{E}-03$ & $1.82 \mathrm{E}-03$ & $8.53 \mathrm{E}-04$ & $4.23 \mathrm{E}-04$ & N/A & $2.57 \mathrm{E}-04$ \\
\hline Gaussian & $8.23 \mathrm{E}-03$ & $4.37 \mathrm{E}-03$ & $1.66 \mathrm{E}-03$ & $9.55 \mathrm{E}-04$ & $4.65 \mathrm{E}-04$ & N/A & $2.97 \mathrm{E}-04$ \\
\hline Large Grooved & $6.27 \mathrm{E}-03$ & $2.72 \mathrm{E}-03$ & $1.50 \mathrm{E}-03$ & $9.57 \mathrm{E}-04$ & $2.58 \mathrm{E}-04$ & N/A & $5.56 \mathrm{E}-05$ \\
\hline Hemisphere & $4.07 \mathrm{E}-03$ & $3.12 \mathrm{E}-03$ & $4.02 \mathrm{E}-04$ & $9.86 \mathrm{E}-05$ & $4.42 \mathrm{E}-05$ & N/A & ------- \\
\hline Fine Grooved & $5.18 \mathrm{E}-03$ & $2.92 \mathrm{E}-03$ & $1.67 \mathrm{E}-03$ & $6.77 \mathrm{E}-04$ & $3.38 \mathrm{E}-04$ & N/A & ------- \\
\hline
\end{tabular}

It is readily identifiable that the two elements with the 'sharpest' peaks (cone and Gaussian) have the highest amount of circulation at the $\mathrm{x} / \mathrm{d}=1.36$ location. By a downstream distance of $\mathrm{x} / \mathrm{d}=40.0$, the circulation has more or less dissipated for all elements. Please note that a dashed line in Table 3.3 refers to a circulation value of zero. In each of these instances, the local $\mathrm{V}$ velocity profile has collapsed to the reference $2 \mathrm{D}$ profile thus giving rise to a zero value of circulation. Figure 3.25 shows the circulation, $\Gamma$, non-dimensionalized by $U_{\tau} \sqrt{A_{f}}$ as a function of streamwise distance $\mathrm{x} / \mathrm{d}$. The cone and Gaussian elements do have the highest circulation values, but they also drop off fairly rapidly and meet up with the other elements around $\mathrm{x} / \mathrm{d}=5.0$ for the Gaussian element and at $\mathrm{x} / \mathrm{d}=10.0$ for the cone. 
The cube elements do not have the same near element trend as the axisymmetric elements. The $\mathrm{x} / \mathrm{d}=1.36$ location for the cubes shows a lower value of circulation than is present at the $\mathrm{x} / \mathrm{d}=2.75$ location. This phenomenon can be explained through brief analysis of the $\mathrm{V}^{+}$plots shown in Figure 3.12. At the $\mathrm{x} / \mathrm{d}=1.36$ location there is a significant amount of downwash near the element height only. This is due to the blockage that the cube elements present to the flow. The measurement location is relatively close to the cube and there is still a significant region of lower velocity fluid present. The $\mathrm{x} / \mathrm{d}=2.75$ profile shows significant downwash through the boundary layer profile as well as above the height of the element. This downstream location not only sees more fluid coming over the top of the cube elements that is being directed toward the wall, but also more of an effect from the horseshoe vortex structures that are present. Subsequently, this larger flow magnitude can be seen in the high circulation values over the rest of the measurement locations. This effect can be seen in particular with the cube at $45^{\circ}$ whose value of circulation is the greatest for all locations between $\mathrm{x} / \mathrm{d}=2.75$ and $\mathrm{x} / \mathrm{d}=10.0$. All elements show a sharp drop in circulation until $\mathrm{x} / \mathrm{d} \approx 10.0$. From this location there is a more gradual decrease in magnitude as the circulation dissipates and eventually reaches zero. The decay of circulation can primarily be attributed to the turbulent diffusion of the vortex structure as it propagates downstream as well as the near wall viscous forces in the spanwise direction that act in retarding the flow. Viscous diffusion is also a contributor as the vortex structure is located in the near-wall region of the turbulent boundary layer.

\subsubsection{Wall Shear Stress}

For information on the scheme used to determine the friction velocity, $\mathrm{U}_{\tau}$, please refer to Section 2.3: Post Processing. In all figures shown, the wall shear stress, $\tau_{w}=U_{\tau}^{2} \rho$, is presented as a ratio of the local wall shear value to the reference $2 \mathrm{D}$ smooth wall boundary layer, $\tau_{\mathrm{o}}$. Figure 3.26 shows the variation of the normalized wall shear $\left(\tau_{\mathrm{w}} / \tau_{\mathrm{o}}\right)$ along the centerline whereas Figures 3.27 and 3.28 show the spanwise variation in wall shear at the $\mathrm{x} / \mathrm{d}=2.75$ and 10.0 planes, respectively. As is discussed in Appendix $\mathrm{C}$, the uncertainty in $\mathrm{U}_{\tau}$ is $\pm 5 \%$. Consequently, it is important to bear in mind that a small percentage of the variations in the presented wall shear stress data is due to uncertainties. 
Focusing on Figure 3.26, the centerline variation in the wall shear stress, the first measurement location after reattachment for all elements is $x / d=2.75$. Therefore, this location is the first available position at which local $\tau_{\mathrm{w}}$ values are available. All axisymmetric elements are very near the same value of $\tau_{\mathrm{w}}$ at $\mathrm{x} / \mathrm{d}=2.75$, whereas the cube elements have a drastically lower value. The lower wall shear stress values exhibited by the cubes are due to the fact that the sharp edges on the cubes create a defined separation point. This separation leads to an area of lower momentum fluid directly behind the elements. Furthermore, this location is just aft of flow reattachment and is in a newly formed shear layer that extends downstream of the reattachment point. All elements show a distinct peak of high local wall shear values at $\mathrm{x} / \mathrm{d}=5.0$. All 'peaked' elements are located at nearly identical values with the hemisphere slightly below and the cube elements at the lowest value. This point of peak wall shear is caused by the horseshoe vortex structures entraining outer layer fluid and creating a wall-ward rush of fluid. A significant drop can be seen in the values between $\mathrm{x} / \mathrm{d}=5.0$ and 10.0 , but the wall shear value is still larger than the reference smooth wall value. This increased wall shear with respect to the reference smooth wall is seen through the remainder of the measurement locations until $\mathrm{x} / \mathrm{d}=40$. At this location, all elements seem to have collapsed back to the reference wall shear value. This indicates that the horseshoe vortices exhibit their presence in the boundary layer, albeit very small, for a significant distance.

Focusing attention on the spanwise wall shear stress variation at the $\mathrm{x} / \mathrm{d}=2.75$ plane, Figure 3.27, we see that all elements exhibit a peak value of wall shear at the centerline location. Again this is due to the outer layer fluid being entrained and brought toward the wall, creating an increase in wall shear relative to the reference wall shear value. In all cases away from the centerline at $\mathrm{z} / \mathrm{d}= \pm 0.28$, there is a decrease in wall shear which leads to a minimum value. From this minimum value, the wall shear gradually increases until around $\mathrm{z} / \mathrm{d} \approx-1.75-2.0$. In this region of increasing wall shear the secondary flow vortices are nearly parallel to the wall. Once the spanwise edge of the wake is reached, the wall shear once again reaches the smooth wall value within the experimental uncertainty of $\pm 5 \%$. The region of lower wall shear is related to the flow being directed away from the 
wall, as is also visible in the oil flow images previously discussed. The cube elements show a significantly lower wall shear value as compared to the rest of the elements due to the previously discussed reasons. There is a sharp change in the wall shear stress value from $\mathrm{z} / \mathrm{d}=-0.55$ to $\mathrm{z} / \mathrm{d}=-0.83$. This dramatic change is due to the effects caused by the sharp edges of the cubes. Once past the sides of the cubes, the wall shear reaches similar values as the other elements.

As the flow proceeds downstream to the $\mathrm{x} / \mathrm{d}=10.0$ plane, there is still fluid being brought toward the wall via the counter-rotating horseshoe vortex structure. For all of the axisymmetric elements we see similar tendencies in the wall shear as we saw at the $\mathrm{x} / \mathrm{d}=2.75$ plane. Although the tendencies are similar, the magnitudes have been reduced due to the diffusion of the horseshoe vortex structure as it progresses downstream. Again, there is a peak of higher wall shear due to the downwash of fluid toward the wall. Directly on either side of this peak we see a minimum value and then an increase to the reference 2D flow wall shear value. As the outer regions of the spanwise direction are reached there is a noticeable difference between the wall shear values of the elements. This difference can be attributed to the differences in the horseshoe vortex structures produced by each element. At the $\mathrm{z} / \mathrm{d}= \pm 0.28$ locations, the large grooved element is seen to have a different wall shear value as compared to the other axisymmetric elements. This difference is attributed to the weaker vortex that is present, at the $\mathrm{x} / \mathrm{d}=10.0$ location, in the large grooved element case as is shown in Table 3.1. There is a noticeable difference in the wall shear behavior of the cube elements around the centerline when the two measurement planes are compared. The $\mathrm{x} / \mathrm{d}=10.0$ plane exhibits a rise in wall shear at the closest off-centerline locations and then a decrease to near smooth wall values. The slightly lower values at the centerline for the cube cases can be related to the fact that the flow diverges from the plane of symmetry in the near wall regions. This divergence in turn causes the reduction in wall shear at the centerline location. As is evidenced by Tables 3.1 and 3.2, the vortices created by the cube elements have not moved as far in the spanwise direction as the other elements. This lack of movement creates a different effect on the centerline wall shear value as more fluid diverges away from the centerline at the downstream measurement plane. This phenomenon is in contrast to the greater 
amount of fluid converging toward the centerline at the upstream measurement plane thus creating a larger wall shear stress at the centerline measurement location.

\subsubsection{Drag}

The drag for each element was calculated using a control volume and momentum balance approach. The undisturbed upstream velocity profile as well as the wake profiles behind each element at the $\mathrm{x} / \mathrm{d}=2.75$ plane are taken into account in this analysis. The derivation of the drag equation and related discussion can be found in Appendix B. Equation 3.4 below was used to calculate the drag increment due to the presence of a roughness element and is taken from Equation (B.11) in Appendix B. The subscripts ' $E$ ' and 'w/oE' refer to boundary layer profiles taken with and without the element being present in the boundary layer, respectively. The numbered subscripts refer to the control volume plane number at which the profile was taken. Similarly, the subscript 'top' refers to the quantity at the top of the control volume. Finally, $-\mathrm{z}_{2 \mathrm{D}}$ is related to the spanwise distance in the (-)z-direction at which the local boundary profile has collapsed to the reference $2 \mathrm{D}$ smooth wall profile.

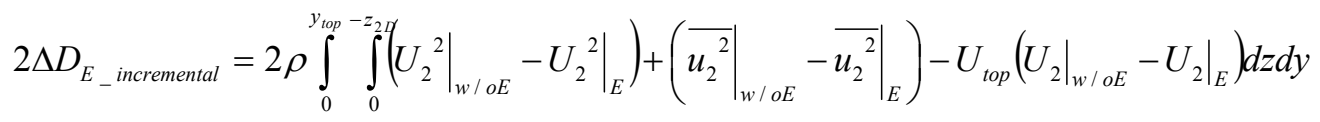

The incremental drag consists of the drag due to the element's presence in the boundary layer as well as the skin friction drag created by the surface area of the floor contained within the control volume. The control volume used in the derivation of Equation 3.4 deems the contribution of the pressure term to form drag to be negligible since the upstream and downstream control volume planes should be at the same uniform pressure. This result stems from the work of Matinuzzi and Tropea (1993) who show a pressure recovery at a downstream distance of $\mathrm{x} / \mathrm{d} \approx 2.25$ for a surface mounted cube. Thus, due to the roughness element geometries under investigation, all elements should experience full pressure recovery by $x / d=2.75$. Please note that the analysis used to calculate the drag also includes the streamwise Reynolds normal stress, $\overline{u^{2}}$, term. Consequently, this approach is more rigorous than a method that only takes into account the mean velocity. The addition of the fluctuating term will cause a slight decrease in the drag as compared to the drag calculated with only the mean velocity. This is due to the fact that some mean 
flow momentum is lost when turbulence is created. As was mentioned previously, it is necessary to account for the drag on the control volume surface in order to obtain the actual drag due to each roughness element. More discussion related to the procedure used to obtain the actual drag can be found in Chapter 4. Table 3.4 presents the actual drag calculated on each element as well as the related elemental areas, where $A_{f}$ and $A_{w}$ correspond to the frontal $\left(\mathrm{A}_{\mathrm{f}}\right)$ and wetted $\left(\mathrm{A}_{\mathrm{w}}\right)$ areas of each roughness element. There are no drag results presented for the fine grooved element due to the fact that measurements were taken at a plane equal to $\mathrm{x} / \mathrm{d}=1.36$ rather than 2.75 .

Table 3.4: Actual experimental drag and elemental areas

\begin{tabular}{|c|c|c|c|c|c|}
\hline Element & $\mathrm{A}_{\mathrm{f}}\left(\mathrm{m}^{2}\right)$ & $\mathrm{A}_{\mathrm{w}}\left(\mathrm{m}^{2}\right)$ & $\mathrm{A}_{\mathrm{w}} / \mathrm{A}_{\mathrm{f}}$ & $\Delta \mathrm{D}_{\mathrm{E} \text { actual }}(\mathrm{N})$ & $\Delta \mathrm{D}_{\mathrm{E} \text { actual }} /\left(\rho \mathrm{U}_{\tau}{ }^{2} \mathrm{~A}_{\mathrm{f}}\right)$ \\
\hline Cone & $3.23 \mathrm{E}-06$ & $1.13 \mathrm{E}-05$ & 3.51 & $1.69 \mathrm{E}-04$ & 52.49 \\
\hline Gaussian & $2.66 \mathrm{E}-06$ & $1.02 \mathrm{E}-05$ & 3.85 & $1.22 \mathrm{E}-04$ & 45.81 \\
\hline Large Grooved & $3.23 \mathrm{E}-06$ & $1.94 \mathrm{E}-05$ & 6.01 & $1.98 \mathrm{E}-04$ & 61.62 \\
\hline Cube at $90^{\circ}$ & $2.98 \mathrm{E}-06$ & $1.49 \mathrm{E}-05$ & 5.00 & $2.52 \mathrm{E}-04$ & 84.63 \\
\hline Cube at $45^{\circ}$ & $4.22 \mathrm{E}-06$ & $1.49 \mathrm{E}-05$ & 3.54 & $2.13 \mathrm{E}-04$ & 50.64 \\
\hline Hemisphere & $3.07 \mathrm{E}-06$ & $1.23 \mathrm{E}-05$ & 4.00 & $1.15 \mathrm{E}-04$ & 37.70 \\
\hline Fine Grooved & $3.23 \mathrm{E}-06$ & $2.12 \mathrm{E}-05$ & 6.58 & N/A & N/A \\
\hline
\end{tabular}

The largest value of drag is seen on the cube at $90^{\circ}$ followed by the cube at $45^{\circ}$, large grooved element, cone, Gaussian element, and the hemisphere. The higher value of drag on the large grooved element can be related not only to its frontal projected area but also its wetted surface area. With the highest wetted surface area, next to the fine grooved element, the large grooved element will experience more viscous drag than the other elements under scrutiny. This result is somewhat obvious and is shown by Table 3.4. The drag on each roughness element appears to be in direct correlation with a combination of the form drag, related to the frontal area, and the viscous drag, which can be related to the wetted surface area, of each element. More discussion related to the drag of each element and possible correlations between the roughness elements is discussed in Chapter 4.

\subsubsection{Eddy Viscosities and Anisotropy Factor}

The eddy viscosities in the $\mathrm{x}$ and $\mathrm{z}$-directions were computed using Equations 3.5 and 3.6 respectively. 
$\varepsilon_{x}=\frac{-\overline{u v}}{\partial U / \partial y}$

$\varepsilon_{z}=\frac{-\overline{v w}}{\partial W / \partial y}$

The centerline variation of normalized streamwise eddy viscosity, $\varepsilon_{x} / v$ vs. $y U_{\tau} / v$ can be found in Figure 3.108. The eddy viscosity is an indicator of how much of the mean flow gradient goes into the production of turbulence. In the near wall region all profiles behave in the same fashion until $\mathrm{y}^{+} \approx 15$. In this region there is an increase in the streamwise eddy viscosities behind the elements due to the high amount of turbulence present in this region. At the closest measurement location to the element, $\mathrm{x} / \mathrm{d}=1.36$, there are a few points that have negative eddy viscosities. These events can be linked to the fact that there is still a significant amount of backflow in this area behind the element. The increase in the streamwise eddy viscosities can be seen until just above the roughness element heights, $\mathrm{y}^{+} \approx 150$, where the profiles tend to collapse back to the reference smooth wall values.

The parameter $\mathrm{N}$, is the anisotropy factor of a flow and is defined as,

$$
N=\frac{\varepsilon_{z}}{\varepsilon_{x}}=\frac{-\overline{v w} /(\partial W / \partial y)}{-\overline{u v} /(\partial U / \partial y)}=\frac{\tan (S S A)}{\tan (F G A)}
$$

where SSA and FGA are the shear stress angle and flow gradient angle. Further discussion related to these parameters can be found in Appendix E. Figures 3.109 through 3.112 show contours of $\mathrm{N}$ in the different measurement planes associated with each element. It is largely assumed that in three dimensional boundary layer flows that the eddy viscosity is isotropic. This assumption is generally not valid and is also shown to be the case in the present study. In order for the flow to be isotropic the eddy viscosity ration $\mathrm{N}$ would be equal to one and any deviation from unity would mean that the flow is anisotropic. As is shown in Figures 3.109 through 3.112, the flow is largely anisotropic and any values larger than unity indicate a more significant spanwise interaction as compared to the streamwise direction. Exceedingly large values in the magnitude of $\mathrm{N}$ in the plots should not be taken as actual results due to the scatter in the $\overline{v w}$ Reynolds 
shearing stress at theses particular locations. It is also important to note that any negative regions of $\mathrm{N}$ can be attributed to the decorrelation of the streamwise and spanwise Reynolds shearing stresses.

\subsection{Reynolds Stresses and Turbulent Kinetic Energy}

An effective means of analyzing the turbulent flow field behind the elements is through the inspection of the Reynolds stress behavior. To this end, the centerline variation of the normalized streamwise Reynolds normal stress, $\overline{u^{2}} / U_{\tau}^{2}$, plotted against $y U_{\tau} / v$ is shown in Figure 3.29. At the $\mathrm{x} / \mathrm{d}=1.36$ location, all elements show a peak in the streamwise Reynolds normal stress at or slightly below the respective element height. All axisymmetric elements are shown to have a greater $\overline{u^{2}}$ Reynolds normal stress throughout the early stages of the profile as compared to the reference smooth wall boundary layer. These elements then show a sharp drop off in the streamwise Reynolds normal stress and collapse to the reference smooth wall profile above the height of the elements near $y^{+} \approx 200$. In contrast to the axisymmetric elements, the cube elements show a reduction in $\overline{u^{2}}$ near the wall. This reduction can be attributed to the blockage that the cubes present, therefore limiting the amount of mixing that occurs directly behind the element. The higher values of normal stresses are due to the intense mixing created by the shear layers that are rolling up and converging toward the centerline of the element. The cube at $45^{\circ}$ shows the highest magnitude of $\overline{u^{2}}$ as compared to the rest of the elements. The cube at $45^{\circ}$ is followed by a close conglomeration of the hemisphere, fine grooved, and cone element data. The lowest value of $\overline{u^{2}}$ at the earliest measurement location is seen in the Gaussian, large grooved, and cube at $90^{\circ}$ elements. At the $\mathrm{x} / \mathrm{d}=2.75$ location the maximum value in $\overline{u^{2}}$ is still from the cube at $45^{\circ}$; the other elements have collapsed to approximately the same peak value. The peak in the streamwise Reynolds normal stress is still seen at or below the element heights with a collapse to the reference boundary layer at approximately the same location as the $\mathrm{x} / \mathrm{d}=1.36$ measurement location. The reduction in peak magnitude in $\overline{u^{2}}$ of about 20$60 \%$ is very large as the flow propagates downstream to the $\mathrm{x} / \mathrm{d}=2.75$ location. As the 
flow continues to proceed farther downstream, there is a small reduction in the streamwise Reynolds normal stress due to slight flow acceleration along the centerline behind the elements. By $\mathrm{x} / \mathrm{d}=10.0$ the profiles have nearly collapsed to the reference boundary layer profile and at $\mathrm{x} / \mathrm{d}=20.0$ and 40.0 there is no effect of the roughness elements' presence in the boundary layer.

Contour plots of the normalized streamwise Reynolds normal stress at the $x / d=2.75$ and 10.0 measurement planes can be found in Figures 3.30 through 3.33. These plots show that for all elements there is a peak value in $\overline{u^{2}}$ at or slightly below the height of the element. All elements show that this peak value is also connected to a higher magnitude pocket located to the side of each element, $\mathrm{z} / \mathrm{d} \approx-1$. This high region of the $\overline{u^{2}}$ Reynolds stress can be attributed to the shear layers that are rolling up and surrounding the trailing leg of the horseshoe vortex structure. Consequently, the vertical locations of the pockets of high $\overline{u^{2}}$ Reynolds stress are similar to the peak $\Omega_{\mathrm{x}}$ locations shown in Table 3.1. The distance from the wall for the peak values of $\overline{u^{2}}$ is near $\mathrm{y}^{+} \approx 20$ whereas the peak $\Omega_{\mathrm{x}}$ locations were found to be near $\mathrm{y}^{+} \approx 13$. The magnitude of this $\overline{u^{2}}$ region is related to the magnitude of the vortex structure produced by each element, in this case the cube at $45^{\circ}$ is the highest followed by, cube at $90^{\circ}$, cone, hemisphere, Gaussian, and large grooved elements. At the $\mathrm{x} / \mathrm{d}=10.0$ plane the values for all elements are nearly equal to the reference two-dimensional boundary layer values. There is some reduction near the center plane due to slight acceleration, but for the most part the influence of the element has drastically dissipated.

Figure 3.34 shows the centerline variation of the normalized normal-to-wall Reynolds normal stress, $\overline{v^{2}} / U_{\tau}^{2}$, plotted with respect to $y U_{\tau} / v$. The same trends can be seen in these plots as was seen in the plots of $\overline{u^{2}} / U_{\tau}^{2}$. There is a peak value at or below the element heights, with the cube at $45^{\circ}$ generally having the largest magnitude. The cube at $90^{\circ}$ at the $\mathrm{x} / \mathrm{d}=1.36$ location shows a significantly lower value of $\overline{v^{2}}$ again due to the lower speed fluid created by the 'blockage effect'. The elements drop off sharply and 
collapse to the reference boundary layer values just above the height of the element near $\mathrm{y}^{+} \approx 200$. At $\mathrm{x} / \mathrm{d}=2.75$, lower magnitudes in the $\overline{v^{2}}$ stress are seen as compared to the upstream location, but there is not as much of a decrease in the $\overline{v^{2}}$ stress as was seen in the $\overline{u^{2}}$ stress. By $\mathrm{x} / \mathrm{d}=5.0$ it appears that the cube element profiles have collapsed nearly to one another, while the axisymmetric elements have done the same. At $\mathrm{x} / \mathrm{d}=10.0$, profiles for all elements have collapsed nearly to the same values and by $\mathrm{x} / \mathrm{d}=20.0$ and 40.0 there is no influence by the elements seen in the profiles.

Contour plots of the normalized $\overline{v^{2}}$ Reynolds normal stress can be seen in Figures 3.35 through 3.38 for the $\mathrm{x} / \mathrm{d}=2.75$ and 10.0 planes, respectively. At the $\mathrm{x} / \mathrm{d}=2.75$ plane, nearly circular contours surround a peak stress value that is located slightly below the height of the element. These peaks in the Reynolds normal stress can again be attributed to the mixing created by shear layer roll-up due to the horseshoe vortex structures. The rush of fluid toward the wall brought in by the common downwash of the vortices is the primary factor that will create and maintain these higher stress values. Consequently, the highest regions of the $\overline{v^{2}}$ Reynolds normal stress are found near the height of each element. The 'peaked' roughness elements tend to see the largest values in the $\overline{v^{2}}$ stress at a y-location near $y^{+} \approx 70$, whereas the hemisphere and cube elements are closer to the wall at $\mathrm{y}^{+} \approx 40-50$. These locations differ from the locations of the peak $\overline{u^{2}}$ stress and peak streamwise vorticity, $\Omega_{\mathrm{x}}$, in that they are along the centerline and significantly farther from the wall. The contour plots at the $\mathrm{x} / \mathrm{d}=10.0$ plane show a small peak of stress that is still located near the element heights. This area is of considerably less magnitude than the upstream location. The higher values tend to be stretched in the spanwise direction as the vortices move away from the centerline and dissipate.

Figure 3.39 shows the centerline variation of the normalized spanwise Reynolds normal stress, $\overline{w^{2}} / U_{\tau}^{2}$, plotted with respect to $y U_{\tau} / v$. These centerline plots are very comparable and show nearly the same trends as do the $\overline{u^{2}} / U_{\tau}^{2}$ and $\overline{v^{2}} / U_{\tau}^{2}$ plots. A few slight differences are that the $\overline{w^{2}}$ stresses are larger in the near-wall region, they keep 
their magnitude for a wider range of y values, and the peak values are seen farther below the element heights as compared to the other stresses. Also, there are no local peaks in $\overline{w^{2}}$ off of the centerline at $\mathrm{x} / \mathrm{d}=2.75$. Contour plots of the normalized $\overline{w^{2}}$ Reynolds normal stress can be seen in Figures 3.40 through 3.43 for the $\mathrm{x} / \mathrm{d}=2.75$ and 10.0 planes, respectively. These plots show the same tendency for the peak stress value below the element height at the $\mathrm{x} / \mathrm{d}=2.75$ plane. There is also a higher region of $\overline{w^{2}}$ stress off of the centerline in the spanwise direction at the $\mathrm{x} / \mathrm{d}=10.0$ plane that can be linked to the propagation of the vortices in the spanwise direction as they dissipate and proceed downstream.

The normalized Reynolds shear stress profiles of $-\overline{u v} / U_{\tau}^{2}$ along the centerline can be found in Figure 3.44. These plots show a significant peak at the $\mathrm{x} / \mathrm{d}=1.36$ location that is an order of magnitude larger for the cube at $45^{\circ}$, hemisphere, and fine grooved element. As the flow proceeds downstream to the $\mathrm{x} / \mathrm{d}=2.75$ location, the cube elements still show the largest Reynolds shear stress values, whereas the $-\overline{u v}$ stress for the other elements has deteriorated to nearly the same values. Overall there is a reduction in the Reynolds shear stress value in the range of 40-50\%. The profiles for all elements have almost collapsed to one another by $\mathrm{x} / \mathrm{d}=5.0$ with the cubes being the outliers. At $\mathrm{x} / \mathrm{d}=10.0$ the centerline plots show no evidence of the roughness element as all the profiles have collapsed to the reference profile.

Contour plots of the normalized Reynolds shear stress, $-\overline{u v}$, can be seen in Figures 3.45 through 3.48 for the $\mathrm{x} / \mathrm{d}=2.75$ and 10.0 planes, respectively. The $\mathrm{x} / \mathrm{d}=2.75$ plane shows large peaks near the element heights. This peak region is due to the ability of the vortex structures to create significant sweeping motions that promote mixing. A lower region of shear stress, as compared to the peak region, is also seen extending away from the elements in the spanwise direction. By the time the flow has reached the $\mathrm{x} / \mathrm{d}=10.0$ plane, the Reynolds shear stress has undergone very substantial decay and is very close to twodimensional values with small regions of higher shear stress extending in the spanwise direction. 
The plots contained in Figures 3.49 through 3.102 are the centerline and contour plots associated with the $\overline{u w}$ and $\overline{v w}$ Reynolds shear stresses as well as the remaining triple products. These figures show good symmetry for all plots involving a single spanwise fluctuating term. This result is expected due to the fact that the spanwise (W) velocity is zero along the centerline of the element. All parameters involving a single normal-towall fluctuating term tend to show peak values along the centerline and near the height of the element. This is the area that the most significant amount of downwash is seen. Other areas of peak triple products can also be seen in the high spanwise velocity regions associated with the secondary flow produced by the horseshoe vortex structures. Consequently, areas near the element heights and in the high secondary flow regions will have significant levels of triple products and stresses due to the intense mixing and the rolling up of shear layers that will be present.

Plots of the centerline variation of the TKE, $\left(\overline{q^{2}} / 2\right) / U_{\tau}^{2}$, with respect to $\mathrm{y}^{+}$can be seen in Figure 3.103. The highest values of TKE are always found below the height of each element. The $\mathrm{x} / \mathrm{d}=1.36$ location shows the cube at $45^{\circ}$ with the highest TKE followed closely by all of the axisymmetric elements and then the cube at $90^{\circ}$. Significant decay between the first and second measurement locations can also be seen with the cube elements now both having the highest amount of TKE. There is still a noteworthy amount of TKE present at $\mathrm{x} / \mathrm{d}=5.0$ for all elements. At $\mathrm{x} / \mathrm{d}=10.0$ there is very little TKE present as was discussed previously. By the time the flow reaches a downstream distance of $\mathrm{x} / \mathrm{d}=20.0$ the TKE has dissipated and reached the reference two-dimensional turbulent boundary layer values.

Figures 3.104 through 3.107 show contour plots of the turbulent kinetic energy (TKE) normalized on $\mathrm{U}_{\tau},\left(\overline{q^{2}} / 2\right) / U_{\tau}^{2}$, where $\overline{q^{2}}=\left(\overline{u^{2}}+\overline{v^{2}}+\overline{w^{2}}\right)$. These plots contain the contours of the $\mathrm{x} / \mathrm{d}=2.75$ and 10.0 measurement planes, respectively. These plots also show the normalized TKE transport velocity vectors, $V_{q} / U_{\tau}=\left(\overline{v q^{2}} / \overline{q^{2}}\right) / U_{\tau}$ and 
$W_{q} / U_{\tau}=\left(\overline{w q^{2}} / \overline{q^{2}}\right) / U_{\tau}$. At the $\mathrm{x} / \mathrm{d}=2.75$ plane, a core of higher TKE can be found below the element height along the centerline. The large regions of TKE near the element heights are due to the horseshoe vortices that not only create sweeping motions toward the wall but also a convergence of associated shear layers toward the center plane. The largest magnitude of TKE is produced by the cube at $45^{\circ}$ followed by the cube at $90^{\circ}$, fine grooved (from Figure 3.103 at the $\mathrm{x} / \mathrm{d}=2.75$ location), cone, large grooved, Gaussian, and hemisphere. The TKE tends to diffuse outward in a radial pattern above the element heights. This radial pattern can only be observed in the plots having log $y$ coordinates. Behind the element and below its height there is still a large amount of TKE present. The larger elements that protrude into the boundary layer and have sharp edges tend to produce more TKE. The hemisphere which is shorter and has smooth sides has the lowest amount of TKE production.

The TKE diffuses outward from the core of highest TKE as is evidenced by the diffusion velocity vectors. The highest diffusion is seen in the cone and cubes whose vectors have very similar magnitudes. The Gaussian, fine grooved, large grooved, and hemisphere elements follow with decreasing diffusion velocities. The basis of putting the fine grooved element ahead of the large grooved element is due to the element's 'peakiness'. As can be seen in Figures 3.104 and 3.105, the diffusion velocities are highest near sharp edges (cubes) and near sharp peaks (cone, Gaussian, etc.). Subsequently, this leads to the conclusion that both the element shape and the orientation play very significant roles in the production and diffusion of TKE. All axisymmetric elements show a higher diffusion of TKE near their respective heights than anywhere else. The cube elements tend to have large diffusion along the sides as well as near the element height. It is also worth noting that even though the hemisphere is smooth, there are still significant diffusion velocities that emanate from the element height.

The $\mathrm{x} / \mathrm{d}=10.0$ measurement plane (Figures 3.106 and 3.107) shows a very extreme decay of the TKE produced by each element. The most noticeable regions of higher TKE are seen away from the elements in the spanwise direction. These areas are mainly due to the 
streamwise vortices that have propagated downstream. The diffusion velocity vectors have also gone through a drastic decay and are for the most part negligible. 


\subsection{Chapter 3 Figures: Single Element}

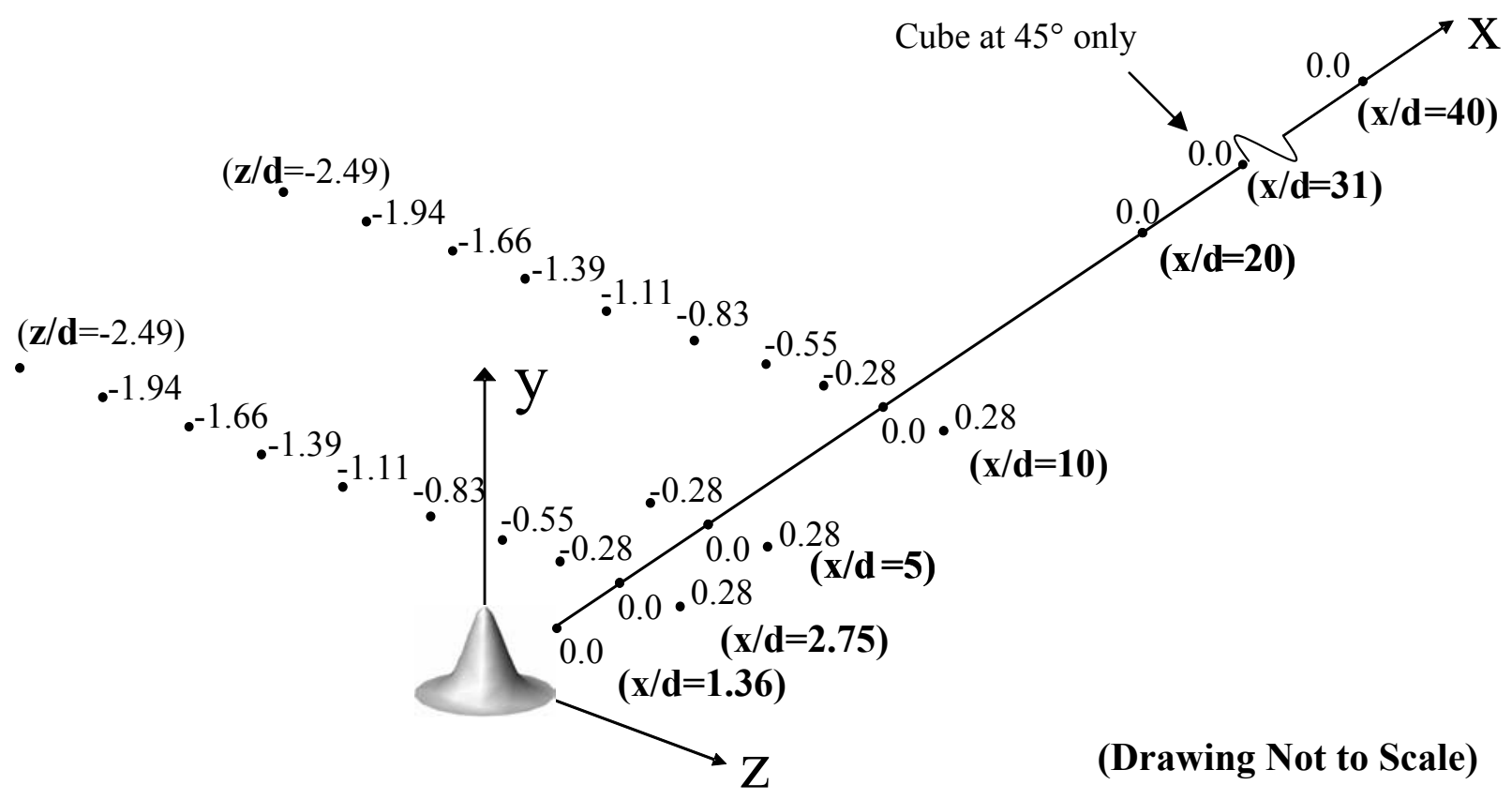

Figure 3.1: Measurement locations for single element cases (except fine grooved)

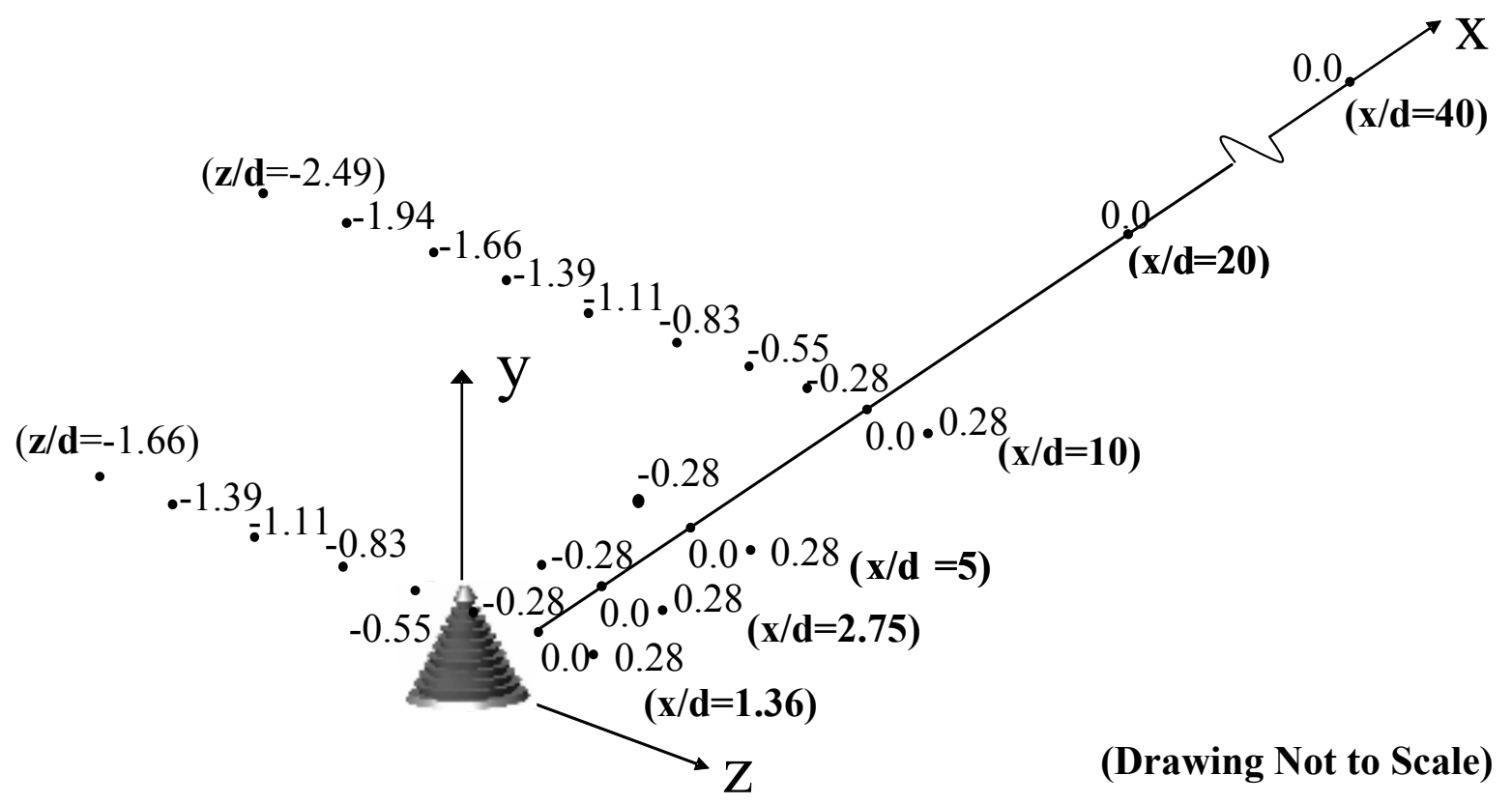

Figure 3.2: Measurement locations for fine grooved element 

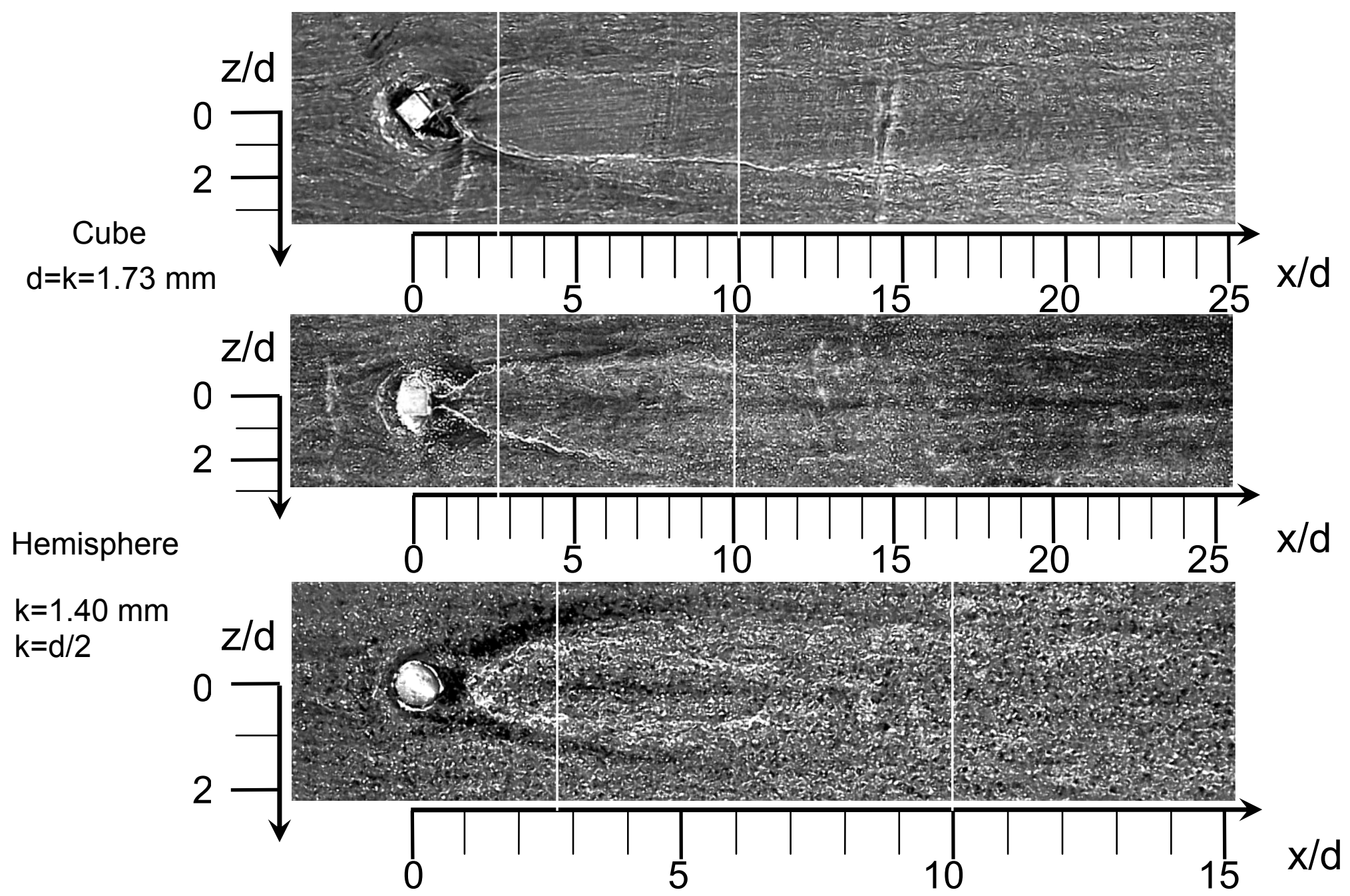

From Top: Cube at $45^{\circ}$, Cube at $90^{\circ}$, Hemisphere

Figure 3.3: Oil flows for the cube and hemisphere elements 


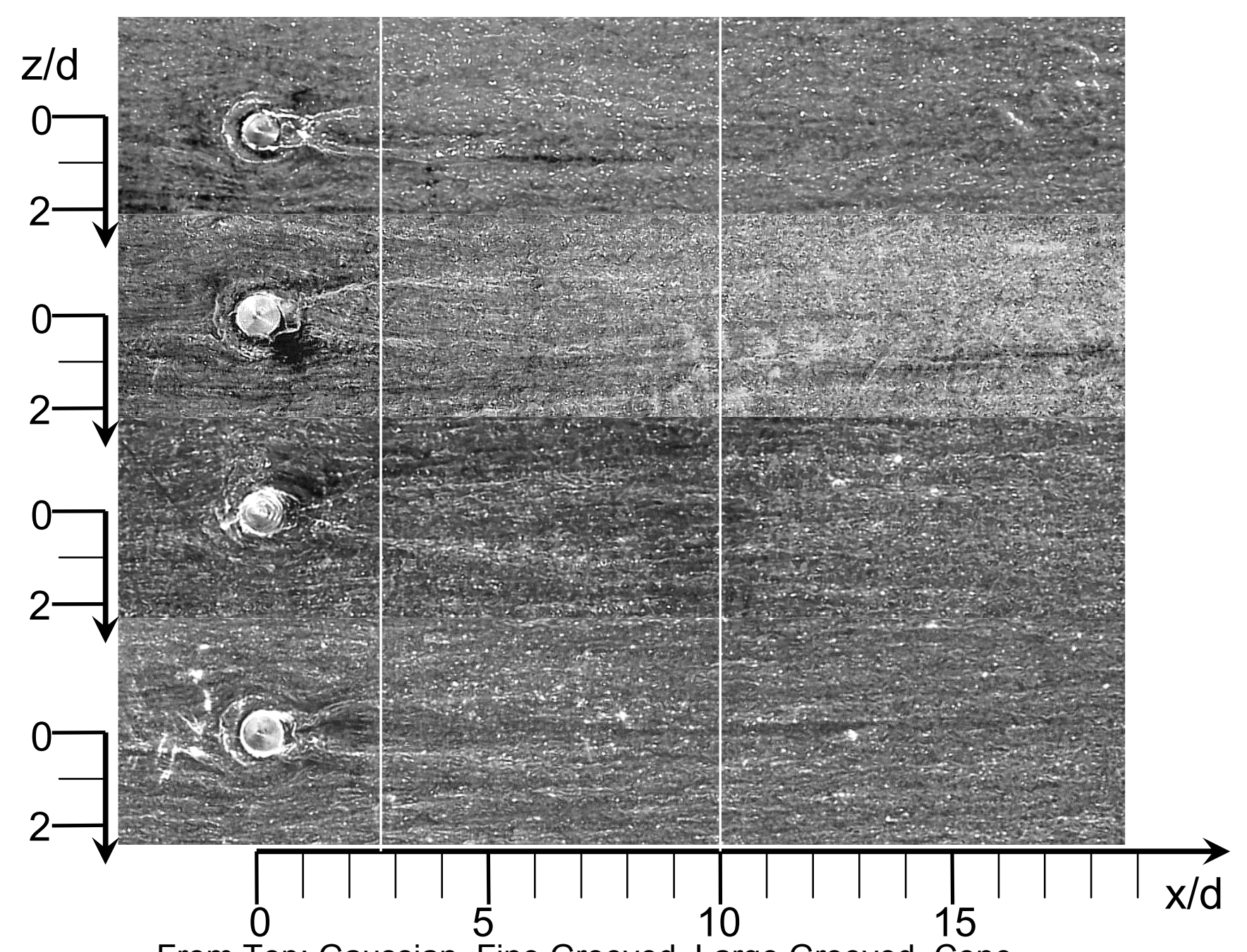

From Top: Gaussian, Fine Grooved, Large Grooved, Cone ( $k=2.54 \mathrm{~mm}$ for all, $\mathrm{d}=2.54,2.69,3.00,2.54 \mathrm{~mm}$, respectively)

Figure 3.4: Oil flows for the Gaussian, fine grooved, large grooved, and cone elements 

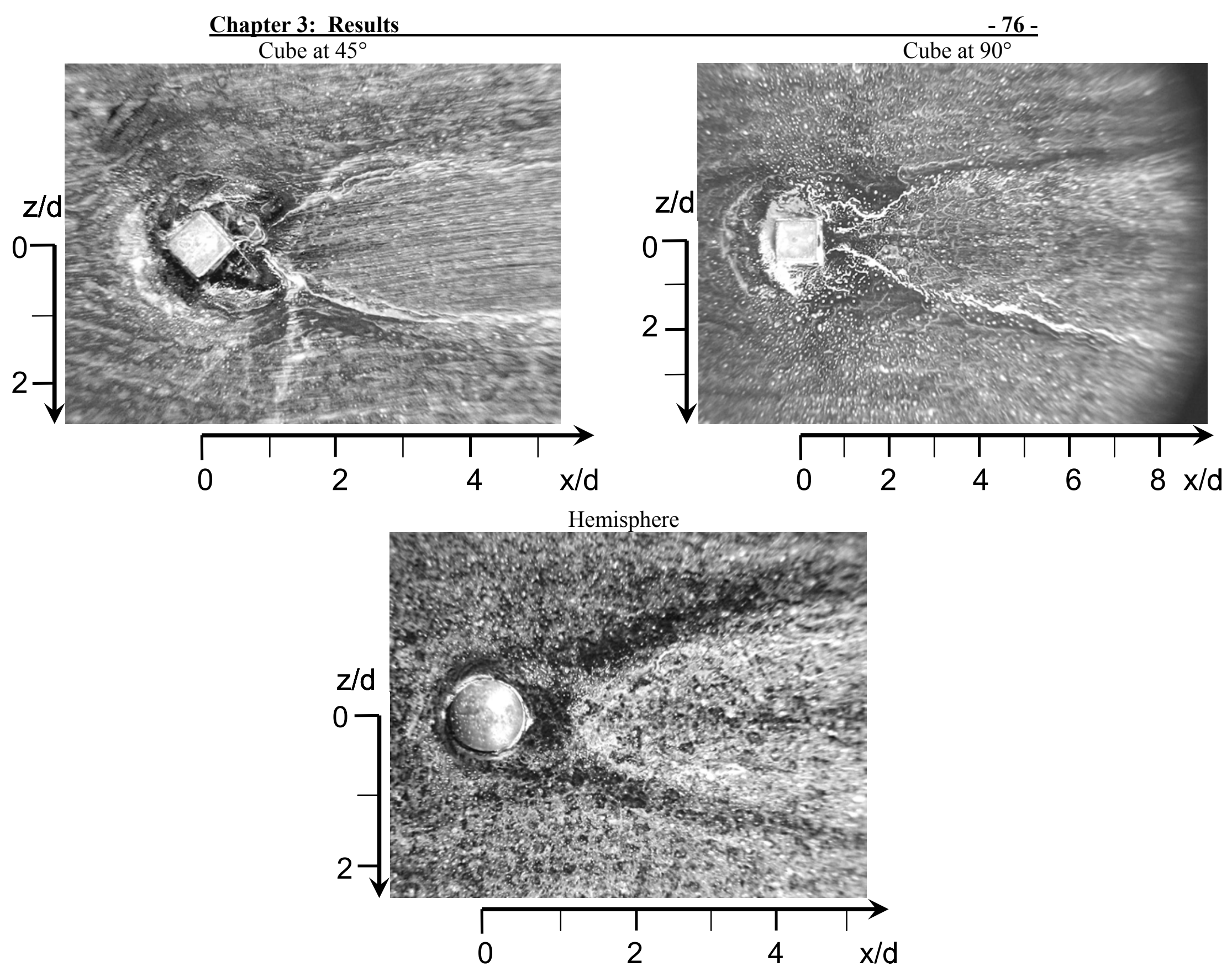

Figure 3.5: Close-up views of oil flows for the cube and hemisphere elements 

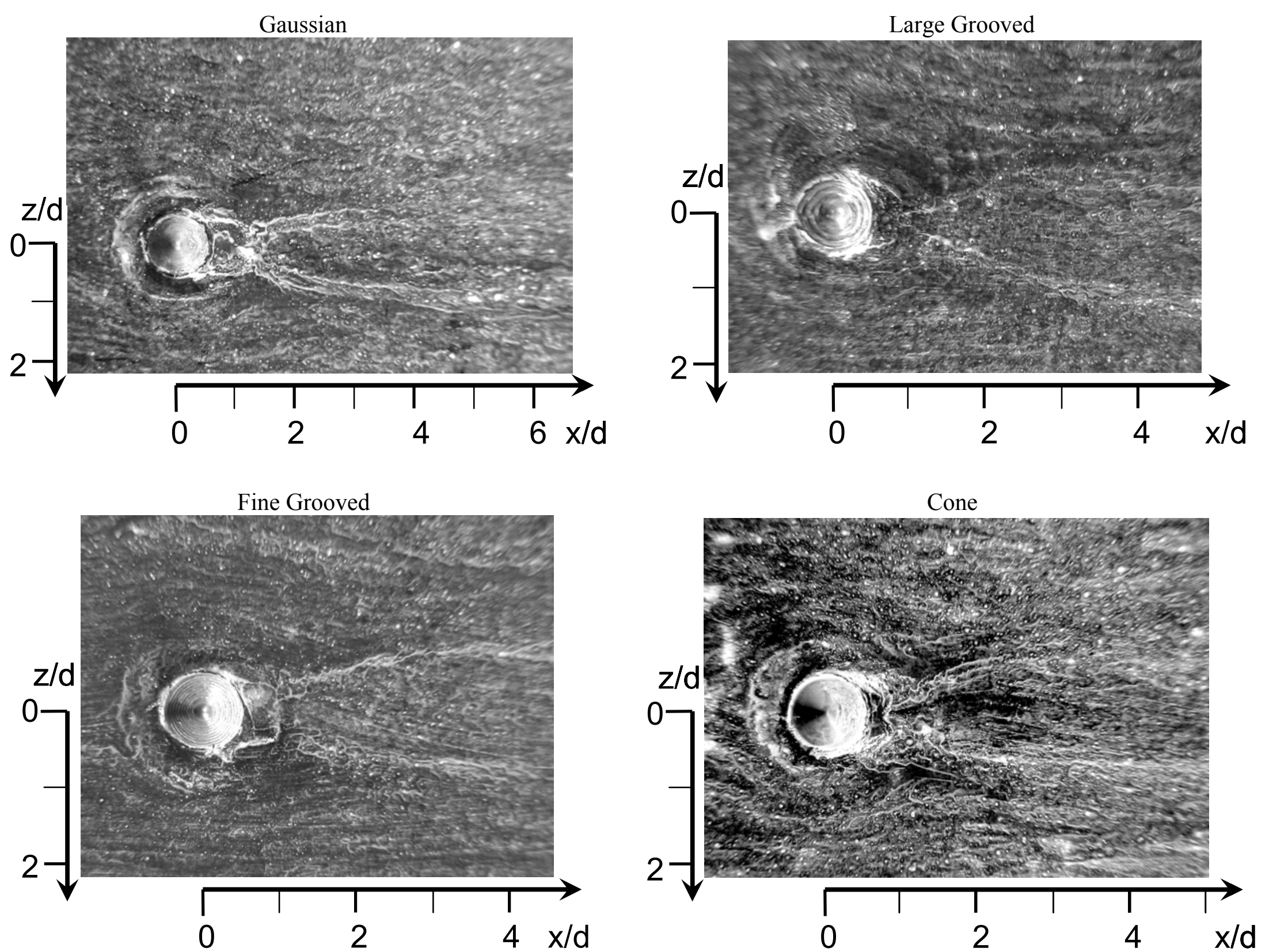

Figure 3.6: Close-up views of the oil flows for the Gaussian, large grooved, fine grooved, and cone elements 


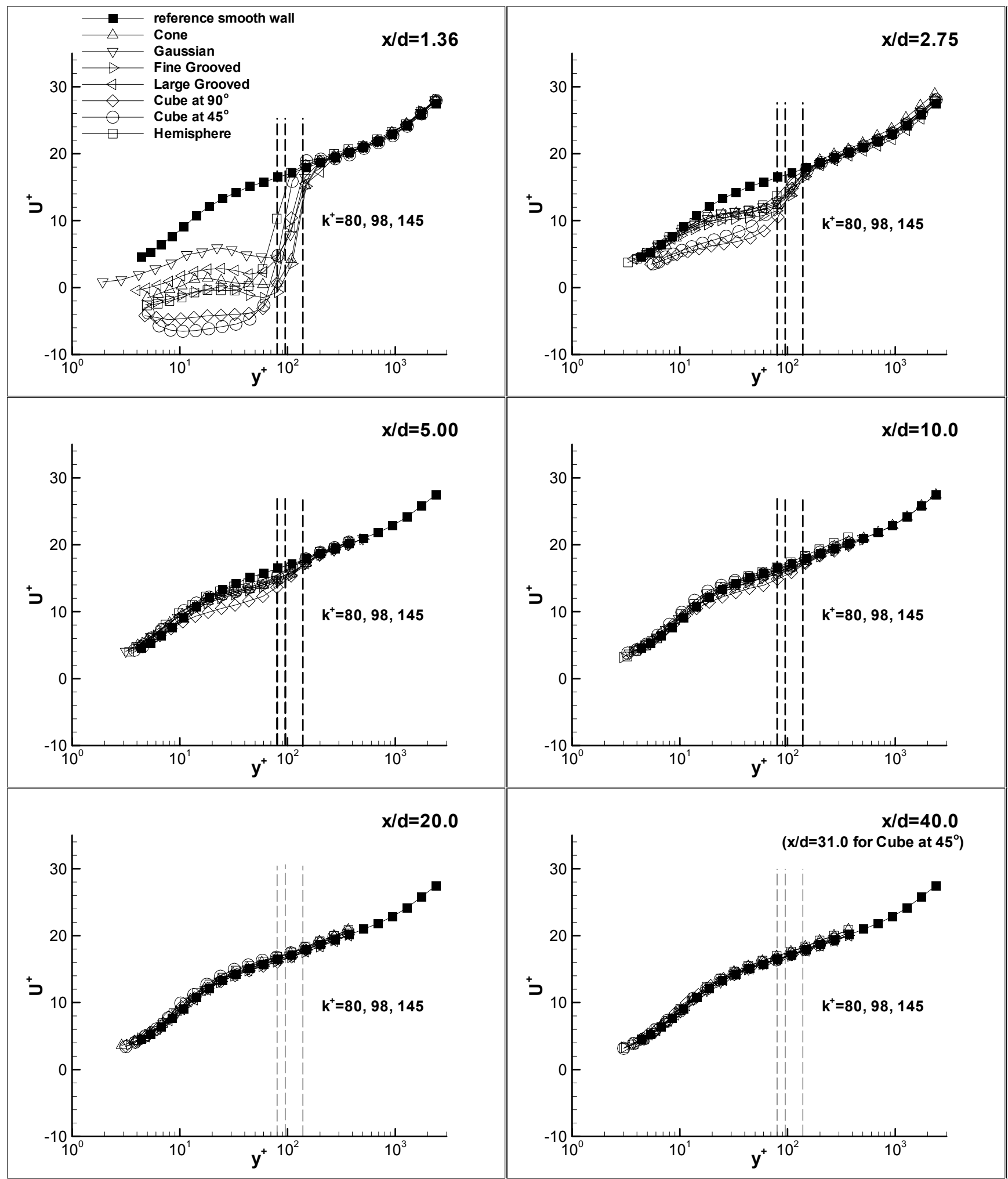

Figure 3.7: $U / U_{\tau}$ vs. ${ }^{y U_{\tau}} / v$, streamwise mean velocity profiles along the centerline 

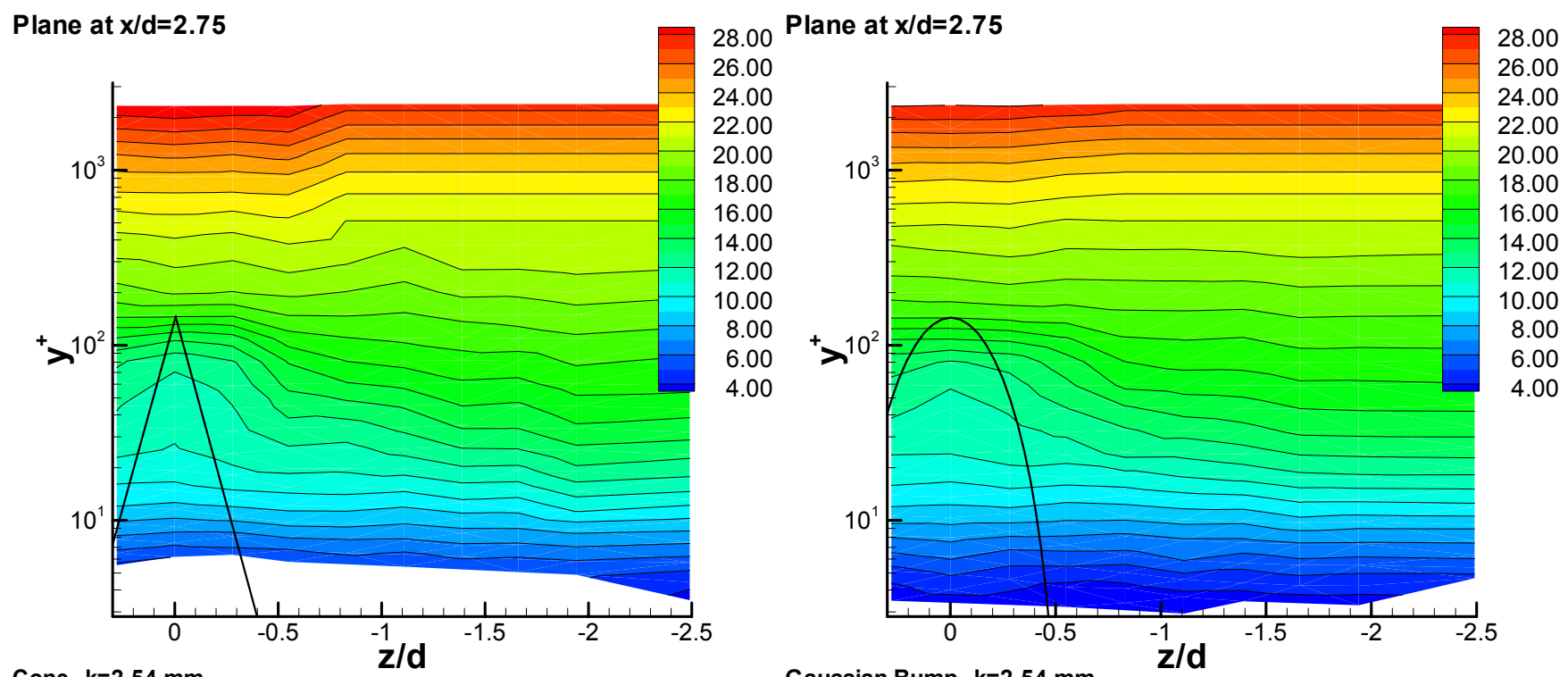

Cone--k=2.54 mm

Gaussian Bump--k=2.54 mm

Plane at $x / d=2.75$

Plane at $x / d=1.36$
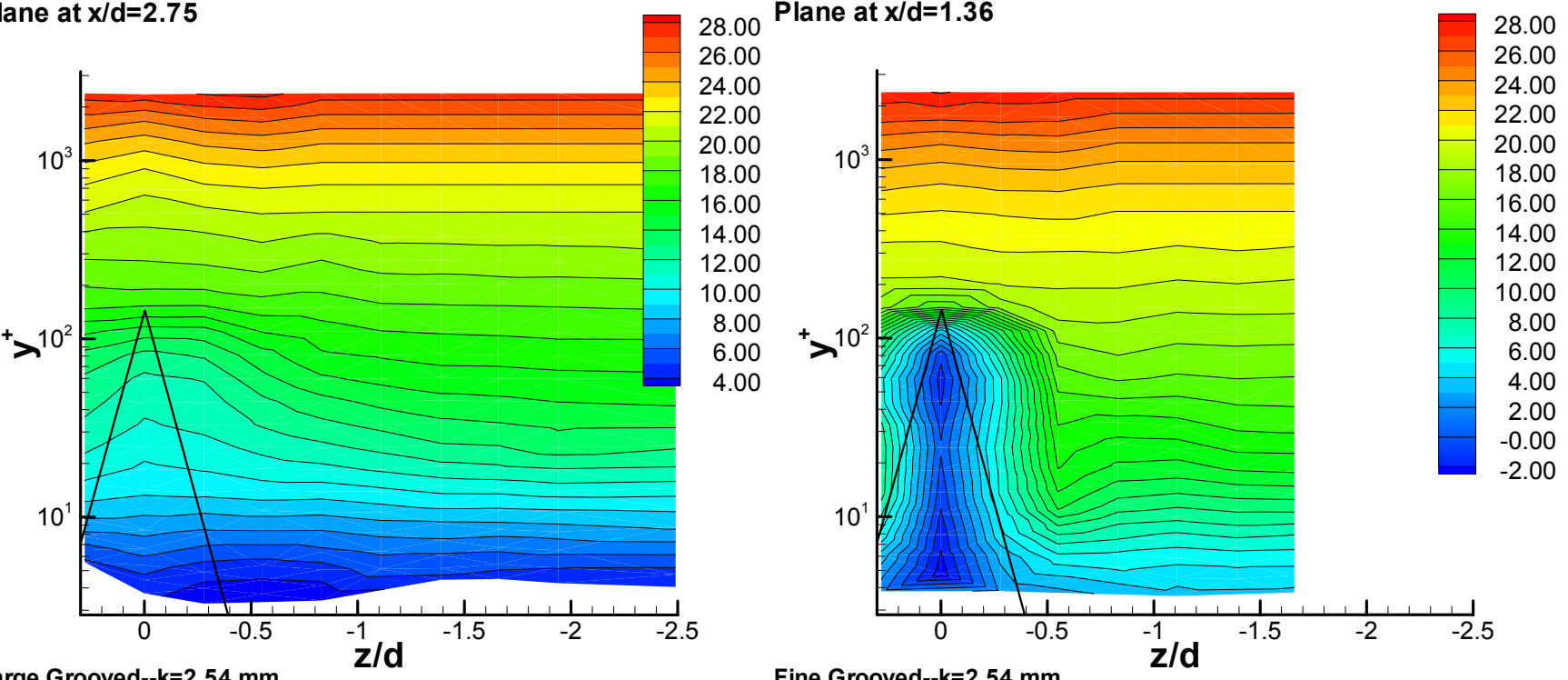

Large Grooved--k=2.54 mm

Fine Grooved--k=2.54 mm

Figure 3.8: Contours of $U / U_{\tau}$ in the $\mathbf{y - z}$ plane at a location of $\mathbf{x} / \mathbf{d}=\mathbf{2 . 7 5}$ (Note: Fine Grooved $\mathbf{x} / \mathbf{d}=1.36$ ) 


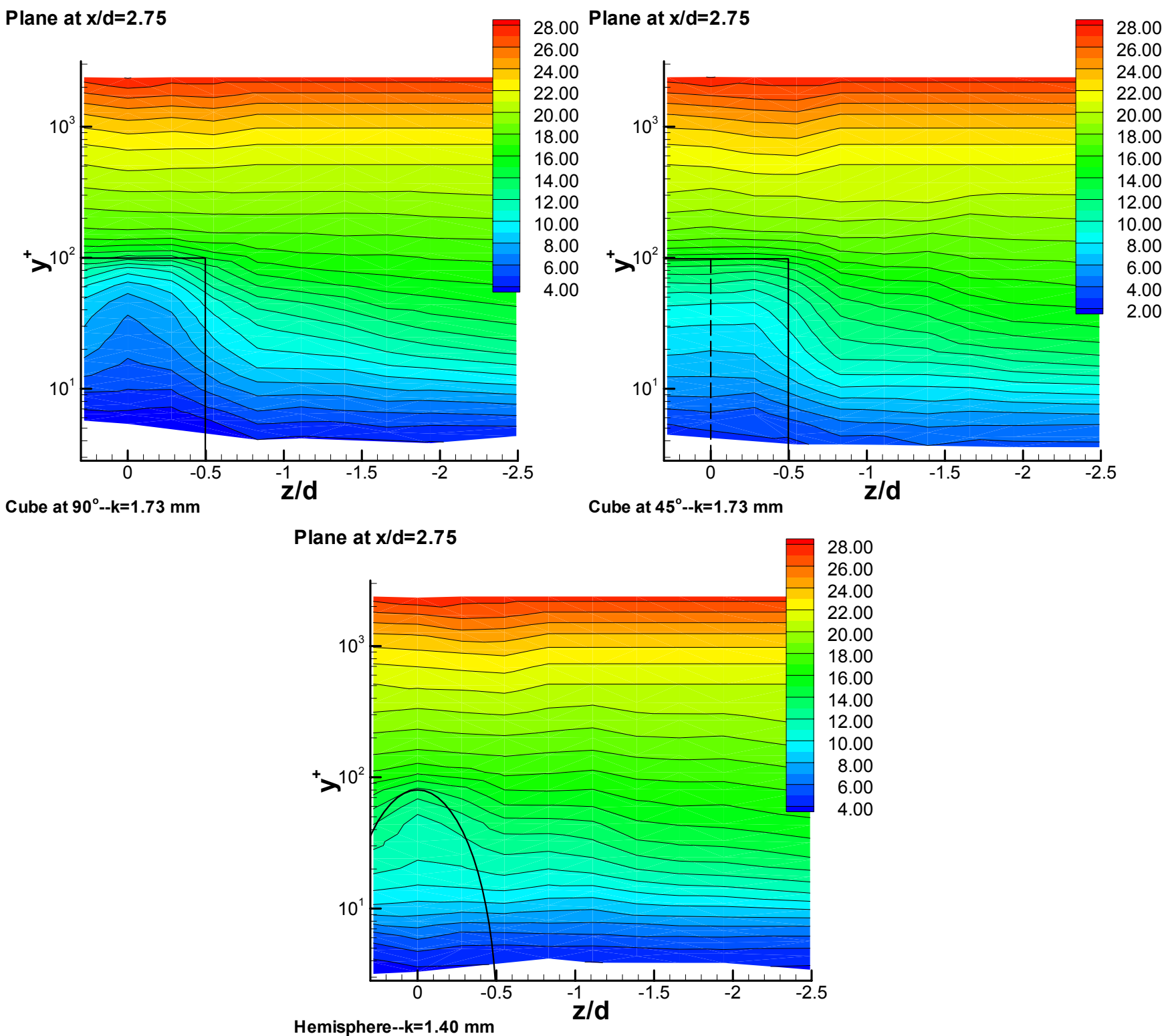

Figure 3.9: Contours of $U / U_{\tau}$ in the $\mathbf{y - z}$ plane at a location of $\mathbf{x} / \mathbf{d}=\mathbf{2 . 7 5}$ 

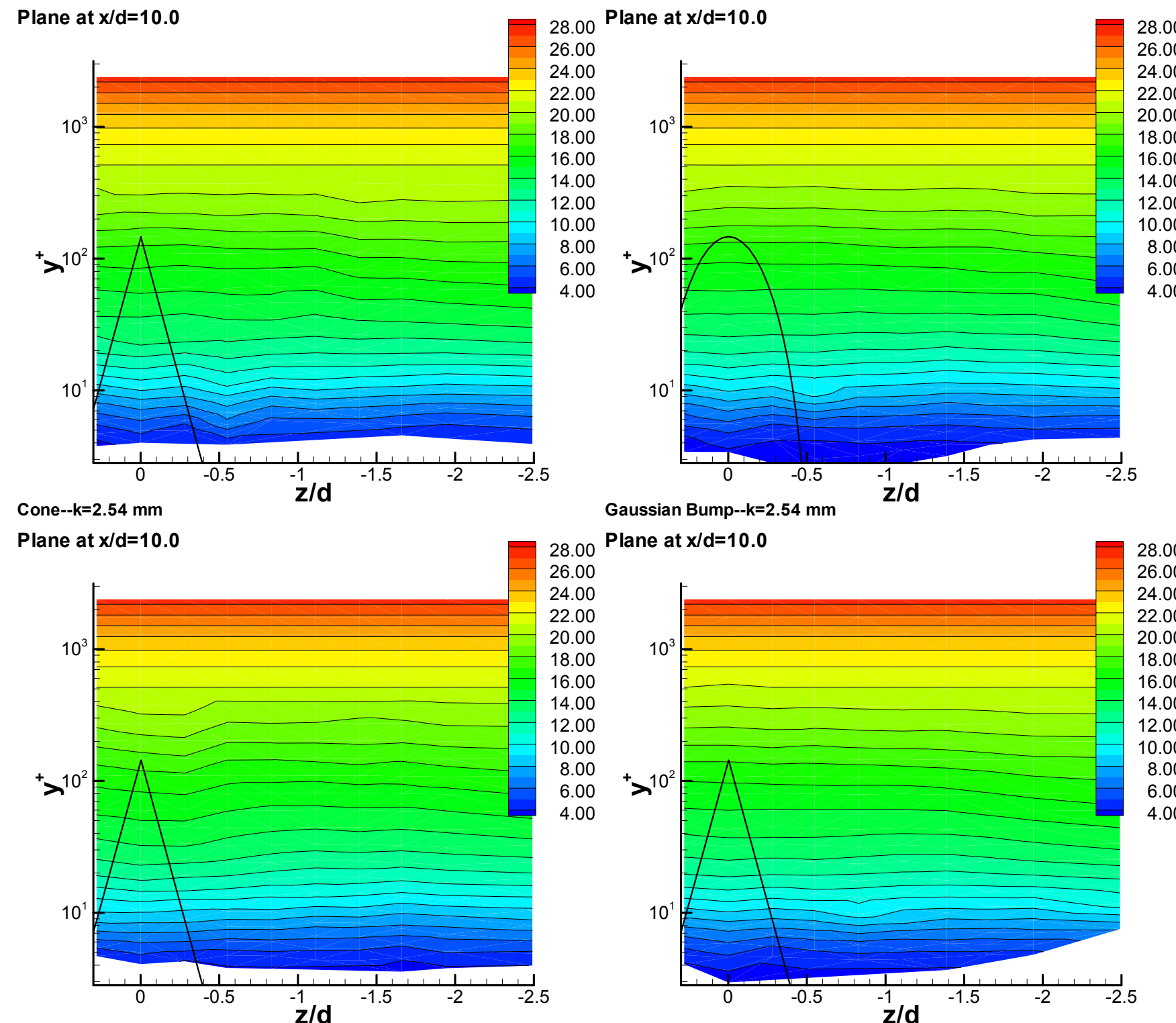

Gaussian Bump--k=2.54 mm

Plane at $x / d=10.0$

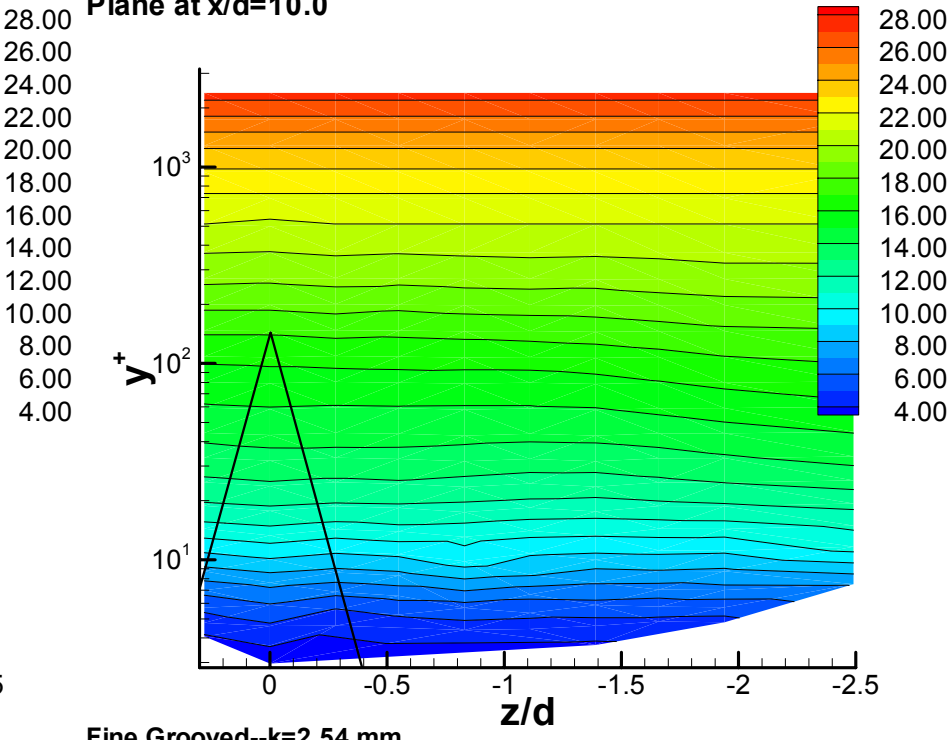

Large Grooved--k=2.54 mm

Fine Grooved--k=2.54 mm

Figure 3.10: Contours of $U / U_{\tau}$ in the $\mathbf{y - z}$ plane at a location of $\mathbf{x} / \mathbf{d}=\mathbf{1 0 . 0}$ 


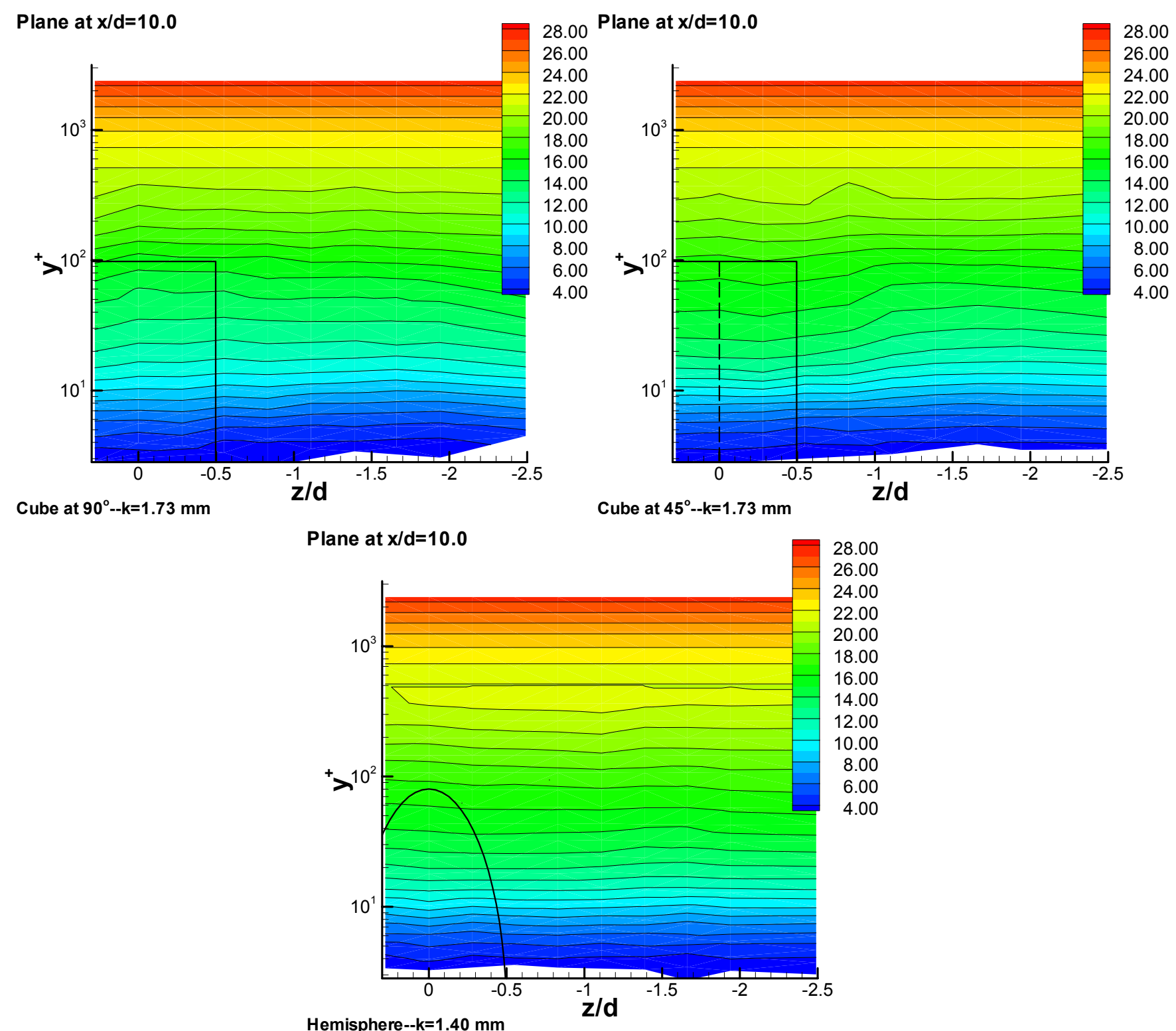

Figure 3.11: Contours of $U / U_{\tau}$ in the $y-z$ plane at a location of $x / d=10.0$ 


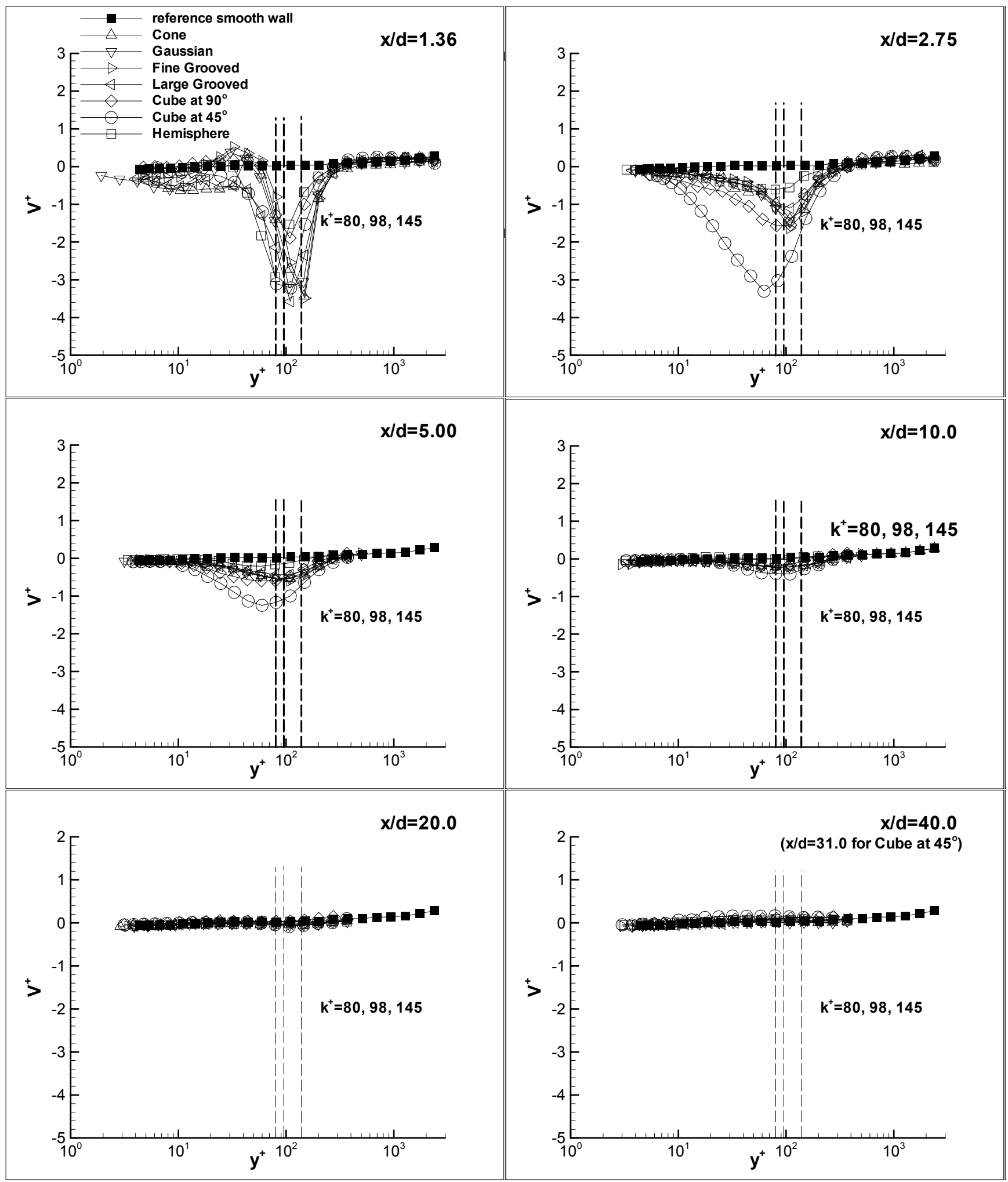

Figure 3.12: $V / U_{\tau}$ vs. ${ }^{y U_{\tau}} / v$, normal to wall mean velocity profiles along the centerline 

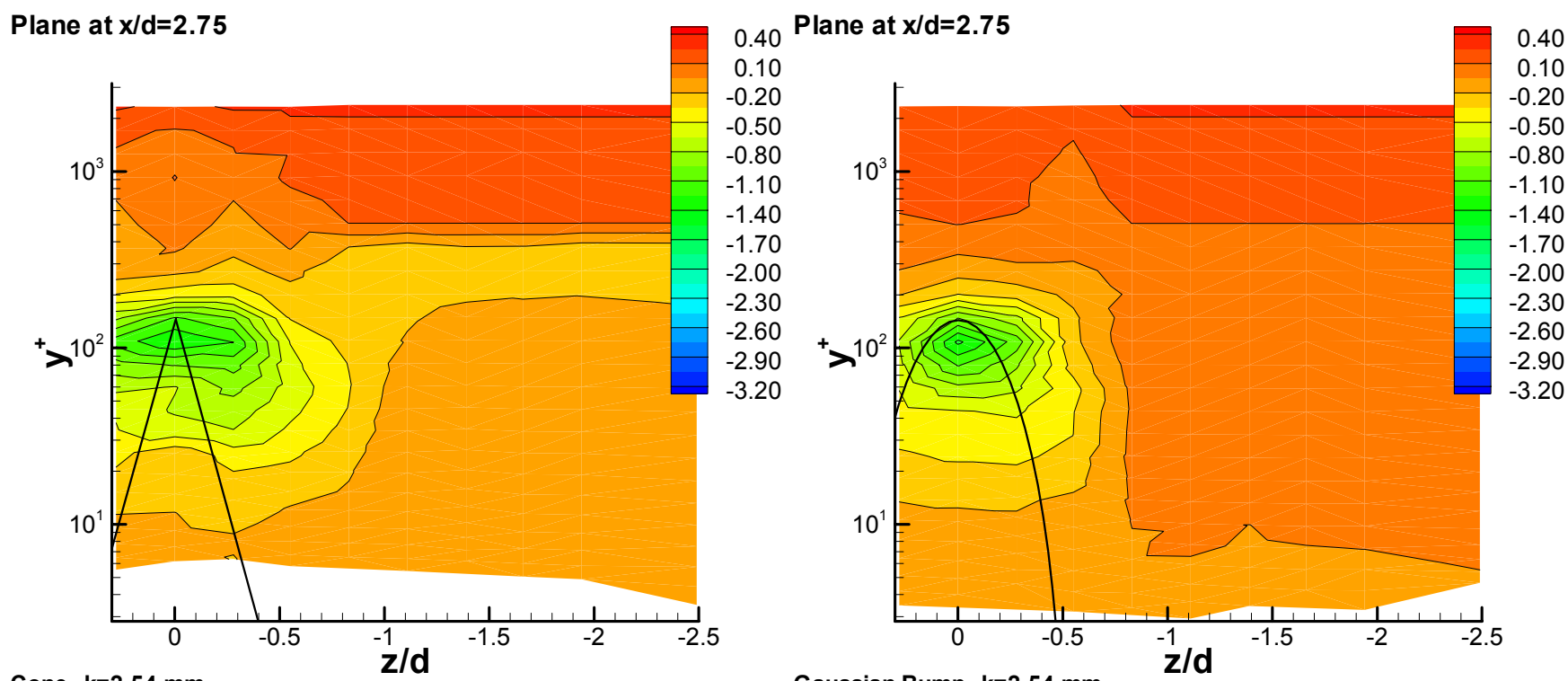

Gaussian Bump--k=2.54 mm

Plane at $x / d=2.75$

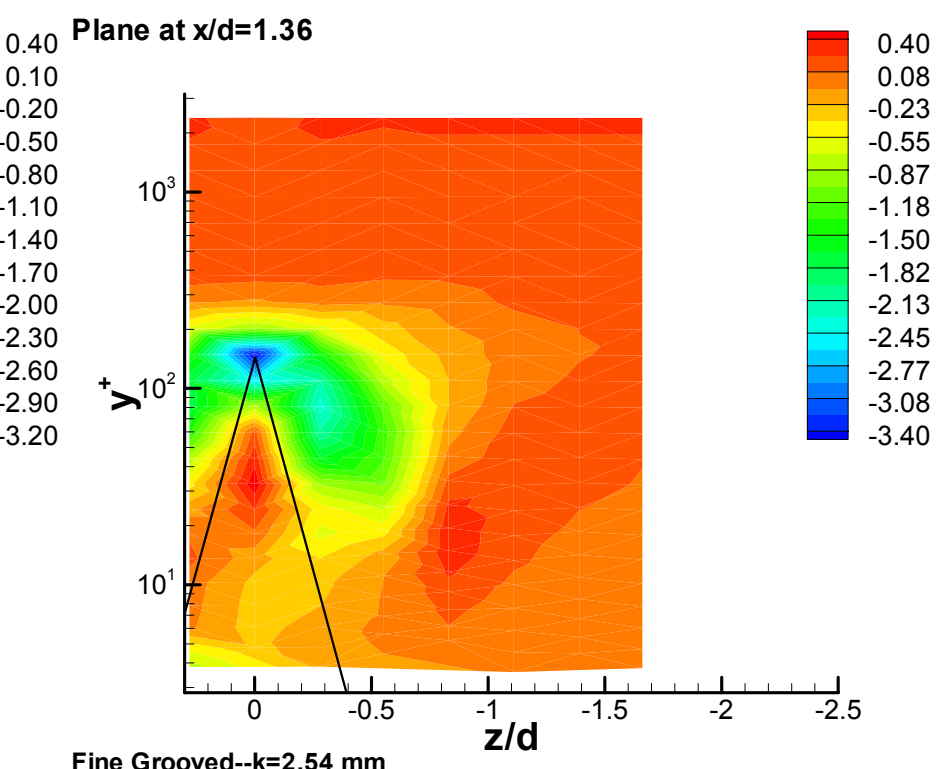

Large Grooved--k=2.54 mm

Fine Grooved--k=2.54 mm

Figure 3.13: Contours of $V / U_{\tau}$ in the $\mathbf{y - z}$ plane at a location of $\mathbf{x} / \mathbf{d}=\mathbf{2 . 7 5}$ (Note: Fine Grooved $\mathbf{x} / \mathbf{d}=1.36$ ) 


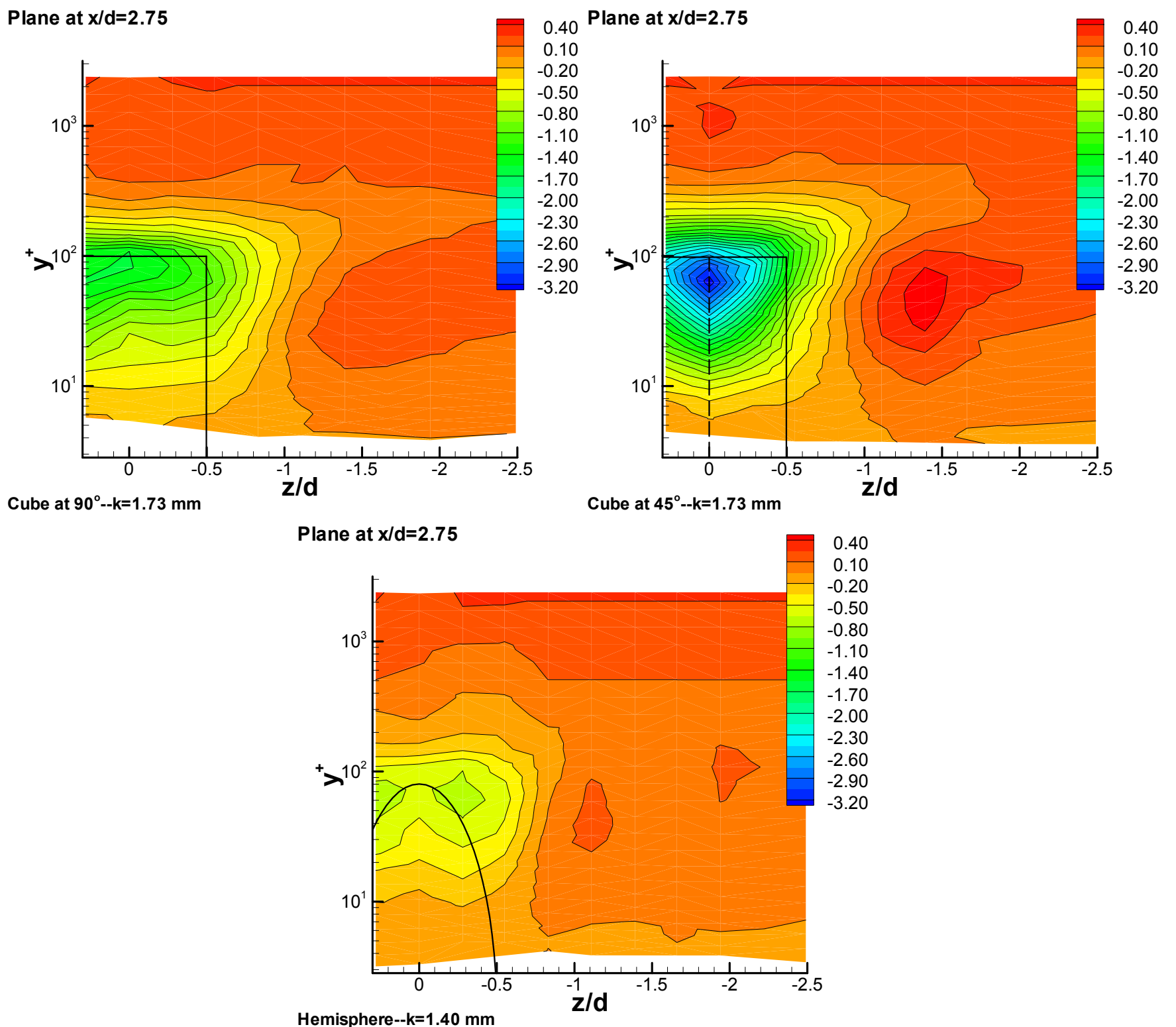

Figure 3.14: Contours of $V / U_{\tau}$ in the $\mathbf{y - z}$ plane at a location of $\mathbf{x} / \mathbf{d}=\mathbf{2 . 7 5}$ 

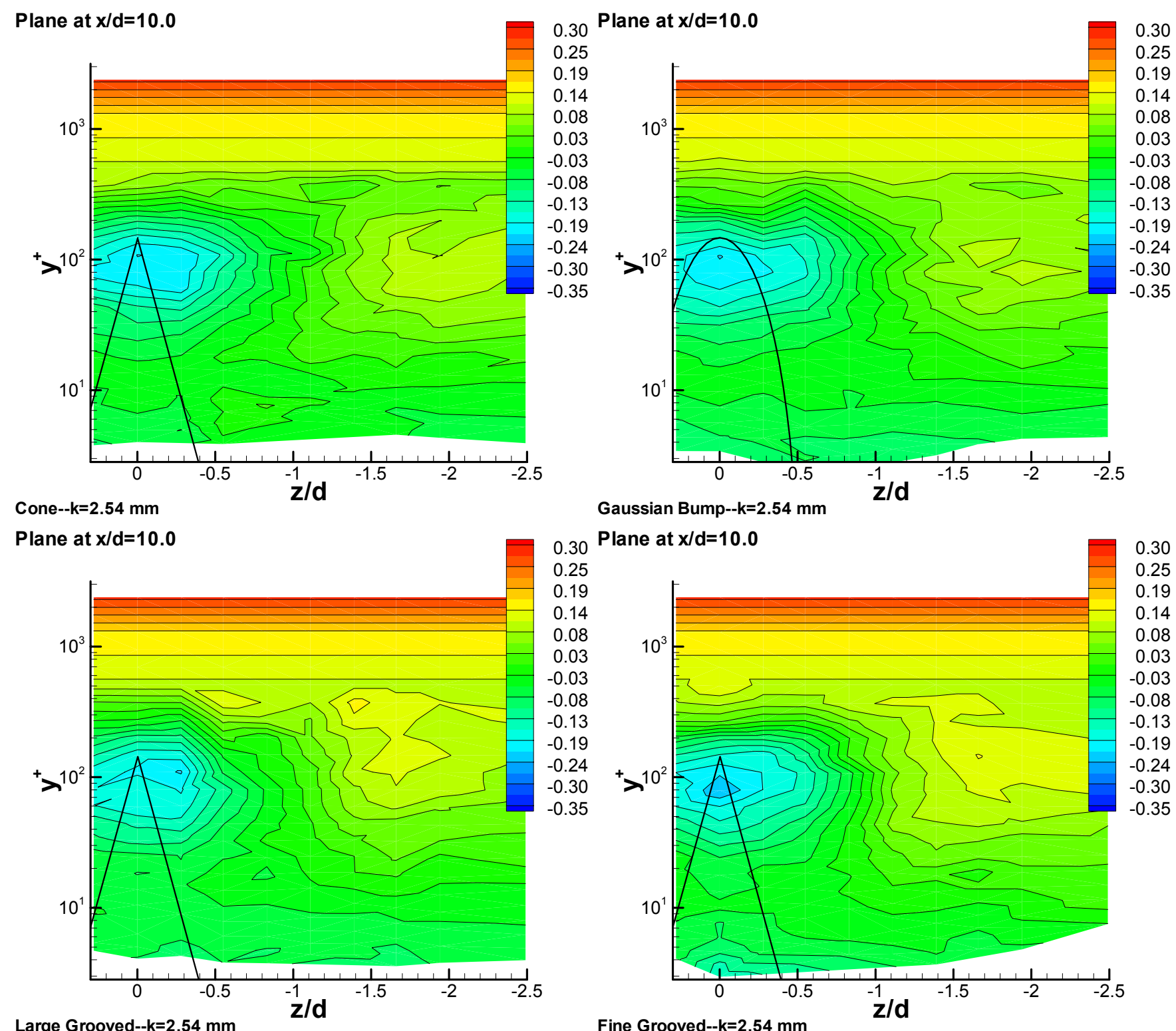

Figure 3.15: Contours of $V / U_{\tau}$ in the $\mathbf{y}-z$ plane at a location of $\mathbf{x} / \mathbf{d}=10.0$ 


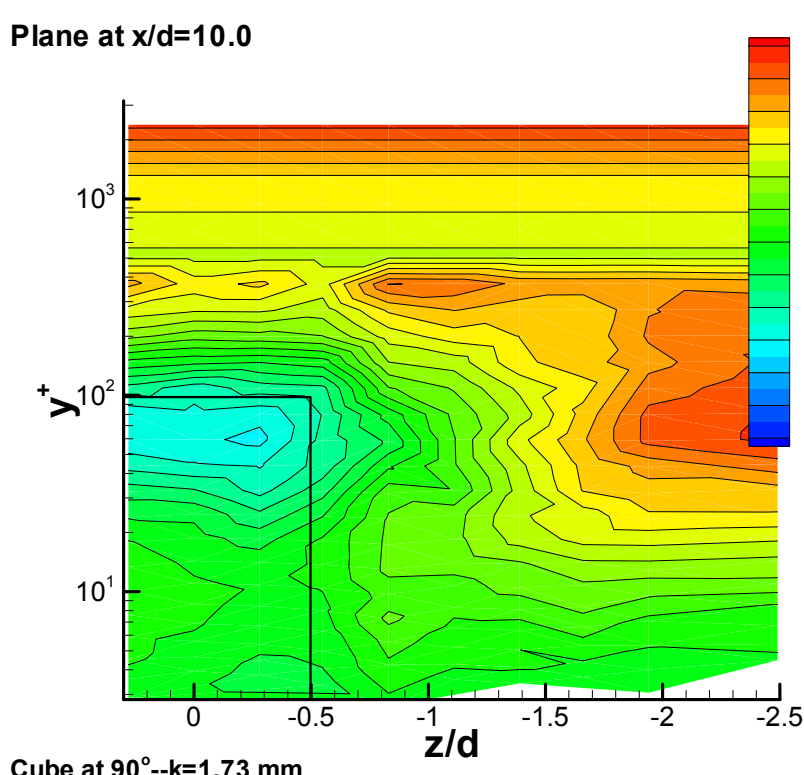

Cube at $90^{\circ}--\mathrm{k}=1.73 \mathrm{~mm}$

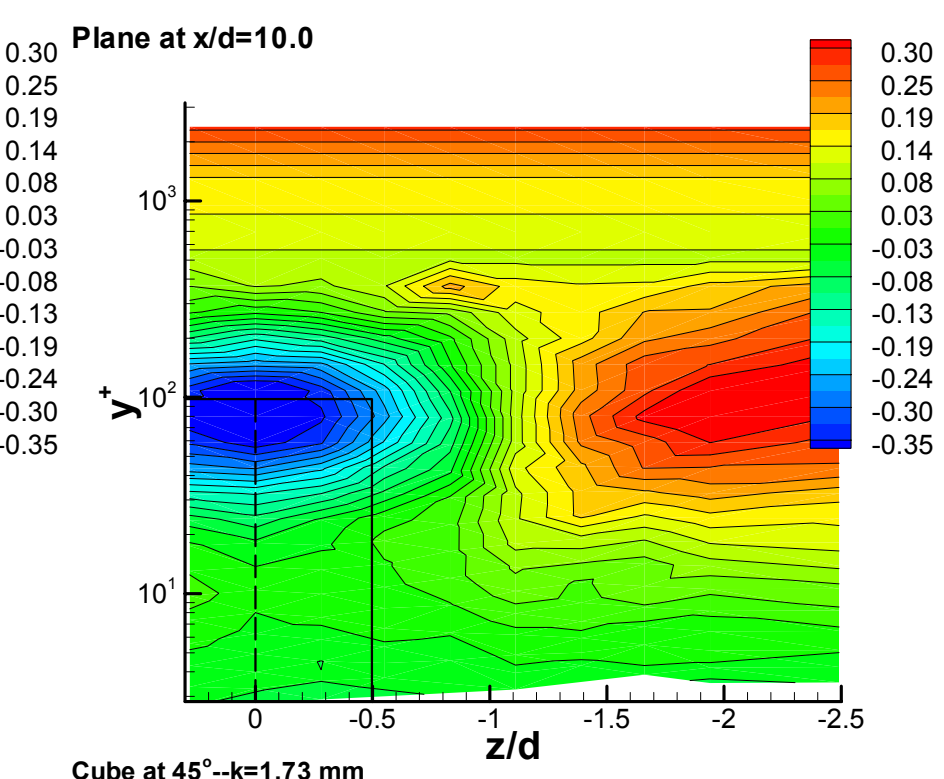

Cube at $45^{\circ}--\mathrm{k}=1.73 \mathrm{~mm}$

Plane at $x / d=10.0$

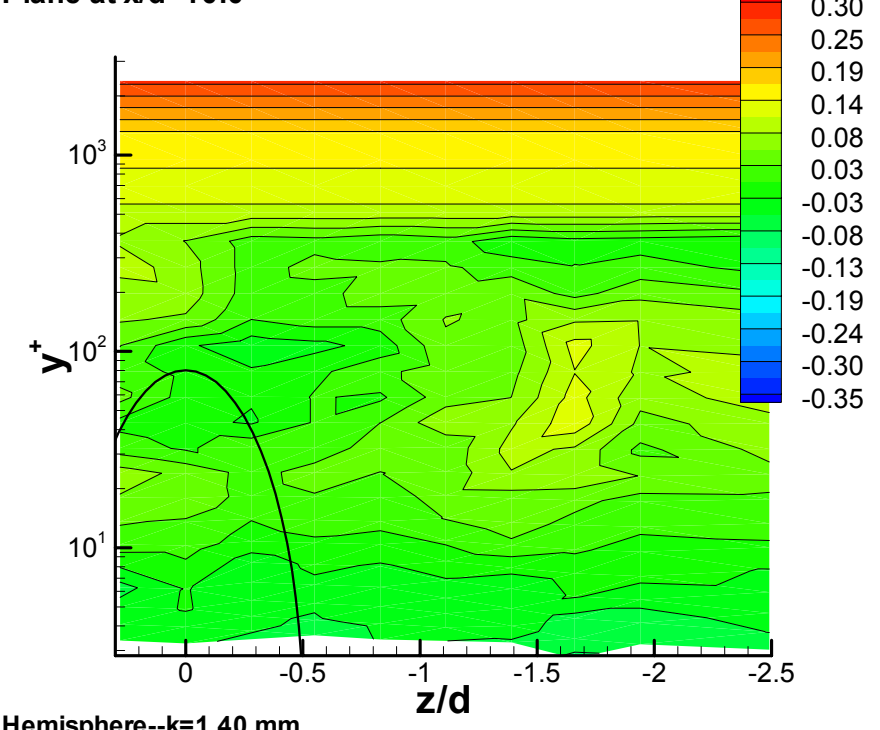

Figure 3.16: Contours of $V / U_{\tau}$ in the $\mathbf{y - z}$ plane at a location of $\mathbf{x} / \mathbf{d}=\mathbf{1 0 . 0}$ 

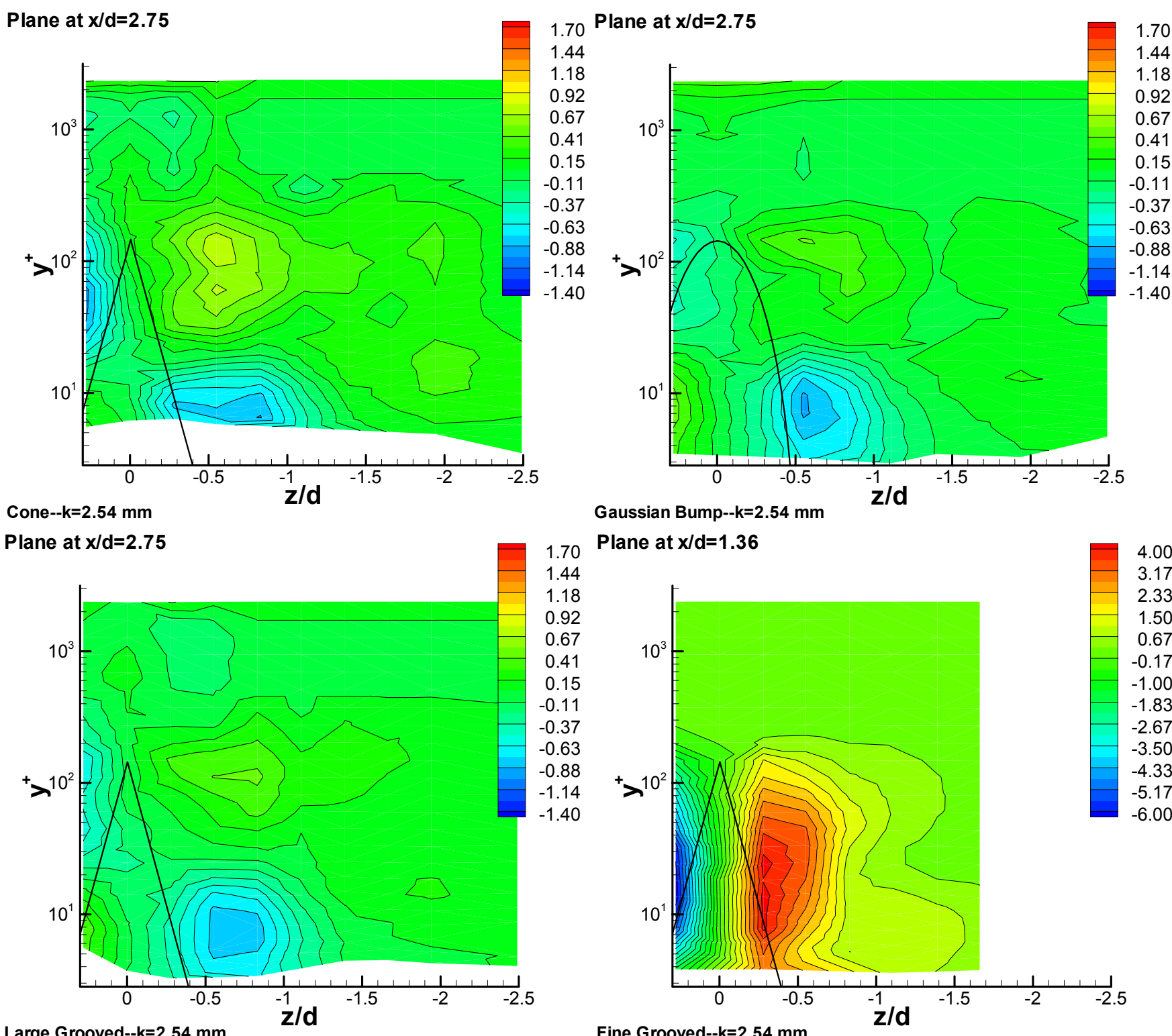

Gaussian Bump--k=2.54 mm

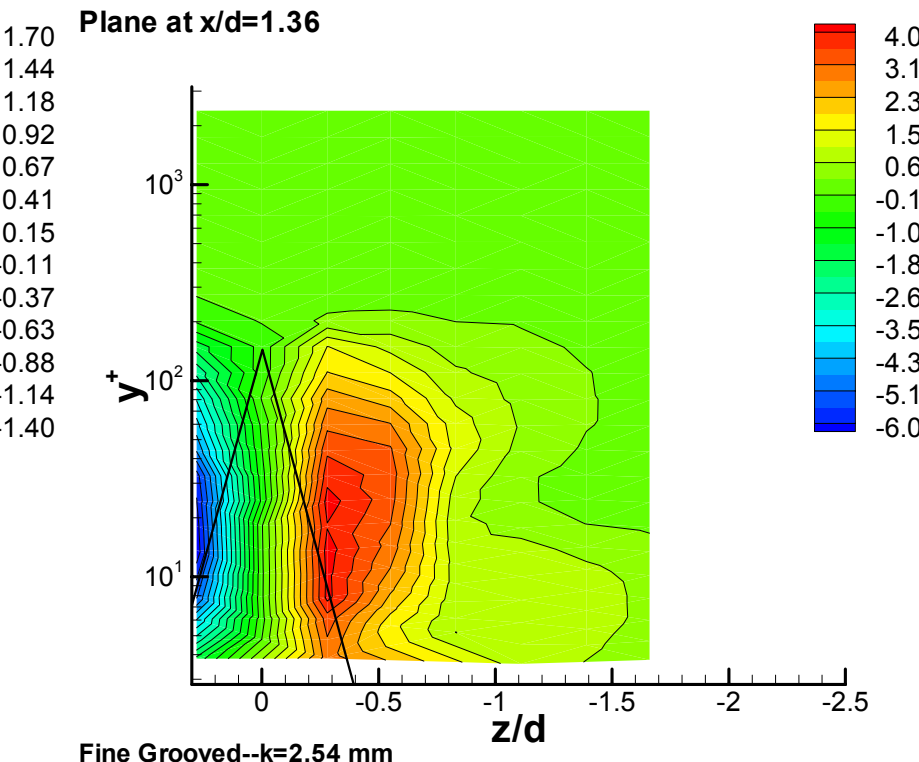

Figure 3.17: Contours of $W / U_{\tau}$ in the $\mathbf{y - z}$ plane at a location of $\mathbf{x} / \mathbf{d}=\mathbf{2 . 7 5}$ (Note: Fine Grooved $\mathbf{x} / \mathbf{d}=\mathbf{1 . 3 6}$ ) 


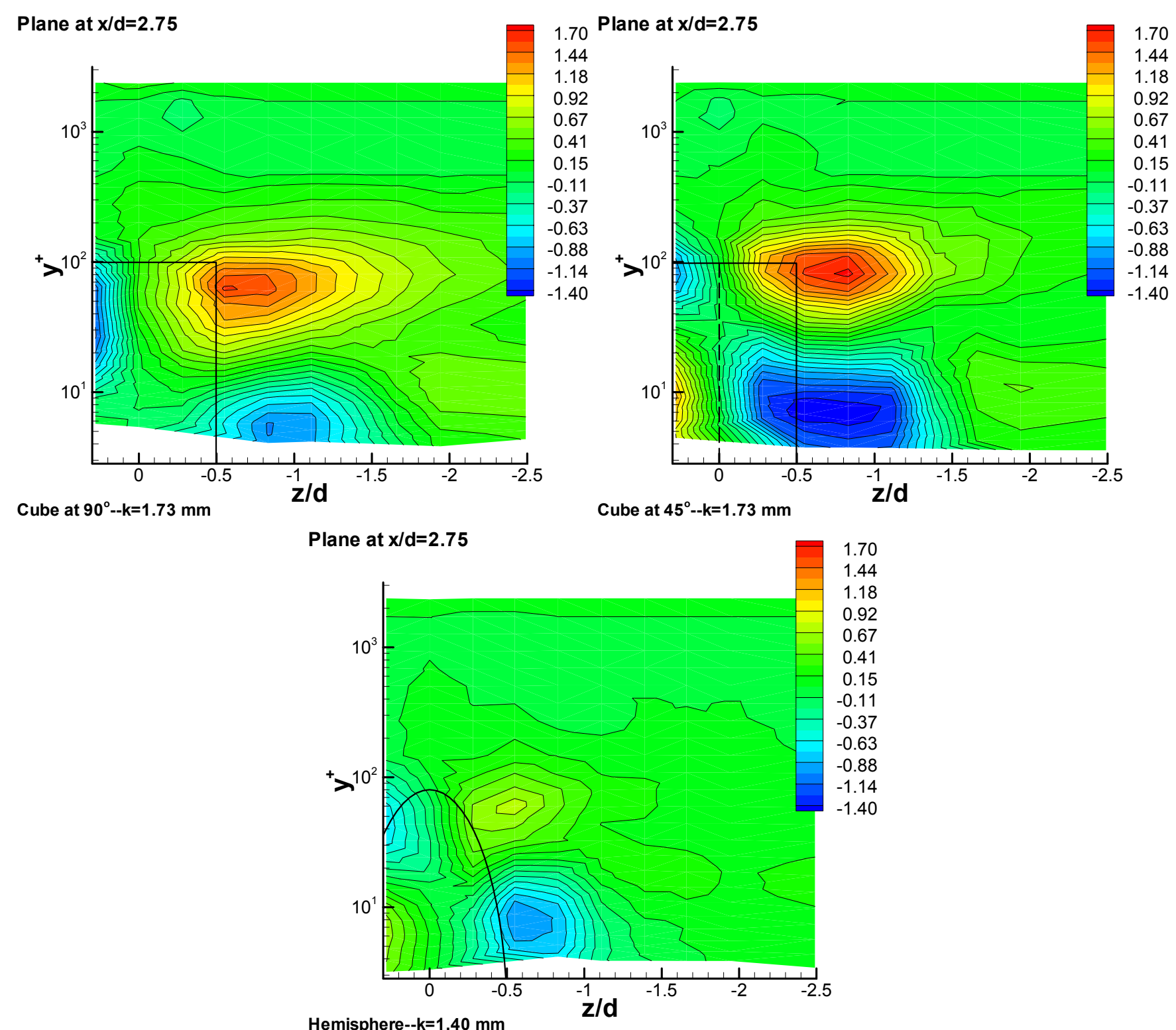

Figure 3.18: Contours of $W / U_{\tau}$ in the $y-z$ plane at a location of $\mathbf{x} / \mathbf{d}=\mathbf{2 . 7 5}$ 

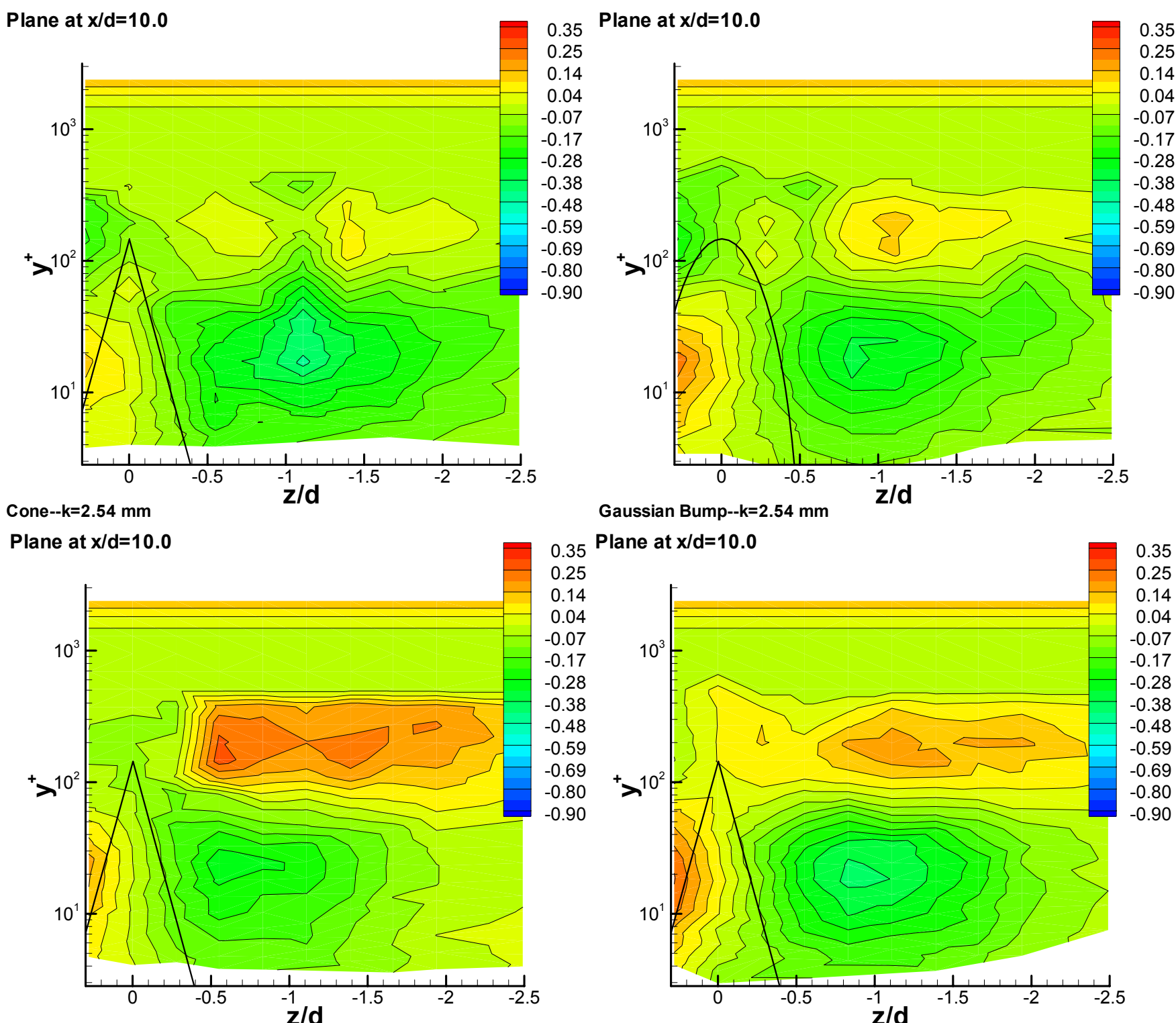

Gaussian Bump--k=2.54 mm

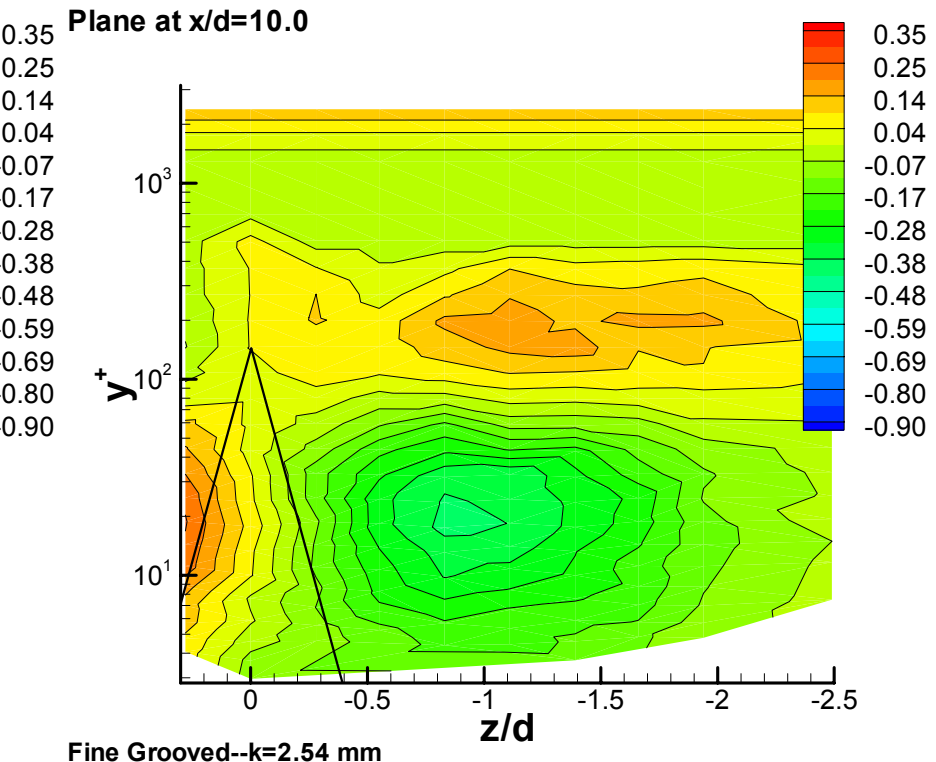

Large Grooved--k=2.54 mm

Fine Grooved--k=2.54 mm

Figure 3.19: Contours of $W / U_{\tau}$ in the $\mathbf{y}-\mathbf{z}$ plane at a location of $\mathbf{x} / \mathbf{d}=\mathbf{1 0 . 0}$ 


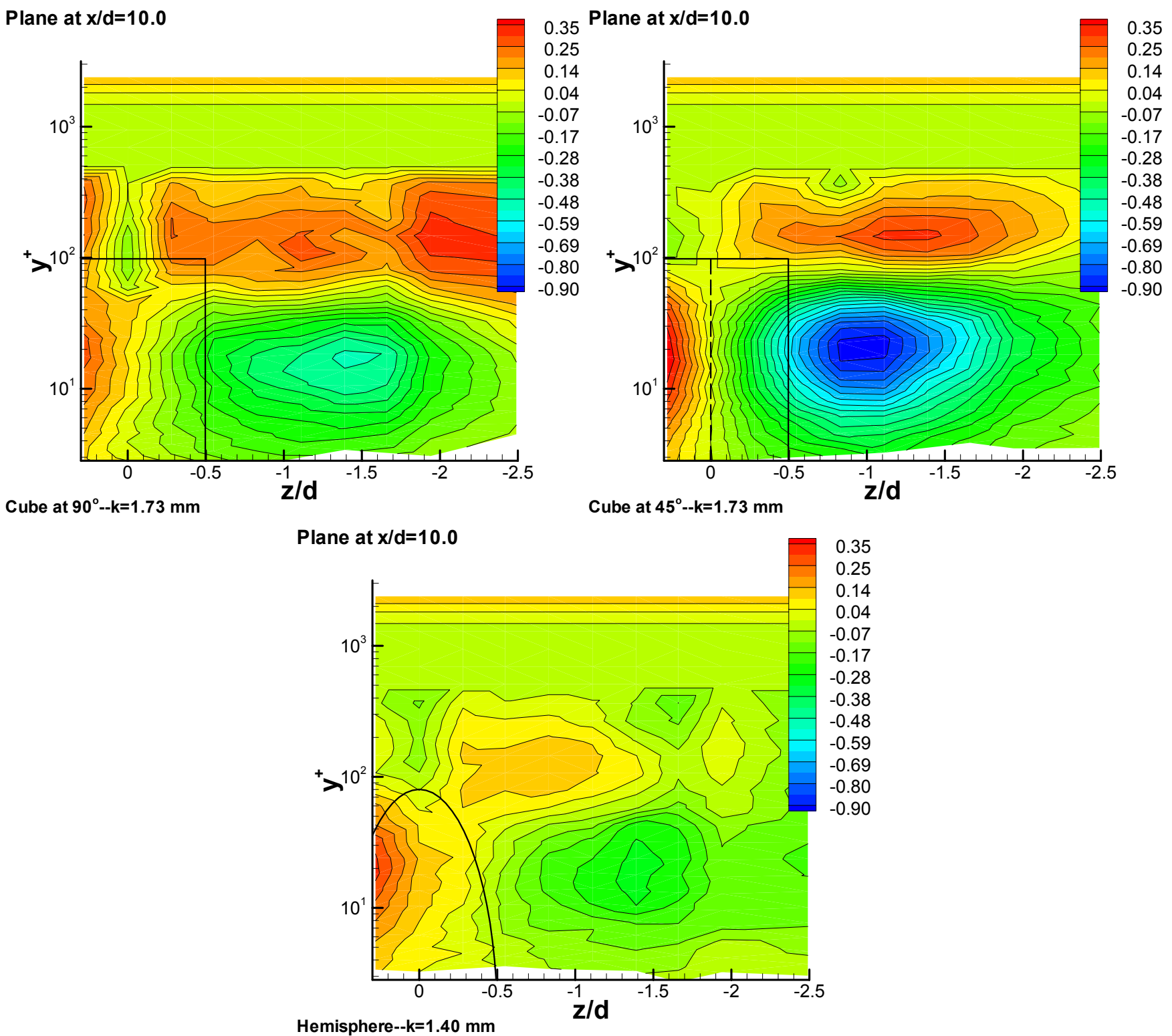

Figure 3.20: Contours of $W / U_{\tau}$ in the $y-z$ plane at a location of $\mathbf{x} / \mathbf{d}=\mathbf{1 0 . 0}$ 

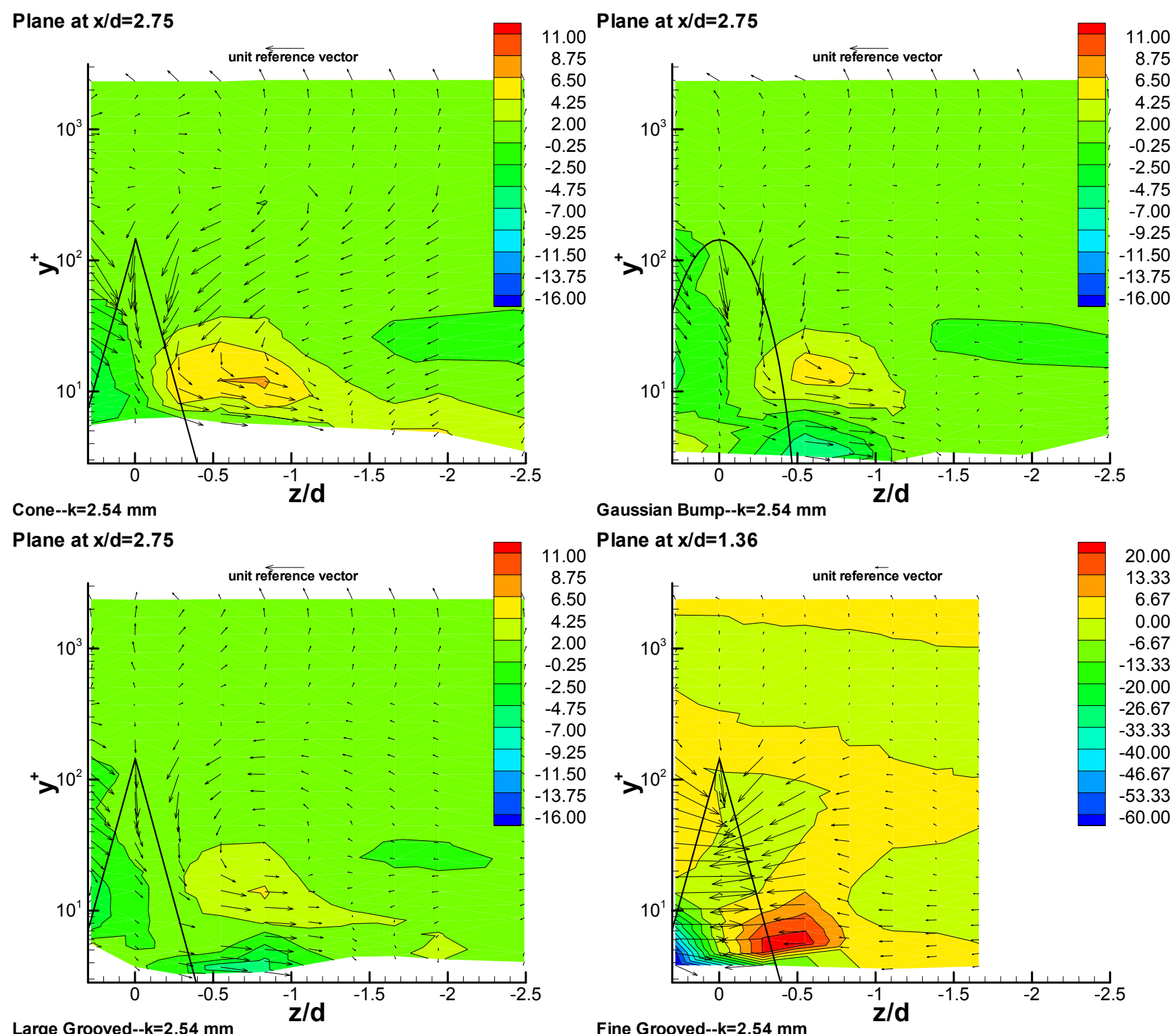

Gaussian Bump--k=2.54 mm

Plane at $x / d=1.36$

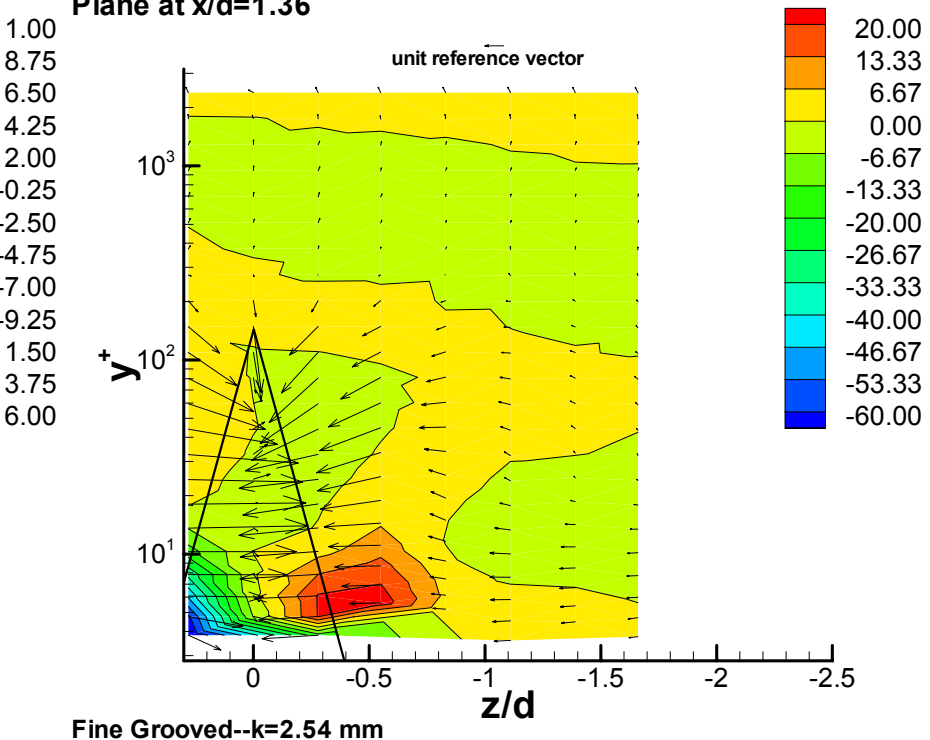

Figure 3.21: Contours of normalized streamwise vorticity, $\Omega_{x} \sqrt{A_{f}} / U_{\tau}$, with normalized secondary velocity vectors $V / U_{\tau}$ and $W / U_{\tau}$ in the $\mathbf{y}-\mathbf{z}$ plane at a location of $\mathbf{x} / \mathbf{d}=\mathbf{2 . 7 5}$ (Note: Fine Grooved $\mathbf{x} / \mathbf{d}=\mathbf{1 . 3 6}$ ) 


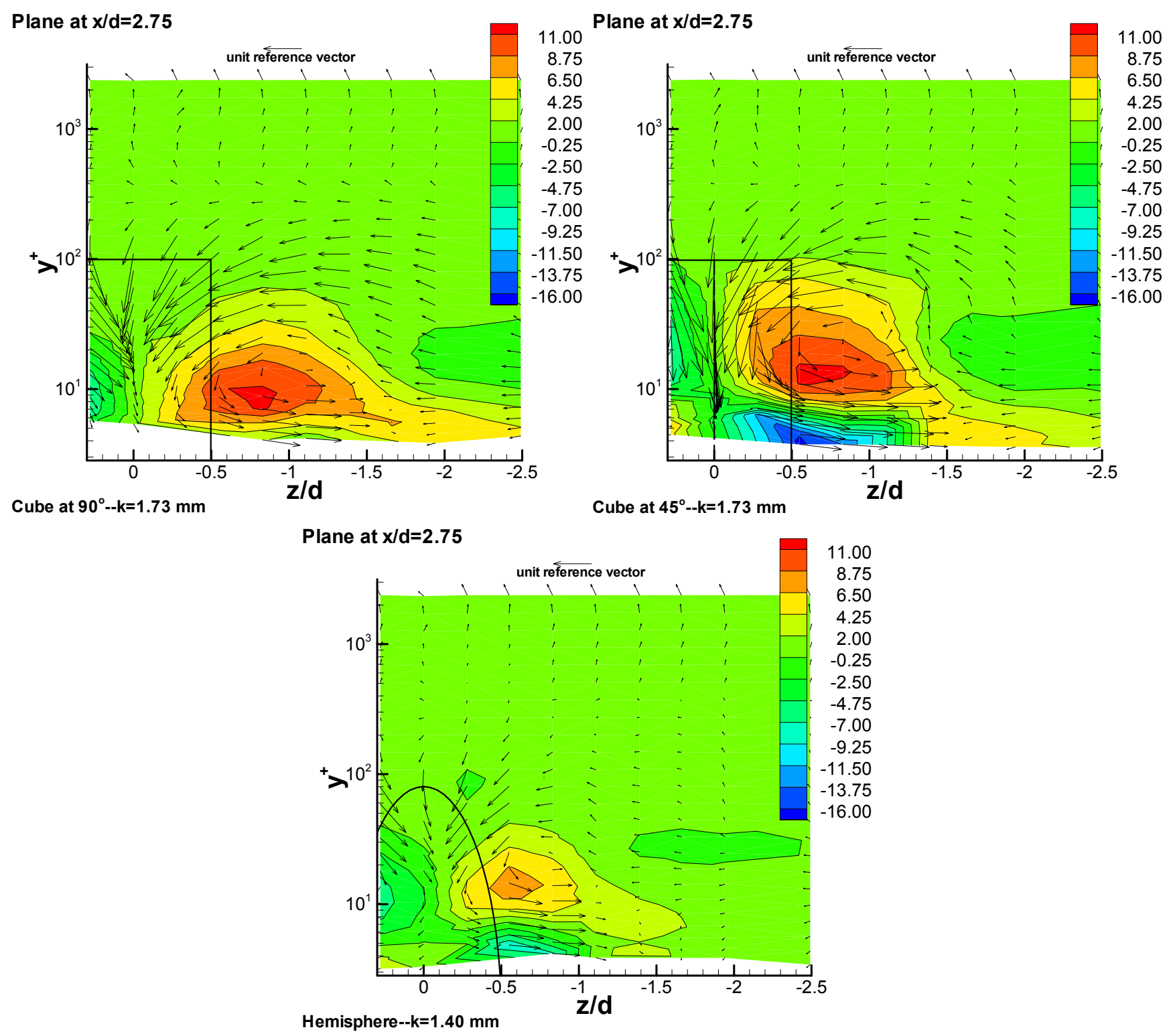

Figure 3.22: Contours of normalized streamwise vorticity, $\Omega_{x} \sqrt{A_{f}} / U_{\tau}$, with normalized secondary velocity vectors $V / U_{\tau}$ and $W / U_{\tau}$ in the $\mathbf{y}-z$ plane at a location of $\mathbf{x} / \mathbf{d}=\mathbf{2 . 7 5}$ 

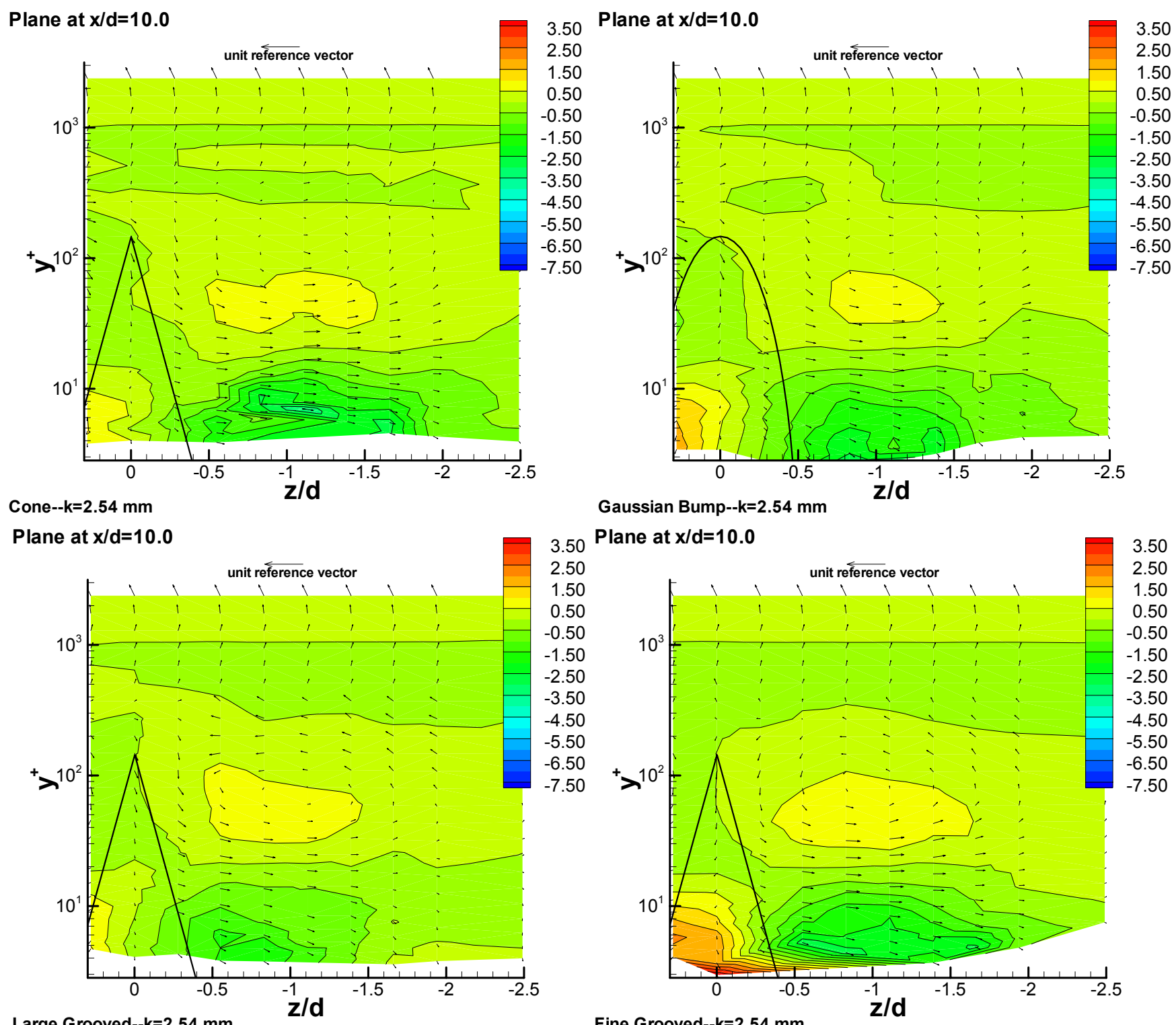

Gaussian Bump--k=2.54 mm

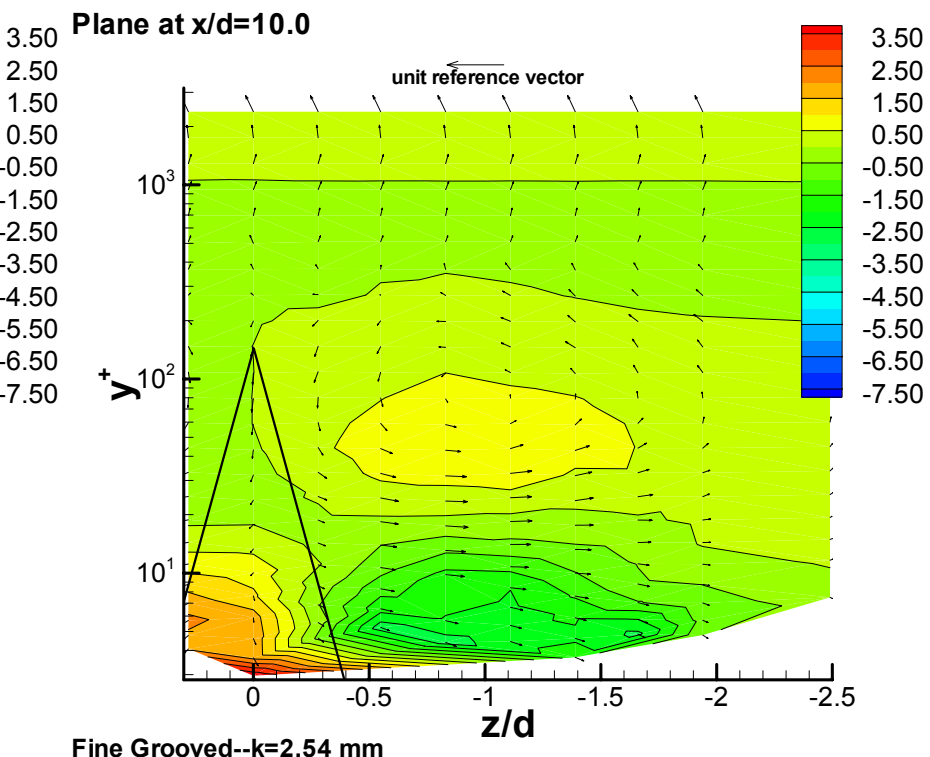

Figure 3.23: Contours of normalized streamwise vorticity, $\Omega_{x} \sqrt{A_{f}} / U_{\tau}$, with normalized secondary velocity vectors $V / U_{\tau}$ and $W / U_{\tau}$ in the $\mathbf{y}-\mathbf{z}$ plane at a location of $\mathbf{x} / \mathbf{d}=\mathbf{1 0 . 0}$ 


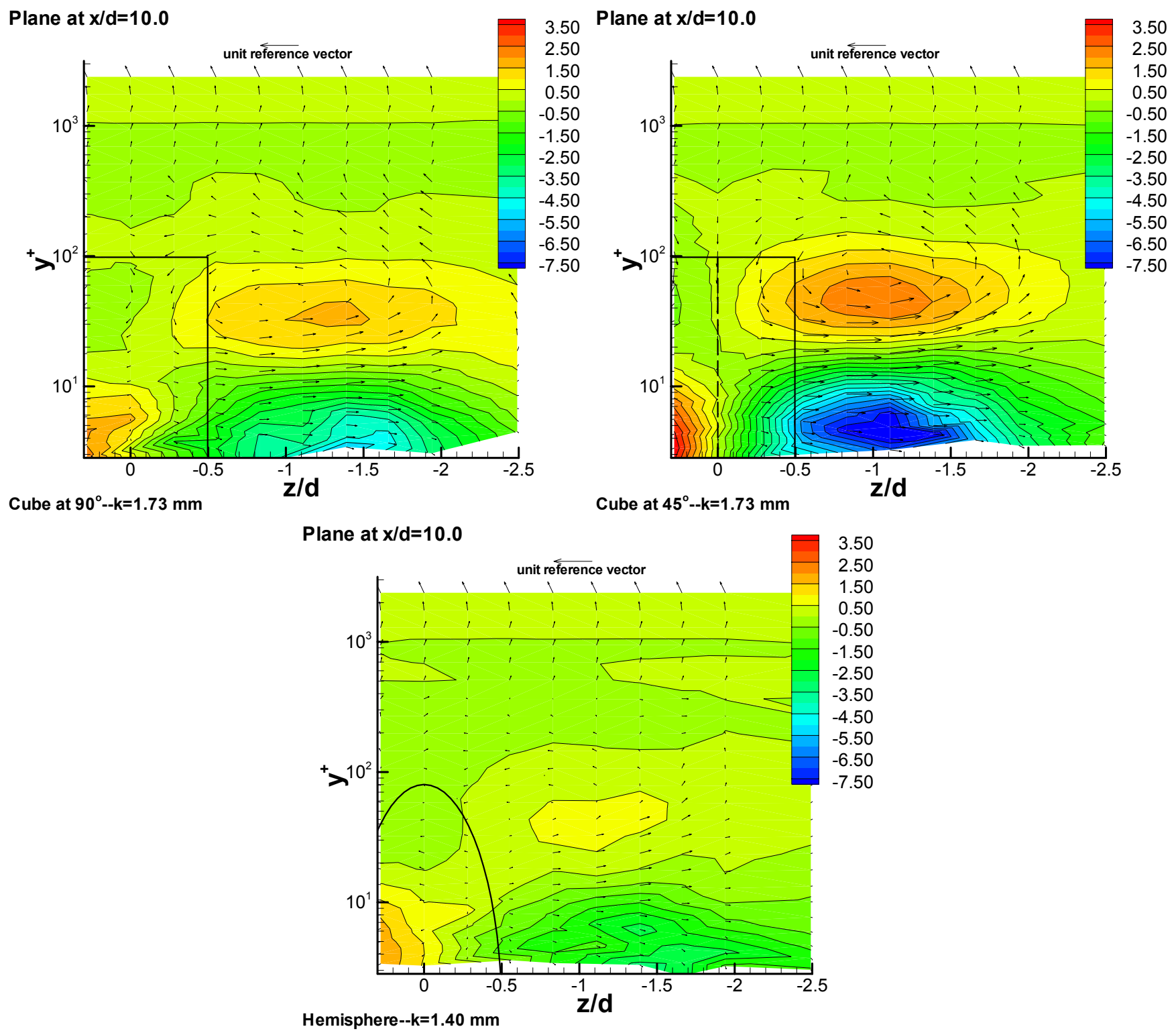

Figure 3.24: Contours of normalized streamwise vorticity, $\Omega_{x} \sqrt{A_{f}} / U_{\tau}$, with normalized secondary velocity vectors $V / U_{\tau}$ and $W / U_{\tau}$ in the $\mathbf{y}-\mathbf{z}$ plane at a location of $\mathbf{x} / \mathbf{d}=\mathbf{1 0 . 0}$ 


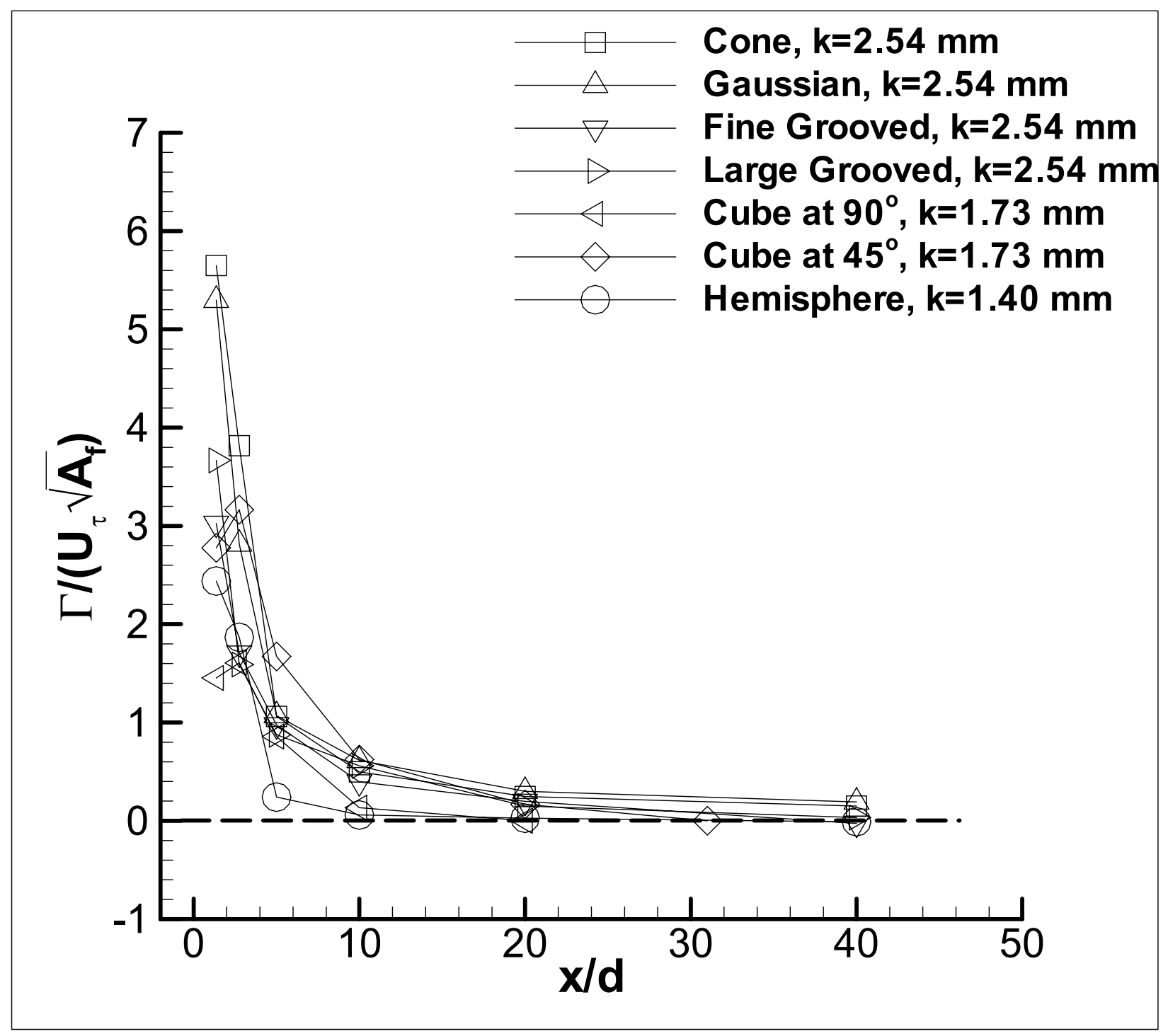

Figure 3.25: Variation of circulation, $\Gamma /\left(U_{\tau} \sqrt{A_{f}}\right)$, with streamwise distance, $x / \mathbf{d}$ 


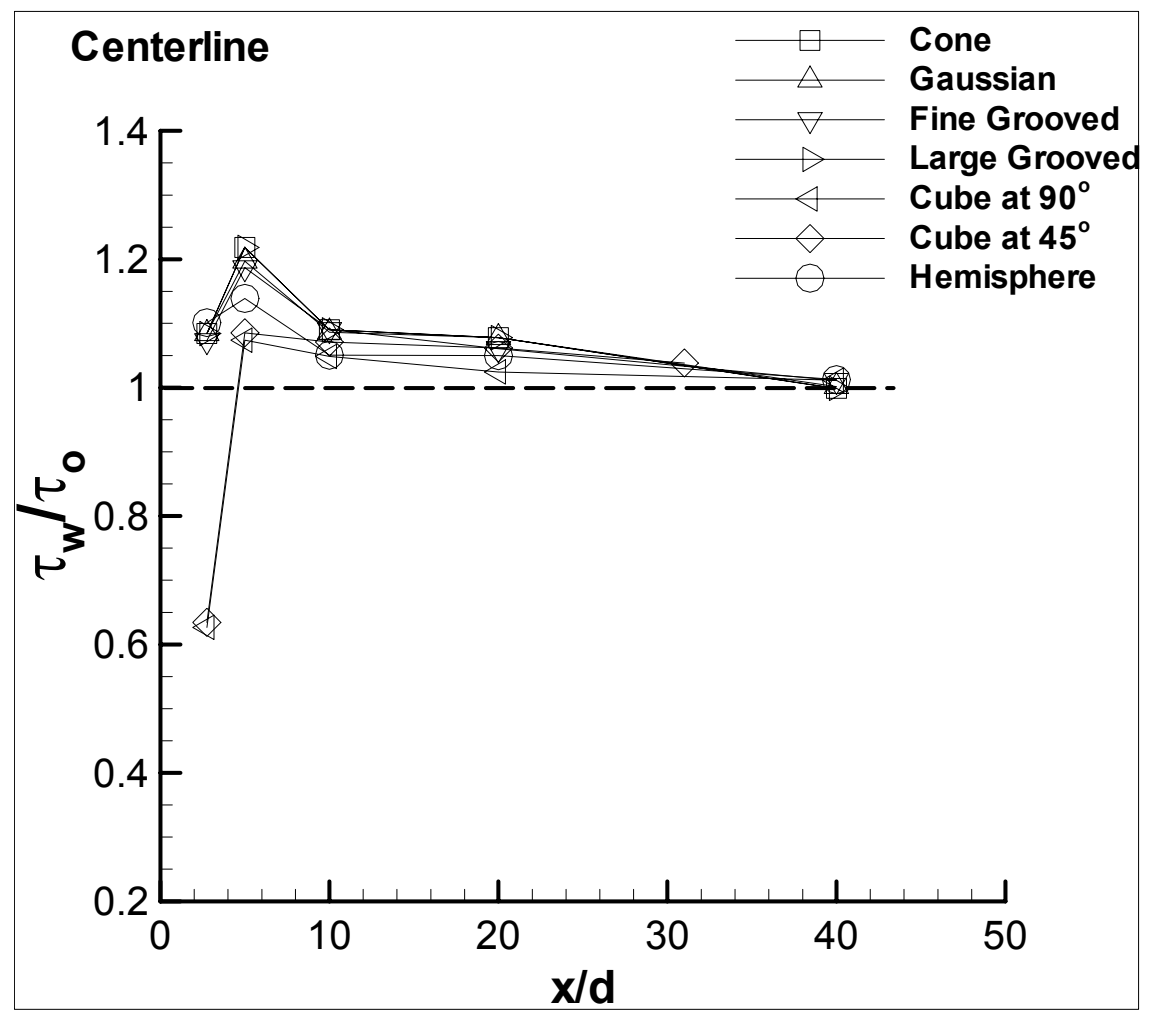

Figure 3.26: Variation of normalized wall shear $\left(\tau_{\mathrm{w}} / \tau_{\mathrm{o}}\right)$ along centerline

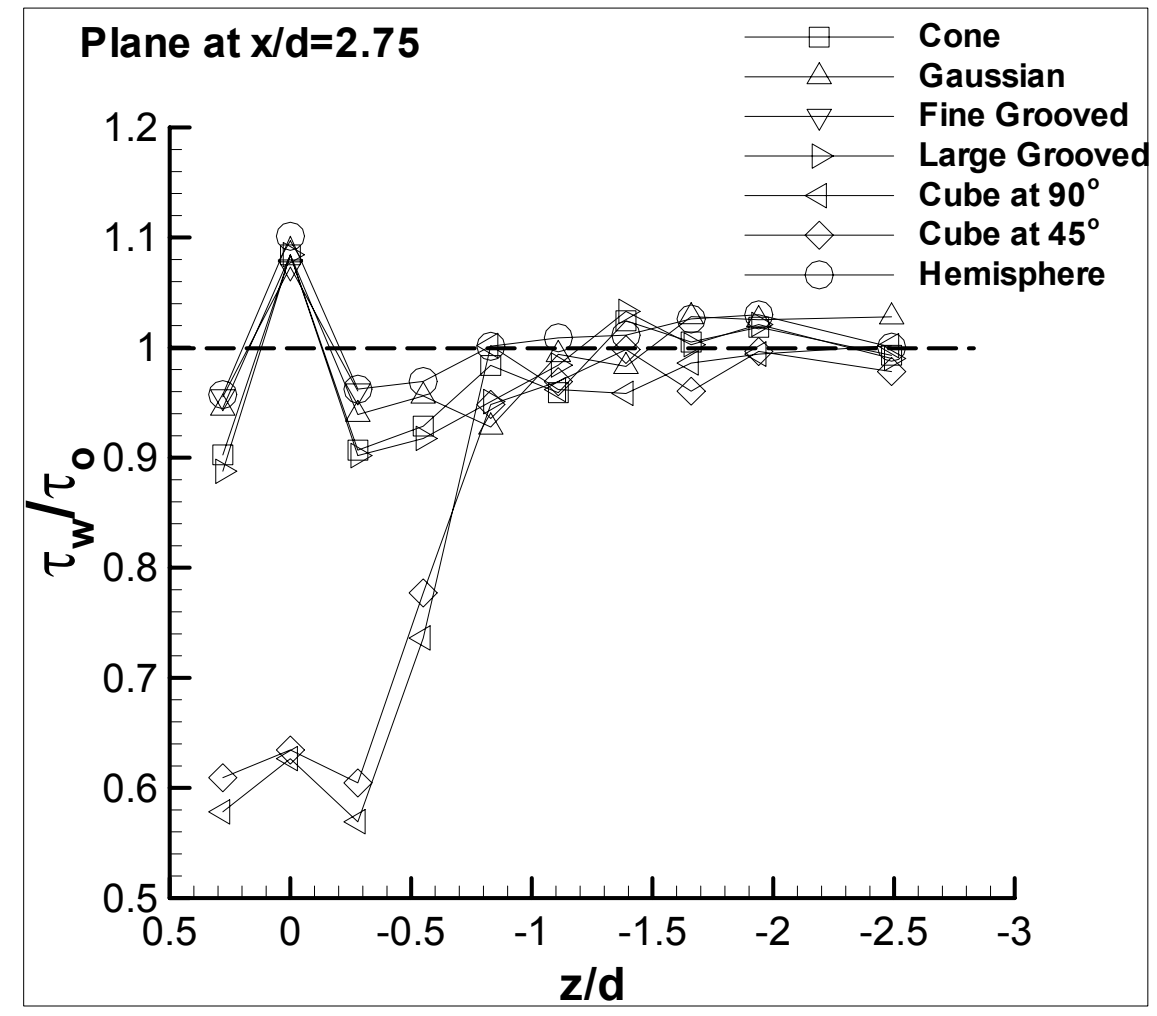

Figure 3.27: Variation of normalized wall shear $\left(\tau_{w} / \tau_{0}\right)$ at $x / d=2.75$ plane 


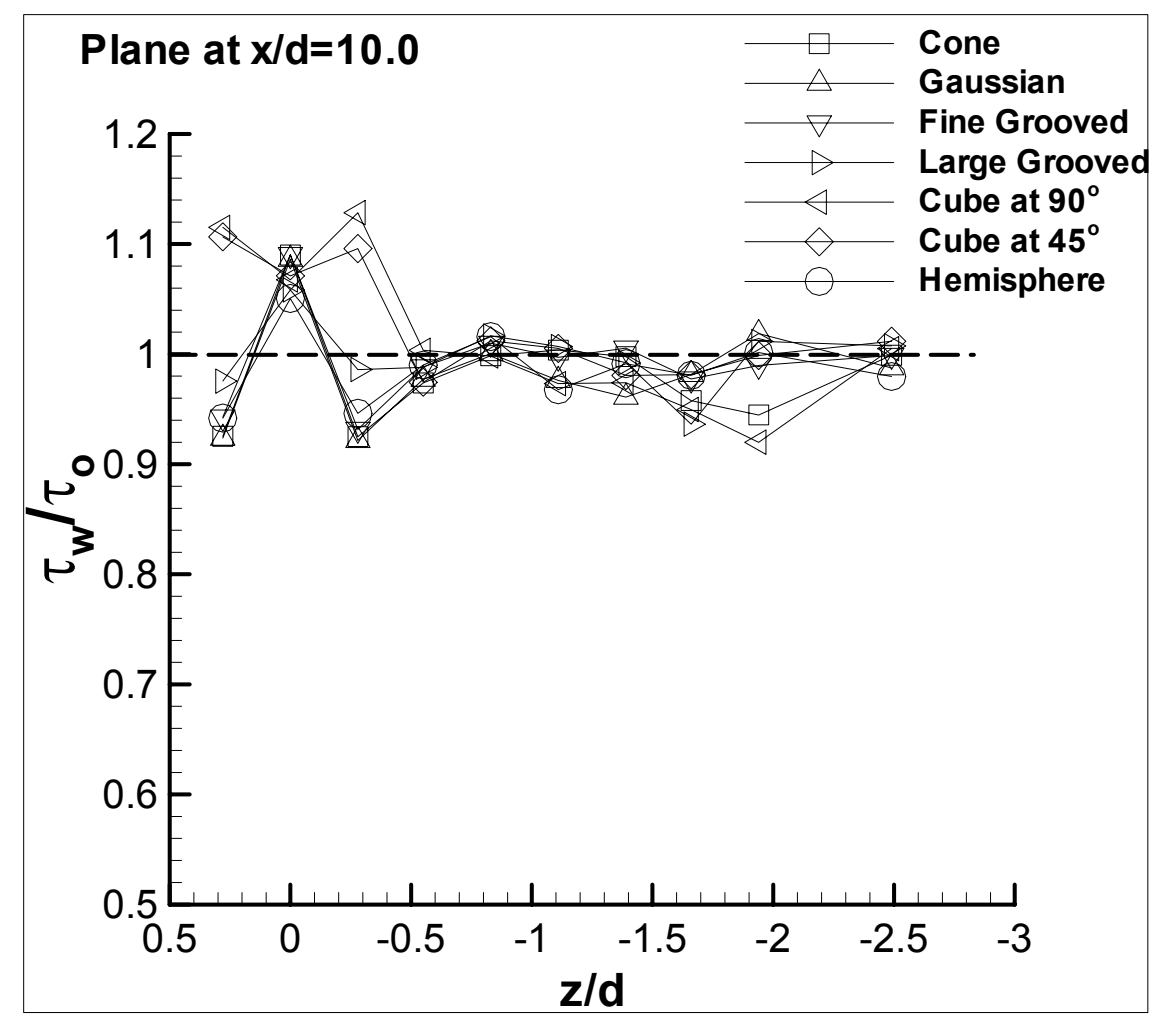

Figure 3.28: Variation of normalized wall shear $\left(\tau_{w} / \tau_{0}\right)$ at $\mathbf{x} / \mathbf{d}=10.0$ plane 


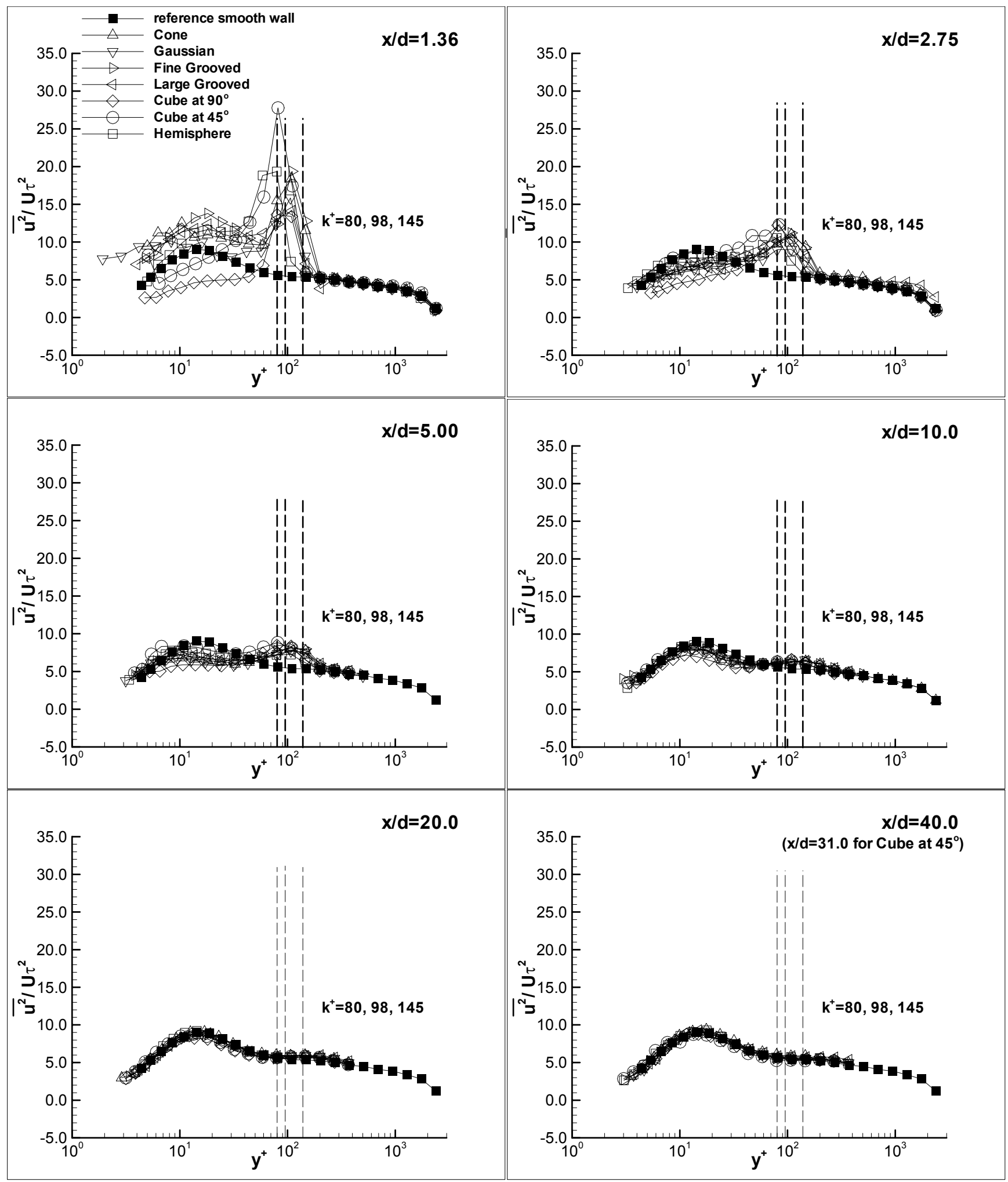

Figure 3.29: $\overline{u^{2}} / U_{\tau}^{2}$ vs. $y U_{\tau} / v$, streamwise Reynolds normal stress profiles along the centerline 

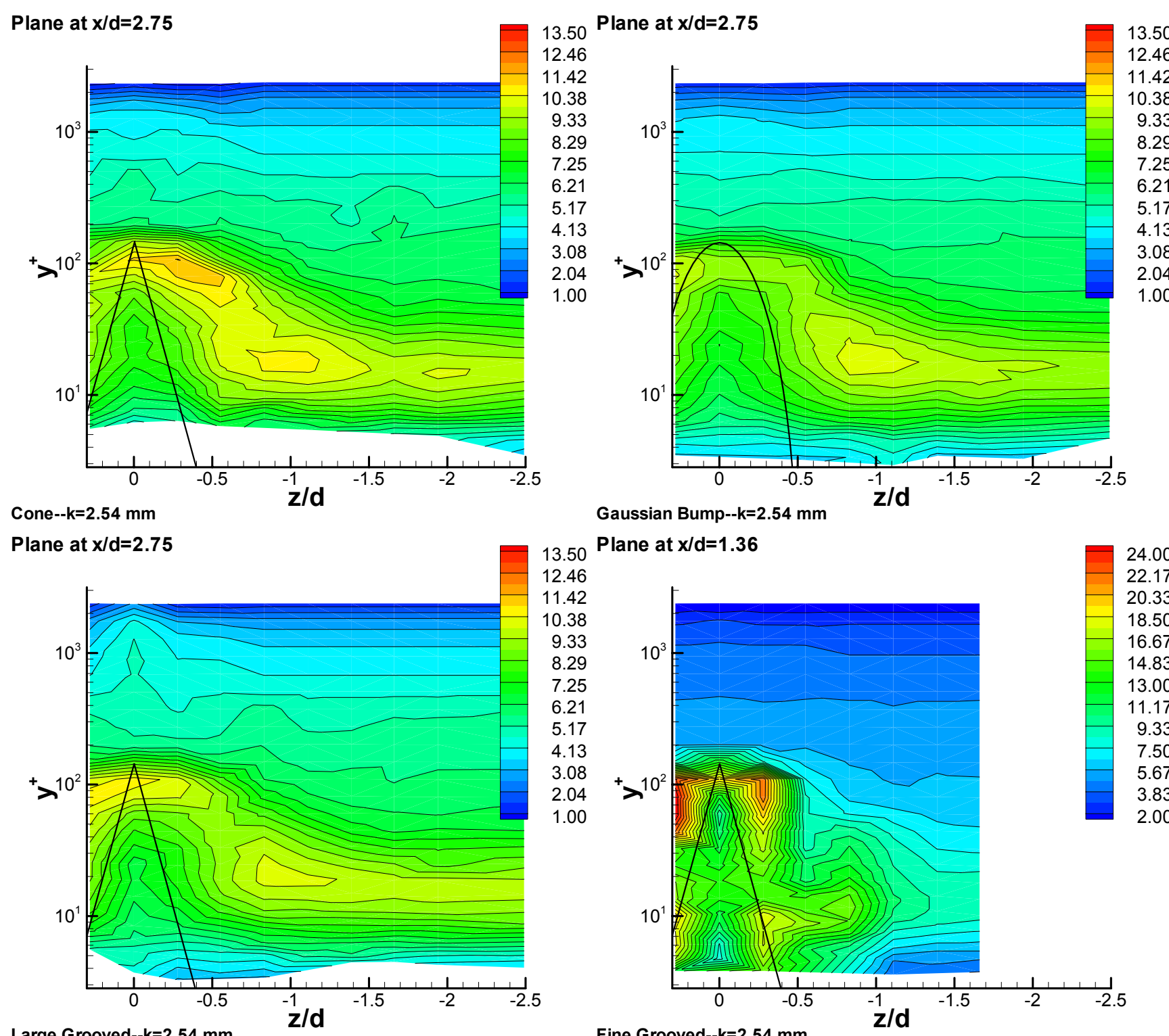

Gaussian Bump--k=2.54 mm

Plane at $x / d=1.36$

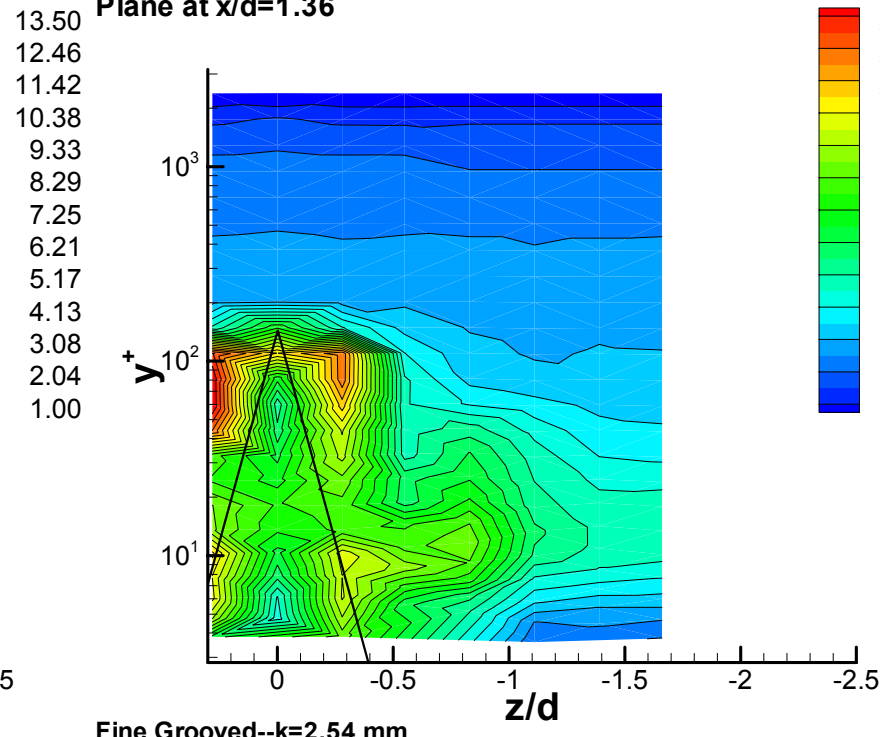

Large Grooved--k=2.54 mm

Fine Grooved--k=2.54 mm

Figure 3.30: Contours of $\overline{u^{2}} / U_{\tau}^{2}$ in the $\mathbf{y}-\mathrm{z}$ plane at a location of $\mathbf{x} / \mathbf{d}=\mathbf{2 . 7 5}$ (Note: Fine Grooved $\mathbf{x} / \mathbf{d}=1.36$ ) 


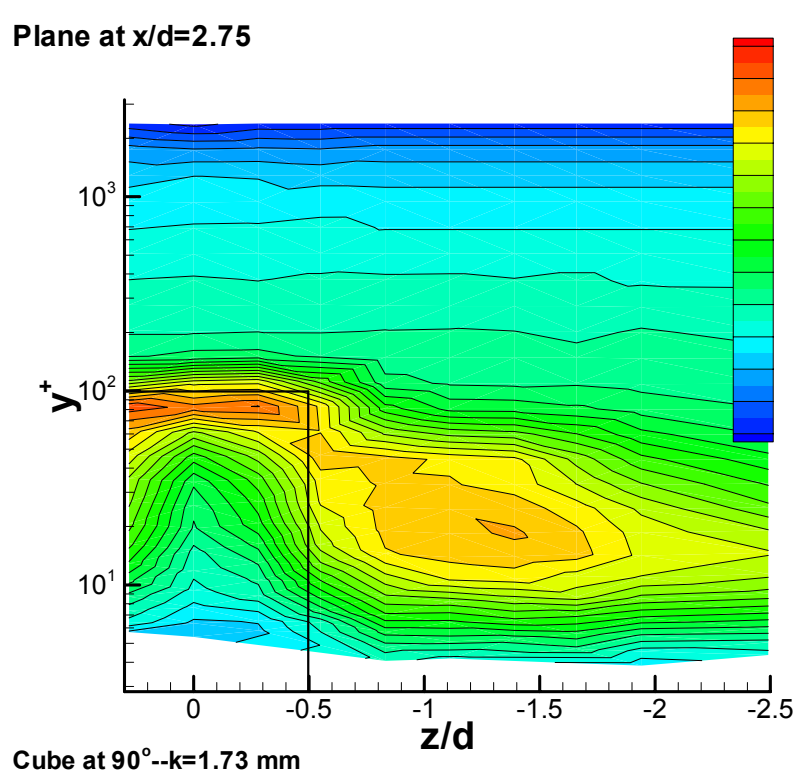

Plane at $\mathrm{x} / \mathrm{d}=\mathbf{2 . 7 5}$
13.50 Plane at $\mathbf{x} / \mathbf{d}=\mathbf{2 . 7 5}$

\begin{tabular}{l|r}
12.46 & 12.46 \\
11.42 & 11.42
\end{tabular}

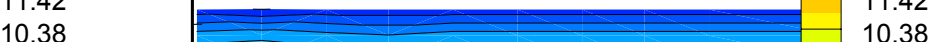

9.33
8.29
7.25

7.25
6.21
5.17

5.17
4.13
3.08

2.04

1.00

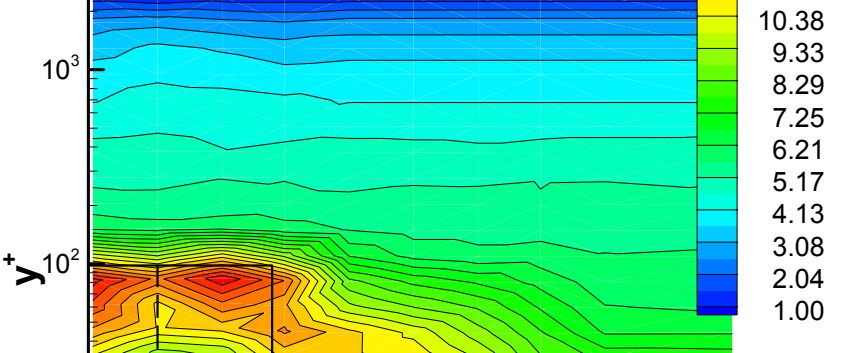

$10^{1}$

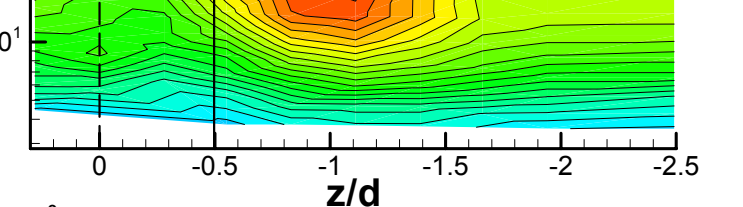

Cube at $45^{\circ}--\mathrm{k}=1.73 \mathrm{~mm}$

$$
\begin{array}{r}
13.50 \\
12.46 \\
11.42 \\
10.38 \\
9.33 \\
8.29 \\
7.25 \\
6.21 \\
5.17 \\
4.13 \\
3.08 \\
2.04 \\
1.00
\end{array}
$$

10

$0^{1}$

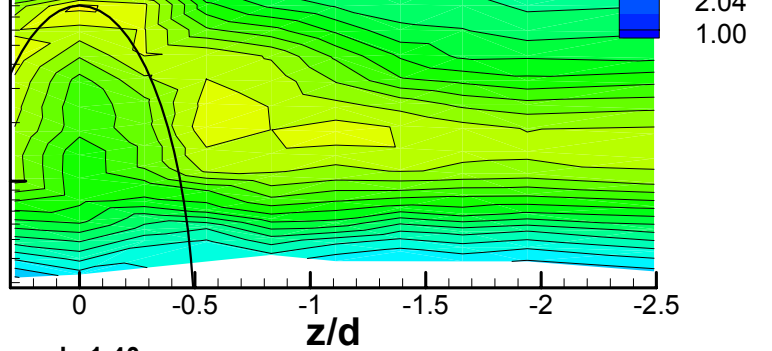

Hemisphere--k=1.40 mm

Figure 3.31: Contours of $\overline{u^{2}} / U_{\tau}^{2}$ in the $\mathbf{y}-\mathbf{z}$ plane at a location of $\mathbf{x} / \mathbf{d}=\mathbf{2 . 7 5}$ 

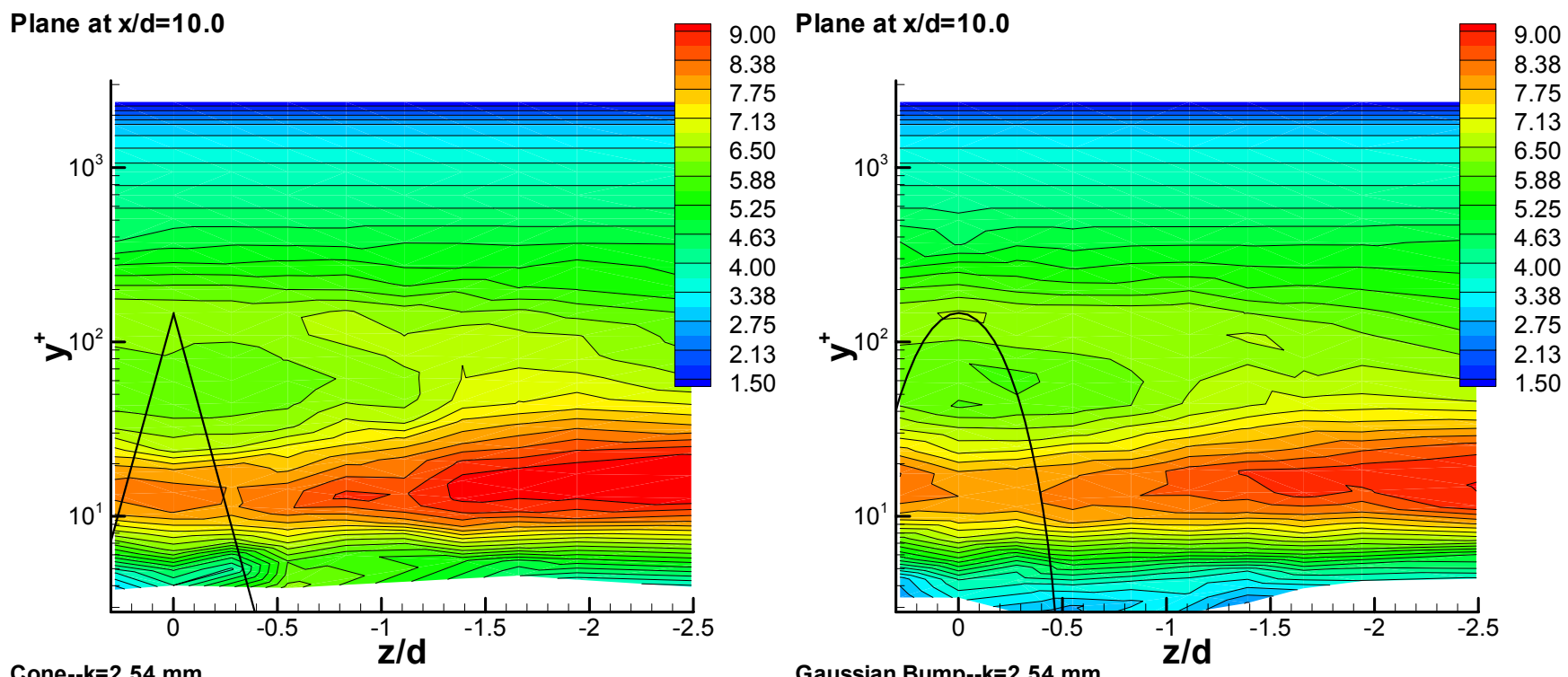

Cone--k=2.54 mm

Gaussian Bump--k=2.54 mm

Plane at $x / d=10.0$
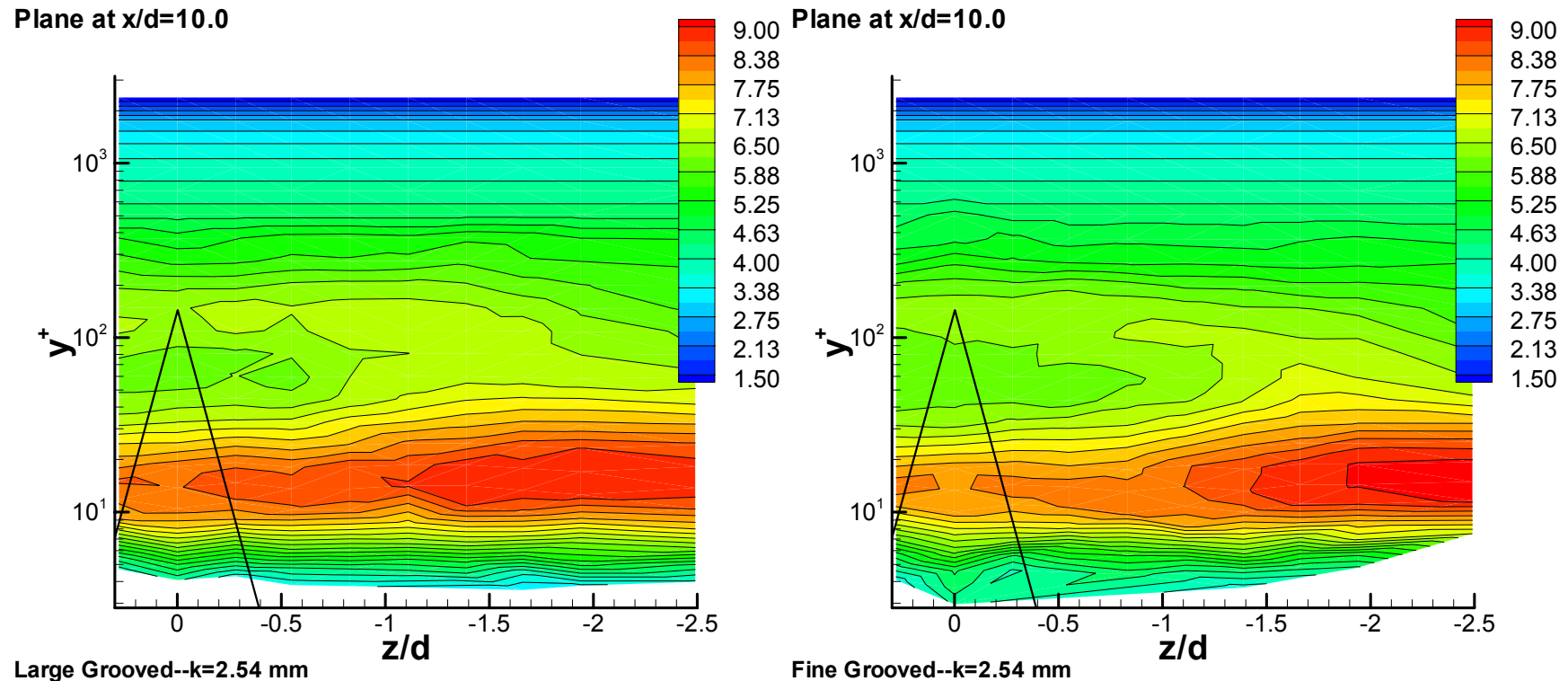

Large Grooved--k=2.54 $\mathrm{mm}$

Fine Grooved--k=2.54 mm

Figure 3.32: Contours of $\overline{u^{2}} / U_{\tau}^{2}$ in the $\mathbf{y}-\mathbf{z}$ plane at a location of $\mathbf{x} / \mathbf{d}=\mathbf{1 0 . 0}$ 


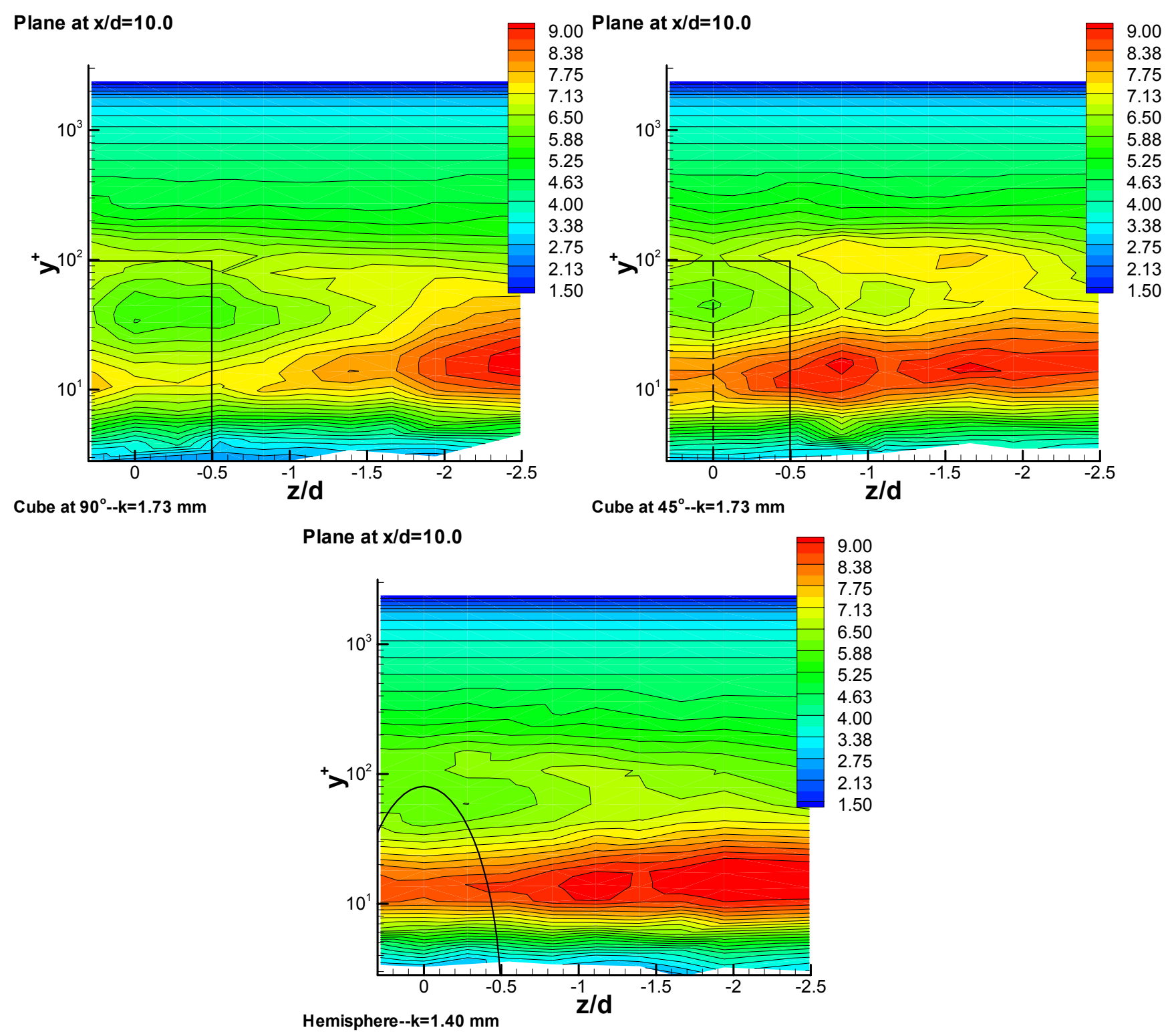

Figure 3.33: Contours of $\overline{u^{2}} / U_{\tau}^{2}$ in the $\mathbf{y}-z$ plane at a location of $\mathbf{x} / \mathbf{d}=10.0$ 


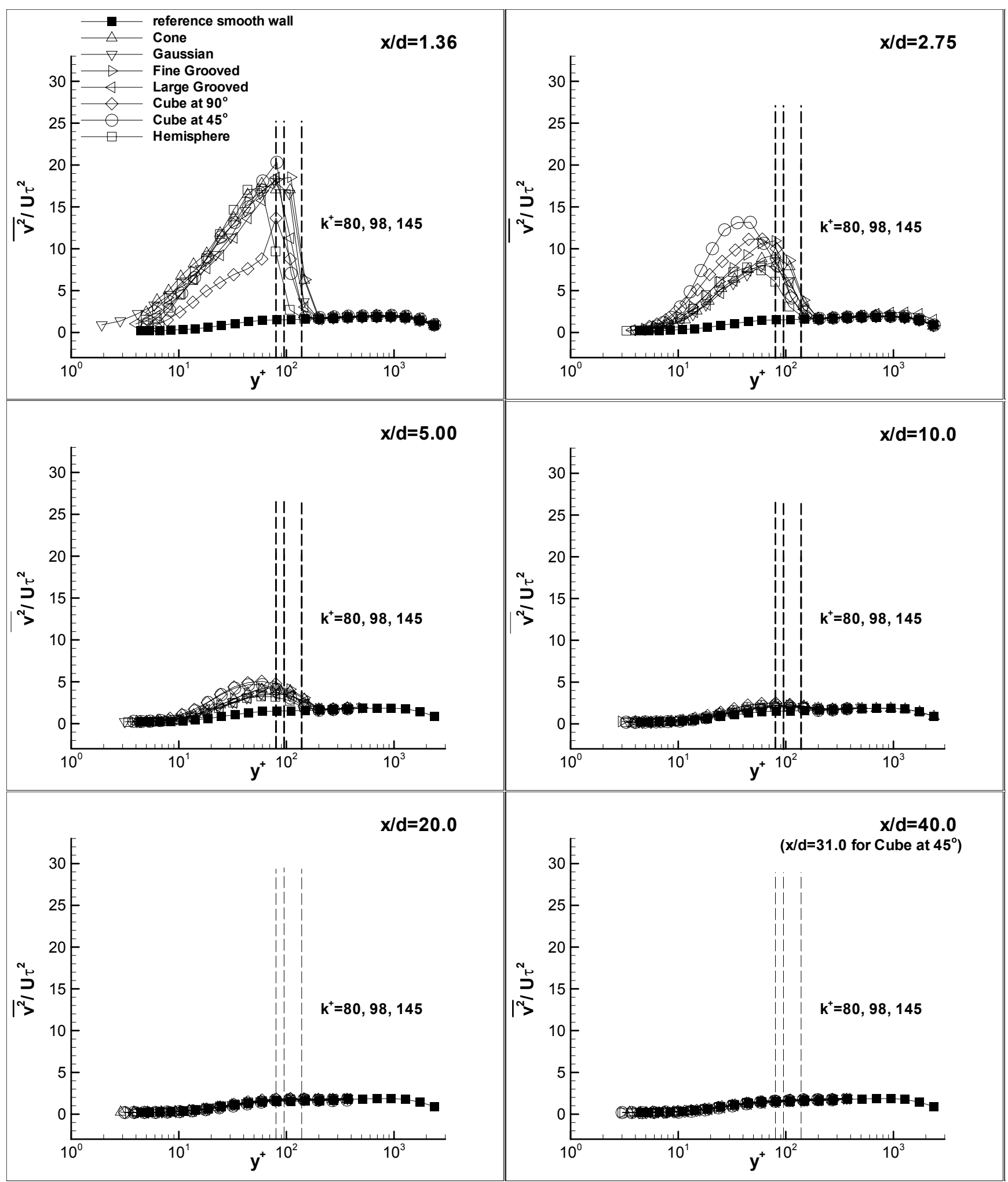

Figure 3.34: $\overline{v^{2}} / U_{\tau}^{2}$ vs. $y U_{\tau} / v$, normal to wall Reynolds normal stress profiles along the centerline 

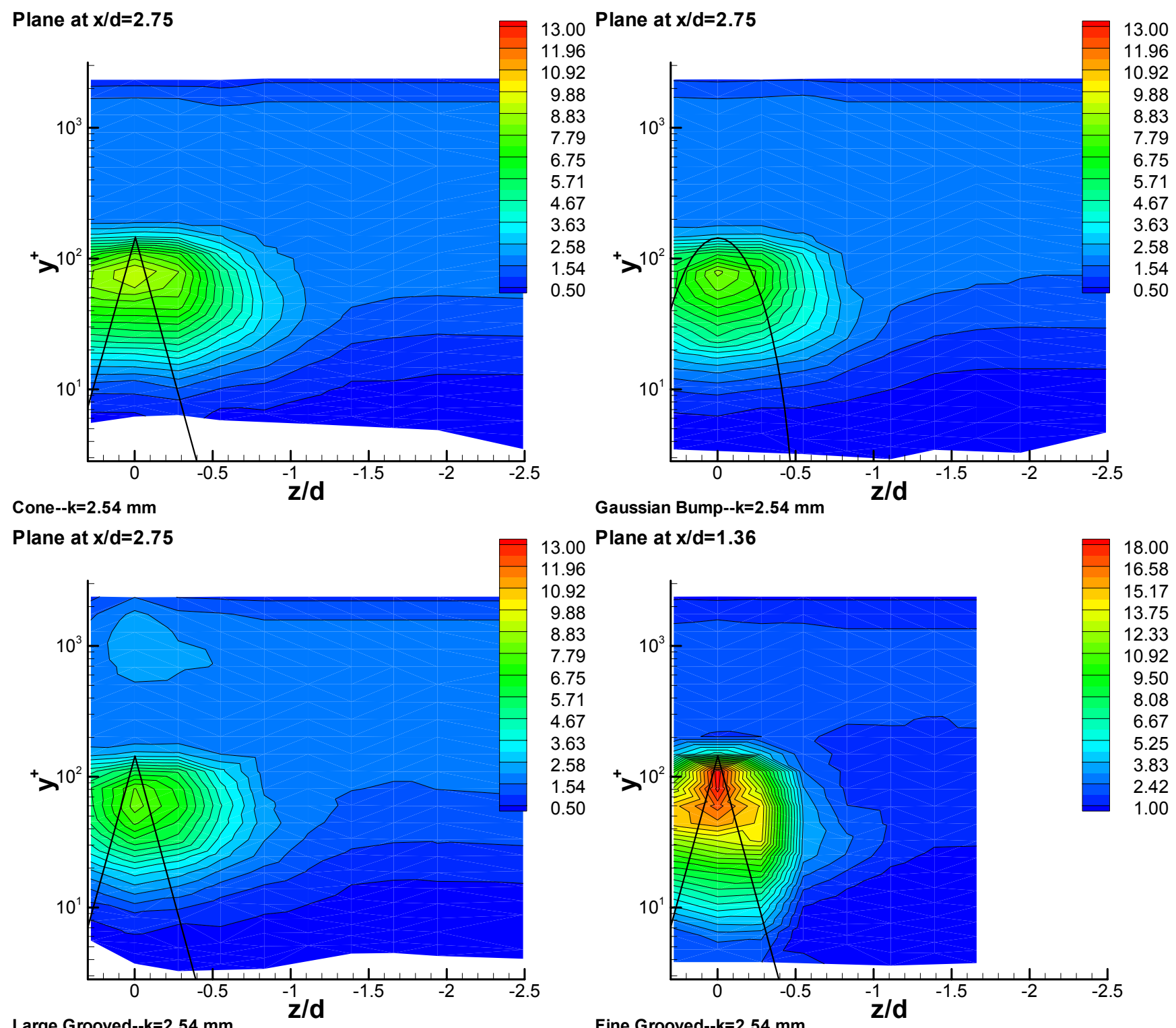

Gaussian Bump--k=2.54 mm

Plane at $x / d=1.36$

11.96

0.92
9.88

9.88
8.83
7.79

8.83
7.79
6.75
5.71

5.71
4.67

4.67
3.63

3.63
2.58
1.54

2.58
1.54
0.50

Large Grooved--k=2.54 mm

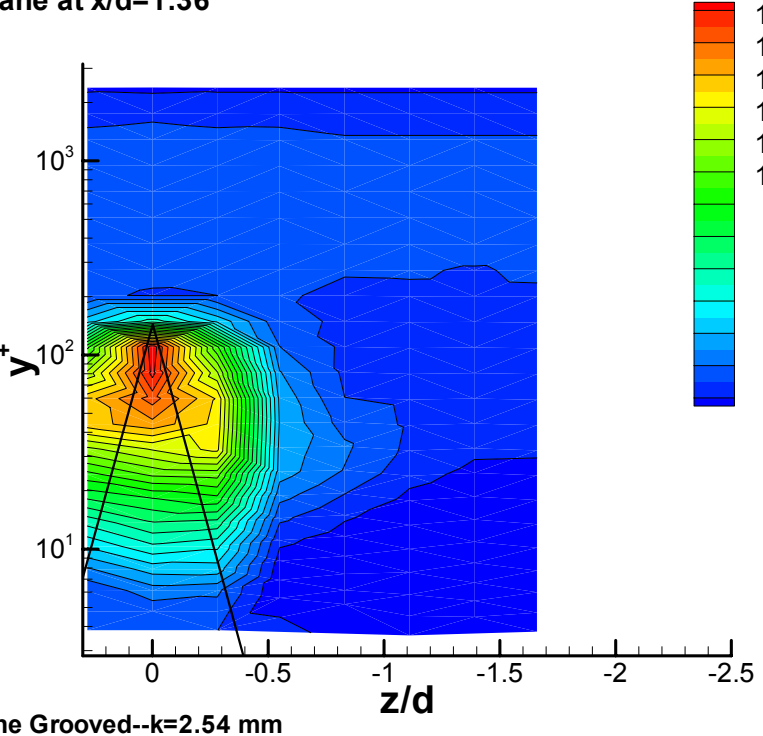

Fine Grooved--k=2.54 mm

Figure 3.35: Contours of $\overline{v^{2}} / U_{\tau}^{2}$ in the $\mathbf{y}-z$ plane at a location of $\mathbf{x} / \mathbf{d}=\mathbf{2 . 7 5}$ (Note: Fine Grooved $\mathbf{x} / \mathbf{d}=1.36$ ) 


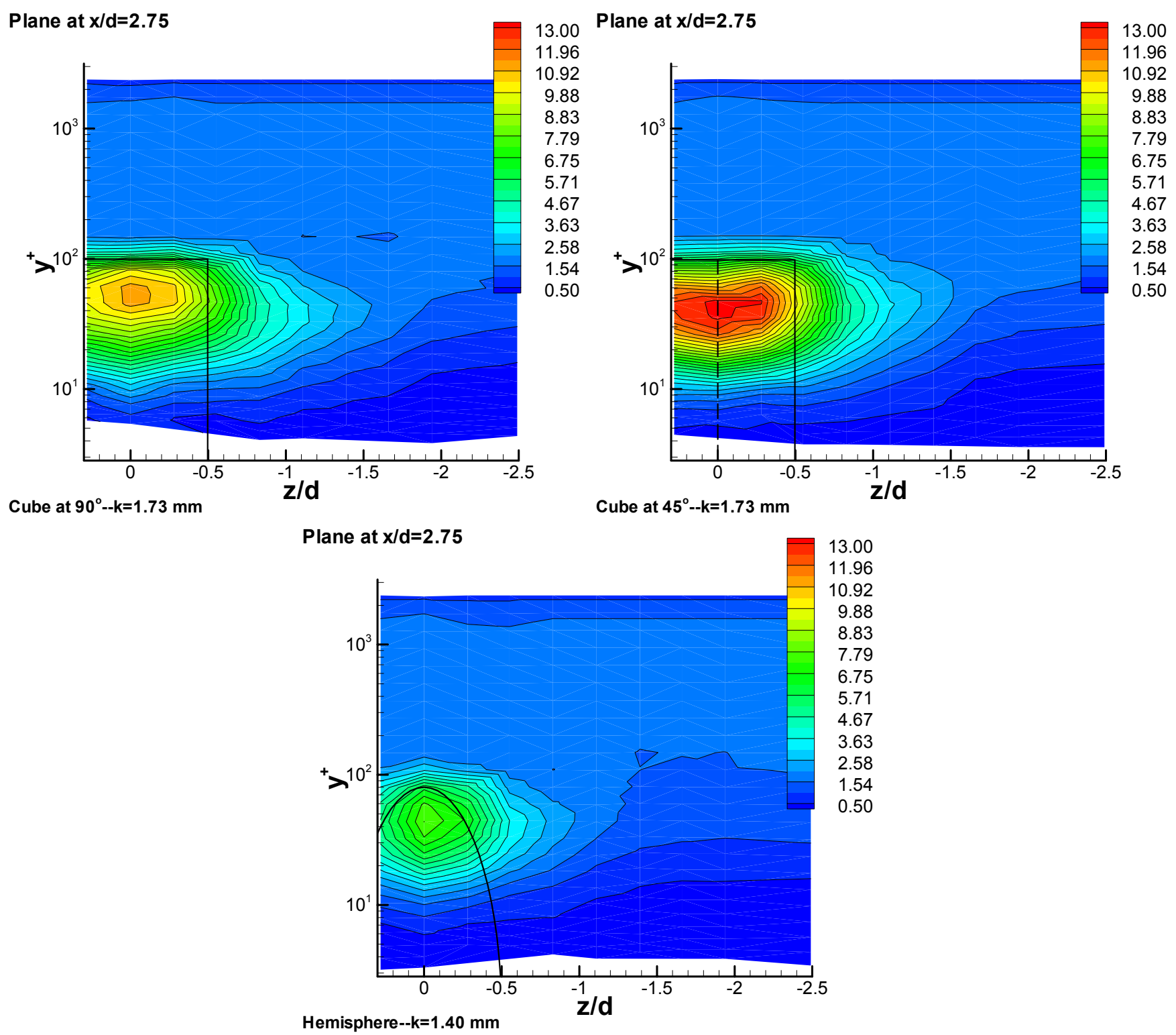

Figure 3.36: Contours of $\overline{v^{2}} / U_{\tau}^{2}$ in the $\mathbf{y}-z$ plane at a location of $\mathbf{x} / \mathbf{d}=\mathbf{2 . 7 5}$ 

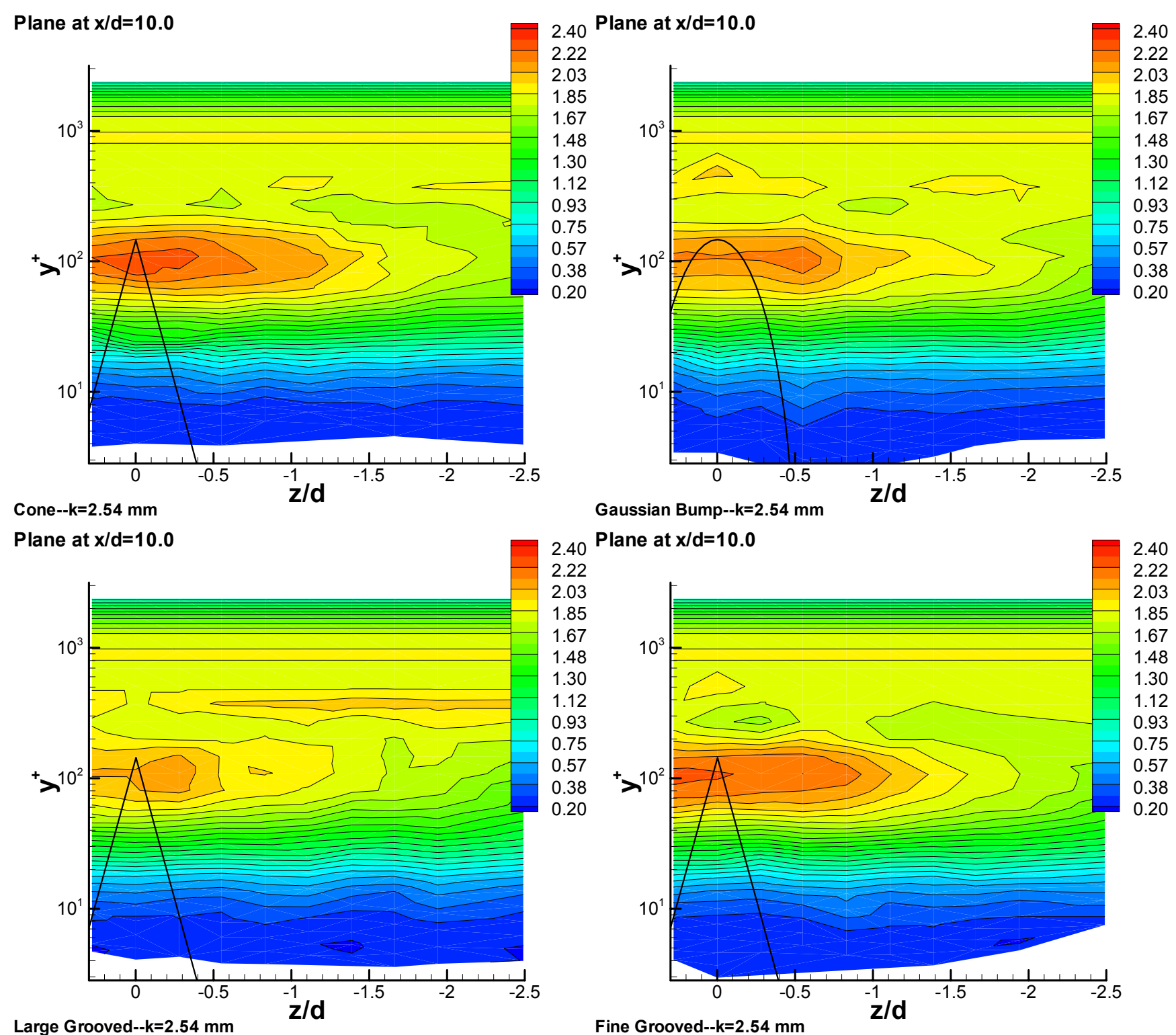

Figure 3.37: Contours of $\overline{v^{2}} / U_{\tau}^{2}$ in the $\mathbf{y}-\mathrm{z}$ plane at a location of $\mathbf{x} / \mathbf{d}=10.0$ 


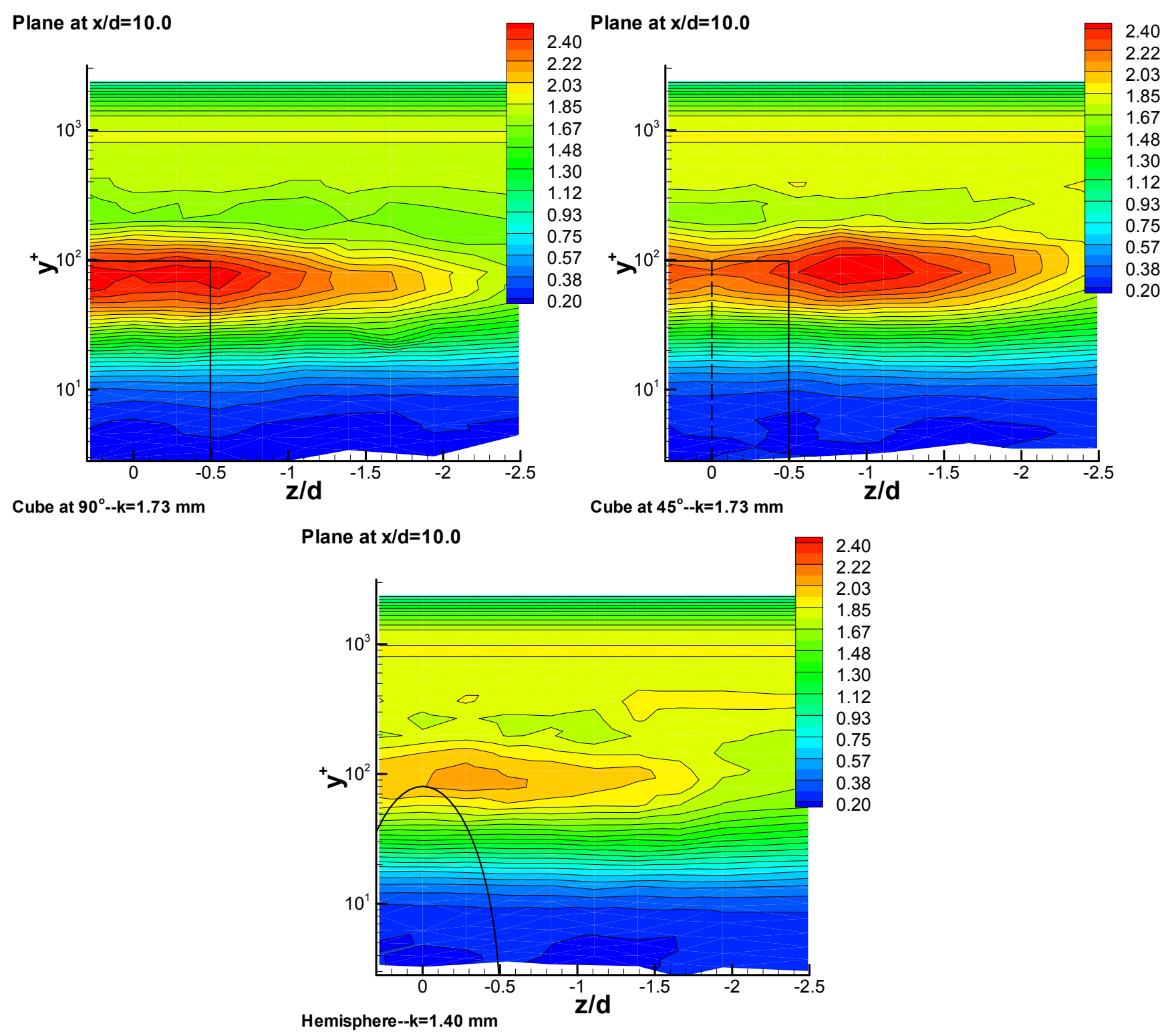

Figure 3.38: Contours of $\overline{v^{2}} / U_{\tau}^{2}$ in the $\mathbf{y}-\mathbf{z}$ plane at a location of $\mathbf{x} / \mathbf{d}=\mathbf{1 0 . 0}$ 


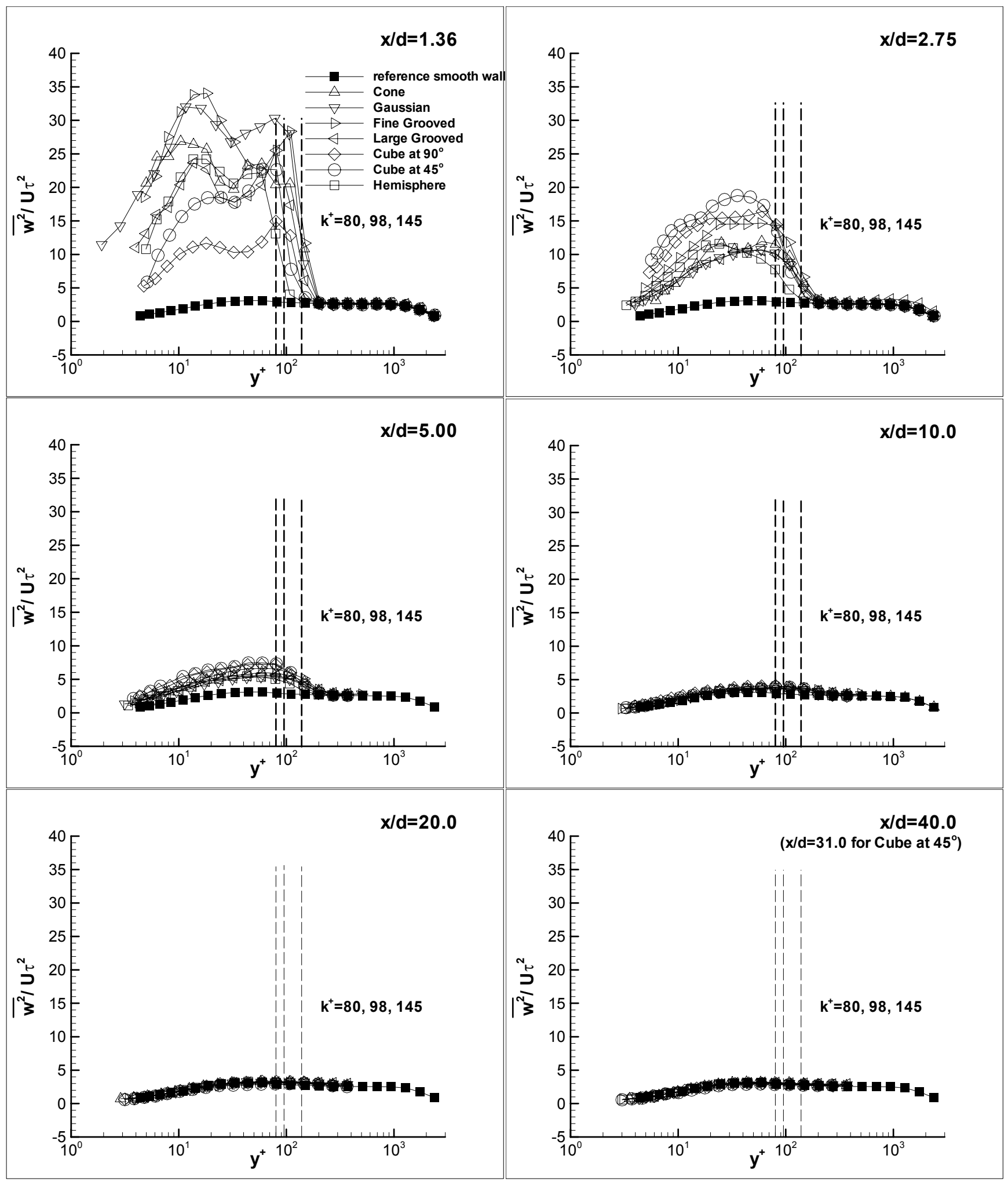

Figure 3.39: $\overline{w^{2}} / U_{\tau}^{2}$ vs. $y U_{\tau} / v$, spanwise Reynolds normal stress profiles along the centerline 


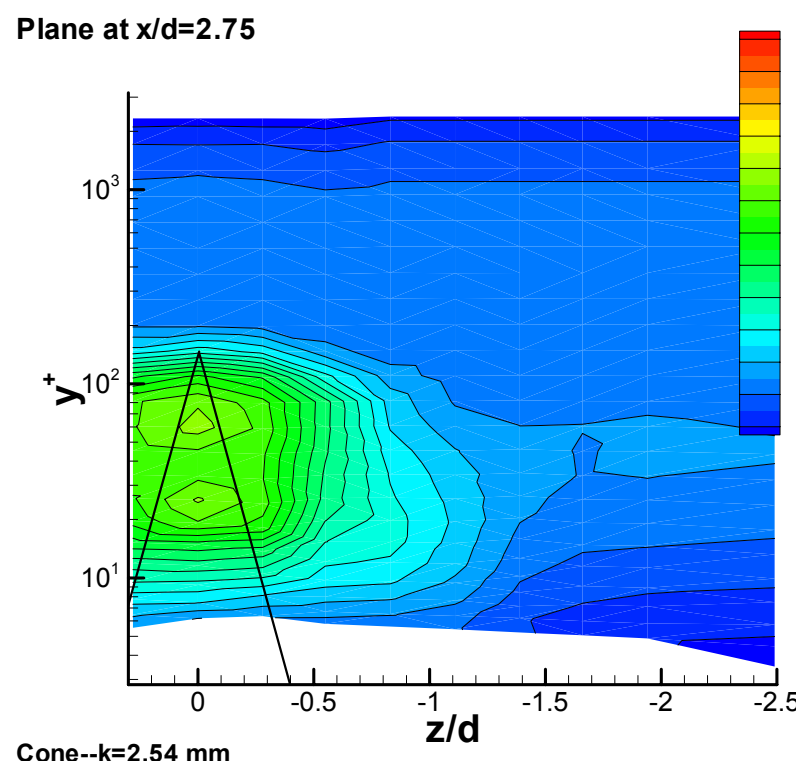

Plane at $x / d=2.75$

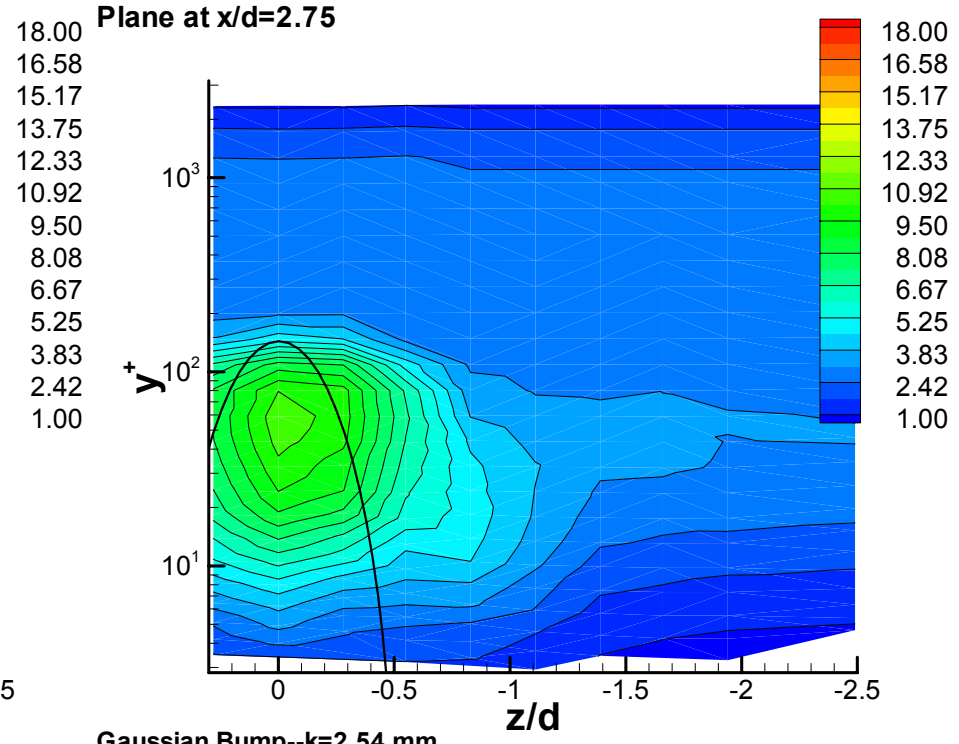

Plane at $x / d=2.75$

Gaussian Bump--k=2.54 mm

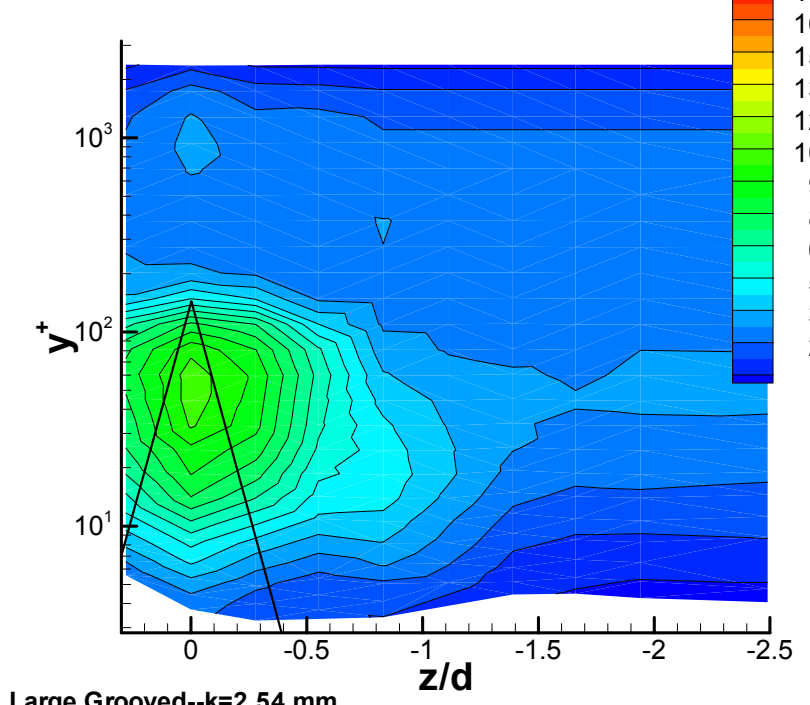
18.00 Plane at $x / d=1.36$

16.58

16.58
15.17

15.17
13.75

13.75
12.33
10.92

10.92
9.50

9.50
8.08

8.08
6.67

6.67
5.25

3.83
2.42

1.00

Large Grooved--k=2.54 mm

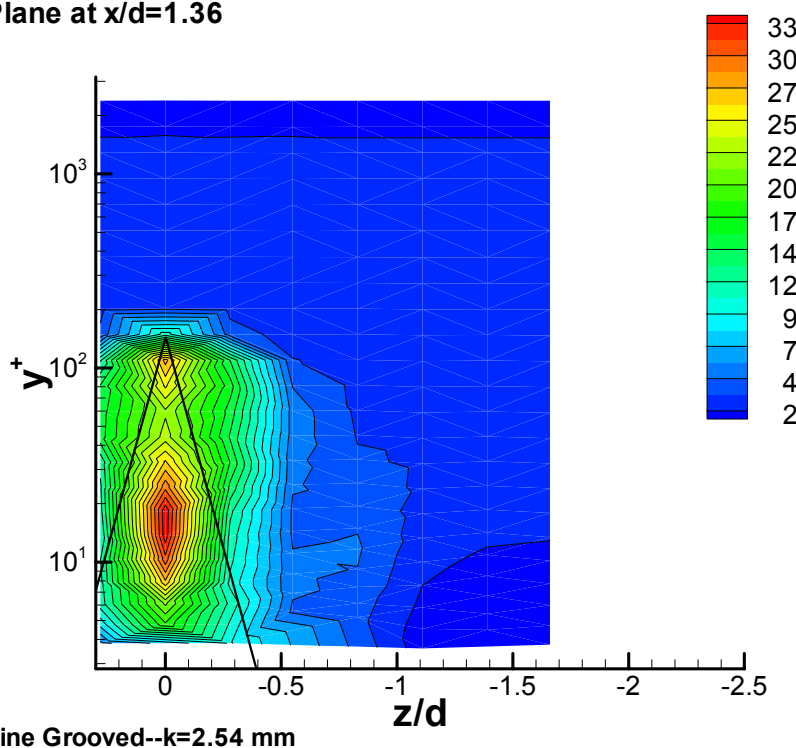

Fine Grooved--k=2.54 mm

Figure 3.40: Contours of $\overline{w^{2}} / U_{\tau}^{2}$ in the $\mathbf{y}-z$ plane at a location of $\mathbf{x} / \mathbf{d}=\mathbf{2 . 7 5}$ (Note: Fine Grooved $\mathbf{x} / \mathbf{d}=1.36$ ) 


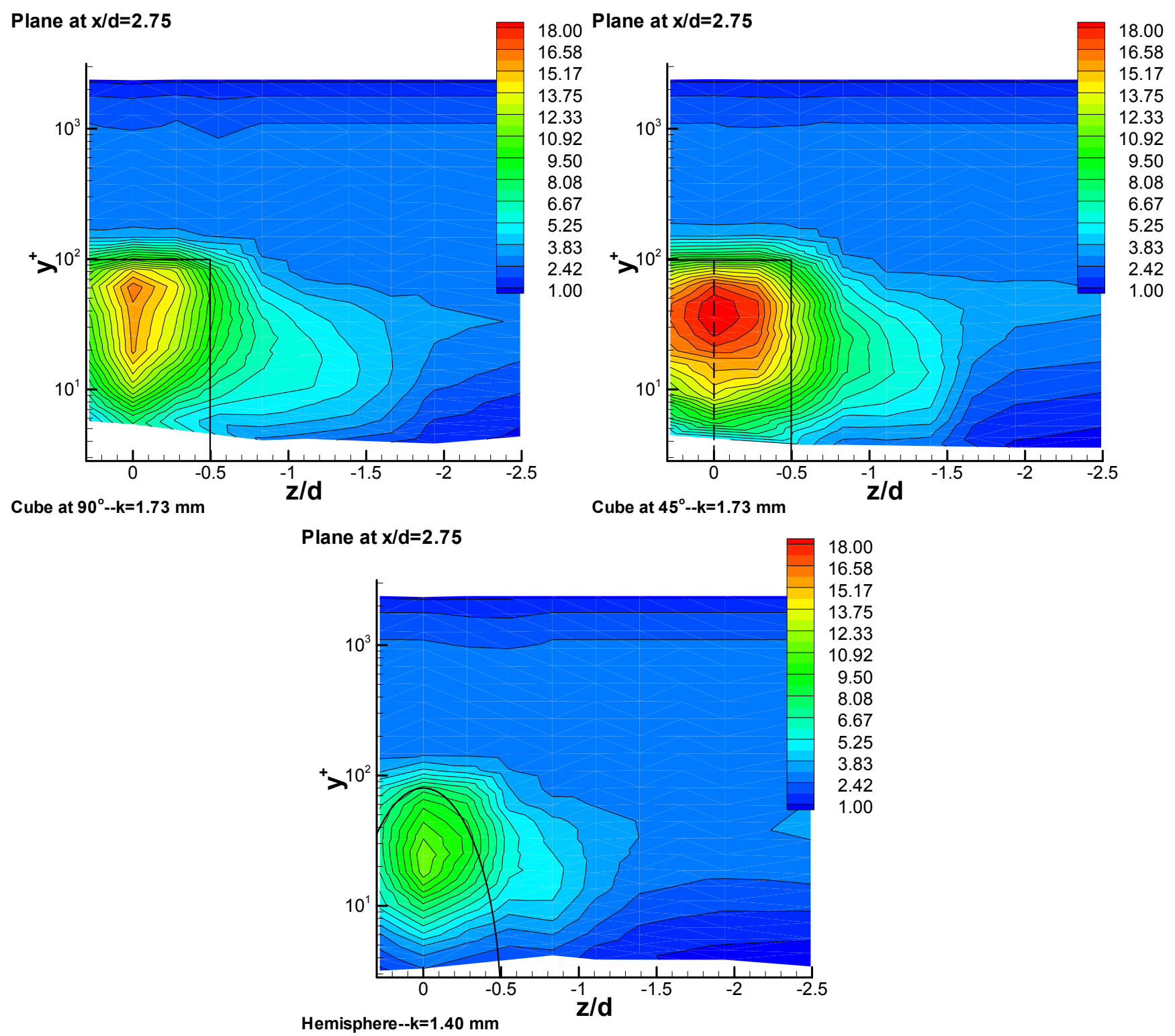

Figure 3.41: Contours of $\overline{w^{2}} / U_{\tau}^{2}$ in the $\mathbf{y}-\mathbf{z}$ plane at a location of $\mathbf{x} / \mathbf{d}=\mathbf{2 . 7 5}$ 

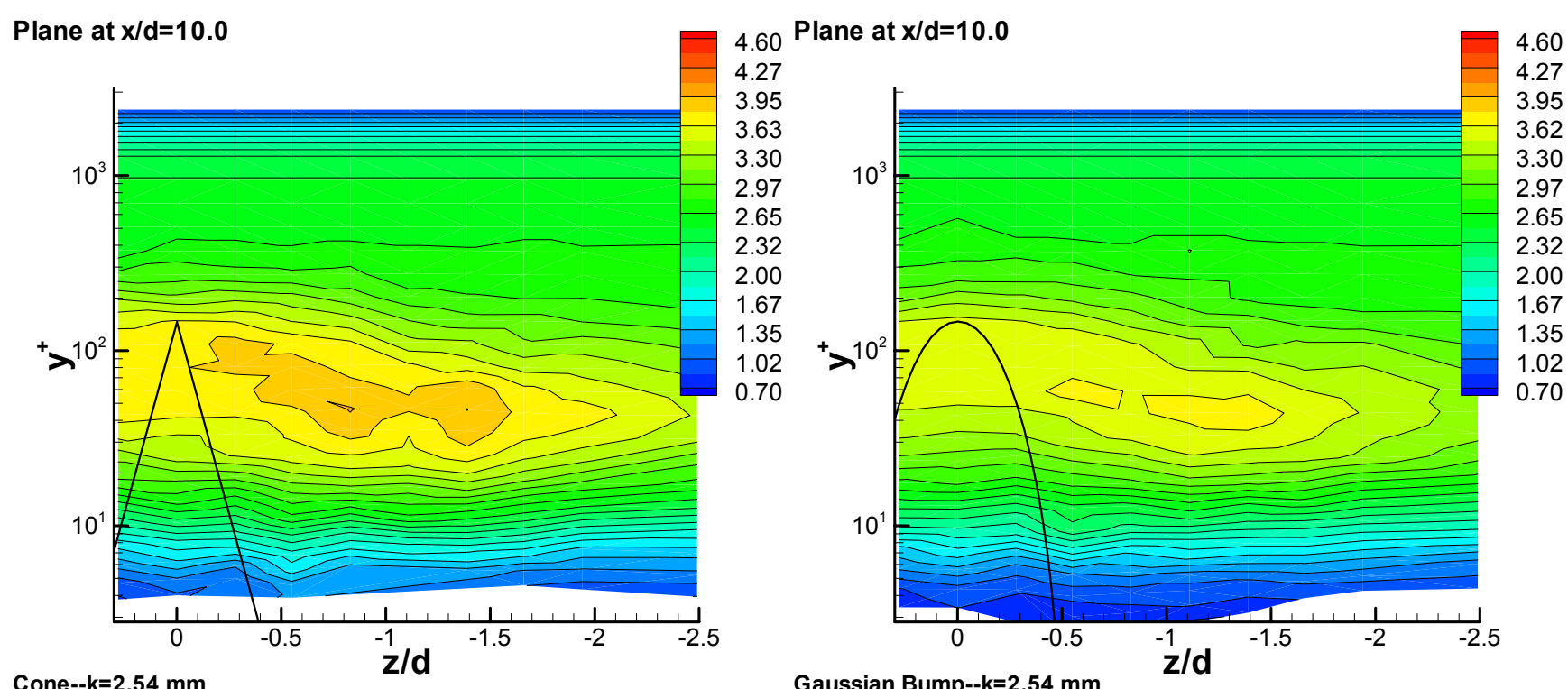

Plane at $x / d=10.0$

Gaussian Bump--k=2.54 mm

Plane at $x / d=10.0$
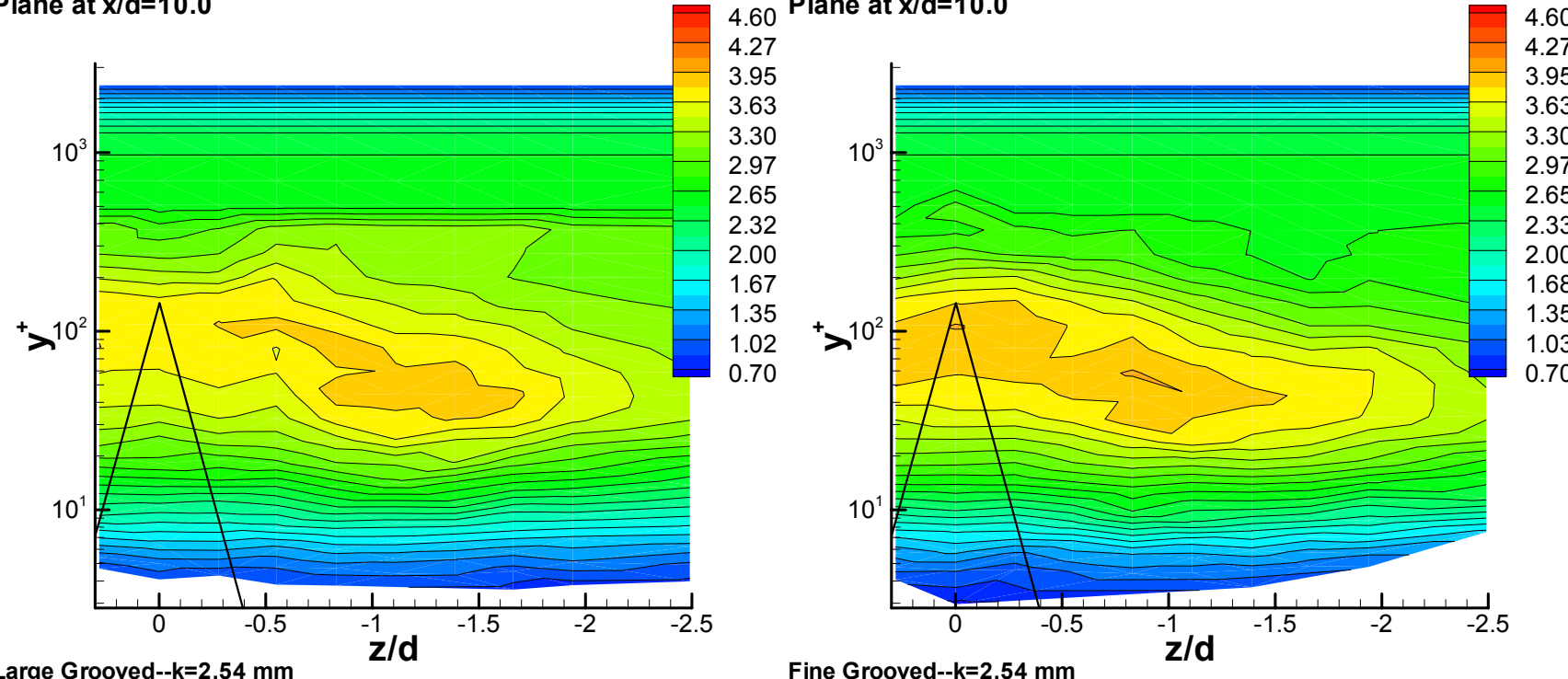

Large Grooved--k=2.54 mm

Fine Grooved--k=2.54 mm

Figure 3.42: Contours of $\overline{w^{2}} / U_{\tau}^{2}$ in the $\mathbf{y}-\mathbf{z}$ plane at a location of $\mathbf{x} / \mathbf{d}=\mathbf{1 0 . 0}$ 


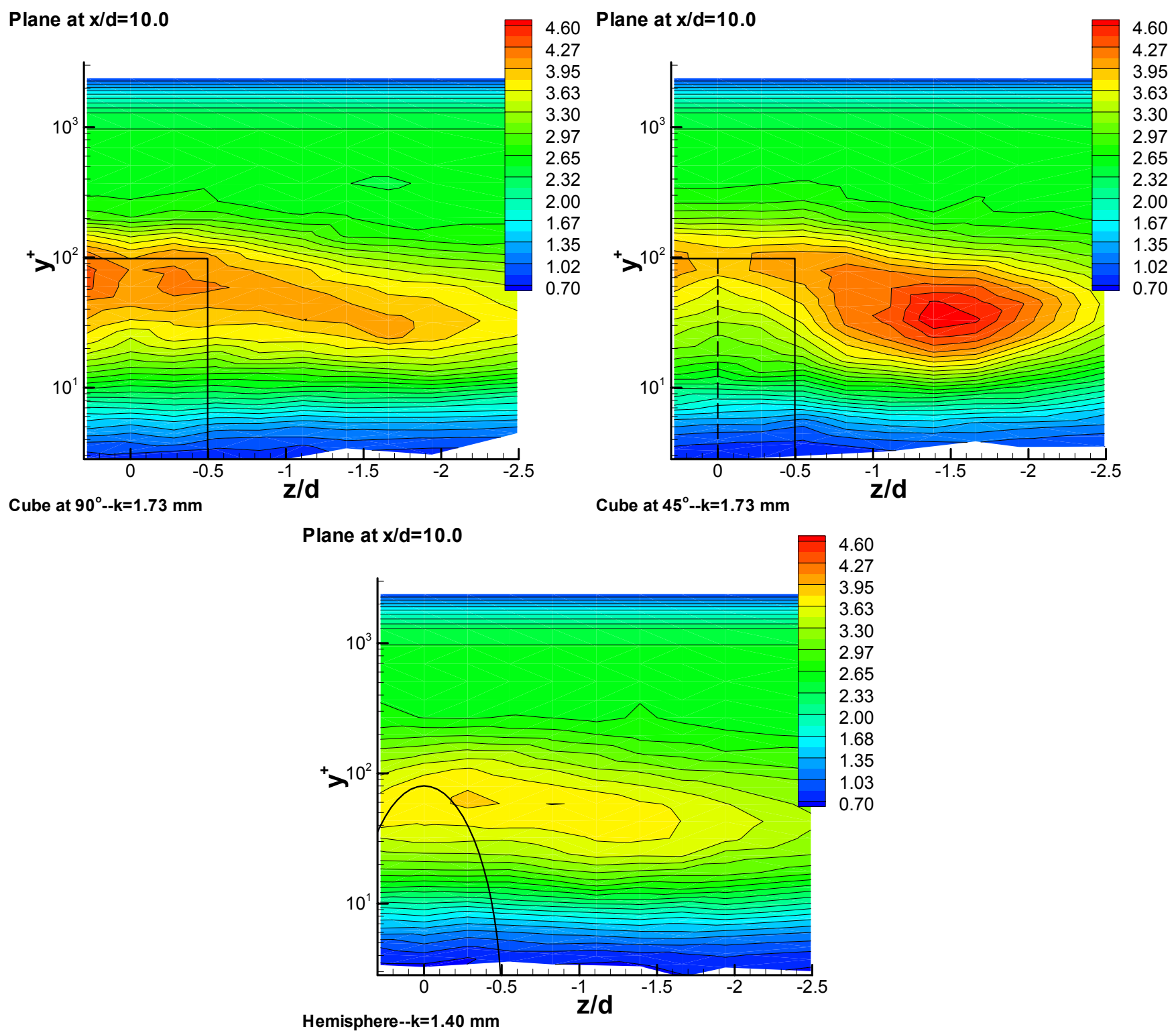

Figure 3.43: Contours of $\overline{w^{2}} / U_{\tau}^{2}$ in the $\mathbf{y}-z$ plane at a location of $\mathbf{x} / \mathbf{d}=10.0$ 


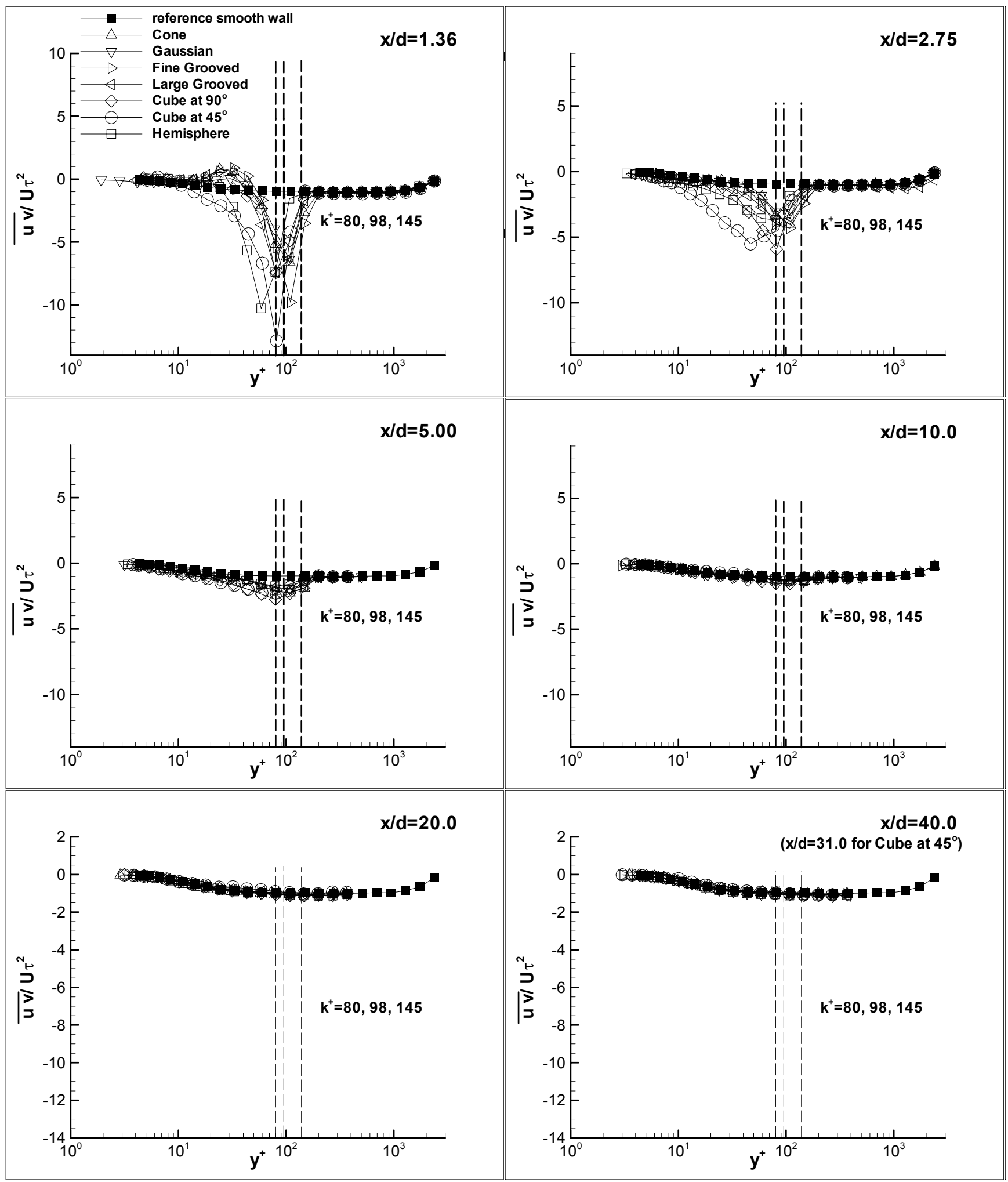

Figure 3.44: $\overline{u v} / U_{\tau}^{2}$ vs. $y U_{\tau} / v$, streamwise Reynolds shear stress profiles along the centerline 

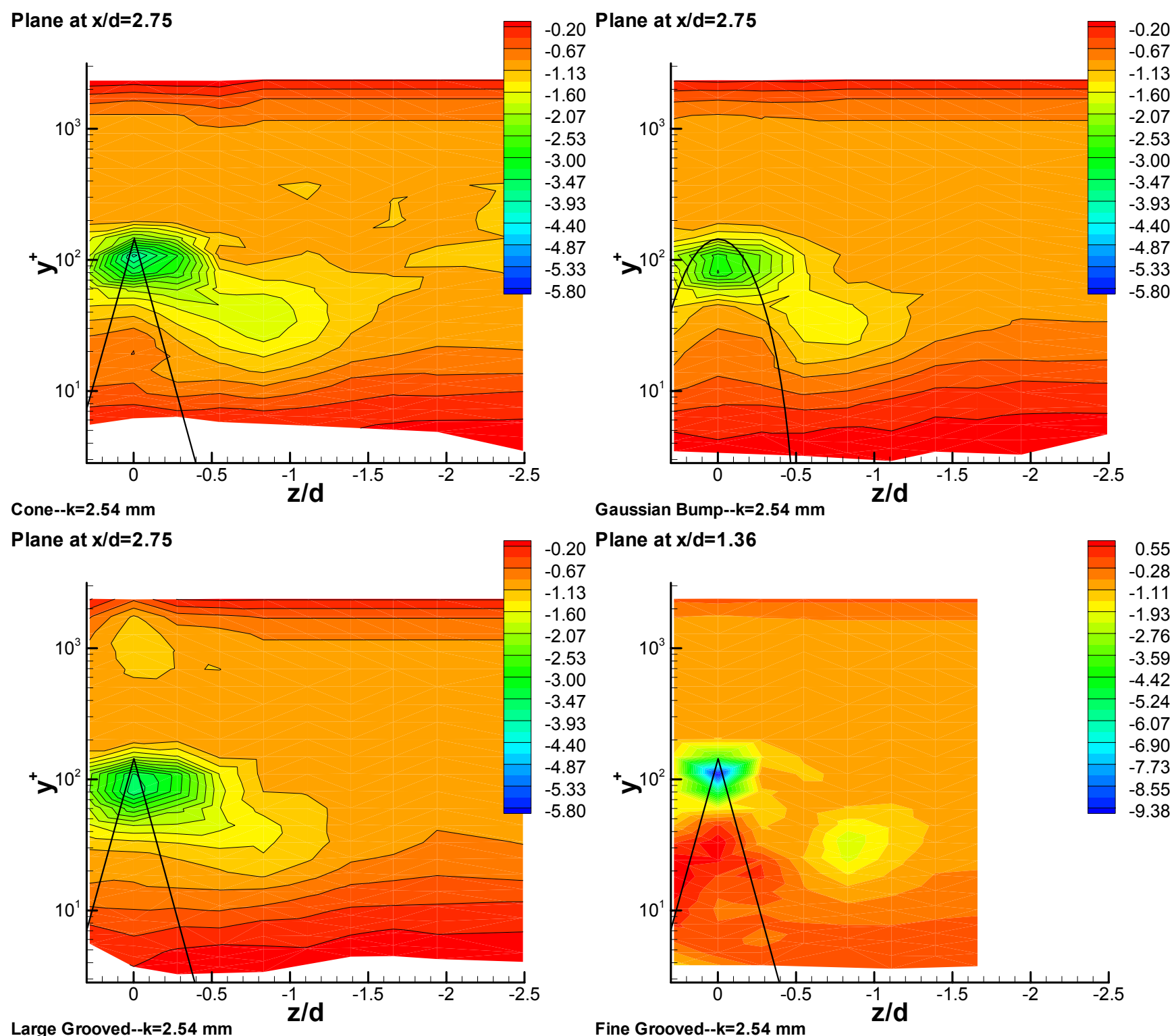

Figure 3.45: Contours of $\overline{u v} / U_{\tau}^{2}$ in the $\mathbf{y}-z$ plane at a location of $\mathbf{x} / \mathbf{d}=\mathbf{2 . 7 5}($ Note: Fine Grooved $\mathbf{x} / \mathbf{d}=1.36)$ 


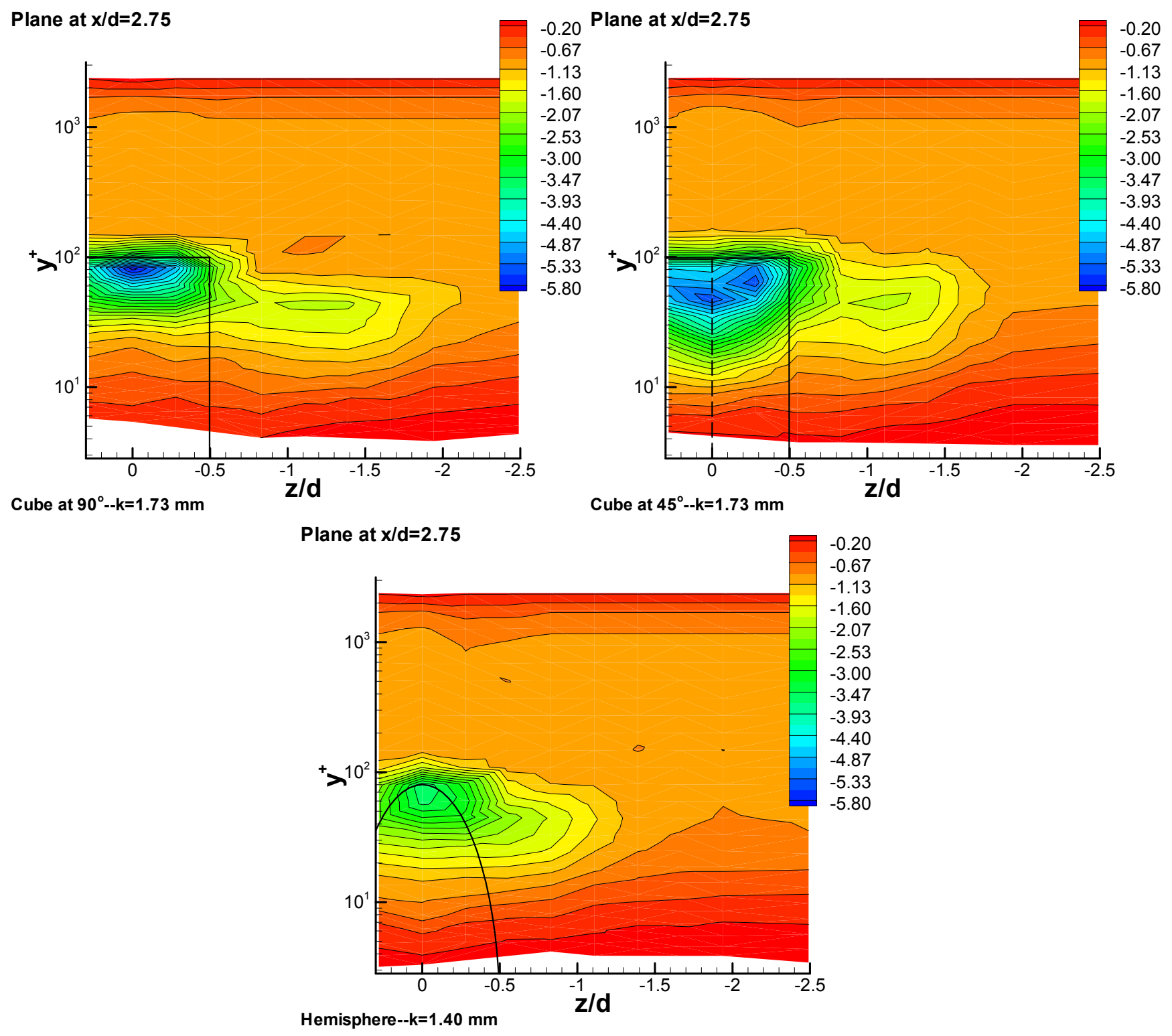

Figure 3.46: Contours of $\overline{u v} / U_{\tau}^{2}$ in the $\mathbf{y}-\mathbf{z}$ plane at a location of $\mathbf{x} / \mathbf{d}=\mathbf{2 . 7 5}$ 

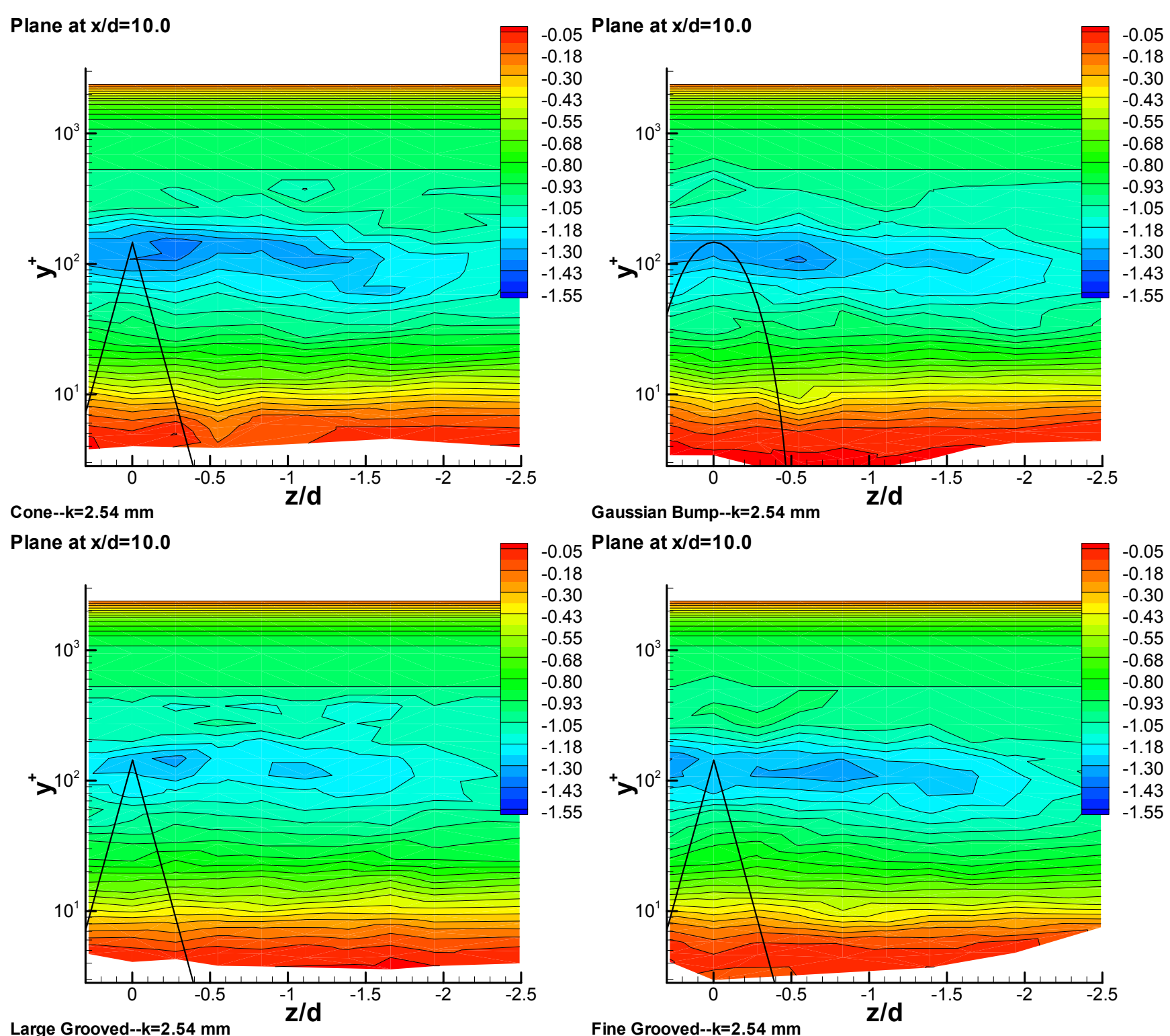

Figure 3.47: Contours of $\overline{u v} / U_{\tau}^{2}$ in the $\mathbf{y}-z$ plane at a location of $\mathbf{x} / \mathbf{d}=10.0$ 


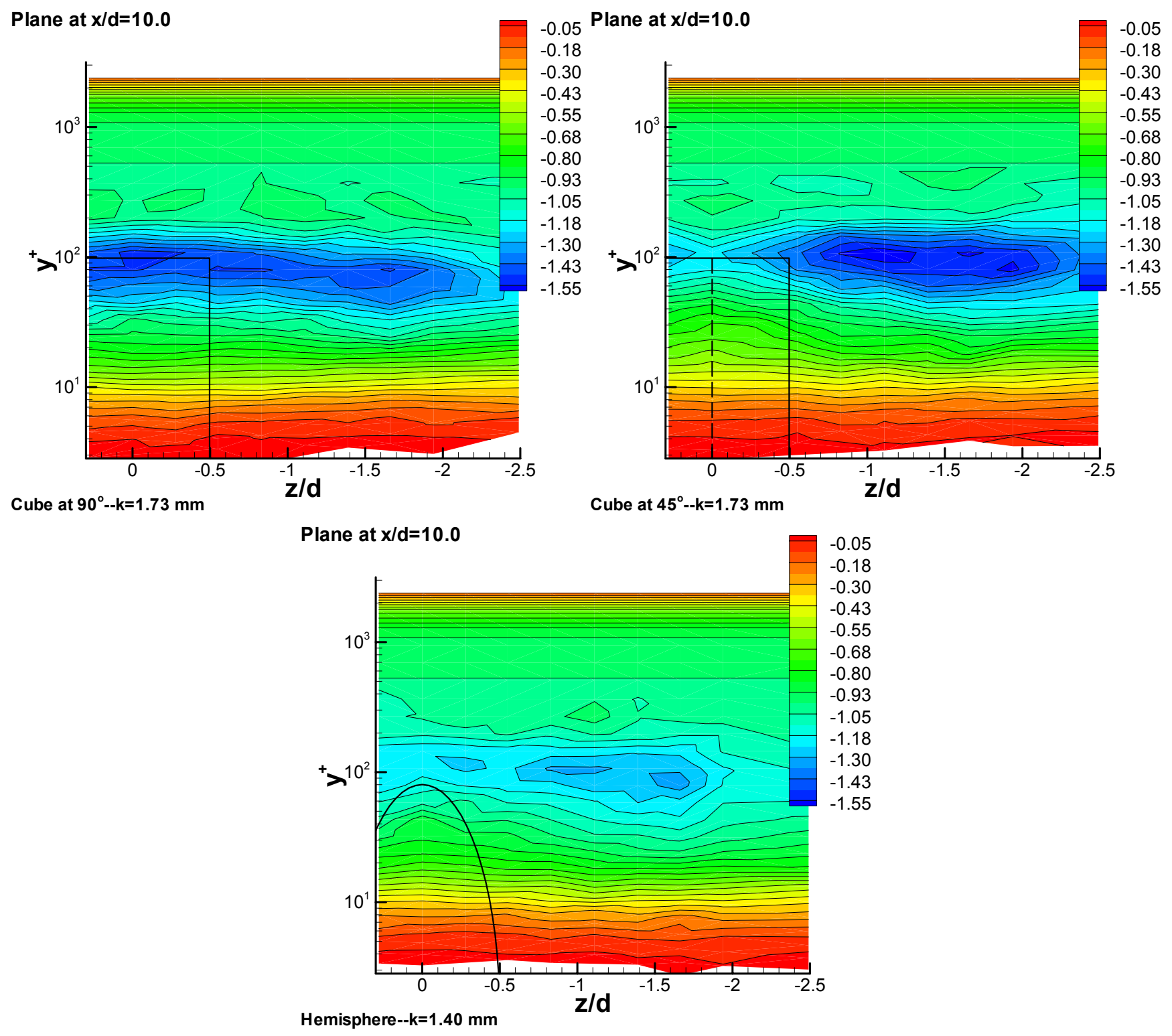

Figure 3.48: Contours of $\overline{u v} / U_{\tau}^{2}$ in the $\mathbf{y}-\mathbf{z}$ plane at a location of $\mathbf{x} / \mathbf{d}=\mathbf{1 0 . 0}$ 

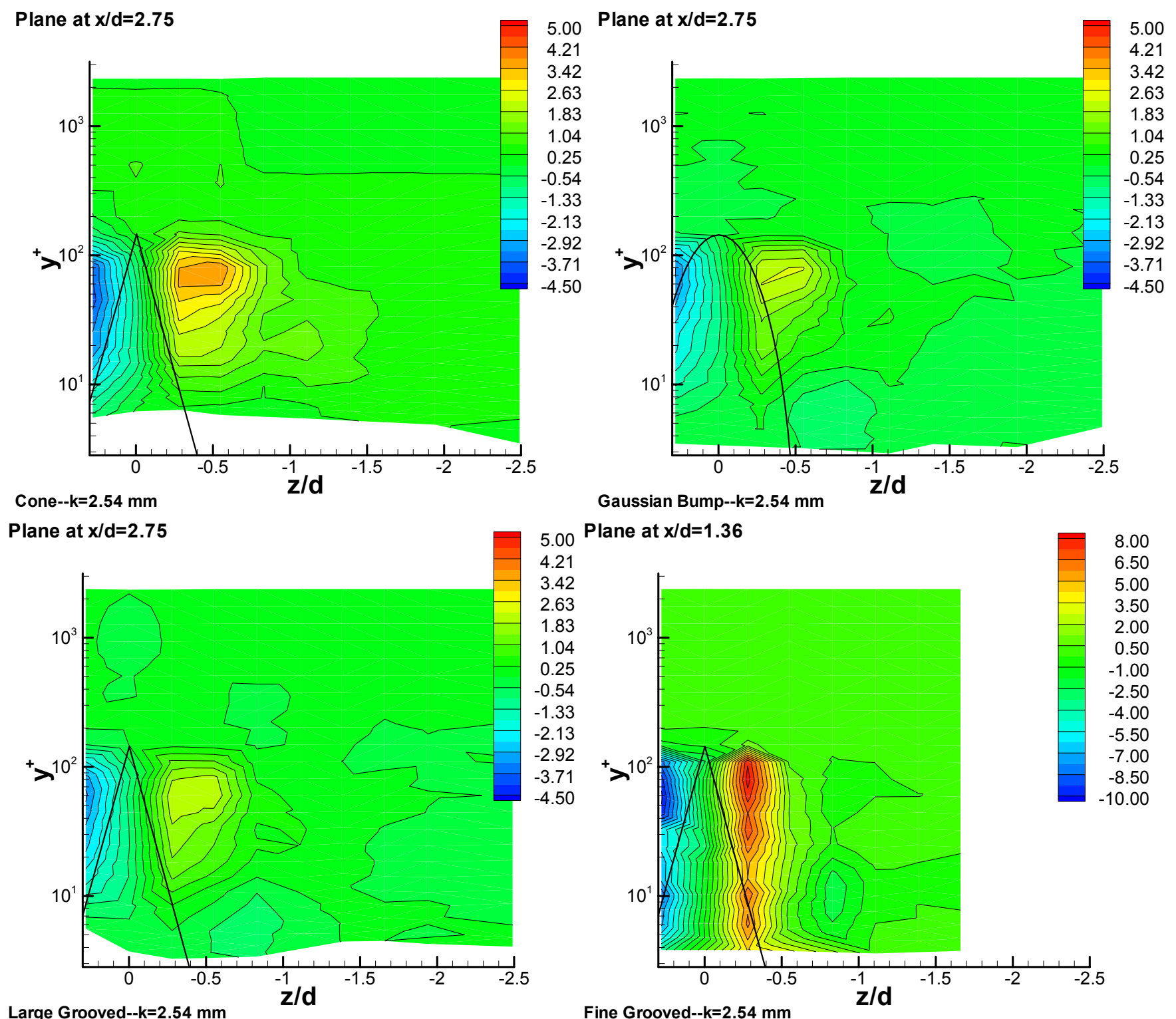

Gaussian Bump--k=2.54 mm

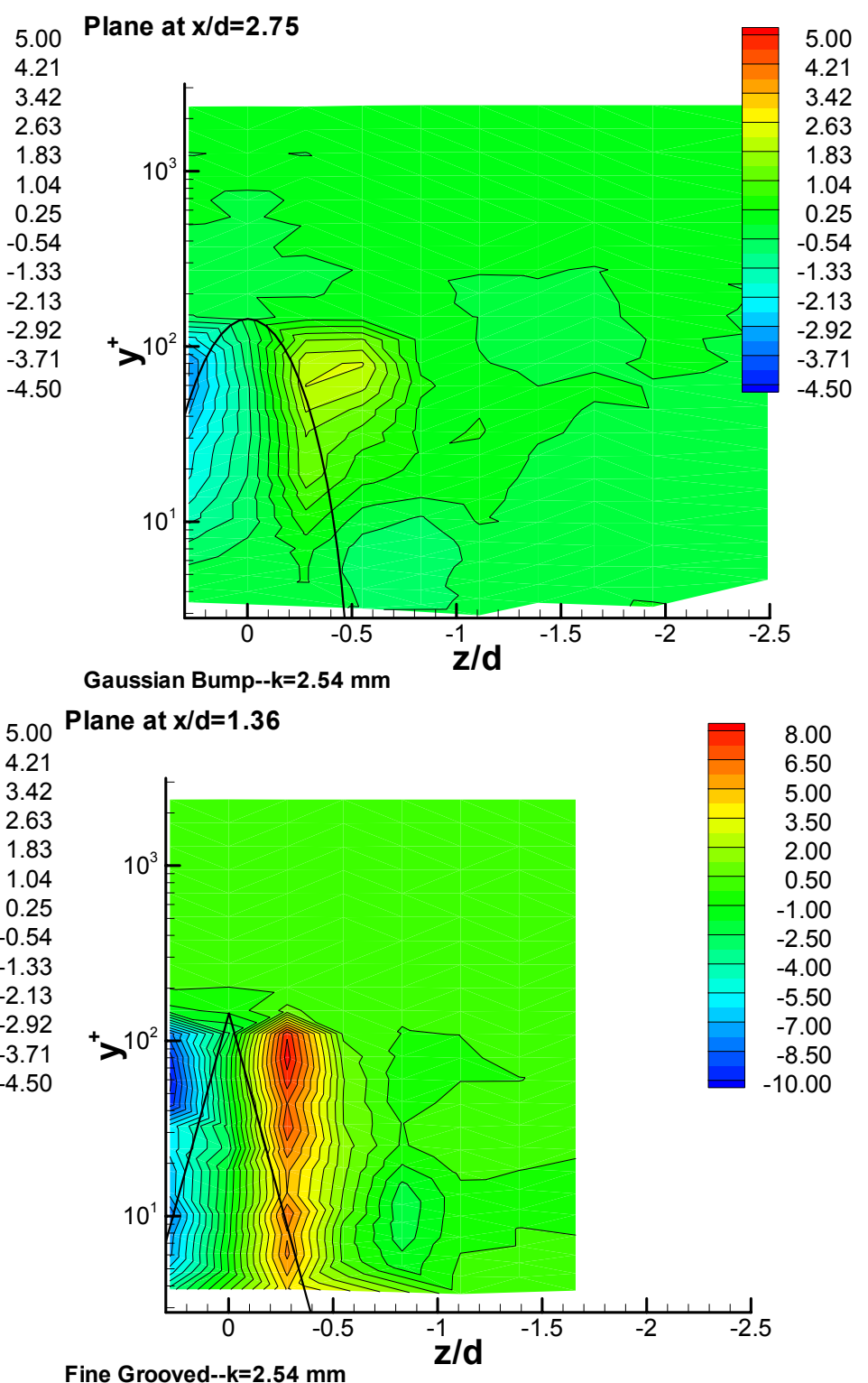

Large Grooved--k=2.54 mm

Figure 3.49: Contours of $\overline{u w} / U_{\tau}^{2}$ in the $\mathbf{y}-\mathbf{z}$ plane at a location of $\mathbf{x} / \mathbf{d}=\mathbf{2 . 7 5}$ (Note: Fine Grooved $\mathbf{x} / \mathbf{d}=\mathbf{1 . 3 6}$ ) 


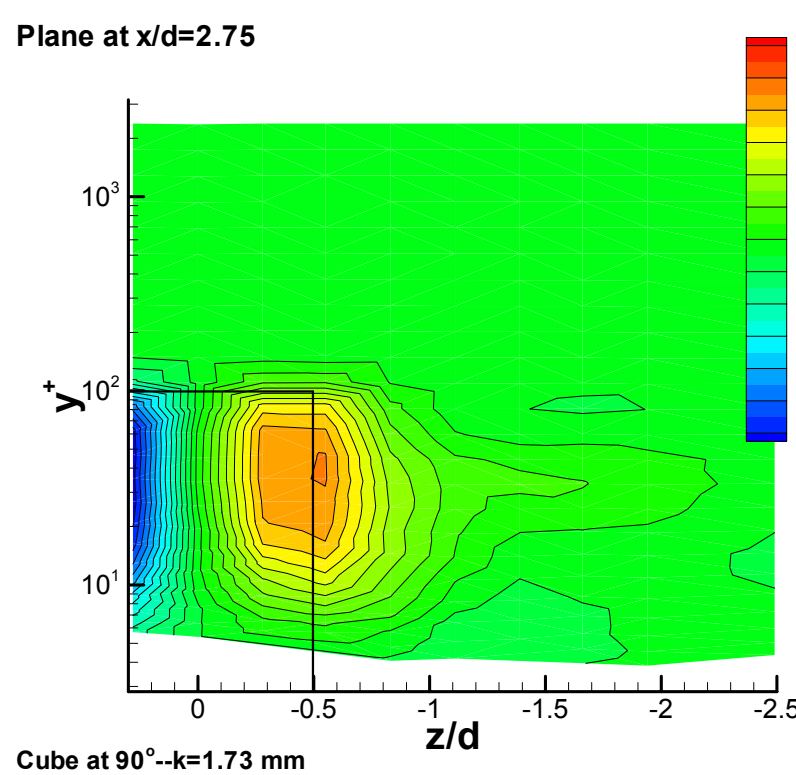

Plane at $x / d=2.75$

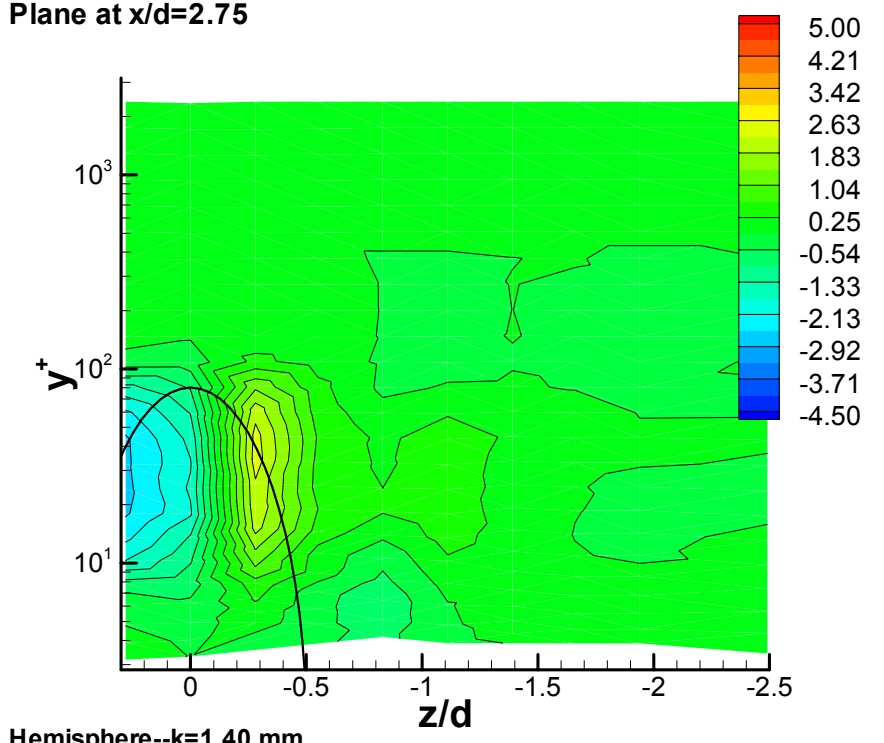

Hemisphere--k=1.40 mm

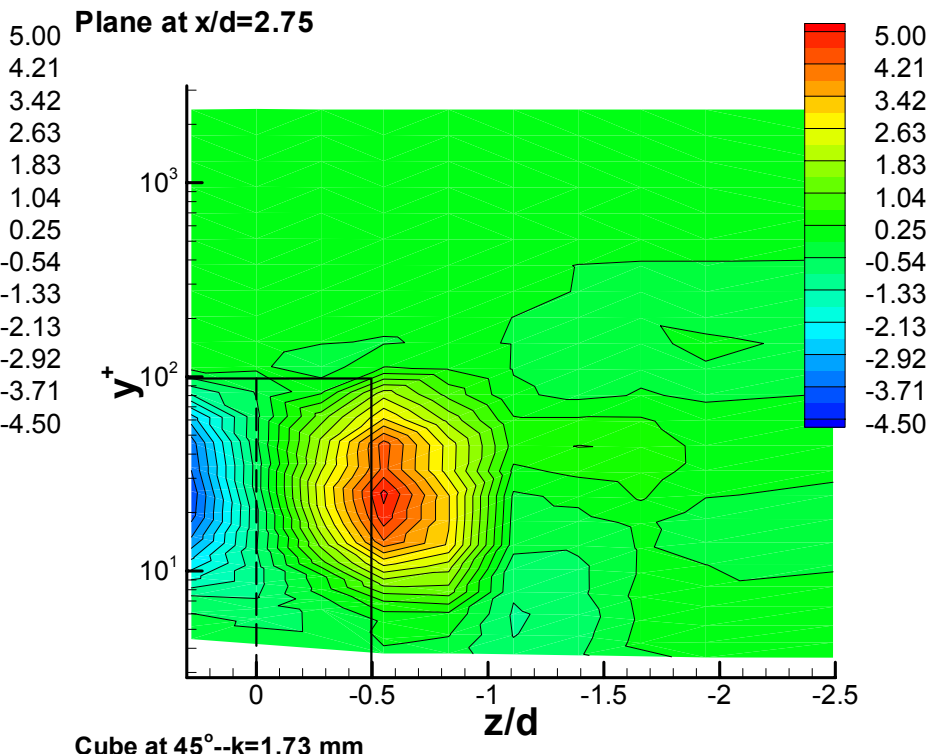

Cube at $45^{\circ}--\mathrm{k}=1.73 \mathrm{~mm}$

Figure 3.50: Contours of $\overline{u w} / U_{\tau}^{2}$ in the $\mathbf{y}-\mathbf{z}$ plane at a location of $\mathbf{x} / \mathbf{d}=\mathbf{2 . 7 5}$ 

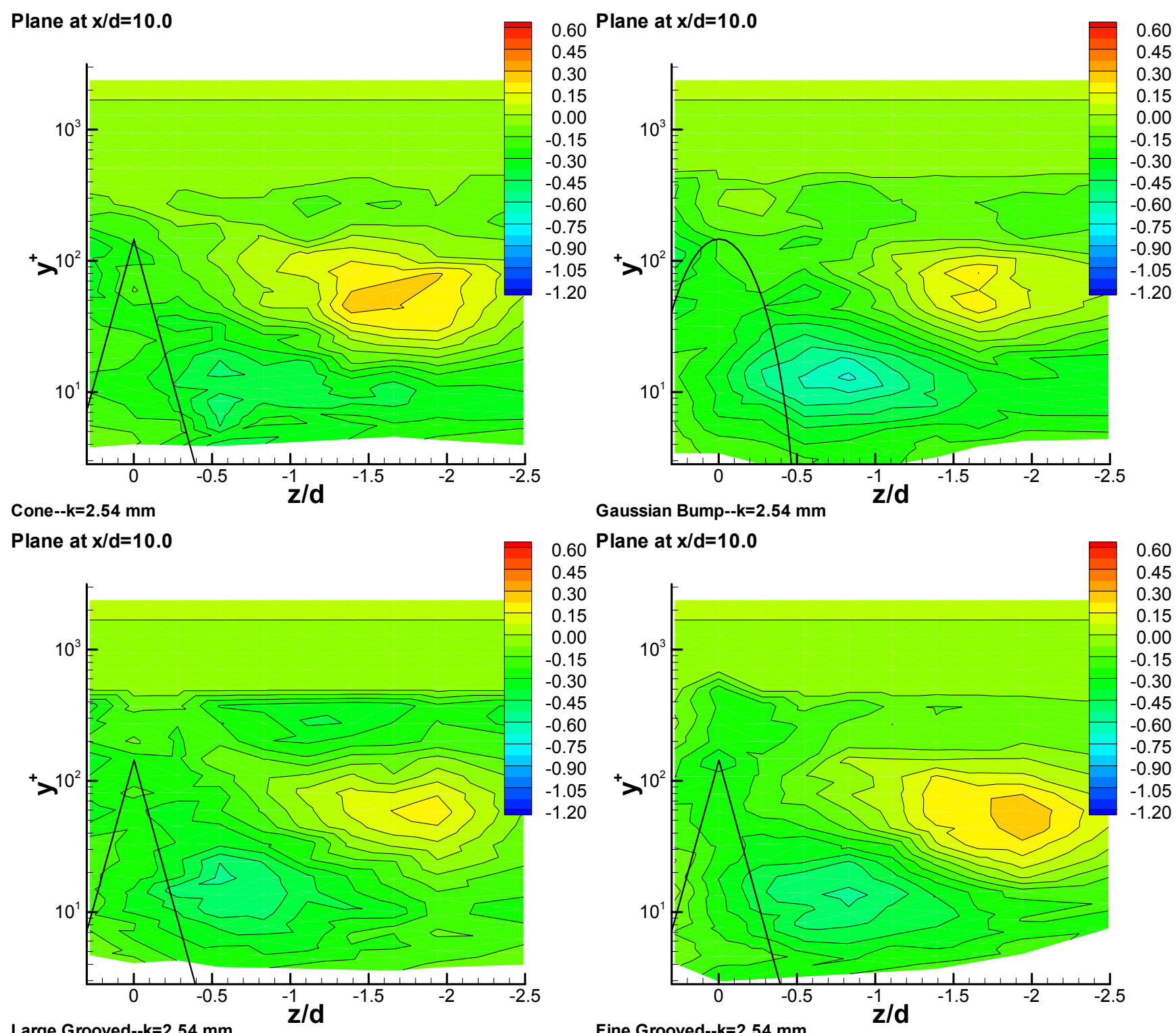

Gaussian Bump--k=2.54 mm

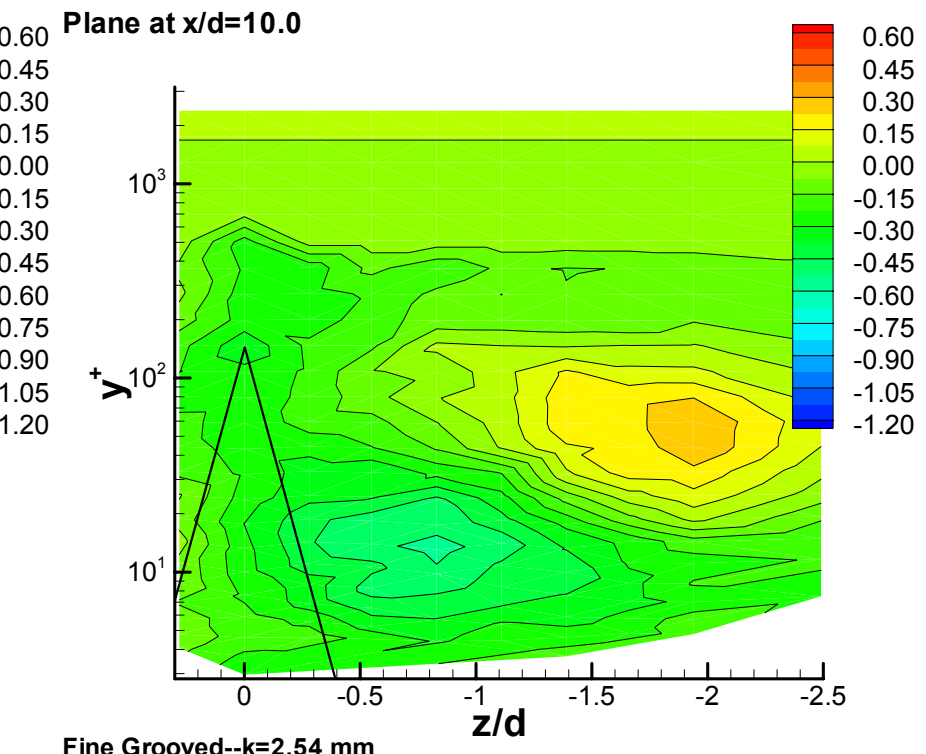

Large Grooved--k=2.54 mm

Fine Grooved--k=2.54 mm

Figure 3.51: Contours of $\overline{u w} / U_{\tau}^{2}$ in the $\mathbf{y}-\mathbf{z}$ plane at a location of $\mathbf{x} / \mathbf{d}=\mathbf{1 0 . 0}$ 


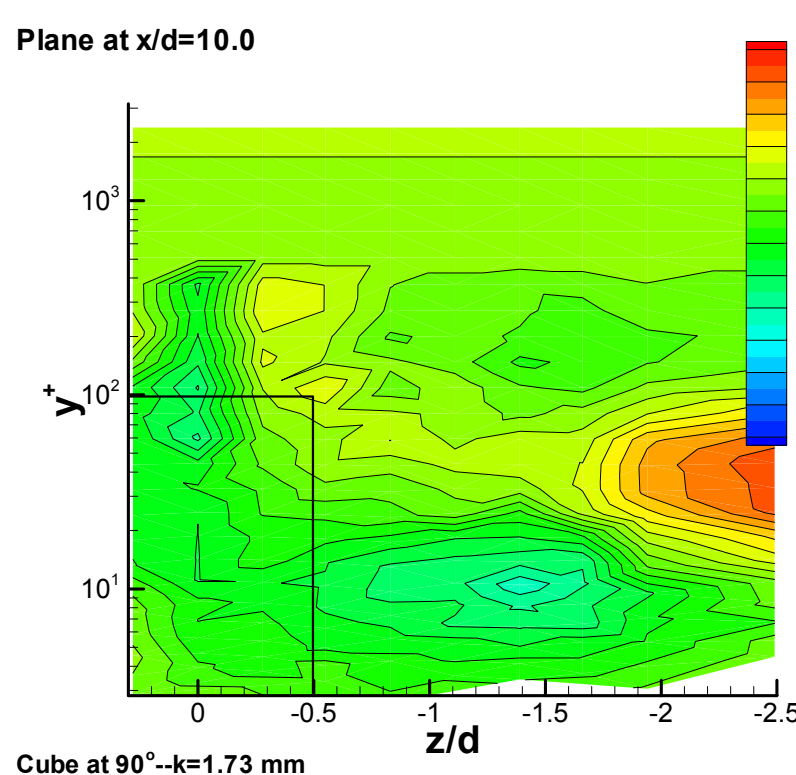

Plane at $x / d=10.0$

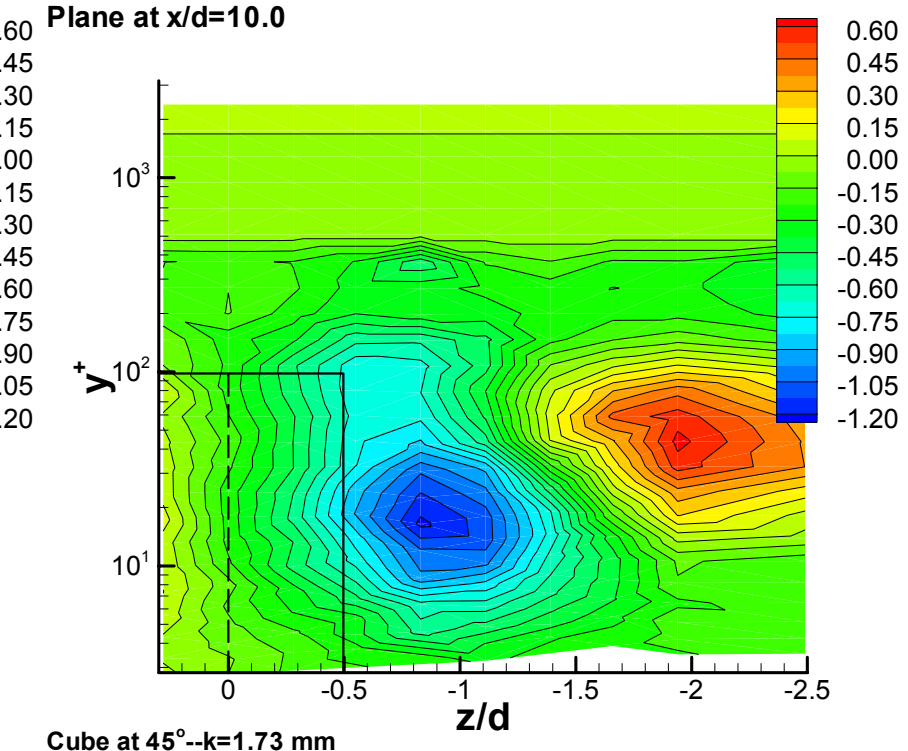

Cube at $45^{\circ}--\mathrm{k}=1.73 \mathrm{~mm}$

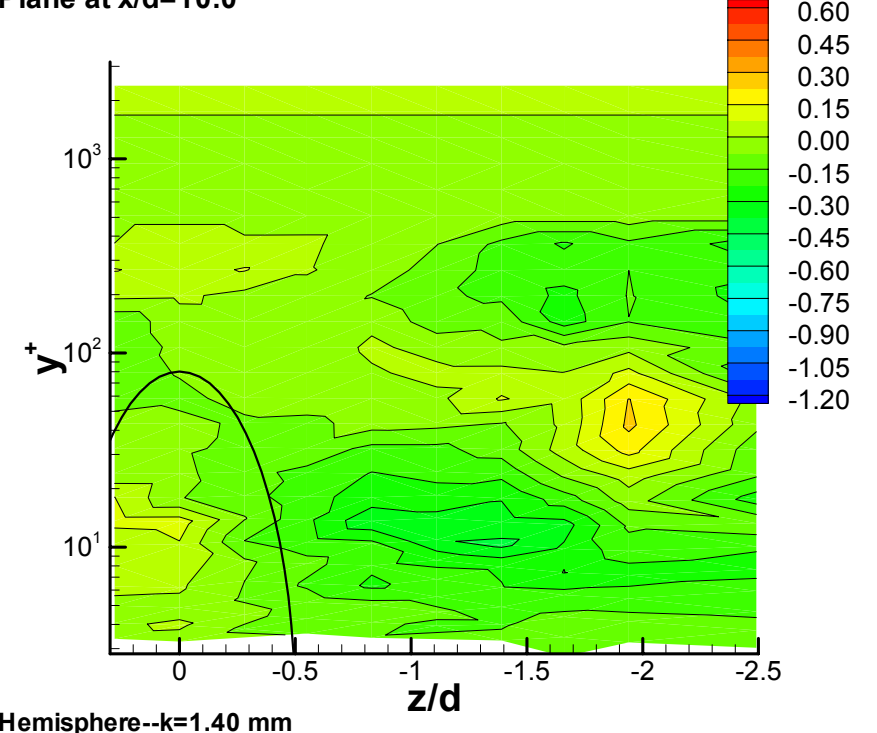

Hemisphere--k=1.40 $\mathrm{mm}$

Figure 3.52: Contours of $\overline{u w} / U_{\tau}^{2}$ in the $\mathbf{y}-\mathbf{z}$ plane at a location of $\mathbf{x} / \mathbf{d}=\mathbf{1 0 . 0}$ 

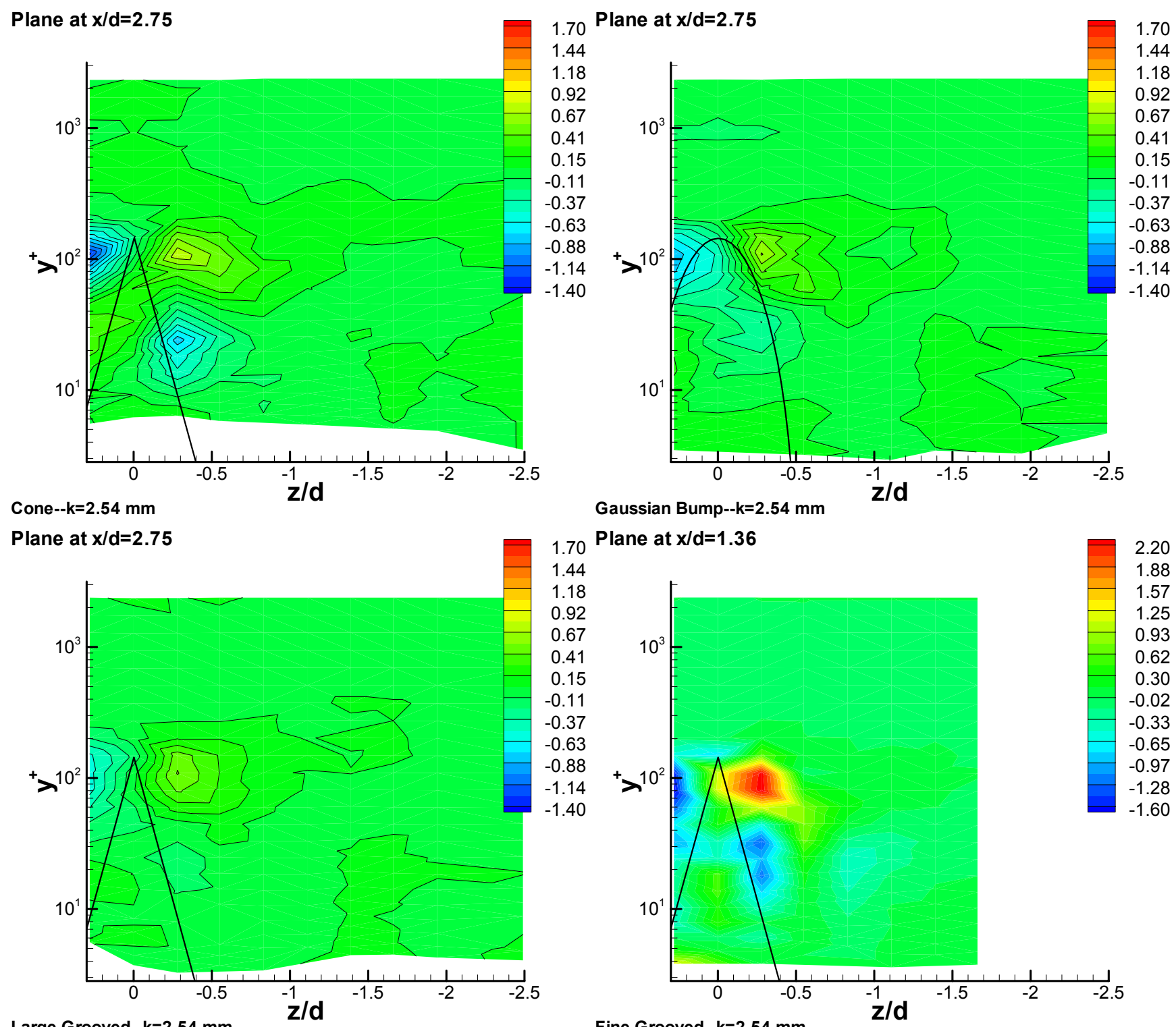

ump--k=2.54 mm

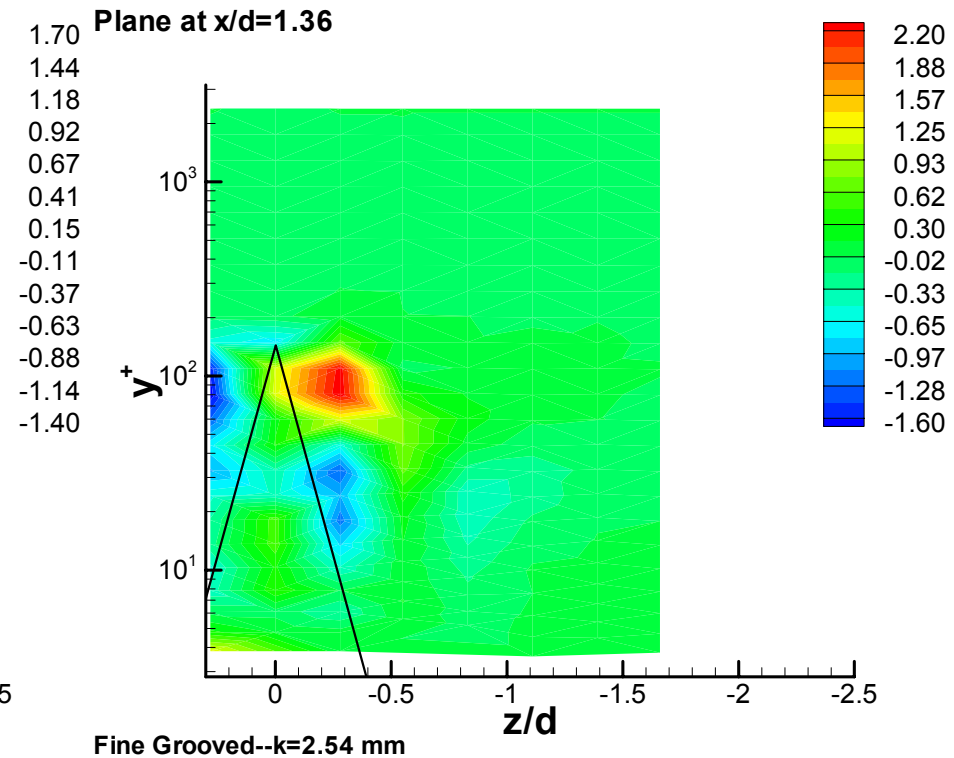

Large Grooved--k=2.54 mm

Figure 3.53: Contours of $\overline{v w} / U_{\tau}^{2}$ in the $\mathbf{y}-\mathbf{z}$ plane at a location of $\mathbf{x} / \mathbf{d}=\mathbf{2 . 7 5}$ (Note: Fine Grooved $\mathbf{x} / \mathbf{d}=\mathbf{1 . 3 6}$ ) 


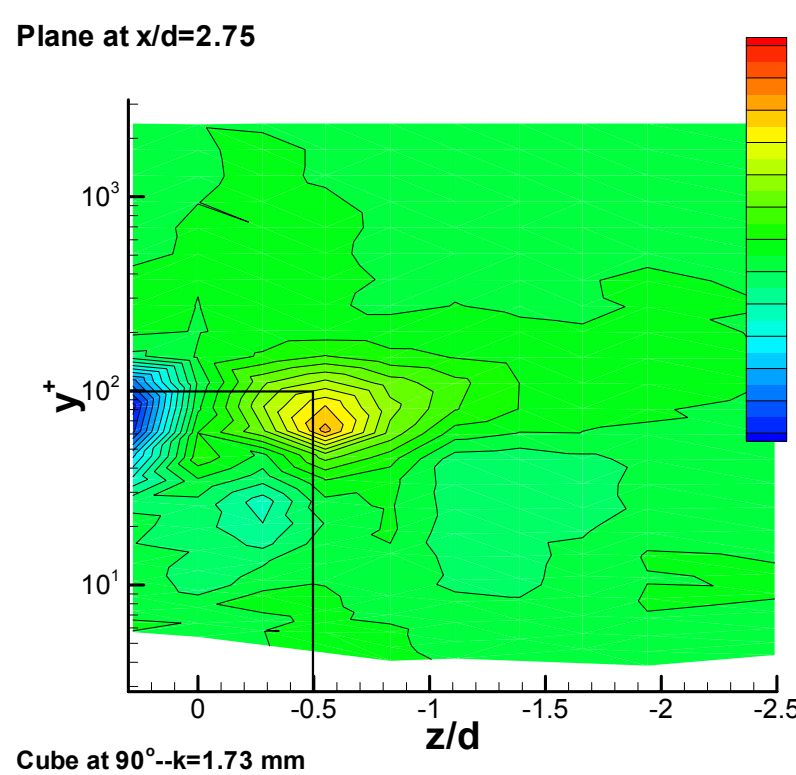

Plane at $x / d=2.75$

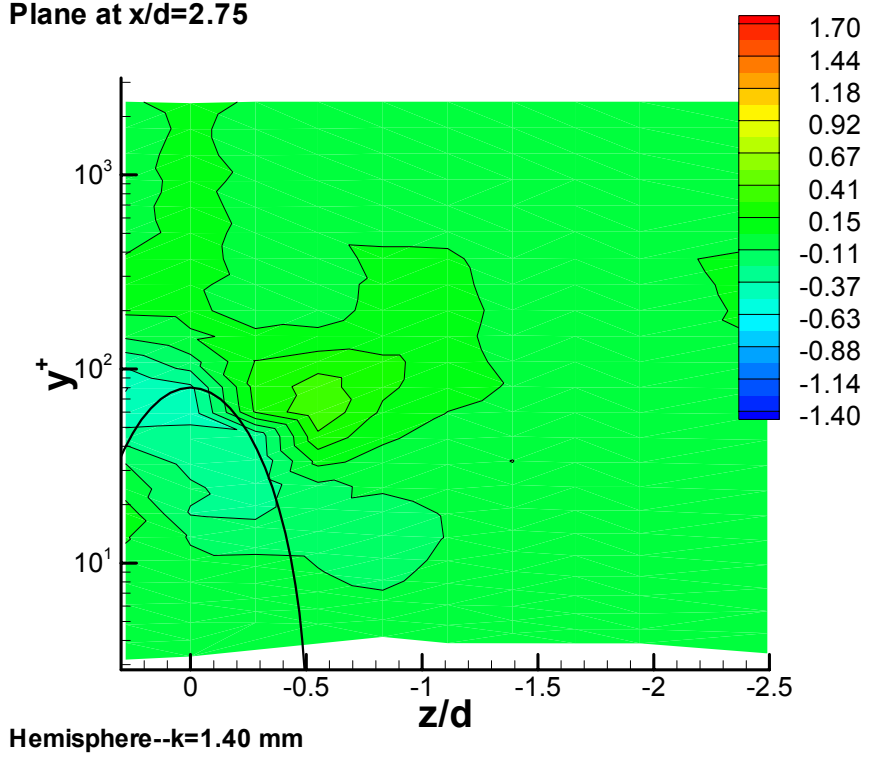

Plane at $x / d=2.75$

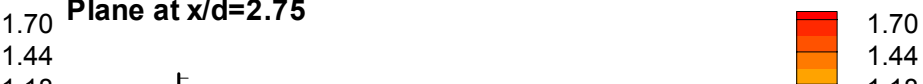

\begin{tabular}{l|l}
1.70 & 1.44 \\
1.18 & 1.18
\end{tabular}

$\begin{array}{ll}0.92 & 0.92 \\ 0.67 & \end{array}$

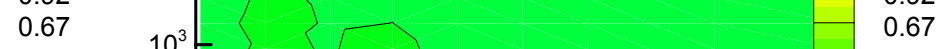

$\begin{array}{ll}0.41 \quad 10^{3} & =2>> \\ 0.15 & 0.41\end{array}$

$0.15 \quad 2 \quad 0.15$

$-0.11$

$-0.37$

$-0.63$

$-0.88$

$-1.14$

$-1.40$
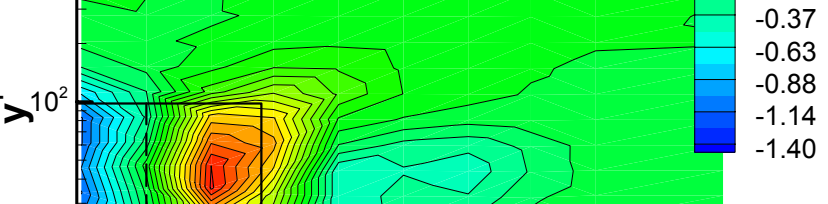

$10^{1}$

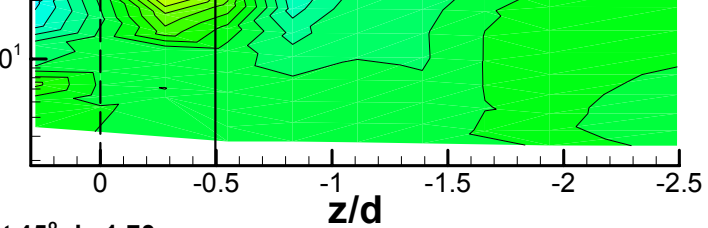

Cube at $45^{\circ}--\mathrm{k}=1.73 \mathrm{~mm}$

$-1.40$

Figure 3.54: Contours of $\overline{v w} / U_{\tau}^{2}$ in the $\mathbf{y}-\mathbf{z}$ plane at a location of $\mathbf{x} / \mathbf{d}=\mathbf{2 . 7 5}$ 


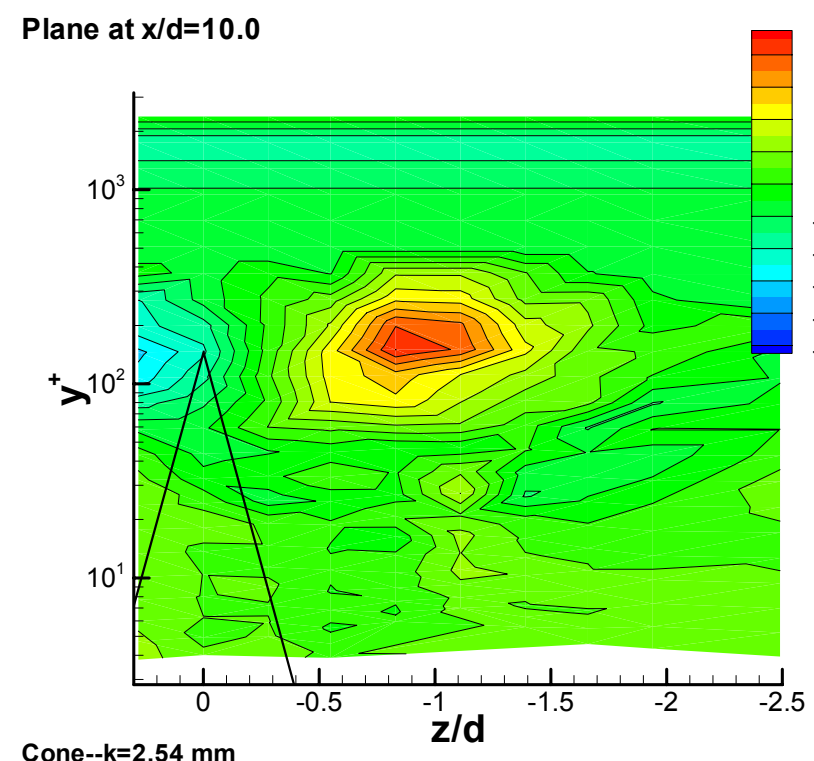

\section{Plane at $x / d=10.0$}

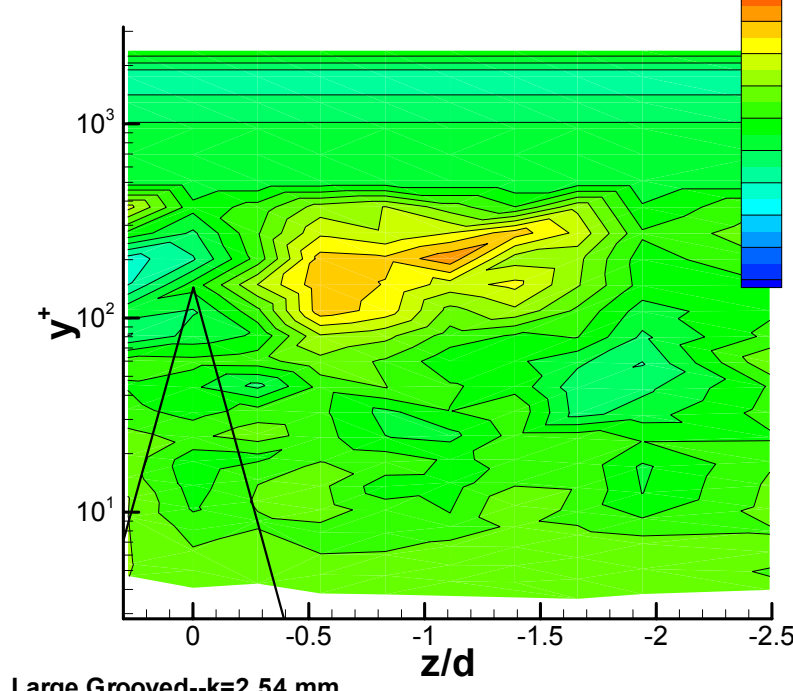

Large Grooved--k=2.54 mm

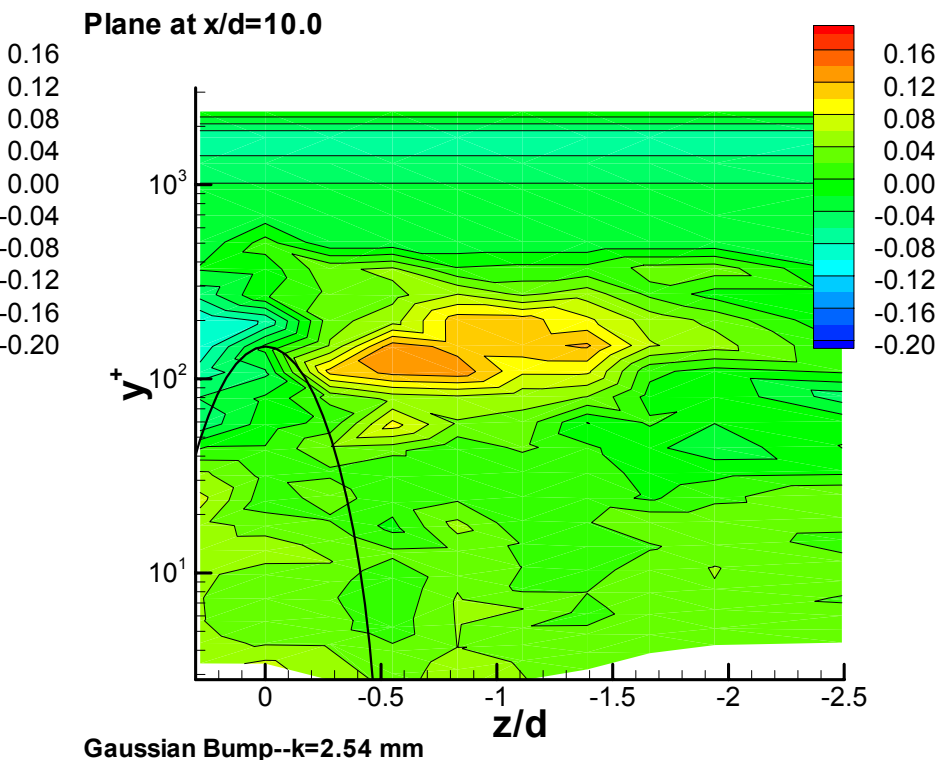

Plane at $x / d=10.0$

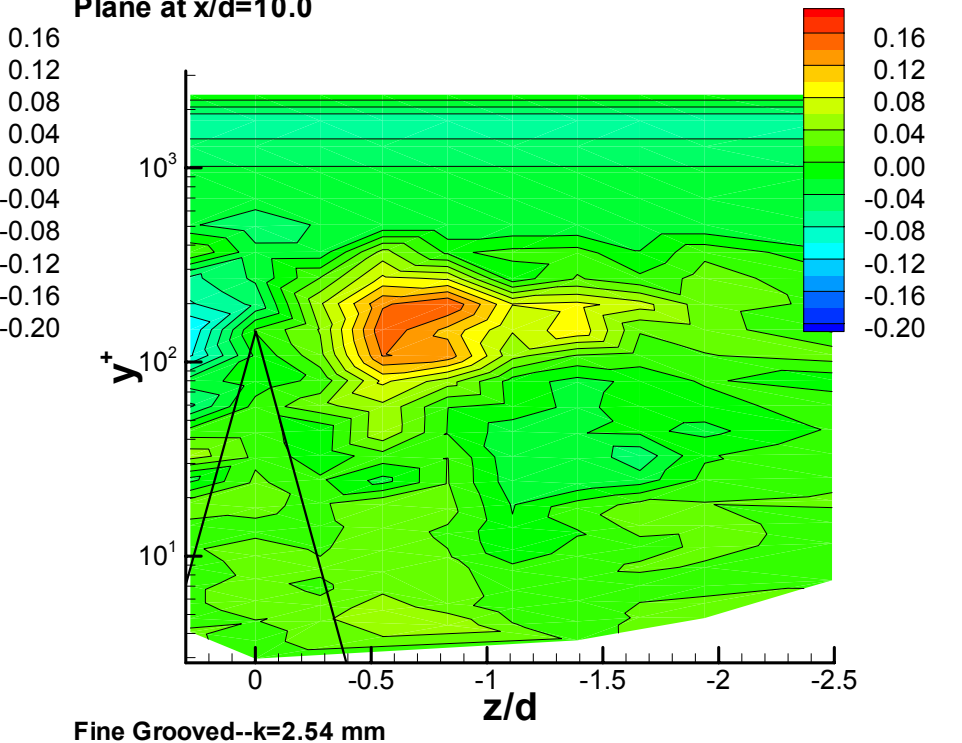

Figure 3.55: Contours of $\overline{v w} / U_{\tau}^{2}$ in the $\mathbf{y}-\mathbf{z}$ plane at a location of $\mathbf{x} / \mathbf{d}=\mathbf{1 0 . 0}$ 


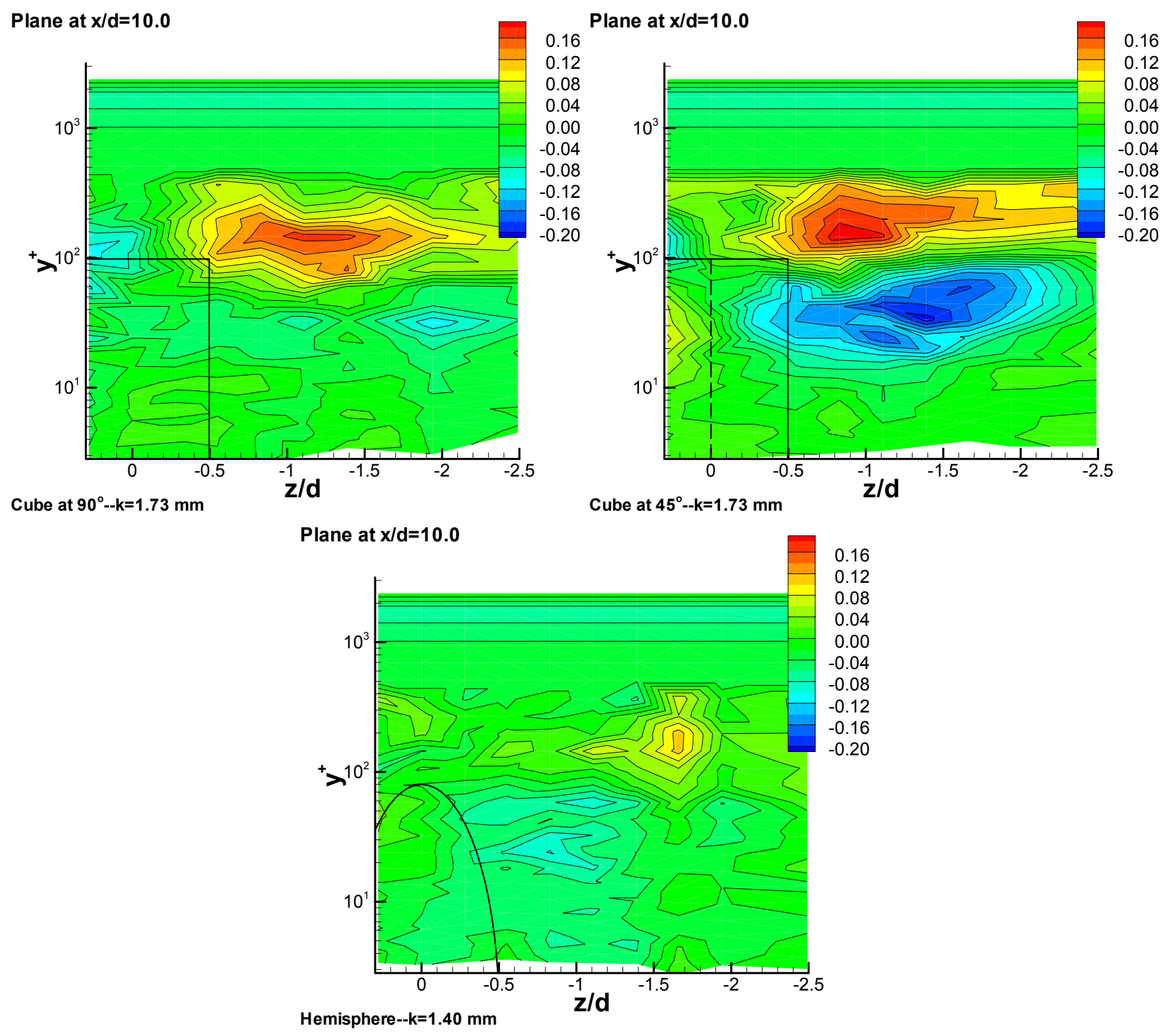

Figure 3.56: Contours of $\overline{v w} / U_{\tau}^{2}$ in the $\mathbf{y}-\mathbf{z}$ plane at a location of $\mathbf{x} / \mathbf{d}=\mathbf{1 0 . 0}$ 


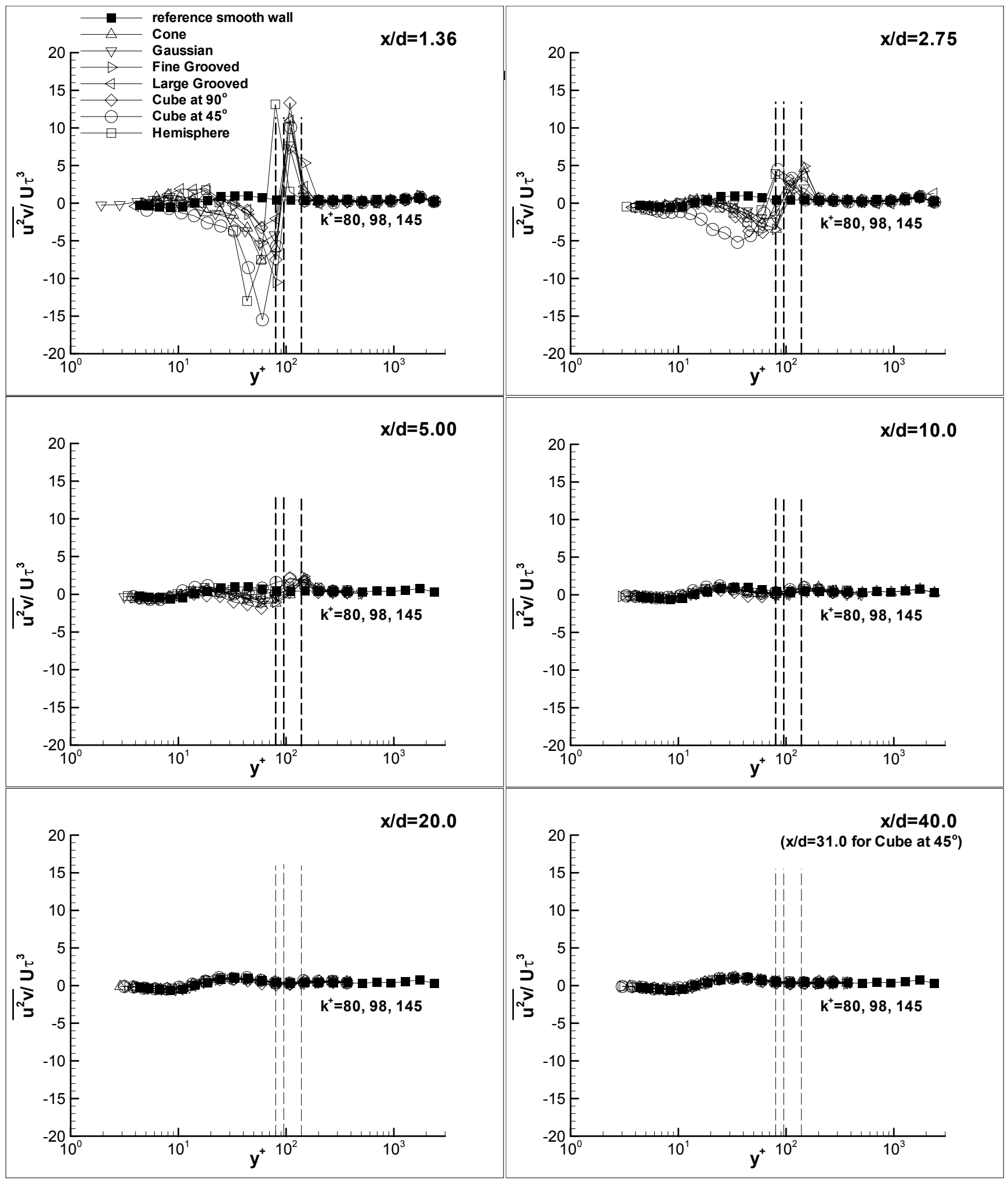

Figure 3.57: $\overline{u^{2} v} /_{U_{\tau}^{3}}$ vs. $y U_{\tau} / v$ profiles along the centerline 

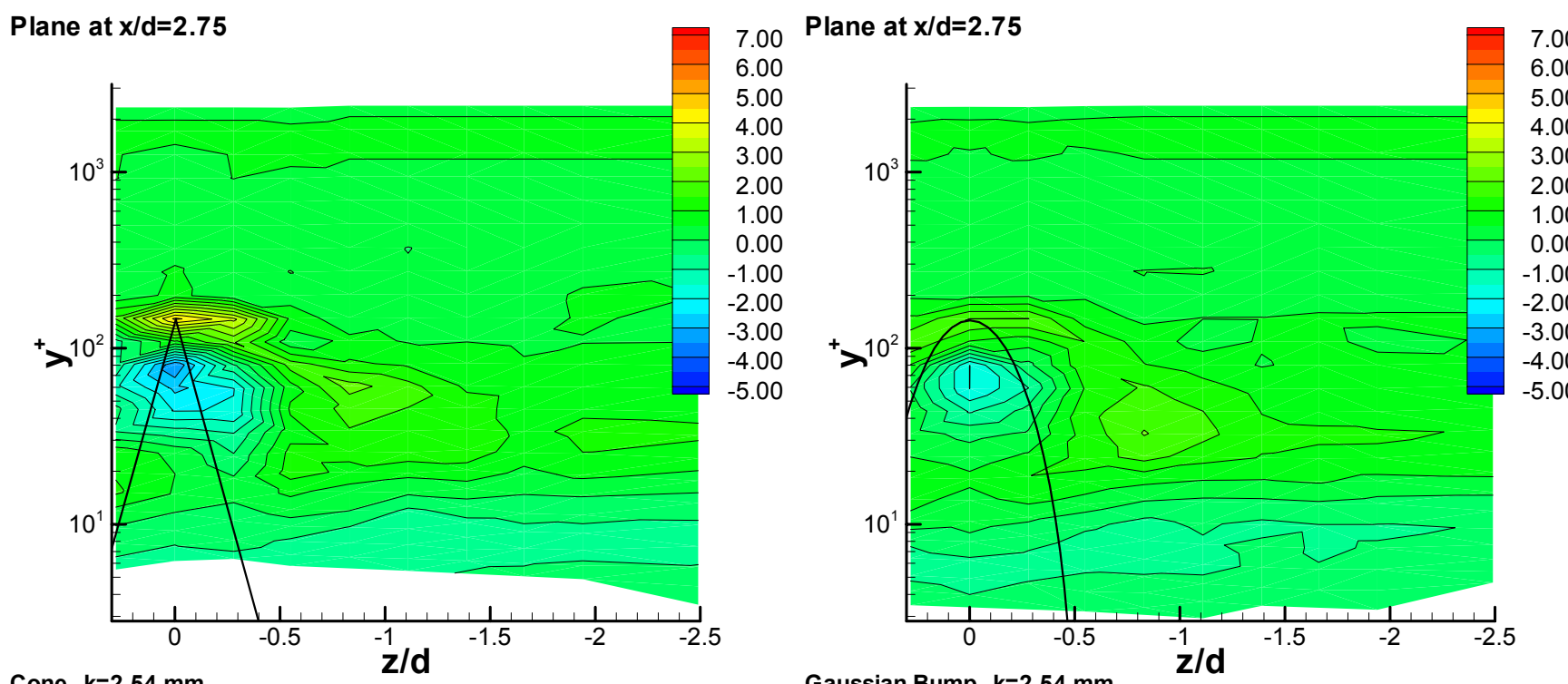

Cone--k=2.54 mm

Gaussian Bump--k=2.54 mm

Plane at $x / d=2.75$

Plane at $x / d=1.36$

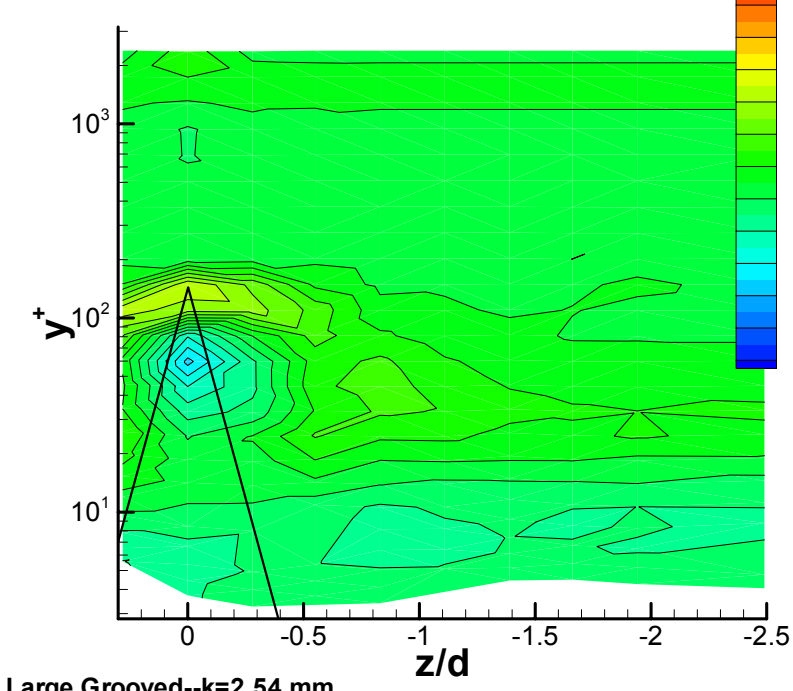

Large Grooved--k=2.54 mm

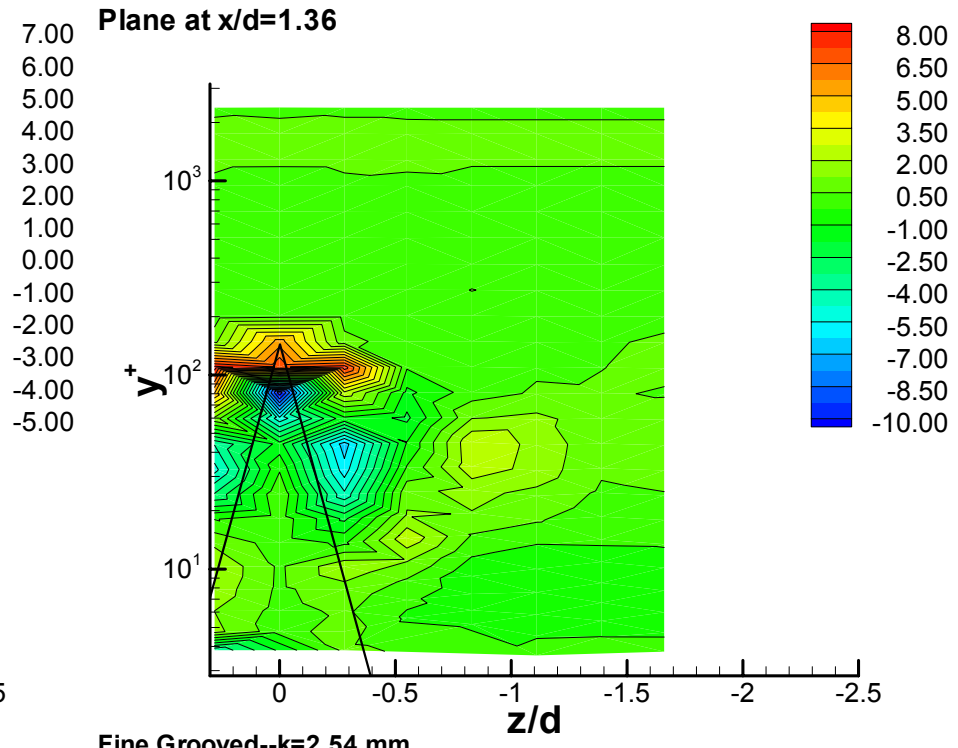

Fine Grooved--k=2.54 mm

Figure 3.58: Contours of $\overline{u^{2} v} / U_{\tau}^{3}$ in the $\mathbf{y}-z$ plane at a location of $\mathbf{x} / \mathbf{d}=\mathbf{2 . 7 5}$ (Note: Fine Grooved $\mathbf{x} / \mathbf{d}=1.36$ ) 


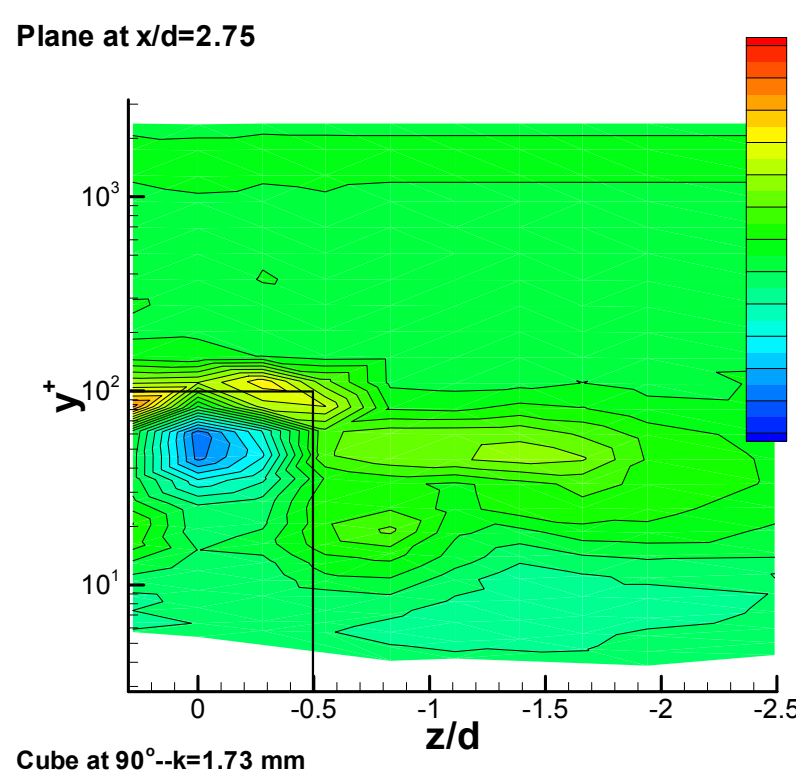

Cube at $90^{\circ}--\mathrm{k}=1.73 \mathrm{~mm}$

\section{Plane at $x / d=2.75$}

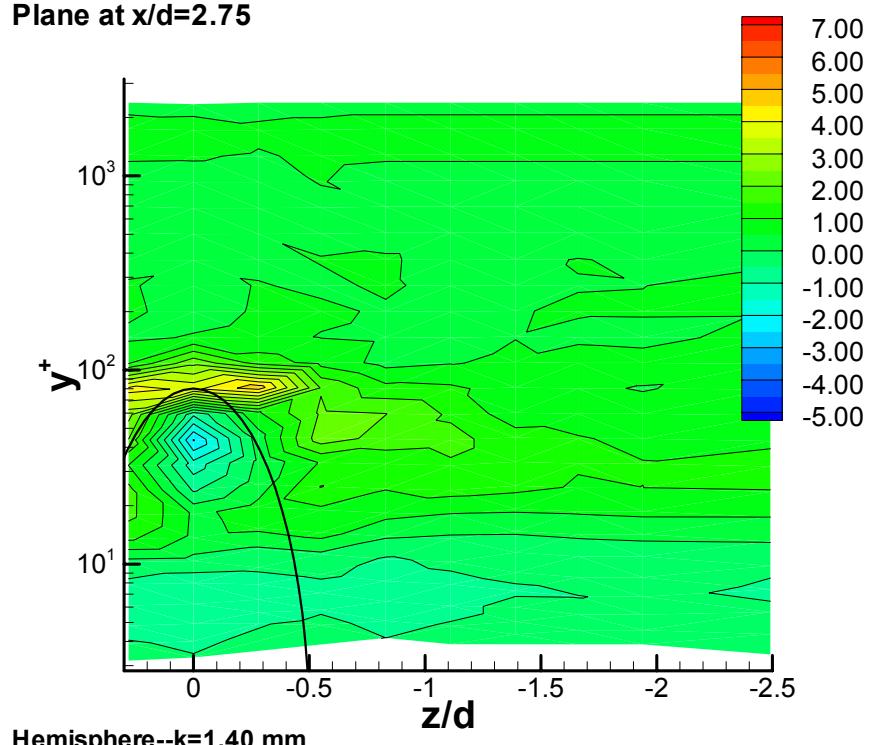

Hemisphere--k=1.40 mm
Plane at $x / d=2.75$

7.00
6.00

6.00
5.00

4.00

3.00

2.00

1.00
0.00

0.00
-1.00

$-2.00$

$-3.00$

$-4.00$

$-5.00$

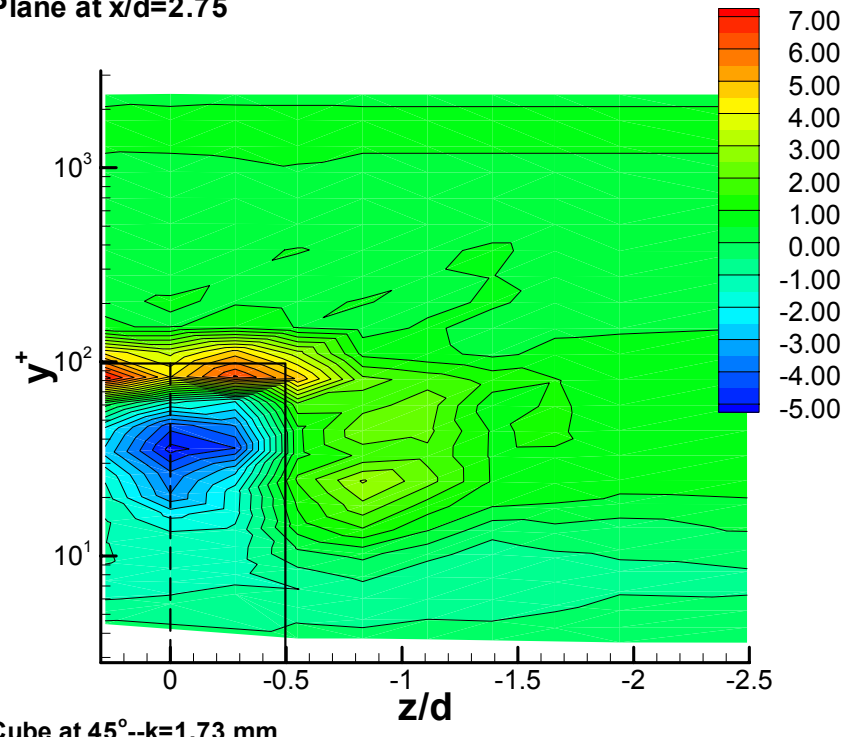

Cube at $45^{\circ}--\mathrm{k}=1.73 \mathrm{~mm}$

2.00

0.00

1.00

$-3.00$

5.00

Figure 3.59: Contours of $\overline{u^{2} v} / U_{\tau}^{3}$ in the $\mathbf{y}-z$ plane at a location of $\mathbf{x} / \mathbf{d}=\mathbf{2 . 7 5}$ 

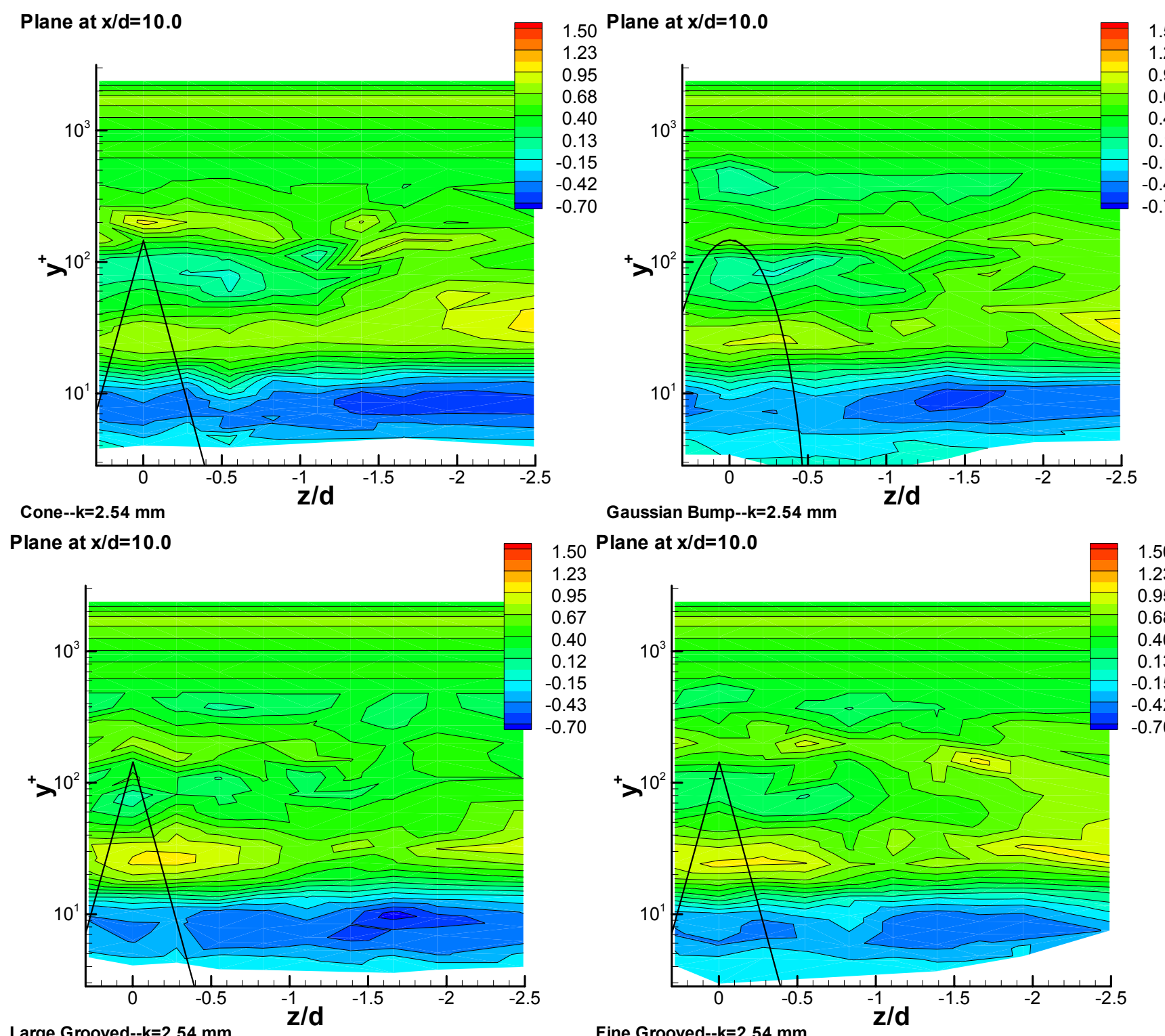

Gaussian Bump--k=2.54 mm

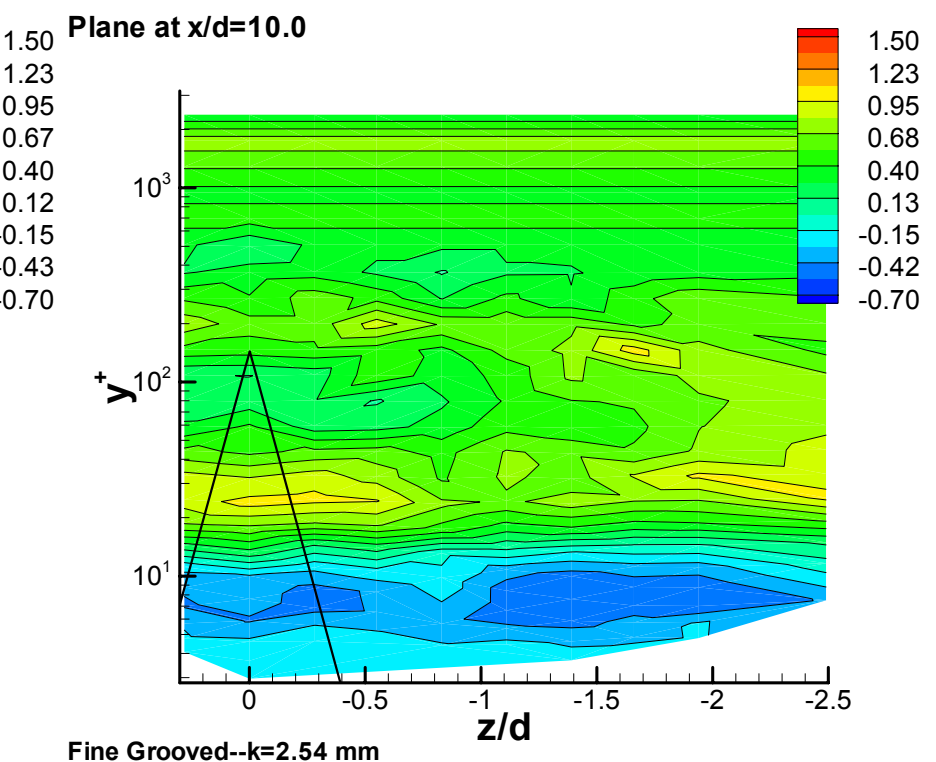

Large Grooved--k=2.54 mm

Fine Grooved--k=2.54 $\mathrm{mm}$

Figure 3.60: Contours of $\overline{u^{2} v} / U_{\tau}^{3}$ in the $\mathbf{y}-z$ plane at a location of $\mathbf{x} / \mathbf{d}=10.0$ 


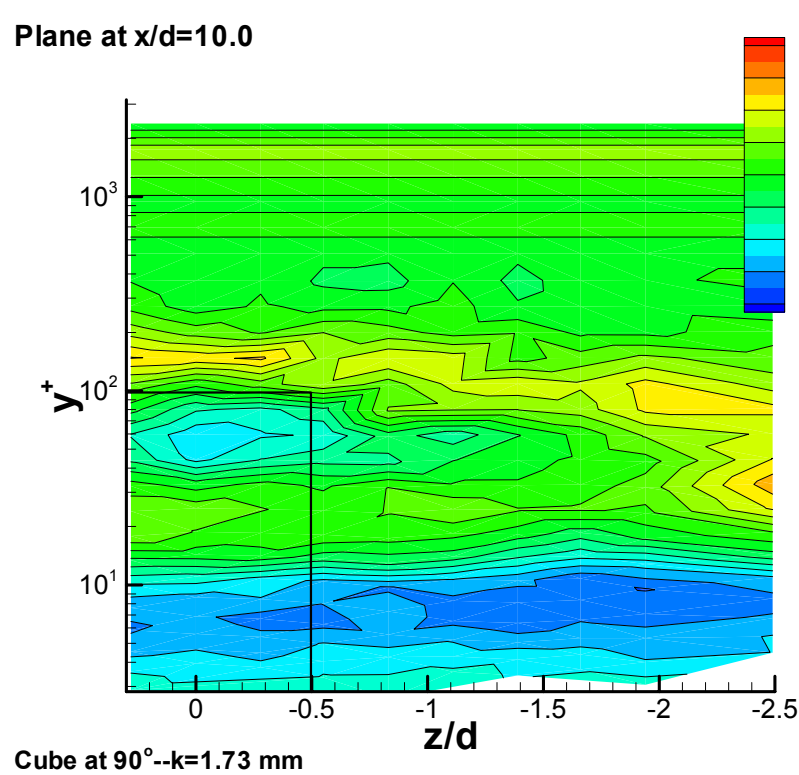

Plane at $x / d=10.0$
Plane at $x / d=10.0$

1.50
1.23
0.95

0.68

0.40

0.13

$-0.15$

-0.42
-0.70

$$
{ }^{+}>^{10^{2}}
$$

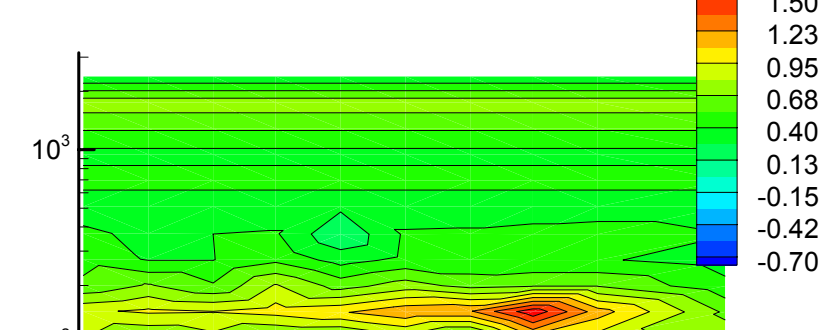

$10^{1}$

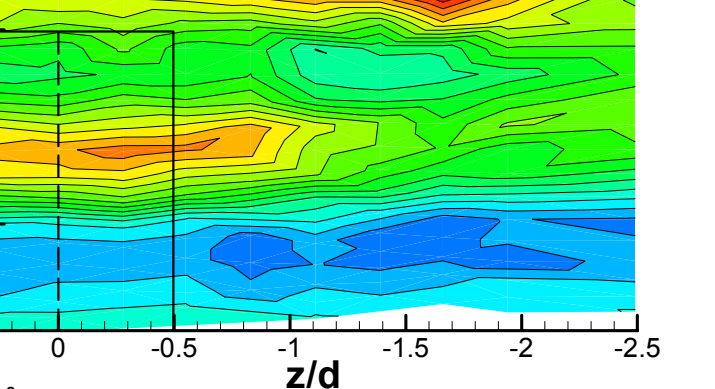

Cube at $45^{\circ}--\mathrm{k}=1.73 \mathrm{~mm}$

$$
1.50
$$

1.23

1.23
0.95

0.68

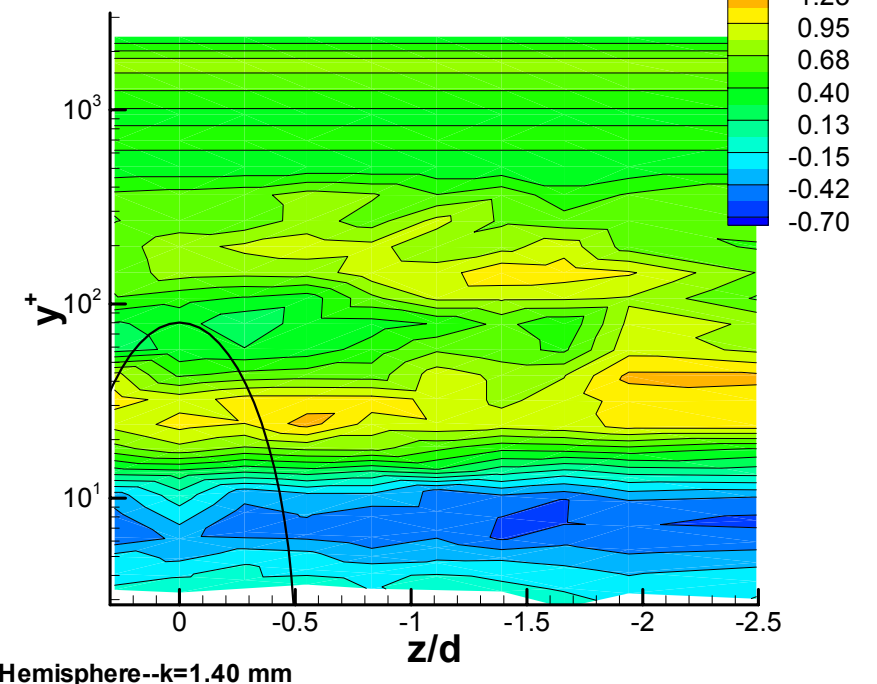

Hemisphere--k=1.40 mm

Figure 3.61: Contours of $\overline{u^{2} v} / U_{\tau}^{3}$ in the $\mathbf{y}-z$ plane at a location of $\mathbf{x} / \mathbf{d}=10.0$ 

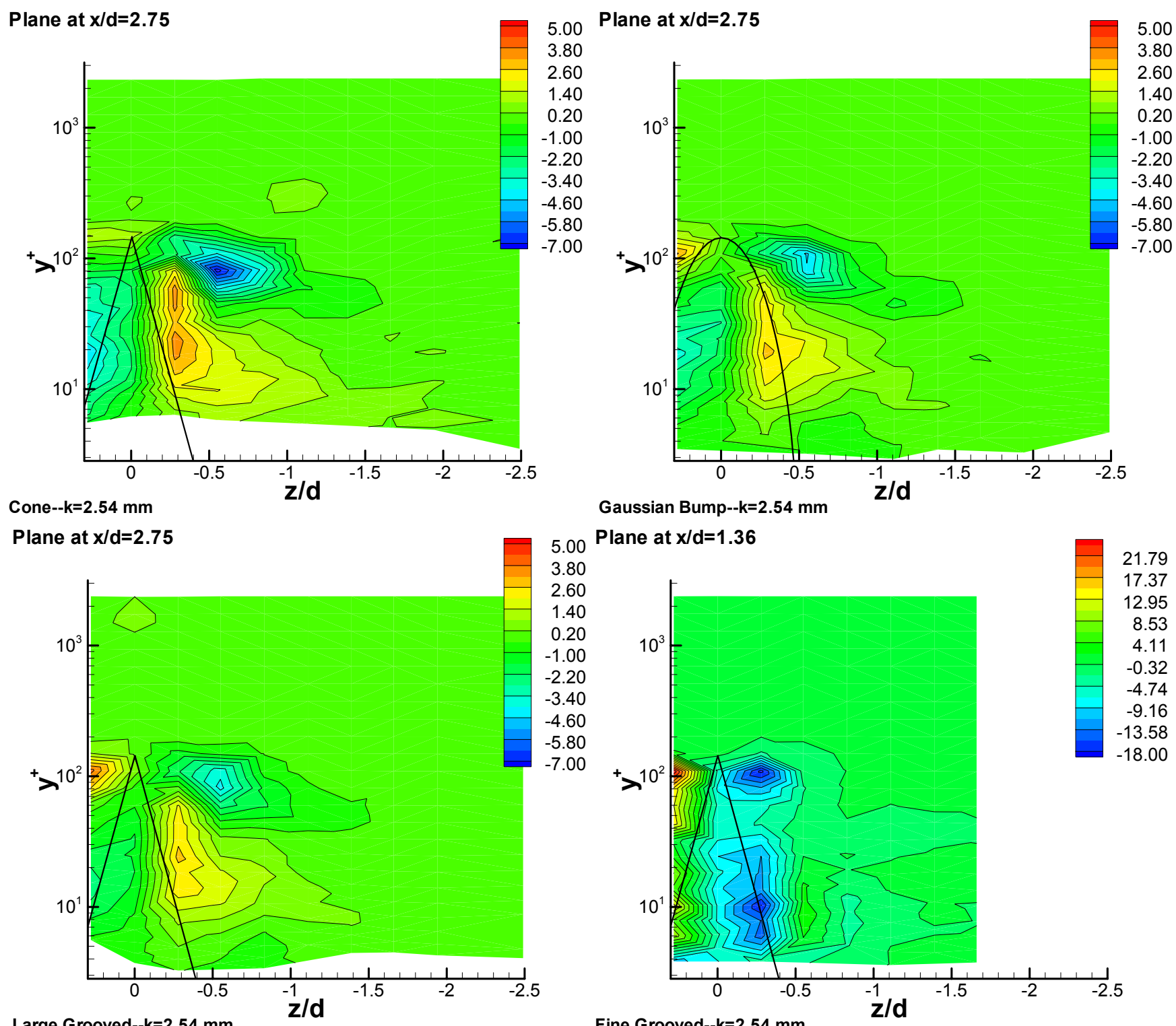

Gaussian Bump--k=2.54 mm

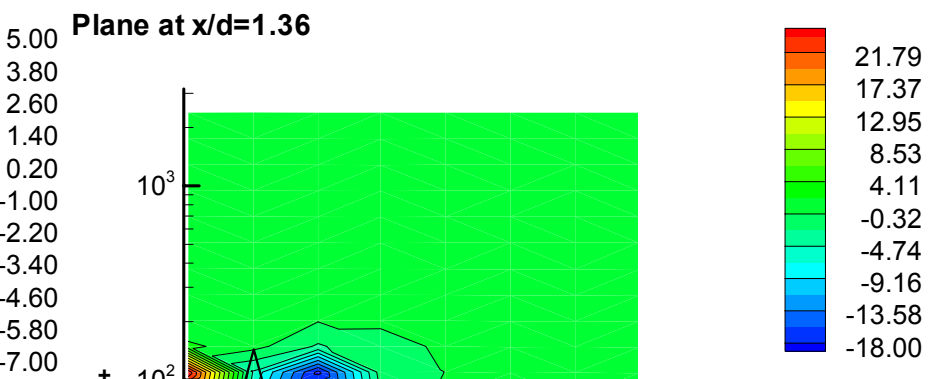

Large Grooved--k=2.54 mm

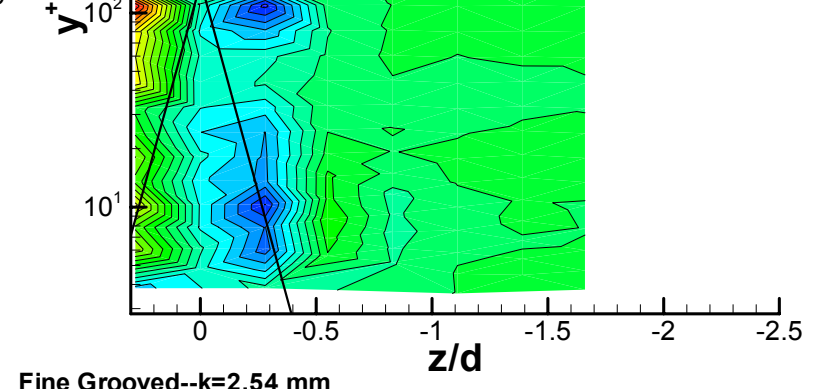

Fine Grooved-k=2.54 mm

Figure 3.62: Contours of $\overline{u^{2} w} / U_{\tau}^{3}$ in the $\mathbf{y}-\mathbf{z}$ plane at a location of $\mathbf{x} / \mathbf{d}=\mathbf{2 . 7 5}$ (Note: Fine Grooved $\mathbf{x} / \mathbf{d}=1.36$ ) 


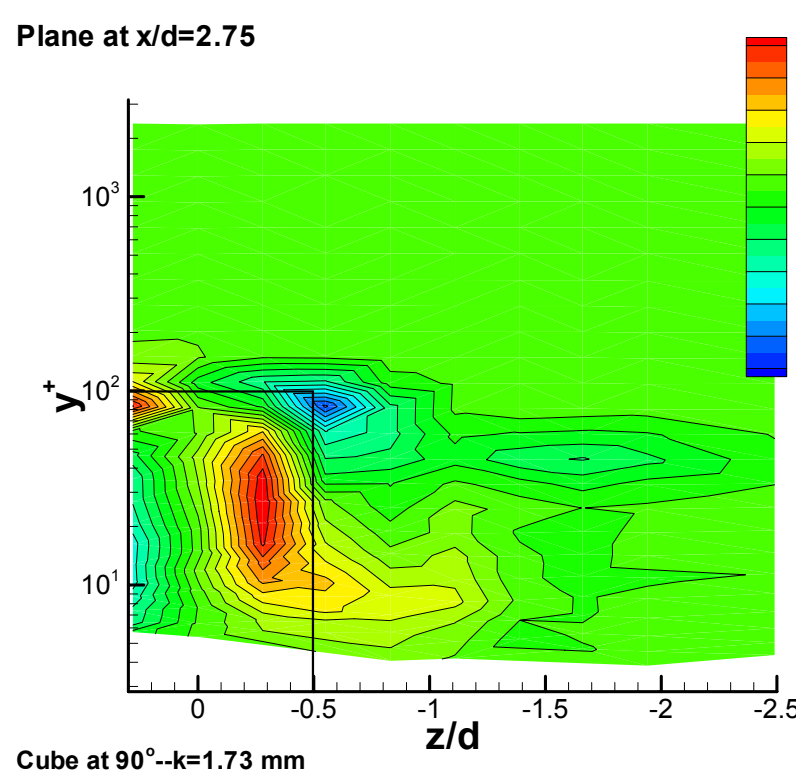

Plane at $x / d=2.75$

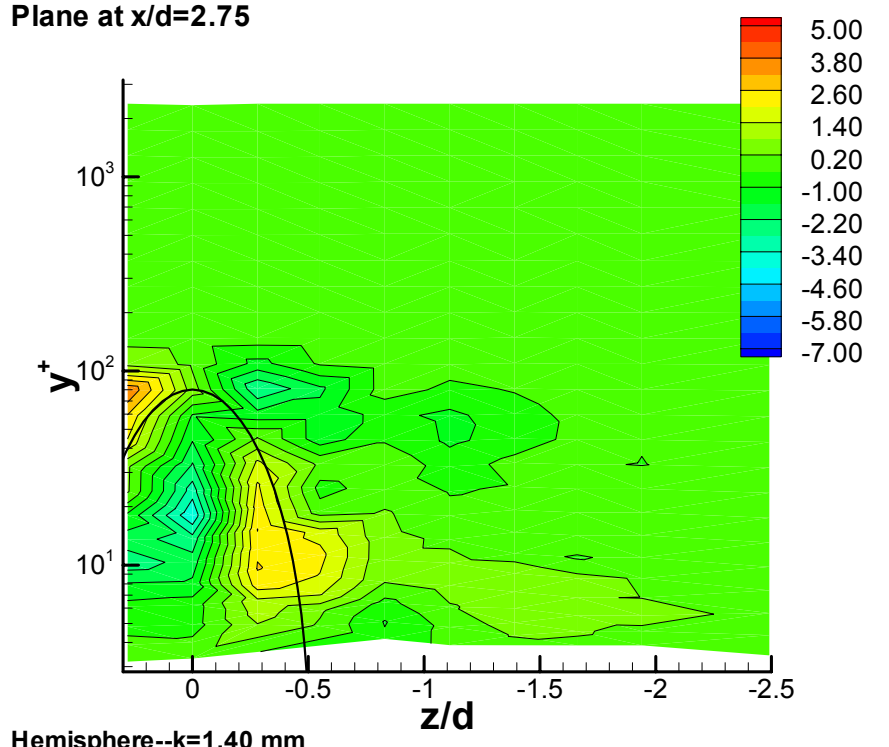

Plane at $x / d=2.75$

5.00
3.80
2.60

2.60

0.20

0.20
-1.00

$-2.20$

$-3.40$

$-5.80$

$-7.00$

$+1$

$10^{1}$

Cube at $45^{\circ}--\mathrm{k}=1.73 \mathrm{~mm}$

.00

8.80

60

0.20

.00

$-60$

$-80$

Hemisphere--k=1.40 mm
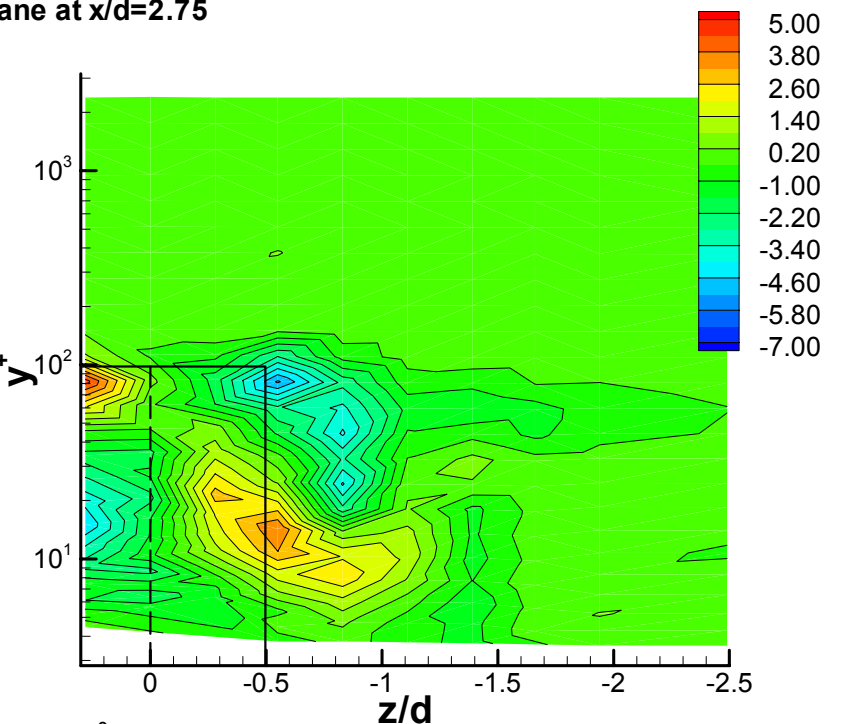

Figure 3.63: Contours of $\overline{u^{2} w} / U_{\tau}^{3}$ in the $\mathbf{y}-\mathbf{z}$ plane at a location of $\mathbf{x} / \mathbf{d}=\mathbf{2 . 7 5}$ 


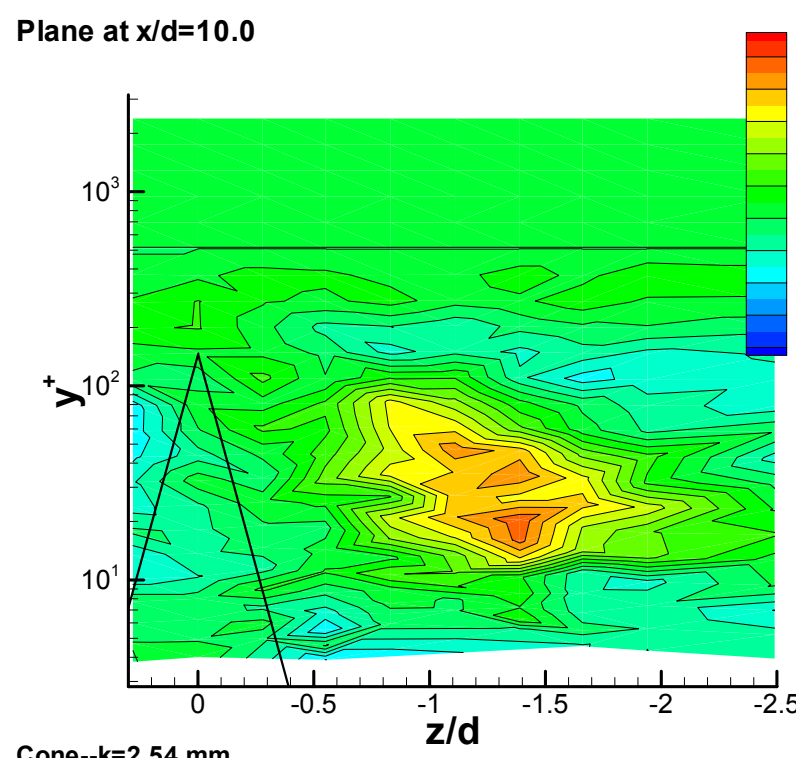

Plane at $x / d=10.0$

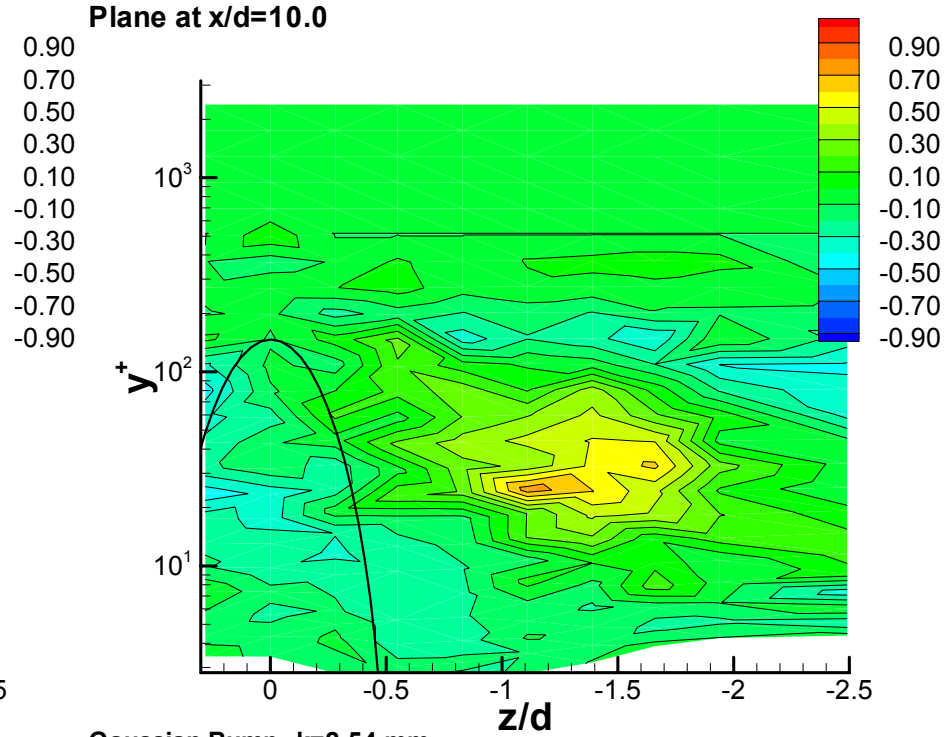

Cone--k=2.54 mm

Gaussian Bump--k=2.54 mm

Plane at $x / d=10.0$

Plane at $x / d=10.0$
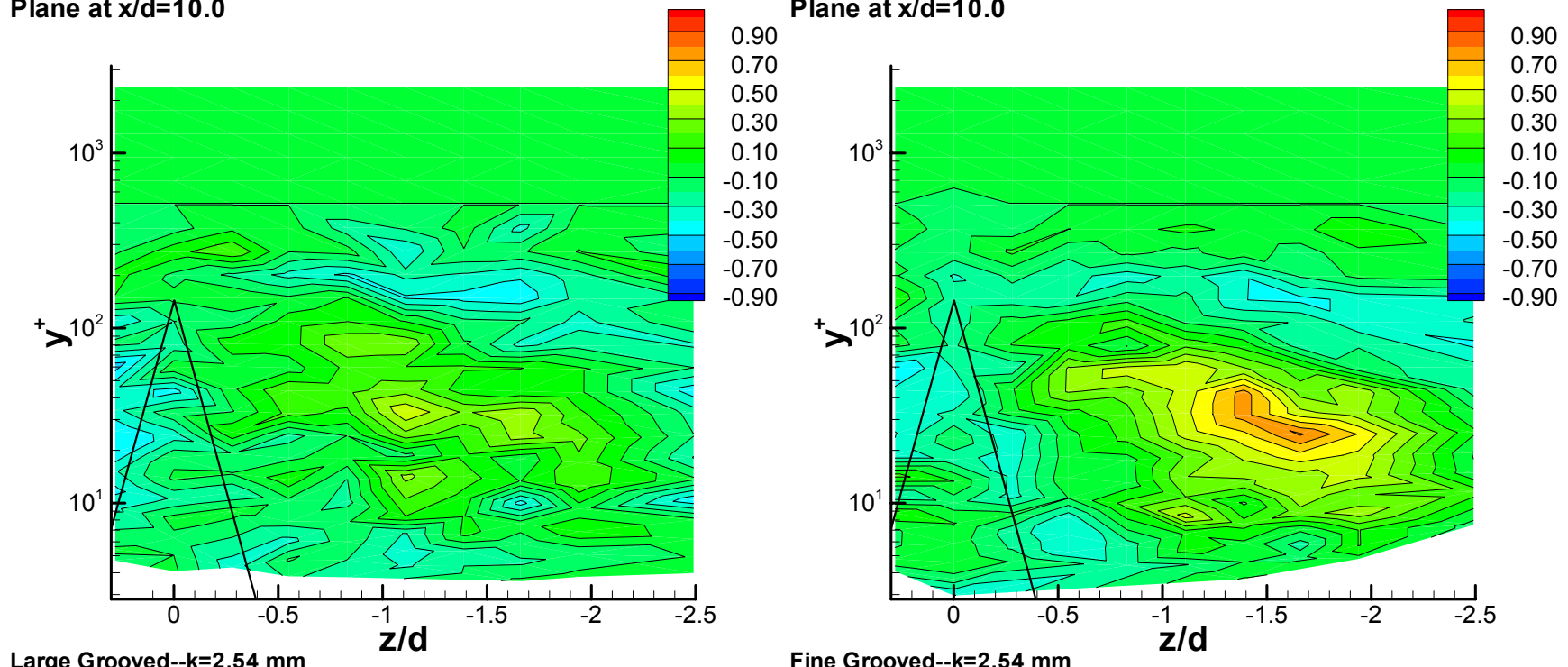

Large Grooved--k=2.54 mm

Fine Grooved--k=2.54 mm

Figure 3.64: Contours of $\overline{u^{2} w} / U_{\tau}^{3}$ in the $\mathbf{y}-\mathbf{z}$ plane at a location of $\mathbf{x} / \mathbf{d}=10.0$ 


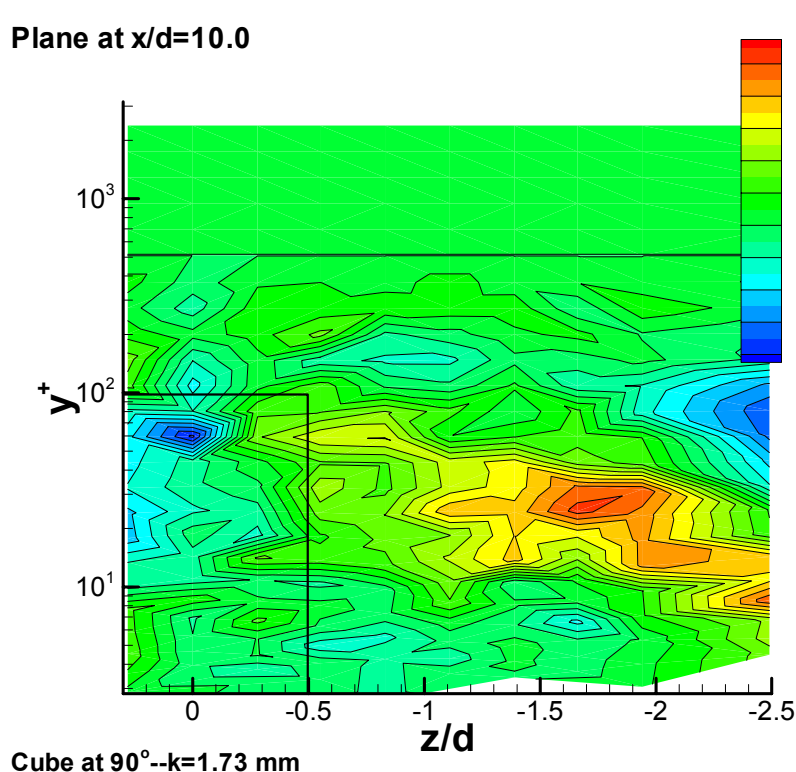

Cube at $90^{\circ}--\mathrm{k}=1.73 \mathrm{~mm}$

\section{Plane at $x / d=10.0$}

Plane at $x / d=10.0$

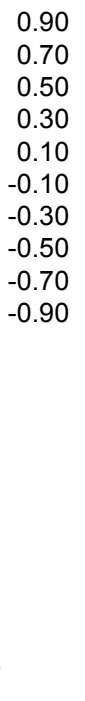

0.90

0.70

0.30

0.30

$-0.50$

0.70

90

$+>^{10}$
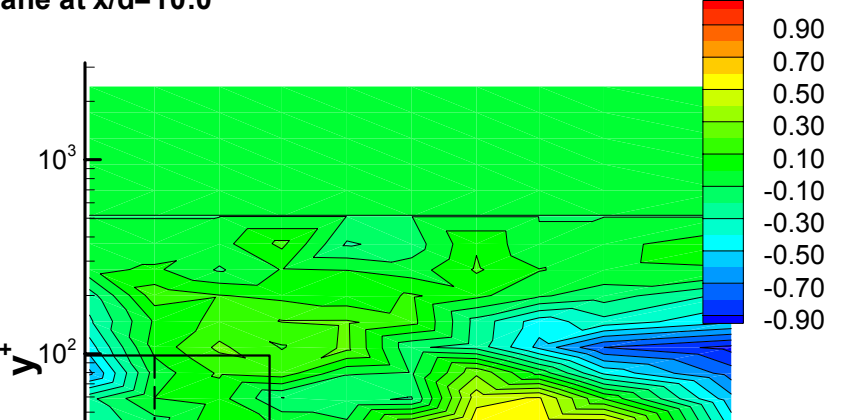

10

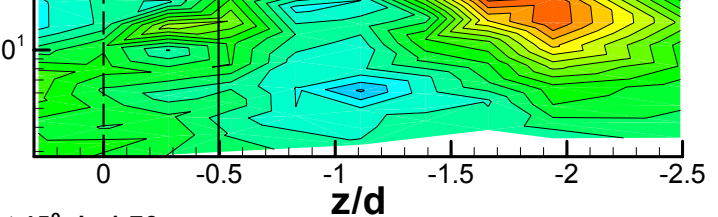

Cube at $45^{\circ}--\mathrm{k}=1.73 \mathrm{~mm}$

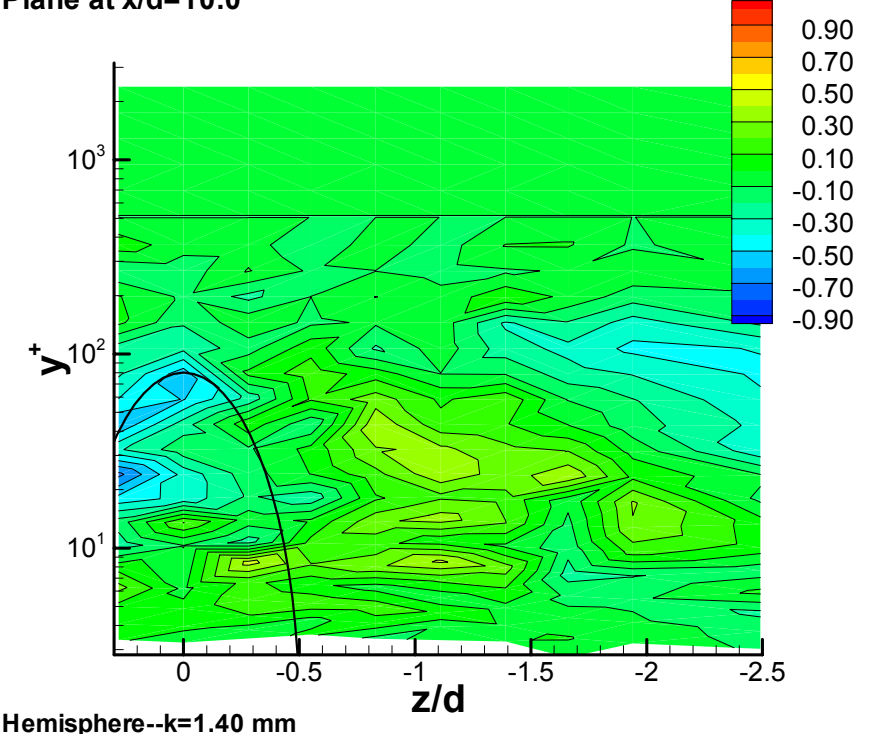

Hemisphere--k=1.40 mm

Figure 3.65: Contours of $\overline{u^{2} w} / U_{\tau}^{3}$ in the $\mathbf{y}-\mathbf{z}$ plane at a location of $\mathbf{x} / \mathbf{d}=10.0$ 

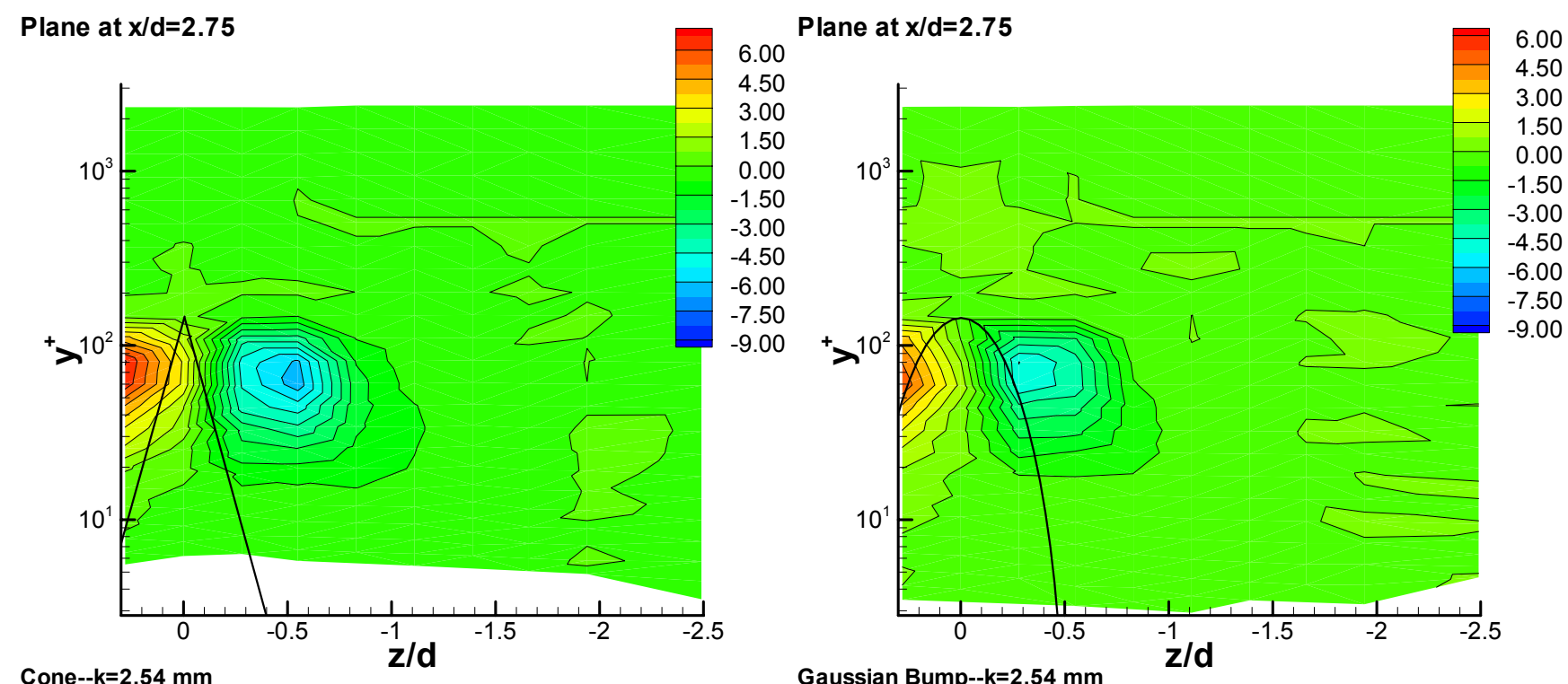

Plane at $\mathrm{x} / \mathrm{d}=\mathbf{2 . 7 5}$

Plane at $x / d=1.36$
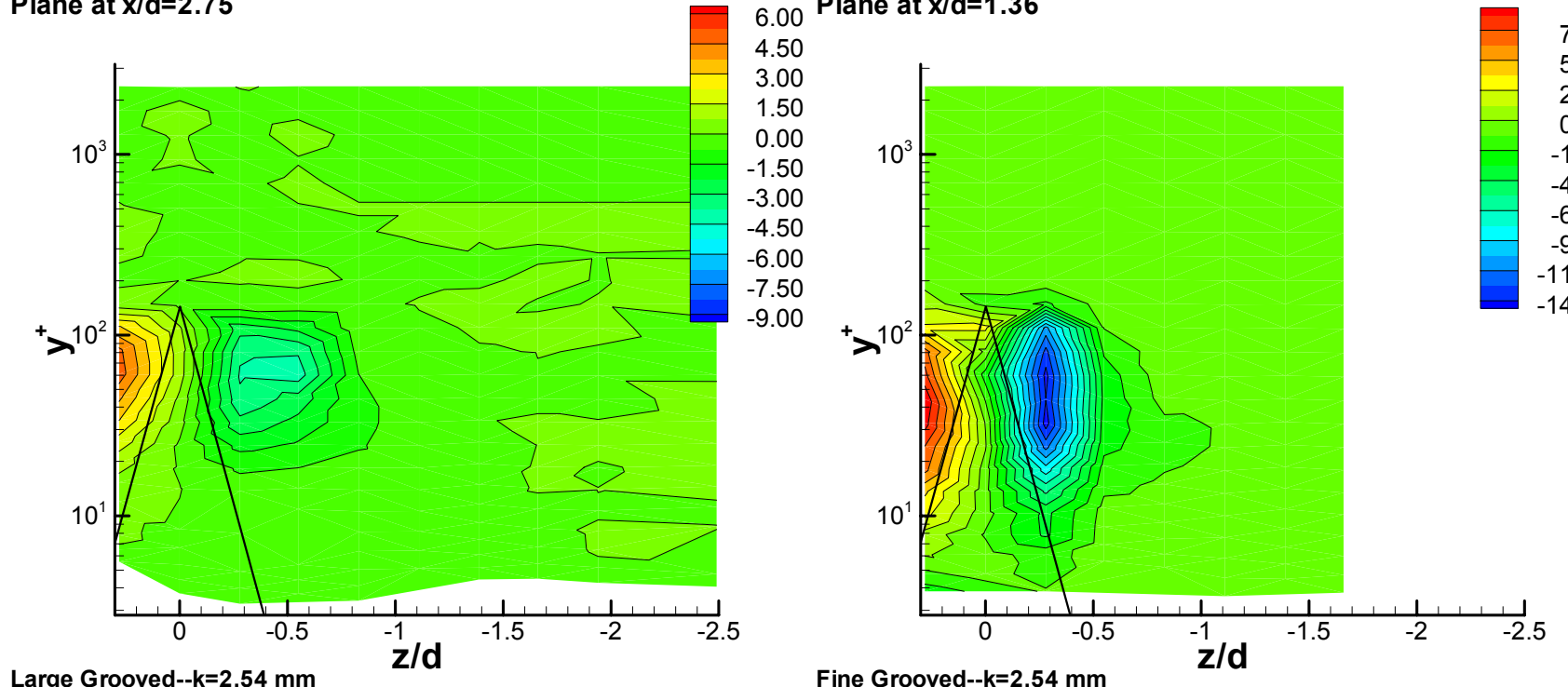

Large Grooved--k=2.54 mm

Fine Grooved--k=2.54 mm

Figure 3.66: Contours of $\overline{v^{2} w} / U_{\tau}^{3}$ in the $\mathbf{y}-\mathbf{z}$ plane at a location of $\mathbf{x} / \mathbf{d}=\mathbf{2 . 7 5}$ (Note: Fine Grooved $\mathbf{x} / \mathbf{d}=\mathbf{1 . 3 6}$ ) 


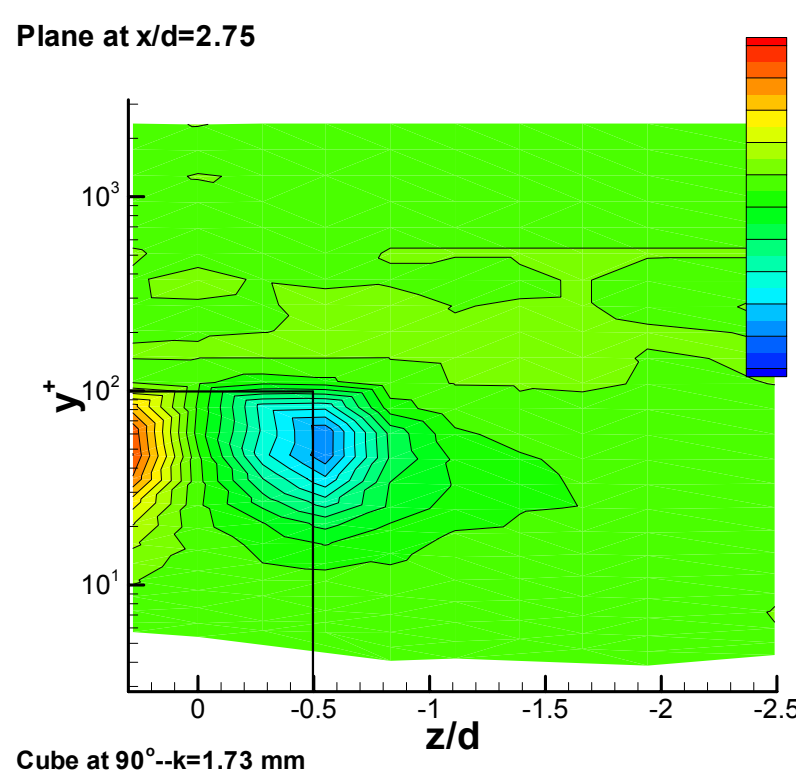

Plane at $x / d=2.75$

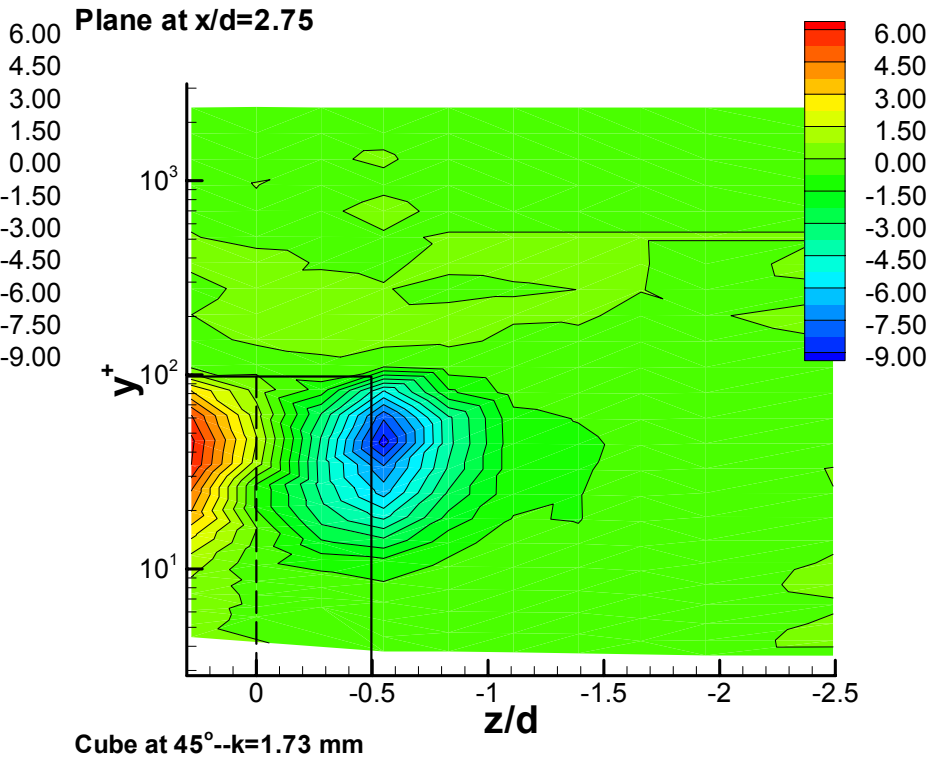

Cube at $45^{\circ}--\mathrm{k}=1.73 \mathrm{~mm}$

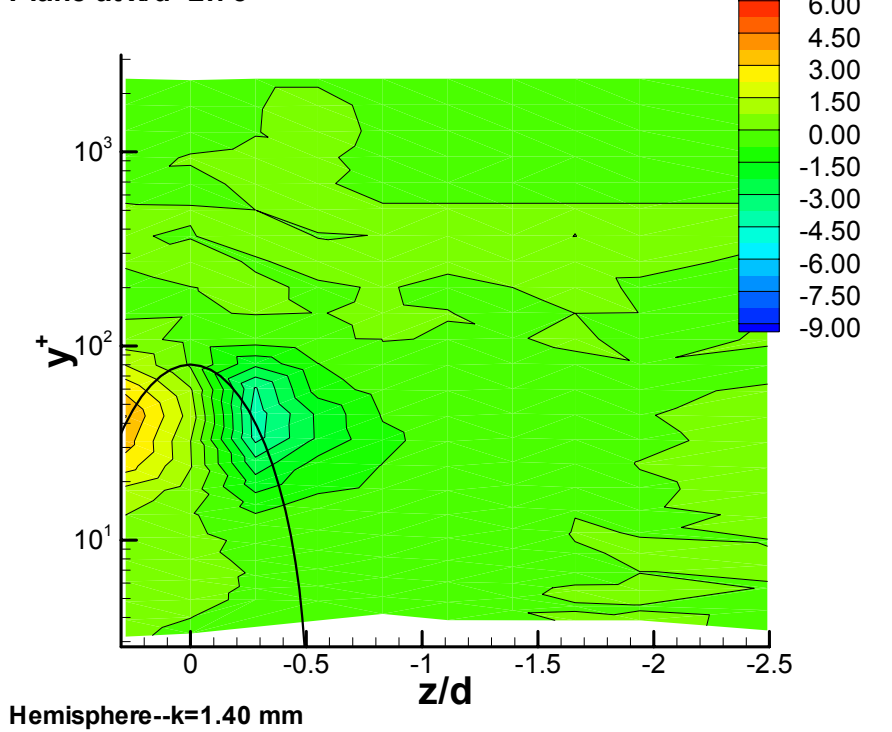

Hemisphere--k=1.40 mm

Figure 3.67: Contours of $\overline{v^{2} w} / U_{\tau}^{3}$ in the $\mathbf{y}-\mathbf{z}$ plane at a location of $\mathbf{x} / \mathbf{d}=\mathbf{2 . 7 5}$ 

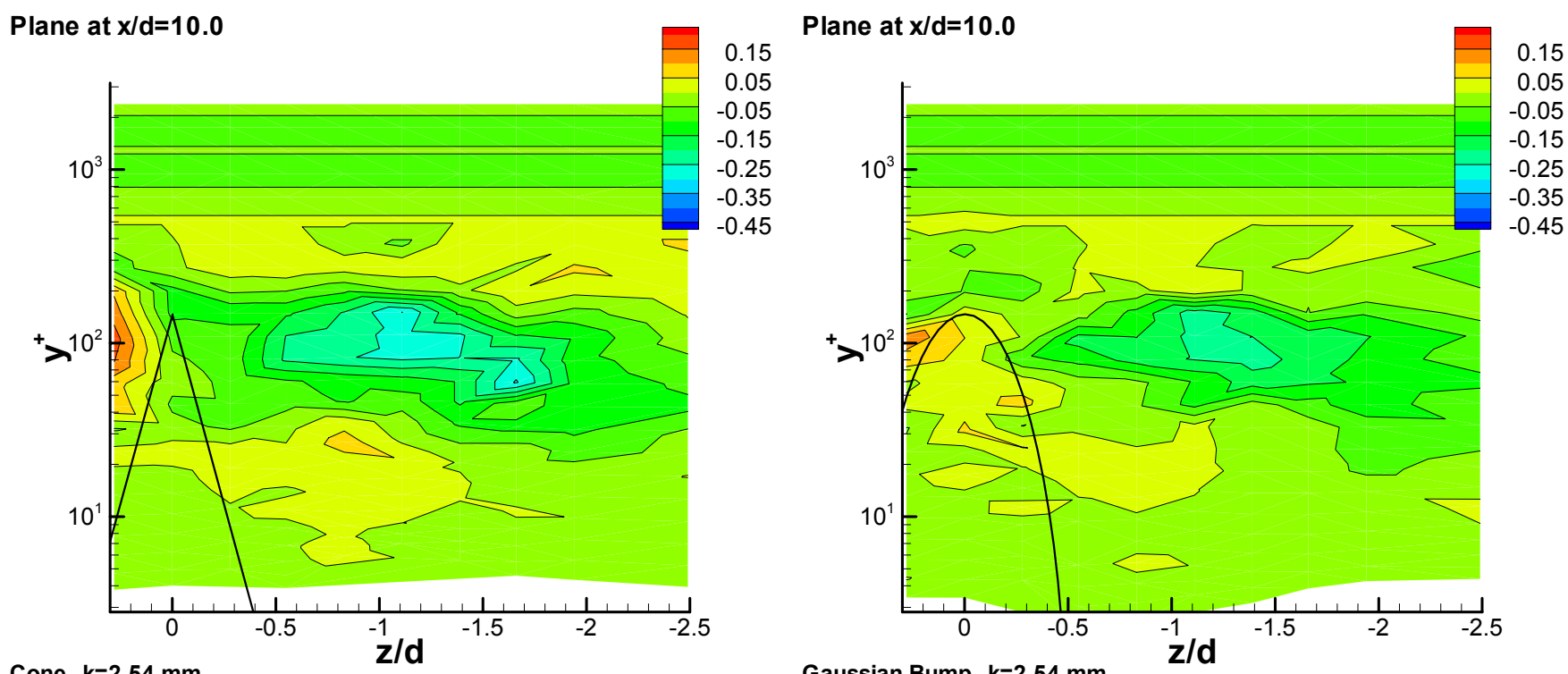

\section{Cone--k=2.54 mm}

Plane at $\mathrm{x} / \mathrm{d}=10.0$
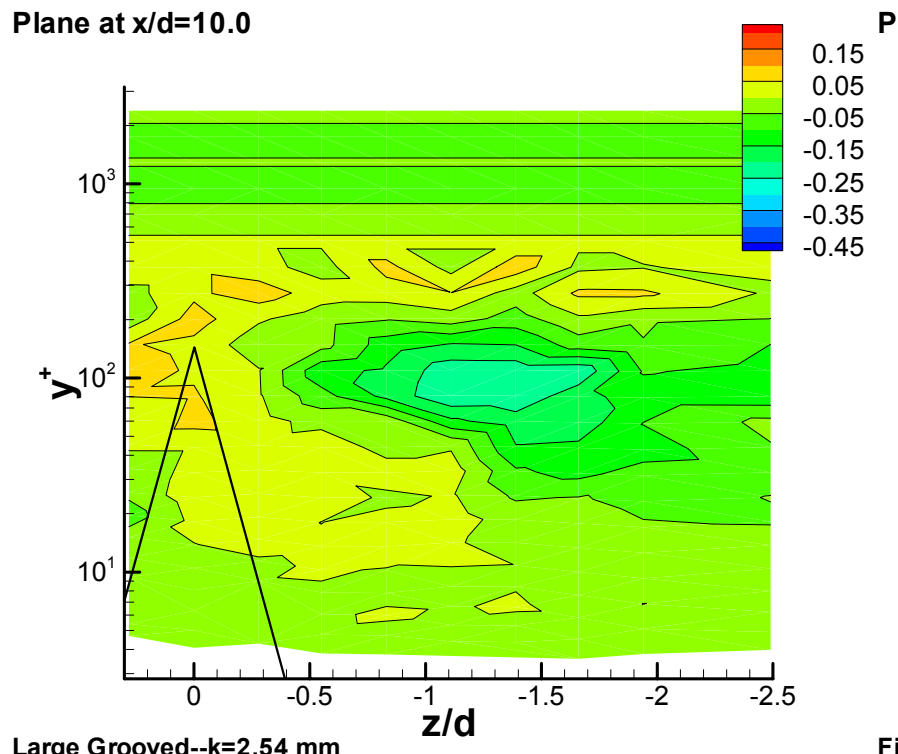

ssian Bump--k=2.54 mm

Plane at $\mathrm{x} / \mathrm{d}=\mathbf{1 0 . 0}$

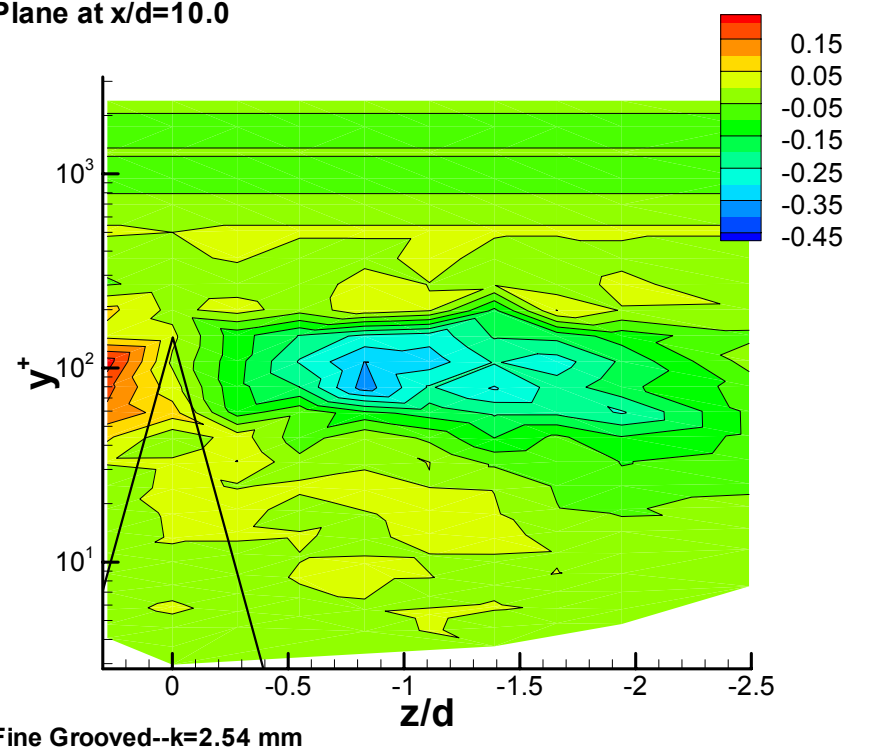

Figure 3.68: Contours of $\overline{v^{2} w} / U_{\tau}^{3}$ in the $\mathbf{y}-z$ plane at a location of $\mathbf{x} / \mathbf{d}=10.0$ 


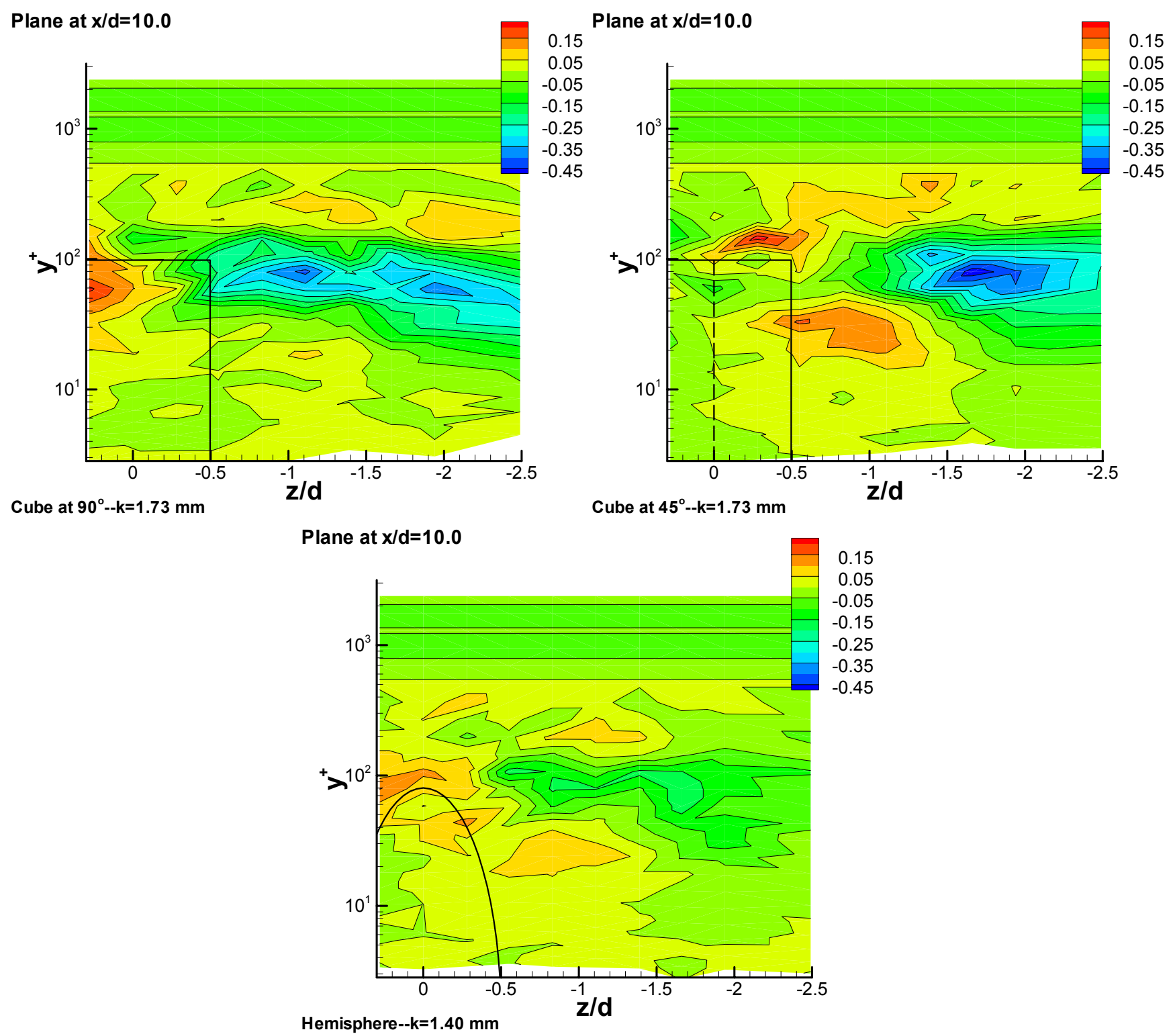

Figure 3.69: Contours of $\overline{v^{2} w} / U_{\tau}^{3}$ in the $\mathbf{y}-\mathbf{z}$ plane at a location of $\mathbf{x} / \mathbf{d}=10.0$ 


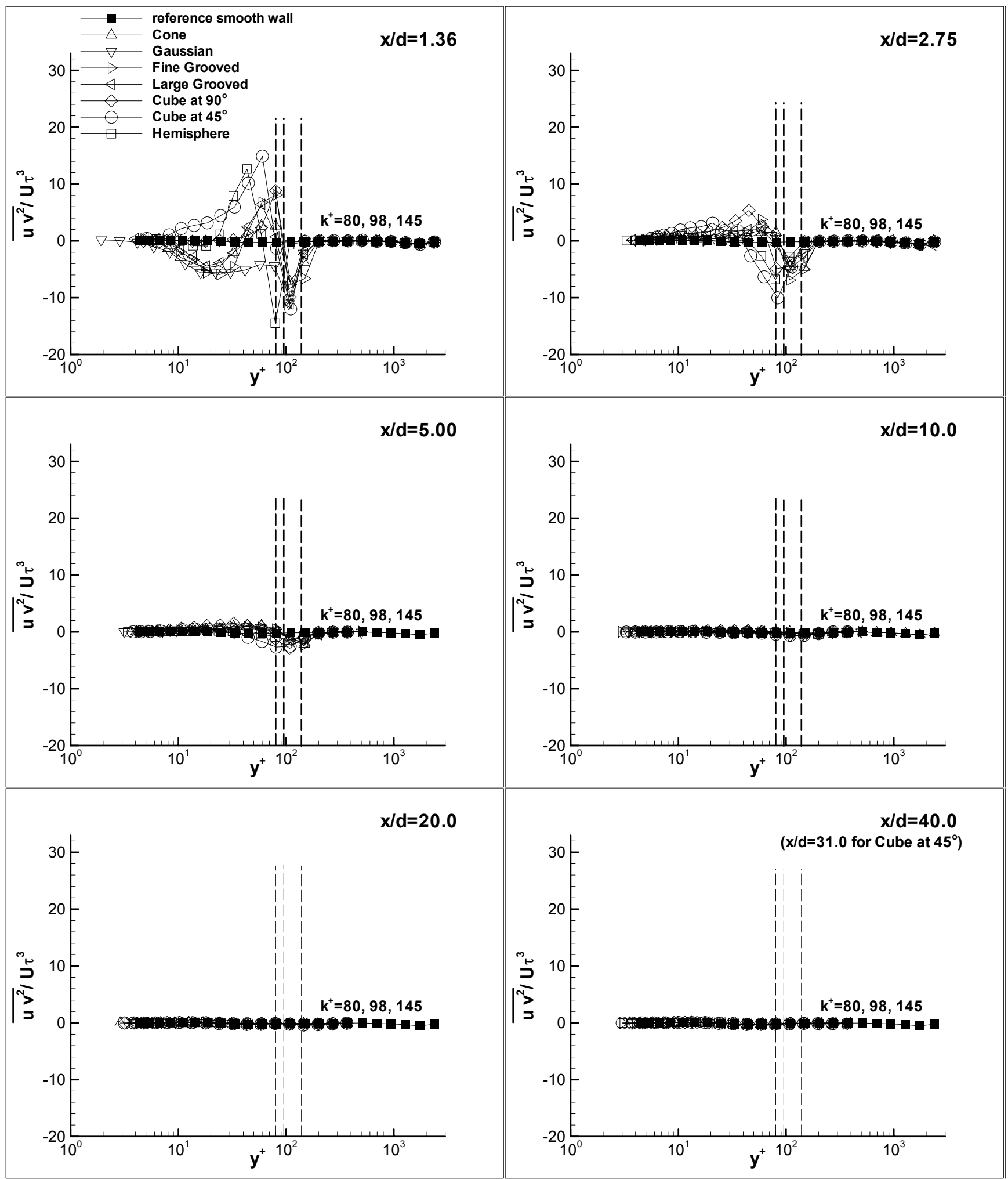

Figure 3.70: $\overline{u v^{2}} /_{U_{\tau}^{3}}$ vs. $y U_{\tau} / v$ profiles along the centerline 

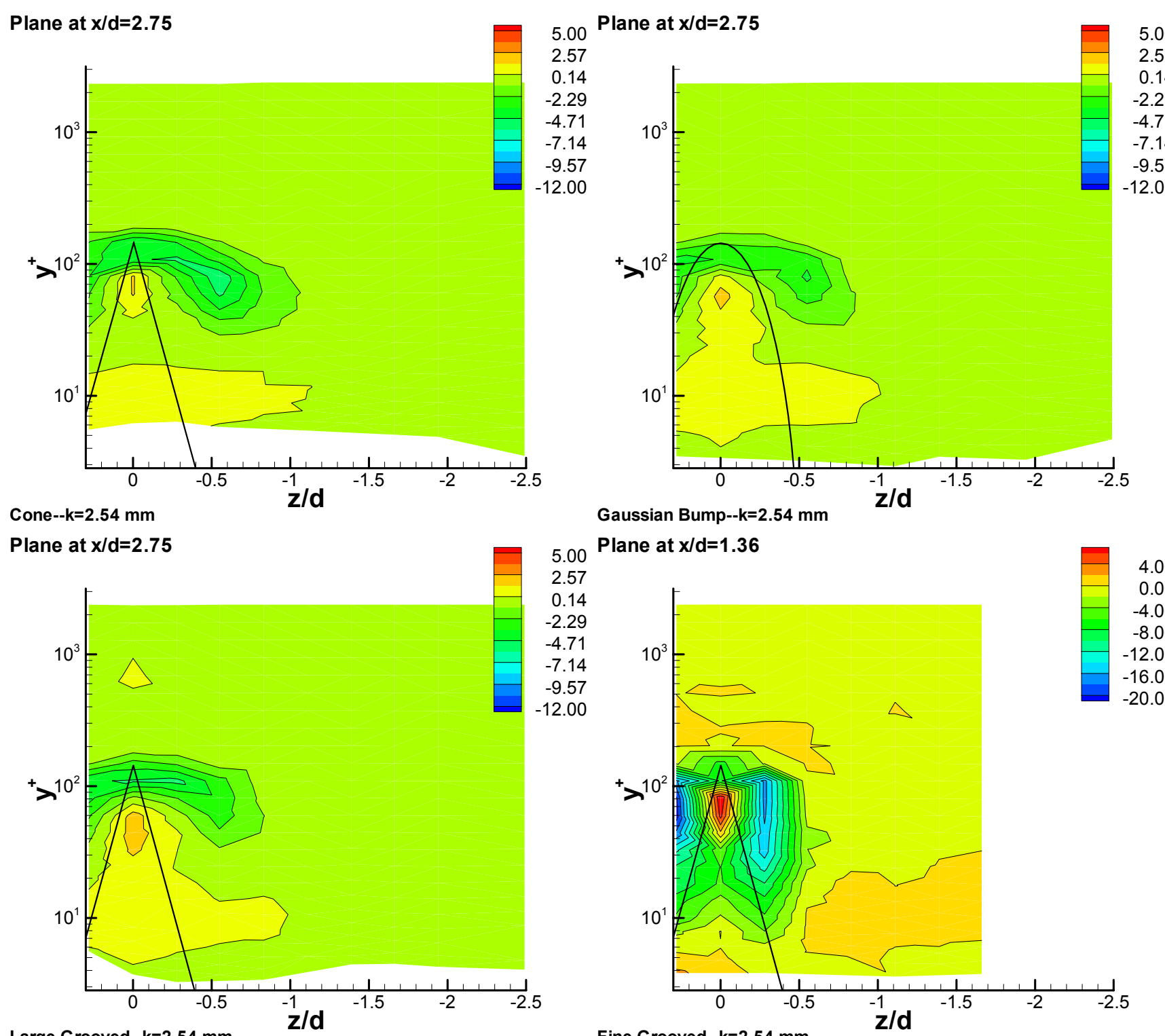

Plane at $x / d=1.36$

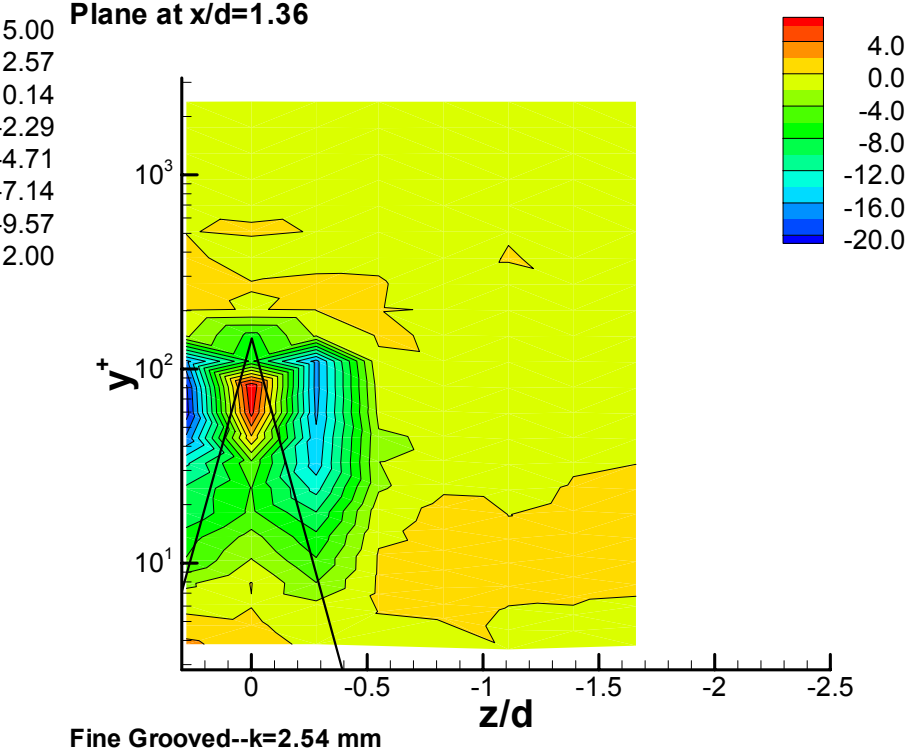

Large Grooved--k=2.54 mm

Fine Grooved--k=2.54 mm

Figure 3.71: Contours of $\overline{u v^{2}} / U_{\tau}^{3}$ in the $\mathbf{y}-\mathbf{z}$ plane at a location of $\mathbf{x} / \mathbf{d}=\mathbf{2 . 7 5}$ (Note: Fine Grooved $\mathbf{x} / \mathbf{d}=1.36$ ) 


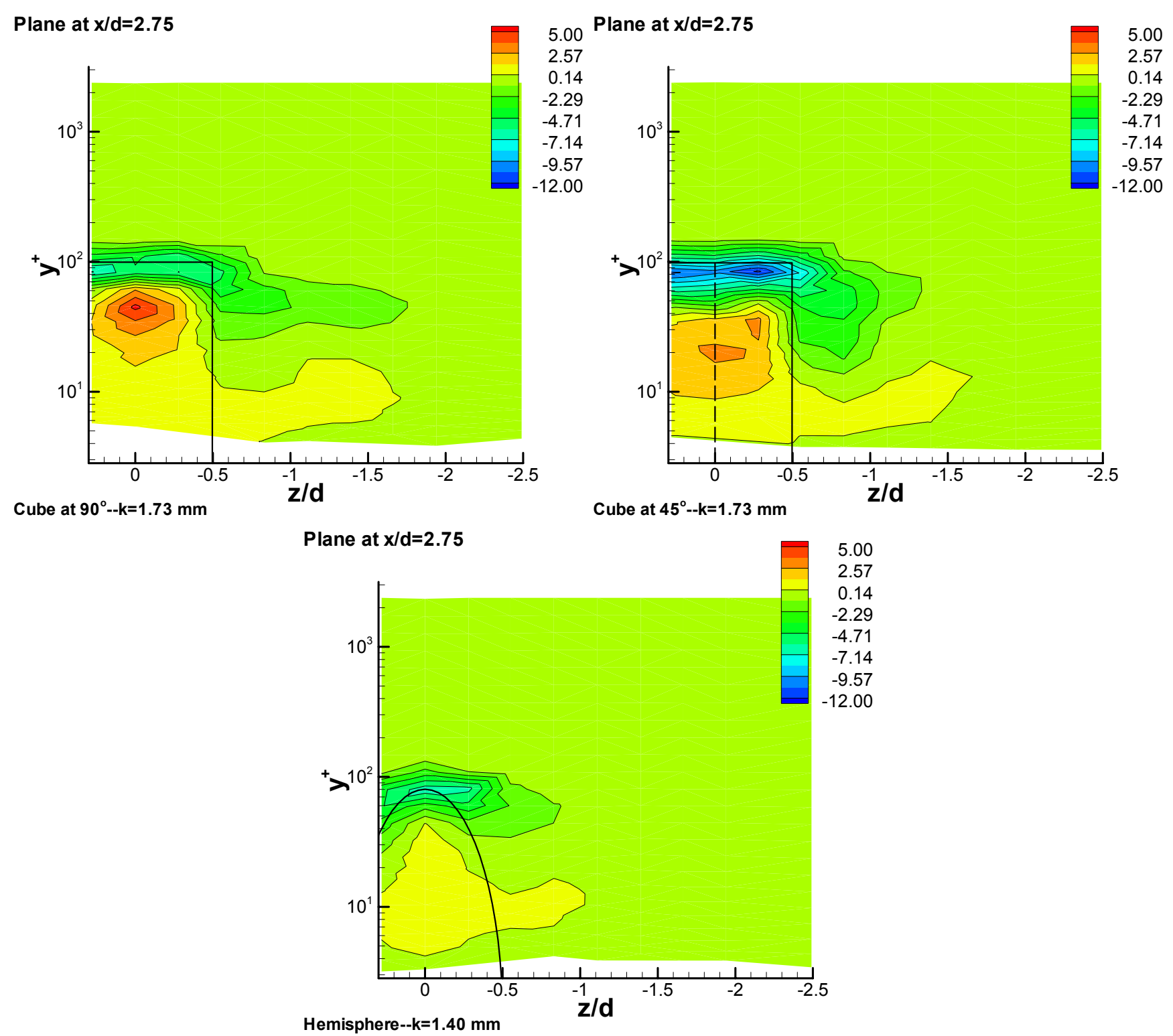

Figure 3.72: Contours of $\overline{u v^{2}} / U_{\tau}^{3}$ in the y-z plane at a location of $\mathbf{x} / \mathbf{d}=\mathbf{2 . 7 5}$ 

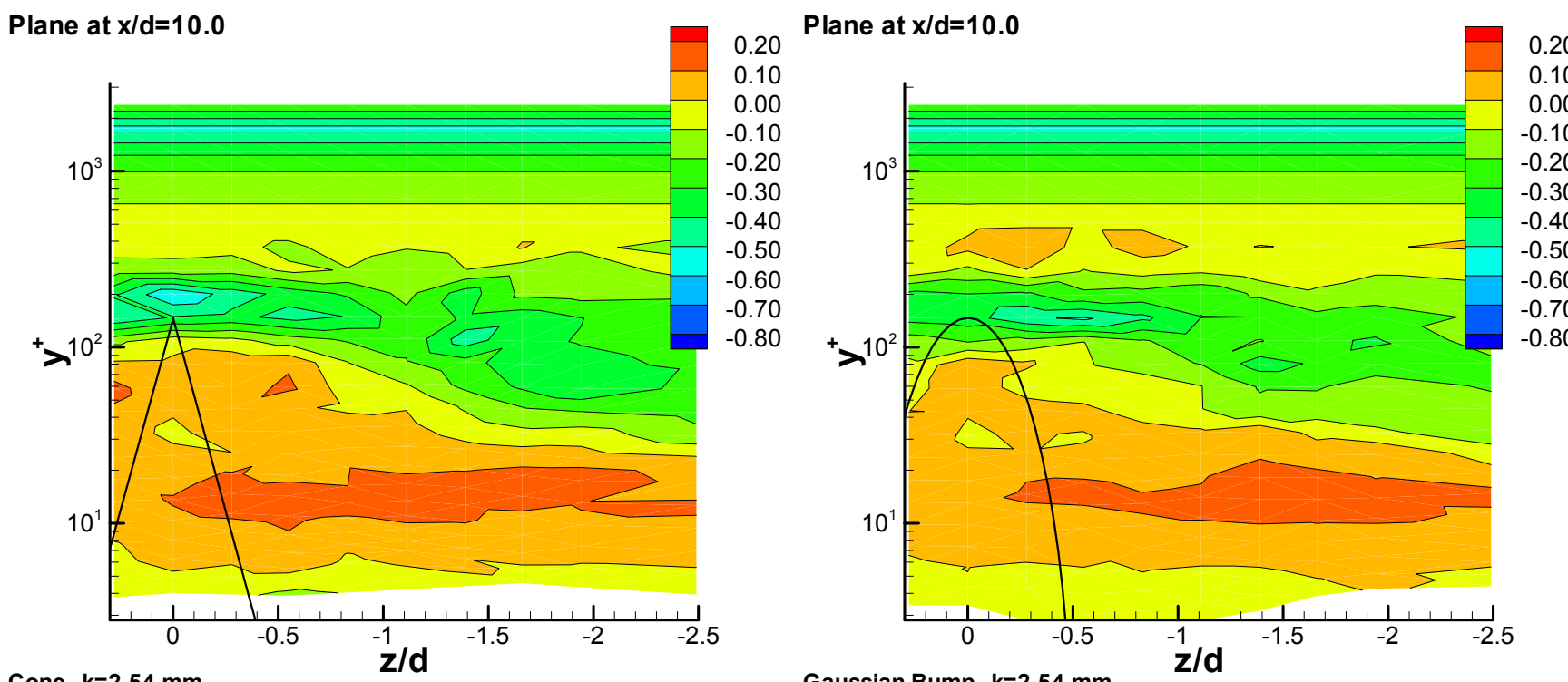

\section{Cone--k=2.54 mm}

Plane at $\mathrm{x} / \mathrm{d}=10.0$
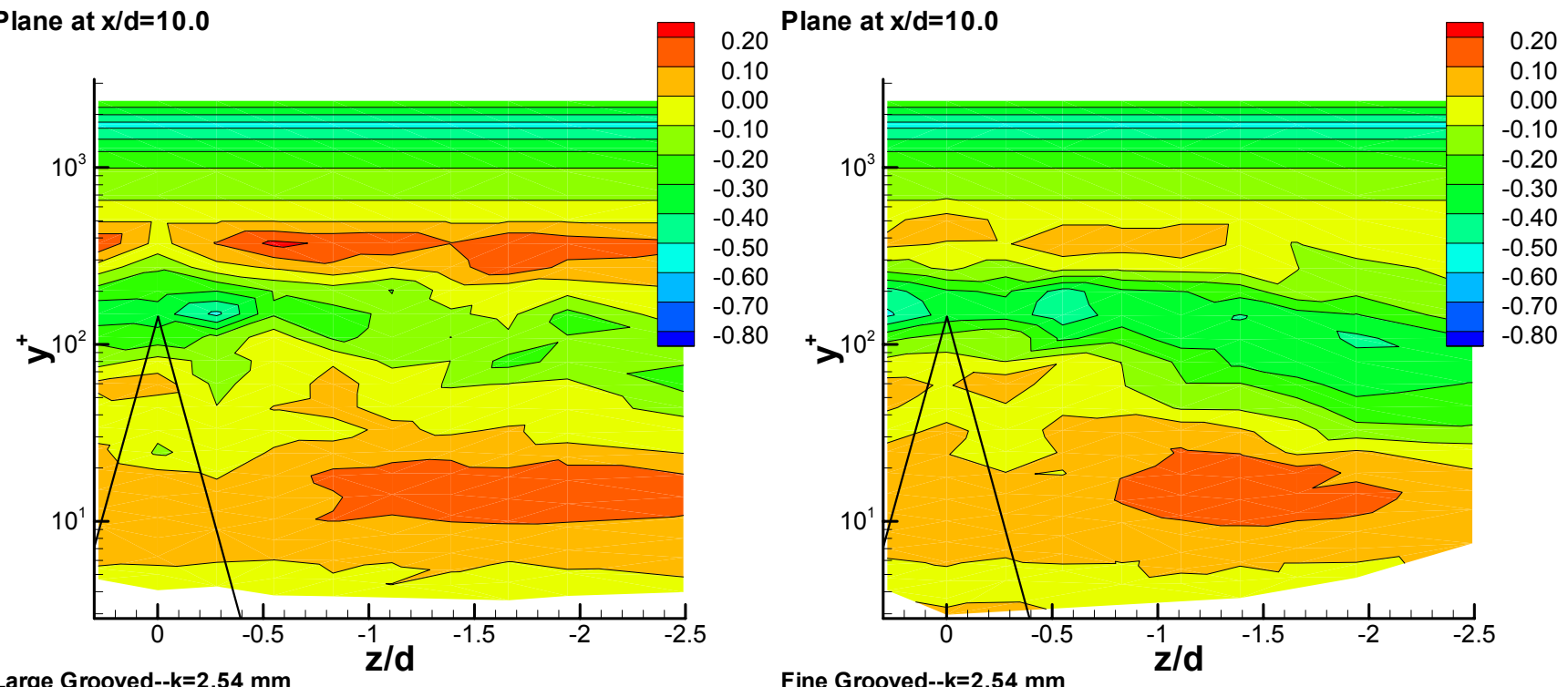

Figure 3.73: Contours of $\overline{u v^{2}} / U_{\tau}^{3}$ in the $\mathbf{y}-z$ plane at a location of $\mathbf{x} / \mathbf{d}=10.0$ 


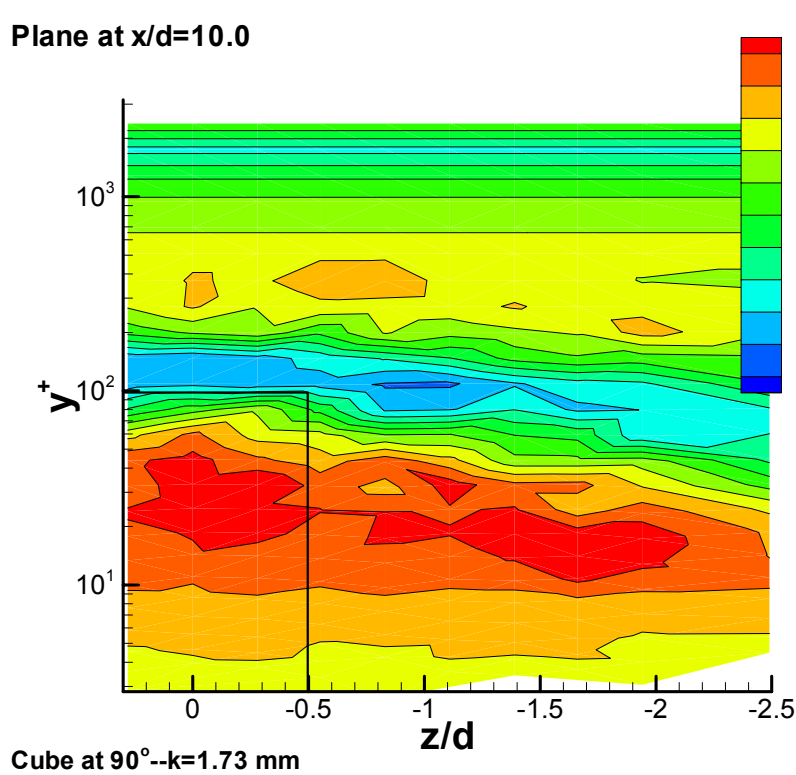

Cube at $90^{\circ}--\mathrm{k}=1.73 \mathrm{~mm}$

\section{Plane at $x / d=10.0$}

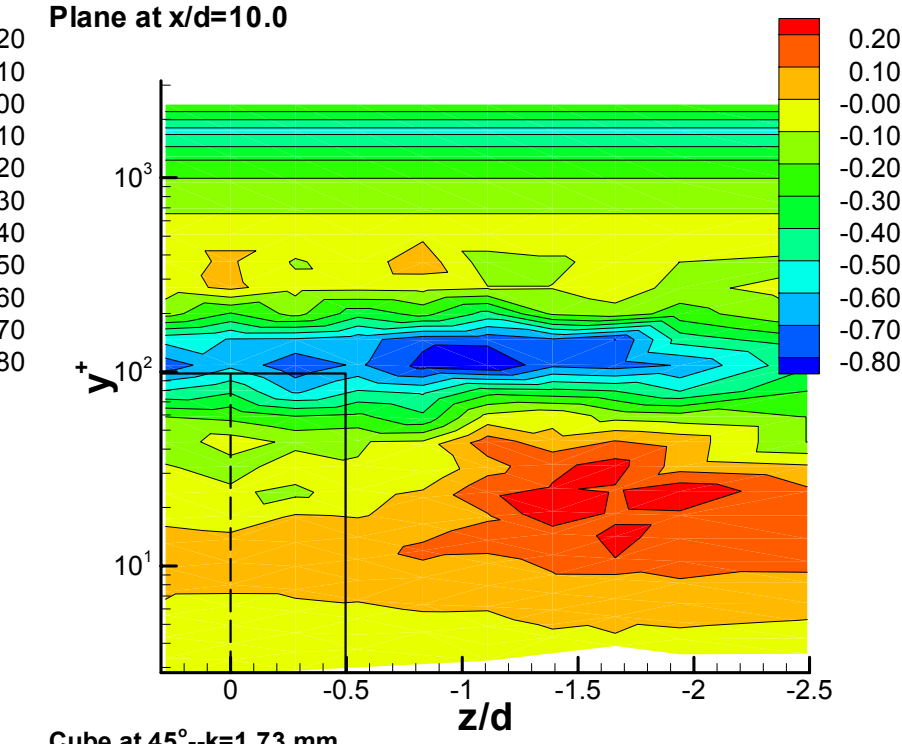

Cube at $45^{\circ}--\mathrm{k}=1.73 \mathrm{~mm}$

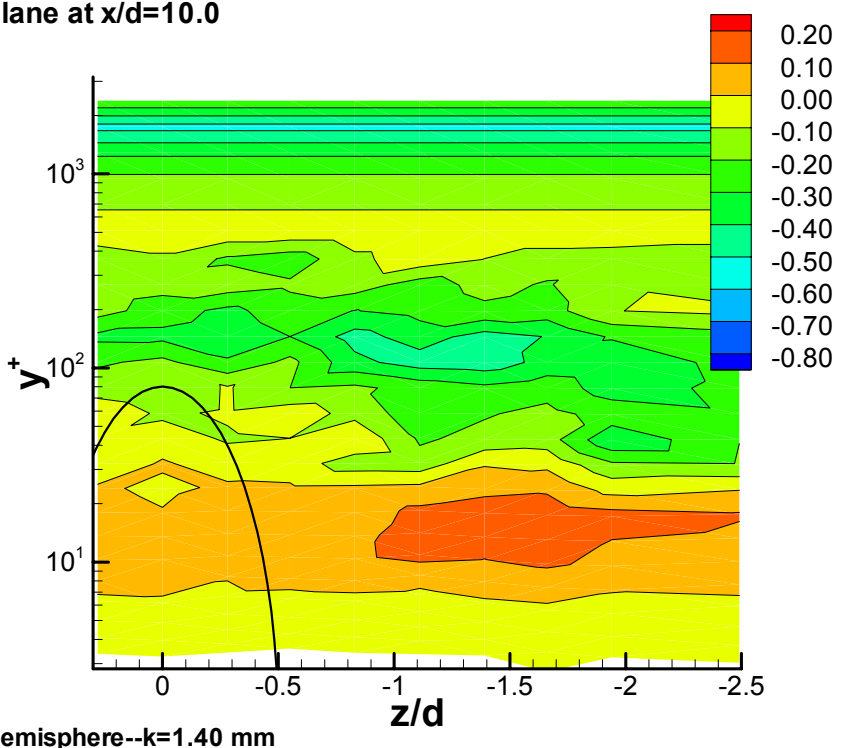

Figure 3.74: Contours of $\overline{u v^{2}} / U_{\tau}^{3}$ in the y-z plane at a location of $\mathbf{x} / \mathbf{d}=10.0$ 


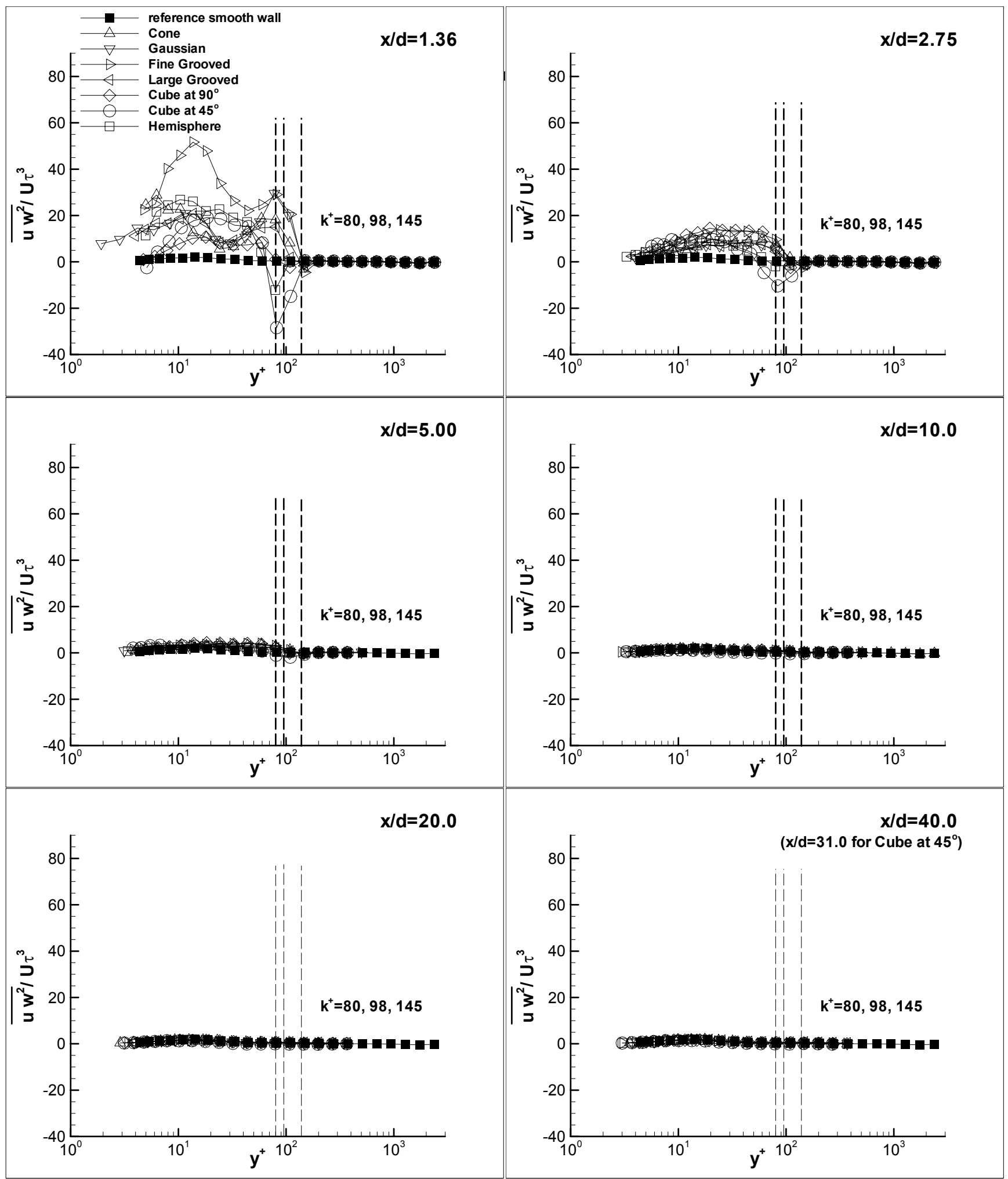

Figure 3.75: $\overline{u w^{2}} /_{U_{\tau}^{3}}$ vs. $y U_{\tau} / v$ profiles along the centerline 

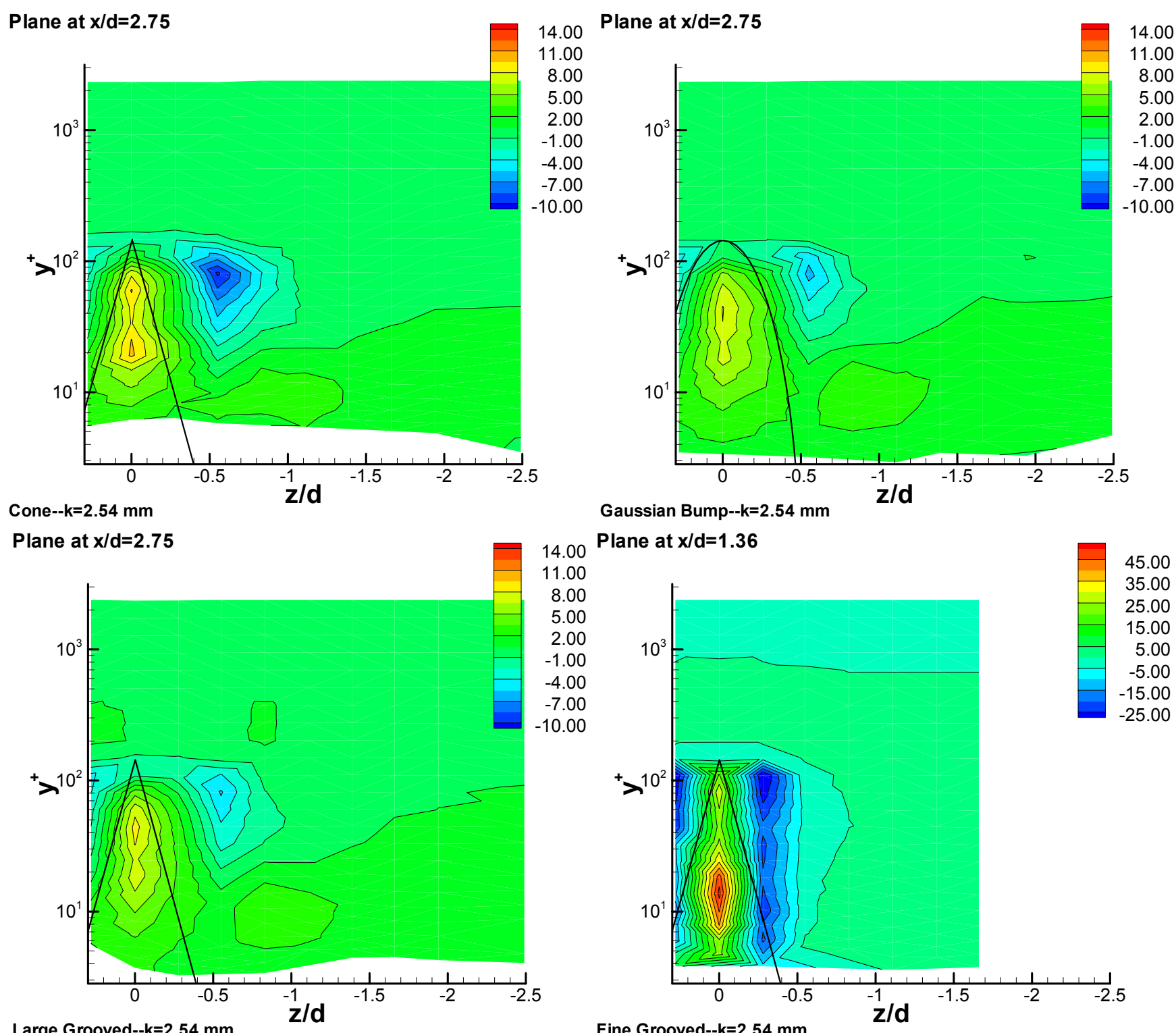

Gaussian Bump--k=2.54 mm

Plane at $x / d=1.36$

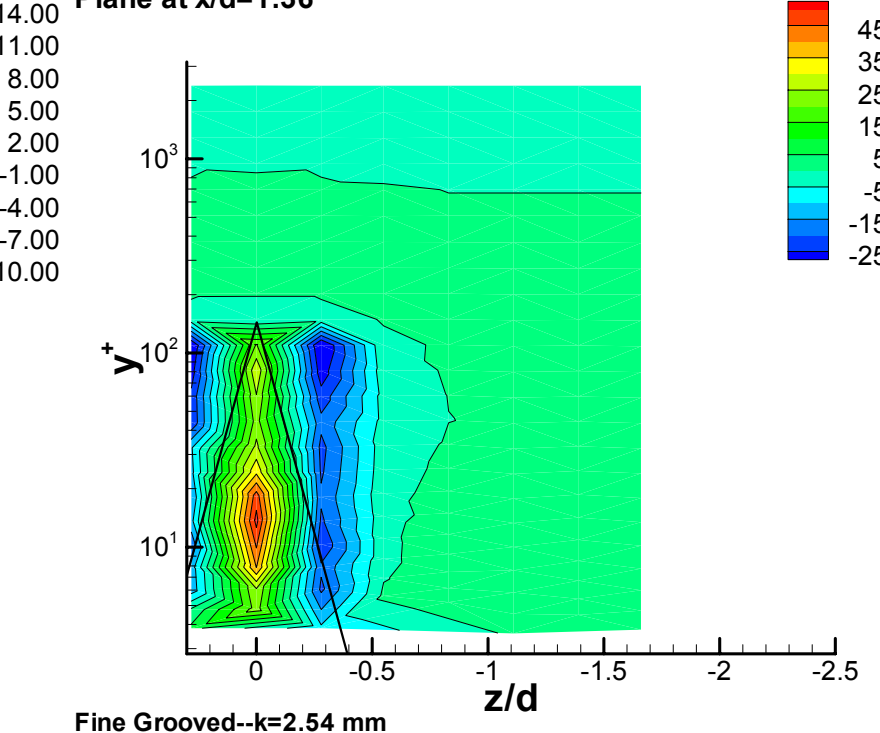

Large Grooved--k=2.54 mm

Fine Grooved--k=2.54 mm

Figure 3.76: Contours of $\overline{u w^{2}} / U_{\tau}^{3}$ in the $\mathbf{y}-\mathbf{z}$ plane at a location of $\mathbf{x} / \mathbf{d}=\mathbf{2 . 7 5}$ (Note: Fine Grooved $\mathbf{x} / \mathbf{d}=\mathbf{1 . 3 6}$ ) 


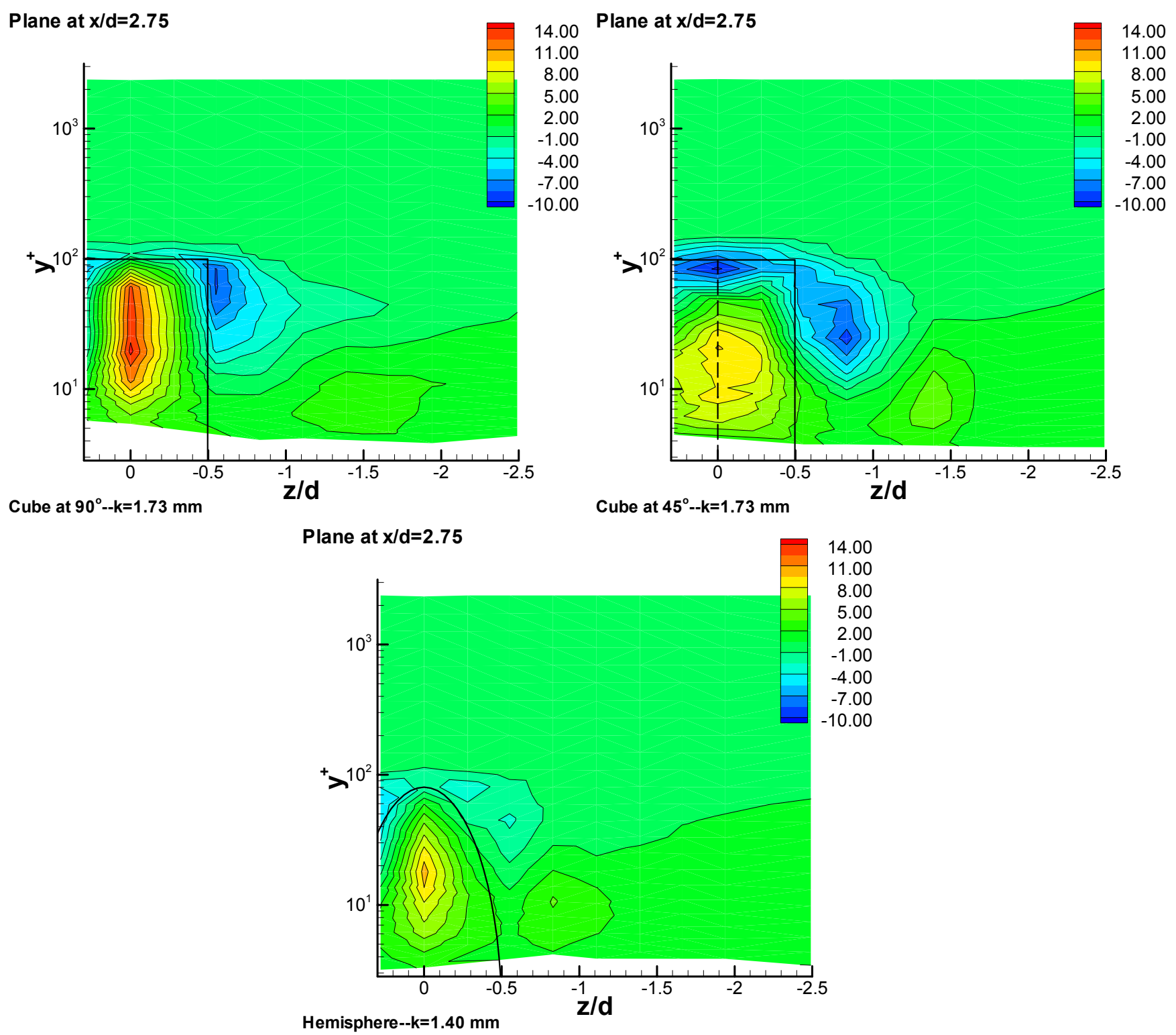

Figure 3.77: Contours of $\overline{u w^{2}} / U_{\tau}^{3}$ in the $\mathbf{y}-z$ plane at a location of $\mathbf{x} / \mathbf{d}=\mathbf{2 . 7 5}$ 


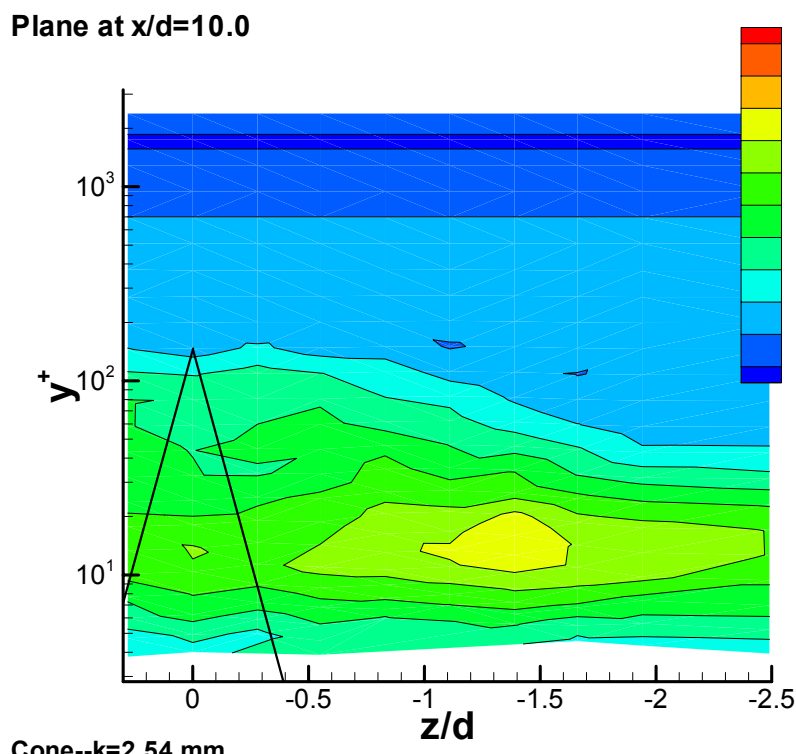

Plane at $x / d=10.0$

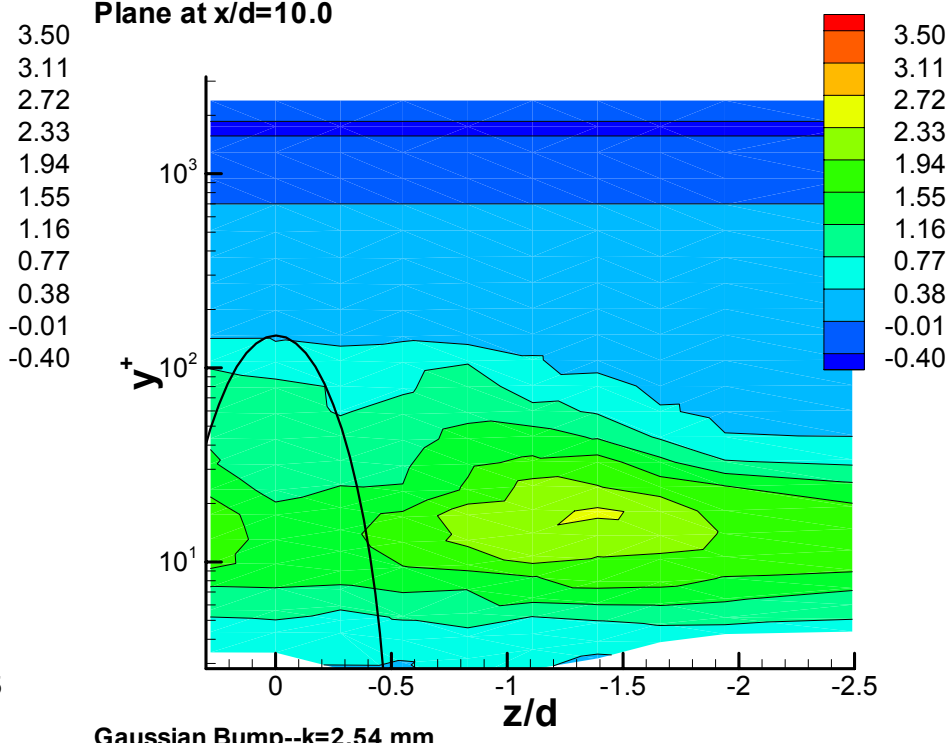

Cone--k=2.54 mm

Gaussian Bump--k=2.54 mm

Plane at $x / d=10.0$

Plane at $x / d=10.0$

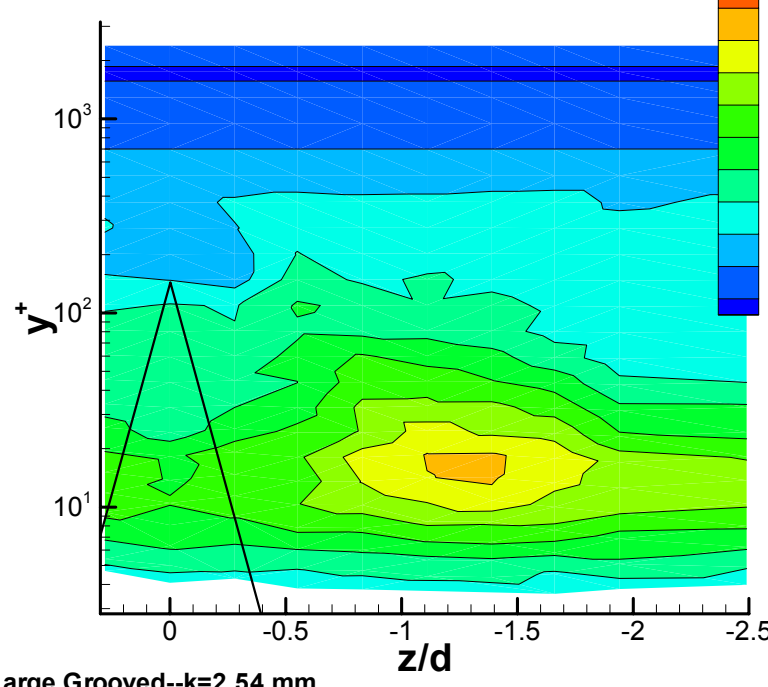

Large Grooved--k=2.54 mm

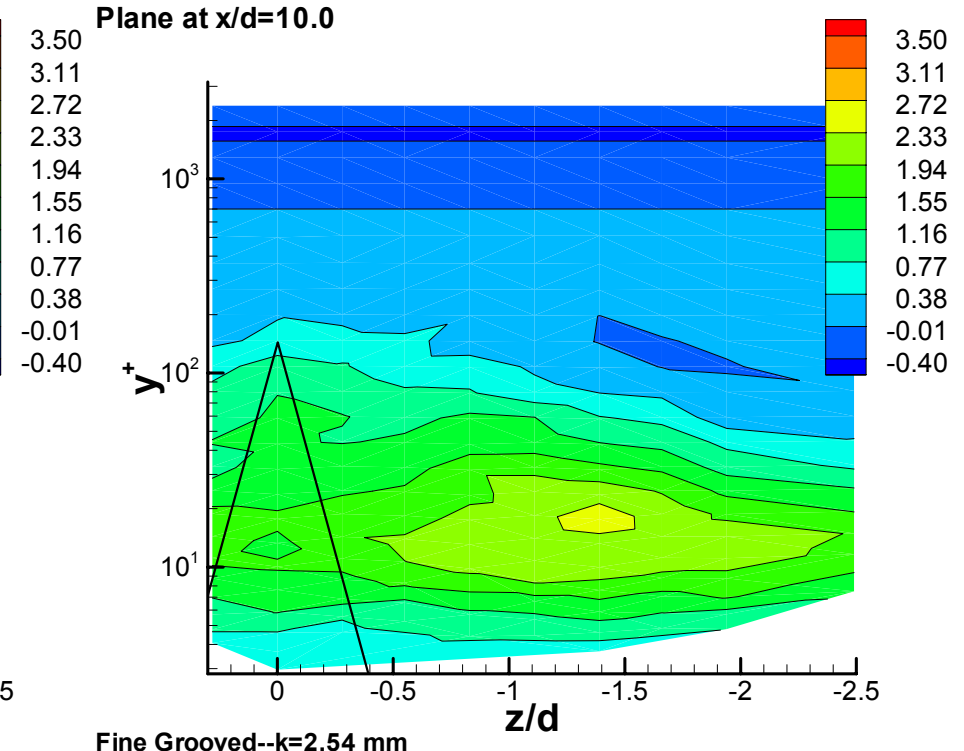

Fine Grooved--k=2.54 mm

Figure 3.78: Contours of $\overline{u w^{2}} / U_{\tau}^{3}$ in the $\mathbf{y}-\mathbf{z}$ plane at a location of $\mathbf{x} / \mathbf{d}=10.0$ 


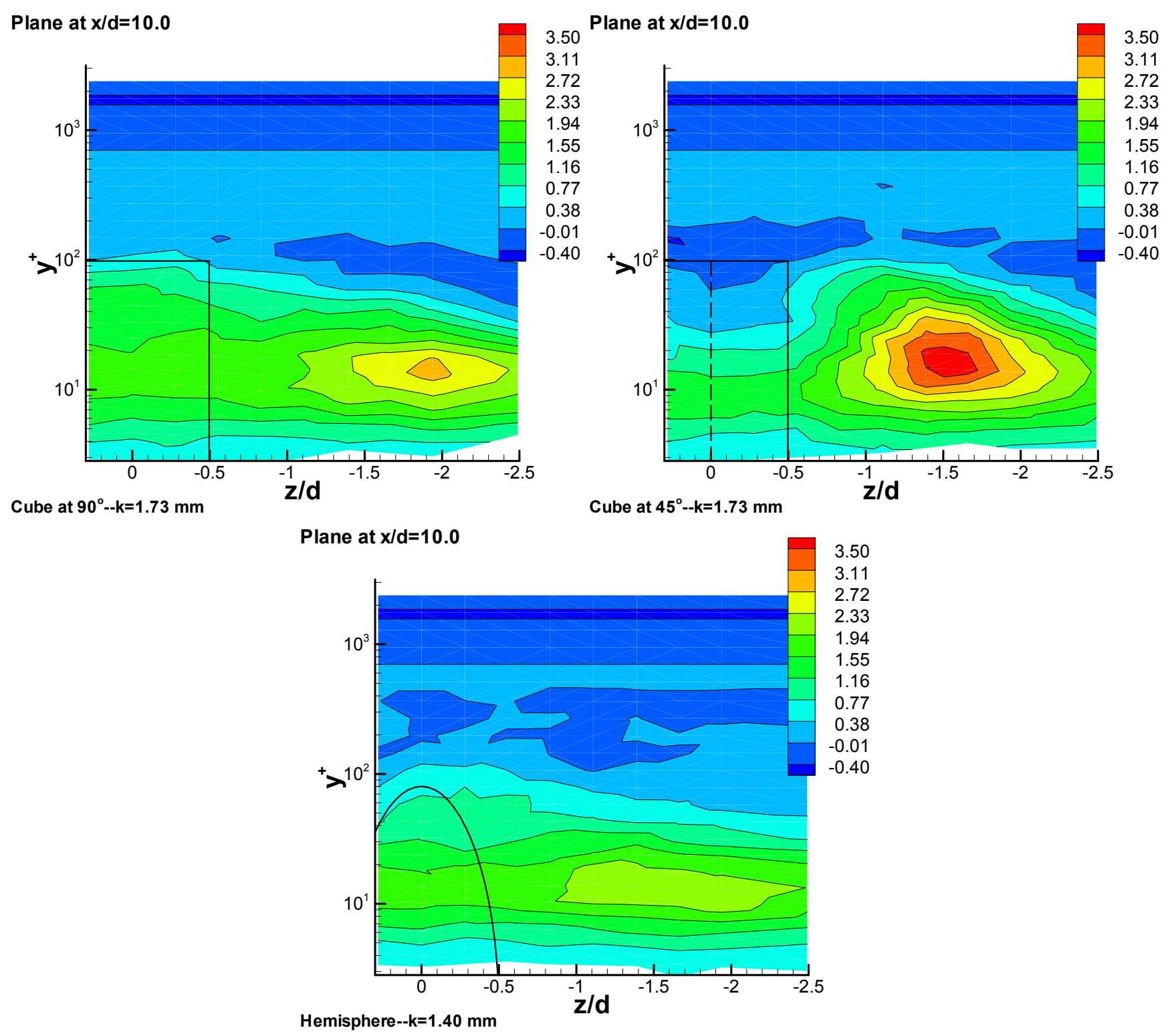

Figure 3.79: Contours of $\overline{u w^{2}} / U_{\tau}^{3}$ in the $\mathbf{y}-\mathbf{z}$ plane at a location of $\mathbf{x} / \mathbf{d}=10.0$ 


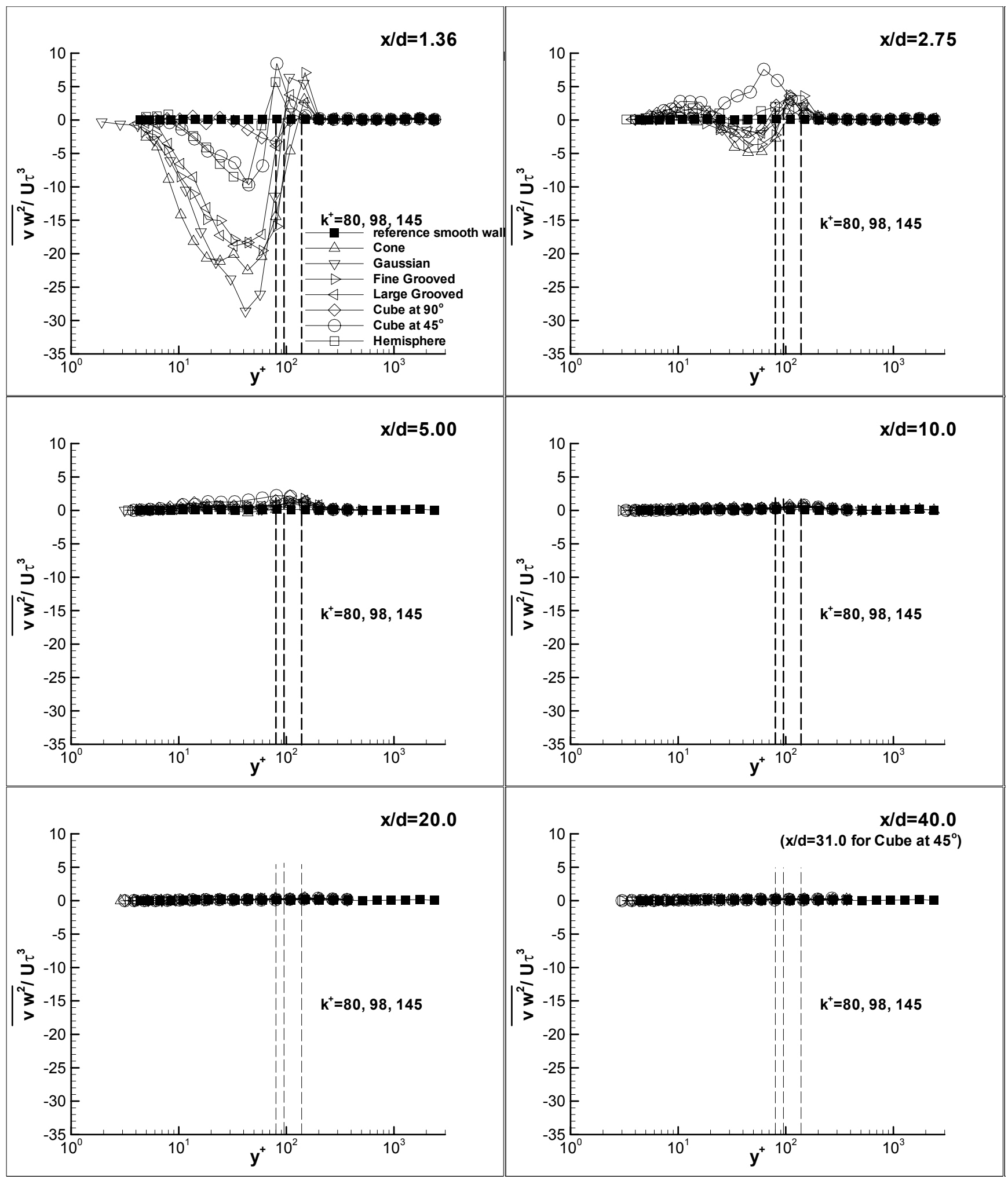

Figure 3.80: $\overline{v w^{2}} /_{U_{\tau}^{3}}$ vs. $y U_{\tau} / v$ profiles along the centerline 

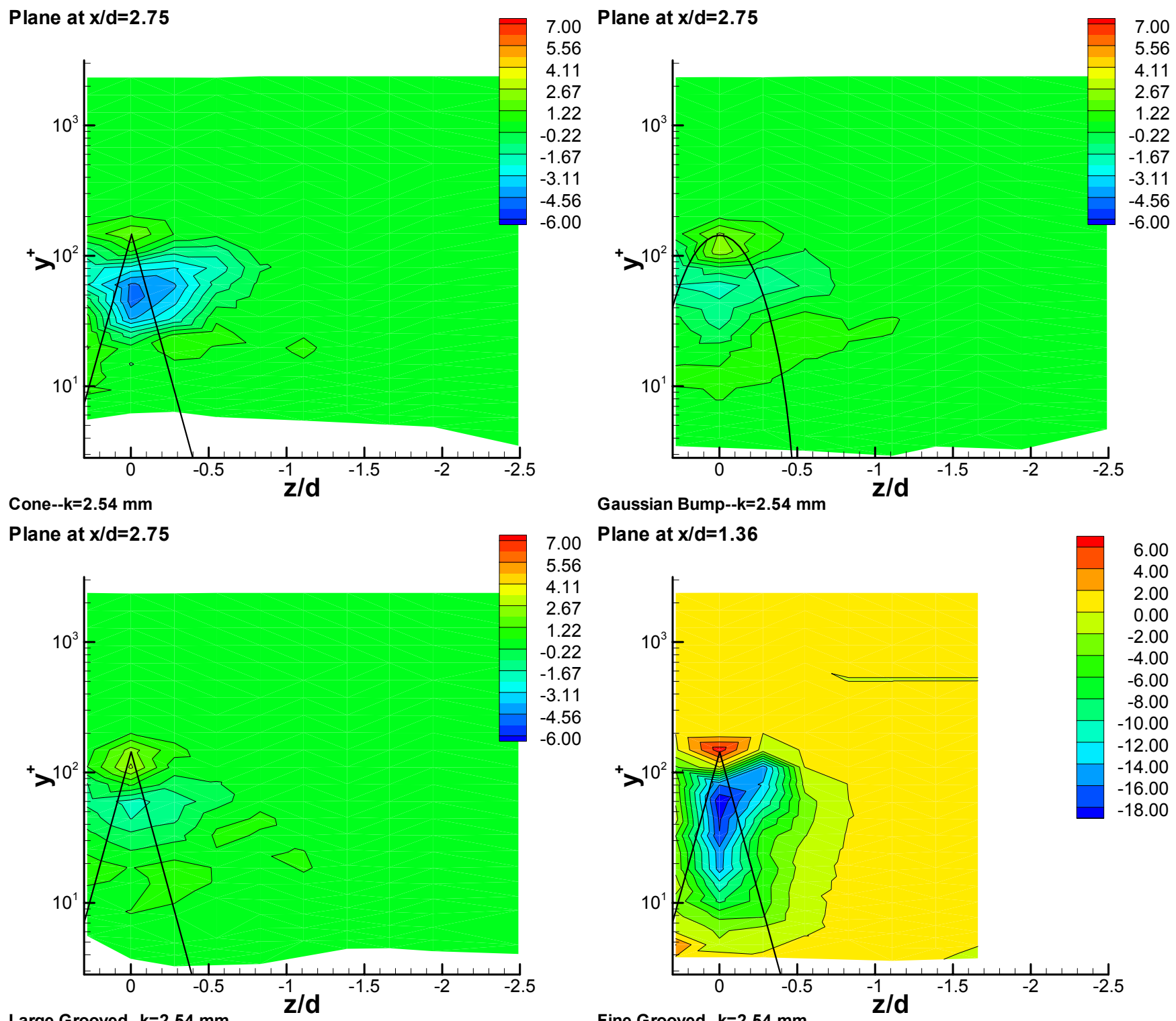

Gaussian Bump--k=2.54 mm

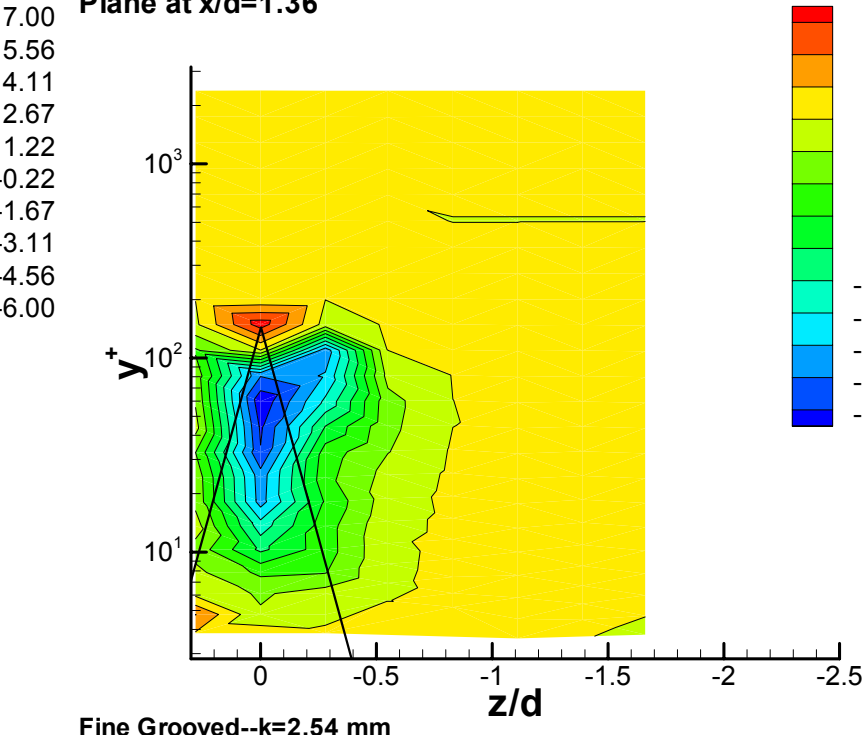

Large Grooved--k=2.54 mm

Fine Grooved--k=2.54 mm

Figure 3.81: Contours of $\overline{v w^{2}} / U_{\tau}^{3}$ in the $\mathbf{y}-z$ plane at a location of $\mathbf{x} / \mathbf{d}=\mathbf{2 . 7 5}$ (Note: Fine Grooved $\mathbf{x} / \mathbf{d}=\mathbf{1 . 3 6}$ ) 


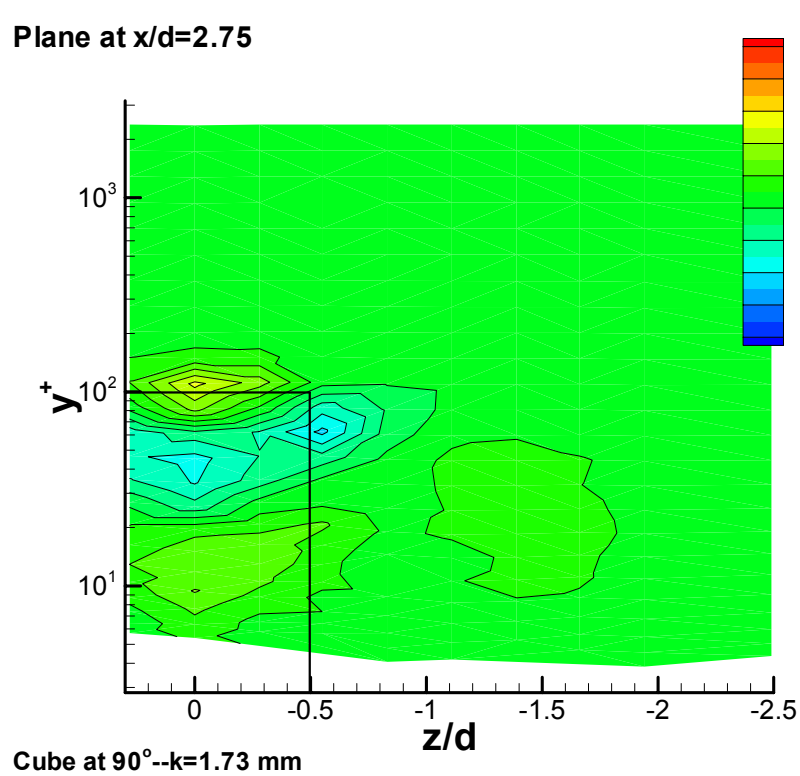

Cube at $90^{\circ}--\mathrm{k}=1.73 \mathrm{~mm}$

\section{Plane at $x / d=2.75$}

Plane at $x / d=2.75$

7.00
5.56

5.56

4.11
2.67

2.67
1.22

1.22
-0.22

-0.22
-1.67

$-3.11$

$-4.56$

$-6.00$

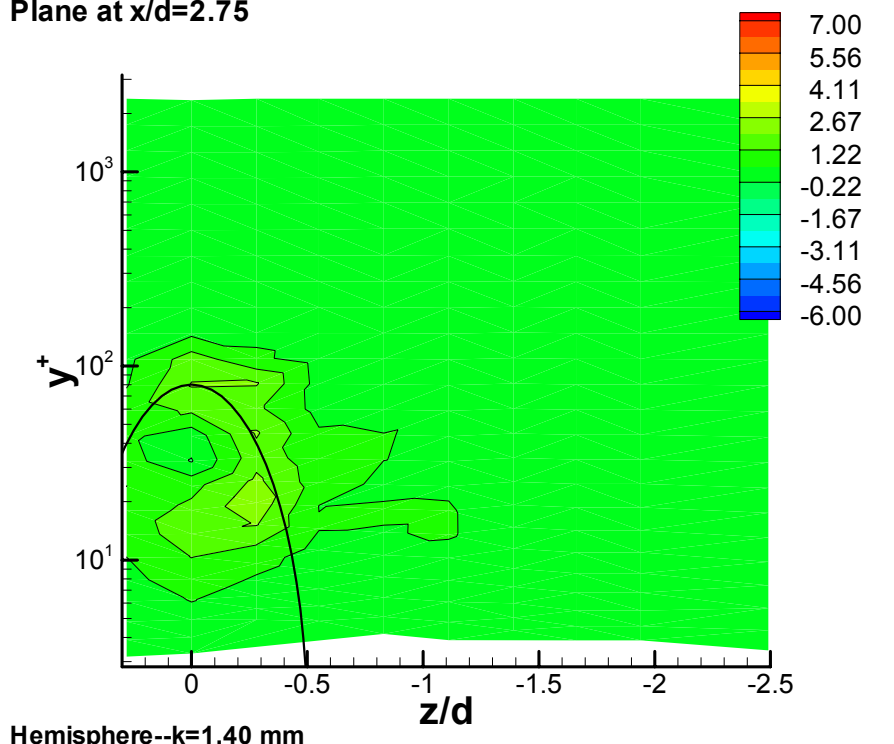

Hemisphere--k=1.40 mm

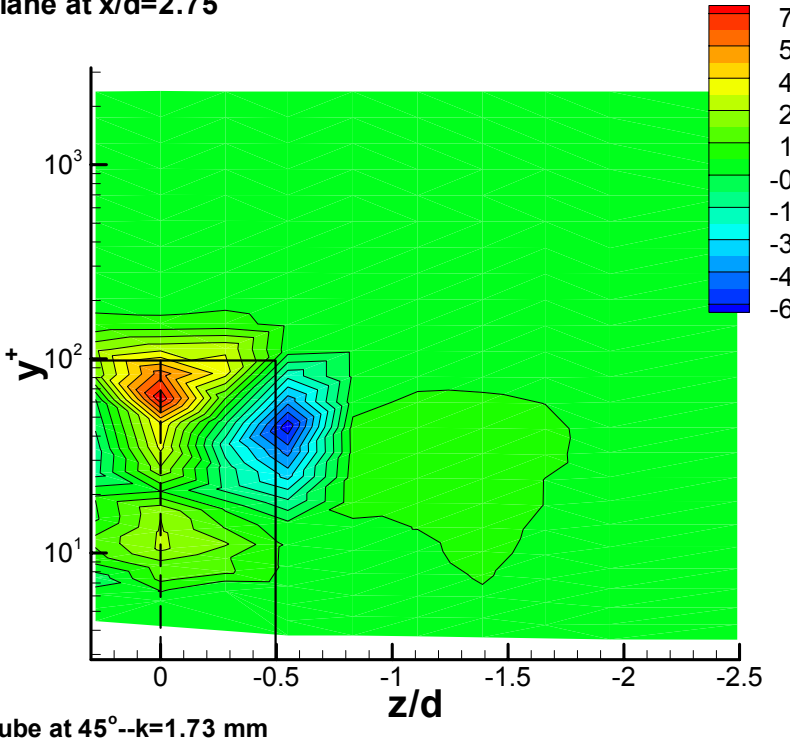

Figure 3.82: Contours of $\overline{v w^{2}} / U_{\tau}^{3}$ in the $\mathbf{y}-\mathbf{z}$ plane at a location of $\mathbf{x} / \mathbf{d}=\mathbf{2 . 7 5}$ 

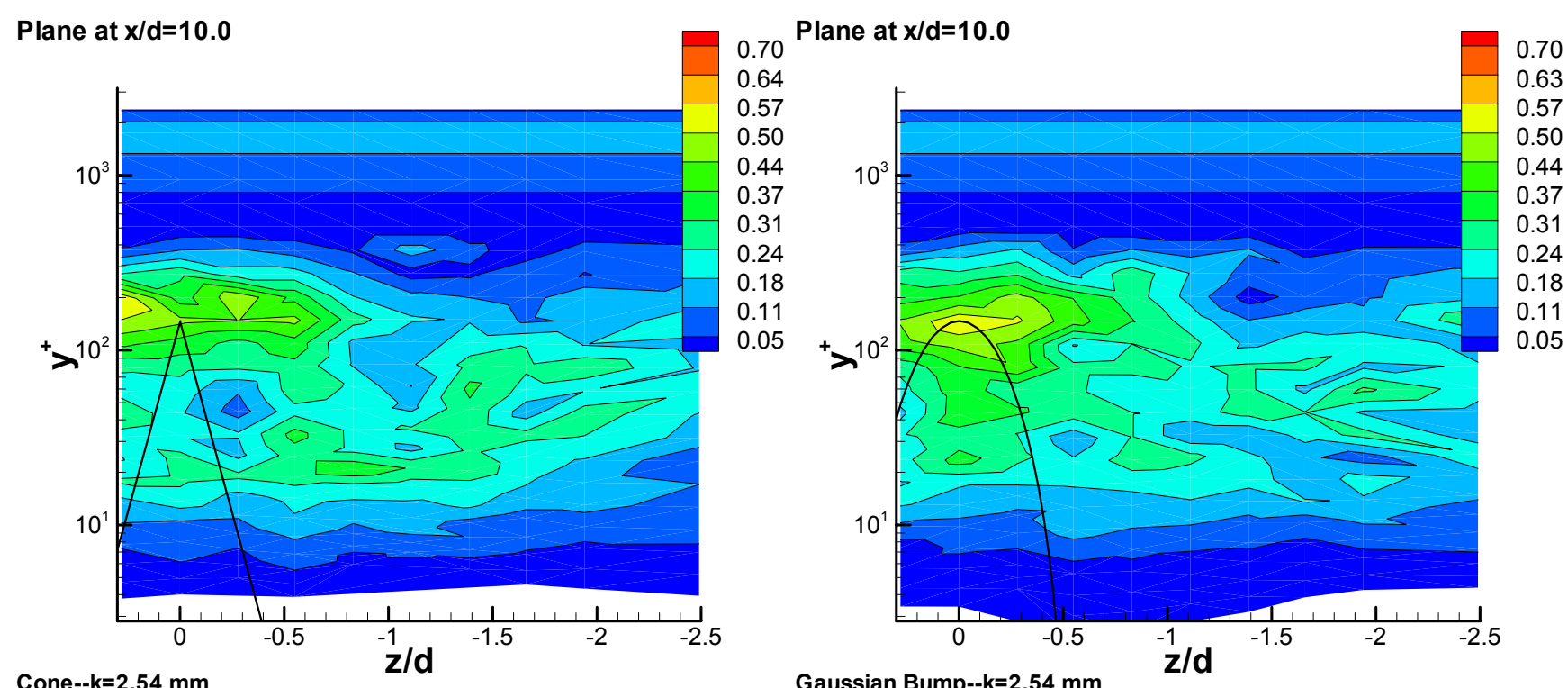

Plane at $x / d=10.0$

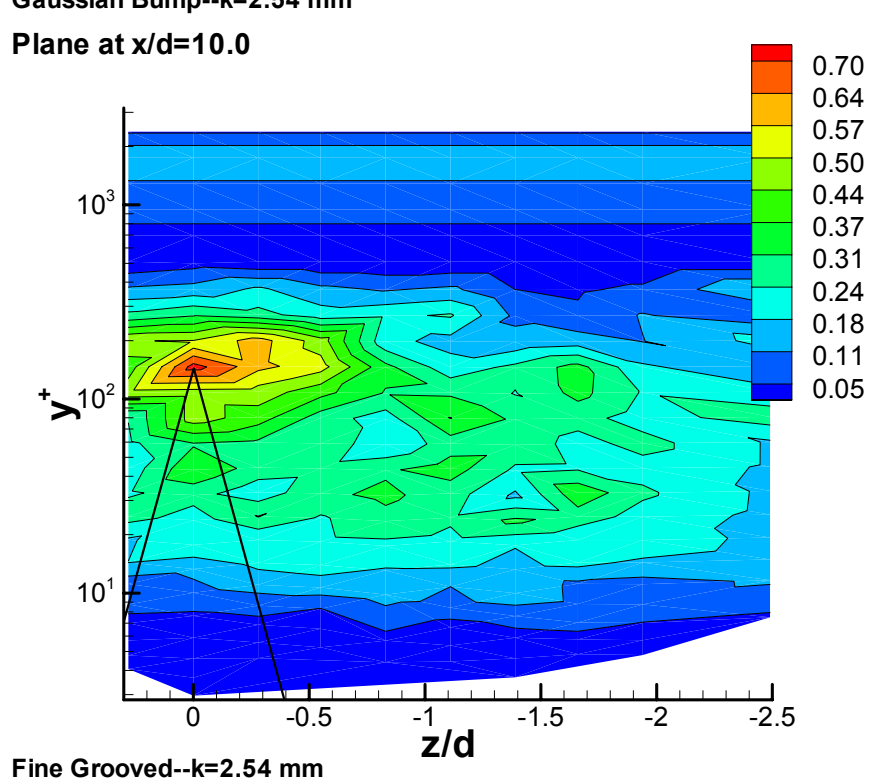

Large Grooved--k=2.54 mm

Fine Grooved--k=2.54 mm

Figure 3.83: Contours of $\overline{v w^{2}} / U_{\tau}^{3}$ in the $\mathbf{y}-\mathbf{z}$ plane at a location of $\mathbf{x} / \mathbf{d}=10.0$ 


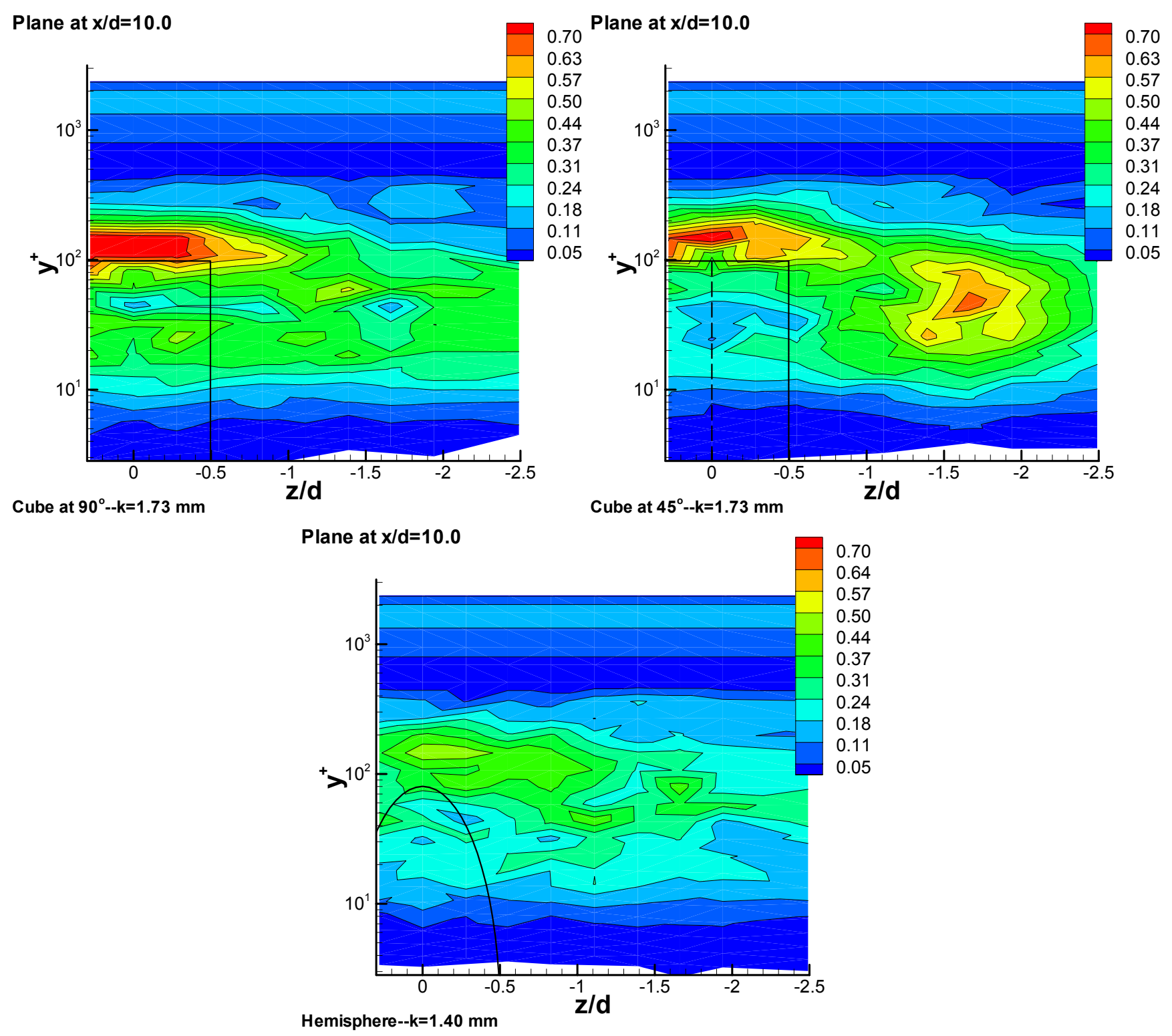

Figure 3.84: Contours of $\overline{v w^{2}} / U_{\tau}^{3}$ in the $\mathbf{y}-\mathbf{z}$ plane at a location of $\mathbf{x} / \mathbf{d}=10.0$ 

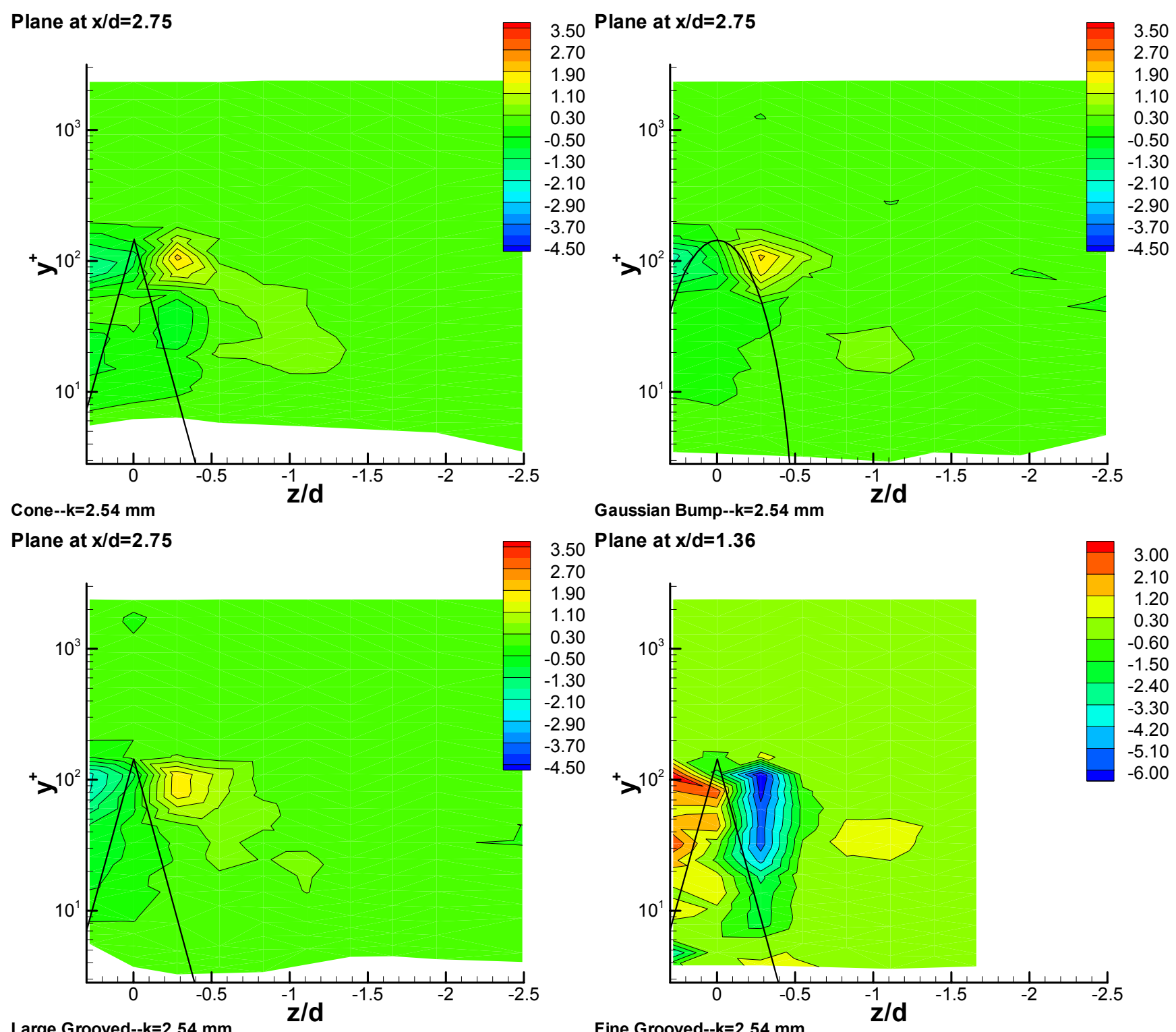

Gaussian Bump--k=2.54 mm

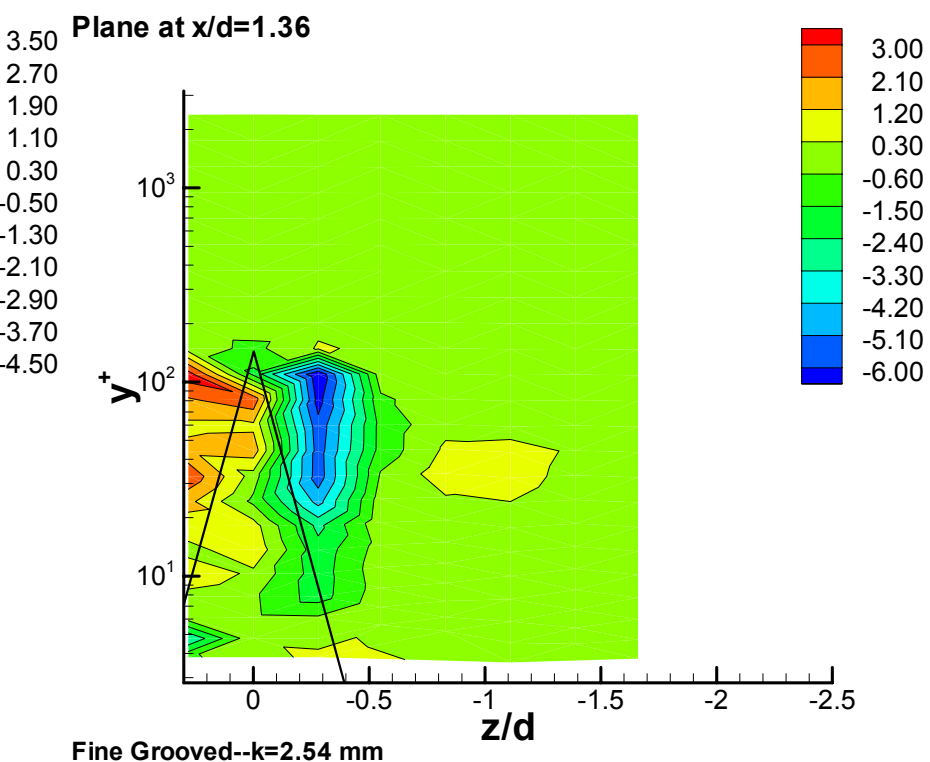

Figure 3.85: Contours of $\overline{u v w} / U_{\tau}^{3}$ in the $\mathbf{y}-z$ plane at a location of $\mathbf{x} / \mathbf{d}=\mathbf{2 . 7 5}$ (Note: Fine Grooved $\mathbf{x} / \mathbf{d}=1.36$ ) 


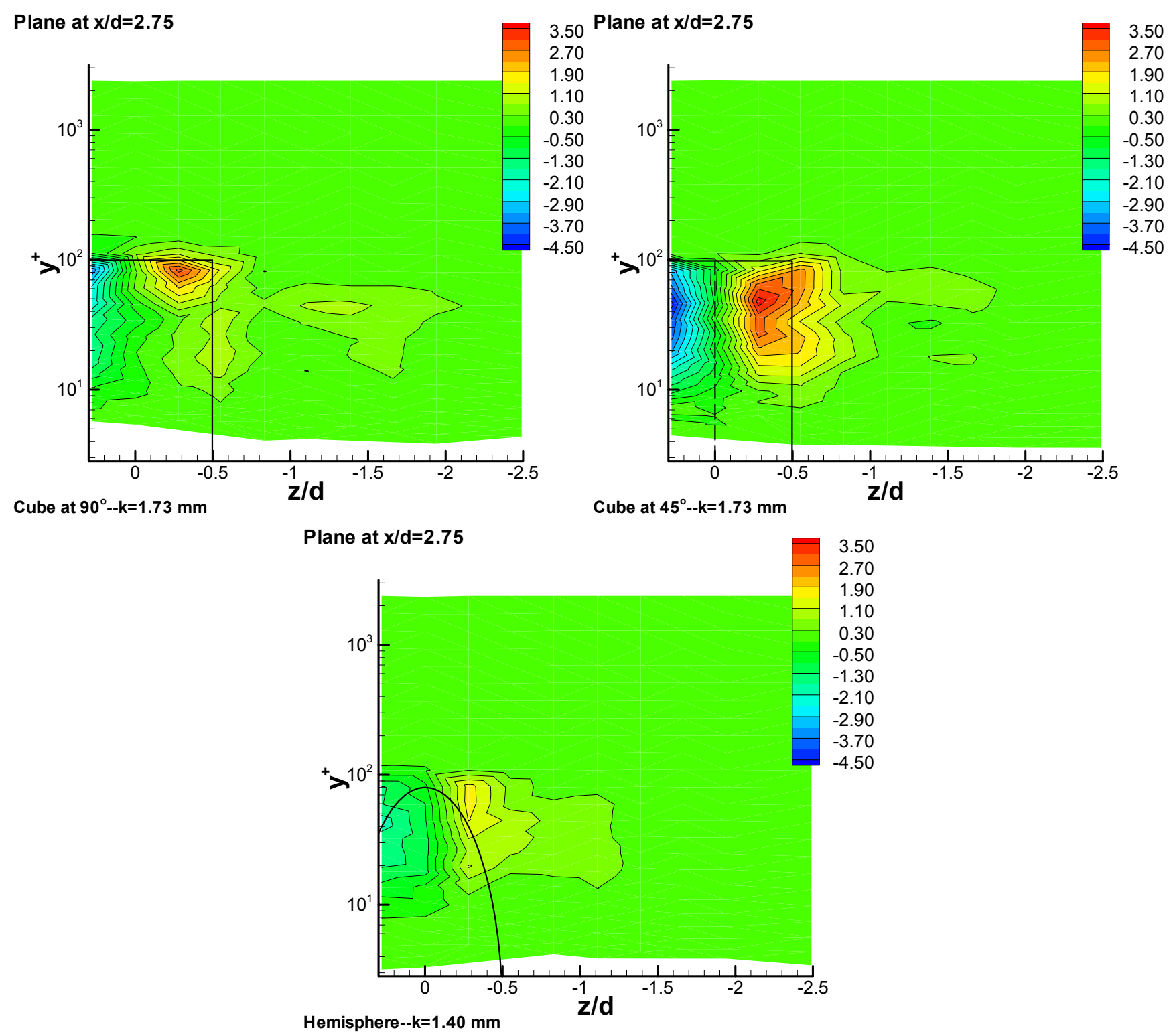

Figure 3.86: Contours of $\overline{u v w} / U_{\tau}^{3}$ in the $\mathbf{y}-z$ plane at a location of $\mathbf{x} / \mathbf{d}=\mathbf{2 . 7 5}$ 

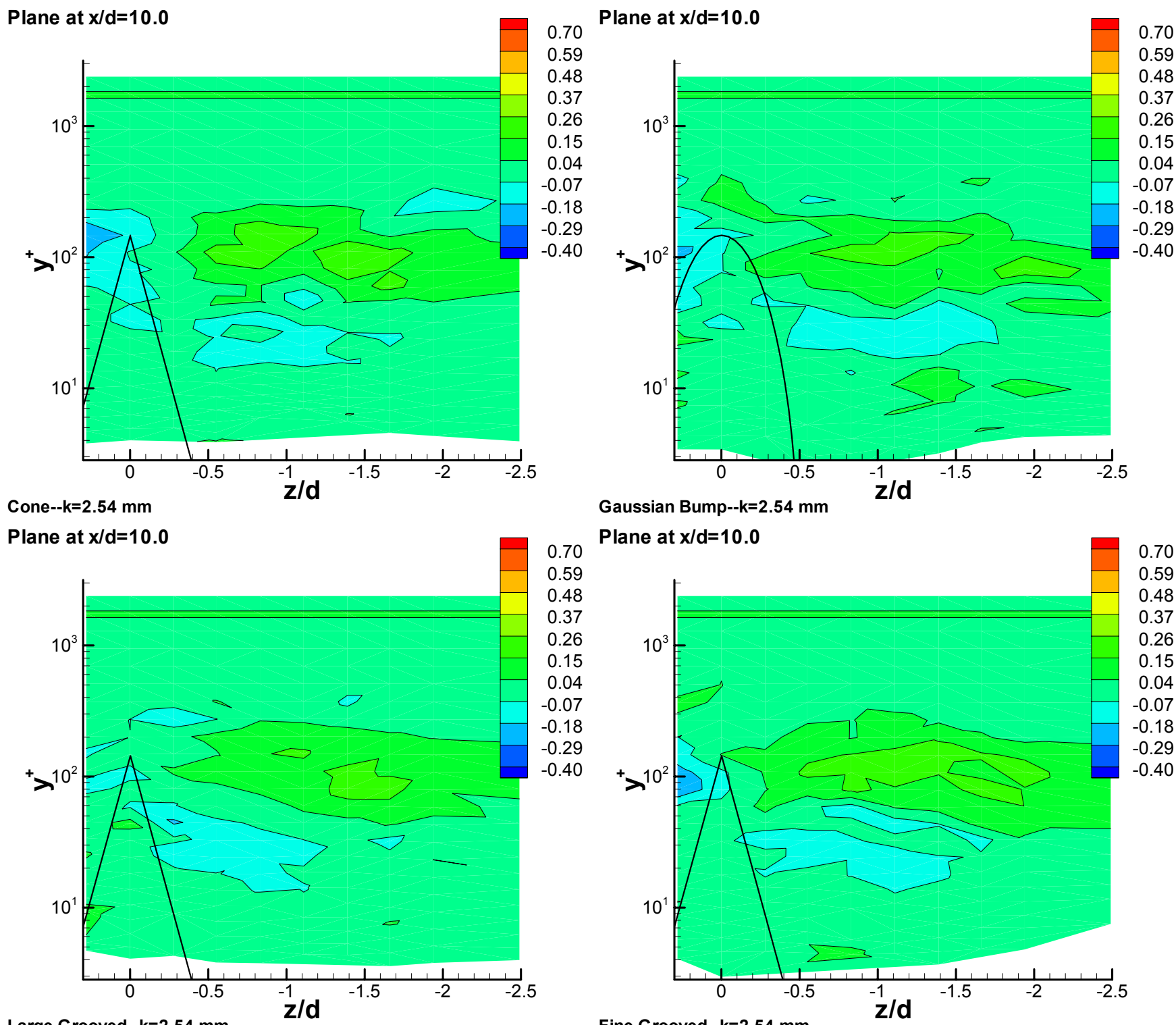

Gaussian Bump--k=2.54 mm

Plane at $x / d=10.0$

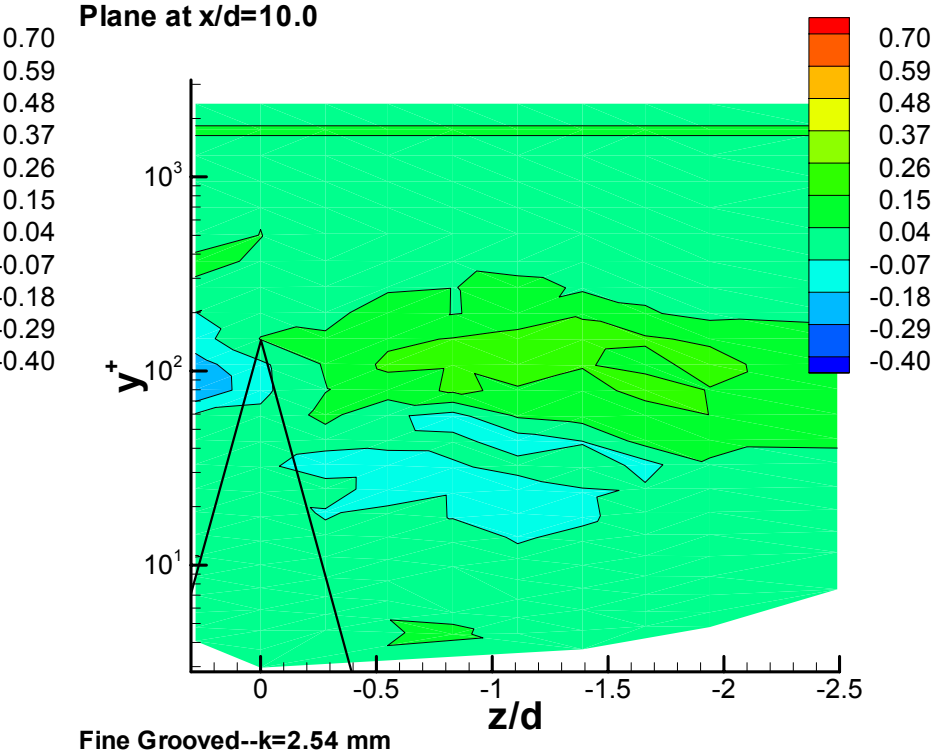

Figure 3.87: Contours of $\overline{u v w} / U_{\tau}^{3}$ in the $\mathbf{y}-z$ plane at a location of $\mathbf{x} / \mathbf{d}=\mathbf{1 0 . 0}$ 


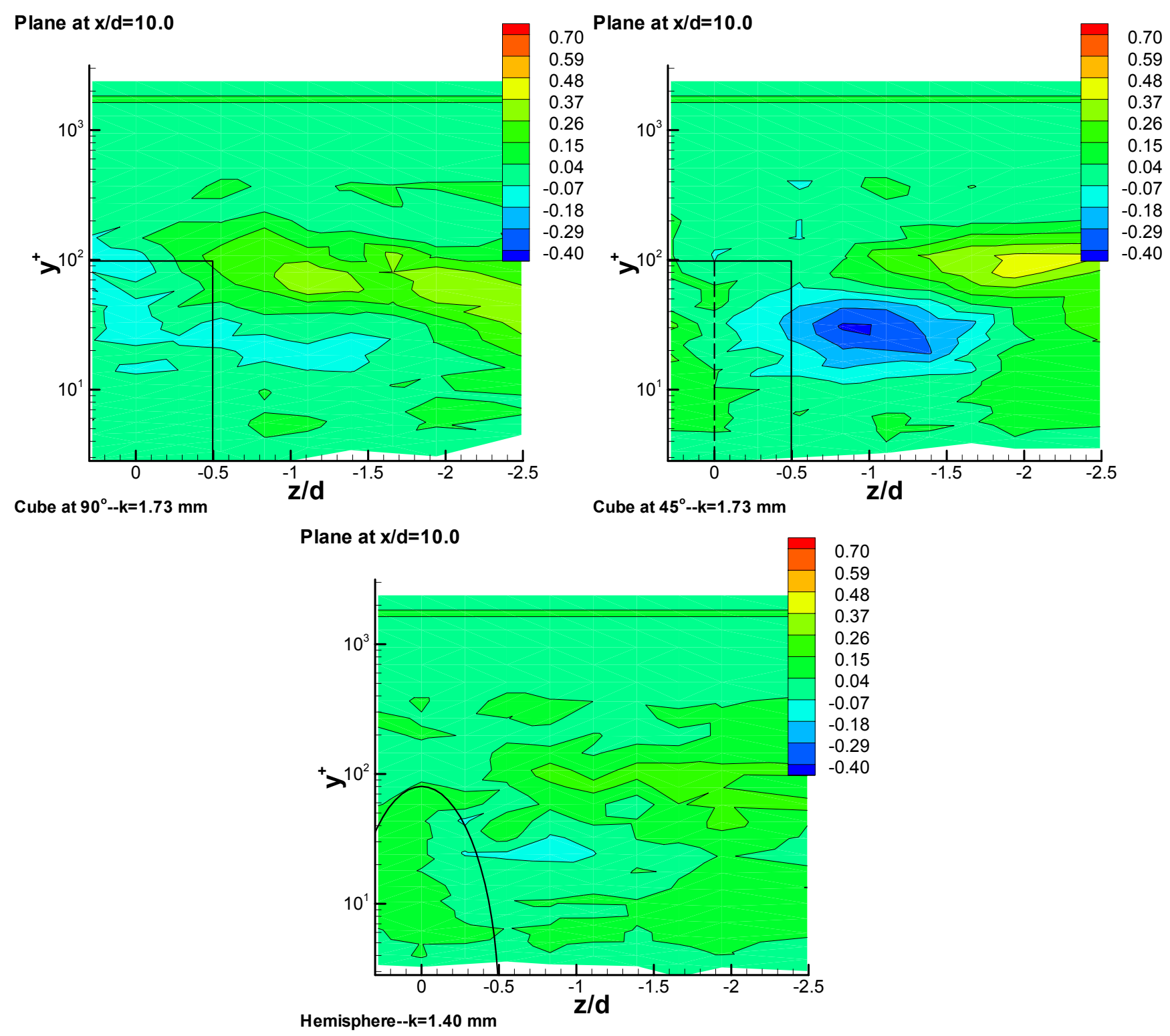

Figure 3.88: Contours of $\overline{u v w} / U_{\tau}^{3}$ in the $\mathbf{y}-z$ plane at a location of $\mathbf{x} / \mathbf{d}=10.0$ 


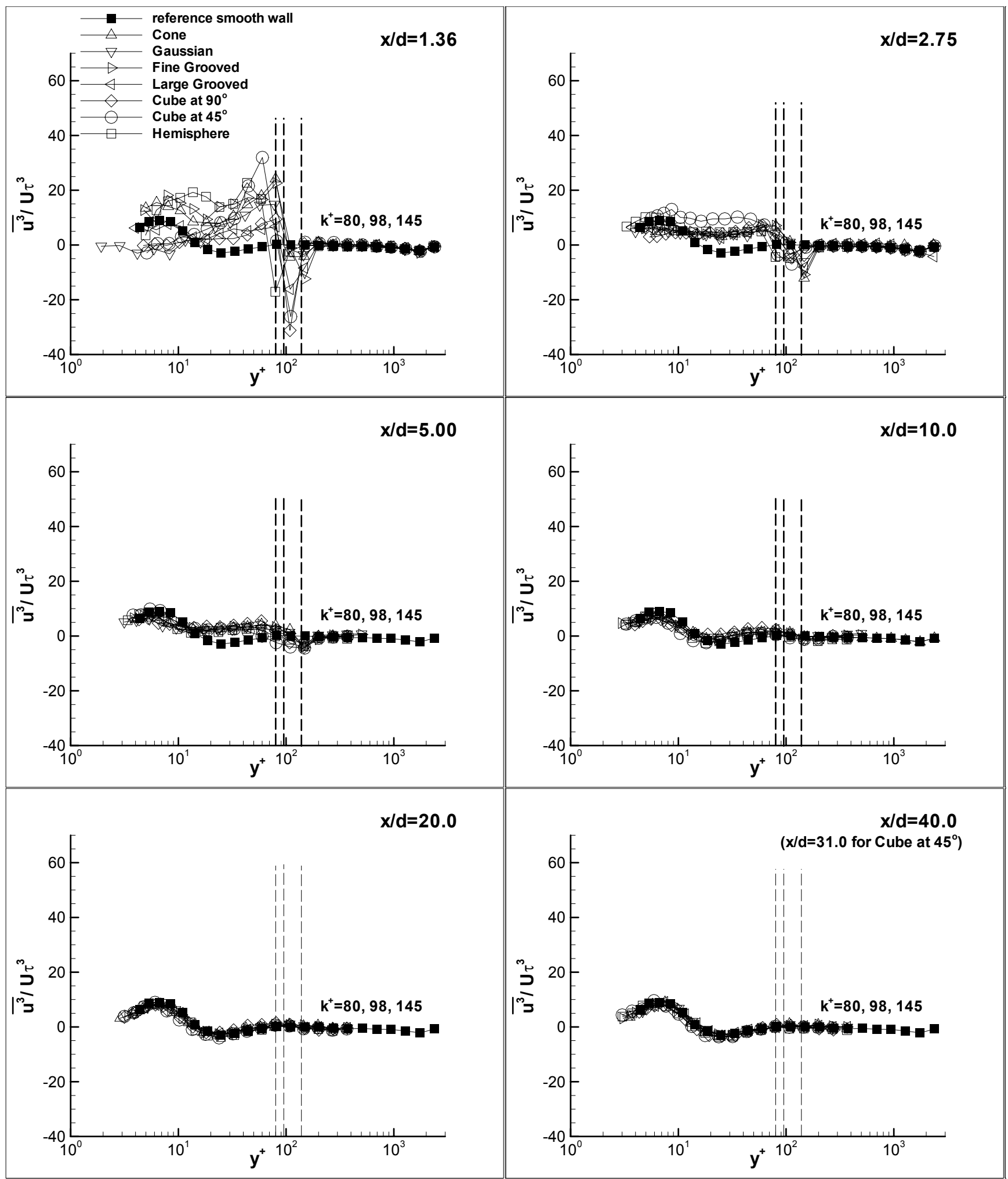

Figure 3.89: $\overline{u^{3}} / U_{\tau}^{3}$ vs. $y U_{\tau} / v$ profiles along the centerline 

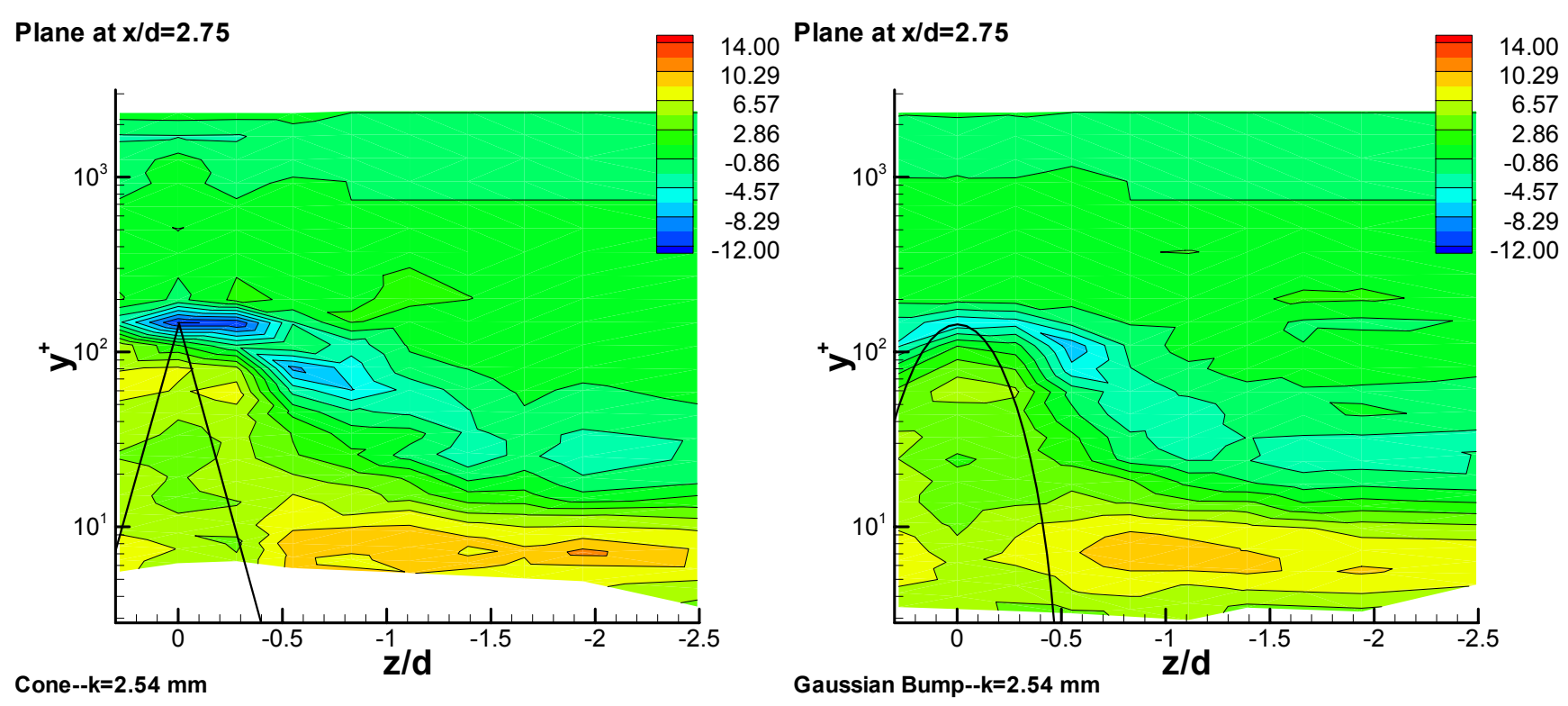

Plane at $\mathrm{x} / \mathrm{d}=\mathbf{2 . 7 5}$

Plane at $x / d=1.36$
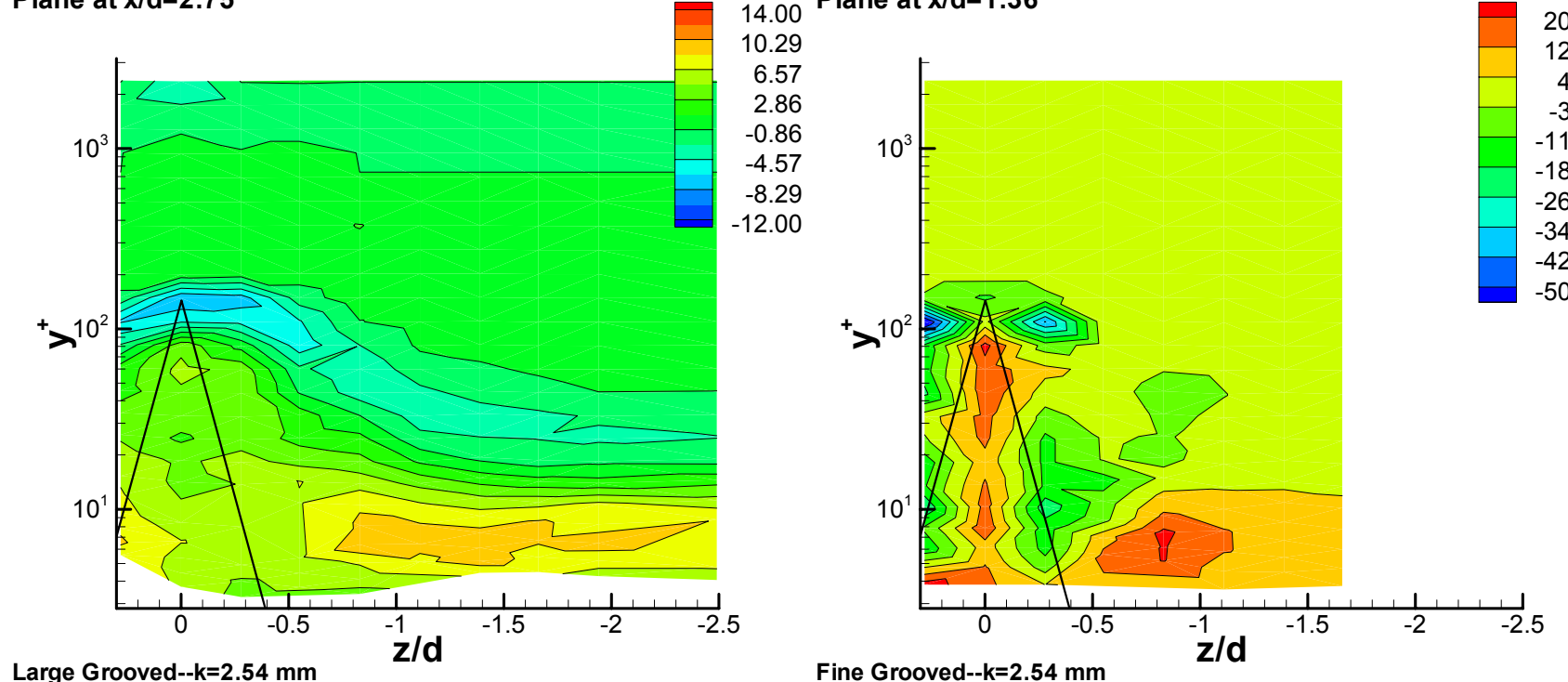

Large Grooved--k=2.54 mm

Fine Grooved--k=2.54 mm

Figure 3.90: Contours of $\overline{u^{3}} / U_{\tau}^{3}$ in the $\mathbf{y}-z$ plane at a location of $\mathbf{x} / \mathbf{d}=\mathbf{2 . 7 5}$ (Note: Fine Grooved $\mathbf{x} / \mathbf{d}=1.36$ ) 


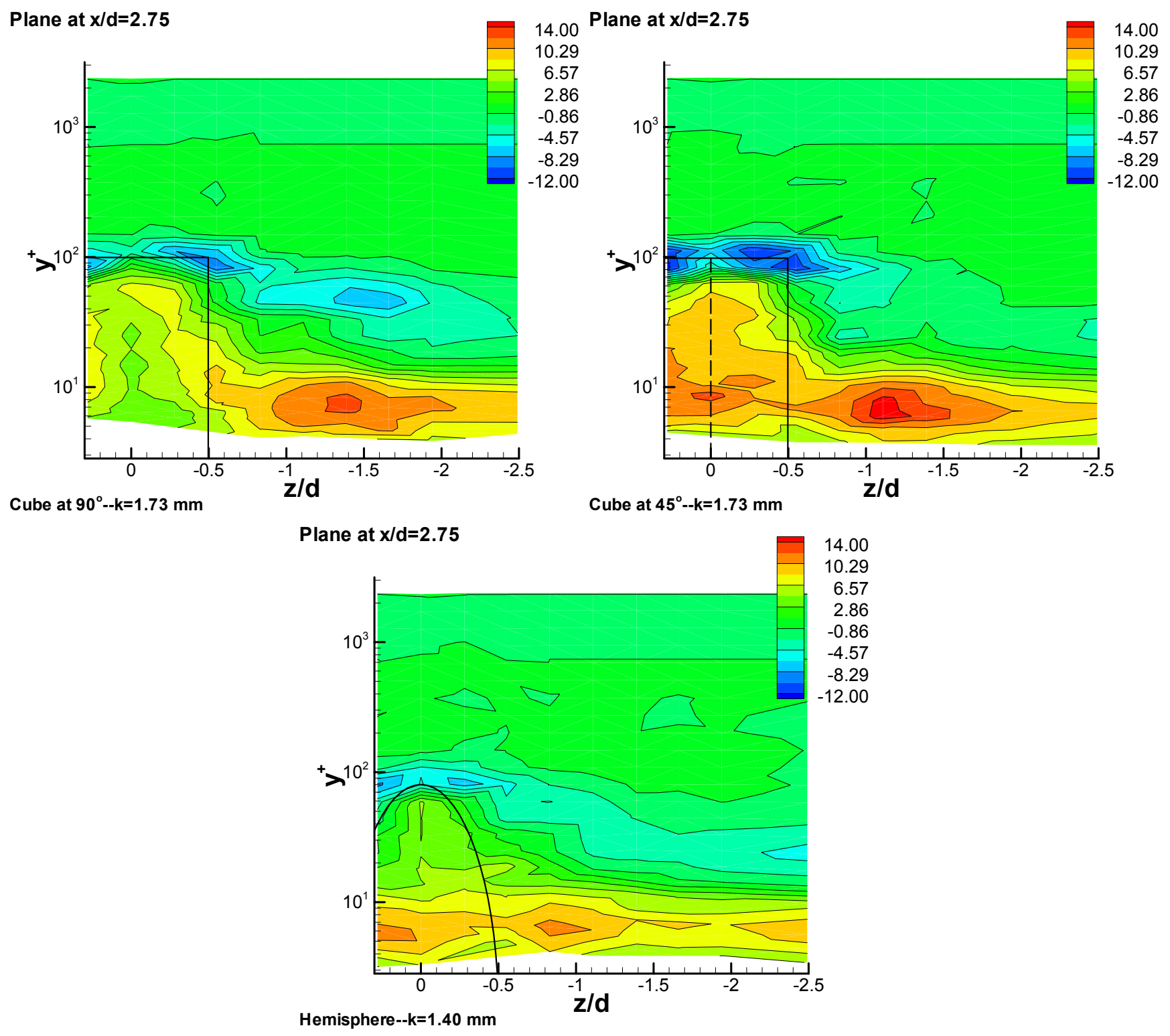

Figure 3.91: Contours of $\overline{u^{3}} / U_{\tau}^{3}$ in the $\mathbf{y}-\mathbf{z}$ plane at a location of $\mathbf{x} / \mathbf{d}=\mathbf{2 . 7 5}$ 

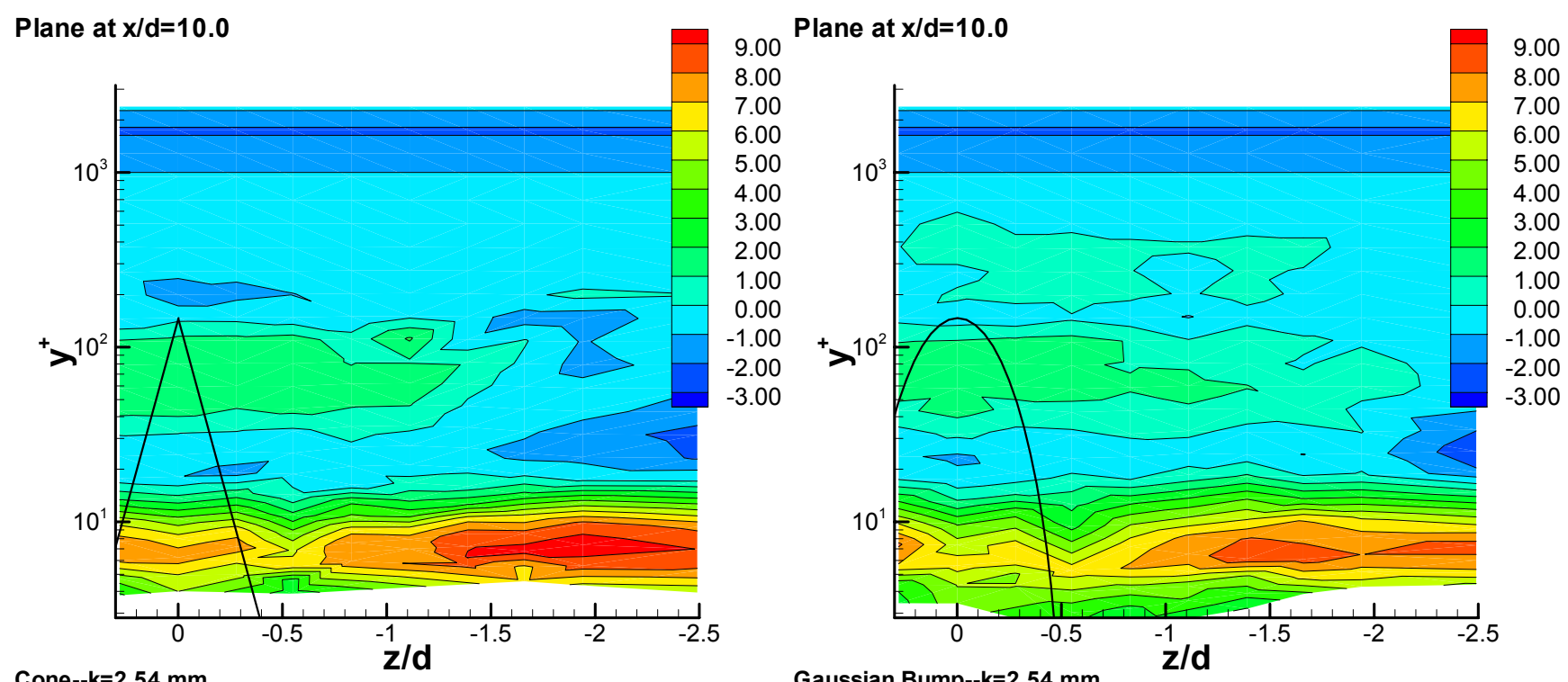

\section{Plane at $x / d=10.0$}

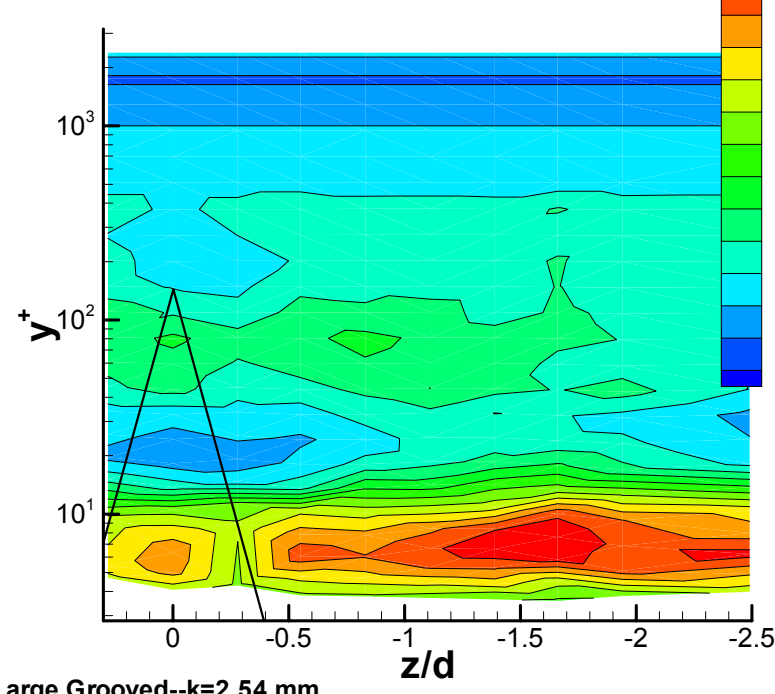

Plane at $x / d=10.0$

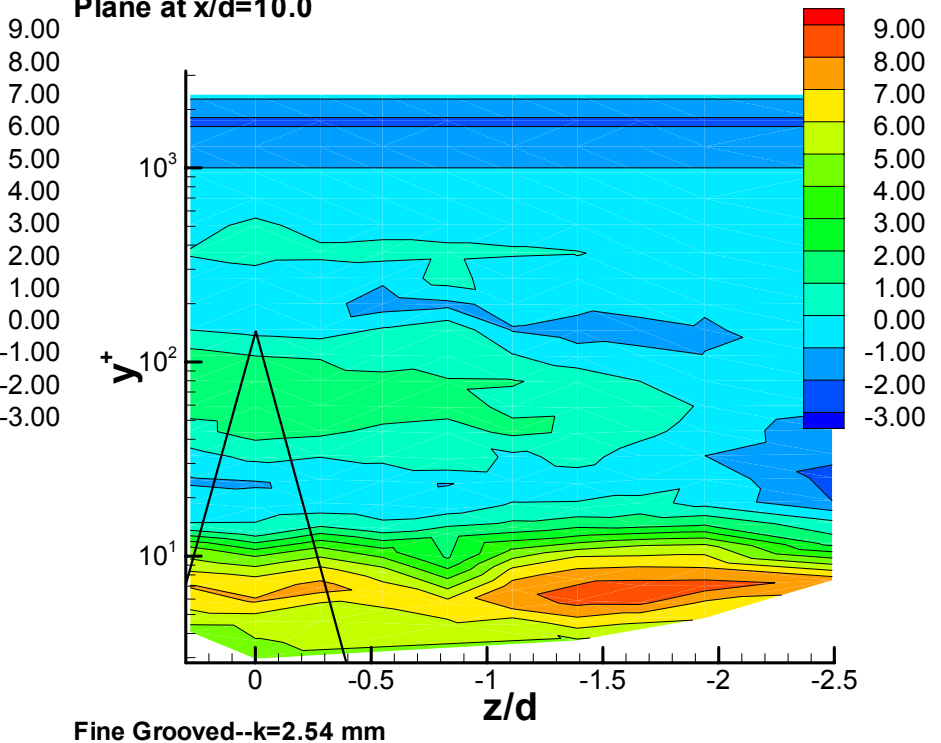

Figure 3.92: Contours of $\overline{u^{3}} / U_{\tau}^{3}$ in the $\mathbf{y}-z$ plane at a location of $\mathbf{x} / \mathbf{d}=10.0$ 


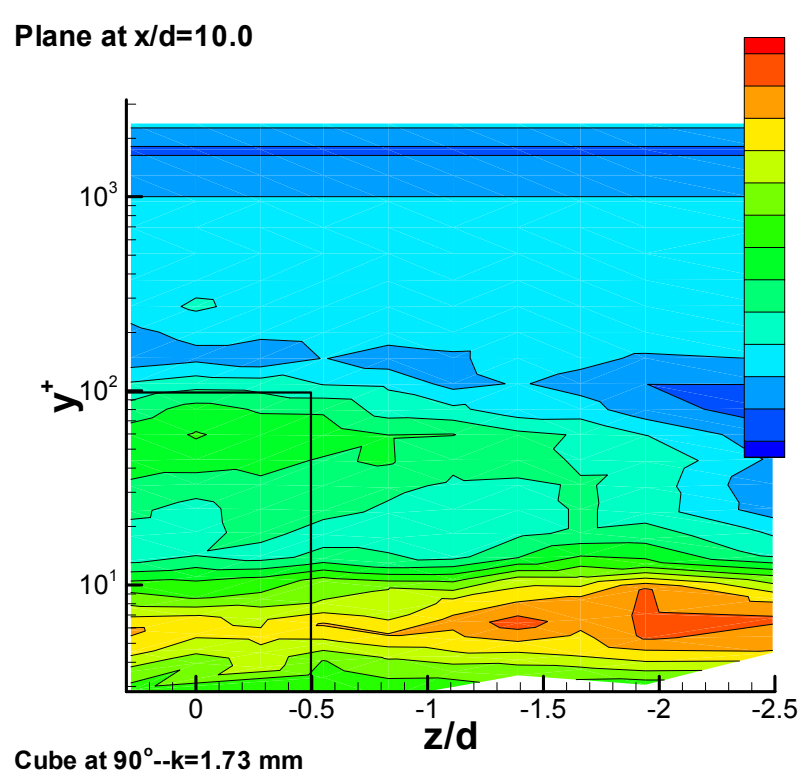

Plane at $x / d=10.0$

\section{Plane at $x / d=10.0$}

9.00
8.00
7.00

7.00
6.00
5.00
4.00

5.00
4.00
3.00
2.00

2.00

2.00
1.00
0.00

$-1.00$

$-2.00$

$-3.00$$$
10^{1}
$$

$10^{1}$

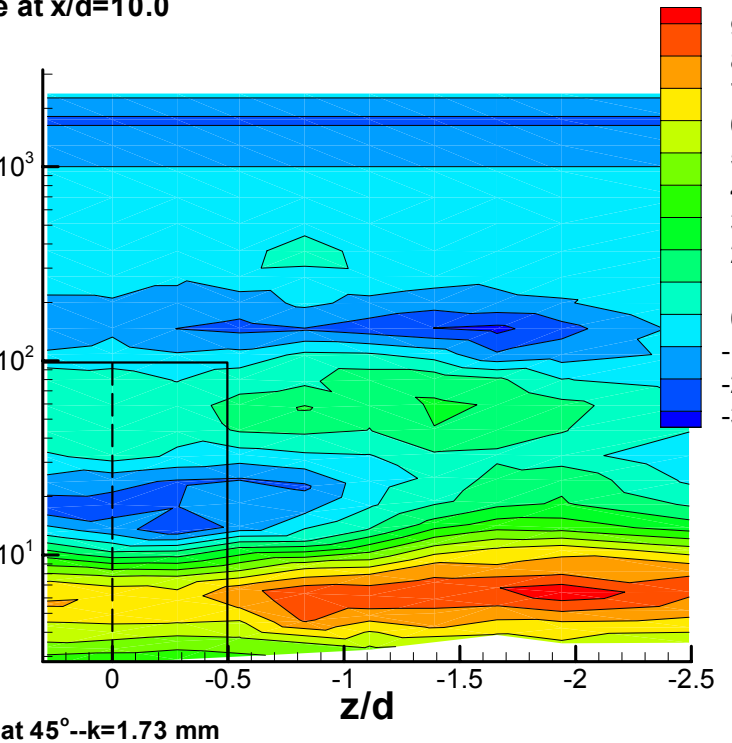

Cube at $45^{\circ}--\mathrm{k}=1.73 \mathrm{~mm}$
9.00

8.00

7.00

(1.00

4.00

3.00

2.00

1.00

0.00

$-2.00$

$-3.00$

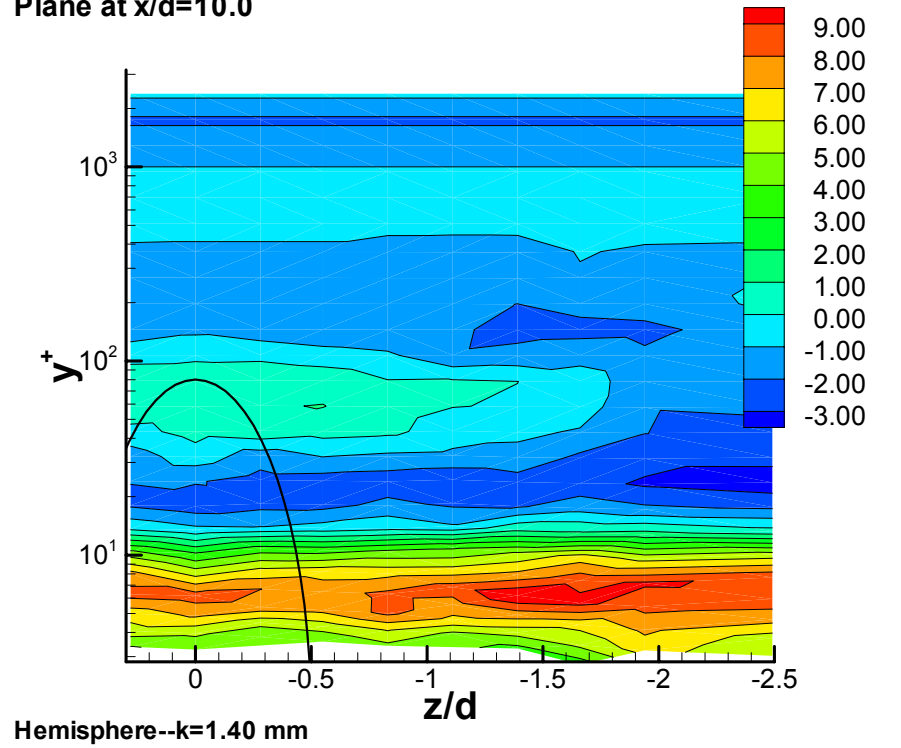

Hemisphere--k $=1.40 \mathrm{~mm}$

Figure 3.93: Contours of $\overline{u^{3}} / U_{\tau}^{3}$ in the $\mathbf{y}-\mathbf{z}$ plane at a location of $\mathbf{x} / \mathbf{d}=10.0$ 


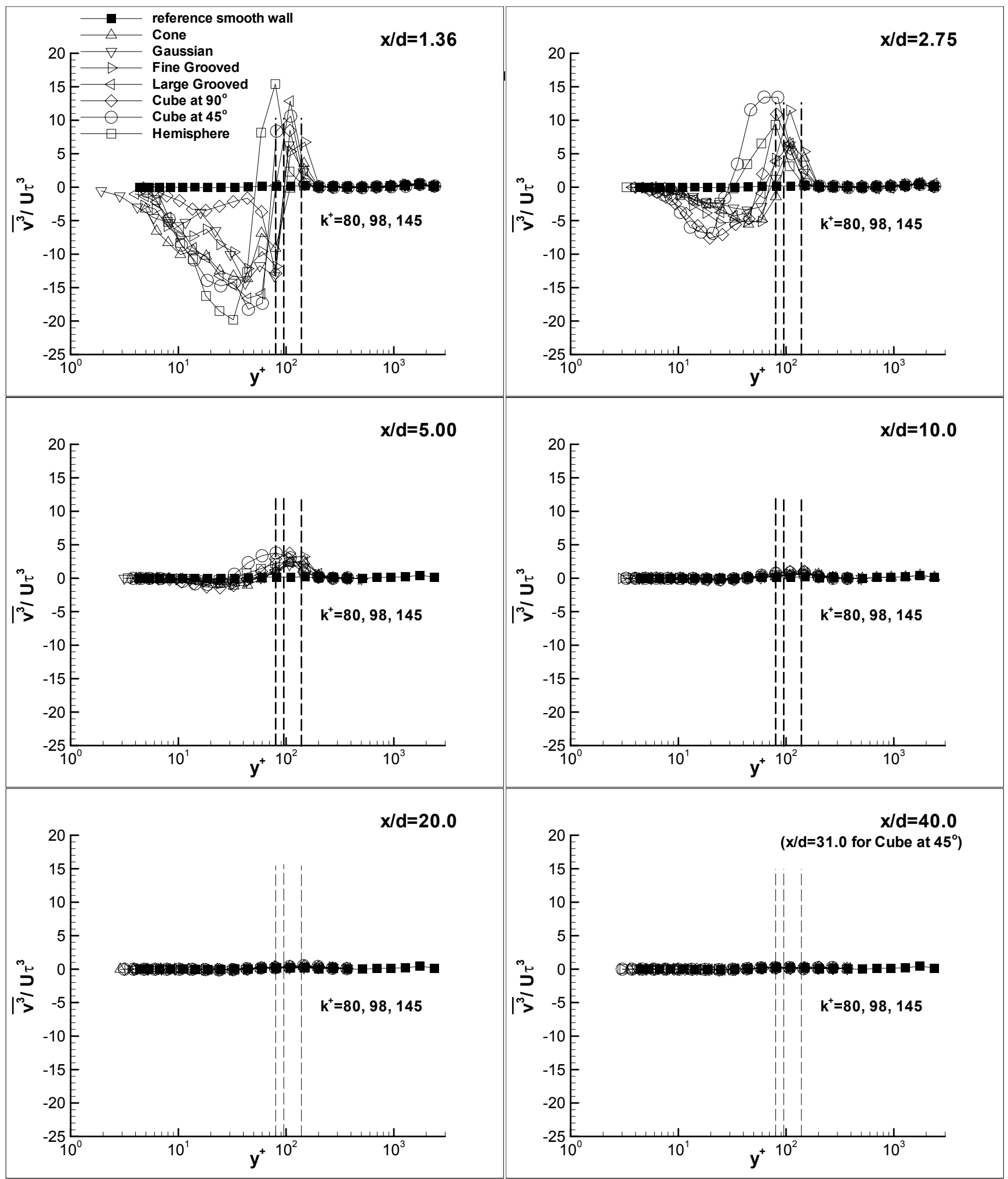

Figure 3.94: $\overline{v^{3}} / U_{\tau}^{3}$ vs. $y U_{\tau} / v$ profiles along the centerline 


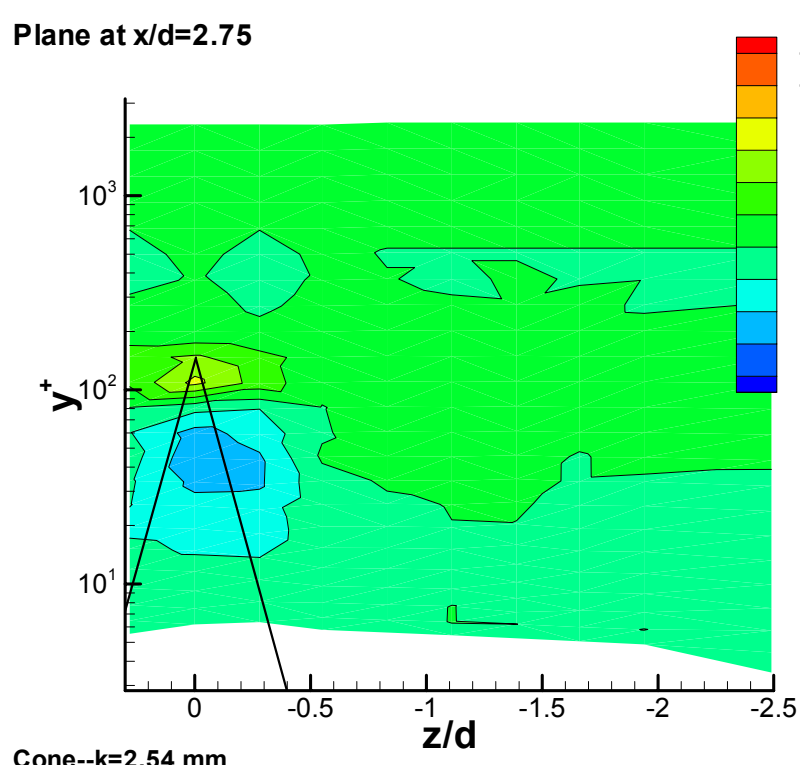

Plane at $x / d=2.75$

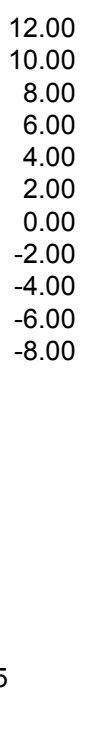

12.00

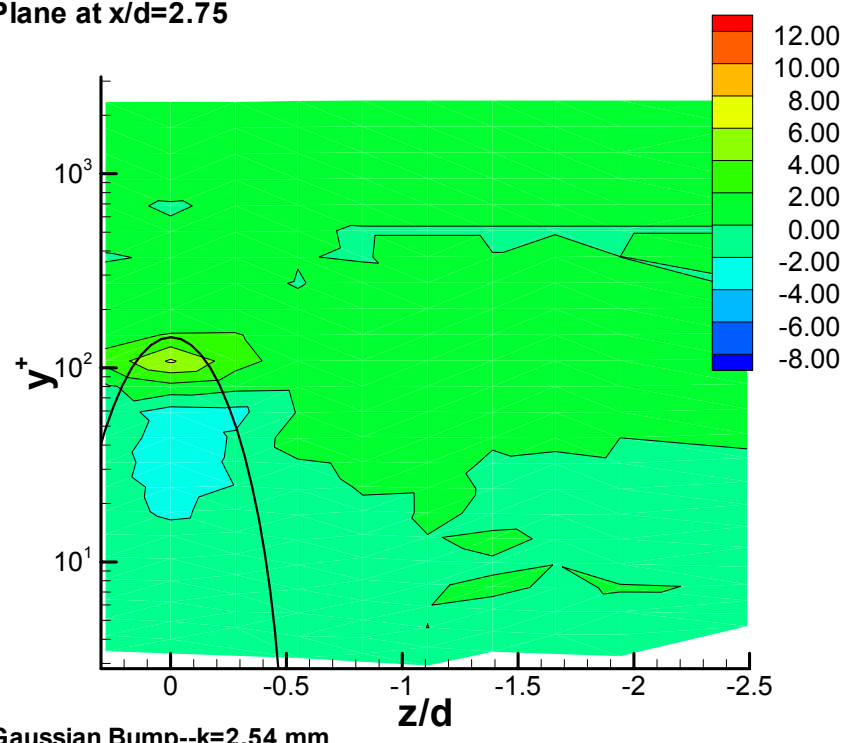

Gaussian Bump--k=2.54 mm

Plane at $\mathbf{x} / \mathrm{d}=\mathbf{2 . 7 5}$

Plane at $x / d=1.36$
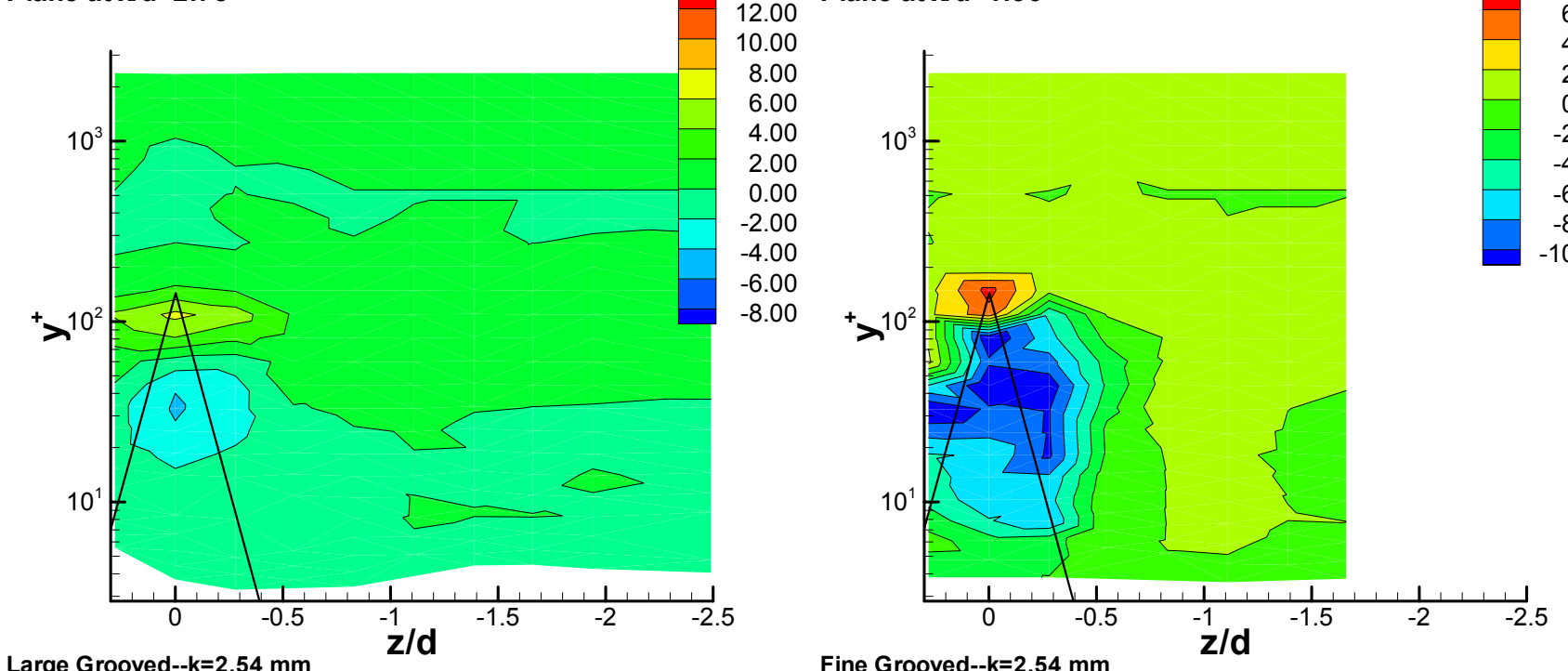

Large Grooved--k=2.54 $\mathrm{mm}$

Fine Grooved--k=2.54 mm

Figure 3.95: Contours of $\overline{v^{3}} / U_{\tau}^{3}$ in the $\mathbf{y}-\mathbf{z}$ plane at a location of $\mathbf{x} / \mathbf{d}=\mathbf{2 . 7 5}$ (Note: Fine Grooved $\mathbf{x} / \mathbf{d}=1.36$ ) 


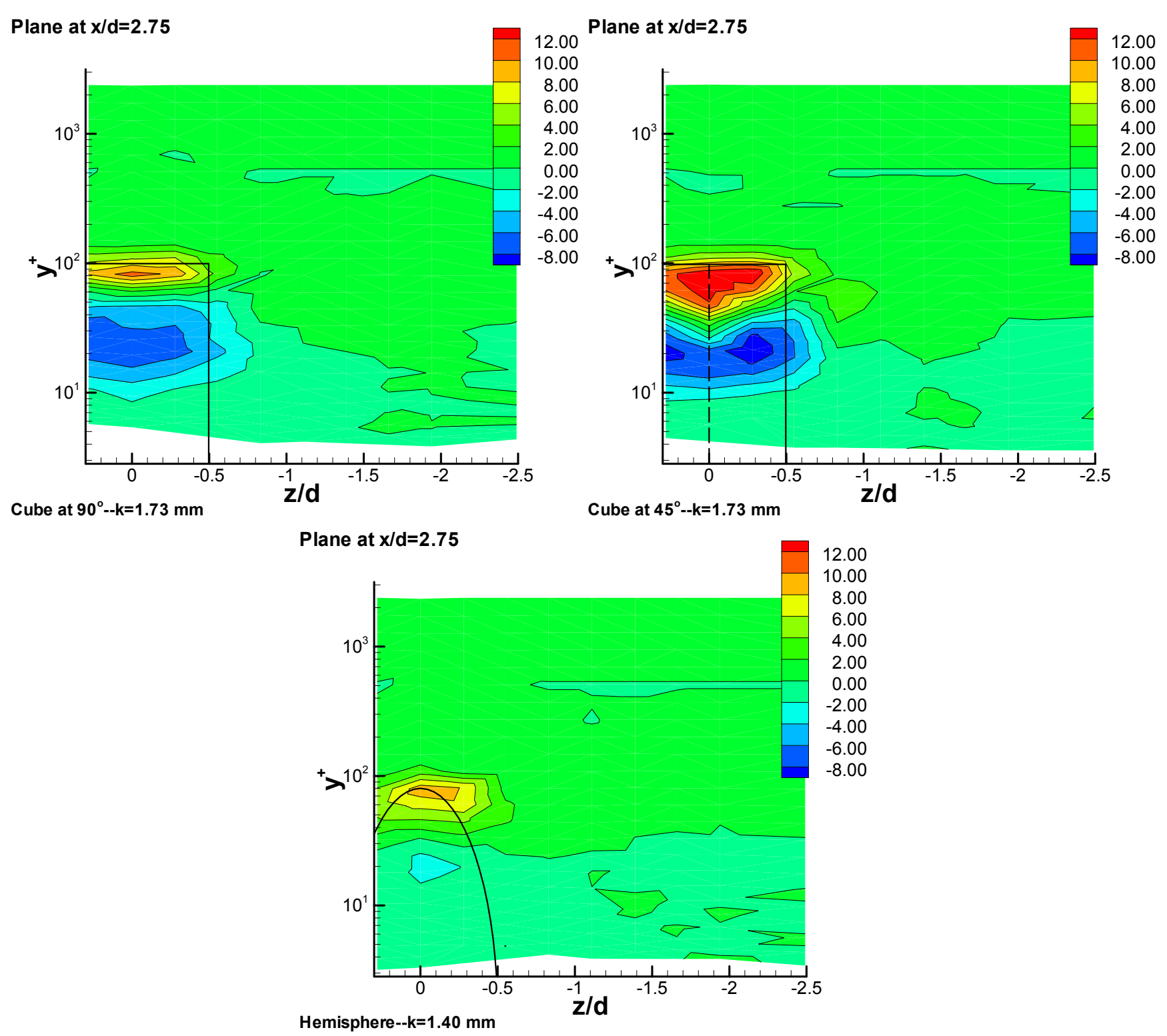

Figure 3.96: Contours of $\overline{v^{3}} / U_{\tau}^{3}$ in the $\mathbf{y}-\mathbf{z}$ plane at a location of $\mathbf{x} / \mathbf{d}=\mathbf{2 . 7 5}$ 

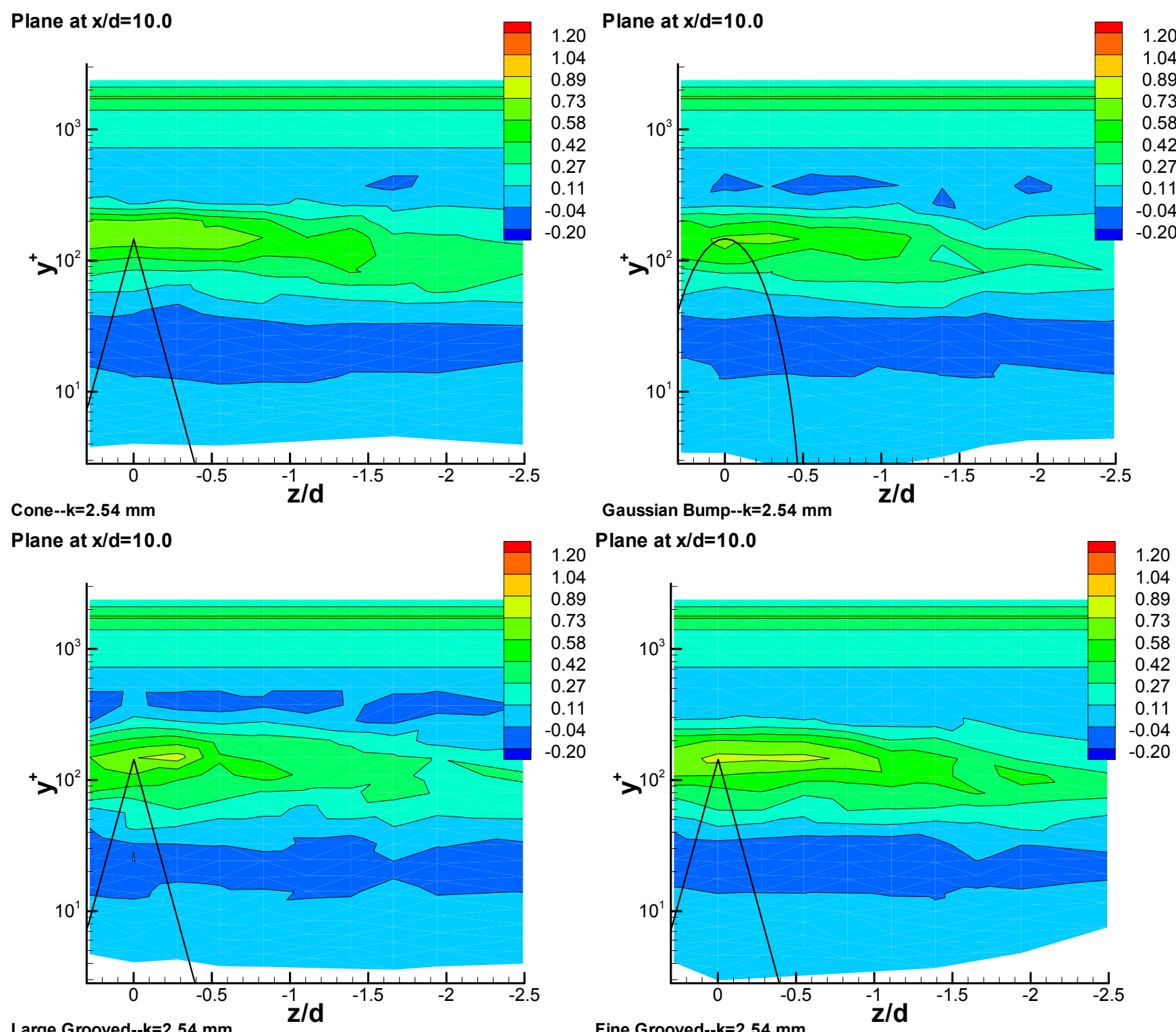

Gaussian Bump--k -2.
Plane at $x / d=10.0$

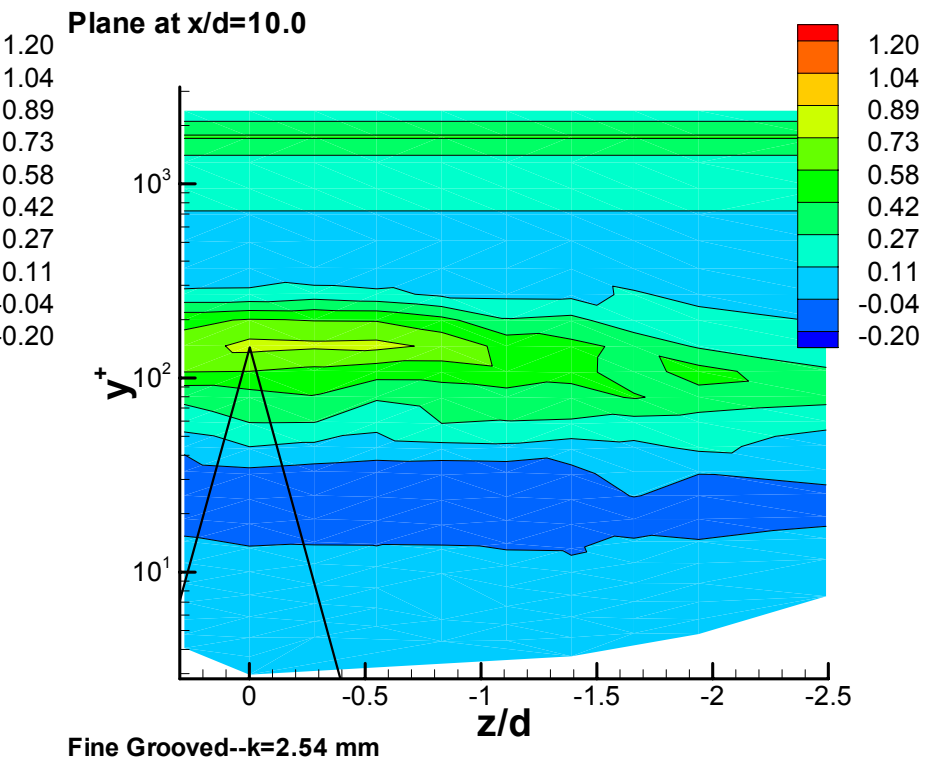

Figure 3.97: Contours of $\overline{v^{3}} / U_{\tau}^{3}$ in the $\mathbf{y}-\mathbf{z}$ plane at a location of $\mathbf{x} / \mathbf{d}=\mathbf{1 0 . 0}$ 


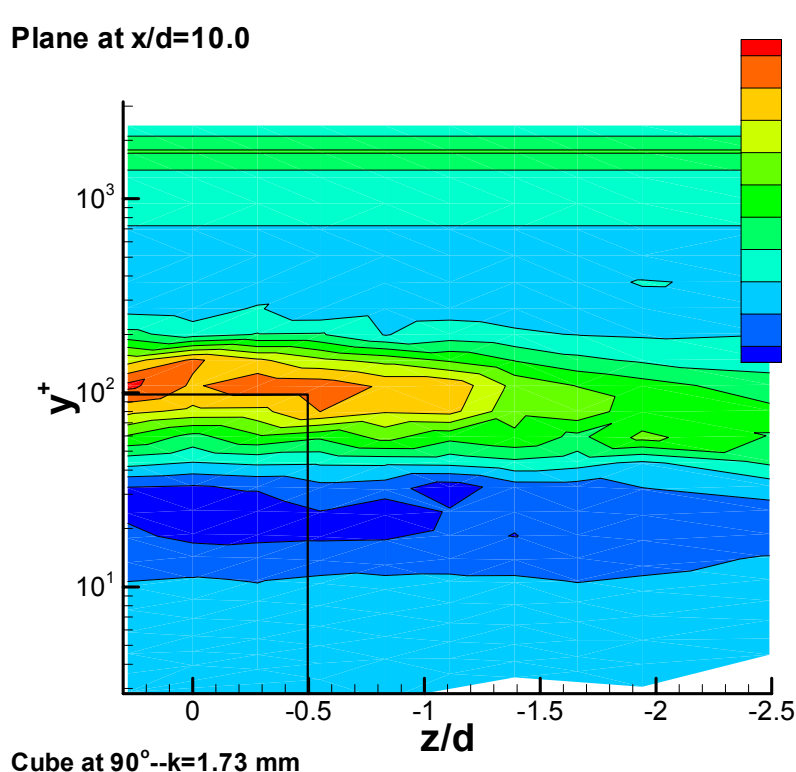

Cube at $90^{\circ}--\mathrm{k}=1.73 \mathrm{~mm}$

\section{Plane at $x / d=10.0$}

Plane at $x / d=10.0$

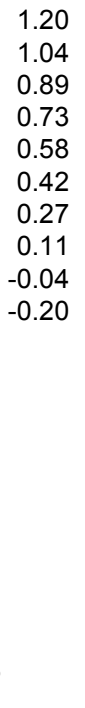

1.20

0.89

0.73

0.58

0.42

0.11

$-0.04$

$+>^{10}$

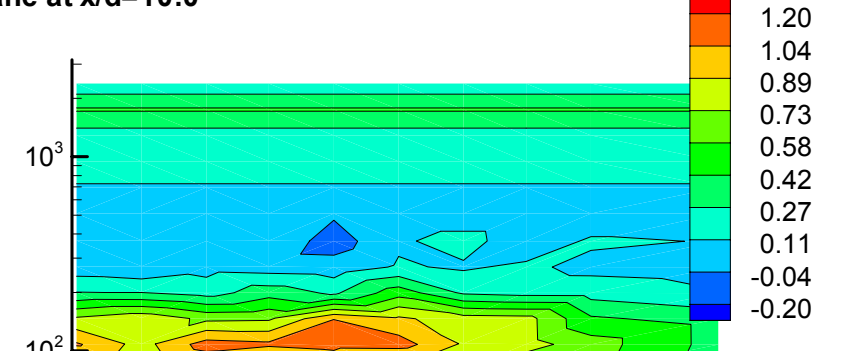

Cube at $45^{\circ}--\mathrm{k}=1.73 \mathrm{~mm}$

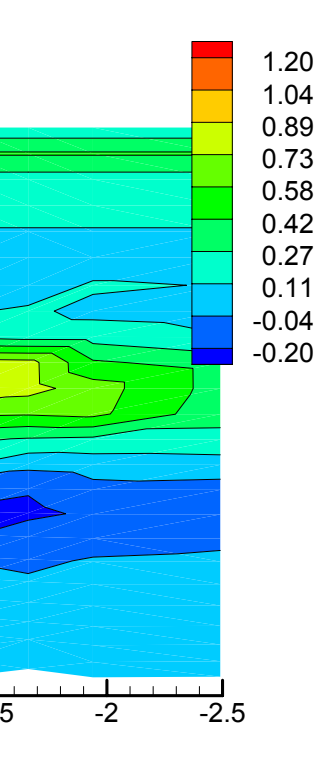

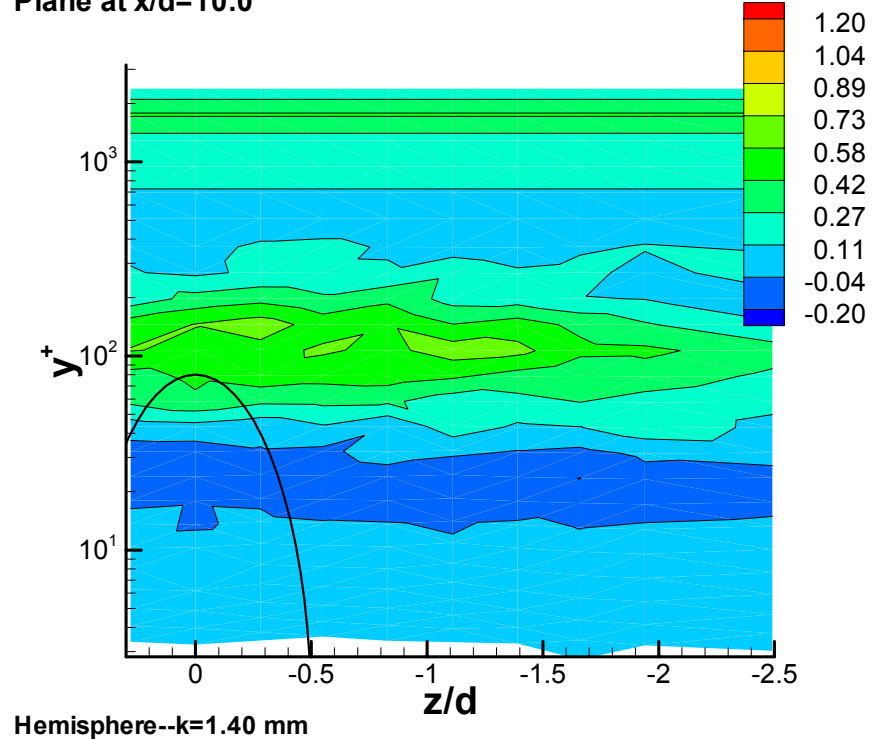

Hemisphere--k=1.40 mm

Figure 3.98: Contours of $\overline{v^{3}} / U_{\tau}^{3}$ in the $\mathbf{y}-\mathbf{z}$ plane at a location of $\mathbf{x} / \mathbf{d}=\mathbf{1 0 . 0}$ 

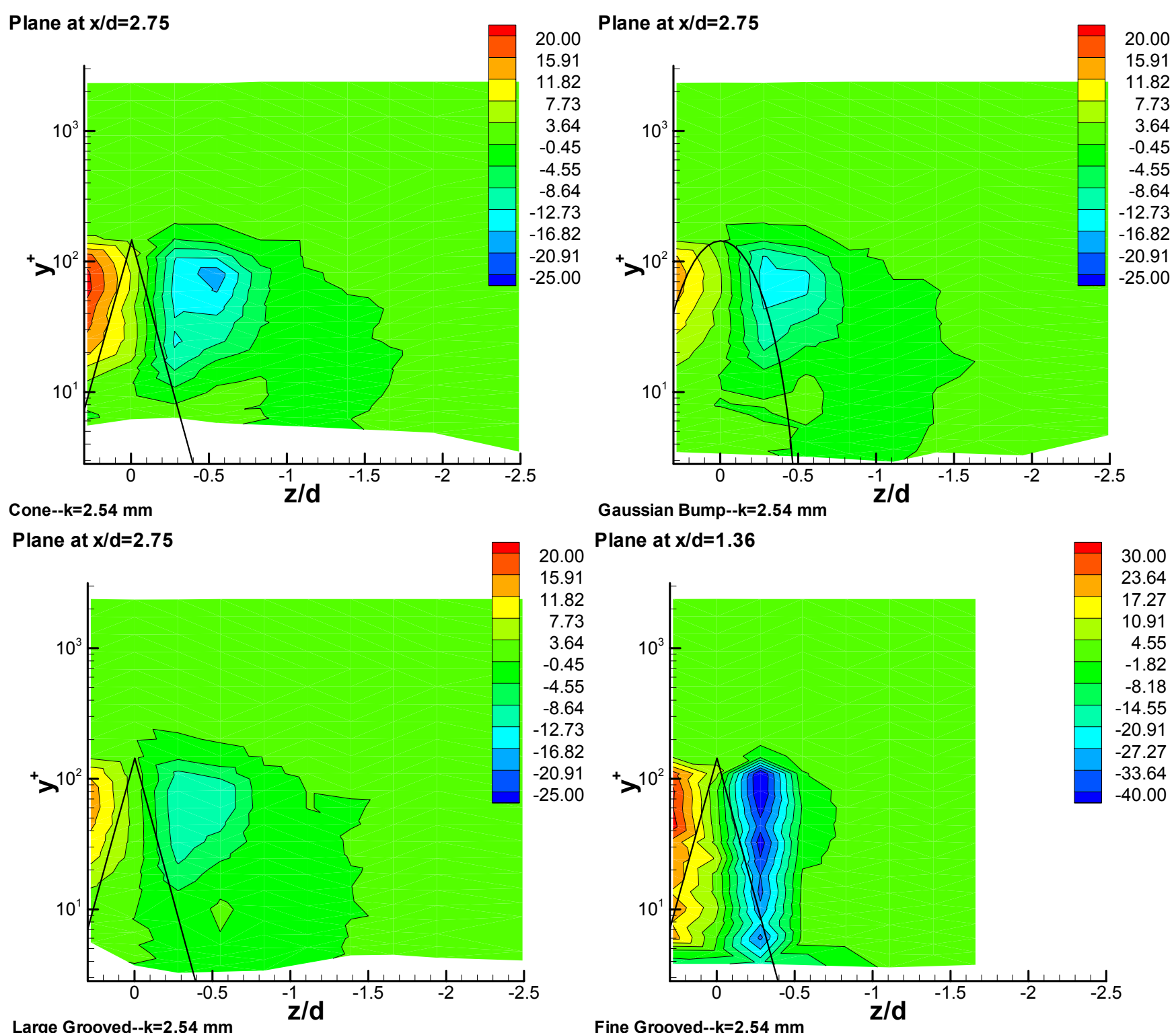

Gaussian Bump--k=2.54 mm

Plane at $x / d=1.36$

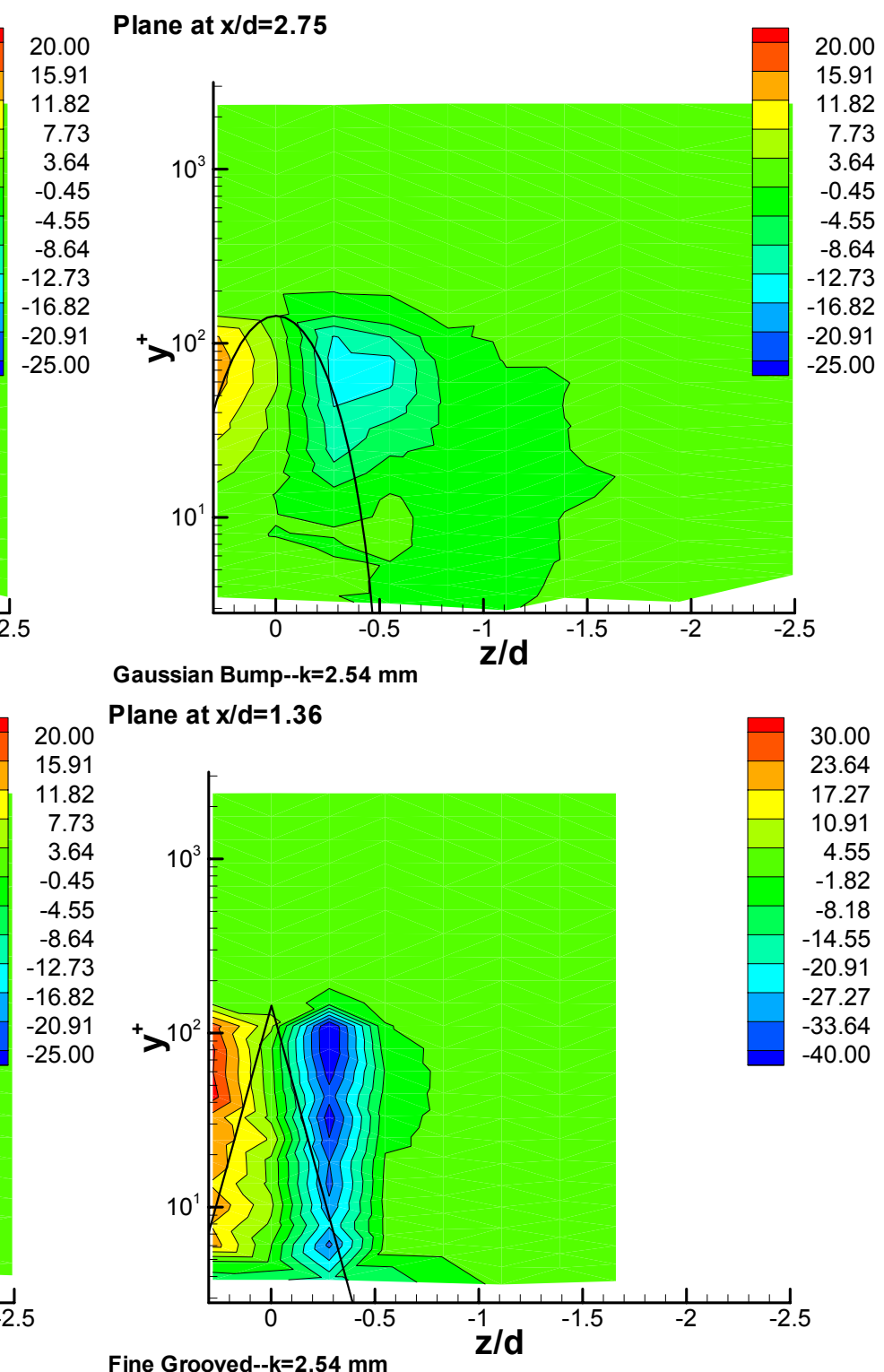

Large Grooved--k=2.54 mm

Figure 3.99: Contours of $\overline{w^{3}} / U_{\tau}^{3}$ in the $\mathbf{y}-z$ plane at a location of $\mathbf{x} / \mathbf{d}=\mathbf{2 . 7 5}$ (Note: Fine Grooved $\mathbf{x} / \mathbf{d}=1.36$ ) 


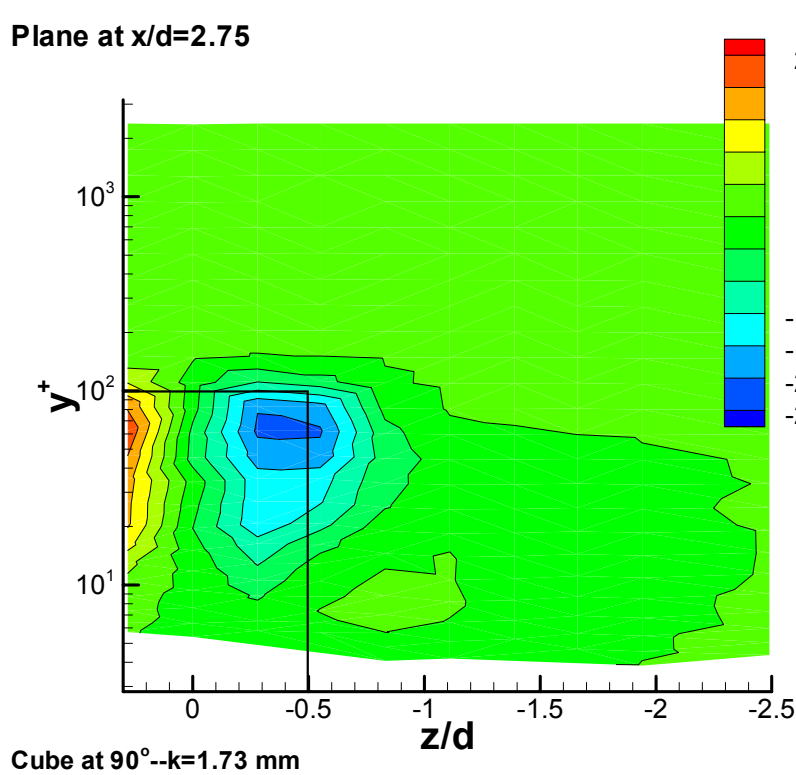

Cube at $90^{\circ}--\mathrm{k}=1.73 \mathrm{~mm}$

\section{Plane at $x / d=2.75$}

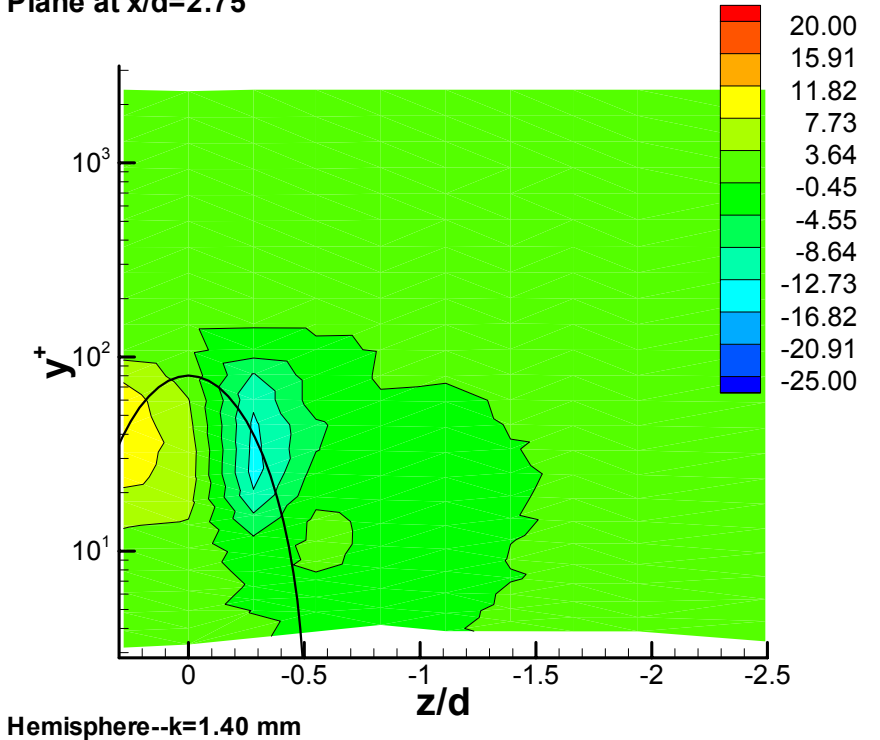

Hemisphere- $k=1.40 \mathrm{~mm}$

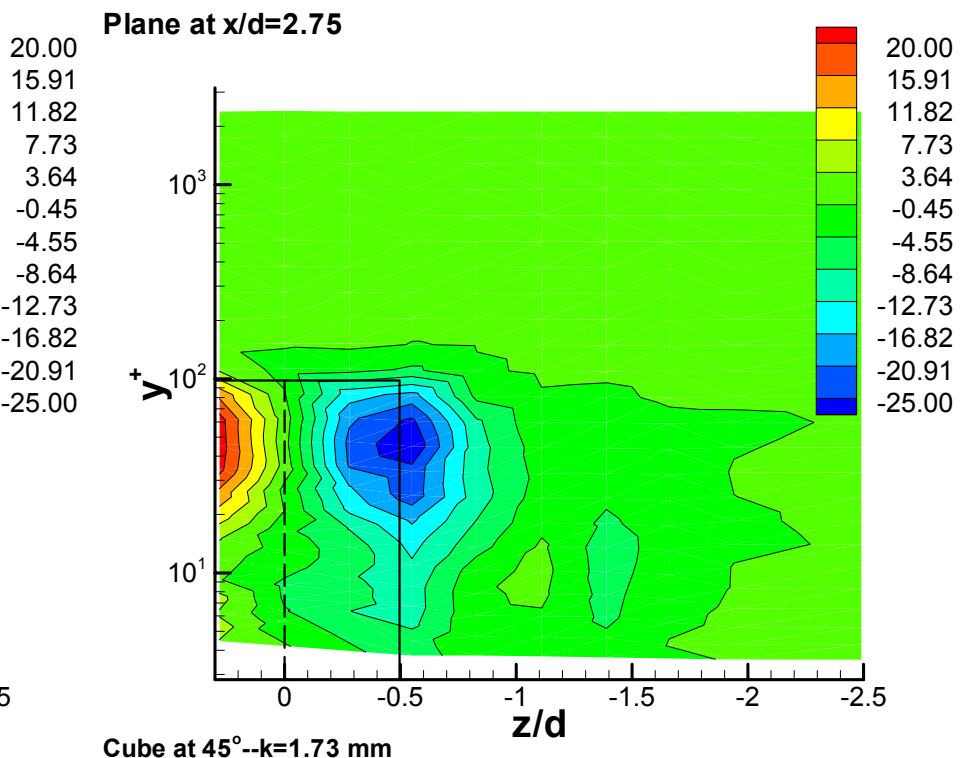

Cube at $45^{\circ}--\mathrm{k}=1.73 \mathrm{~mm}$

$$
\begin{array}{r}
20.00 \\
15.91 \\
11.82 \\
7.73 \\
3.64 \\
-0.45 \\
-4.55 \\
-8.64 \\
-12.73 \\
-16.82 \\
-20.91 \\
-25.00
\end{array}
$$

Figure 3.100: Contours of $\overline{w^{3}} / U_{\tau}^{3}$ in the $\mathbf{y}-\mathbf{z}$ plane at a location of $\mathbf{x} / \mathbf{d}=\mathbf{2 . 7 5}$ 


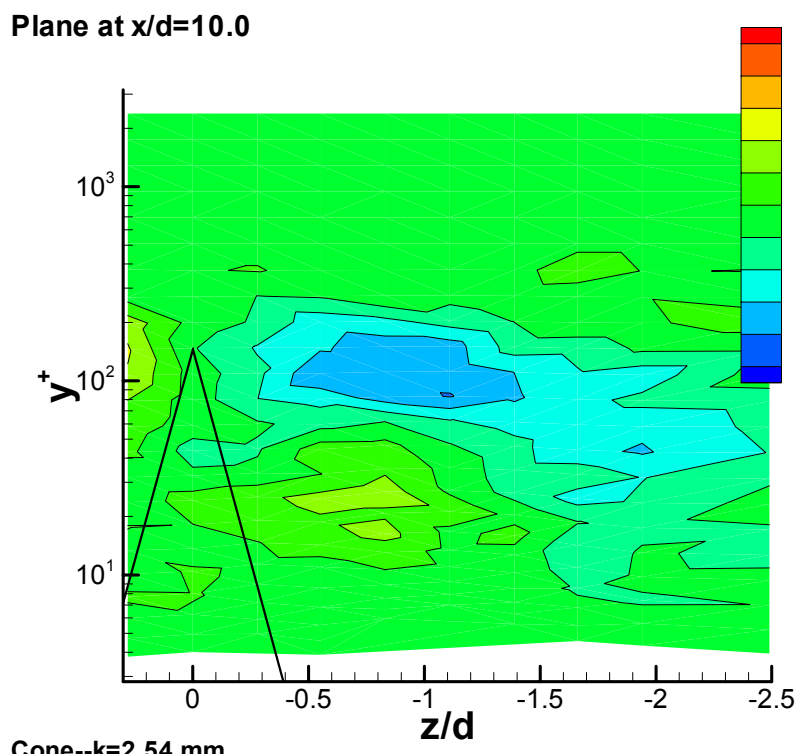

Plane at $x / d=10.0$

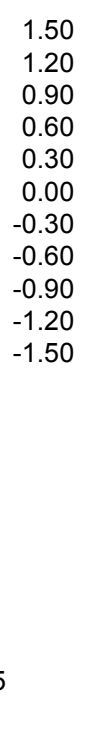

Cone--k=2.54 mm

Plane at $\mathrm{x} / \mathrm{d}=10.0$

Gaussian Bump--k=2.54 mm
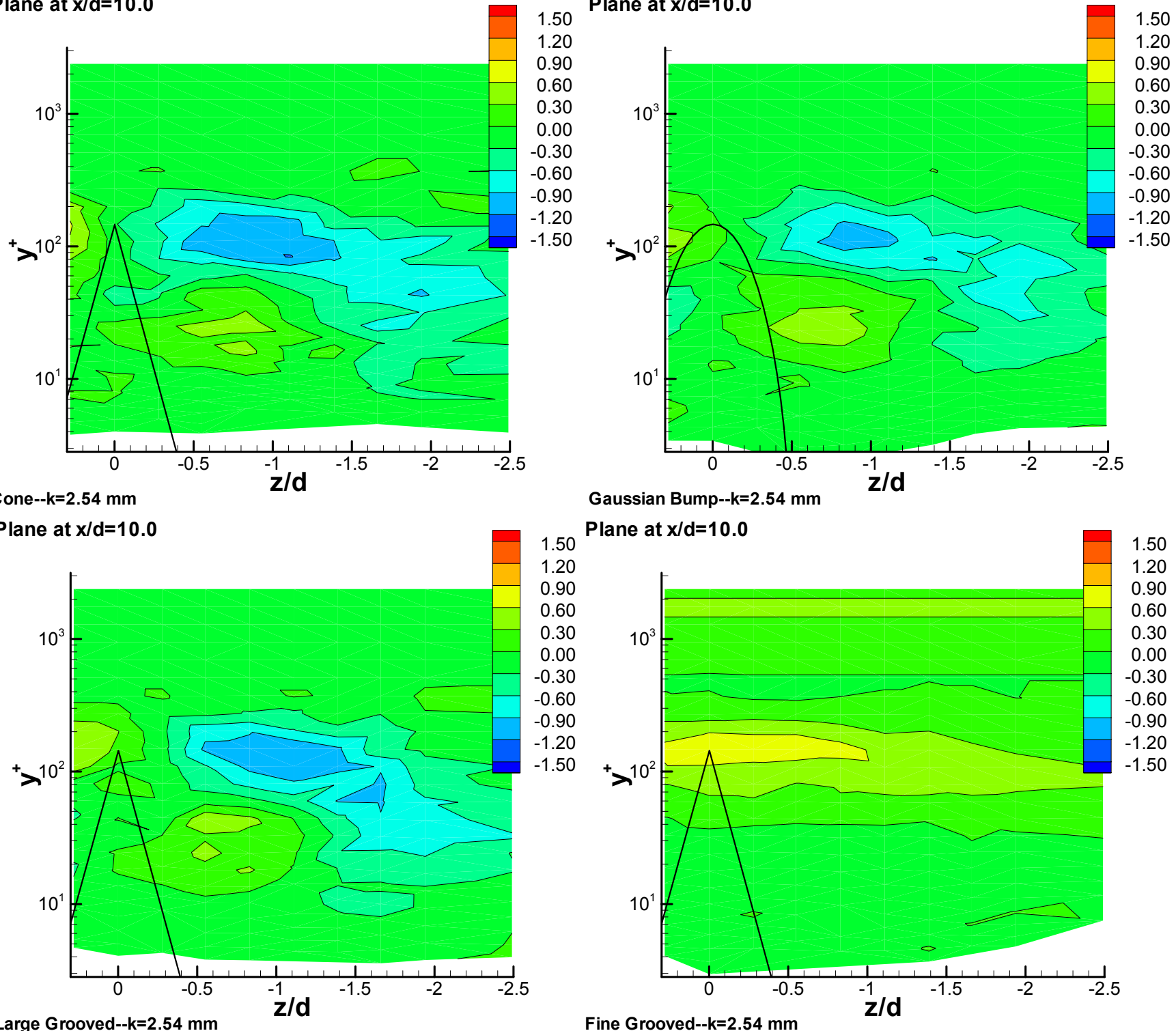

Large Grooved--k=2.54 mm

Fine Grooved--k=2.54 mm

Figure 3.101: Contours of $\overline{w^{3}} / U_{\tau}^{3}$ in the $\mathbf{y}-\mathbf{z}$ plane at a location of $\mathbf{x} / \mathbf{d}=10.0$ 


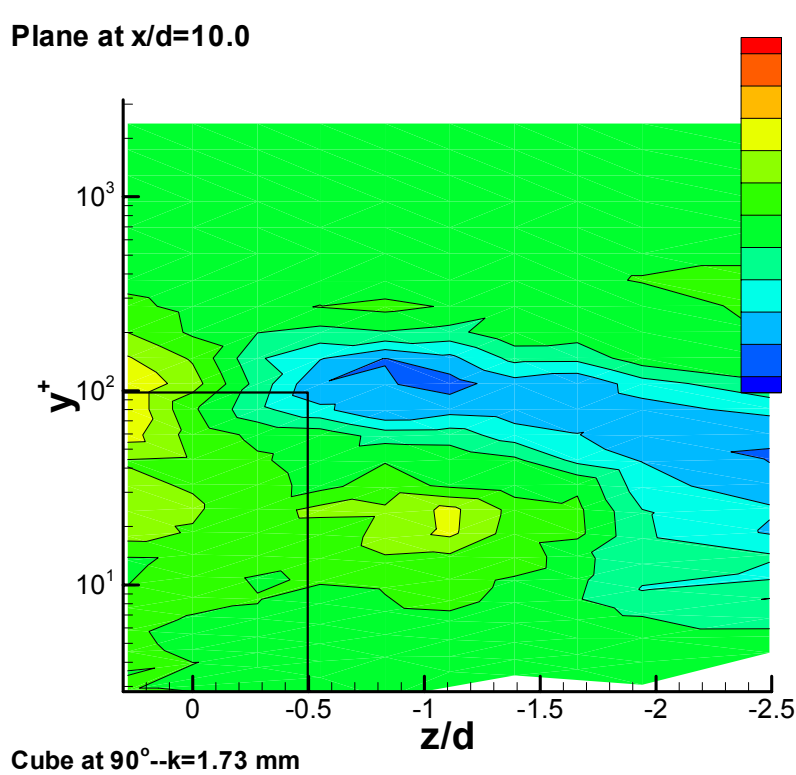

Cube at $90^{\circ}--\mathrm{k}=1.73 \mathrm{~mm}$

\section{Plane at $x / d=10.0$}

Plane at $x / d=10.0$

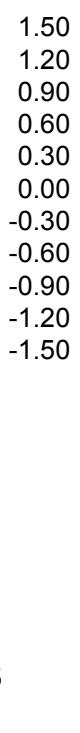

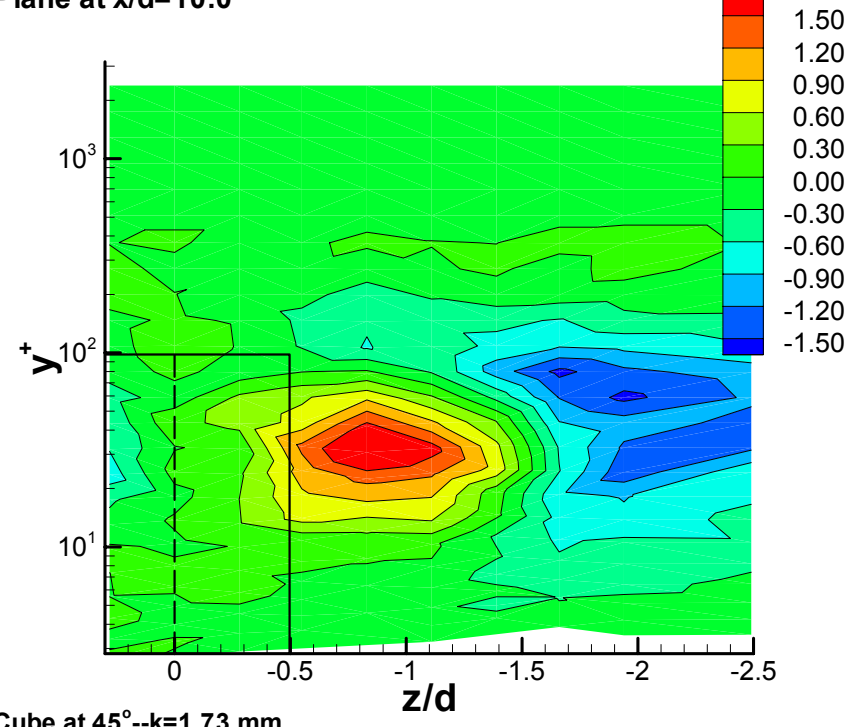

Cube at $45^{\circ}--\mathrm{k}=1.73 \mathrm{~mm}$

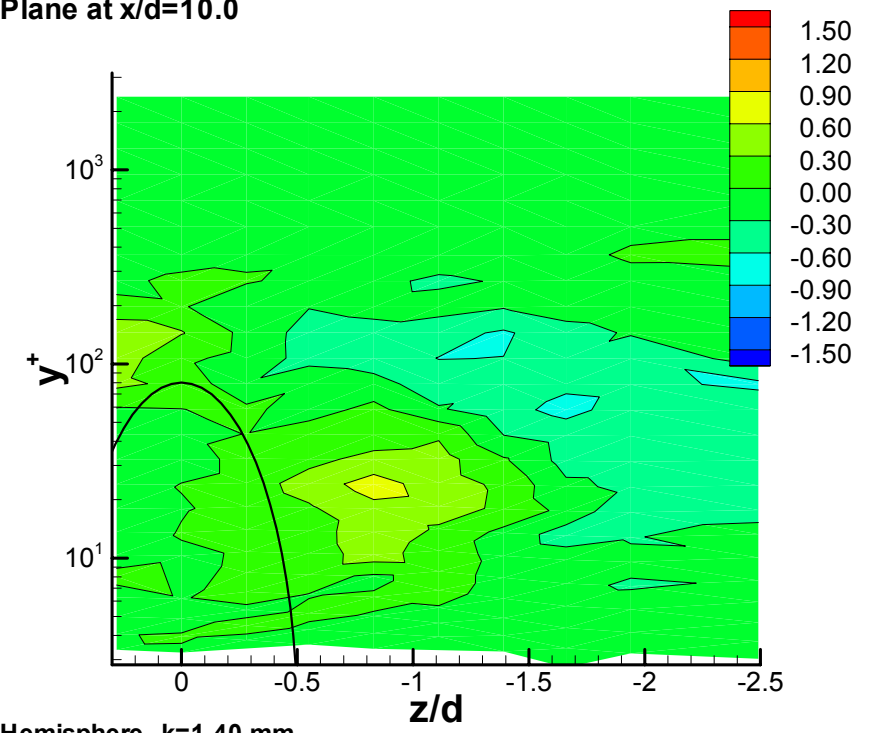

Hemisphere--k=1.40 $\mathrm{mm}$

Figure 3.102: Contours of $\overline{w^{3}} / U_{\tau}^{3}$ in the $\mathbf{y}-z$ plane at a location of $\mathbf{x} / \mathbf{d}=\mathbf{1 0 . 0}$ 


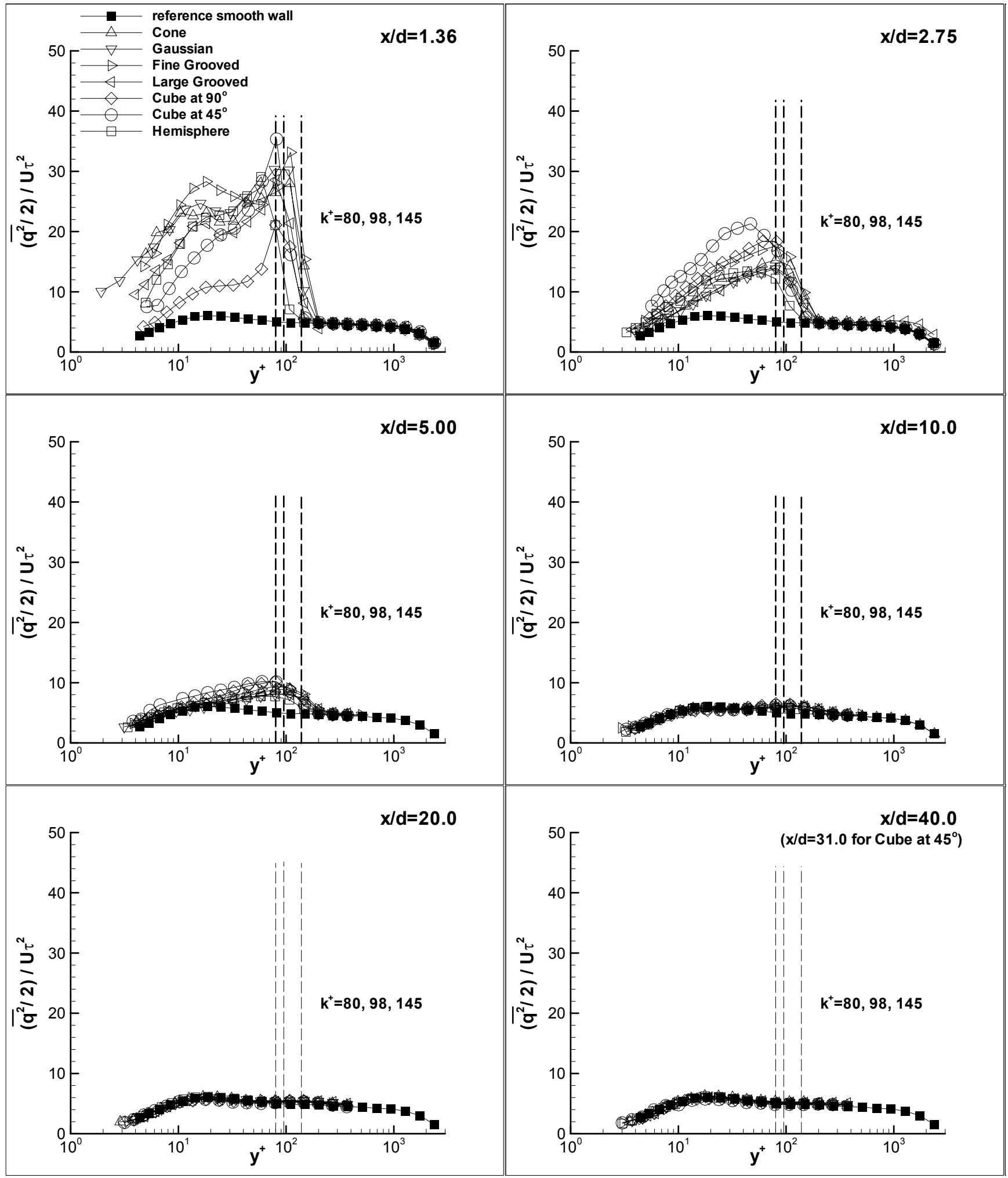

Figure 3.103: Turbulent Kinetic Energy (TKE), $\left[\left(\overline{q^{2}} / 2\right) / U_{\tau}^{2}\right\rfloor$ vs. $y U_{\tau} / v$ profiles along the centerline 

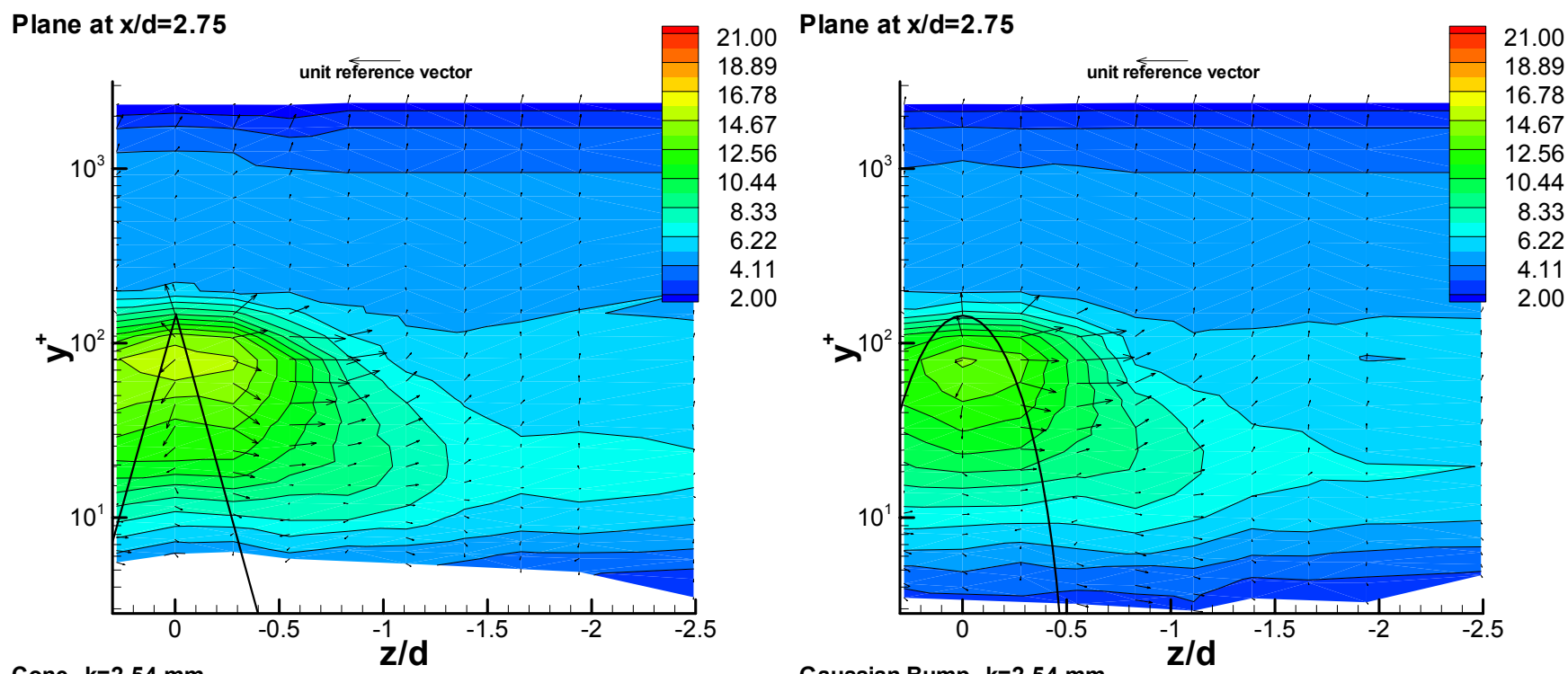

Cone--k=2.54 mm

Gaussian Bump--k=2.54 mm

Plane at $\mathrm{x} / \mathrm{d}=\mathbf{2 . 7 5}$

Plane at $x / d=1.36$
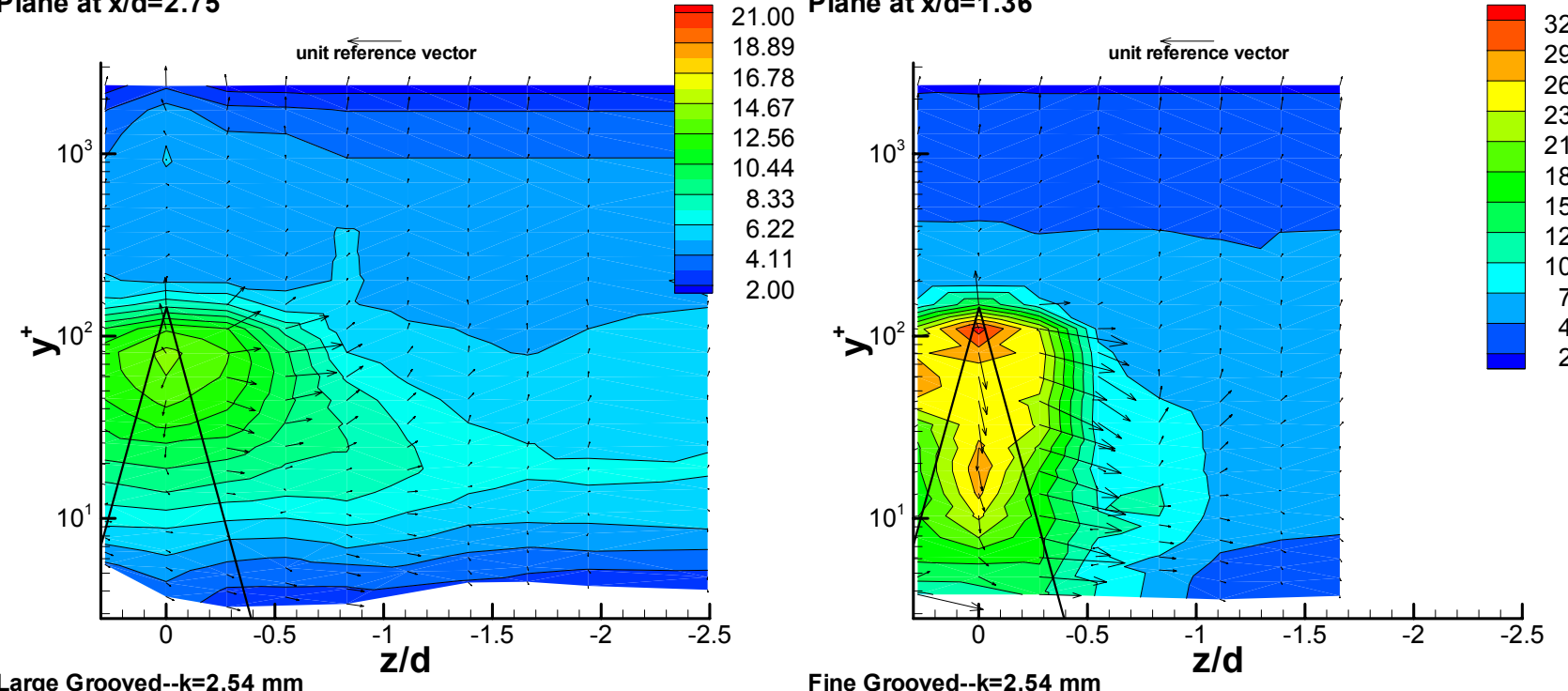

Large Grooved--k=2.54 mm

Fine Grooved--k=2.54 $\mathrm{mm}$

Figure 3.104: Contours of TKE $\left[\left(\overline{q^{2}} / 2\right) / U_{\tau}^{2}\right]$ with diffusion velocity vectors, $V_{q} / U_{\tau}=\left(\overline{v q^{2}} / \overline{q^{2}}\right) / U_{\tau}$ and $W_{q} / U_{\tau}=\left(\overline{w q^{2}} / \overline{q^{2}}\right) / U_{\tau}$, in the $y-z$ plane at a location of $x / d=2.75$ (Note: Fine Grooved $x / d=1.36$ ) 


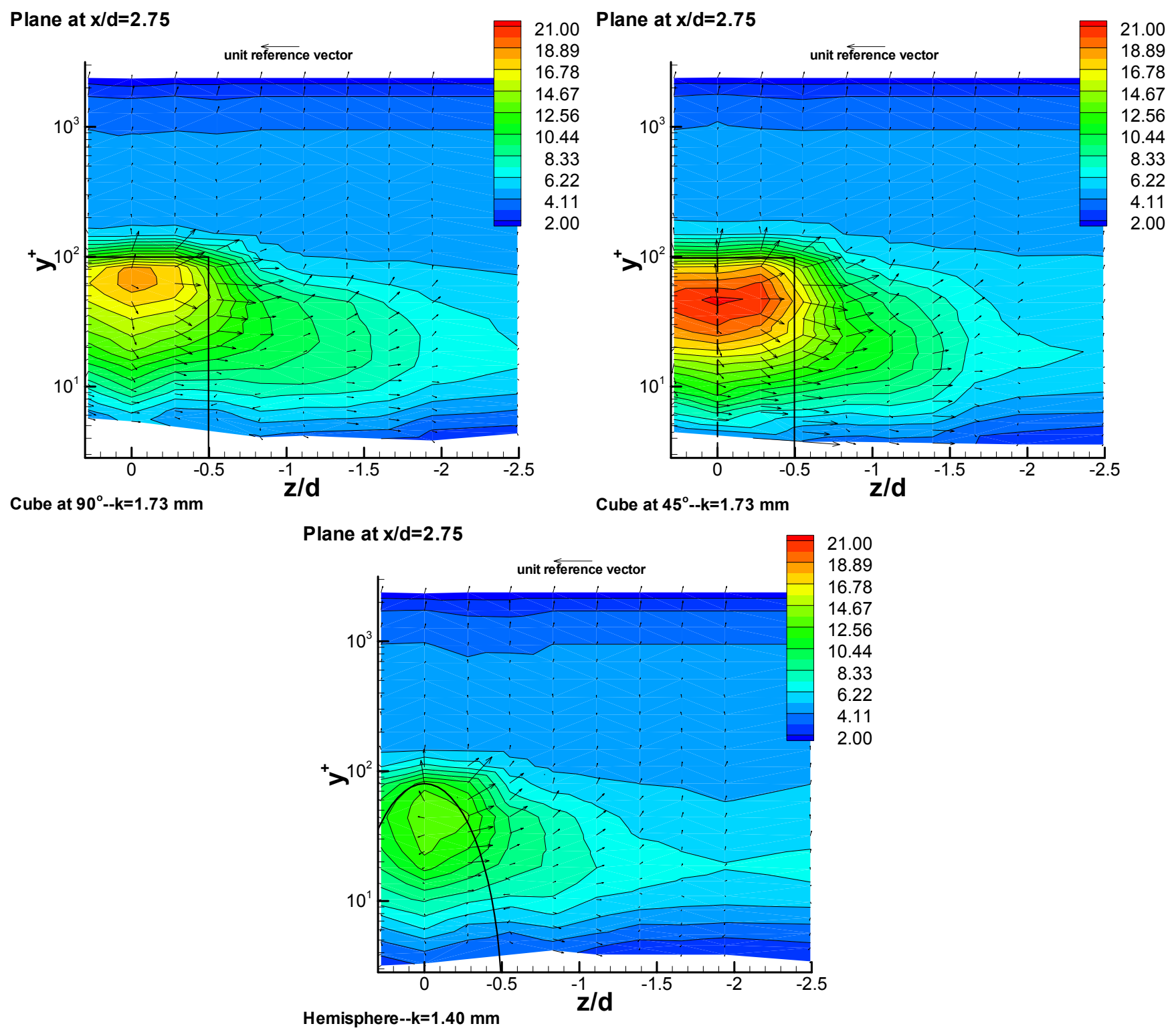

Figure 3.105: Contours of TKE $\left.\|\left(\overline{q^{2}} / 2\right) / U_{\tau}^{2}\right]$ with diffusion velocity vectors, $V_{q} / U_{\tau}=\left(\overline{v q^{2}} / \overline{q^{2}}\right) / U_{\tau}$ and $W_{q} / U_{\tau}=\left(\overline{w q^{2}} / \overline{q^{2}}\right) / U_{\tau}$, in the $y-z$ plane at a location of $x / d=2.75$ 

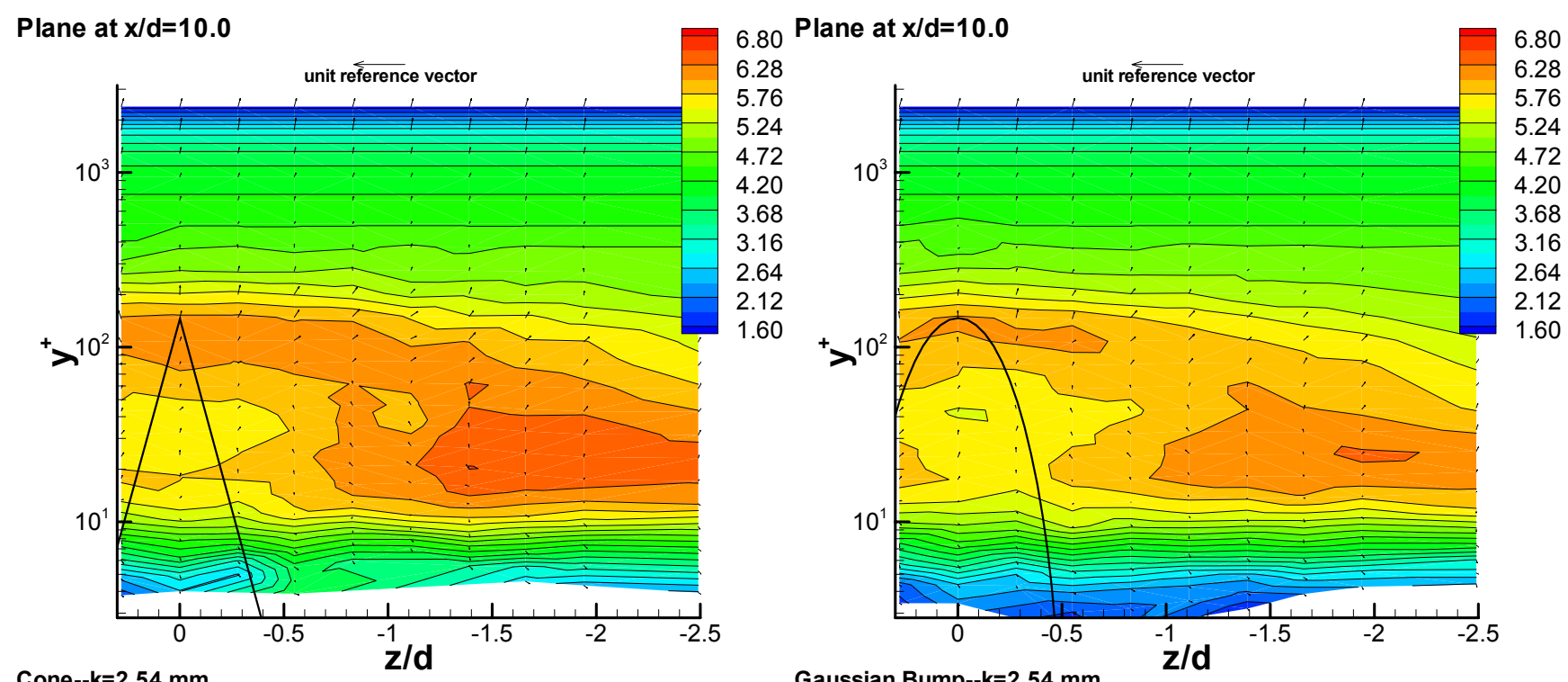

Plane at $\mathrm{x} / \mathrm{d}=10.0$

Gaussian Bump--k=2.54 mm
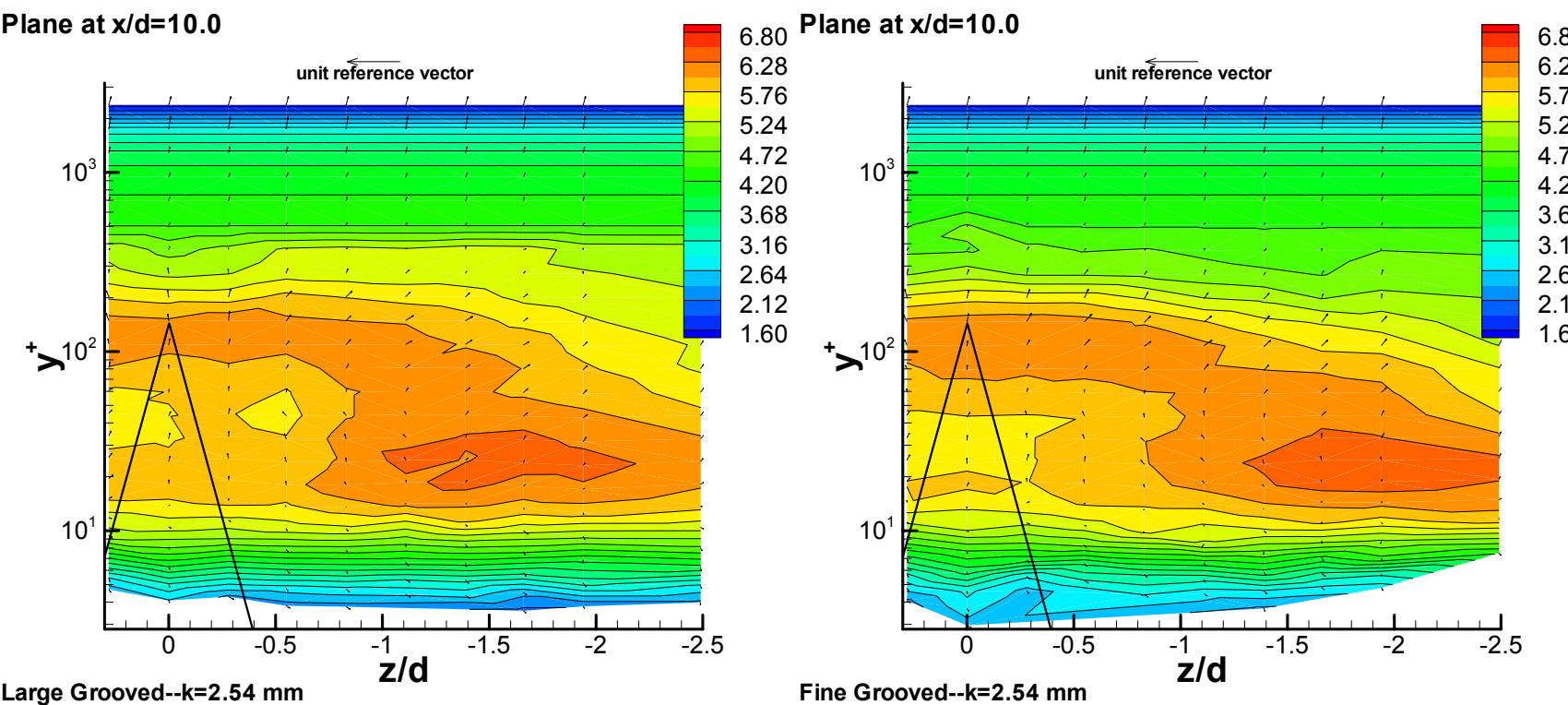

Large Grooved--k=2.54 mm

Fine Grooved--k=2.54 mm

Figure 3.106: Contours of TKE $\left[\left(\overline{q^{2}} / 2\right) / U_{\tau}^{2}\right]$ with diffusion velocity vectors, $V_{q} / U_{\tau}=\left(\overline{v q^{2}} / \overline{q^{2}}\right) / U_{\tau}$ and $W_{q} / U_{\tau}=\left(\overline{w q^{2}} / \overline{q^{2}}\right) / U_{\tau}$, in the $y-z$ plane at a location of $x / d=10.0$ 


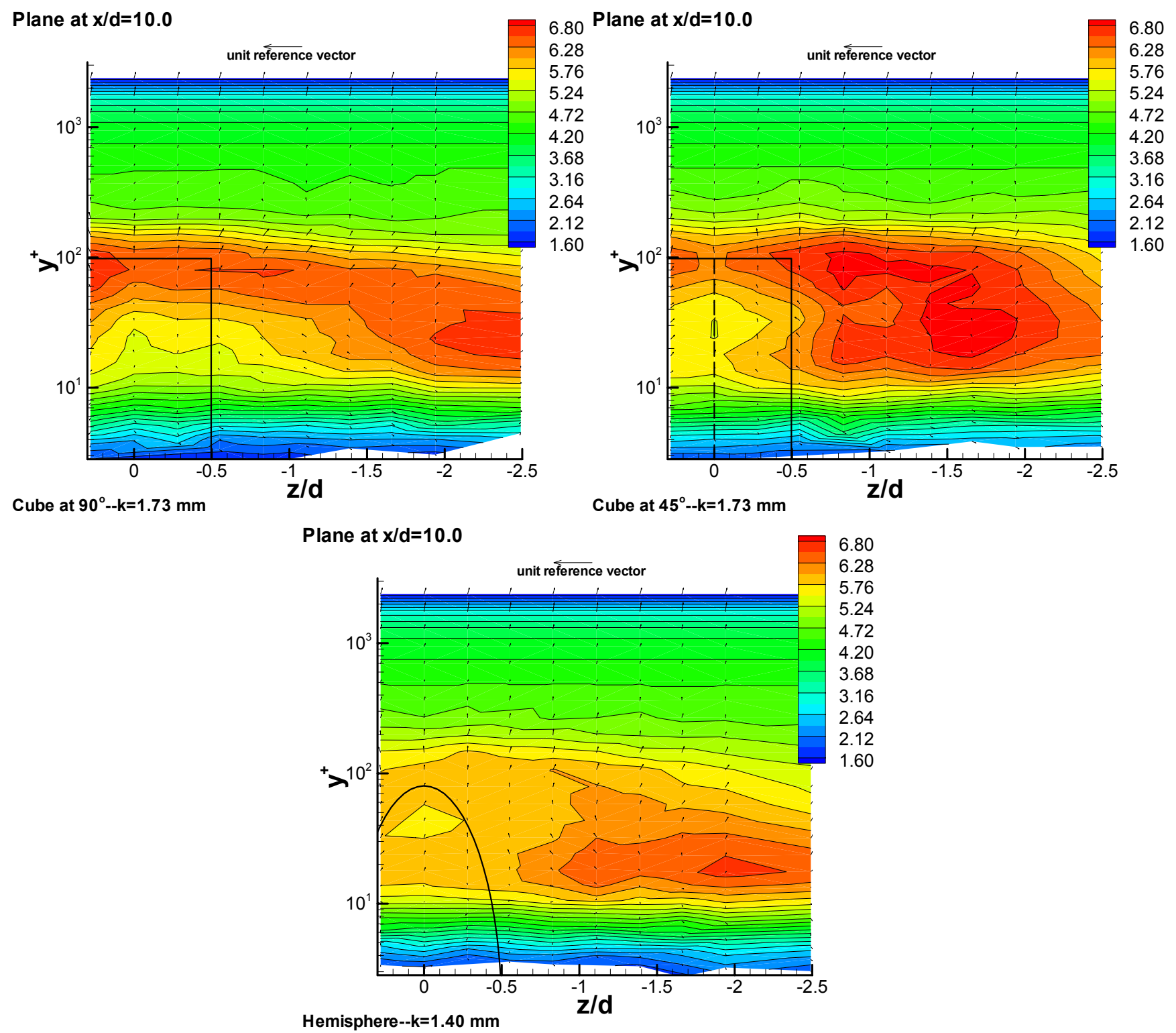

Figure 3.107: Contours of TKE $\left[\left(\overline{q^{2}} / 2\right) / U_{\tau}^{2}\right\rfloor$ with diffusion velocity vectors, $V_{q} / U_{\tau}=\left(\overline{v q^{2}} / \overline{q^{2}}\right) / U_{\tau}$ and $W_{q} / U_{\tau}=\left(\overline{w q^{2}} / \overline{q^{2}}\right) / U_{\tau}$, in the $y-z$ plane at a location of $x / d=10.0$ 


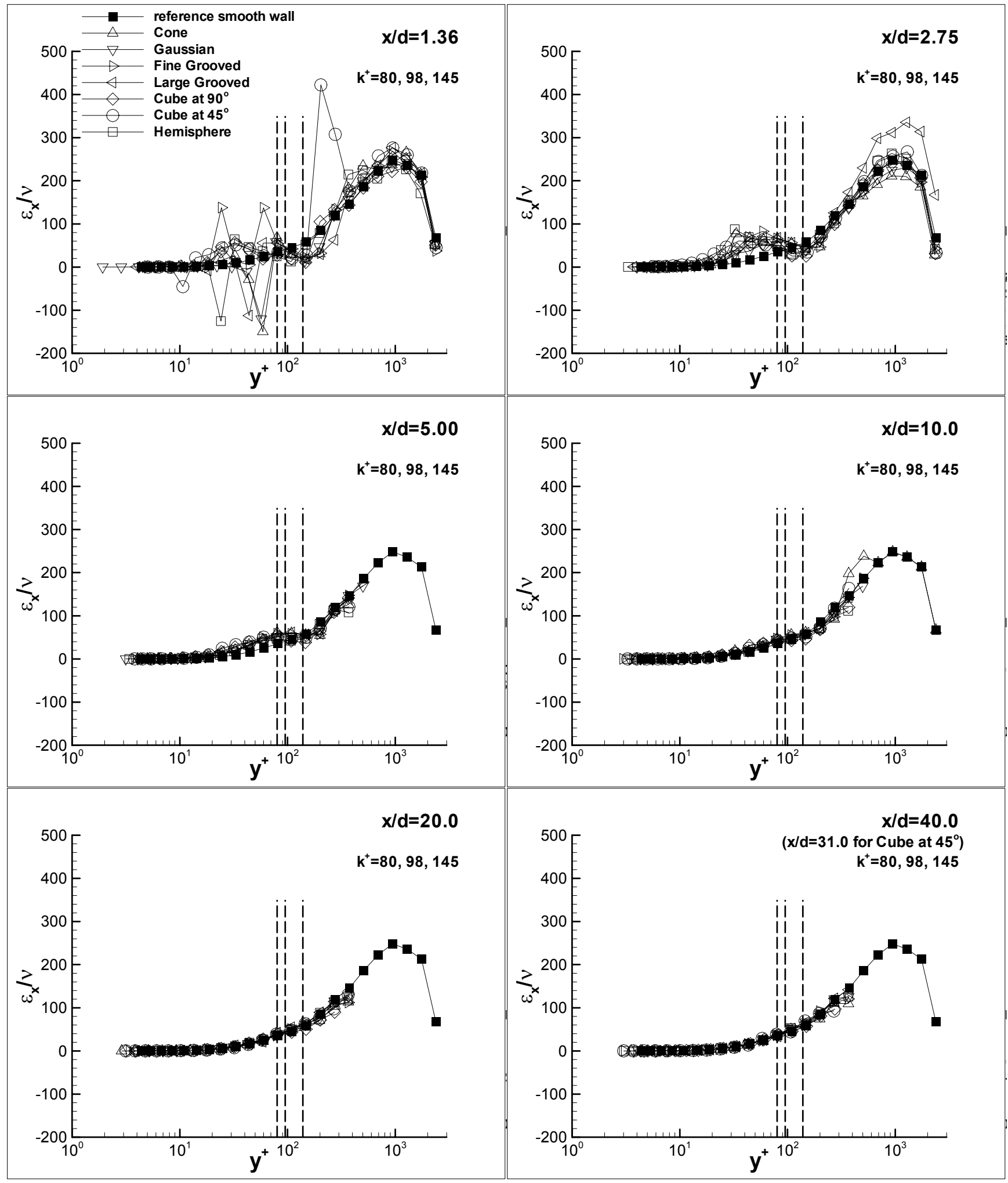

Figure 3.108: Centerline variation of normalized streamwise eddy viscosity, $\varepsilon_{x} / v$ vs. $y U_{\tau} / v$ 


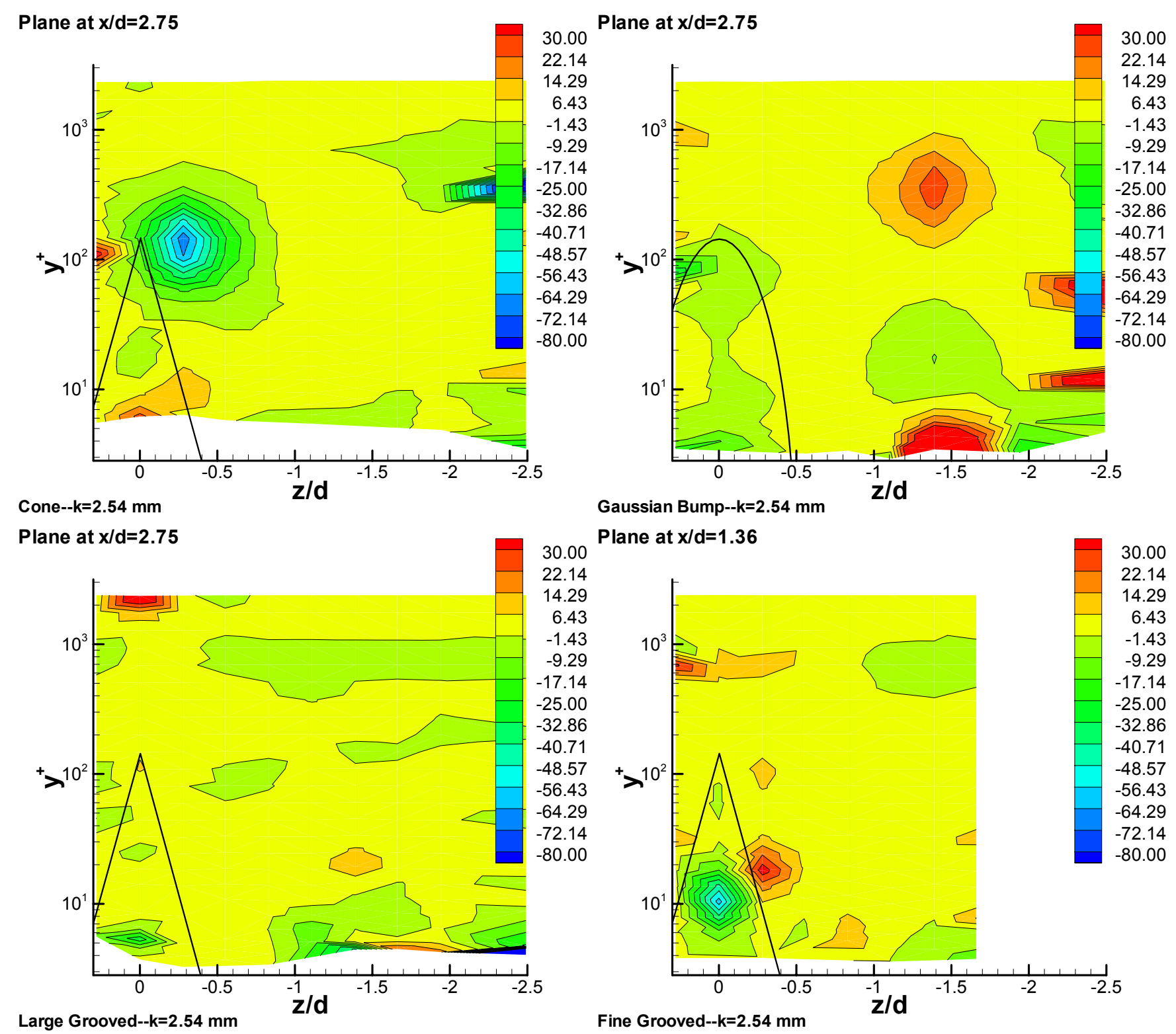

Figure 3.109: Contours of eddy viscosity ratio, $N_{\varepsilon}=\varepsilon_{z} / \varepsilon_{x}$, in the $\mathbf{y}-\mathbf{z}$ plane at $\mathbf{x} / \mathbf{d}=\mathbf{2 . 7 5}$ (Fine Grooved $\mathbf{x} / \mathbf{d}=\mathbf{1 . 3 6}$ ) 

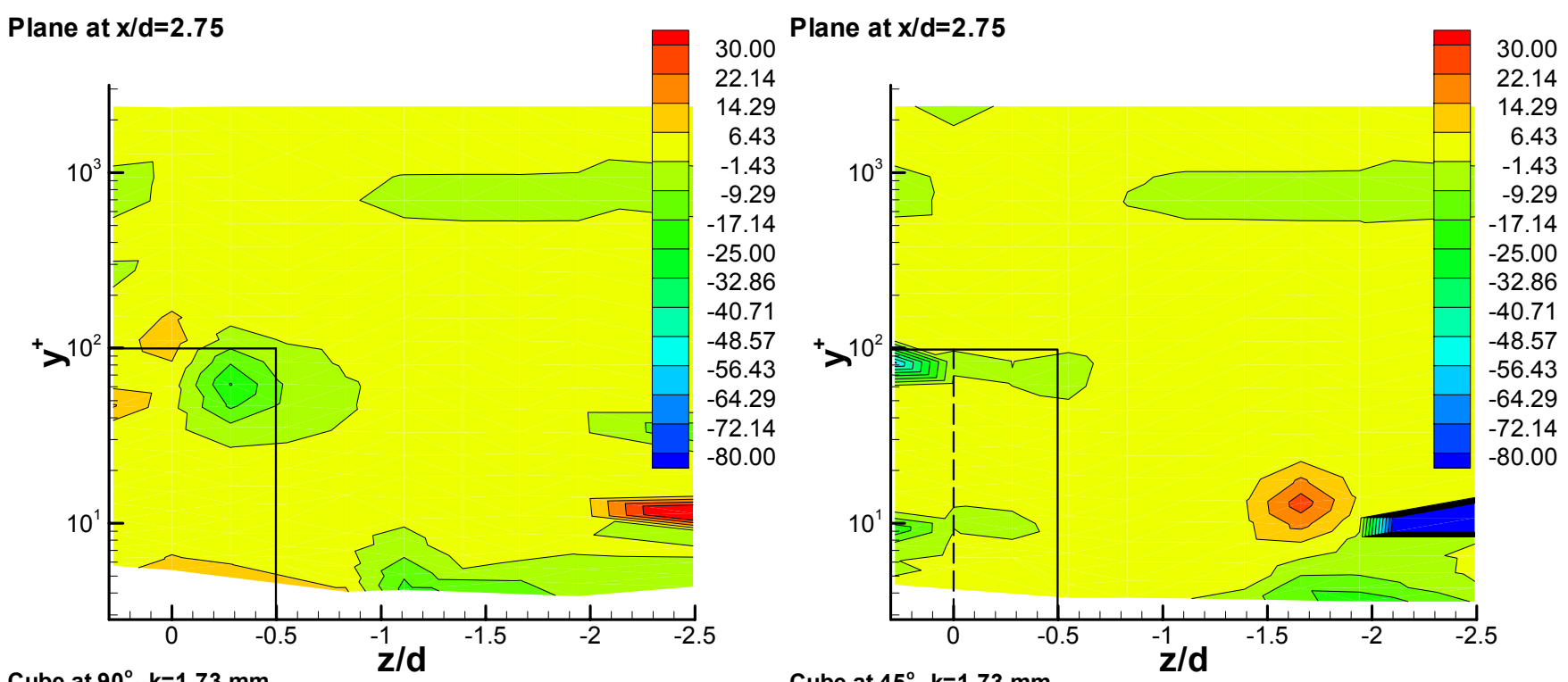

Cube at $90^{\circ}--\mathrm{k}=1.73 \mathrm{~mm}$

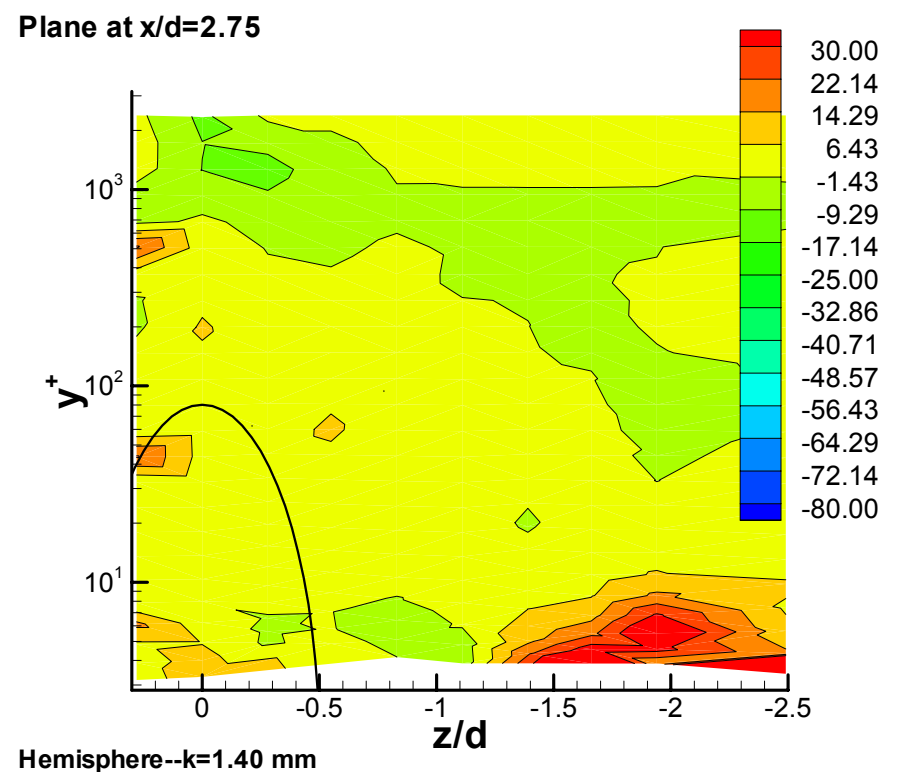

Figure 3.110: Contours of eddy viscosity ratio, $N_{\varepsilon}=\varepsilon_{z} / \varepsilon_{x}$, in the $\mathbf{y}-\mathbf{z}$ plane at $\mathbf{x} / \mathbf{d}=\mathbf{2 . 7 5}$ 


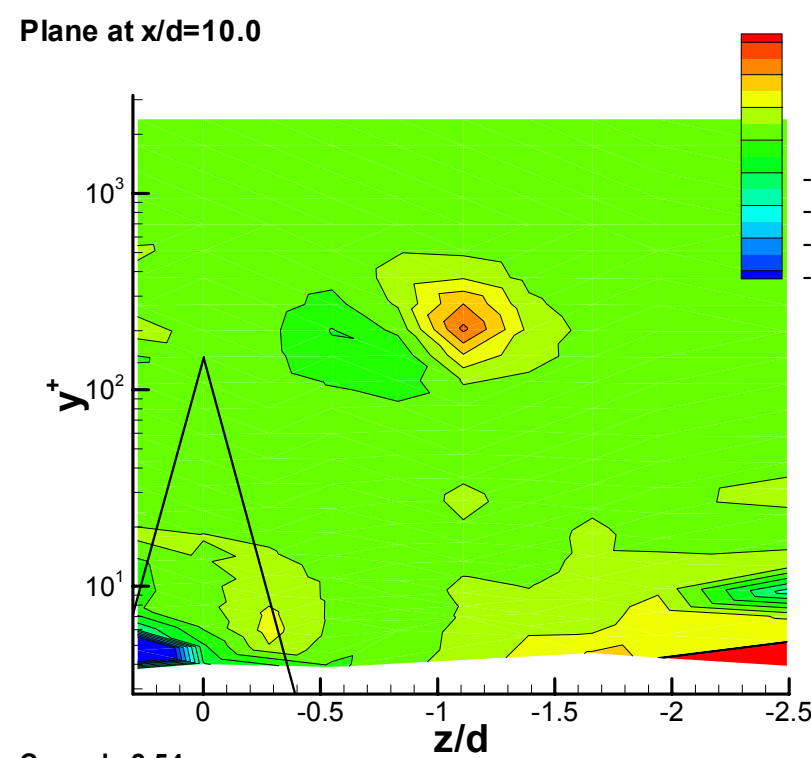

Cone--k=2.54 mm Plane at $x / d=10.0$

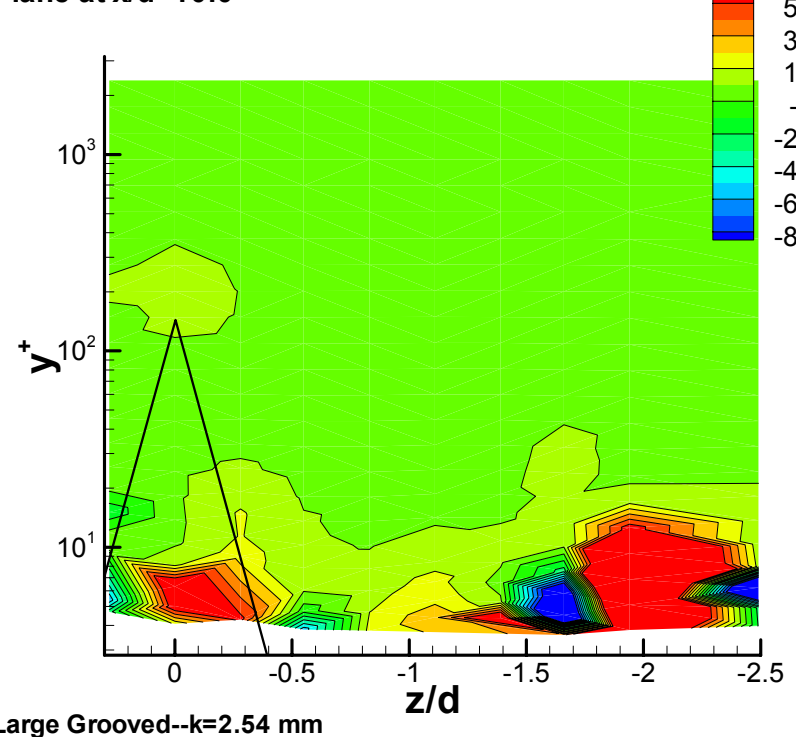

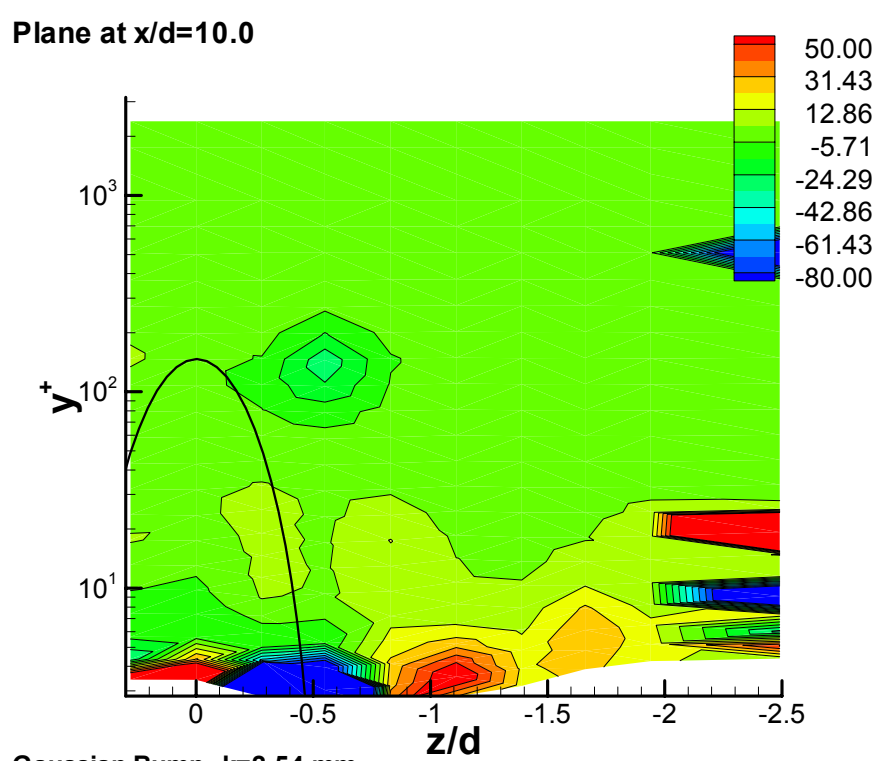

Gaussian Bump--k=2.54 mm

Plane at $x / d=10.0$

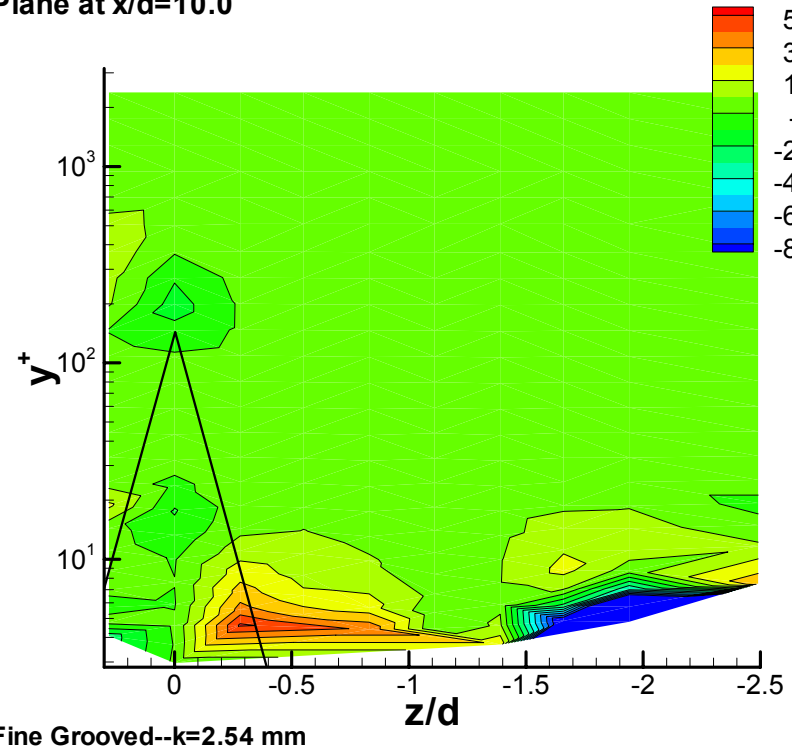

Figure 3.111: Contours of eddy viscosity ratio, $N_{\varepsilon}=\varepsilon_{z} / \varepsilon_{x}$, in the $\mathbf{y}-\mathbf{z}$ plane at $\mathbf{x} / \mathbf{d}=10.0$ 


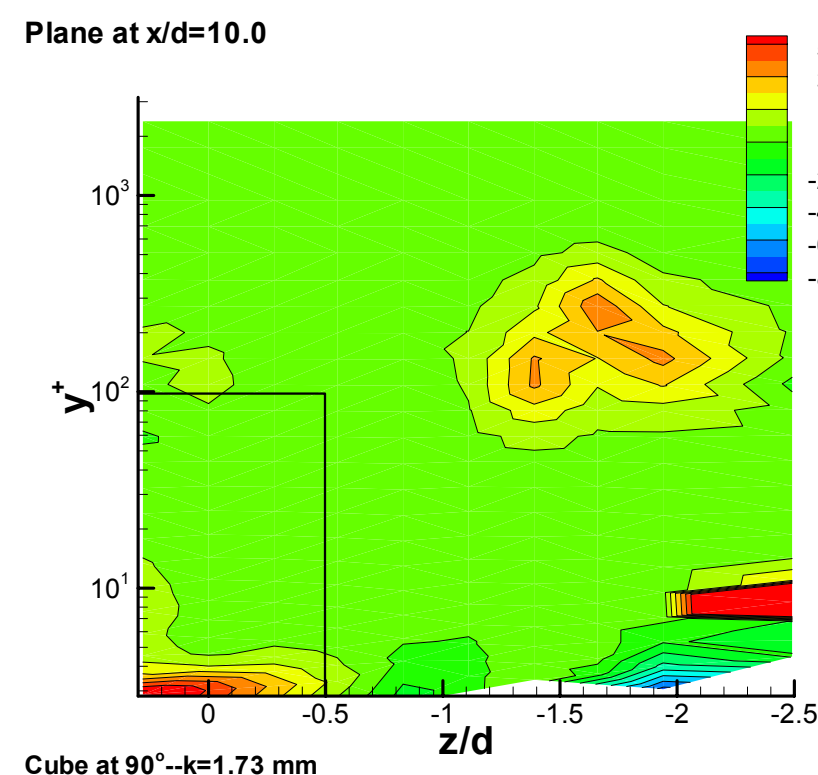

Cube at $90^{\circ}--\mathrm{k}=1.73 \mathrm{~mm}$
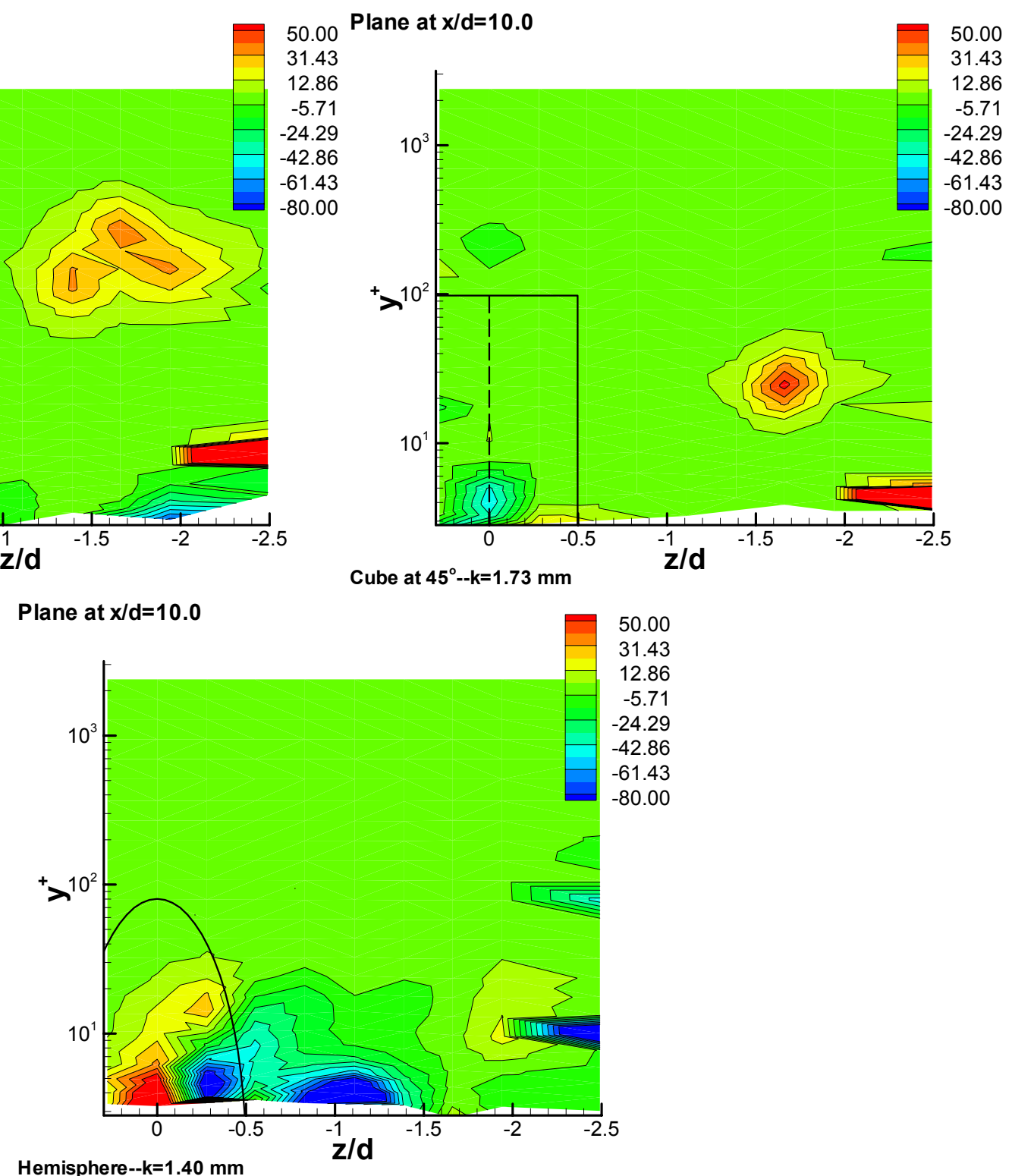

Figure 3.112: Contours of eddy viscosity ratio, $N_{\varepsilon}=\varepsilon_{z} / \varepsilon_{x}$, in the $\mathbf{y}-\mathbf{z}$ plane at $\mathbf{x} / \mathbf{d}=\mathbf{1 0 . 0}$ 


\subsection{Chapter 3 Figures: Gaussian Fetch}

Note:

A single profile at $\mathrm{x} / \mathrm{d}=1.08, \mathrm{z} / \mathrm{d}=0.0$ was also taken at a location eight (8) cells downstream of the shown configuration

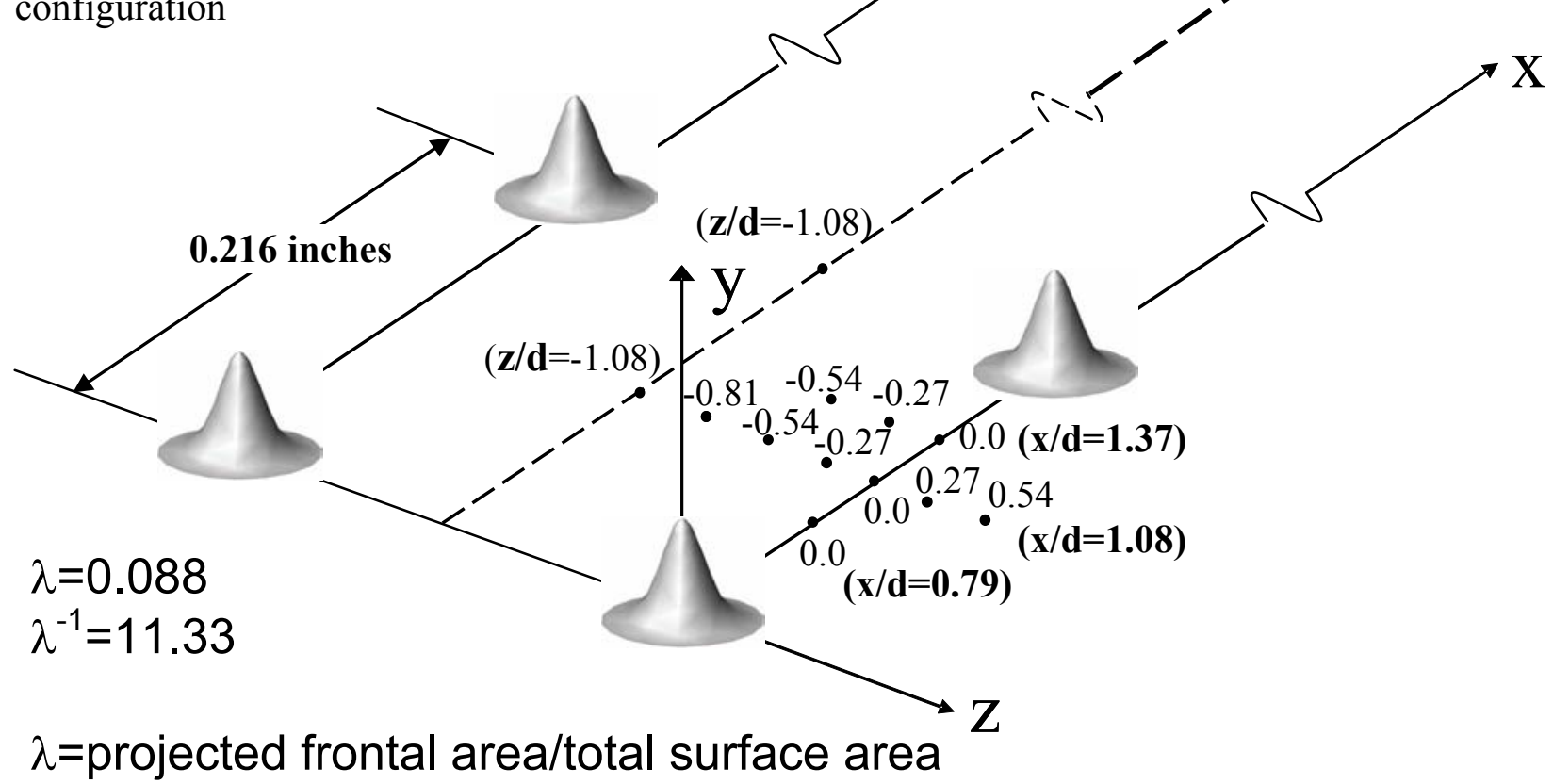

Figure 3.113: Measurement locations for the Gaussian fetch of roughness 


\section{Chapter 4 Discussion of Results}

\subsection{Introduction}

The purpose of this chapter is to unify the results from the previous chapters into a useful and applicable understanding of the experimental results. Schematics of the turbulent flow field behind each roughness element are presented and comparisons are made with respect to each element. Work associated with possible correlations between the drag of all elements is examined. An effort related to the implementation of a circulation correlation to connect an element's circulation to its respective 'bluntness factor' is also presented. The streamwise decay of circulation for each element is discussed along with possible relationships to all tested roughness elements. Through this analysis and discussion, a more complete understanding of the interrelation between each flow field and its associated physics will be brought to light. It is of significance to note that all experimental data acquired throughout this investigation meet the realizability conditions set forth by Schumann (1977). The requirements for a realizable data set as stated by Schumann, where $R_{\alpha \beta}=\overline{u_{\alpha} u_{\beta}}$ are the appropriate Reynolds stresses, are $\mathrm{R}_{\alpha \beta} \geq 0$ for $\alpha=$ $\beta,\left(R_{\alpha \beta}\right)^{2} \leq R_{\alpha \alpha} R_{\beta \beta}$ for $\alpha \neq \beta$, and the matrix $R_{\alpha \beta}$ be positive semi-definite. Meeting these requirements signifies that the data are realizable in the current coordinate system, as well as in any orthogonal coordinate system.

\subsection{Drag Correlations}

As was discussed briefly in Chapter 3, the drag for each element was calculated using a control volume and momentum balance approach. The differences between the undisturbed upstream velocity profile and the wake profiles at a downstream location of $\mathrm{x} / \mathrm{d}=2.75$ were taken into account throughout the investigation. Thus, the fine grooved element is the only element not analyzed due to its plane of measurements being taken at $\mathrm{x} / \mathrm{d}=1.36$. The derivation of the drag equation and related discussion can be found in Appendix B. Equation 4.1 below was used to calculate the drag increment due to the presence of a roughness element and is taken from Equation (B.11) in Appendix B. 
$2 \Delta D_{E_{-} \text {incremental }}=2 \rho \int_{0}^{y_{\text {top }}-z_{2}} \int_{0}^{(}\left(\left.U_{2}^{2}\right|_{w / o E}-\left.U_{2}^{2}\right|_{E}\right)+\left(\left.\overline{u_{2}^{2}}\right|_{w / o E}-\left.\overline{u_{2}^{2}}\right|_{E}\right)-U_{\text {top }}\left(\left.U_{2}\right|_{w / o E}-\left.U_{2}\right|_{E}\right) d z d y$

The incremental drag consists of the drag due to the element's presence in the boundary layer as well as the skin friction drag created by the surface area of the floor contained within the control volume. The control volume used in the derivation of Equation 4.1 assumes that the contribution of the pressure forces on the control volume surface to be negligible. This assumption stems from the work of Martinuzzi and Tropea (1993) who show a pressure recovery at a downstream distance of $\mathrm{x} / \mathrm{d} \approx 2.25$ for a surface mounted cube. Thus, due to the roughness element geometries under investigation, all elements should experience full pressure recovery by $\mathrm{x} / \mathrm{d}=2.75$. It is also of significance to be aware that the analysis used to calculate the drag also includes the stream-wise Reynolds normal stress, $\overline{u^{2}}$, term. Consequently, this approach is more rigorous than a method which only takes into account the mean stream-wise velocity. The addition of the fluctuating term will cause a slight decrease in the calculated drag as compared to the drag calculated using only the mean velocity. This is due to the fact that some mean flow momentum is lost when turbulence is created. As a result, the total momentum loss is due to the drag on the element as well as the production of turbulence caused by the element's presence in the boundary layer.

In order to obtain the drag due only to the element's presence within the boundary layer one must take into account the drag due to the floor surface area of the control volume. To this end, it is necessary to subtract off the drag due to the floor without the element being present. There are a few critical factors to keep in mind when performing this part of the analysis. First, it is imperative that knowledge of the wake decay in the spanwise direction be known. The edge of the control volume is determined to be the location where the wake region has decayed to the two-dimensional smooth wall conditions. If this location is not considered, it is quite possible that too large or too small a quantity will be subtracted from the incremental drag calculation. The other important aspect is that the separated flow in the wake region directly behind the roughness element will create a drastically different value of wall shear as compared to the regions farther away from the centerline of the element and the reference smooth wall. In order to take this 
phenomenon into account, the area of the wake region directly behind the roughness element is approximated by a triangular section extending from the base of the roughness element to the $x / d=2.75$ measurement plane. Due to the fact that wall shear data within this region is extremely difficult to obtain, a reasonable approach would be to assume a linear trend in the wall shear value from the downstream edge of the roughness element to the $\mathrm{x} / \mathrm{d}=2.75$ measurement plane. An average wall shear value within this range would then be used to find the drag due to the floor in this triangular section with the roughness element being present. This area is in turn subtracted from the entire area of the control volume so as not to be accounted for twice. The drag on each separate area of the floor is then calculated and subtracted from the incremental drag result. Equation 4.2 below was used to obtain the drag due only to the element, $\Delta \mathrm{D}_{\mathrm{E} \_ \text {actual, }}$

$$
\Delta D_{E_{-} \text {actual }}=\Delta D_{E_{-} \text {incremental }}-[\underbrace{C_{f_{-} \text {outer }}\left(\frac{1}{2} \rho U_{e}^{2}\right) A_{\text {proj_outer }}}_{A}+\underbrace{C_{f_{-} \text {inner }}\left(\frac{1}{2} \rho U_{e}^{2}\right) A_{\text {proj_inner }}}_{B}]
$$

where $\mathrm{C}_{\mathrm{f}_{-} \text {outer }}$ and $\mathrm{C}_{\mathrm{f}_{-} \text {inner }}$ are the skin friction coefficients in the far spanwise wake and near element centerline wake regions within the control volume, respectively. Here $\mathrm{C}_{\mathrm{f}_{-} \text {outer }}$ is taken to be the reference smooth wall value and $\mathrm{C}_{\mathrm{f}_{-} \text {inner }}$ is found using the averaging method previously discussed. Similarly, $\mathrm{A}_{\text {proj_outer }}$ and $\mathrm{A}_{\text {proj_inner }}$ are the projected floor surface areas of the respective wake regions. Table 4.1 below gives the contributions of the various terms used to calculate the actual drag on each roughness element as well as the $\bar{q}$ and $\mathrm{C}_{\mathrm{WwA}}$ terms in the model equation used to correlate the experimental drag data. A perturbation analysis was employed to calculate the uncertainty associated with $\Delta \mathrm{D}_{\mathrm{E}_{-} \text {incremental. }}$. The uncertainty in $\Delta \mathrm{D}_{\mathrm{E}_{-} \text {incremental }}$ was found to be $\pm 6 \%$. Discussion related to this uncertainty analysis can be found in Appendix C. 
Table 4.1: Contributions of terms to related drag equations

\begin{tabular}{|c|c|c|c|c|c|c|c|}
\hline Element & $\begin{array}{c}\Delta \mathrm{D}_{\mathrm{E} \text { incremental }} \\
(\mathrm{N})\end{array}$ & $\begin{array}{c}\mathrm{A}_{\text {proj inner }} \\
\left(\mathrm{m}^{2}\right)\end{array}$ & $\begin{array}{c}\mathrm{A}_{\text {proj outer }} \\
\left(\mathrm{m}^{2}\right)\end{array}$ & $\begin{array}{c}\text { Term A } \\
(\text { Eqn. 4.2) }\end{array}$ & $\begin{array}{c}\text { Term B } \\
(\text { Eqn. 4.2) }\end{array}$ & $\bar{q}$ & $\mathrm{C}_{\text {WwA }}$ \\
\hline Cone & $2.66 \mathrm{E}-04$ & $1.69 \mathrm{E}-05$ & $8.76 \mathrm{E}-05$ & $8.74 \mathrm{E}-05$ & $9.12 \mathrm{E}-06$ & 124.19 & 0.60 \\
\hline Gaussian & $1.96 \mathrm{E}-04$ & $1.51 \mathrm{E}-05$ & $6.63 \mathrm{E}-05$ & $6.62 \mathrm{E}-05$ & $8.16 \mathrm{E}-06$ & 124.25 & 0.60 \\
\hline Large Grooved & $3.32 \mathrm{E}-04$ & $2.47 \mathrm{E}-05$ & $1.21 \mathrm{E}-04$ & $1.20 \mathrm{E}-04$ & $1.34 \mathrm{E}-05$ & 129.79 & 0.55 \\
\hline Cube at $90^{\circ}$ & $3.02 \mathrm{E}-04$ & $1.23 \mathrm{E}-05$ & $4.59 \mathrm{E}-05$ & $4.58 \mathrm{E}-05$ & $3.85 \mathrm{E}-06$ & 128.61 & N/A \\
\hline Cube at $45^{\circ}$ & $3.13 \mathrm{E}-04$ & $2.46 \mathrm{E}-05$ & $9.17 \mathrm{E}-05$ & $9.16 \mathrm{E}-05$ & $7.79 \mathrm{E}-06$ & 128.61 & N/A \\
\hline Hemisphere & $2.02 \mathrm{E}-04$ & $2.68 \mathrm{E}-05$ & $7.16 \mathrm{E}-05$ & $7.15 \mathrm{E}-05$ & $1.47 \mathrm{E}-05$ & 114.35 & 0.50 \\
\hline
\end{tabular}

In order to obtain a more complete understanding of the effects that each roughness element presents to a turbulent boundary layer flow, efforts have been made in devising a scheme that will correlate the calculated drag data from all of the elements. This scheme takes into account the form (pressure) drag related to the frontal area of each roughness element as well as the viscous (friction) drag which corresponds to the windward wetted surface area of each element. This scheme is not designed to be an a priori drag calculation method or model due the fact that, as discussed earlier, knowledge of the wake region is necessary in order to obtain the actual drag due to each element. Equation 4.3 presents the model equation utilized to correlate the drag results where $C_{d p}$ and $C_{d v}$ are coefficients acquired via a least squares fit.

$$
\Delta D_{E_{-} \text {calculated }}=\underbrace{C_{d p} \bar{q} A_{f}}_{1}+\underbrace{C_{d \nu} C_{W W A} \frac{\rho U_{\tau}}{12} \int_{0}^{k} U(y) E(y) f d y}_{2}
$$

Term 1 takes into account the pressure form drag on the element where $\bar{q}$ is the stagnation pressure averaged over the frontal area, $\mathrm{A}_{\mathrm{f}}$, of the roughness element. The $\bar{q}$ term is taken from previous studies done by Wieghardt (1946) and is defined as,

$$
\bar{q}=\frac{1}{A_{f}} \int_{0}^{k} \frac{\rho}{2}\left[\left(U^{2}+\overline{u^{2}}\right) E(y)\right] d y
$$

where, $\left(U^{2}+\overline{u^{2}}\right)$ is the approach streamwise two-dimensional mean-square velocity profile, and $\mathrm{E}(\mathrm{y})$ is the $\mathrm{y}$-variation of the frontal projected width of the roughness element. The $\mathrm{E}(\mathrm{y})$ function is known for each element and is integrated over the 
roughness height, $\mathrm{k}$, in order to account for the projected frontal area of the roughness element.

Term 2 in Equation 4.3 considers the viscous contribution to the total drag. A viscous stress is taken to be of the form $\mu \Delta U / \Delta n$ where $\Delta \mathrm{n}$ in this instance is an incremental distance. Furthermore, if the smallest scale spanwise structures for a smooth wall are taken into account, the following relationship can be obtained $\Delta U / \Delta n=U(y) /\left(12 v / U_{\tau}\right)$. Rearrangement of this relation leads to the following, $\mu \Delta U / \Delta n=\rho U(y) U_{\tau} / 12$, which is what is present in Term 2 of Equation 4.3. The $E(y) f d y$ factor in Term 2 is the variation in the y-direction of the wetted area. In order to obtain the wetted surface area of each axisymmetric element it is necessary to integrate the variation of the incremental circumference over the roughness height $(\mathrm{k})$. Term 2 for the axisymmetric elements would then be $\underbrace{C_{d V} C_{W W A} \frac{\rho U_{\tau} \pi}{12} \int_{0}^{k} U(y) E(y) f d y}_{\text {Term_2 _axisymmetric }}$, where $\mathrm{C}_{\mathrm{WWA}}$ is a coefficient that is associated with the fraction of total surface area that sees attached flow, i.e. the windward wetted area. Here, $f$ is a dimensionless function particular to each roughness element's incremental variation in the length of its surface as a function of y. For example, the cone would have a function equal to $f_{\text {cone }}=\sqrt{1+\left(d_{\text {cone }} /\left(2 k_{\text {cone }}\right)^{2}\right)}$ in order to obtain the incremental variation in its surface area. To determine the values of $\mathrm{C}_{\mathrm{WWA}}$, it is necessary to know the separation locations on the surface of the axisymmetric elements. The extremely small size of the roughness elements under scrutiny make it very hard, if not impossible, to obtain these exact separation locations. Consequently, it is necessary to glean information from previous studies.

No previous study has been performed on roughness elements having comparative size and shape characteristics to the ones under current investigation. An investigation was done by Okamoto et al. (1977) who performed experiments on surface mounted cones with varying vertex angles $(\alpha)$. These experiments investigate surface pressure distributions, wake properties and separation lines associated with each cone. 
Experiments were carried out on a similarly shaped cone as in the current research. The Okamoto investigation examined a cone with a vertex angle $2 \alpha=60^{\circ}$ whereas the current research considers a cone with $2 \alpha=53.1^{\circ}$. The $60^{\circ}$ vertex angle cone had a height of $86.43 \mathrm{~mm}$ and was placed in a $7.6 \mathrm{~mm}$ thick turbulent boundary layer. Although the parameters in the Okamoto investigation are not the same as the current setup, which has a $2.54 \mathrm{~mm}$ tall cone placed in a $39.3 \mathrm{~mm}$ thick turbulent boundary layer, useful comparisons can still be gathered. From the data gather by Okamoto et al., it was found that the flow stayed attached to approximately $45 \%$ of the total surface area of the $60^{\circ}$ vertex angle cone. It is reasonable to assume that a roughness element of the present size (height $<7 \%$ of the boundary layer thickness), fully immersed in a turbulent boundary would experience a higher percentage of attached flow as compared to the Okamoto study. This assumption stems from the fact that a turbulent boundary layer will have more 'energy' and thus be able to stay attached to the surface longer as compared to the uniform flow seen in the freestream. After thoroughly scrutinizing the close-up oil flows in Figure 3.6, a value of approximately $55-60 \%$ of the total surface area was selected to be the attached flow surface area for the peaked elements. Consequently, $\mathrm{C}_{\mathrm{WWA}}$ is taken to be $0.55-0.6$ for the peaked elements.

A series of experiments performed by Gaudet (1987) on rivet heads immersed in a turbulent boundary layer provide some information on the separation location on the hemisphere. As in the Okamoto case above for the cones, the work done by Gaudet was on elements that were much larger than the boundary layer thickness and the elements tested were more 'flat' having a height to diameter ratio of 1:5. Gaudet, however, did show a trend in his data that leads to $\approx 50 \%$ of the total surface area having attached flow in similar flow conditions to the current research. The oil flow pattern of the near wall flow around the hemisphere, Figure 3.5, also shows the flow on the wall staying attached only to the front half of the hemisphere. The combination of these results leads one to use $\mathrm{C}_{\mathrm{WWA}}=0.5$ for the drag analysis done on the hemisphere in Equation 4.3.

When performing the drag analysis using Equation 4.3, the cube elements are much simpler due to the fact that the width of each element is constant. Therefore there is no 
need for an $f$ function as described above. The wetted surface area of the cube at $90^{\circ}$ is taken to be the surface area of three sides and the top whereas the wetted surface area of the cube at $45^{\circ}$ is taken as the surface area of two sides and the top. The surfaces on the downstream side, or sides, of the elements will not contribute to the viscous drag and were deemed negligible in this analysis.

Following the examination and application of the previously discussed issues, a least squares fit of the $\Delta \mathrm{D}_{\mathrm{E} \_ \text {actual }}$ data to Equation 4.3 was employed to correlate the incremental drag of the six elements under scrutiny. The two coefficients $\mathrm{C}_{\mathrm{dp}}$ and $\mathrm{C}_{\mathrm{dv}}$, were obtained in this fashion and found to be 0.1 and 14.25, respectively. Figure 4.1 shows the results of the incremental drag analysis. The solid symbols are the calculated values from the least squares fit, whereas the open symbols are the actual experimental values calculated from Equation 4.2. The open symbols are plotted with respect to themselves in order to give an idea of where the ideal calculated values should fall. The calculated drag value for each element, from Equation 4.3, is in turn plotted vs. its respective experimental data point. The dashed line is a linear fit to these calculated data points and has an $\mathrm{R}^{2}$ correlation coefficient value equal to 0.96 . A better way to analyze how well the calculated values compare to the experimental values is to look at the percent difference between the two values. The uncertainty bars presented on the open experimental symbols are $\pm 10 \%$ of the respective experimental value. All calculated drag values are within this $\pm 10 \%$ range except for the hemisphere which is $\approx 11 \%$ higher than its respective experimental value. Table 4.2 presents the results for the experimental (actual) and the calculated drag on each roughness element from Equation 4.2 and Equation 4.3, respectively. The percent difference between the actual and calculated drag value associated with each roughness element is also given. The discrepancies between the calculated and experimental values can primarily be attributed to the uncertainties associated with the actual separation locations on the surface of the elements as well as the area of the inner wake region and its associated value of wall shear. 
Table 4.2: Comparison of actual and calculated drag values

\begin{tabular}{|c|c|c|c|}
\hline Element & $\Delta \mathrm{D}_{\mathrm{E} \text { actual }}(\mathrm{N})$ & $\Delta \mathrm{D}_{\mathrm{E} \text { calculated }}(\mathrm{N})$ & $\%$ Difference \\
\hline Cone & $1.69 \mathrm{E}-04$ & $1.55 \mathrm{E}-04$ & $8 \%$ \\
\hline Gaussian & $1.22 \mathrm{E}-04$ & $1.17 \mathrm{E}-04$ & $4 \%$ \\
\hline Large Grooved & $1.98 \mathrm{E}-04$ & $1.84 \mathrm{E}-04$ & $7 \%$ \\
\hline Cube at $90^{\circ}$ & $2.52 \mathrm{E}-04$ & $2.39 \mathrm{E}-04$ & $5 \%$ \\
\hline Cube at $45^{\circ}$ & $2.13 \mathrm{E}-04$ & $2.05 \mathrm{E}-04$ & $4 \%$ \\
\hline Hemisphere & $1.15 \mathrm{E}-04$ & $1.28 \mathrm{E}-04$ & $11 \%$ \\
\hline
\end{tabular}

The results obtained from Equation 4.3 help to bring a certain sense of unity to the drag values obtained from the experiments. The largest value of drag is seen on the cube at $90^{\circ}$ followed by the cube at $45^{\circ}$, large grooved cone element, cone, Gaussian element, and the hemisphere. The cube at $90^{\circ}$ presents more of a blockage effect to the flow and is the least 'streamlined' element being tested. Thus, it is not surprising that it has the highest value of drag. The cube at $45^{\circ}$ does have a larger amount of frontal area than the cube at $90^{\circ}$ by a factor of $\sqrt{2}$, but its orientation is more aerodynamic than that of the cube at $90^{\circ}$. The larger value of drag on the large grooved element, as compared to the other peaked elements, can be related not only to its frontal projected area but also its wetted surface area. With the highest wetted surface area, next to the fine grooved element, the large grooved element will experience more viscous drag than the other elements under scrutiny. The rest of the elements follow an apparent trend in that the cone has the next largest amount of frontal and wetted area of the peaked elements followed by the Gaussian element. The hemisphere has the smoothest surface of all the elements and is also submerged the lowest in the boundary layer. Consequently, the lower speed fluid moving over the hemisphere in conjunction with the hemisphere's smooth shape will generate the least amount of drag of all elements.

Other efforts have been made to find possible drag correlations using just the frontal and wetted areas of the elements. The results of these efforts can be seen in Figures 4.2 and 4.3. The drag coefficient $\mathrm{C}_{\mathrm{d}}$ is denoted by a (frontal) or a (wetted) subscript indicating that either the frontal or wetted area of the element was used in the normalization process. The drag coefficient is obtained using the following equation. 


$$
C_{d(\text { frontal_or_wetted })}=\frac{\Delta D_{E_{-} \text {actual }}}{\frac{1}{2} \rho U_{e}^{2} A_{f_{-} o r_{-} w}}
$$

Similarly, results are also plotted in Figure 4.2 using a drag coefficient defined by Equation 4.6. This drag coefficient was first used by Wieghardt (1946).

$$
C_{d_{-} \text {Wieghardt }}=\frac{\Delta D_{E_{-} \text {actual }}}{\bar{q} A_{\text {frontal }}}
$$

Each drag coefficient is presented as a ratio with respect to the reference smooth wall skin friction coefficient, $\mathrm{C}_{\mathrm{f}}$. These drag ratios are plotted vs. the normalized circulation $\Gamma /\left(U_{\tau}\left(A_{f_{-} o r_{-} w}\right)^{1 / 2}\right)$ which utilizes the circulation, $\Gamma$, from the $\mathrm{x} / \mathrm{d}=2.75$ plane and the reference smooth wall value of $U_{\tau}$.

The relationship between the various drag data normalized with respect to the element frontal area show a fairly flat trend as $\mathrm{C}_{\mathrm{d}(\text { frontal })} / \mathrm{C}_{\mathrm{f}}$ is somewhat constant with increasing $\Gamma /\left(U_{\tau}\left(A_{f_{-} o r_{-} w}\right)^{1 / 2}\right)$. The cube at $90^{\circ}$ tends to be the outlier in view of the fact that it has the highest amount of drag with the next to lowest (Gaussian has least) frontal area. This result is not extremely surprising due to the fact that the drag of the roughness element is related to both the viscous drag and the form drag as was discussed earlier. With this in mind, looking at Figure 4.3 reveals that the cube at $90^{\circ}$ is still separated from the other elements even though the wetted area of the cube is the third largest behind both of the grooved elements. The blockage created by the cube at $90^{\circ}$ decreases the amount of circulation produced, thus offsetting it from the other values once more. A somewhat linear trend can be seen in the data of Figure 4.3 as the normalized drag increases so does the normalized circulation. After studying Figures 4.1 through 4.3, it is obvious that in order to grasp the physics of what is happening related to the drag of each element it is essential that the analysis encompass both the form drag of the element as well as the viscous drag. 


\subsection{Downstream Flow Field Comparisons}

In an attempt to bring the immense amount of data gathered in this study into a more condensed and useful form, drawings for the flow around each roughness element were completed and can be seen in Figures 4.4 through 4.10. For conciseness, a legend common to all drawings can be found in Figure 4.4. Each drawing shows the measured features at the $\mathrm{x} / \mathrm{d}=2.75$ plane for all elements except the fine grooved element where the plane at $\mathrm{x} / \mathrm{d}=1.36$ is shown. Regions of vorticity and turbulent kinetic energy (TKE) maxima are shown at these measurement planes. The TKE diffusion velocity vectors, $V_{q} / U_{\tau}=\left(\overline{v q^{2}} / \overline{q^{2}}\right) / U_{\tau}$ and $W_{q} / U_{\tau}=\left(\overline{w q^{2}} / \overline{q^{2}}\right) / U_{\tau}$ are also presented in the region nearest the maximum TKE. These vectors are only presented in the peak TKE region for each element in order for one to grasp the magnitude and direction of the greatest diffusion of TKE.

As is evidenced by the drawings, all elements produce a streamwise horseshoe vortex structure that is on the order of the size of the roughness element from which it emanates. At the $\mathrm{x} / \mathrm{d}=2.75$ plane, the secondary velocity produced by the horseshoe vortex structures is very significant. The magnitude of the secondary velocity at this location causes a large amount of fluid to be brought toward the wall. All elements at this location show a significant normal-to-wall (V) velocity at the centerline (Figure 3.12). The highest magnitude of negative $\mathrm{V}$ can be seen in the two cube elements, followed by the hemisphere, cone, Gaussian, and the large grooved element. From Figure 3.12, the fine grooved element's $\mathrm{V}$ at the $\mathrm{x} / \mathrm{d}=2.75$ plane is very near the magnitude of the Gaussian element. Thus it is reasonable to assume that the horseshoe vortex structure produced by the fine grooved element at $\mathrm{x} / \mathrm{d}=2.75$ is close to the same strength as the structure produced by the Gaussian element. All counter rotating horseshoe vortices move away from the wall and centerline and dissipate as they propagate downstream. The movement and strength of the vortex structure will be affected a great deal by the generation of secondary vorticity near the wall. In this region the near wall viscous forces will cause the loss of strength in the primary vortex. 
Figure 4.7 for the fine grooved element shows that at the $\mathrm{x} / \mathrm{d}=1.36$ measurement plane there is fluid being swept in around the sides of the element. This location precedes the reattachment point and the horseshoe vortex structure has not yet taken over the secondary flow field. Therefore, the dominant secondary flow structure appears to be the flow coming around the sides of the element rather than the counter-rotating structure shown at the $\mathrm{x} / \mathrm{d}=2.75$ plane. All peaked elements, Figures 4.4 to 4.7 , are thought to have weak vortices emanating from the top of the element. Although the presence of these vortices could not be determined from the experimental data, they have been shown to be present in a study by Fontaine and Deutsch (1996) on a smaller Gaussian shaped element. The strength of these smaller 'peak' vortices is orders of magnitude less than the primary horseshoe vortex structure.

Regions of positive mean streamwise vorticity, $\Omega_{\mathrm{x}}$, can be located in all drawings. The magnitude of maximum streamwise vorticity is given in each drawing as well as its associated location. The location of the vortex center is taken to be the point of maximum vorticity as was also done in previous studies by Wendt and Greber (1992). All spanwise $(\mathrm{z})$ position values are normalized by the parameter $\mathrm{d}$ for each element. This parameter is simply the element's base diameter for all axisymmetric elements, the edge length for the cube at $90^{\circ}$, and the length of the diagonal for the cube at $45^{\circ}$. The vertical (y) position values are normalized by the height, $h$, of each element.

The location of maximum streamwise vorticity for all elements is quite close to the wall at $\mathrm{x} / \mathrm{d}=2.75$. The cube at $45^{\circ}$ and the hemisphere show the largest normalized $\mathrm{y} / \mathrm{k}$ locations from the wall at $\mathrm{y} / \mathrm{k}=0.138$ and 0.183 respectively where the rest of the elements are in the range of $y / k \approx 0.83-0.96$. It is worth noting that the distance of maximum streamwise vorticity for the fine grooved element at $\mathrm{x} / \mathrm{d}=1.36$ is very near the wall at $\mathrm{y} / \mathrm{k}=0.039$. These results show the significance of having near wall data due to the large amount of activity that is seen in this locale. The major source of vorticity at the $\mathrm{x} / \mathrm{d}=2.75$ location is below $\mathrm{y}^{+}=15$ for all elements. The spanwise distance from the wall for most elements is similar and around $\mathrm{z} / \mathrm{d} \approx-0.83$. This is not the case for the Gaussian element and the hemisphere which have maximum vorticity locations in the 
spanwise direction equal to $\mathrm{z} / \mathrm{d}=-0.552$ and -0.551 respectively. This result is expected due the shape of these elements. The Gaussian element is fairly slender along its height, thus causing the vortex structures to be closer to the centerline. The hemisphere on the other hand is very smooth and has more flow coming over its top and rushing toward the wall; thus bringing the horseshoe vortex structures closer to the centerline in the near element regions.

The magnitude of the vorticity is directly related to the strength of the horseshoe vortex structure. As a result, the maximum value of normalized streamwise vorticity, $\left(\Omega_{x} \sqrt{A_{f}} / U_{\tau}\right)_{\max }$, at $\mathrm{x} / \mathrm{d}=2.75$ is observed in the cube elements. The cube at $90^{\circ}$ shows the largest magnitude equal to 11.59 and the cube at $45^{\circ}$ is slightly less at 11.35 . The rest of the elements tend to be fairly close to one another with the large grooved element having the smallest normalized vorticity maximum at 4.44 . The presence of the larger grooves (2.5 times the smallest sublayer structure length scale) seems to have a noticeable effect on the flow field around the roughness element. The irregularities that are 2.5 times the smallest sublayer structure length scale not only increase the viscous drag on the element, but they also tend to 'straighten' the flow, produce weaker vortices and less vorticity than the cone element with equal frontal area and similar shape. The finer grooves that are the size of the smallest sublayer structure length scale also increase the viscous drag on the element, but do not have as much effect on the production of vorticity as the larger grooves. Due to the fact that the vortices produced by the elements are located very close to the wall, as was discussed above, viscous forces will have a major contribution in the decay of the vortices as they propagate downstream. Another aspect that is related to the diffusion and transport of the vortices is the entrainment of fluid from the boundary layer.

The zone of separated flow for each element is shown in Figures 4.4 through 4.10 to be directly behind each roughness element and of varying breadth for the different cases. The defined separation points on the sharp edges of the cube elements create a larger region of separated flow as compared to the axisymmetric elements. The cube elements also present a significant 'blockage' effect to the flow. This blockage creates a region of 
stagnant fluid and tends to delay flow reattachment. The presence of an arch vortex can be seen in the flow field directly downstream of the cube elements. This vortex is formed from the separation and shear layer roll-up created near the sharp edges of the element. The horseshoe vortex structure tends to bring fluid in toward the element along the centerline. Smaller vortices can also be found on the sides and top of the cube at $90^{\circ}$ due to the shear layers rolling up at these edges. The separated flow regions behind the axisymmetric elements are smaller and not as well defined as those associated with the cube elements. The size of the separated flow region obviously depends on the size and shape of the element. The more slender shape of the Gaussian element lends itself to the creation of the smallest region of separated flow. Thus, the Gaussian element is the only element not to show backflow at the $\mathrm{x} / \mathrm{d}=1.36$ measurement location. The large grooved element has the second to smallest portion of separated flow due to the fact that the base of the element does not extend all the way to the wall. The first groove machined into the element starts at the base of the element, thus creating an opening for the flow to proceed through. The hemisphere, cone and fine grooved elements have comparable separated regions but the hemisphere reattaches sooner due to the flow accelerating over the top of the element and being directed toward the wall. The near wall separation point for all peaked elements tends to be on the downstream side of the element. Accordingly, it is a reasonable assumption due to the size of the element and associated boundary layer parameters that the flow stays attached to approximately $55-60 \%$ of the element's surface. The hemisphere element on the other hand seems to have a separation point nearer to its midpoint owing to the reasoning that approximately $50 \%$ of the surface sees attached flow. As was discussed in Section 4.2, these conclusions stem from the oil flow visualizations done on the roughness elements as well as the work done by Okamato et al. (1977) and Gaudet (1987).

The magnitude and location of the peak TKE at each element's respective measurement plane is presented in the flow field drawings as well. Although all maximum values occur along the centerline of the element, the normalized height ratios vary significantly between the axisymmetric elements and the cube elements. The large regions of TKE are due to the horseshoe vortices that not only create sweeping motions toward the wall but 
also a convergence of associated shear layers toward the center plane. The largest magnitude of normalized TKE at the $\mathrm{x} / \mathrm{d}=2.75$ measurement plane is produced by the cube at $45^{\circ}$ with a value equal to 21.29 . The cube at $90^{\circ}$ follows with a peak value of 18.36. The fine grooved element also has a rather large peak value (17.59), as shown in Figure 3.103 at the $\mathrm{x} / \mathrm{d}=2.75$ location. The cone, large grooved, Gaussian, and hemisphere elements follow with peak TKE values of 15.31, 13.96, 13.82, and 13.42, respectively. The larger elements that protrude higher into the boundary layer and have sharp edges tend to produce more TKE. The hemisphere which is shorter and has smooth sides has the lowest amount of TKE production. The fine grooved element shows a peak value at the $\mathrm{x} / \mathrm{d}=1.36$ plane equal to 33.15 which is nearly twice as much as the peak value at the $\mathrm{x} / \mathrm{d}=2.75$ centerline measurement location.

The diffusion velocity vectors are shown in the immediate area surrounding the peak value of TKE for all elements. The highest diffusion velocities are produced by the cone and cubes whose vectors have very similar magnitudes. The Gaussian, fine grooved, large grooved, and hemisphere elements follow with decreasing diffusion velocities. The fine grooved element is more 'peaky' than the large grooved element and has greater TKE diffusion. As was discussed in Chapter 3, the diffusion velocities are highest near sharp edges (cubes) and near sharp peaks (cone, Gaussian, etc.). Subsequently, this leads to the conclusion that both the element shape and the orientation play very significant roles in the production and diffusion of TKE. Although the hemisphere has a smoother surface than the other elements, there are still significant diffusion velocities near the element height. The height at which the greatest magnitude of TKE exists at the $\mathrm{x} / \mathrm{d}=2.75$ location is very similar for the peaked elements as they all are within the range $0.550 \leq \mathrm{y} / \mathrm{k} \leq 0.555$. This result can be attributed to the fact that the horseshoe vortex structures produced by the peaked elements are nearly the same size at this location. Therefore, the sweeping motions toward the wall as well as the convergence of associated shear layers toward the center plane by the horseshoe vortices will be at similar positions within the boundary layer. The normalized y-location of the maximum TKE associated with the hemisphere is $\mathrm{y} / \mathrm{k}=0.555$, thus making it nearly the same as the peaked elements. The normalized $\mathrm{y}$-location of the maximum TKE production at the $\mathrm{x} / \mathrm{d}=1.36$ plane for the 
fine grooved element is $\approx 38 \%$ higher than its value at $\mathrm{x} / \mathrm{d}=2.75$. The high TKE value seen at the $\mathrm{x} / \mathrm{d}=1.36$ measurement location is significantly influenced by the peak of the element as it causes intense mixing near the element. As the flow progresses downstream, the influence of the horseshoe vortices tends to have more of an effect on the magnitude of the TKE. The cube elements have normalized height values that differ from the axisymmetric elements. The cube at $90^{\circ}$ has its maximum TKE location at $\mathrm{y} / \mathrm{k}=0.714$, whereas the cube at $45^{\circ}$ sees its maximum value at $\mathrm{y} / \mathrm{k}=0.479$. These differences can be linked to the size and shape of the cube elements as they obviously have sharp edges and are more blunt than the axisymmetric elements. These facets will in turn produce horseshoe vortex structures that are significantly different in size, shape and strength.

\subsection{Circulation}

\subsubsection{Circulation Decay}

The decay of circulation is mainly due to near wall viscous forces in the spanwise direction that act in retarding the secondary flow (Wendt and Greber, 1992). Viscous diffusion also plays a role in the dissipation of the circulation as the vortex structure is located in the near-wall region of the turbulent boundary layer. The streamwise decay of circulation, $\Gamma /\left(U_{\tau} \sqrt{A_{f}}\right)$, plotted using a logarithmic scale can be seen in Figure 4.11. The peaked elements show a common trend in the decay of circulation with streamwise distance $\mathrm{x} / \mathrm{d}$. By fitting a curve to the data it is possible to obtain the general behavior of the decay of circulation with streamwise distance. Consequently, the peaked elements have a streamwise decay in circulation that is proportional to $\approx(x / d)^{-1.12}$. The exponent -1.12 was obtained by taking an average value from all peaked elements. All actual values obtained from the individual decay of each element's circulation are all relatively close to the stated average value. The cone is the farthest from the average value with a difference of $11 \%$. The fine grooved element, Gaussian element, and large grooved element follow with respective $\%$ differences of $7 \%, 3 \%$, and $0.4 \%$. These rather small percent differences lead to a confidence in the general trend related to the streamwise 
decay of circulation. Knowing a general trend in the decay circulation is of great benefit in practical applications. Such knowledge can give a qualitative idea of the behavior of the downstream secondary flow field and strength of the horseshoe vortex structure. The hemisphere and cube elements do not have a discernible trend that can be related to one another. Starting at the $\mathrm{x} / \mathrm{d}=5.0$ measurement location and continuing downstream, the hemisphere tends to have lower values of circulation as compared to the other elements. This in turn will lead to the faster decay of circulation due to the near wall viscous effects taking over as the vortex structure propagates downstream. On the other hand, the cube elements have a totally different near element behavior as was discussed in Chapter 3. Once the $\mathrm{x} / \mathrm{d}=2.75$ measurement location is reached though, the cube elements show a decay more along the lines of the other elements.

\subsubsection{Streamwise and Spanwise Circulation Relationship}

There is a significant flux in vorticity between the upstream and downstream regions of the roughness element in the turbulent boundary layer. This change can be attributed to the pressure gradient induced by the roughness element which in turn generates the additional vorticity seen in the wake of the element. The vorticity in the x-y plane along the centerline of the element can be related to the generation of additional vorticity in the $\mathrm{y}-\mathrm{z}$ plane at the $\mathrm{x} / \mathrm{d}=2.75$ measurement location. In order to discuss this phenomenon, it is necessary to define two planar control volumes. The $x-y$ planar control volume $(\mathrm{CV})$ is defined with an upstream location at the front of the roughness element and a downstream location at $\mathrm{x} / \mathrm{d}=2.75$. The height of the $\mathrm{x}-\mathrm{y}$ planar $\mathrm{CV}$ is at the location where the mean streamwise, $\mathrm{U}$, velocity is constant (i.e. at $\mathrm{y}=\delta$ ). The $\mathrm{y}-\mathrm{z}$ planar $\mathrm{CV}$ is located at $\mathrm{x} / \mathrm{d}=2.75$ and has the same dimensions as the $\mathrm{x}-\mathrm{y} \mathrm{CV}$. The $\mathrm{y}-\mathrm{z} \mathrm{CV}$ has a side located along the centerline (x-axis) of the element. It is possible to arrive at the following equations below if the upstream and far spanwise $\mathrm{V}$ velocity profiles are taken to be negligible along with the observable fact of flow symmetry along the centerline $(\mathrm{W}=0)$. The circulation in the $\mathrm{x}-\mathrm{y}$ plane, $\Gamma_{\mathrm{z}}$, is defined as

$$
\Gamma_{z}=\int_{\Delta y} V(y) d y-\int_{\Delta x} U(y) d x
$$

where $\Delta \mathrm{y}$ and $\Delta \mathrm{x}$ are taken to be the edges of the control volume circuit in the $\mathrm{y}$ and $\mathrm{x}$ directions. Similarly the circulation in the $y-z$ plane, $\Gamma_{x}$, can then be defined as 


$$
\Gamma_{x}=-\int_{\Delta y} V(y) d y
$$

where $\Delta y$ is taken to be the same as above.

From Equations 4.7 and 4.8 it is clear that the streamwise and spanwise circulation are directly related through the $\mathrm{V}$ velocity profile at the $\mathrm{x} / \mathrm{d}=2.75$ centerline measurement location. The only difference between the streamwise and spanwise circulation is seen in the mean streamwise (U) velocity term that is present in the spanwise circulation. This tells us that a significant amount of the $\Gamma_{z}$ in this control volume is due to the strong downwash produced by the horseshoe vortex structures created by presence of the roughness element. Knowing this relationship is beneficial due to the fact that it provides a deeper understanding of how $\Gamma_{\mathrm{z}}$ is related to $\Gamma_{\mathrm{x}}$. Similarly, the streamwise and spanwise vorticity values, $\Omega_{\mathrm{x}}$ and $\Omega_{\mathrm{z}}$, can be related. This in turn allows one to have an understanding of the connection between the additional vorticity created in the wake of the element as compared to the incoming spanwise vorticity.

\subsection{Future Work}

Future plans that will be done with the knowledge gained from the current research will include the examination of various fetches of roughness. These fetches will have varying values of lambda $(\lambda)$ where $\lambda=($ projected frontal area)/(total surface area) in order to acquire a more complete data base for different roughness regimes. Sparsely distributed roughness (small $\lambda$ values) as well as more densely packed roughness (large $\lambda$ values) will be examined. Figure 4.12 presents two examples of smaller spacing between roughness elements which in turn would create larger values of $\lambda$. Once a fairly complete data base is compiled, the 'effectiveness' of a particular roughness element can be determined. The 'effectiveness' of a roughness element or a roughness regime will provide a summary of how a given roughness element geometry affects the boundary layer flow conditions. This 'effectiveness' parameter is based on the size, shape, and spacing of the roughness elements as well as the on-coming flow characteristics upstream 
of the elements. Once the 'effectiveness' factor is known, it could be superimposed into a certain flow field in order to more closely calculate the flow characteristics. 


\subsection{Chapter 4 Figures}

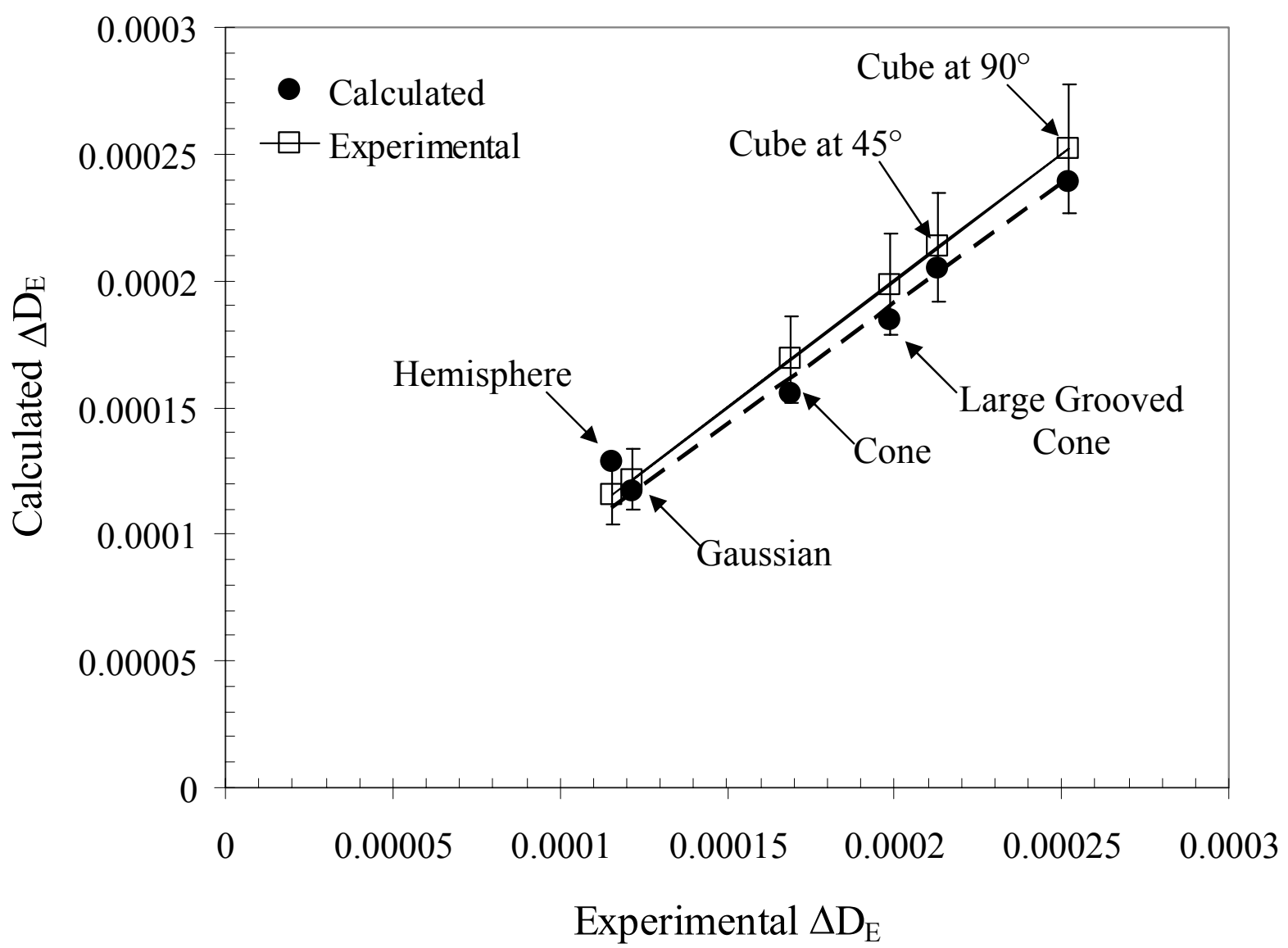

Figure 4.1: Drag correlation results 


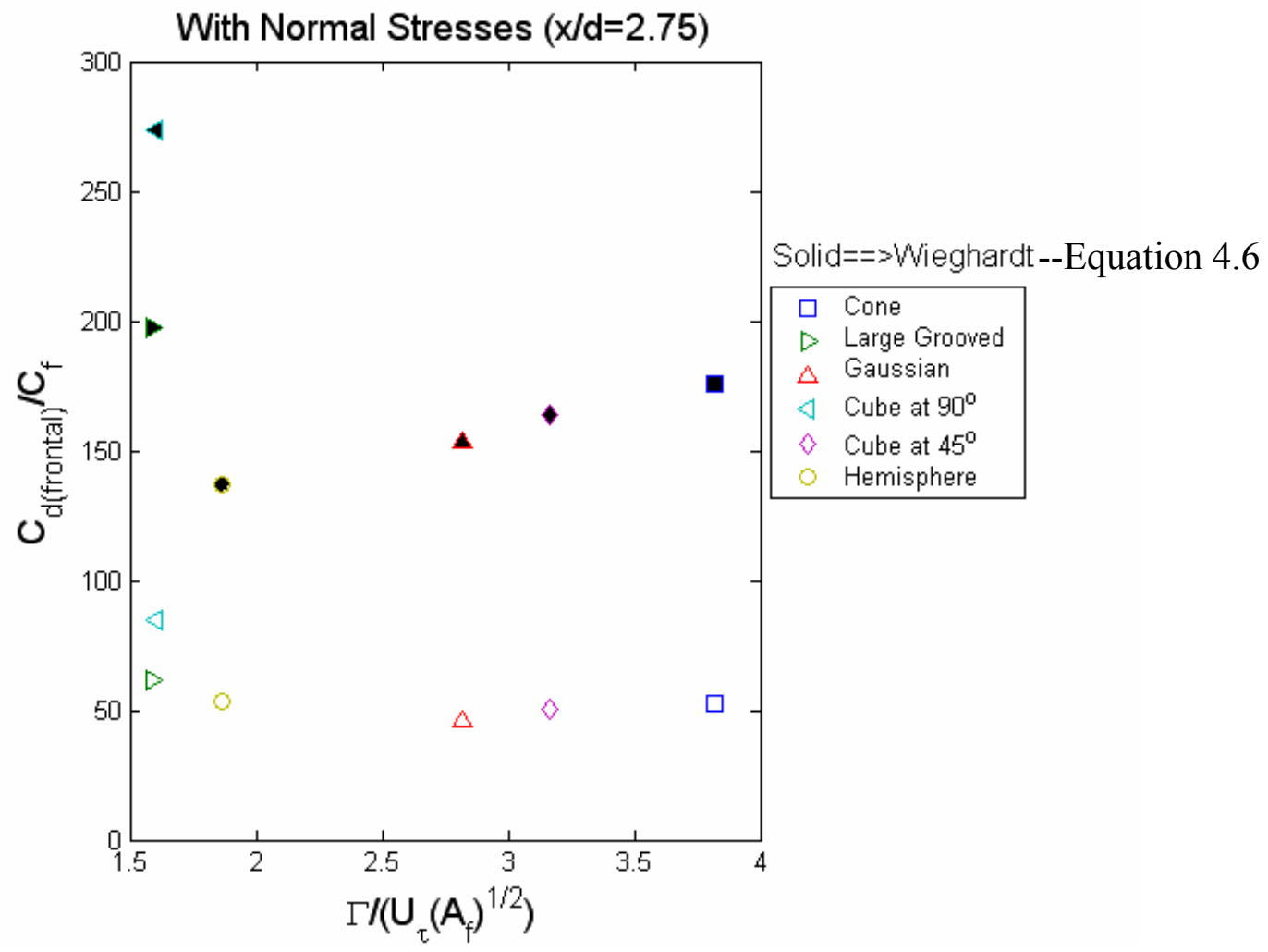

Figure 4.2: Variation of normalized drag coefficient based on frontal area $C_{d(f r o n t a l)} / C_{f}$

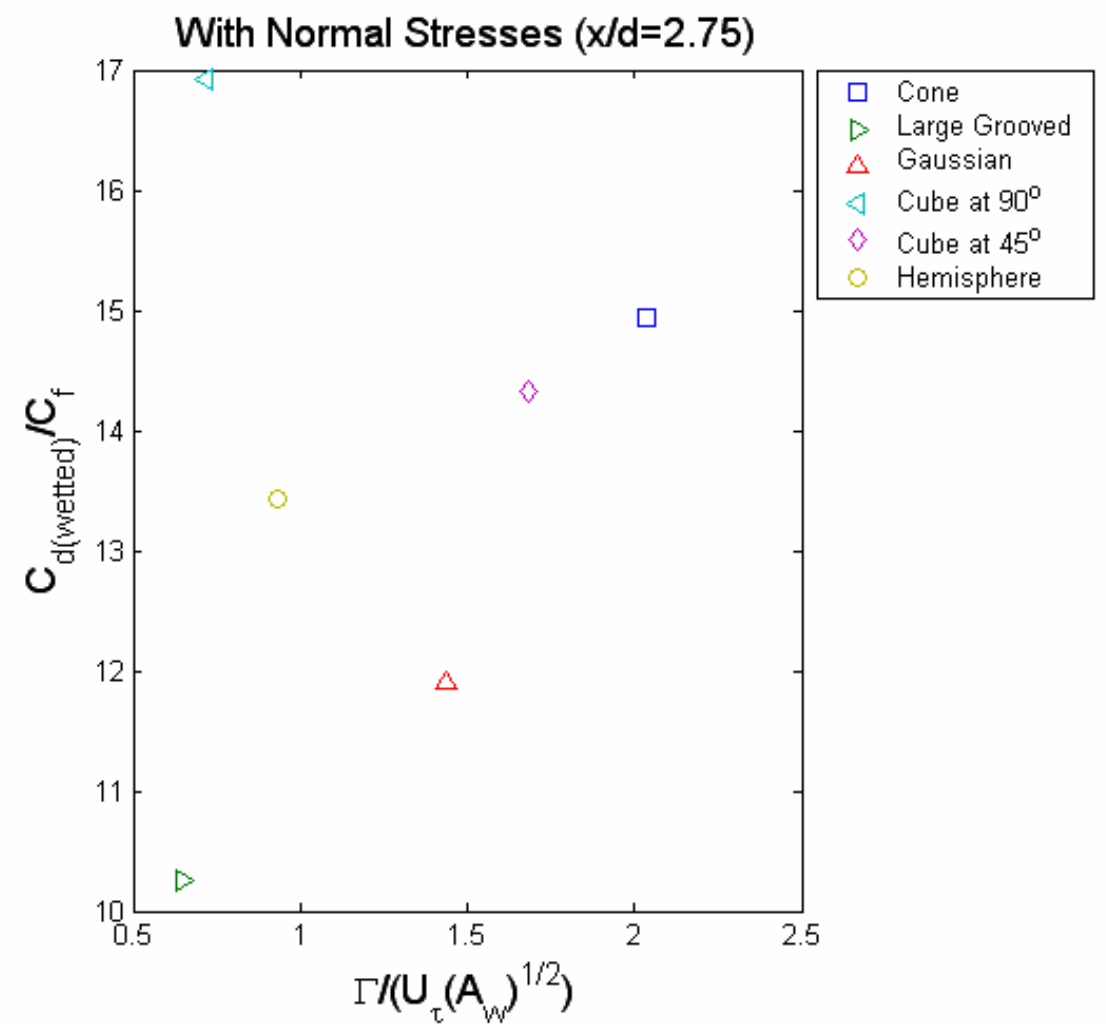

Figure 4.3: Variation of normalized drag coefficient based on wetted area $C_{d(w e t t e d)} / C_{f}$ 

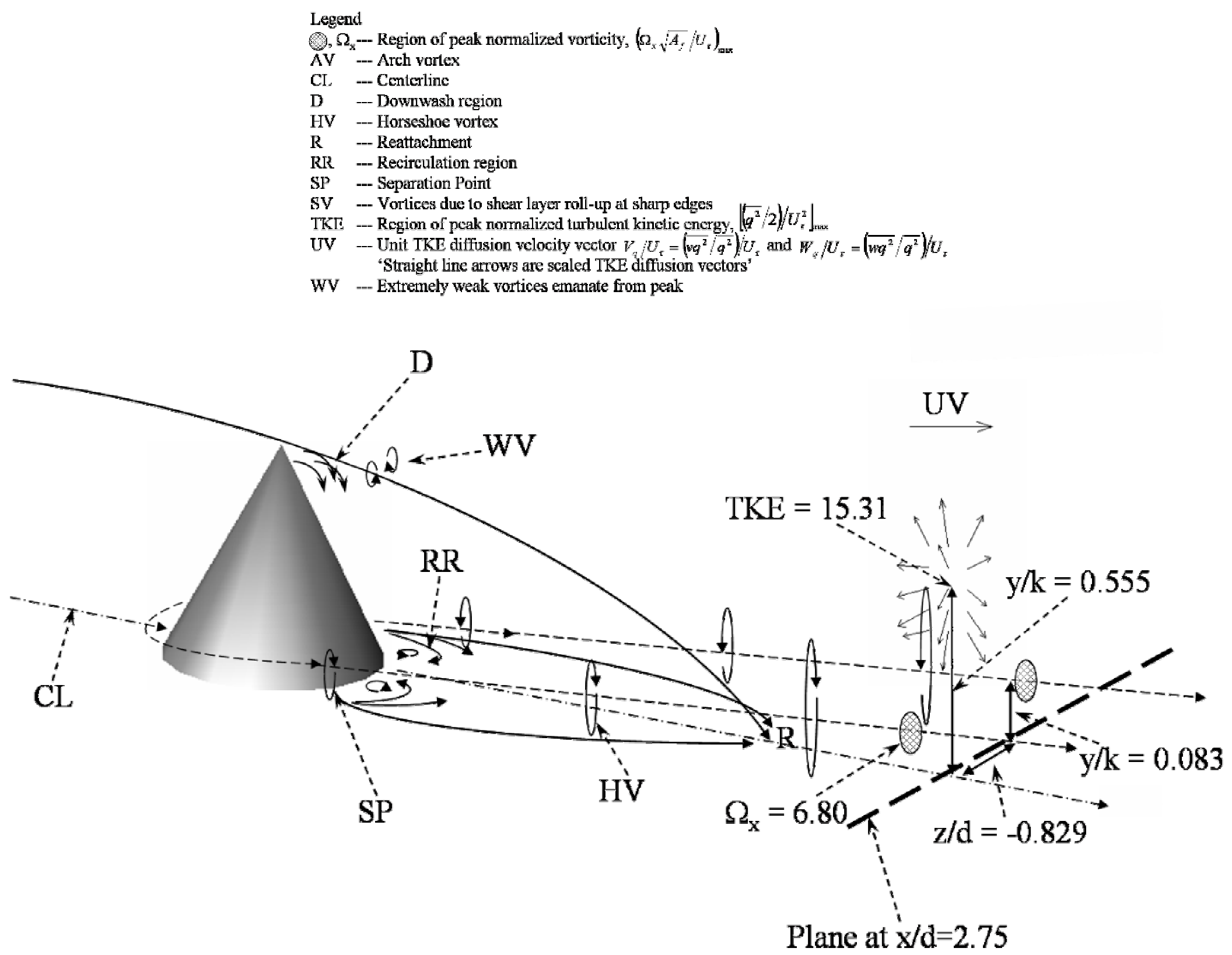

Figure 4.4: Drawing of the flow around the cone element 


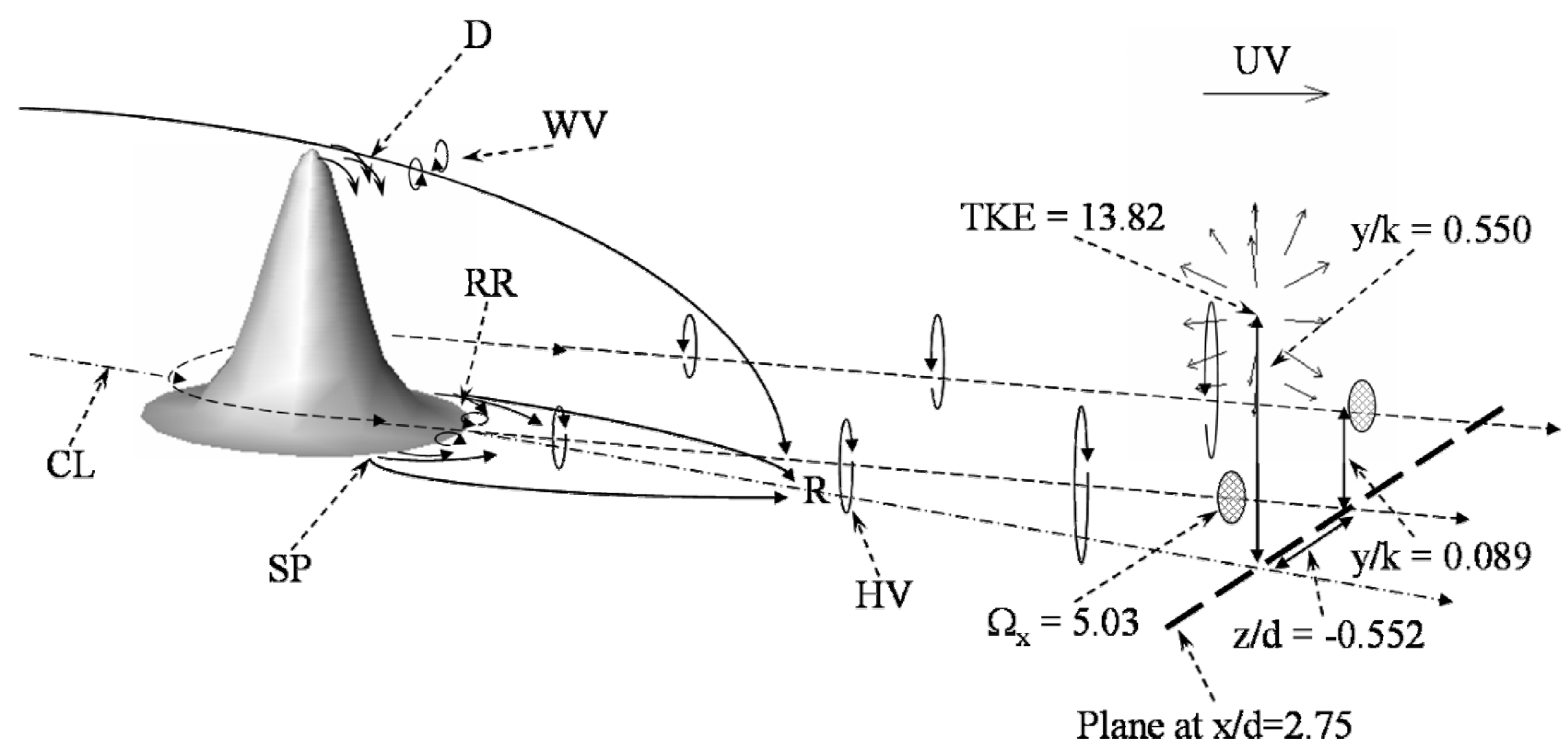

Figure 4.5: Drawing of the flow around the Gaussian element 
Note: Larger Grooves Tend to

'Straighten' Flow

(produce less vorticity)

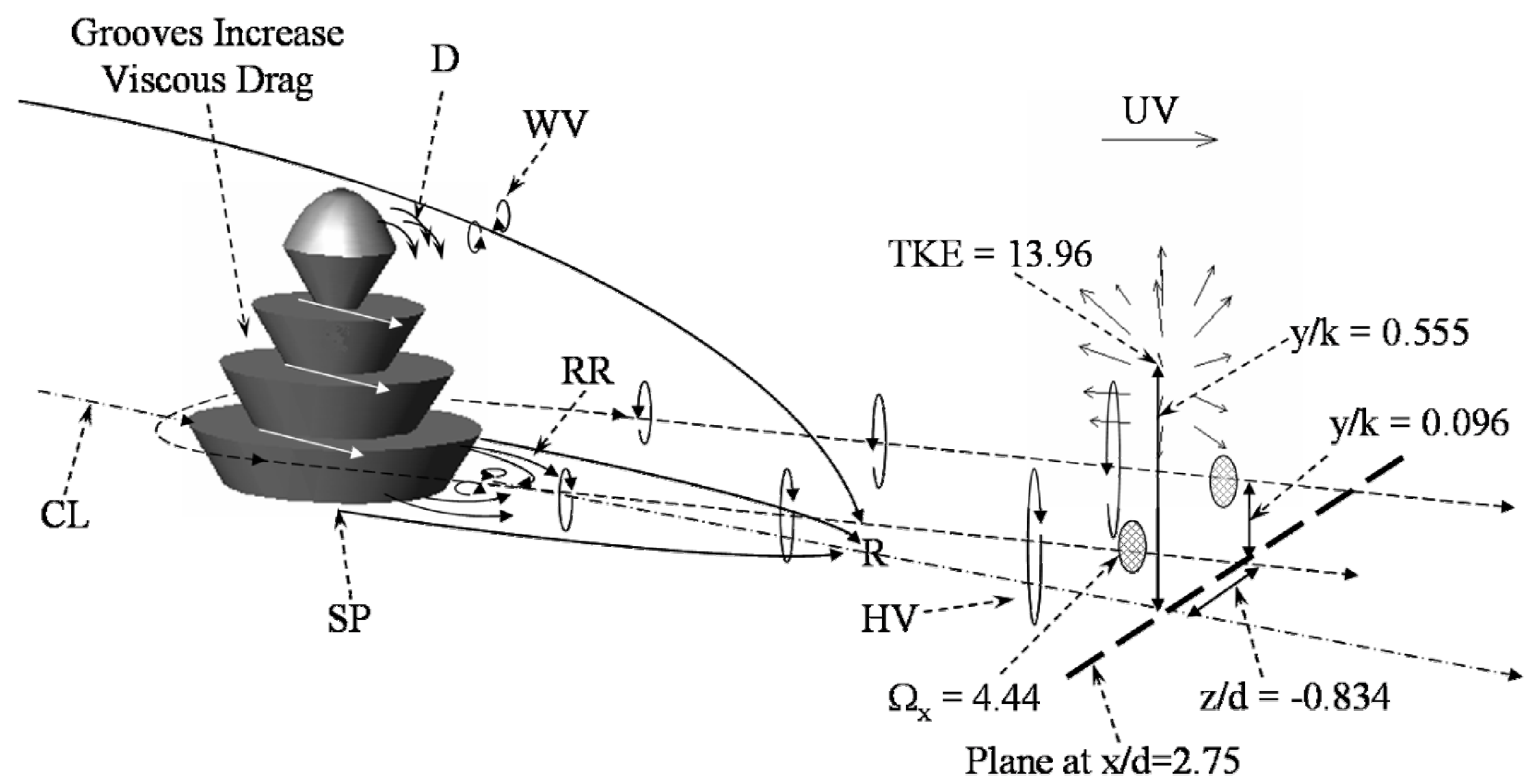

Figure 4.6: Drawing of the flow around the large grooved element 


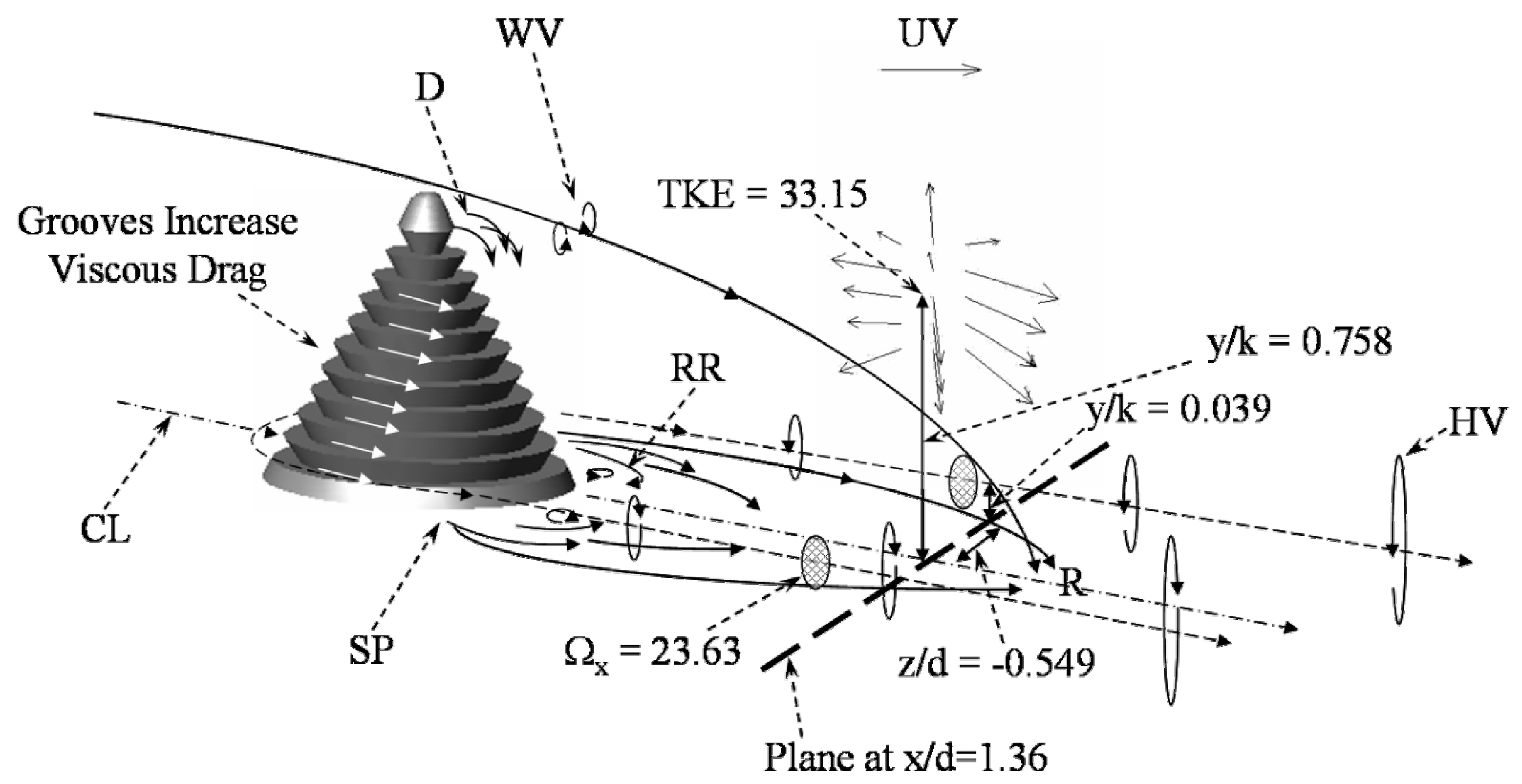

Figure 4.7: Drawing of the flow around the fine grooved element 


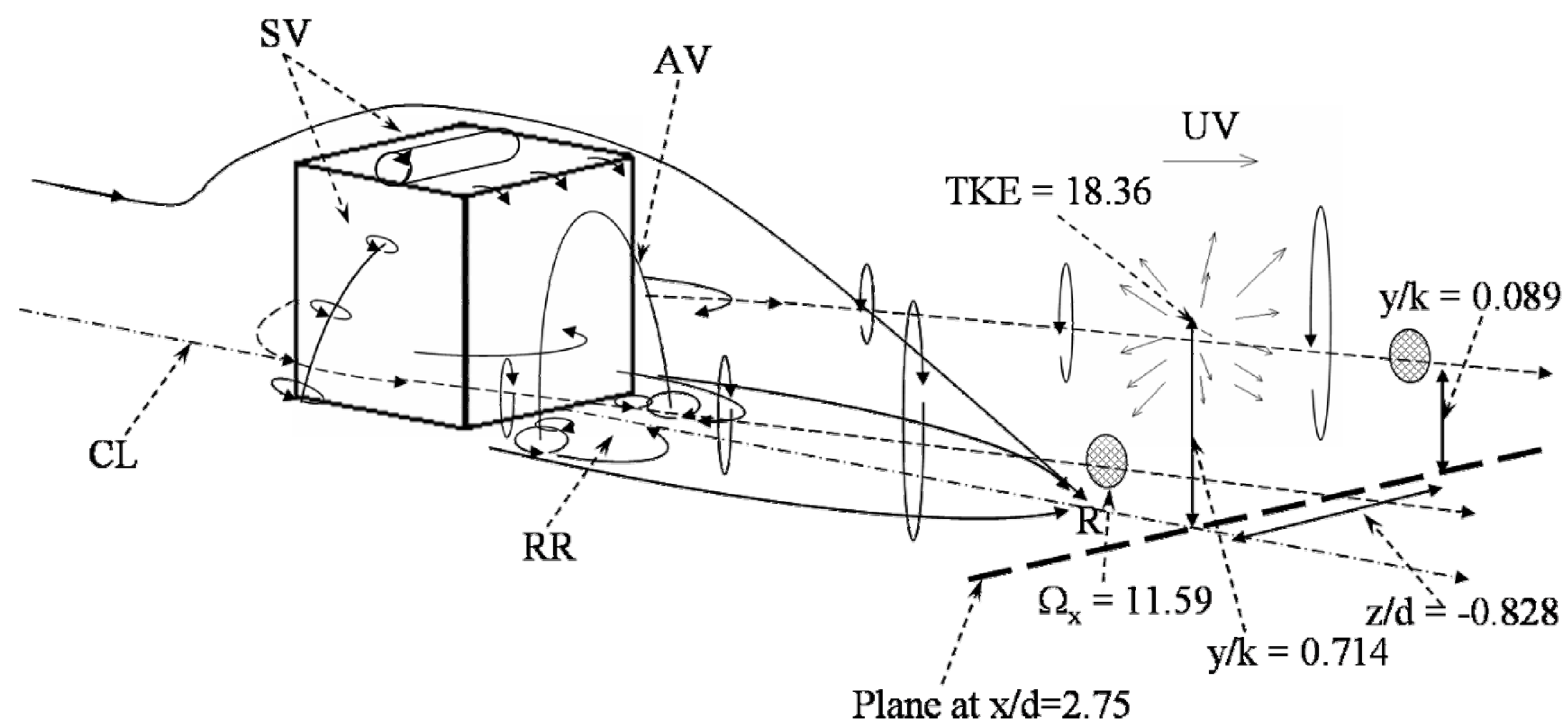

Figure 4.8: Drawing of the flow around the cube at the $90^{\circ}$ orientation 


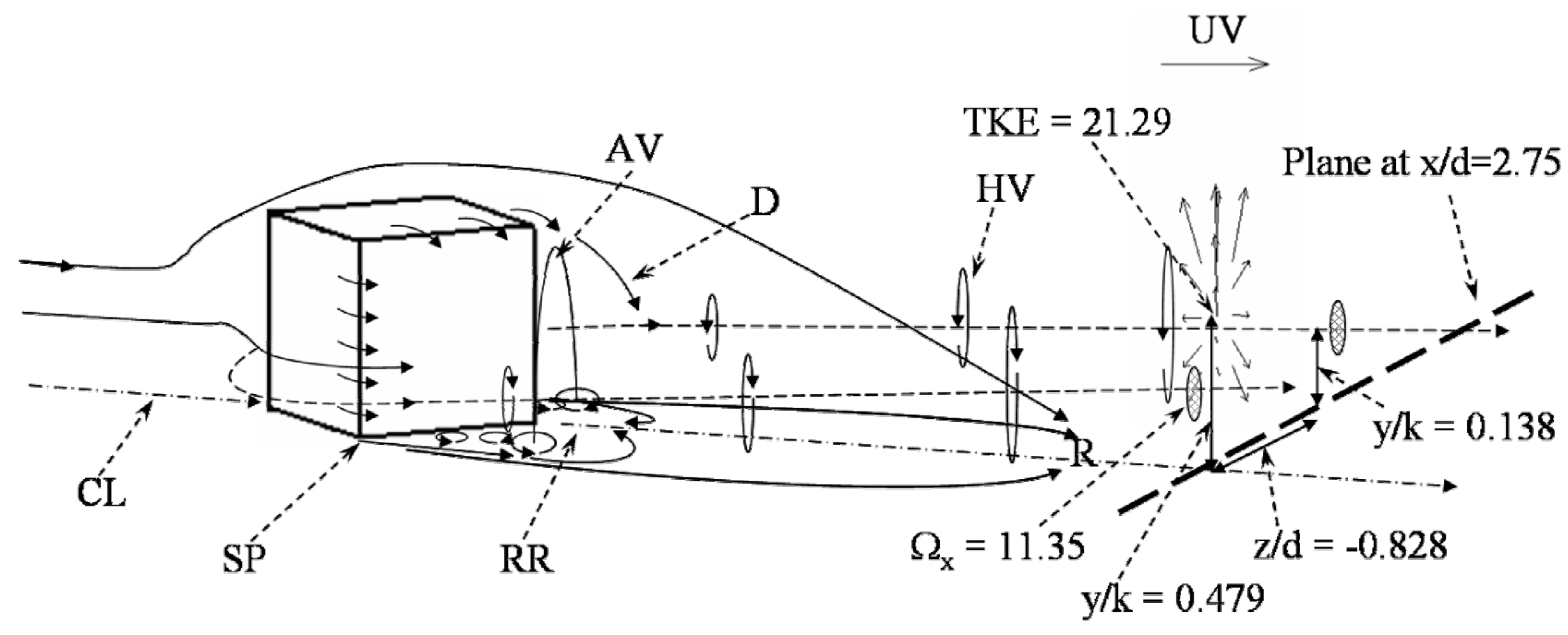

Figure 4.9: Drawing of the flow around the cube at the $45^{\circ}$ orientation 


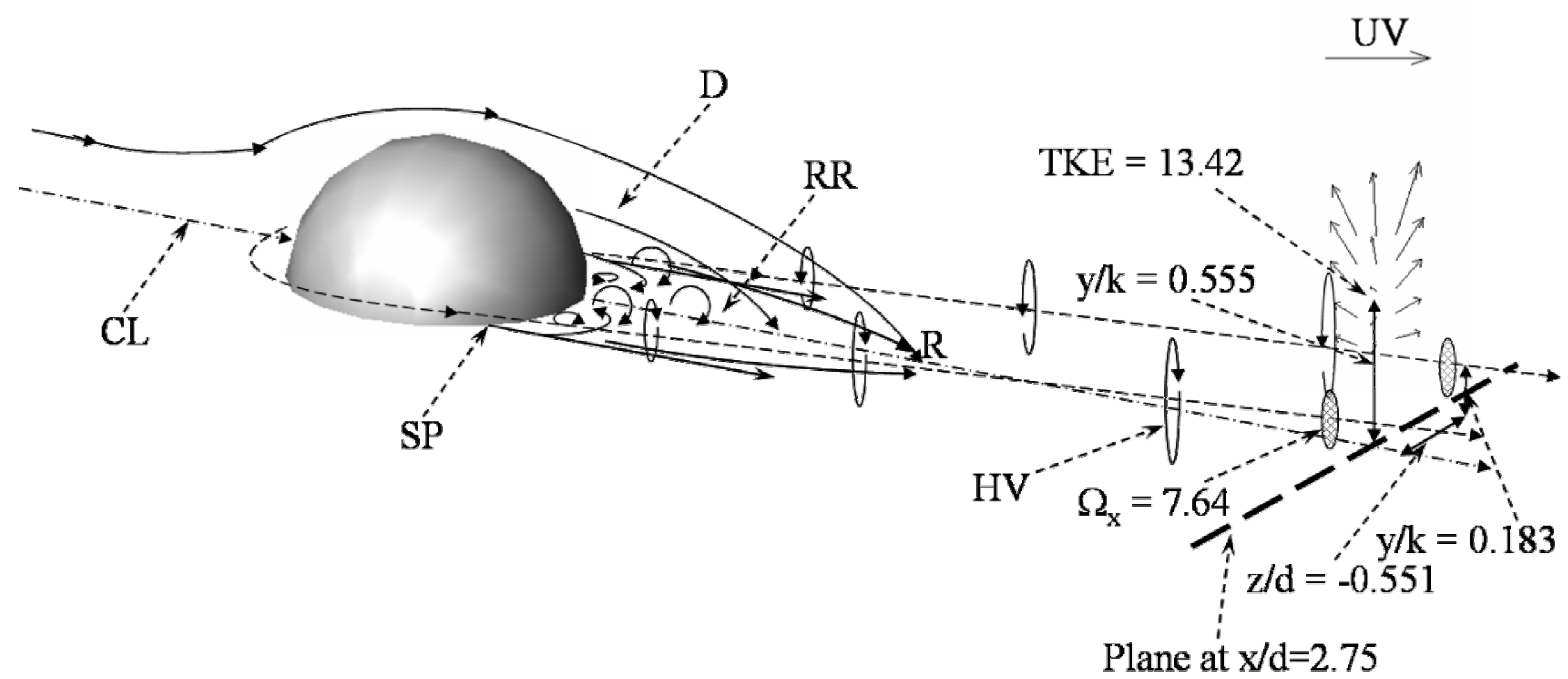

Figure 4.10: Drawing of the flow around the hemisphere 


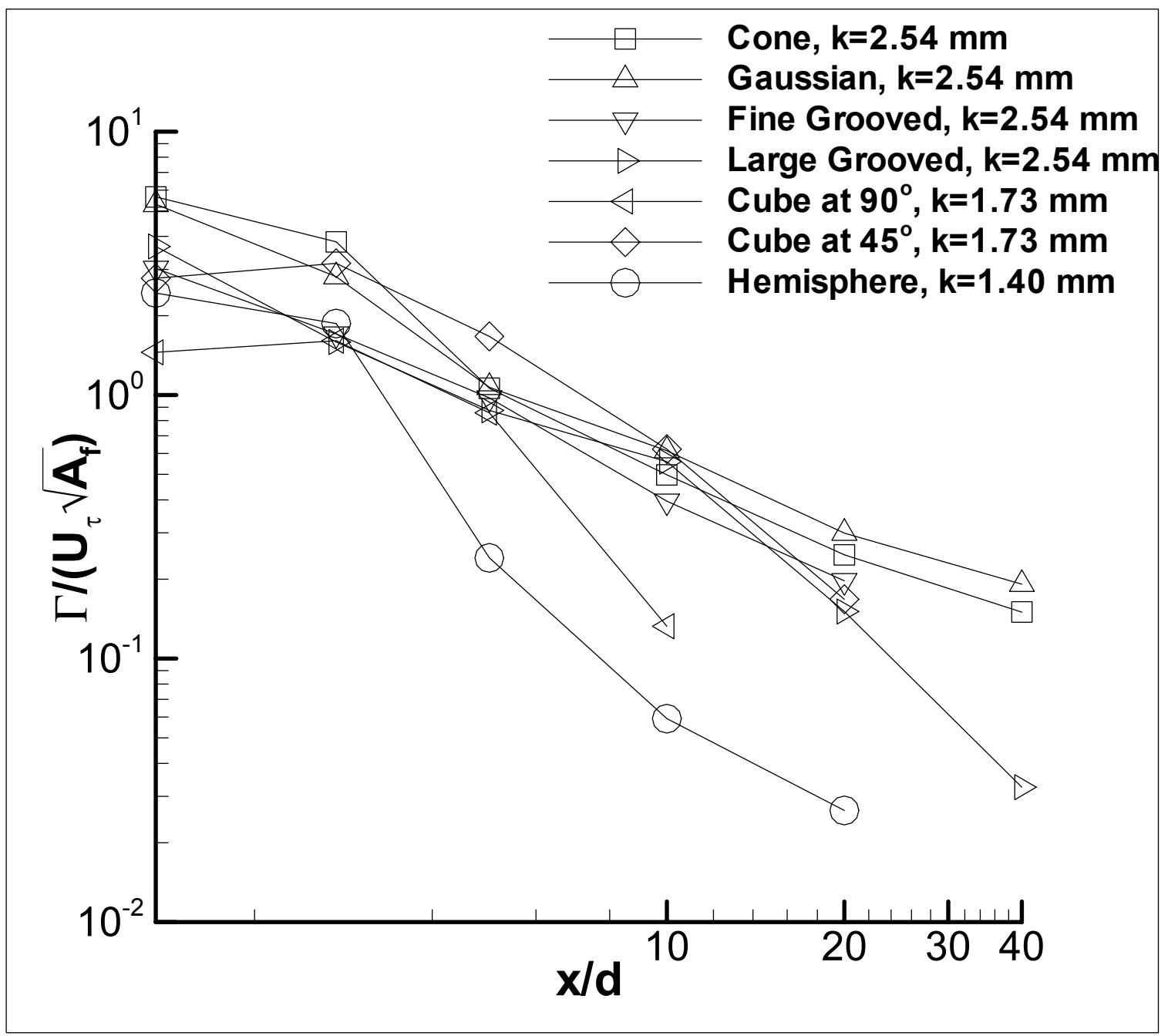

Figure 4.11: Log-log plot of the decay of circulation, $\Gamma /\left(U_{\tau} \sqrt{A_{f}}\right)$, with streamwise distance, $x / d$ 


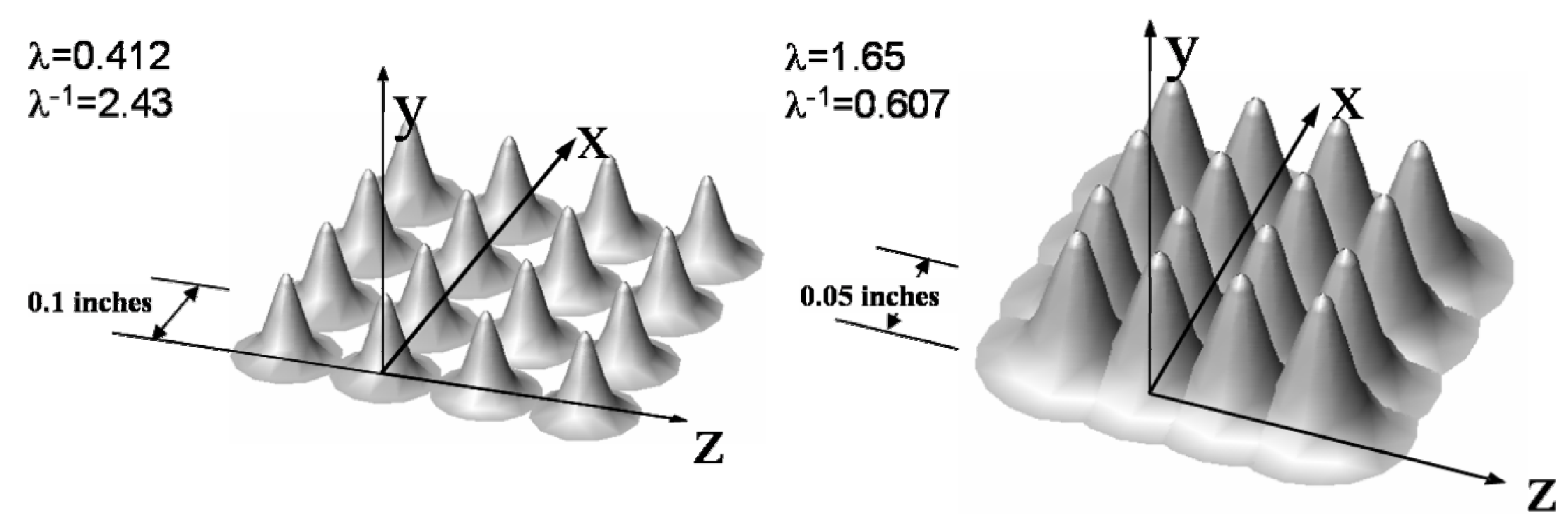

Figure 4.12: Various roughness regimes that could be investigated in the future 


\section{Chapter 5 Conclusions}

A detailed study has been conducted in the Virginia Tech Aerospace and Ocean Engineering Department small boundary layer wind tunnel with the primary intent to fully measure and understand the effects of various shaped roughness elements in a twodimensional high Reynolds number turbulent boundary layer. The roughness elements under scrutiny were less than $7 \%$ of the boundary layer thickness in height and are as follows: cone, cone with spatial variations equal to the smallest sublayer structure length scale, cone with spatial variations equal to 2.5 times the smallest sublayer structure length scale, Gaussian-shaped element, hemisphere, cube aligned perpendicular to the flow (cube at $90^{\circ}$ ), and a cube rotated $45^{\circ}$ relative to the flow. The roughness element heights, $\mathrm{k}^{+}$, non-dimensionalized by the friction velocity $\left(\mathrm{U}_{\tau}\right)$ of the approaching turbulent boundary layer, are 145, 145, 145, 145, 80, 98, and 98, respectively. A brief analysis of a three-dimensional fetch of the same Gaussian-shaped elements described previously was also undertaken. In order to analyze the complex flow fields, detailed measurements were obtained using a fine-measurement-volume (50 micron diameter) three-velocity component laser-Doppler velocimetry (LDV) system. The LDV measurement system was used to obtain boundary layer profiles along the centerline of the element at varying downstream distances. Two planes of data were also acquired at different downstream locations for each element. Surface oil flow patterns were taken for each isolated roughness element in order to give a qualitative idea of the near wall flow structure. This is the first time that a detailed fundamental study has been done on roughness elements of these shapes and sizes. Moreover, it is essential to note that no detailed analysis exists concerning the effects of spatial smoothing related to these types of roughness elements.

Through this research it was found that a horseshoe vortex structure forms in front of all elements and is on the order of the size of the roughness element from which it emanates. Evidence of the upstream formation of these vortices can be seen in the oil flow visualizations performed on each isolated roughness element. As the horseshoe vortex structure proceeds downstream, its presence induces the downwash of higher momentum 
fluid toward the wall. This 'sweep' motion not only creates high Reynolds stresses $\left(\overline{v^{2}}, \overline{w^{2}},-\overline{u v}\right)$ downstream of the element, but also leads to higher skin-friction drag. Triple products of velocity fluctuation components were also found to be very significant near the height of the element. These parameters are important in regards to the turbulent diffusion of the turbulent kinetic energy in the flow. The larger elements that protrude into the boundary layer and have sharp edges tend to produce more TKE. The largest magnitude of TKE was produced by the cube at $45^{\circ}$ followed by the cube at $90^{\circ}$, the fine grooved element, cone, large grooved, Gaussian, and finally the hemisphere element. The cone and cubes have the highest diffusion velocities with their respective vectors having very similar magnitudes. This indicates that sharp edges and peaks produce greater diffusion of TKE. Likewise, both the shape of a roughness element as well as its orientation play vital roles in the diffusion of TKE.

The oil flow patterns indicate the size of the separated flow regions directly behind each element, as well as a qualitative idea of the horseshoe vortex behavior in the near wall region. The cube elements were determined to have an arch vortex that formed directly downstream of the element. The spanwise variation in wall shear was very similar for all of the axisymmetric elements, whereas the cube elements showed similar trends as compared to one another. A distinct peak value in the centerline variation in wall shear occurs for the axisymmetric elements at $\mathrm{x} / \mathrm{d}=5.0$ as the flow from around the elements as well as the flow from over the top converged on the centerline. The cube elements produced drastic reductions in wall shear in the near element measurement locations due to the significant blockage created by the bluntness of the cubes. At the $\mathrm{x} / \mathrm{d}=2.75$ plane, all elements had the spanwise variation in wall shear decrease directly on either side of the centerline and gradually climb to the smooth wall value. This is due to the horseshoe vortex structure causing significant downwash in the centerline region and then an associated upwash region at the off-centerline locations as fluid is directed away from the wall. This phenomenon was confirmed by the oil flow patterns as dark high shear scoured zones near the centerline followed by deposits of tracer particles in the offcenterline lower shear regions. 
It was discovered that the cube oriented $45^{\circ}$ relative to the flow produced a stronger downstream counter-rotating horseshoe vortex pair than the cube oriented $90^{\circ}$ relative to the flow. As was previously discussed, the same result was also found to be the case in the study performed by Castro and Robins (1977). In the present research, the highest magnitude of secondary flow can be seen in the two cube elements, followed by the hemisphere, cone, Gaussian, and the large grooved element.

The strengths of the time mean horseshoe vortex structures are evaluated through the investigation of the vorticity and circulation of each vortex. The major source of vorticity at the $\mathrm{x} / \mathrm{d}=2.75$ location is below $\mathrm{y}^{+}=15$ for all elements. It was found that the maximum value of streamwise vorticity, at the $\mathrm{x} / \mathrm{d}=2.75$ measurement plane, is produced by the cube elements. The cube at $90^{\circ}$ shows the largest magnitude with the cube at $45^{\circ}$ slightly less. The rest of the elements tend to be fairly close to one another with the large grooved element having the smallest vorticity maximum. The presence of the larger grooves (2.5 times the smallest sublayer structure length scale) seems to have a noticeable effect on the flow field around the roughness element. The irregularities that are 2.5 times the smallest sublayer structure length scale not only facilitate the increase in viscous drag on the element, but they also tend to 'straighten' the flow and produce weaker vortices than the cone element with equal frontal area and similar shape. The fine grooved element (spatial variations equal to the smallest sublayer structure length scale) also increase the viscous drag on the element but do not seem to have near the effect on the production of vorticity as the larger grooves have shown.

The upstream measurement planes also reveal the presence of negative vorticity in the near wall region. The presence of these negative regions originates from the convection of wall induced vorticity caused by the interaction of the horseshoe vortex with the noslip condition at the wall. These negative regions of vorticity are smaller and of less magnitude as compared to their corresponding positive vorticity regions. These regions tend to grow in size and magnitude as the vortex structure propagates downstream. The movement and strength of the vortex structure is affected a great deal by the generation 
of secondary vorticity near the wall. In this region the near wall viscous forces cause the loss of strength in the primary vortex.

The 'peakiness' of the roughness element was found to have a direct correlation to the production of circulation, whereas the spatial smoothing does not have an immense effect on this parameter. A trend in the streamwise decay of circulation, $\Gamma /\left(U_{\tau} \sqrt{A_{f}}\right)$, was observed for the peaked elements. These elements showed a general trend in their decay that is proportional to $\approx(x / d)^{-1.12}$. Knowledge of a trend in the decay of circulation can give a qualitative idea of how the downstream flow field is behaving regarding the strength of the horseshoe vortex structure and its related secondary flow field.

A model equation has been proposed to correlate the drag of the roughness elements under scrutiny. This equation is not an a priori method for calculating the drag due to roughness. However, the development of the equation has provided a deeper understanding of the various parameters that affect the drag on roughness elements in a turbulent boundary layer. 


\section{References}

AGARD, Advisory Group for Aerospace Research and Development, 1996, "Turbulent Boundary Layers in Subsonic and Supersonic Flow," AGARD-AG-335, North Atlantic Treaty Organization, pp.25-28.

Allen, T., Brown, A.R., (2002), "Large-Eddy Simulation of Turbulent Separated Flow Over Rough Hills,” Boundary-Layer Meteorology, 102:177-198.

Arie, M., Kiya, M., Tamura, H., and Kanayama, Y., (1975a), "Flow over rectangular cylinders immersed in a turbulent boundary layer (Part 1, Correlation between pressure drag and boundary-layer characteristics),” Bull. JSME, 18, 1260-1268.

Arie, M., Kiya, M., Tamura, H., Kosugi, M., and Takaoka, K., (1975b), "Flow over rectangular cylinders immersed in a turbulent boundary layer (Part 2, Flow patterns and pressure distributions)," Bull. JSME, 18:1269-1276.

Ashill, P.R., Fulker, J.L., and Hackett, K.C., (2002), "Studies of flows induced by Sub Boundary Layer Vortex Generators (SBVGs)," AIAA Paper 2002-0968.

Ballio, F., Bettoni, C., and Franzetti, S., (1998), "A Survey of Time-Averaged Characteristics of Laminar and Turbulent Horseshoe Vortices," J. of Fluids Engineering, 120:233-242.

Bandyopadyay, P.R., and Watson, R.D., (1998), "Structure of rough-wall turbulent boundary layers," Phys. Fluids, 31:1877-1883.

Barrett, R.V., Rickards, J., Swales, C., and Brake, C.J., (1993), "Enhanced performance of a cross-coupled three-dimensional laser-Doppler anemometer for small scale flow surveys using improved alignment and operational procedures," Proc. ICIASF '93, St. Louis, USA, Sep 1993.

Castillo, L., Seo, J., Hangan, H., and Johansson, T.G., (2004), "Smooth and rough turbulent boundary layers at high Reynolds number," Experiments in Fluids, 36:759-774.

Castro, I.P., and Robins, A.G., (1977), "The flow around a surface-mounted cube in uniform and turbulent streams," J. Fluid Mech., 79:307-335.

Chesnakas, C.J., and Simpson, R.L., (1994), "Full three-dimensional measurements of the cross-flow separation region of a 6:1 prolate spheroid," Experiments in Fluids, 17:6874.

Chesnakas, C.J. (1995), "The Miniature, 3-D, Fiber-Optic, Laser Doppler Velocimeter," Internal Report, Aerospace and Ocean Engineering, Virginia Tech, November. 
Chou, J.H., and Chao, S.Y., (2000), "Branching of a horseshoe vortex around surfacemounted rectangular cylinders," Exp Fluids, 28:394-402.

Coleman, H.W., Hodge, B.K., and Taylor, R.P., (1984), "A Re-Evaluation of Schlichting's Surface Roughness Experiment,” J. Fluids Eng., 106:60-65.

Corino, C.R., and Brodkey, R.S., (1969), "A visual investigation of the wall region in turbulent flow,” J. Fluid Mech., 37 (1):1-30.

Cui, J., Patel, V.C., and Lin, C.L., (2003), "Prediction of Turbulent Flow Over Rough Surfaces Using a Force Field in Large Eddy Simulation,” J. Fluids Eng., 125:2-9.

Cutler, A.D., and Bradshaw, P., (1986), "The interaction between a strong longitudinal vortex and a turbulent boundary layer," AIAA Paper 86-1071.

Cutler, A.D., and Bradshaw, P., (1993a), "Strong vortex/boundary layer interactions, Part I. Vortices high,” Exp. Fluids, 14:321-332.

Cutler, A.D., and Bradshaw, P., (1993b), "Strong vortex/boundary layer interactions, Part II. Vortices low," Exp Fluids, 14:393-401.

Durst, F., Melling, A., and Whitelaw, J.H., (1981), "Principles and Practice of LaserDoppler Anemometry," Second ed., Acad. Press, p.32 and p. 207.

Durst, F., Jovanovic, J., and Sender, J., (1995), "LDA measurements in the near-wall region of a turbulent pipe flow," Journal of Fluid Mechanics, 295:305-335.

Dvorak, F.A., (1969), “Calculation of Turbulent Boundary Layers on Rough Surfaces in Pressure Gradient,” AIAA J., 7(9):1752-1759.

Echols, W.H. and Young, J.A., (1963), "Studies of portable air-operated aerosol generators," NRL Report, 5929.

Esmaili, H., and Piomelli, U., (1992), "Large-eddy simulation of turbulent boundary layers with embedded streamwise vortices," AIAA Paper 92-0552.

Fontaine, A. A., and Deutsch, S., (1996), "Structure of near wall turbulence downstream of a wall mounted protrusion: an interesting Reynolds stress suppression phenomena," Exp. Fluids, 20:365-376.

Gaudet, L. and Winter, K.G., (1973), "Measurement of the drag of some characteristic aircraft excrescences immersed in turbulent boundary layers," RAE Tech. Memo Aero. 1538

Gaudet, L., (1987), "Measurement of the drag of various three-dimensional excrescences in turbulent boundary layers,” Aeronautical J., April 1987, 170-182. 
George, J., (2004), "Some effects of sparsely distributed three-dimensional roughness elements on two and three-dimensional turbulent boundary layers," Ph.D. Dissertation (in progress), Aerospace and Ocean Engineering, Virginia Tech.

George, J. and Simpson, R.L., (2000), "Some effects of sparsely distributed threedimensional roughness elements on two-dimensional turbulent boundary layers," AIAA Paper 2000-0915.

George, J. and Simpson, R.L., (2001), "Structure of a two-dimensional turbulent boundary layer over a submerged cylindrical protuberance," $2^{\text {nd }}$ Turbulent Shear Flow Phenomena Symposium, Royal Institute of Technology (KTH), Stockholm, Sweden, June 27-29.

George, J. and Simpson, R.L., (2002), "Some Three-Dimensional Rough-Wall Turbulent Boundary Layers," AIAA Paper 2002-0580.

George, J. and Simpson, R.L., (2003), "Roughness Effects on Turbulent Boundary Layers-Contributions to a Conceptual Model," AIAA Paper 2003-0644.

George, J. and Simpson, R.L., (2004), "Transport-rate budgets of Reynolds stresses in 2D and 3-D rough-wall turbulent boundary layers," AIAA Paper 2004-1286.

Granville, P.S., (1987), "Three Indirect Methods for the Drag Characterization of Arbitrarily Rough Surfaces on Flat Plates," Journal of Ship Research, 31:70-77.

Gregory, N., and Walker, W.S., (1955), "The effect of transition of isolated surface excrescences in the boundary layer," R. \& M. 2779, Pt. I, 1955, Aeronautical Research Council, England.

Han, L.S., (1991), “A Mixing Length Model for Turbulent Boundary Layers Over Rough Surfaces,” Int. J. Heat/Mass Transfer, 34(8):2053-2062.

Heist, D.K., and Gouldin, F.C., (1997), "Turbulent flow normal to a triangular cylinder," J. Fluid Mech., 331:107-125.

Hoerner, S.F., (1958), “Fluid Dynamic Drag,” Published by the Author.

Hunt, J.C.R., Abell, C.J., Peterka, J.A., and Woo, H., (1978), "Kinematical studies of the flows around free or surface-mounted obstacles, applying topology to flow visualization," J. Fluid Mech. 86:179-200.

Hussein, H.J., and Martinuzzi, R.J., (1996), "Energy balance for turbulent flow around a surface mounted cube placed in a channel," Phys. Fluids, 8(3):764-780. 
Ichimaya, M., (1999), "The effect of a single roughness element on a flat plate boundary layer transition," Engineering Turbulence Modeling and Experiments - 4, W. Rodi and D. Laurence (Eds.), Elsevier Science Ltd.

Jacobson, S.A., and Reynolds, W.C., (1998), "Active control of streamwise vortices and streaks in boundary layers," J. Fluid Mech. 360:179-211.

Jimenez, J., and Moin, P., (1991), “The minimal flow unit in near-wall turbulence," J. Fluid Mech., 225:213-240.

Jimenez, J., (2004), “Turbulent Flows Over Rough Walls," Annu. Rev. Fluid Mech., 36:173-196.

Kays, W.M., (1966), "Convective Heat and Mass Transfer," McGraw-Hill Book Company, pp. 92-95.

Klebanoff, P.S., Cleveland, W.G., and Tidstrom, K.D., (1992), "On the evolution of a turbulent boundary layer induced by a three-dimensional roughness element," J. Fluid Mech. 237:101-187.

Kline S.J., Reynolds, W.C., Schraub, F.A., and Runstadler, P.W., (1967), “The structure of turbulent boundary layers," J. Fluid Mech. 30 (4):741-773.

Krogstad, P.A., (1991), "Modification of the van Driest Damping Function to Include the Effects of Surface Roughness,” AIAA Journal, 29:888-894.

Krogstad, P.A., and Antonia, R.A., (1994), "Structure of turbulent boundary layers on smooth and rough walls," J. Fluid Mech., 277:1-21.

Krogstad, P.A., and Antonia, R.A., (1999), "Surface roughness effects in turbulent boundary layers," Experiments in Fluids, 27:450-460.

Kuhl, D., (2001), "Near Wall Investigation of Three Dimensional Turbulent Boundary Layers," M.S. Thesis, Aerospace and Ocean Engineering, Virginia Tech.

Larousse, A., Martinuzzi, R., and Tropea, C., (1992), "Flow Around Surface-Mounted, Three-Dimensional Obstacles," Selected Papers of the $8^{\text {th }}$ Turbulent Shear Symposium, Springer-Verlag, Berlin.

Lee, J., (1996), "Modeling Boundary Layer Flows Over Rough Surfaces Using a Modified Chien k-e Turbulence Model, “ AIAA Paper 96-0384.

Logan, E., and Lin, S-H., (1982), "Wakes from arrays of buildings," NASA CR-170666, 1982. 
Lowe, K.T., (2004), “The Comprehensive Laser-Doppler Velocimeter for Fully-Resolved Instantaneous Gradient Measurements in Turbulent Flows," Ph.D. Dissertation (in progress), Aerospace and Ocean Engineering, Virginia Tech.

Martinuzzi, R.J., and AbuOmar, M., (2003), "Study of the flow around surface-mounted pyramids," Experiments in Fluids, 34:379-389.

Martinuzzi, R.J., and Havel, B., (2000), "Turbulent Flow Around Two Interfering Surface-Mounted Cubic Obstacles in Tandem Arrangement," J. Fluids Eng., 122:24-31.

Martinuzzi, R.J., and Tropea, C., (1993), "The flow around surface-mounted, prismatic obstacles placed in a fully developed channel flow," Trans. ASME, J. Fluids Eng., 115:85-92.

Mason, P.J., and Morton, B.R., (1987), "Trailing vortices in the wakes of surfacemounted obstacles," J. Fluid Mechanics, 175:247-293.

Mazouz, A., Labraga, L., and Tournier, C., (1994), "Behaviour of the Reynolds stress on rough walls," Experiments in Fluids, 17:39-44.

Mehta, R.D., Shabaka, I.M.M.A., Shibl, A., and Bradshaw, P., (1983), "Longitudinal vortices imbedded in turbulent boundary layers," AIAA Paper 83-0378.

Miyake, Y., Tsujimoto, K., and Agata, Y., (2000), "A DNS of a Turbulent Flow in a Rough-Wall Channel Using Roughness Elements Model,” JSME Int. J., 43(2):233-242.

Nikuradse, J., (1933), "Stromunsgesetze in rauhen rohen," Forschungshefte, Vol. 36, VDI, also NACA TM 1292.

Okamoto, T., Yagita, M., and Kataoka, S., (1977), "Flow past cone placed on flat plate," Bull. JSME, 20:329-336.

Olcmen, S.M., and Simpson, R.L., (1995), "A five-velocity component laser-Doppler velocimeter for measurements of a three-dimensional turbulent boundary layer," Measurement Science and Technology, 6:702-716.

Olcmen, S.M., Simpson, R.L., and Goody, M. (1998), “An Experimental Investigation of Two-Point Correlations in Two and Three-Dimensional Turbulent Boundary Layers," AIAA paper $98-0427,36^{\text {th }}$ Aerospace Sciences Meeting and Exhibit, Reno, NV, January 12-15.

Olcmen, S.M., Simpson, R.L., and George, J., (1999), "Experimental study of high Reynolds number $\left(\mathrm{Re}_{\theta}=23200\right)$ two and three-dimensional turbulent boundary layers," AIAA Paper 99-0553. 
Papangelou, A., (1992), "Vortex shedding from slender cones at low Reynolds numbers," J. Fluid Mechanics, 242:299-321.

Patel, V.C., (1998), "Perspective: Flow at High Reynolds Number and Over Rough Surfaces-Achilles Heel of CFD,” J. Fluids Eng., 120:434-444.

Pauley, W. R., and Eaton, J. K., (1988), "Experimental study of the development of longitudinal vortex pairs embedded in a turbulent boundary layer," AIAA J., 26(7):816823.

Pauley, W. R., and Eaton, J. K., (1989), "Boundary layer turbulence structure in the presence of embedded streamwise vortex pair," Proc. Seventh Symposium on Turbulent Shear Flows, Stanford University, August 21-23, 1989.

Perry, A.E., Lim, K.L., and Henbest, S.M., (1987), "An experimental study of the turbulence structure in smooth and rough-wall boundary layers," J. Fluid Mech., 177:437-466.

Perry, A.E., Scholfield, W.H., and Joubert, P.N., (1969), "Rough wall turbulent boundary layers," J. Fluid Mech., 37:383-413.

Pope, S.B., (2000), “Turbulent Flows," Cambridge University Press, The Edinburgh Building, Cambridge, CB2 2RU, UK.

Raupach, M.R., Antonia, R.A., and Rajagopalan, S., (1991), "Rough-wall turbulent boundary layers," Appl. Mech. Rev., 44(1):1-25.

Robinson, S.K., (1990), Sakamoto, H., (1985), "Aerodynamic forces acting on a rectangular prism placed vertically in a turbulent boundary layer," J. Wind Eng. Ind. Aero., 18:131-151.

Sakamoto, H., Moriya, M., Taniguchi, S., and Arie, M., (1982), "The form drag of threedimensional bluff bodies immersed in turbulent boundary layers," Trans. ASME J. Fluids Eng. 104:326-334.

Saripalli, K. R., Simpson, R. L., (1980), "Investigation of Blown Boundary Layers With an Improved Wall Jet System,” NASA Contractor Report 3340, pp. 15-27.

Savory, E., and Toy, N., (1988), "The Separated Shear Layers Associated with Hemispherical Bodies in Turbulent Boundary Layers," Journal of Wind Engineering and Industrial Aerodynamics, 28:291-300.

Schetz, J.A., (1993), "Boundary Layer Analysis," Prentice-Hall, Inc., A Pearson Education Company, Upper Saddle River, NJ. 
Schofield, W.H., and Logan, E., (1990), "Turbulent shear flow over surface mounted obstacles," Trans. ASME J. Fluids Eng., 112, 376-385.

Schumann, U., (1977), "Realizability of Reynolds-stress turbulence models," The Physics of Fluids, 20(5):721-725.

Sedney, R., (1973), "A survey of the effects of small protuberances on boundary-layer flows,” AIAA J., June 1973, 782-792.

Shabaka, I.M.M.A., Mehta R.D., and Bradshaw P., (1985), "Longitudinal vortices imbedded in turbulent boundary layers. Part 1," Single vortex. J. Fluid Mech. 155, 37.

Shim, J., Chung, J., and Lee, K.D., (2000), “A Comparison of Turbulence Modeling in Flow Analysis of Iced Airfoils,” AIAA Paper, A00-39840.

Shim, J., Chung, J., and Lee, K.D., (2001), "A Computational Investigation of Ice Geometry Effects on Airfoil Performances," AIAA Paper, A01-0540.

Sigal, A., and Danberg, J.E., (1990), "New correlation of roughness density effect on the turbulent boundary layer," AIAA J., 28:554-556.

Simpson, R.L., (1973), “A Generalized Correlation of Roughness Density Effects on the Turbulent Boundary Layer,” AIAA J., 11(2):242-244.

Smith, E. J., Rife, M. C., and Devenport, W. J., (1990), "Investigation of the Small Boundary Layer Tunnel," Aerospace and Ocean Engineering Department, Virginia Polytechnic Institute and State University Report, VPI-AOE-175, July17.

Souders, D.T., and Hirt, C.W., (2002), "Modeling Roughness Effects in Open Channel Flows," Flow Science, Inc., TN60, pp.1-10.

Sousa, J.M.M., (2002), “Turbulent flow around a surface-mounted obstacle using 2D-3C DPIV,” Experiments in Fluids 33:854-862.

Spaid, F.W., (1967), "The Drag of Small Protuberances Immersed in a Turbulent Boundary Layer," AIAA J., 7(4):780-782.

Spalart, P.R., (1988), "Direct simulation of a turbulent boundary layer up to $\mathrm{Re}_{\theta}=1410$," Journal of Fluid Mechanics, 187:61-98.

Stukel, J.J., Hopke, P.K., and Nourmohammadi, K., (1984), "Turbulent Air Flow Over Rough Surfaces: Mean Flow Parameters,’ J. Fluids Eng., 106:405-409.

Sutton, O.G., (1953), "Micrometeorology-A Study of Physical Processes in the Lowest Layers of the Earth's Atmosphere,” McGraw-Hill Book Company, pp.276-295. 
Stewart, D. (2004), "Effects of Sparsely Distributed Three-dimensional Roughness Elements in a High Reynolds Number Turbulent Boundary Layer," M.S. Thesis (in progress), Aerospace and Ocean Engineering, Virginia Tech.

Tani, I., (1961), "Effect of two-dimensional and isolated roughness on laminar flow," Boundary Layer and Flow Control, its Principles and Applications, 2, Lachmann, G.V. (Ed.), Pergamon Press, 1961, 637-656.

Tian, Q., (2003), "Some Features of Tip Gap Flow Fields of a Linear Compressor Cascade," M.S. Thesis, Aerospace and Ocean Engineering, Virginia Tech.

Tang, G., (2004), "Measurements of the Tip-Gap Turbulent Flow Structure In a Low Speed Compressor Cascade," Ph.D. Dissertation, Aerospace and Ocean Engineering, Virginia Tech.

Tani, I., (1961), "Effect of two-dimensional and isolated roughness on laminar flow. Boundary Layer and Flow Control, its Principles and Applications. 2," Lachmann, G. V. (Ed.), Pergamon Press, 1961, 637-656.

Taniguchi, S., Sakamoto, H., and Arie, M., (1981), "Flow around circular cylinders of finite height placed vertically in turbulent boundary layers," Bull. JSME, 24, 37-44.

Taylor, R.P., Scaggs, W.F., and Coleman, H.W., (1988), "Measurement and Prediction of the Effects of Nonuniform Surface Roughness on Turbulent Flow Friction Coefficients," J. Fluids Engineering, 110:380-384.

Vosper, S.B., Castro, I.P., Snyder, W.H., and Mobbs, S.D., (1999), "Experimental studies of strongly stratified flow past three-dimensional orography," J. Fluid Mech., 390:223249.

Waigh, D.R., and Kind, R.J., (1998), "Improved Aerodynamic Characterization of Regular Three-Dimensional Roughness,” AIAA J., 36(6):1117-1119.

Wendt, B.J., Greber, I., and Hingst, W.R., (1992), “The structure and development of streamwise vortex arrays embedded in a turbulent boundary layer," AIAA Paper 920551 .

Westphal, R. V., Eaton, J. K., and Pauley, W. R., (1985), "Interaction between a vortex and a turbulent boundary layer in a streamwise pressure gradient," Turbulent Shear Flows 5, F. Durst, B.E. Launder, J.L. Lumley, F.W. Schmidt, and J.H. Whitelaw (Eds.), Springer Verlag, New York, 266-277.

Westphal, R. V., Pauley, W. R., and Eaton, J. K., (1987), "Interaction between a vortex and a turbulent boundary layer, Part 1: Mean flow evolution and turbulence properties," NASA TM-88361. 
Wieghardt, K., (1946), "Increase in turbulent skin friction caused by surface irregularities," MAP R \& T No. 103, Translation of FB 1563, Zentralle Wissenschaftliche Berifte, 1942.

Wooding, R.A., Bradley, E.F., and Marshall, J.K., (1973), "Drag Due to Regular Arrays of Roughness Elements of Varying Geometry," Boundary Layer Methodology, 5:285308.

Yao, C. S., and Lin, J.C., (2002), "Flow-field measurement of device-induced embedded streamwise vortex on a flat plate," AIAA Paper 2002-3162.

Yoshizawa, A., Itoh, S., and Itoh, K., (2003), "Plasma and fluid turbulence: theory and modeling, Series in plasma physics," Institute of Physics Publishing, Bristol and Philadelphia.

Young, A.D., and Paterson J.H., (1981), “Aircraft excrescence drag," AGARDograph No. 264. 


\section{Appendix A Trip Arrangements}

\section{A.1 Introduction}

In order to obtain the desired momentum thickness Reynolds number, $\operatorname{Re}_{\theta} \approx 7500$, and boundary layer thickness, $\delta \approx 39 \mathrm{~mm}$, at the tunnel measurement location, various trip arrangements were studied and carefully considered. The purpose of investigating the numerous and varied trip arrangements was to match the flow conditions in the Virginia Tech Aerospace and Ocean Engineering (AOE) Department small boundary layer tunnel with the conditions already present in the Virginia Tech AOE Department larger boundary layer tunnel. A few important aspects to note about the trip arrangement selection process are as follows. First, momentum thickness, $\theta$, needs to be large enough, yet the boundary layer thickness, $\delta$, and shape factor, $\mathrm{H}=\delta^{*} / \theta$, also need to fit into reasonable bounds. Another factor to consider is that the boundary layer needs to be relaxed enough downstream of the trip to be a two dimensional equilibrium flow at the measurement location.

All boundary layer profiles were taken by means of a flattened-mouth boundary layer impact probe with a y-direction probe opening equal to $0.127 \mathrm{~mm}$. Profiles were taken in even logarithmically spaced $\mathrm{y}$-direction increments; the nearest wall reading was assumed to be at half of the outside diameter of the probe, $0.165 \mathrm{~mm}$ from the wall. The entire setup was fastened on top of the wind tunnel to alleviate any wandering during the measurements. The vertical traverse system is powered by an electric motor which turns a screw with a thread ratio equal to 40 threads/inch. Pressure readings were taken with a Series 475 Mark III Digital Manometer by the Dwyer Co. with an accuracy of \pm 0.01 inches of water. This manometer was calibrated and checked for accuracy with an actual inclined manometer before measurements were taken. The flow conditions in the tunnel are the same as those described in Chapter 2; a zero pressure gradient flow with a freestream speed equal to $27.5 \mathrm{~m} / \mathrm{s}$ at a temperature of $25^{\circ} \mathrm{C} \pm 0.56^{\circ} \mathrm{C}$. Since this is a nominally zero-pressure-gradient flow, the location of the static pressure port will not have a significant effect on the data. Thus, all profiles used a single static pressure port 
that is described in Chapter 2. All profiles are measured in the center of the tunnel at port \#20 unless otherwise specified. Please see Chapter 2 for actual measurement locations related to Port \#'s.

The results of the measurement system used, although good and very repeatable, should only be taken as a solid starting point in order to obtain more refined data with the LDV system. At least one point in the viscous sublayer was obtained in all boundary layer profiles taken. A y-shift value was applied to each profile and was found in an iterative fashion by matching the viscous sublayer relation of $\mathrm{U}^{+}=\mathrm{y}^{+}$for $\mathrm{y}^{+}<10$ with the measured data. The experimental setup with the boundary layer probe and traverse system can be seen in Figure A.1.

A total of twelve trip configurations were studied including various combinations of a grooved plate, sandpaper, and various sized square bars. Figures of the trip arrangements and their related abbreviations can be found in Figure A.2 and Table A.1, respectively. Please note that in Table A.1, the word sandpaper is abbreviated and denoted by s.p. for brevity. The same sandpaper was used in all trip arrangements. The sandpaper was 20 grit silicon-carbide, manufactured by Norton, with a width equal to 8 inches. The final selected trip arrangement was Trip Arrangement 11 (TA 11). This configuration and its relation to the flow field is discussed in more detail in Chapter 2. 
Table A.1: Trip arrangements and related abbreviations

\begin{tabular}{|c|c|}
\hline Description of Trip Arrangement (TA) & Abbreviation \\
\hline 13" s.p. on floor & TA 1 \\
\hline 13" s.p. on floor + 5" s.p. in contraction & TA 2 \\
\hline 18" s.p. on floor + 5" s.p. in contraction & TA 3 \\
\hline Slotted Plate (19 slots) & TA 4 \\
\hline Slotted Plate (19 slots) + 0.25" bar & TA 5 \\
\hline Slotted Plate (19 slots) + 0.125" bar & TA 6 \\
\hline Slotted Plate (19 slots) + 0.1875" bar & TA 7 \\
\hline 12" s.p. on floor + 0.1875" bar & TA 8 \\
\hline 13" s.p. on floor + 0.382" bar & TA 9 \\
\hline 18" s.p. on floor + 0.125" bar & TA 10 \\
\hline 18" s.p. on floor + two 0.125" bars & TA 11 \\
\hline 18" s.p. on floor + 0.125" and 0.1875" bars & TA 12 \\
\hline
\end{tabular}

\section{A.2 Configurations and Related Data}

For each trip arrangement, the boundary layer thickness, $\delta$, momentum thickness, $\theta$, shape factor, $\mathrm{H}=\delta^{*} / \theta$, and the friction velocity, $\mathrm{U}_{\tau}$, are calculated and presented in table format. Here $\delta$ is defined as the height at which the local streamwise velocity is equal to $0.99 \mathrm{U}_{\mathrm{e}}$. For each measured profile the momentum thickness, $\theta$, and the displacement thickness, $\delta^{*}$, were calculated using the following equations, respectively (Kays, 1966).

$\theta=\int_{0}^{\delta}\left(1-\frac{U}{U_{e}}\right) \frac{U}{U_{e}} d y$

$\delta^{*}=\int_{0}^{\delta}\left(1-\frac{U}{U_{e}}\right) d y$

In order to obtain the friction velocity, the use of the Ludwieg-Tillman equation (Equation A.3) from Pope (2000) for skin friction, $\mathrm{C}_{\mathrm{f}}$, was employed,

$$
\frac{C_{f}}{2}=0.123(10)^{-0.678 H}\left(\frac{U_{e} \theta}{v}\right)^{-0.268}
$$


This equation is well known to give good results in favorable and adverse pressure gradient flows with no separation for $10^{3}<\operatorname{Re}_{\theta}<30\left(10^{3}\right)$. As is discussed in Chapter 2 this flow is a zero pressure gradient flow with an $\mathrm{Re}_{\theta}$ within these bounds. The friction velocity in a zero-pressure-gradient $2 \mathrm{D}$ boundary layer can then be calculated using the following relation.

$U_{\tau}=U_{e} \sqrt{\frac{C_{f}}{2}}$

In order to alleviate unnecessary measurements for each trip arrangement, it was helpful to calculate the momentum thickness along the streamwise direction using known data for $\mathrm{U}_{\mathrm{e}}(\mathrm{x})$ and $\theta$ at one $\mathrm{x}$ location. Kays (1966) method for finding the momentum thickness at various streamwise, $\mathrm{x}$, locations within a turbulent boundary layer was therefore employed and can be seen below.

$\left.\theta^{\frac{5}{4}} U_{e}^{4.11}\right|_{x_{o}} ^{x_{1}}=\int_{x_{o}}^{x_{1}}(0.016) U_{e}^{3.86} v^{\frac{1}{4}} d x$

If $\mathrm{U}_{\mathrm{e}}(\mathrm{x})$ is measured between the downstream end of the roughness $\left(\mathrm{x}_{\mathrm{o}}\right)$ and the desired location of boundary layer measurements $\left(\mathrm{x}_{1}\right)$, then one can calculate $\theta$ at any point $\mathrm{x}$ between $\mathrm{x}_{0}$ and $\mathrm{x}_{1}$, due to the fact that $\theta$ is known at $\mathrm{x}_{1}$.

Please note in the following trip arrangements with bars: The first bar listed is located at the contraction exit and the second bar is always located 2 inches downstream of the contraction exit. In all trip arrangements with square bars, the bars extend the entire way across the tunnel floor and are fastened to the floor with super glue.

\section{A.2.1 Trip Arrangements 1-3}

A piece of silicon-carbide Norton 20 grit sandpaper, 8 inches wide, was used as the only method for tripping the boundary layer in the first three cases analyzed. This 8 inch wide section of sandpaper was placed in the center of the 9.5 inch wide test section. The TA 1 was simply a section of sandpaper, 13 inches in streamwise length, attached to the test section floor at the contraction exit. Rubber cement was employed to attach the sandpaper to the center of the tunnel floor for its durability and easy cleanup 
characteristics. The TA 2 and TA 3 used the same sandpaper as TA 1 but the sandpaper was extended 5 inches into the contraction and, in the case of TA 3, 5 inches farther into the test section. The results for these trip arrangements can be seen in Table A.2 and Figure A.3. For actual x-locations associated with the Port \#'s please refer to Chapter 2.

Table A.2: Results from trip arrangements 1-3

\begin{tabular}{|c|c|c|c|c|c|}
\hline Trip Arrangement & Location (Port \#) & $\delta(\mathbf{m})$ & $\mathbf{R e}_{\theta}$ & $\mathbf{H}=\delta^{* / \boldsymbol{\theta}}$ & $\mathbf{U}_{\tau}(\mathbf{m} / \mathbf{s})$ \\
\hline TA 1 (Run 1) & 20 & 0.0391 & 6632 & 1.36 & 0.90 \\
\hline TA 1 (Run 2) & 20 & 0.0403 & 6782 & 1.37 & 0.89 \\
\hline TA 2 & 20 & 0.0339 & 6339 & 1.34 & 1.01 \\
\hline TA 3 & 20 & 0.0376 & 6817 & 1.33 & 1.02 \\
\hline
\end{tabular}

It is important to note that the boundary layer thickness for TA 2 and TA 3 actually dropped from the values shown by TA 1 . This is due to the fact that there is a thickness to the sandpaper which in turn acts as a trip itself when placed at the beginning of the test section. Whereas for TA 2 and TA 3, the sandpaper was extended into the contraction and actually provided a smoother transition into the test section; thus the initial 'front edge trip' was not felt, which decreased the boundary layer thickness. The shape of all boundary layer profiles do match nicely with what is expected thus giving added confidence in the measurement scheme.

\section{A.2.2 Trip Arrangements 4-7}

Trip arrangements TA4-TA7 made use of an aluminum plate located in the spanwise center of the test section floor with the following dimensions: 12 inches long, 8 inches wide, and 0.125 inches thick. Spanwise slots, 0.25 inches wide by .0625 inches deep, were machined across the entire width of the plate. These 19 slots started 3 inches from the front of the plate and continued downstream with a spacing equal to 0.3125 inches. At the front and rear sections of the plate, a slope of $7.13^{\circ}$ by 1 inch long was machined across the width of the plate. This ensured smooth transition between the tunnel floor and the slotted plate. This trip arrangement was placed at the contraction exit and was fastened to the tunnel floor via countersunk screws. TA 4 was simply the slotted plate itself. TA 5-7 were the slotted plate along with a 0.25 inch, 0.125 inch, and 0.1875 inch 
square bar, respectively, placed directly in front of the plate. Results from the boundary measurements are given in Tables A.3 and A.4 and Figures A.4 and A.5.

Table A.3: Results from trip arrangements 4-6

\begin{tabular}{|c|c|c|c|c|c|}
\hline Trip Arrangement & Location (Port \#) & $\delta(\mathbf{m})$ & $\mathbf{R e}_{\theta}$ & $\mathbf{H}=\delta^{*} / \boldsymbol{\theta}$ & $\mathbf{U}_{\tau}(\mathbf{m} / \mathbf{s})$ \\
\hline TA 4 & 20 & 0.0307 & 5683 & 1.34 & 1.04 \\
\hline TA 5 & 20 & 0.0516 & 8466 & 1.26 & 1.04 \\
\hline TA 6 & 20 & 0.0429 & 7462 & 1.31 & 1.02 \\
\hline
\end{tabular}

Table A.4: Results from trip arrangement 7

\begin{tabular}{|c|c|c|c|c|c|}
\hline Trip Arrangement & Location (Port \#) & $\delta(\mathbf{m})$ & $\mathbf{R e}_{\theta}$ & $\mathbf{H}=\delta^{*} / \boldsymbol{\theta}$ & $\mathbf{U}_{\tau}(\mathbf{m} / \mathbf{s})$ \\
\hline TA 7 & 20 & 0.0493 & 8271 & 1.31 & 1.01 \\
\hline TA 7 & 13 & 0.0412 & 7049 & 1.28 & 1.07 \\
\hline TA 7 & 15 & 0.0486 & 7620 & 1.28 & 1.06 \\
\hline
\end{tabular}

As would be expected, TA 5 has the greatest boundary layer thickness due the larger bar placed in the flow. Figure A.4 also shows that there is not a wake region present for this particular flow regime. Measurements were taken at ports 13 and 15 because the actual measurement location for the LDV measurements is between these two ports; see Chapter 2. The TA 7 is close to the desired boundary layer properties, but the shape factor is not as close to the desired value of $\mathrm{H} \approx 1.34$ and the boundary layer thickness is slightly too high.

\section{A.2.3 Trip Arrangements 8-9}

Trip arrangements 8-9 both utilized the same sandpaper as discussed previously. The TA 8 has 12 inches of sandpaper attached to the tunnel floor along with a 0.1875 inch bar placed directly in front of the sandpaper at the contraction exit. The TA 9 is slightly different in that it has 13 inches of sandpaper attached to the tunnel floor along with a 0.382 inch bar placed directly in front of the sandpaper at the contraction exit. Results from TA 8-9 can be seen in Table A.5 and Figure A.6.

Table A.5: Results from trip arrangements 8-9

\begin{tabular}{|c|c|c|c|c|c|}
\hline Trip Arrangement & Location (Port \#) & $\boldsymbol{\delta}(\mathbf{m})$ & $\mathbf{R e}_{\theta}$ & $\mathbf{H}=\delta^{* / \boldsymbol{\theta}}$ & $\mathbf{U}_{\tau}(\mathbf{m} / \mathbf{s})$ \\
\hline TA 8 & 13 & 0.0405 & 6934 & 1.28 & 1.07 \\
\hline TA 9 & 20 & 0.0546 & 7379 & 1.23 & 1.06 \\
\hline
\end{tabular}


Again, these configurations yielded a significantly lower shape factor than desired along with a larger boundary layer. As can be seen in Figure A.6, TA 9 does not exhibit a normal wake region, thus indicating a possible merging of the ceiling and floor boundary layers.

\section{A.2.4 Trip Arrangements 10-11}

In order to ensure a relaxed and two-dimensional turbulent boundary layer in equilibrium, more sandpaper was utilized as well as smaller initial bar trips. Sandpaper is an excellent boundary layer trip due to the fact that it has a significant amount of small randomly distributed roughness elements that produce drag and ensure good mixing. To this end, TA 10 consists of 18 inches of the same sandpaper discussed previously along with a 0.125 inch square bar placed directly in front of the sandpaper at the contraction exit. TA 11 is exactly the same as TA 10 but with another 0.125 inch square bar placed 2 inches downstream of the contraction exit on the tunnel floor. The placement of the second 0.125 inch square bar was determined by giving the flow sufficient distance to reattach before applying another step. Results from the boundary measurements can be seen in Table A.6 and Figure A.7.

Table A.6: Results from trip arrangements 10-11

\begin{tabular}{|c|c|c|c|c|c|}
\hline Trip Arrangement & Location (Port \#) & $\boldsymbol{\delta}(\mathbf{m})$ & $\mathbf{R e}_{\theta}$ & $\mathbf{H}=\delta^{* / \boldsymbol{\theta}}$ & $\mathbf{U}_{\tau}(\mathbf{m} / \mathbf{s})$ \\
\hline TA 10 & 14 & 0.0313 & 6181 & 1.33 & 1.04 \\
\hline TA 11 & 14 & 0.0350 & 6500 & 1.33 & 1.04 \\
\hline
\end{tabular}

Measurements of these trip arrangements were made at Port \#14 due to the fact that this location is the closest to the actual measurement location for the LDV data. Figure A.7 shows the boundary layer profiles for TA 10-11 to be smooth as well as have well defined $\log$ and wake regions. The shape factor for these configurations is very near the desired value of $H \approx 1.34$. The boundary layer thickness and $\operatorname{Re}_{\theta}$ are slightly low, but as can be seen in Chapter 2, more refined LDV measurements give the desired results in all areas of describing the boundary layer. Thus, TA 11 was the final trip arrangement that was selected for use in all measurements of the roughness elements. 


\section{A.2.5 Trip Arrangement 12}

The TA 12 is identical to TA 11 except the second square bar in TA 11 is replaced with a 0.1875 inch square bar in TA 12 . The results from the TA 12 boundary layer profiles can be seen in Table A.7 and Figure A.8.

Table A.7: Results from trip arrangement 12

\begin{tabular}{|c|c|c|c|c|c|}
\hline Trip Arrangement & Location (Port \#) & $\delta(\mathbf{m})$ & $\mathbf{R e}_{\theta}$ & $\mathbf{H}=\delta * / \theta$ & $\mathbf{U}_{\tau}(\mathbf{m} / \mathbf{s})$ \\
\hline TA 12 & 14 & 0.0383 & 6728 & 1.31 & 1.05 \\
\hline TA 12 & 15 & 0.0379 & 6610 & 1.31 & 1.05 \\
\hline TA 12 & 20 & 0.0428 & 7892 & 1.32 & 1.01 \\
\hline
\end{tabular}

TA 12 gave results that were very similar to TA 11, the most noticeable differences being in the shape factor, $\mathrm{H}$, and boundary layer thickness, $\delta$. Considering all effects, TA 11 and TA 12 are remarkably close as should be expected due to the fact that only a slight difference exists in the second bar for each case. 


\section{A.3 Appendix A Figures}

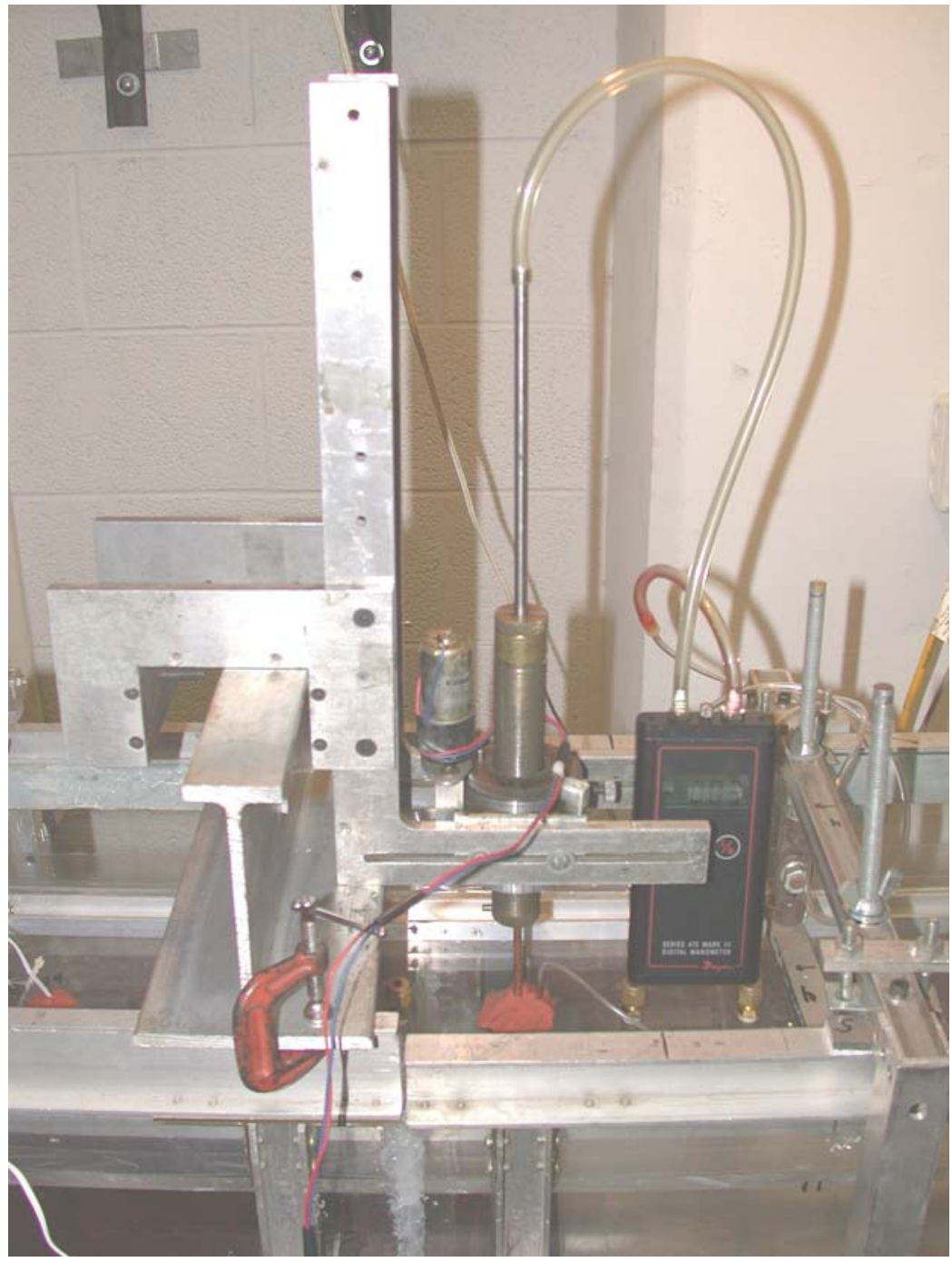

Figure A.1: Boundary layer impact probe and traverse system 


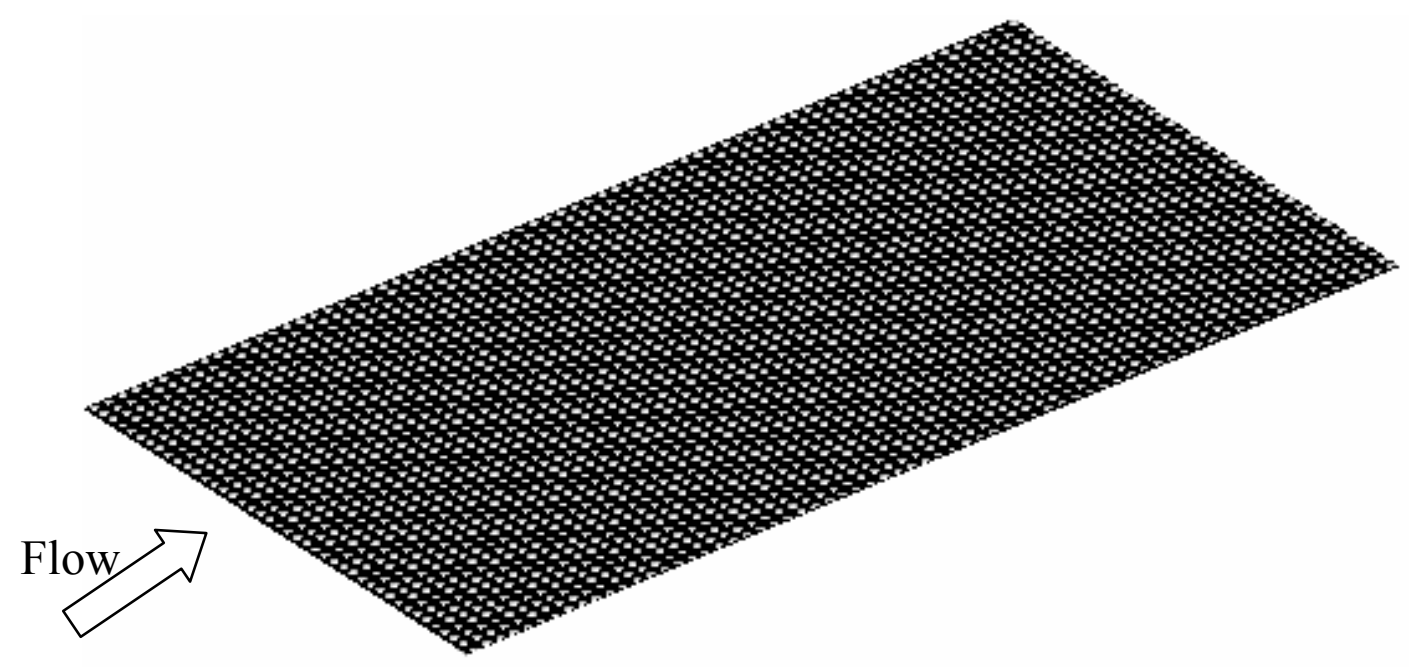

13 inches of sandpaper (TA 1)
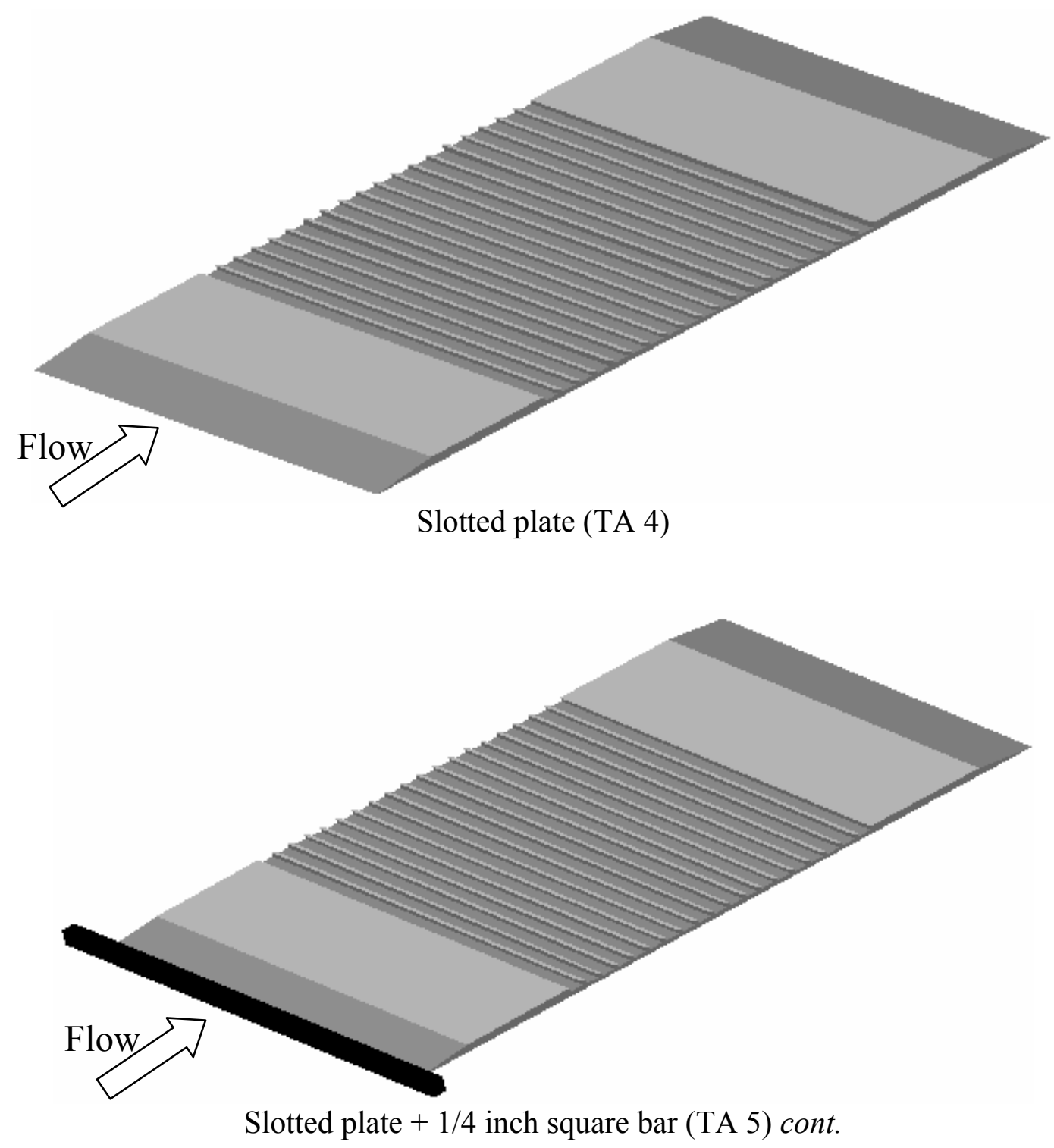

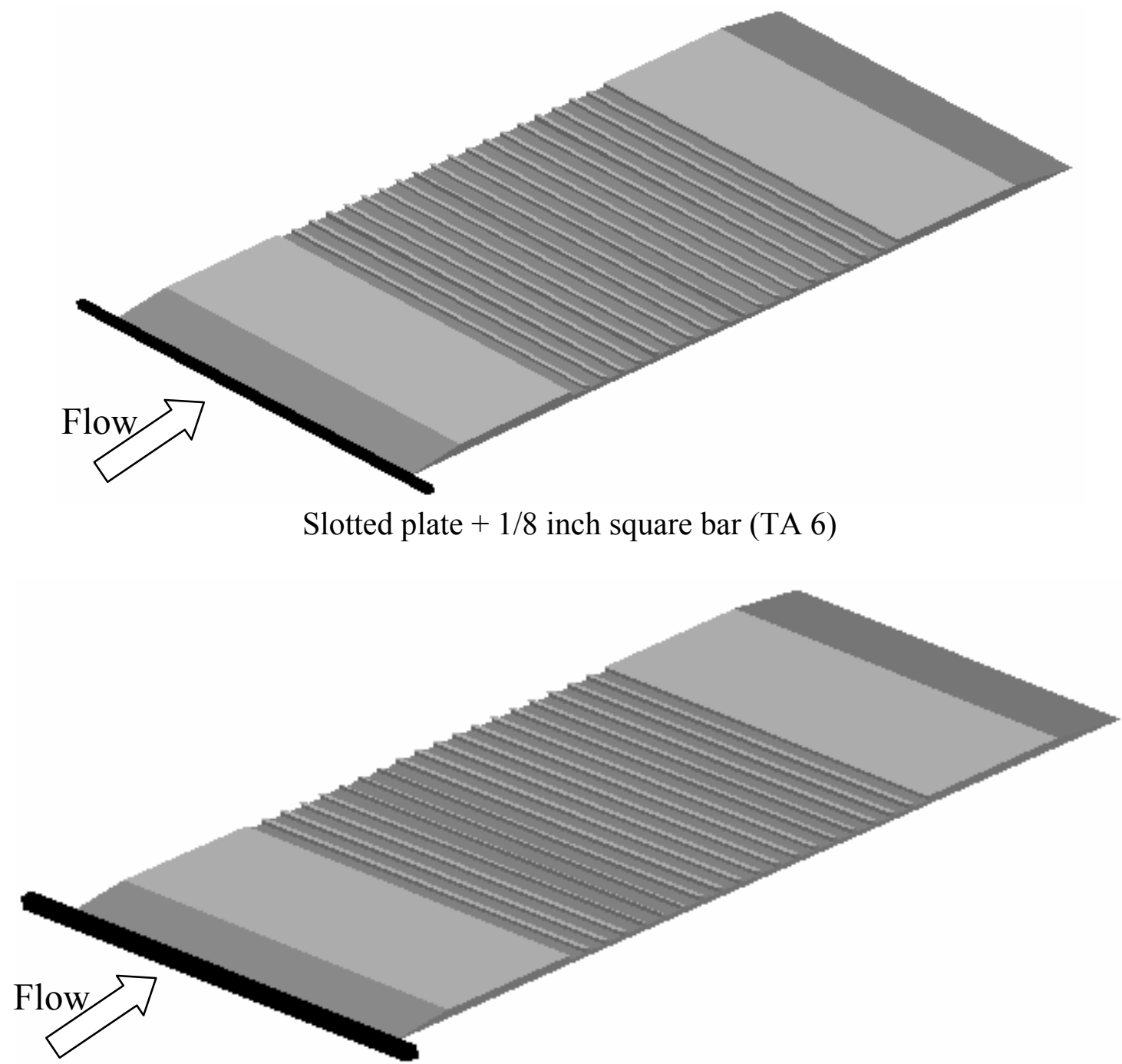

Slotted plate $+3 / 16$ inch square bar (TA 7)

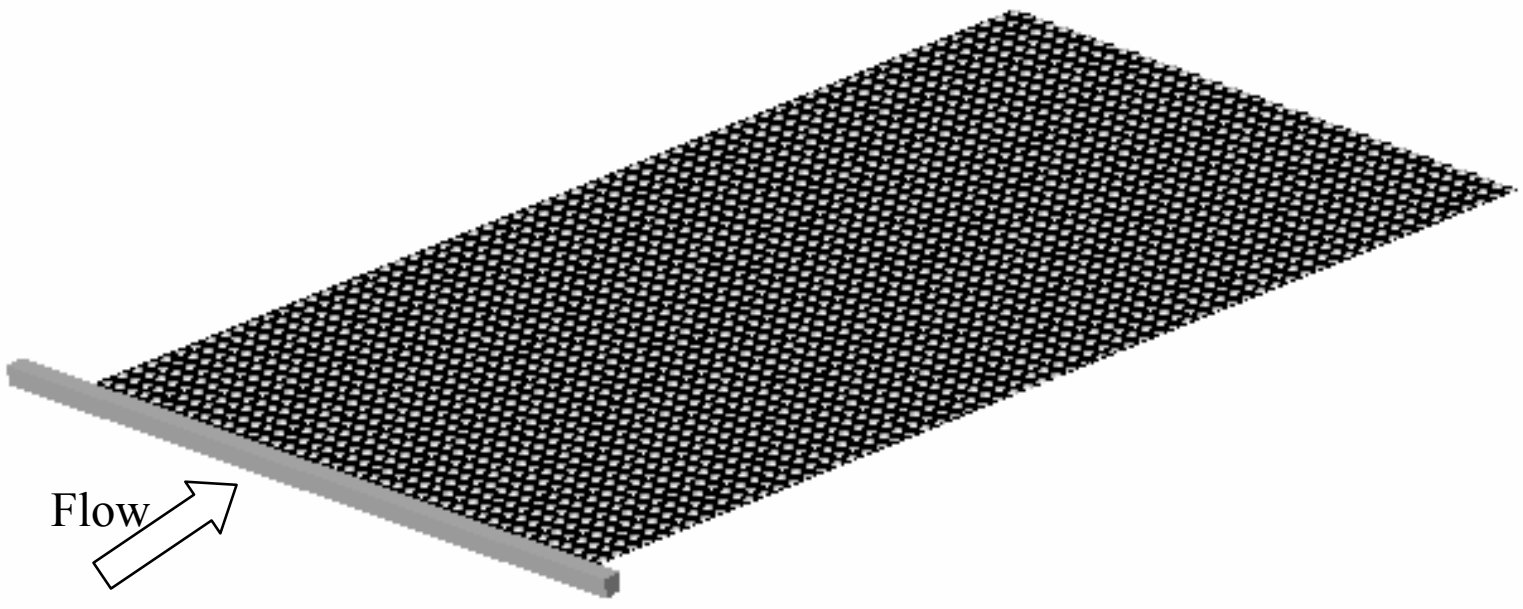

12 inches of sandpaper $+3 / 16$ inch square bar (TA 8) cont. 


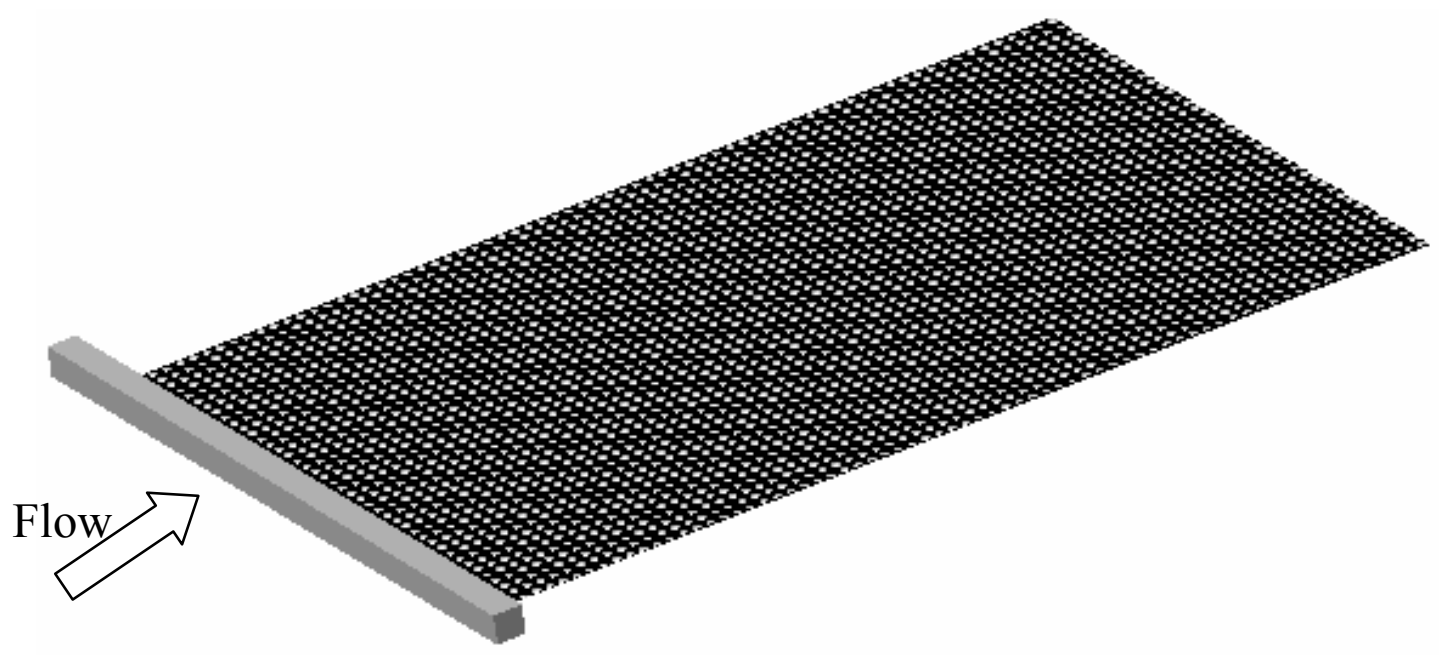

13 inches of sandpaper +0.382 inch square bar (TA 9)
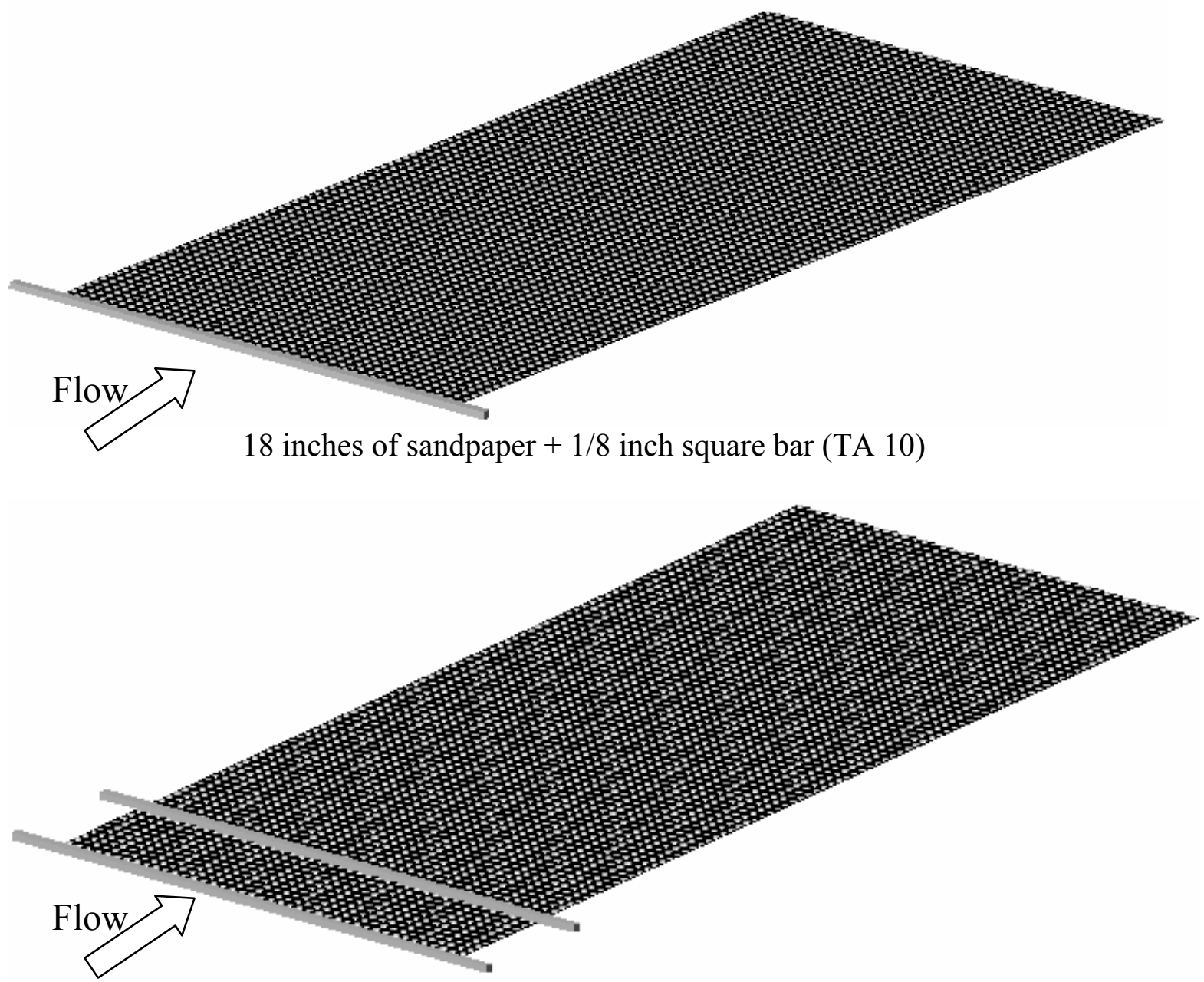

18 inches of sandpaper + two 1/8 inch square bars (TA 11) cont. 


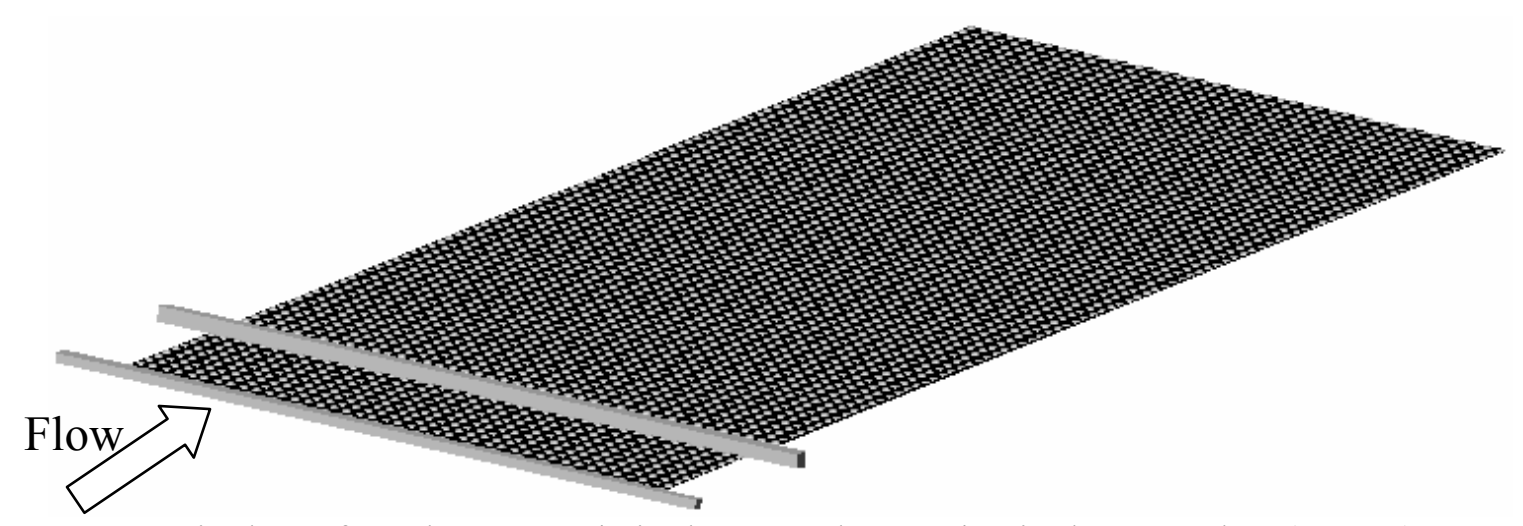

18 inches of sandpaper $+1 / 8$ inch square bar $+3 / 16$ inch square bar (TA 12)

Figure A.2: Tested trip arrangements: TA 1-TA 12 (Note that TA 2 and TA 3 are not shown)

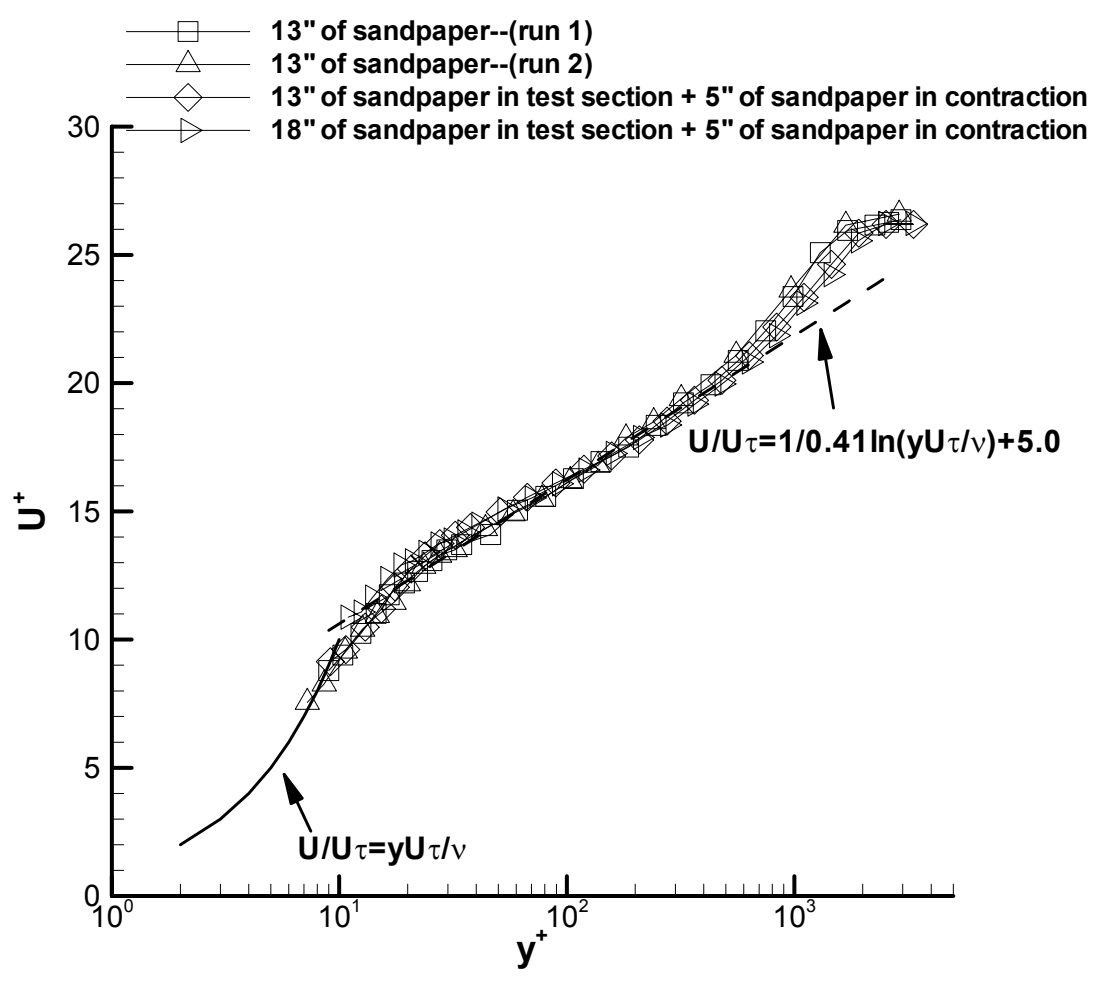

Figure A.3: Streamwise velocity profiles of trip arrangements TA 1 - TA 3 


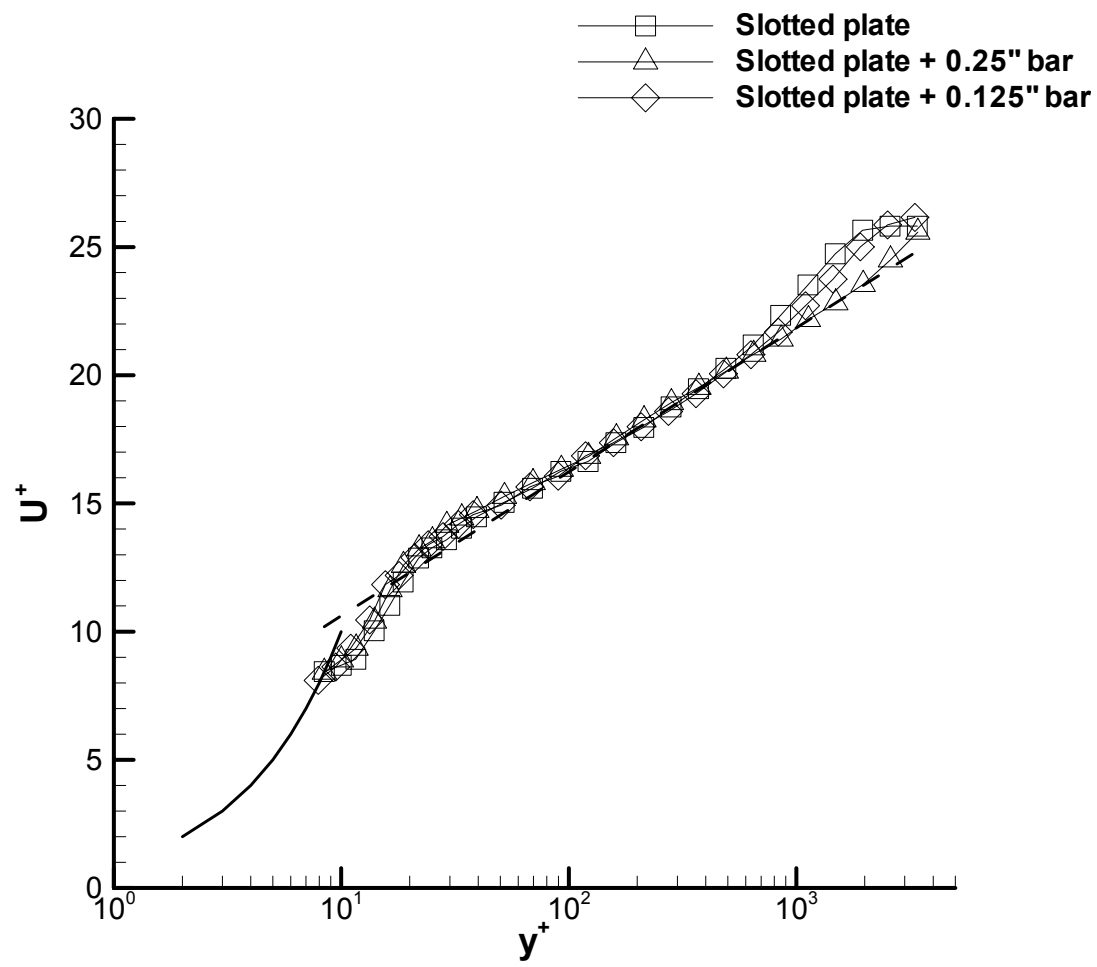

Figure A.4: Streamwise velocity profiles of trip arrangements TA 4 - TA 6

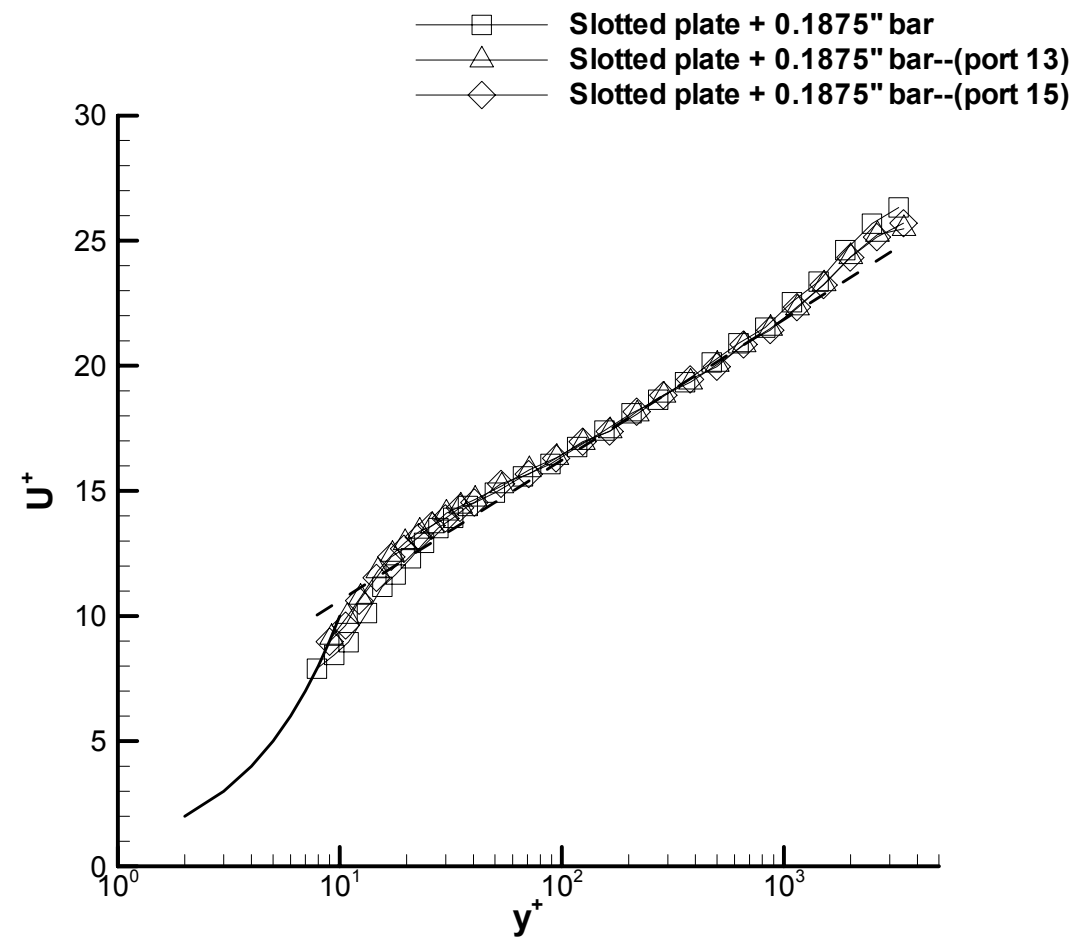

Figure A.5: Streamwise velocity profiles of trip arrangement TA 7 


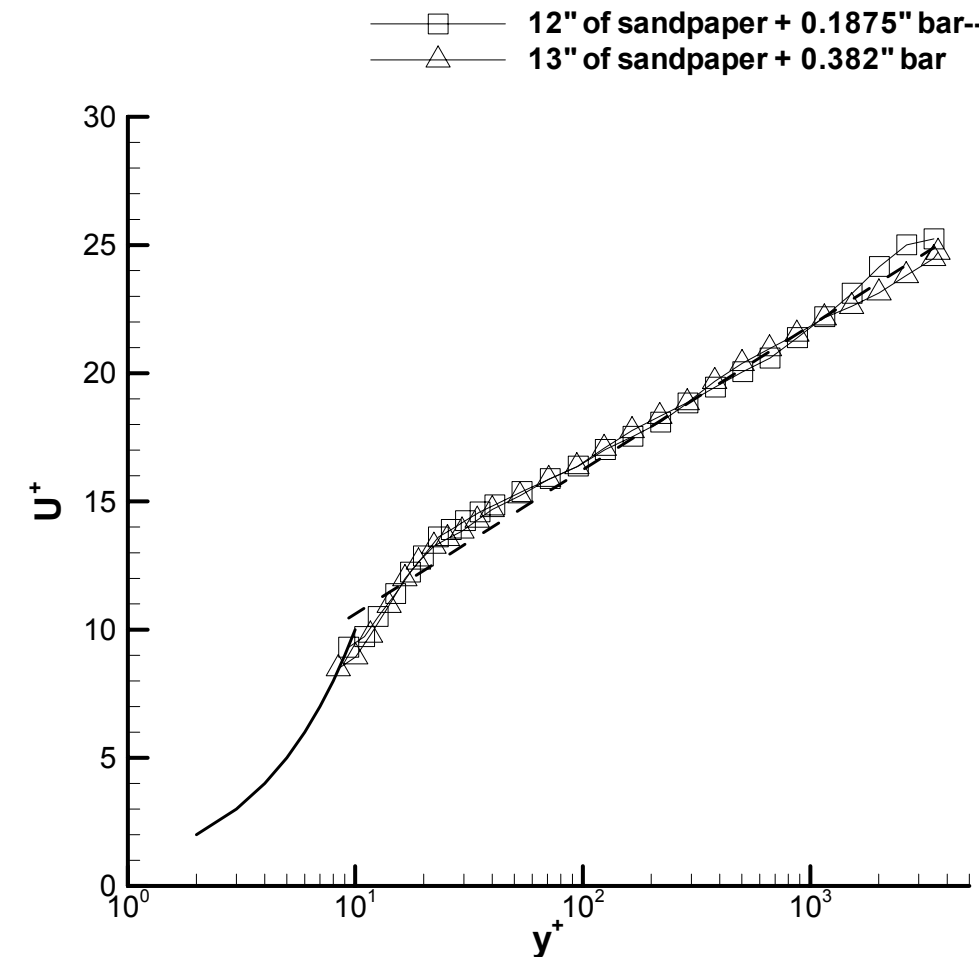

Figure A.6: Streamwise velocity profiles of trip arrangement TA 8 - TA 9

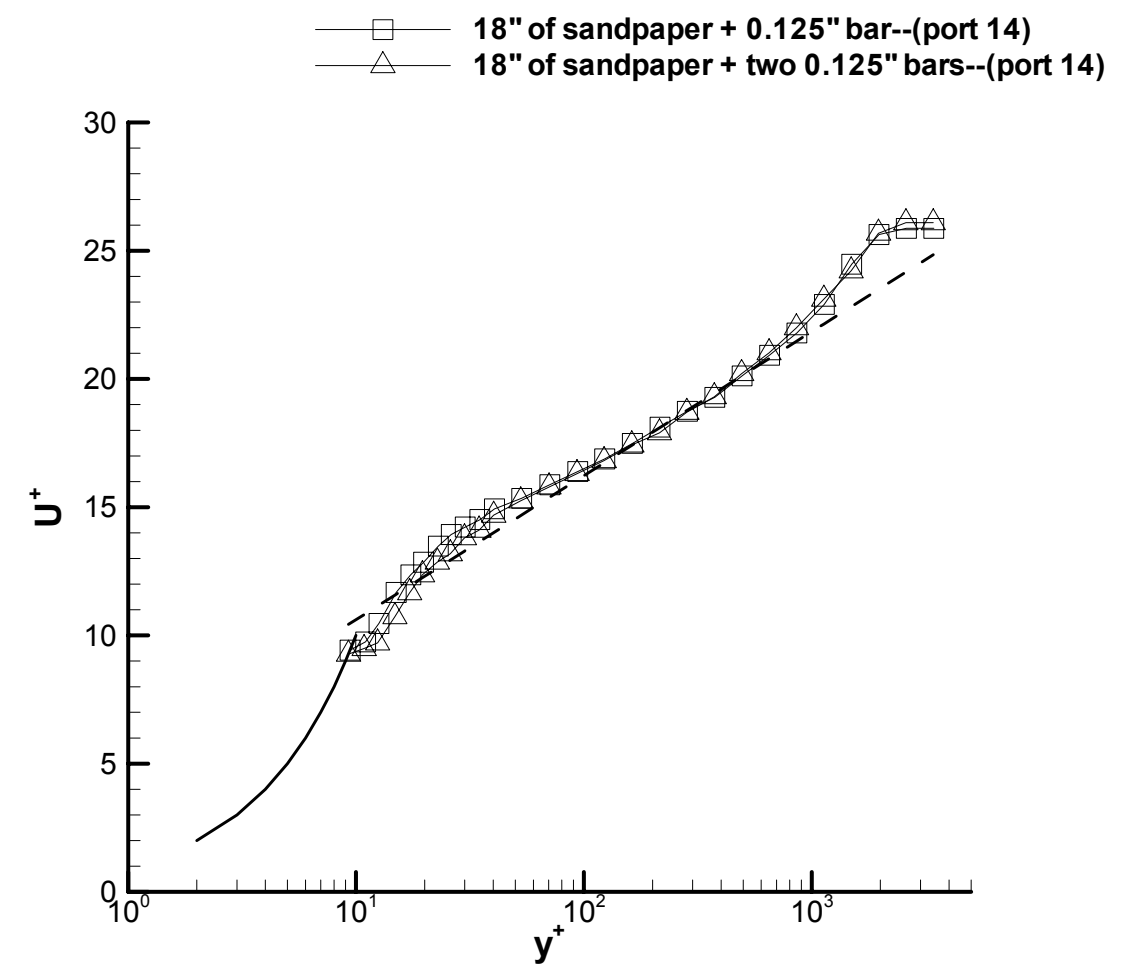

Figure A.7: Streamwise velocity profiles of trip arrangements TA 10 - TA 11 


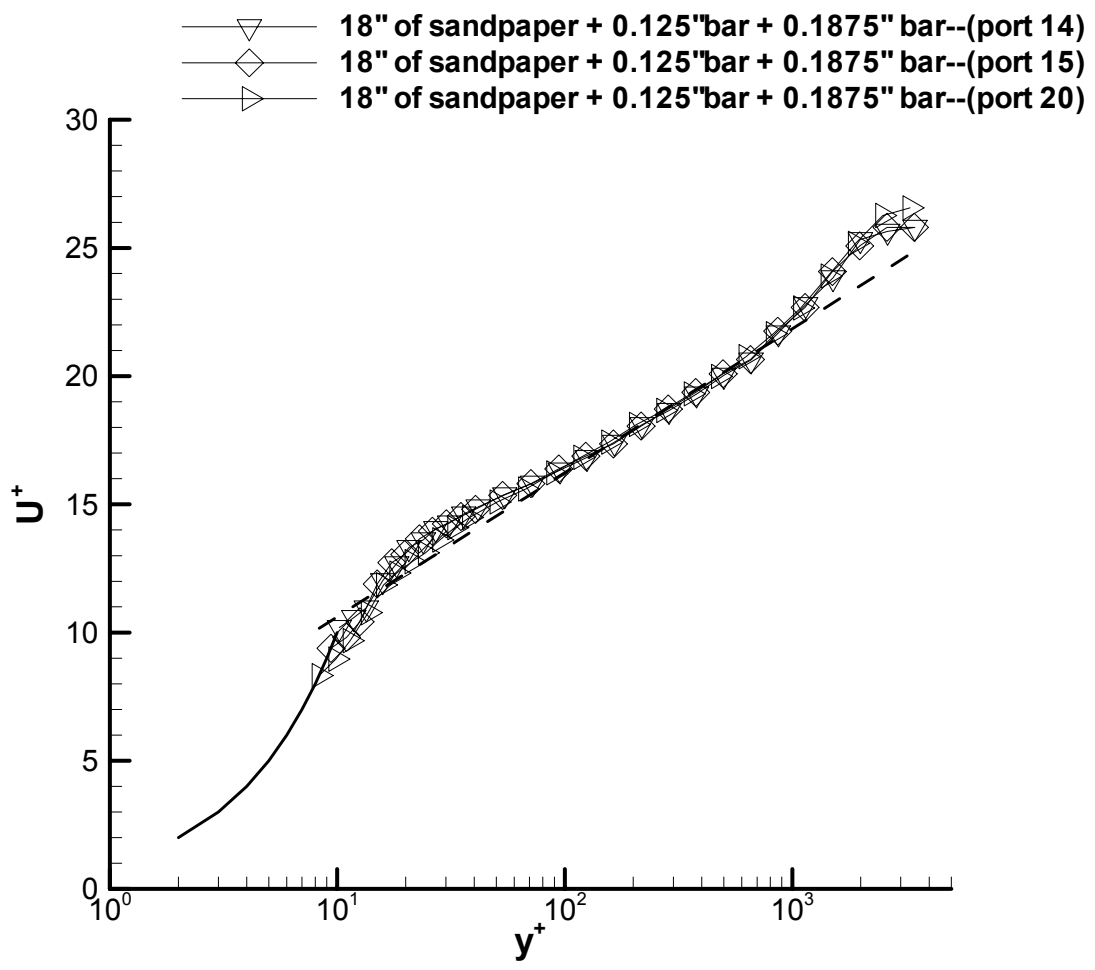

Figure A.8: Streamwise velocity profiles of trip arrangement TA 12 


\section{Appendix B Single Element Drag Analysis}

\section{B.1 Derivation of Drag Equation}

The following derivation of the equation used to compute the drag on the single isolated roughness elements is taken directly from George and Simpson (2004). The control volume $(\mathrm{CV})$ used in this analysis can be found in Figure B.1. The CV encloses one half of the element domain; thus, it is important to keep in mind that the drag obtained will be $1 / 2$ of the total drag due to the element. In order to obtain the drag due to the element, one needs to start from the mass conservation and momentum principles. Each principle is applied to the CV with the element (E) and again without the element (w/oE). The drag due to the element is then determined to be the difference between the individual drag terms with and without the element. Please note that the subscripted numbers in the equations refer to the respective face of the control volume.

The CV is selected so that one side, Face 4, is along the line of symmetry of the element in order to obtain a spanwise velocity component equal to zero on this face. Consequently, $\vec{V} \cdot \vec{n}=0$ on this particular face which simplifies the analysis, where $\vec{V}=(U+u) \dot{i}+(V+v) \vec{j}+(W+w) \vec{k}$. The length of the $\mathrm{CV}$ is taken to be $7.75 \mathrm{~d}$, where Face 2 is located a distance of 2.75 d downstream of the element and Face 1 is taken to be at the upstream side of the element and is referred to as $-2 \mathrm{D}$, i.e. twodimensional flow at a negative x-direction location (see Figure B.1). Full pressure recovery is expected for all elements at this downstream distance based on previous work done by Martinuzzi and Tropea (1993). From this analysis it was shown that in the flow field downstream of a cube, there was full pressure recovery at a distance equal to $2.25 \mathrm{~d}$. Therefore, full pressure recovery can be expected in all of the cases under investigation due to the fact that the other elements should recover even sooner than the cube. The location of Face 3, $-\mathrm{Z}_{2} \mathrm{D}$, is taken as the distance at which the boundary layer sees no effect from the element's presence and is the same as the smooth wall 2D boundary layer. 
The location of the top face of the $\mathrm{CV}, \mathrm{y}_{\text {top }}$, is located at a distance far enough from the wall where the $\mathrm{U}$ and $\mathrm{V}$ velocity values show no effect of the element being present. For consistency, this distance is taken to be the boundary layer thickness, $\delta$.

Start by applying the steady flow conservation of mass principle to the CV with and without the element.

$\oiint \rho(\vec{V} \cdot \vec{n}) d A=0$,

where $\mathrm{dA}$ is the elemental area.

After applying Equation (B.1) to the CV with and without the element, the time averaged results are Equations (B.2) and (B.3) below respectively.

With the element present, Eqn. (B.2):

$-\left.\rho \int_{0}^{y_{\text {top }}} \int_{0}^{-z_{2 D}} U_{1} d z d y\right|_{E, 1}+\left.\rho \int_{0}^{y_{\text {top }}} \int_{0}^{-z_{2 D}} U_{2} d z d y\right|_{E, 2}+\left.\rho \int_{0}^{y_{t o p}} \int_{-2 D}^{2.75 d} W_{3} d x d y\right|_{E, 3}+\left.\rho \int_{0}^{-z_{2 D}} \int_{-2 D}^{2.75 d} V_{t o p} d x d z\right|_{E, \text { top }}=0$

Without the element present, Eqn. (B.3):

$-\left.\rho \int_{0}^{y_{\text {top }}} \int_{0}^{-z_{2 D}} U_{1} d z d y\right|_{w / o E, 1}+\left.\rho \int_{0}^{y_{\text {top }}} \int_{0}^{-z_{2} D} U_{2} d z d y\right|_{w / o E, 2}+\left.\rho \int_{0}^{y_{\text {top }}} \int_{-2 D}^{2.75 d} W_{3} d x d y\right|_{w / o E, 3}+\left.\rho \int_{0}^{-z_{2 D}} \int_{-2 D}^{2.75 d} V_{t o p} d x d z\right|_{w / o E, t o p}=0$

Next, apply the momentum principle to the CV knowing that the change in the $\mathrm{x}$ momentum is balanced with the sum of the forces in the x-direction.

$\oiint \rho(\vec{V} \cdot \vec{i})(\vec{V} \cdot \vec{n}) d A=-\oiiint \frac{d p}{d x} d \sigma-D_{x}$

Note that $\mathrm{dA}$ is the elemental area, $\mathrm{d} \sigma$ is the elemental volume, and $\mathrm{D}_{\mathrm{x}}$ is the $\mathrm{x}-$ component of the drag acting on the control volume. Following the time-averaging of Eqn. (B.4), we get:

With the element present, Eqn. (B.5):

$$
\begin{aligned}
& -\left.\rho \int_{0}^{y_{\text {top }}} \int_{0}^{-z_{2} D}\left(U_{1}^{2}+u_{1}^{2}\right) d z d y\right|_{E, 1}+\left.\rho \int_{0}^{y_{\text {top }}} \int_{0}^{-z_{2 D}}\left(U_{2}^{2}+u_{2}^{2}\right) d z d y\right|_{E, 2}+\left.\rho \int_{0}^{y_{\text {top }}} \int_{-2 D}^{2.75 d}\left(U_{3} W_{3}+\overline{u_{3} w_{3}}\right) d x d y\right|_{E, 3} \\
& +\left.\rho \int_{0}^{-z_{2 D}} \int_{-2 D}^{2.75 d}\left(U_{\text {top }} V_{\text {top }}+\overline{u_{\text {top }} v_{\text {top }}}\right) d x d z\right|_{E, \text { top }}=-\left.\frac{d p}{d x}\left[\text { Volume }_{C V}\right]\right|_{E}-D_{x_{-} E}
\end{aligned}
$$

Without the element present, Eqn. (B.6): 


$$
\begin{aligned}
& -\left.\rho \int_{0}^{y_{\text {top }}} \int_{0}^{-z_{2} D}\left(U_{1}^{2}+u_{1}^{2}\right) d z d y\right|_{w / o E, 1}+\left.\rho \int_{0}^{y_{\text {top }}-z_{2}} \int_{0}^{2}\left(U_{2}{ }^{2}+u_{2}^{2}\right) d z d y\right|_{w / o E, 2}+\left.\rho \int_{0}^{y_{\text {top }}} \int_{-2 D}^{2.75 d}\left(U_{3} W_{3}+\overline{u_{3} w_{3}}\right) d x d y\right|_{w / o E, 3} \\
& +\left.\rho \int_{0}^{-z_{2 D}} \int_{-2 D}^{2.75 d}\left(U_{t o p} V_{t o p}+\overline{u_{t o p} v_{t o p}}\right) d x d z\right|_{w / o E, t o p}=-\left.\frac{d p}{d x}\left[\text { Volume }_{C V}\right]\right|_{w / o E}-D_{x_{-} w / o E}
\end{aligned}
$$

Now, subtract Eqn. (B.6) from Eqn. (B.5) to obtain the following equation, Eqn. (B.7).

Keep in mind that the $\mathrm{U}$ and $\overline{u^{2}}$ profiles are identical on the front face of the CV, Face 1.

Thus, the two instances with and without the element will cancel each other. For reasons discussed previously, the pressure gradient in the streamwise direction is also negligible.

This in turn, reduces the respective term to zero.

$$
\begin{aligned}
& -\left.\rho \int_{0}^{y_{t o p}} \int_{0}^{-z_{2}}\left(\left.U_{2}{ }^{2}\right|_{w / o E}-\left.U_{2}^{2}\right|_{E}\right) d z d y\right|_{2}-\left.\rho \int_{0}^{y_{t o p}} \int_{0}^{-z_{2} D}\left(\left.\overline{u_{2}^{2}}\right|_{w / o E}-\left.\overline{u_{2}^{2}}\right|_{E}\right) d z d y\right|_{2}-\left.\rho \int_{0}^{y_{t o p}} \int_{-2 D}^{2.75 d}\left(\left.\left(U_{3} W_{3}\right)\right|_{w / o E}-\left.\left(U_{3} W_{3}\right)\right|_{E}\right) d x d y\right|_{3} \\
& \left.-\left.\rho \int_{0}^{y_{\text {top }}} \int_{-2 D}^{2.75}\left(\left.(\overline{u w})_{3}\right|_{w / o E}-\left.(\overline{u w})_{3}\right|_{E}\right) d x d y\right|_{3}-\rho \int_{0}^{-z_{2 D}} \int_{-2 D}^{2.75}\left(\left.\left(U_{\text {top }} V_{\text {top }}\right)\right|_{w / o E}-\left(U_{\text {top }} V_{\text {top }}\right)\right)_{E}\right)\left.d x d z\right|_{\text {top }} \\
& =\left.\rho \int_{0}^{-z_{2 D}} \int_{-2 D}^{2.75 q}\left(\left.(\overline{u v})_{t o p}\right|_{w / o E}-\left.(\overline{u v})_{t o p}\right|_{E}\right) d x d z\right|_{t o p}-D_{x_{-} E}+D_{x_{-} w / o E}
\end{aligned}
$$

The next step in the analysis is to multiply Eqns. (B.2) and (B.3) by their respective $U$ velocity at the top face of the $\mathrm{CV}, \mathrm{U}_{\text {top. }}$ Following this step, rearrange to obtain Eqns. (B.8) and (B.9) below.

$$
\begin{aligned}
& \left.U_{t o p}\right|_{E}\left[\left.\rho \int_{0}^{-z_{2 D}} \int_{-2 D}^{2.75 d} V_{t o p} d x d z\right|_{E, t o p}=\left.\rho \int_{0}^{y_{\text {top }}} \int_{0}^{-z_{2} D} U_{1} d z d y\right|_{E, 1}-\left.\rho \int_{0}^{y_{\text {top }}} \int_{0}^{-z_{2 D}} U_{2} d z d y\right|_{E, 2}-\left.\rho \int_{0}^{y_{\text {top }}} \int_{-2 D}^{2.75 d} W_{3} d x d y\right|_{E, 3}\right] \\
& \left.U_{\text {top }}\right|_{w / o E}\left[\left.\rho \int_{0}^{-z_{2 D}} \int_{-2 D}^{2.75 d} V_{t o p} d x d z\right|_{w / o E, \text { top }}=\left.\rho \int_{0}^{y_{\text {top }}} \int_{0}^{-z_{2 D}} U_{1} d z d y\right|_{w / o E, 1} ^{y_{\text {top }}}-\left.\rho \int_{0}^{-z_{2} D} \int_{0}^{y_{2}} U_{2} d z d y\right|_{w / o E, 2}-\left.\rho \int_{0}^{y_{\text {top }}} \int_{-2 D}^{2.75 d} W_{3} d x d y\right|_{w / o E, 3}\right]
\end{aligned}
$$

Due to the fact that the respective streamwise velocities at the top of the $\mathrm{CV}, \mathrm{U}_{\text {top }}$, are constant, we can substitute Eqns. (B.8) and (B.9) into the fifth term on the LHS of Eqn. (B.7) above. It is also of importance to note that $\left.U_{t o p}\right|_{w / o E}=\left.U_{t o p}\right|_{E}$ for all faces of the CV 
and for the remainder of the derivation will simply be called $U_{\text {top }}$. In doing so, the following result is obtained:

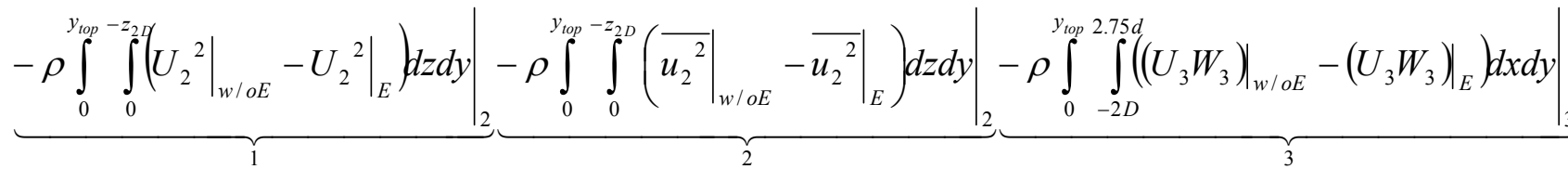

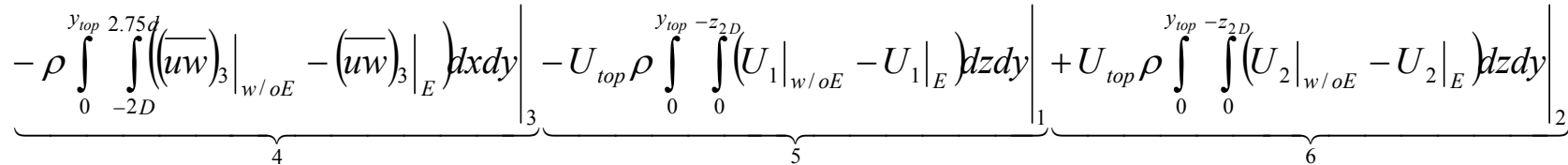

$\underbrace{\left.U_{t o p} \rho \int_{0}^{y_{t o p}} \int_{-2 D}^{2.75 d}\left(\left.W_{3}\right|_{w / o E}-\left.W_{3}\right|_{E}\right) d x d y\right|_{3}}_{7}=\underbrace{\left.\rho \int_{0}^{-z_{2 D}} \int_{-2 D}^{2.75 q}\left(\left.(\overline{u v})_{t o p}\right|_{w / o E}-\left.(\overline{u v})_{t o p}\right|_{E}\right) d x d z\right|_{t o p}}_{8} \underbrace{-D_{x_{-} E}+D_{x_{-} w / o E}}_{-\Delta D_{E}}$

In order to simplify Eqn. (B.10), it is significant to note that for large distances away from the centerline, the flow will be two-dimensional and thus there will be no spanwise velocity component. Therefore, terms 3,4 , and 7 will be zero. Term 5 will also reduce to zero due to the fact that the $\mathrm{U}$ velocity profiles on Face 1 are identical for both cases as was discussed previously. Term 8 will also reduce to zero due to the fact that at the top of the CV, $\left.(\overline{u v})_{t o p}\right|_{w / o E}=\left.(\overline{u v})_{t o p}\right|_{E}$. Thus, it can be seen then that the only velocity profiles needed to calculate the drag are the profiles located at Face 2. Following the simplification we arrive at Eqn. (B.11) below:

$$
\begin{aligned}
& \Delta D_{E}=\rho \int_{0}^{y_{\text {top }}} \int_{0}^{-z_{2}}\left(\left.U_{2}{ }^{2}\right|_{w / o E}-\left.U_{2}{ }^{2}\right|_{E}\right) d z d y+\rho \int_{0}^{y_{\text {top }}-z_{2 D}} \int_{0}^{y_{\text {top }}-z_{2 D}}\left(\left.\overline{u_{2}^{2}}\right|_{w / o E}-\left.\overline{u_{2}^{2}}\right|_{E}\right) d z d y \\
& -U_{t o p} \rho \int_{0}^{2} \int_{0}^{2}\left(\left.U_{2}\right|_{w / o E}-\left.U_{2}\right|_{E}\right) d z d y
\end{aligned}
$$

As discussed previously, the total drag due to the element's presence in the boundary layer can now be defined as twice that of Eqn. (B.11). The drag due to the element can also be presented as a drag coefficient in the following way.

$$
C_{d}=\frac{2 \Delta D_{E}}{\frac{1}{2} \rho U_{e}^{2} A}=\frac{2 \rho \int_{0}^{y_{t o p}} \int_{0}^{-z_{2}}\left(\left.U_{2}{ }^{2}\right|_{w / o E}-\left.U_{2}^{2}\right|_{E}\right)+\left(\left.\overline{u_{2}^{2}}\right|_{w / o E}-\overline{\left.u_{2}^{2}\right|_{E}}\right)-U_{t o p}\left(\left.U_{2}\right|_{w / o E}-\left.U_{2}\right|_{E}\right) d z d y}{\frac{1}{2} \rho U_{e}^{2} A}
$$


This drag coefficient is normalized by the free-stream dynamic pressure multiplied by an elemental area term. This area is taken to be either the frontal or wetted area of the element, $A_{f}$ or $A_{w}$. Note that a designation is made for the frontal or wetted area when presented. 


\section{B.2 Appendix B Figures}

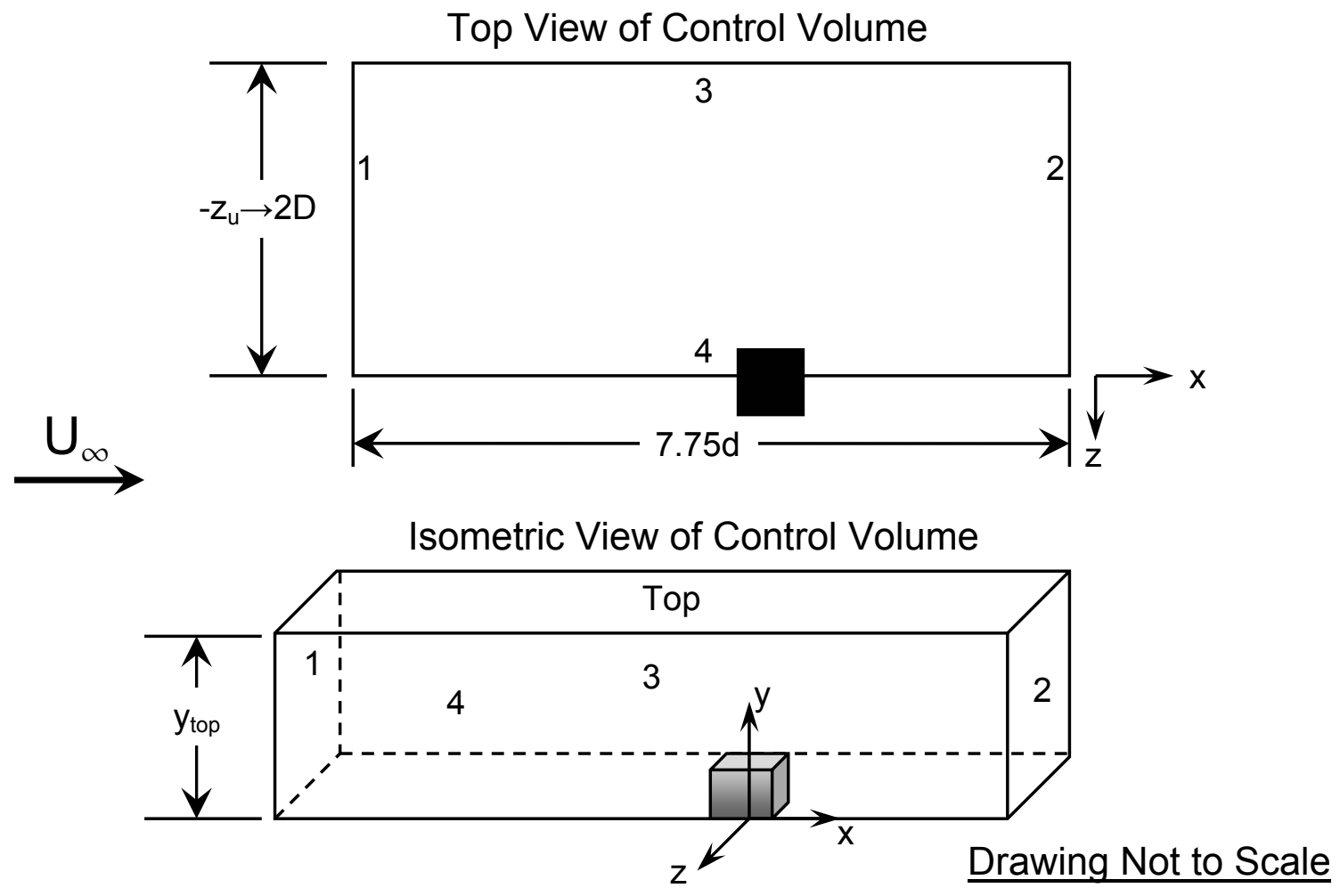

Figure B.1: Views of control volume used in drag analysis 


\section{Appendix C Uncertainty Analysis}

\section{C.1 Wall Location}

An iterative procedure was utilized in order to find the location of the wall for each profile. A spectrum analyzer and an oscilloscope were used to find the wall position with respect to the measurement volume. When the measurement volume is centered on the surface of the glass disc (wall) a strong signal is sent to the spectrum analyzer and subsequently to the oscilloscope for viewing. Careful examination of the calculated yshift values from all profiles, the traverse system, and the size of the measurement volume, leads to the conclusion that the uncertainty is on the order of the measurement volume diameter. Consequently, the uncertainty is $\pm 50 \mu \mathrm{m}$.

\section{C.2 Alignment of Probe with Element}

The alignment of the LDV probe with the center of each element was done in an iterative fashion, and the procedure is discussed in Chapter 2, Section 2.6.2. By watching the beam profiles change along the sides of the element and the wall of the tunnel (when the measurement volume was higher than the element height) it was possible to have an uncertainty in positioning of \pm 60 microns in the $\mathrm{x}$ and $\mathrm{z}$ directions.

\section{C.3 Velocities}

The uncertainties associated with this LDV system have been evaluated by Kuhl (2001) and Tang (2004). Through these previous investigations, the biases associated with the alignment of the probe and the measurement of the beam angles were found to be negligible. The fringe bias, geometric bias, angular bias, and velocity gradient bias are negligible due to the fact that the measurement volume of the LDV is small and nearly spherical. These prior studies also show that the velocity bias is negligible for this system. The measured signal and related data processing of that signal can include different uncertainty effects. Likewise, Tang (2004) discovered that the effect of finite 
transit time broadening on the LDV was negligible. Olcmen et al. (1998) investigated the instrument broadening effects of the LDV system. The same Macrodyne signal processors that were used in this research were analyzed and proven to have negligible bandwidth broadening effects.

Once the beam angles were measured for the current setup, a two-dimensional boundary layer profile was taken in order to validate the LDV system. Near-wall DNS data as well as the law of the wall for a two-dimensional turbulent boundary layer were used to validate the LDV measurements. As is shown in Figure 2.11, the systematic error for the LDV system has been taken into account and alleviated. Consequently, the uncertainty associated with the LDV system was analyzed by looking at the measurement procedure as a whole. The uncertainties in the velocities obtained from the LDV system are calculated following the same procedure used by Olcmen and Simpson (1995). Two profiles were taken on two consecutive days at the same location inside the wind tunnel. The location was $116.72 \mathrm{~cm}$ downstream of the contraction exit and at the spanwise center of the wind tunnel test section. The profiles taken were in a smooth wall turbulent boundary with no roughness element present. The uncertainties of the velocity values are calculated using Chauvenet's criterion to calculate the standard deviation. Equation C.1 was used to give 21:1 odds uncertainties in the data.

$$
\frac{d_{\max }}{\sigma}=1.15
$$

Here, $d_{\max }$ is the average of the half of the differences between two data values and $\sigma$ is the standard deviation. The constant 1.15 is chosen to give 21:1 odds uncertainties calculated as $\pm 2 \sigma$. The calculated uncertainties can be seen in Table C. 1 below. 
Table C.1: Uncertainties in measured quantities

\begin{tabular}{|ll|ll|}
\hline$U / U_{\tau}$ & \pm 0.28 & $\overline{u^{2} v} / U_{\tau}^{3}$ & \pm 0.19 \\
\hline$V / U_{\tau}$ & \pm 0.07 & $\overline{u^{2} w} / U_{\tau}^{3}$ & \pm 0.19 \\
\hline$W / U_{\tau}$ & \pm 0.17 & $\overline{v^{2} w} / U_{\tau}^{3}$ & \pm 0.03 \\
\hline$\overline{u^{2}} / U_{\tau}^{2}$ & \pm 0.51 & $\overline{u v^{2}} / U_{\tau}^{3}$ & \pm 0.07 \\
\hline$\overline{v^{2}} / U_{\tau}^{2}$ & \pm 0.06 & $\overline{u w^{2}} / U_{\tau}^{3}$ & \pm 0.18 \\
\hline$\overline{w^{2}} / U_{\tau}^{2}$ & \pm 0.11 & $\overline{v w^{2}} / U_{\tau}^{3}$ & \pm 0.04 \\
\hline$\overline{u v} / U_{\tau}^{2}$ & \pm 0.05 & $\overline{u v w} / U_{\tau}^{3}$ & \pm 0.05 \\
\hline$\overline{u w} / U_{\tau}^{2}$ & \pm 0.07 & $\overline{u^{3}} / U_{\tau}^{3}$ & \pm 0.79 \\
\hline$\overline{v w} / U_{\tau}^{2}$ & \pm 0.03 & $\overline{v^{3}} / U_{\tau}^{3}$ & \pm 0.06 \\
\cline { 3 - 4 } & & $\overline{w^{3}} / U_{\tau}^{3}$ & \pm 0.64 \\
\cline { 3 - 4 } & & &
\end{tabular}

\section{C.4 Turbulent Kinetic Energy}

The turbulent kinetic energy (TKE) is calculated using the equation $T K E=\left(\overline{q^{2}} / 2\right)=\left\lfloor\left(\overline{u^{2}}+\overline{v^{2}}+\overline{w^{2}}\right) / 2\right\rfloor$, where $\overline{u^{2}}, \overline{v^{2}}, \overline{w^{2}}$ are the streamwise, normal-to-wall, and spanwise Reynolds normal stresses respectively. Through the application of the rootmean-square (RMS) error analysis technique, the uncertainties are calculated using Equation. C.2,

$\delta T K E= \pm \sqrt{\left(\frac{\partial T K E}{\partial \overline{u^{2}}}\right)^{2} \delta \overline{u^{2}}+\left(\frac{\partial T K E}{\partial \overline{v^{2}}}\right)^{2} \delta \overline{v^{2}}+\left(\frac{\partial T K E}{\partial \overline{w^{2}}}\right)^{2} \delta \overline{w^{2}}}$

where $\delta \overline{u^{2}}, \delta \overline{v^{2}}, \delta \overline{w^{2}}$ are the associated uncertainties of the respective Reynolds normal stresses. These uncertainty values can be found in Table 1 above. Taking the derivatives and substituting the necessary uncertainties gives $\delta \mathrm{TKE}=\delta\left(\overline{q^{2}} / 2\right)= \pm 0.265 \mathrm{~m}^{2} / \mathrm{s}^{2}$.

\section{C.5 Skin Friction Velocity}

The error in the skin friction velocity, $U_{\tau}$, at 20:1 odds was obtained using the following equation, Equation C.3, 


$$
\delta U_{t}=\frac{2.365 \sigma}{\sqrt{n}}
$$

where $\mathrm{n}$ is the number of identical profiles taken and $\sigma$ is the standard deviation between profiles. The total number of two-dimensional profiles taken that were used to evaluate the uncertainty was 7 . The profiles were all taken at the same location on different days. The value of 2.365 was obtained using the student's t-distribution table for a $95 \%$ confidence interval. Following the substitution of values, the uncertainty in $U_{\tau}= \pm 5 \%$.

\section{C.6 Flow Angle Data}

The mean flow angle (FA) is calculated via $F A=\tan ^{-1}(W / U)$, where $\mathrm{W}$ is the mean spanwise velocity and $U$ is the mean streamwise velocity. The uncertainties in the FA can be calculated using the RMS error analysis technique seen in Equation C.4,

$$
\delta F A= \pm \sqrt{\left(\frac{\partial F A}{\partial U}\right)^{2} \delta U^{2}+\left(\frac{\partial F A}{\partial W}\right)^{2} \delta W^{2}}
$$

where $\delta U$ and $\delta \mathrm{W}$ are the uncertainties in the mean velocities. In this case $\delta U=0.266 \mathrm{~m} / \mathrm{s}$ and $\delta \mathrm{W}=0.165 \mathrm{~m} / \mathrm{s}$. The $\mathrm{U}$ and $\mathrm{W}$ values are taken to be $\mathrm{U}=13.582 \mathrm{~m} / \mathrm{s}$ and $\mathrm{W}=0.034$ $\mathrm{m} / \mathrm{s}$ and were obtained by taking an average over all single element profiles. Therefore, taking the necessary derivatives and substituting in the proper values the uncertainty in the flow angle $\mathrm{FA}$ is $\delta \mathrm{FA}= \pm 0.695^{\circ}$. Thus, the flow angle uncertainty is taken to be $\delta \mathrm{FA}$ $\approx \pm 0.7^{\circ}$.

The shear stress angle (SSA) is calculated using the equation, $S S A=\tan ^{-1}(-\overline{v w} /-\overline{u v})$. Following the same principal as above, the uncertainties in the SSA are calculated using Equation C.5 below.

$$
\delta S S A= \pm \sqrt{\left(\frac{\partial S S A}{\partial \overline{v w}}\right)^{2} \delta \overline{v w}^{2}+\left(\frac{\partial S S A}{\partial \overline{u v}}\right)^{2} \delta \overline{u v}^{2}}
$$

The uncertainties in $\overline{u v}$ and $\overline{v w}$ are as follows, $\delta \overline{u v}=0.050(\mathrm{~m} / \mathrm{s})^{2}$ and $\delta \overline{v w}=0.030$ $(\mathrm{m} / \mathrm{s})^{2}$. Similar to above, the $\overline{u v}$ and $\overline{v w}$ values are taken to be $\overline{u v}=-0.725(\mathrm{~m} / \mathrm{s})^{2}$ and 
$\overline{v w}=-0.008(\mathrm{~m} / \mathrm{s})^{2}$ and were obtained by taking an average over all single element profiles. After performing the necessary steps, $\delta \mathrm{SSA}= \pm 2.37^{\circ} \approx \pm 2.4^{\circ}$.

\section{C.7 Drag}

The uncertainty of the drag on an isolated roughness element was calculated via a perturbation (jitter) analysis performed on the data reduction code used to calculate Equation 4.1. The standard deviations of the mean streamwise U velocity as well as the streamwise Reynolds normal stress $\overline{u^{2}}$ were calculated using, $\delta() / 1.96$, where $\delta()$ is the uncertainty associated with either $\mathrm{U}$ or $\overline{u^{2}}$. Vectors of random numbers were generated from a normal distribution with a mean equal to zero and a standard deviation equal to one. These random vectors had lengths that were equal to the interpolated velocity profiles. These vectors were then multiplied by the respective standard deviations of $\mathrm{U}$ and $\overline{u^{2}}$ and added to the original $\mathrm{U}$ and $\overline{u^{2}}$ velocity vectors in order to obtain a 'perturbed' vector. The uncertainty in the measurement location was also taken into account via the same process as above, with one exception. The uncertainty associated with the $\mathrm{y}$ and $\mathrm{z}$ positions is only relative to the wall and centerline, respectively. Thus, all $\mathrm{y}$ and $\mathrm{z}$ position values were perturbed as a whole. This is in contrast to the above mentioned method in that all of the velocity values were perturbed with respect to each other.

The same procedure that was used to calculate Equation 4.1 was performed using the new 'perturbed' vectors. This process was repeated ten times and an average and standard deviation value were obtained from these runs. From this analysis, the uncertainty in the calculated drag was determined to be $\pm 6 \%$. 


\section{Appendix D Gaussian Fetch Data}

\section{D.1 Introduction}

This appendix contains a brief analysis and discussion of the data obtained on the Gaussian fetch of roughness. More in depth analysis will be conducted in the future. Figures of the related roughness data can be found in the figures at the end of the appendix.

\section{D.2 Brief Analysis}

The flow conditions within the tunnel for both the fetch of Gaussian roughness and the isolated roughness element cases were the same, i.e. $\mathrm{dp} / \mathrm{dx}=0, \mathrm{U}_{\mathrm{e}}=27.5 \mathrm{~m} / \mathrm{s}$. The boundary layer thickness, $\delta$, at the measurement region for the fetch of Gaussian roughness was $52.2 \mathrm{~mm}$. The friction velocity, $\mathrm{U}_{\tau}$, was found by assuming a constant shear stress region and calculated using Equation D.1.

$U_{\tau}=\sqrt{-\overline{u v_{\text {avg }}}}$

The constant Reynolds shear stress values in the log region are averaged to give $\overline{u v_{\text {avg }}}$. The actual value obtained for the Gaussian fetch regime was $U_{\tau}=1.43 \mathrm{~m} / \mathrm{s}$. This value of $\mathrm{U}_{\tau}$ was used to non-dimensionalize the measured velocity quantities presented for the Gaussian fetch of roughness. The non-dimensionalized roughness height based on $U_{\tau}$ for the Gaussian fetch is $\mathrm{k}^{+}=217$.

As can be seen in Figure 1, the $\mathrm{U}^{+}$velocity profiles along the centerline of the element show a drastic difference as compared to the two-dimensional smooth wall case. There is not only a $\Delta \mathrm{U}^{+}$shift downward, but also a reduction in the log layer as well. The $\mathrm{U}^{+}$ profiles tend to collapse to one another just after the height of the roughness. A pitot tube boundary layer profile was also taken at the centerline location of $\mathrm{x} / \mathrm{d}=1.08$ in order to obtain the boundary layer thickness and to compare with the LDV data. The $\mathrm{V}^{+}$profiles show a slight peak in downwash just above the element heights. The profiles closest to 
the element show that there is a positive $\mathrm{V}^{+}$velocity directing fluid away from the wall below the height of the element. The Reynolds normal and shearing stresses, $\overline{u^{2}}, \overline{v^{2}}, \overline{w^{2}}, \overline{u v}$ show peaks in magnitudes at or just above the height of the element. This is due to the fact that there is lower momentum fluid near the wall and around the roughness elements. Thus, higher Reynolds stresses will be produced just above the height of the element where the slower near wall fluid meets the faster out region fluid. This interaction will create a shear layer at the interface of these two regions that will increase turbulence quantities. The related contour plots of the Reynolds normal and shear stresses at the center plane between elements $(\mathrm{x} / \mathrm{d}=1.08)$, Figures D.5 through D.8, also show this same phenomenon. These plots show a core of peak values near the roughness element height which radiate outward. Although the magnitudes are smaller for the fetch of roughness, this trend is comparable to the single element case.

The secondary velocity vectors at the center plane location between elements, $\mathrm{x} / \mathrm{d}=1.08$, are presented on the $\mathrm{U}^{+}$contour plot in Figure D.2. These flow vectors show fluid being swept in towards the wall and around the element at this center plane location. Contours of normalized turbulent kinetic energy (TKE), $\left.\left(\overline{q^{2}} / 2\right) / U_{\tau}^{2}\right\rfloor$, at the $\mathrm{x} / \mathrm{d}=1.08$ plane can be seen in Figure D.21. There is a high region of TKE near the element height. These peak TKE values for the fetch are significantly lower than the values for the single element case. The normalized diffusion velocity vectors $V_{q} / U_{\tau}=\left(\overline{v q^{2}} / \overline{q^{2}}\right) / U_{\tau}$ and $W_{q} / U_{\tau}=\left(\overline{w q^{2}} / \overline{q^{2}}\right) / U_{\tau}$ are also presented in Figure D.21. These vectors show the transport of TKE to be much smaller than the single element case. There is still dissipation of TKE away from the core value near the height of the element but the vector magnitudes and directions are not nearly as distinct as they are for the single Gaussian element case. This incident is not surprising due to the fact that there is lower momentum fluid down near the roughness elements in the fetch regime. Although there are significant differences between the single roughness element case and the fetch of roughness, there are also distinct similarities. This leads to the conclusion that a thorough knowledge of each case, single element and fetch, is necessary in order to completely 
understand the effects that roughness has on the flow physics in a turbulent boundary layer. 


\section{D.3 Appendix D Figures}
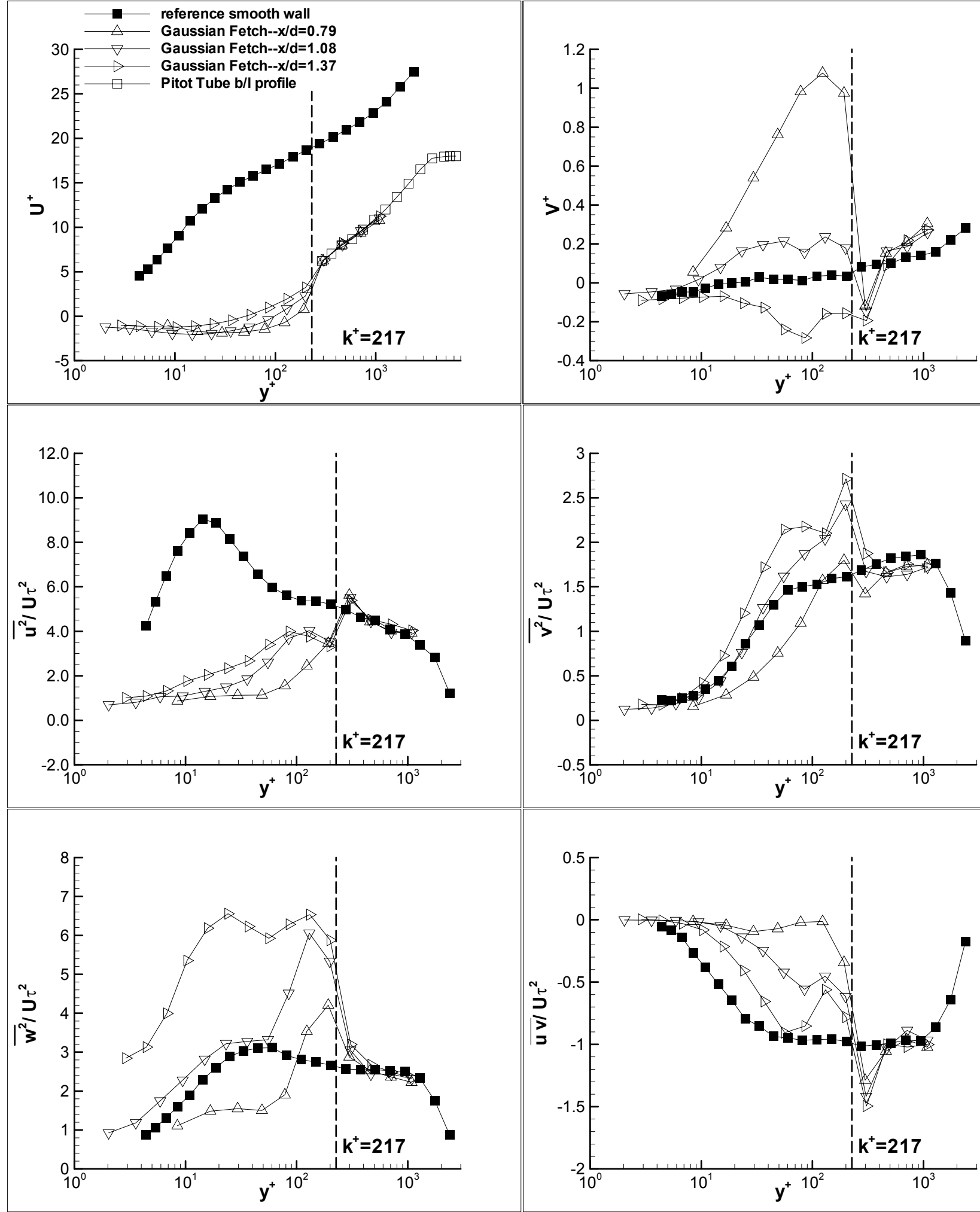

(cont.) 


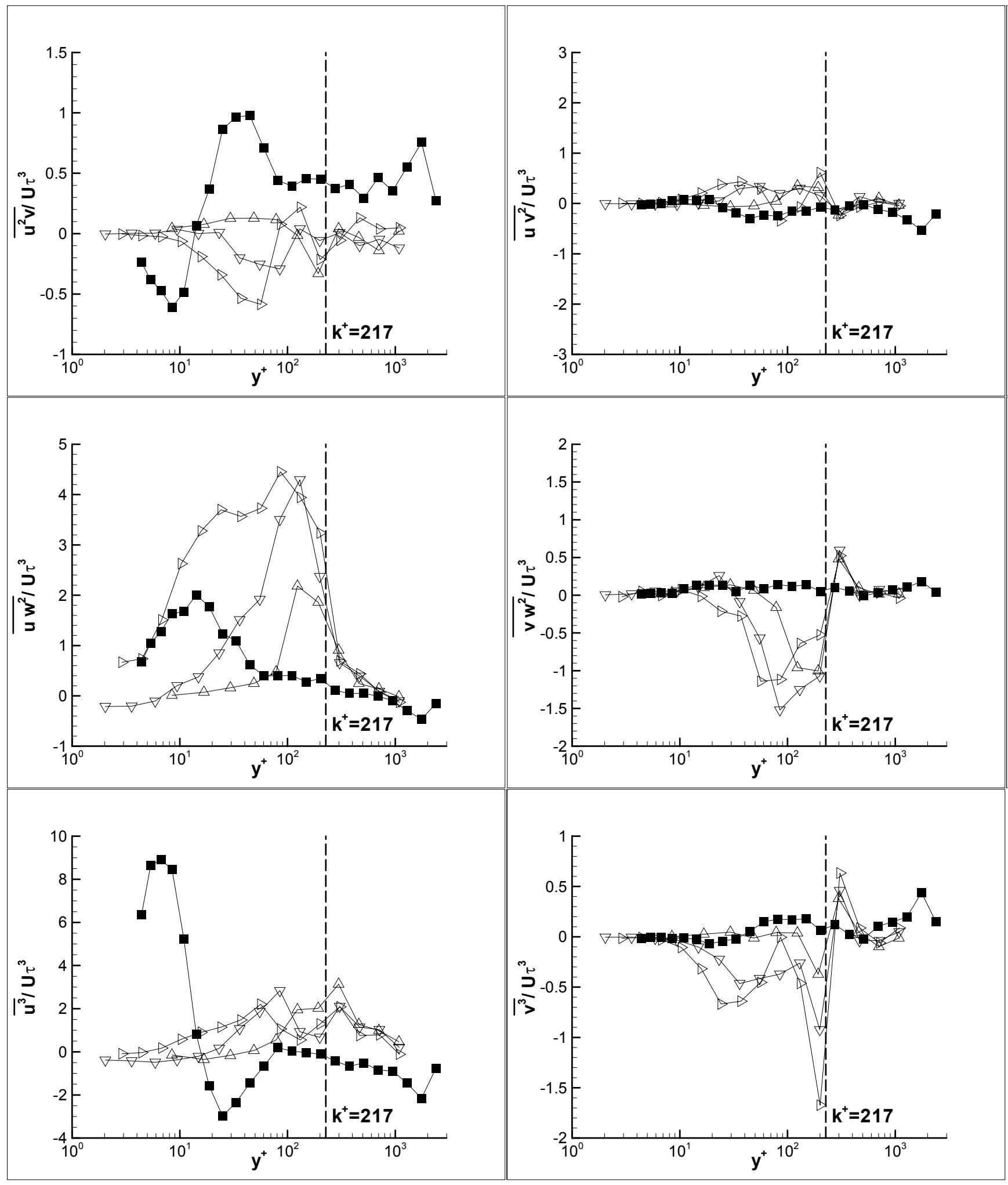

Figure D.1: Centerline profiles of Gaussian fetch (mean velocities, Reynolds stresses, and triple products) 


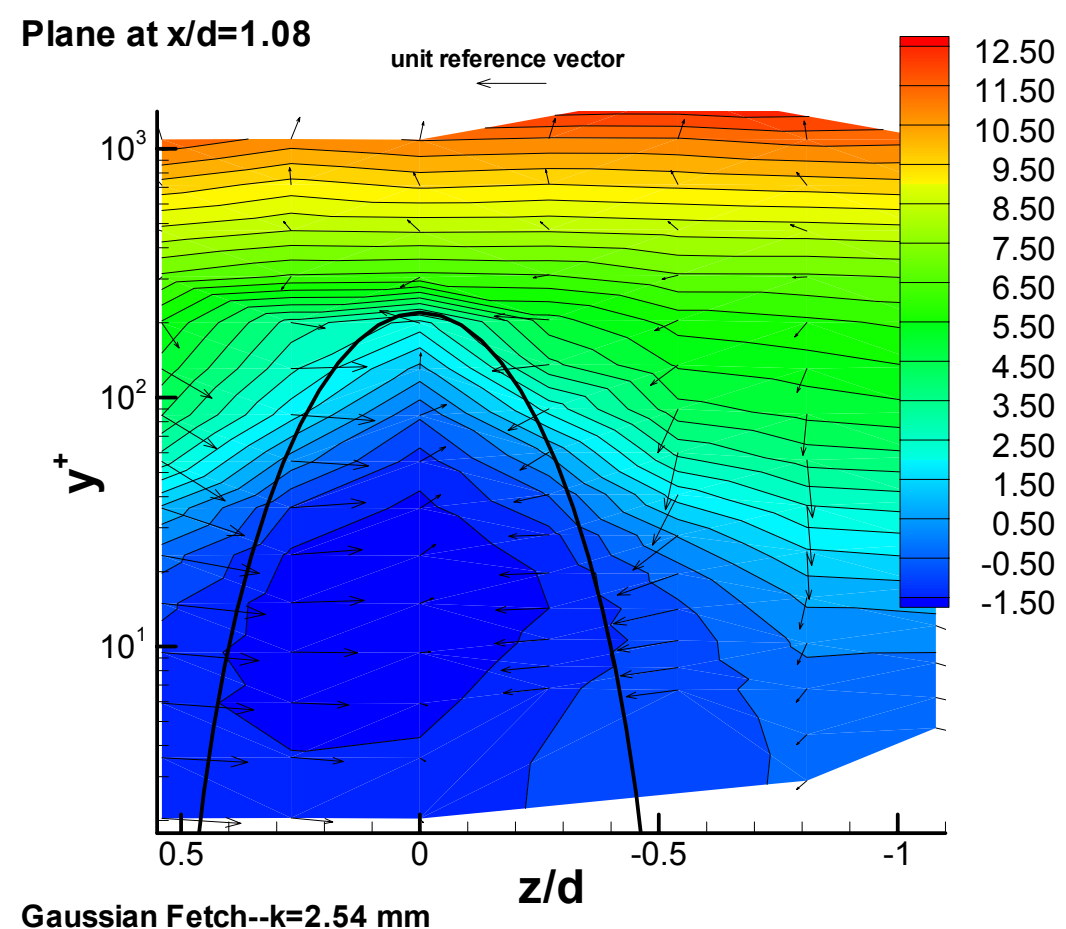

Figure D.2: Contours of $U / U_{\tau}$ in the $y-z$ plane at a location of $x / d=1.08$ with normalized secondary velocity vectors $V / U_{\tau}$ and $W / U_{\tau}$

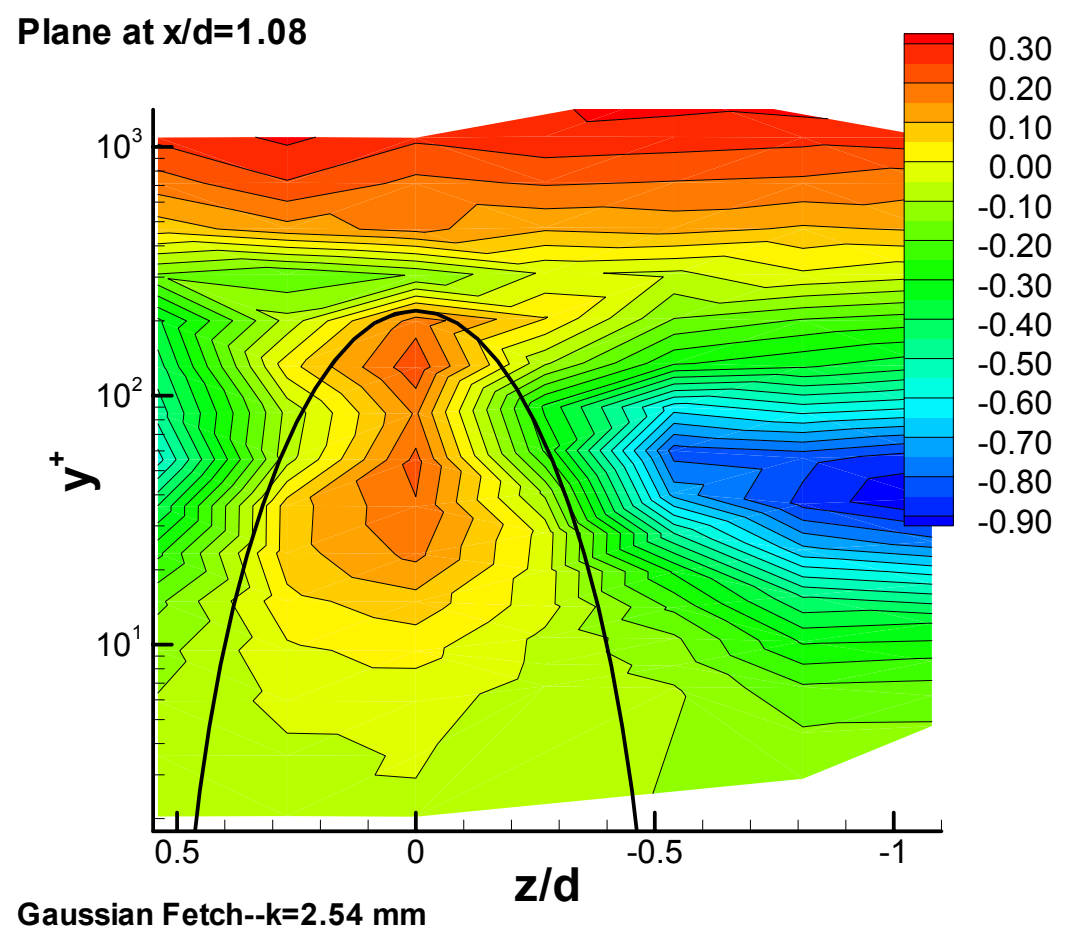

Figure D.3: Contours of $V / U_{\tau}$ in the $y-z$ plane at a location of $\mathbf{x} / \mathbf{d}=\mathbf{1 . 0 8}$ 


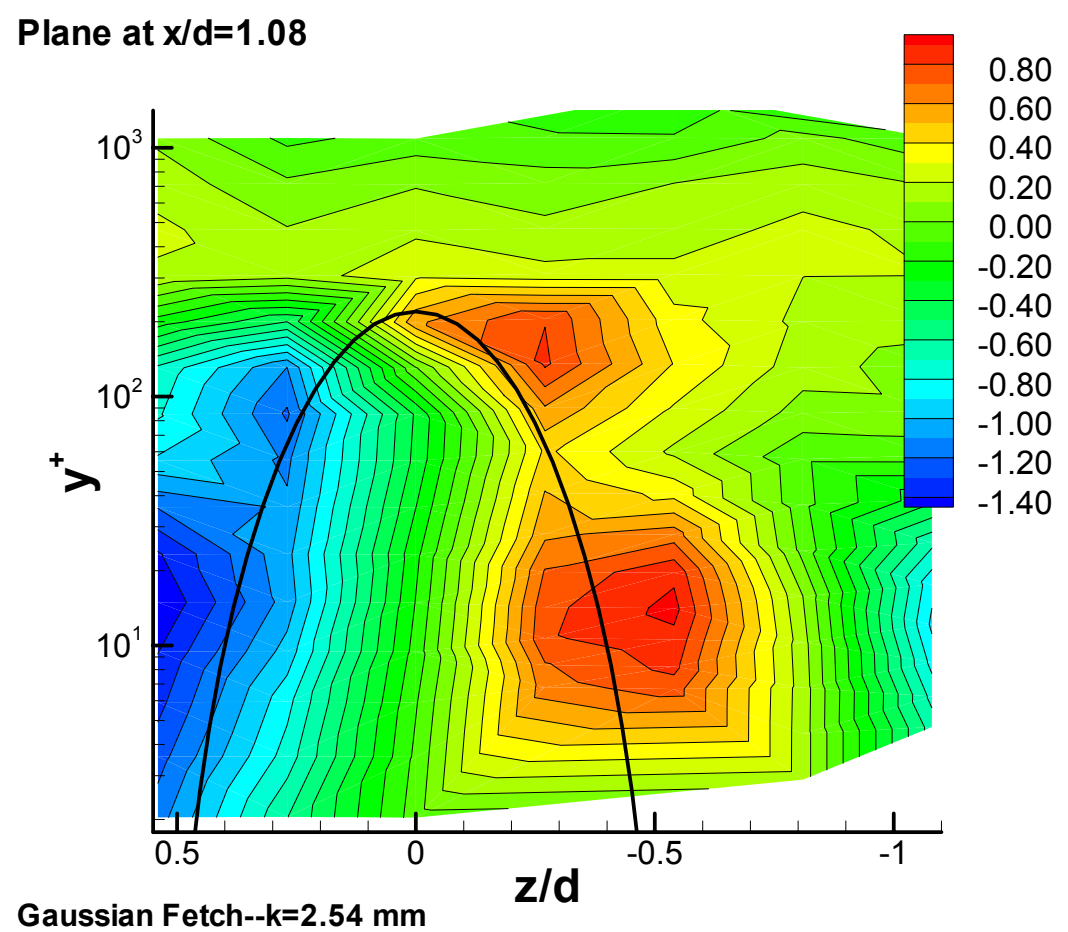

Figure D.4: Contours of $W / U_{\tau}$ in the $\mathbf{y - z}$ plane at a location of $\mathbf{x} / \mathbf{d}=1.08$

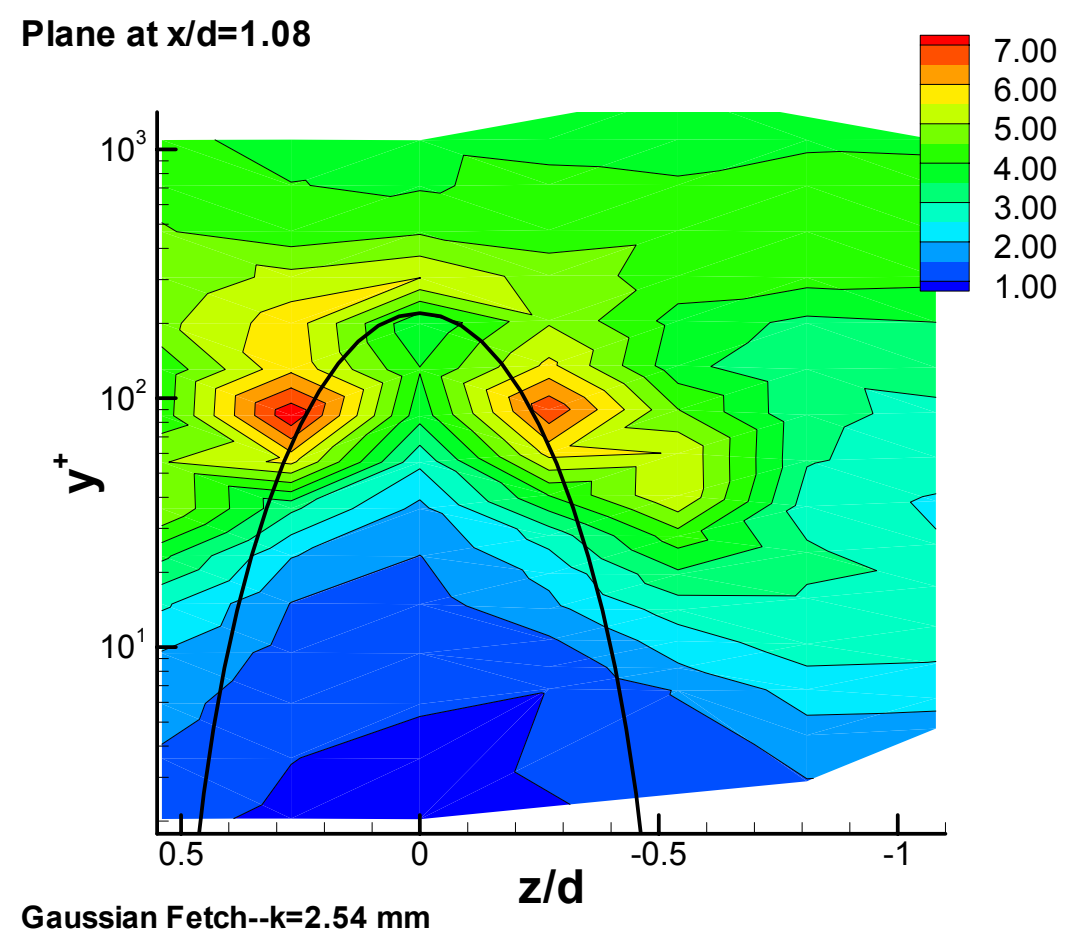

Figure D.5: Contours of $\overline{u^{2}} / U_{\tau}^{2}$ in the $y-z$ plane at a location of $x / d=1.08$ 


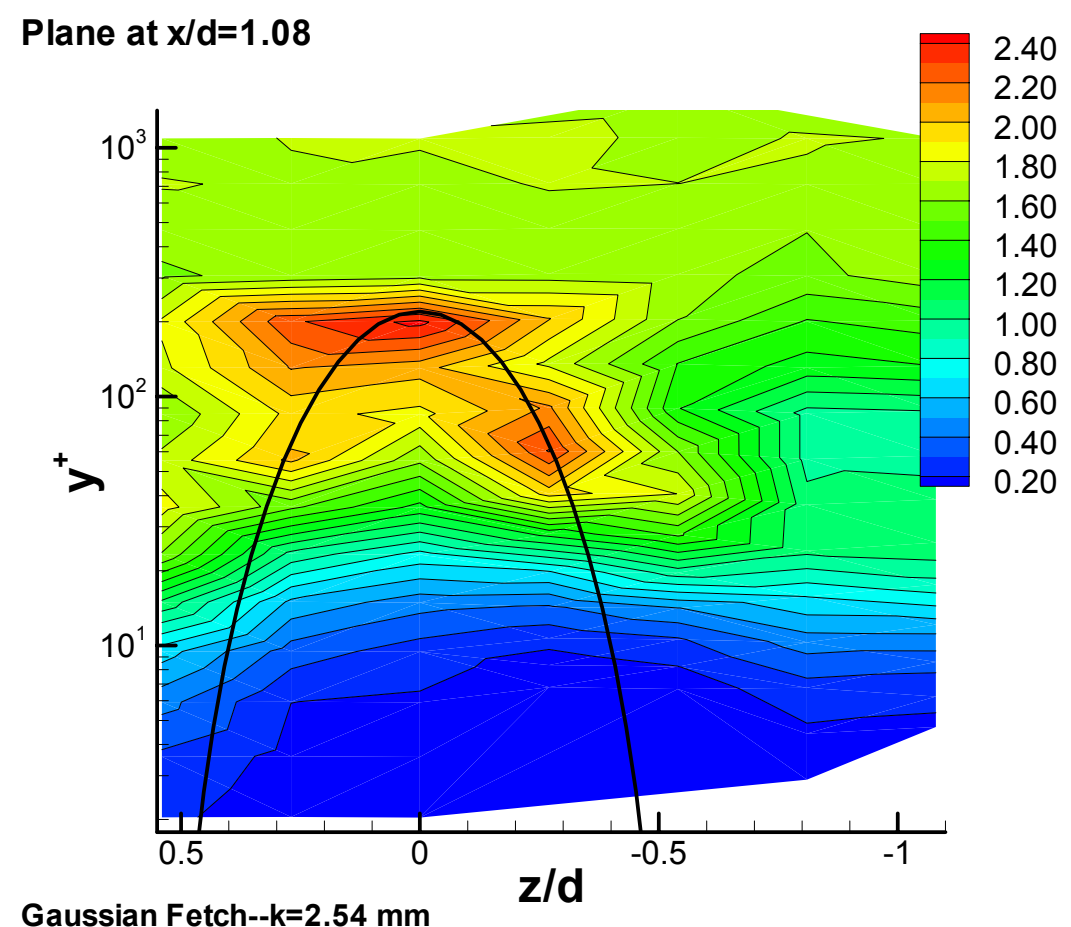

Figure D.6: Contours of $\overline{v^{2}} / U_{\tau}^{2}$ in the $\mathbf{y}-\mathrm{z}$ plane at a location of $\mathbf{x} / \mathbf{d}=1.08$

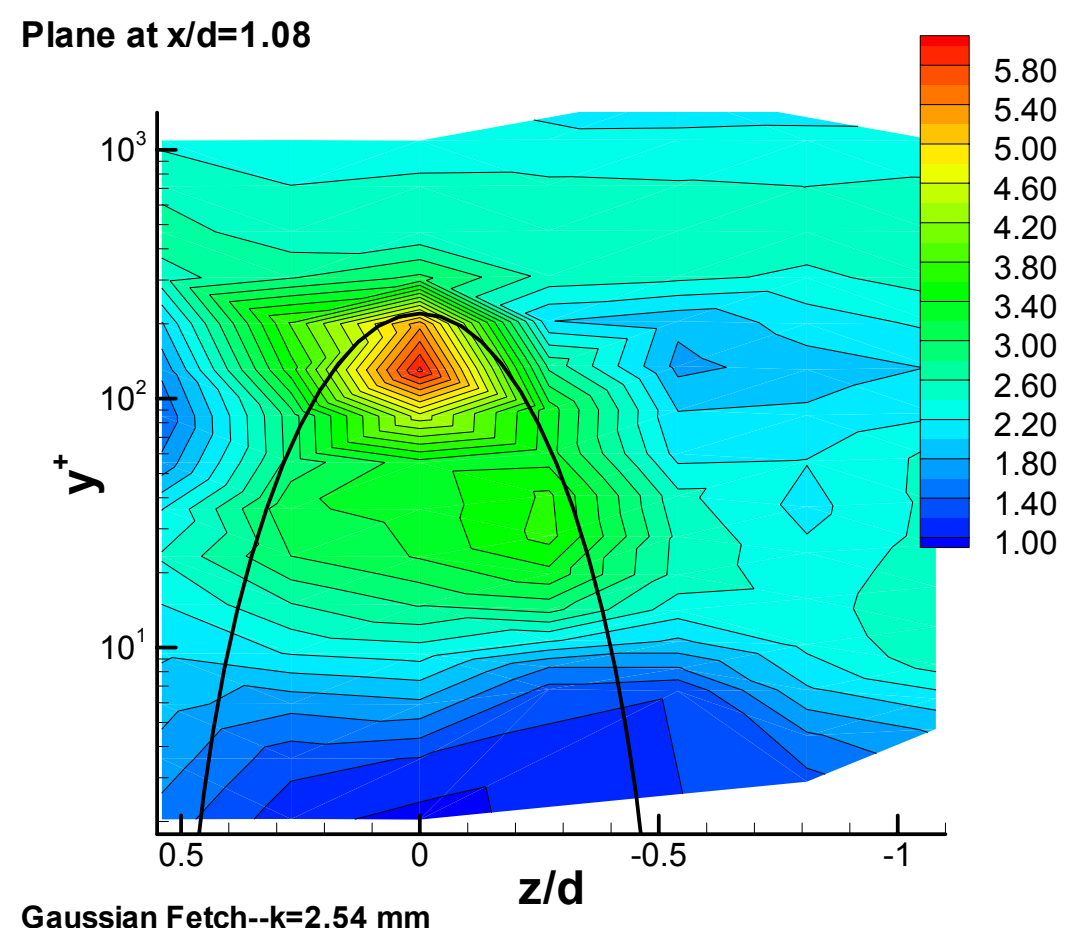

Figure D.7: Contours of $\overline{w^{2}} / U_{\tau}^{2}$ in the $\mathbf{y}-\mathbf{z}$ plane at a location of $\mathbf{x} / \mathbf{d}=1.08$ 


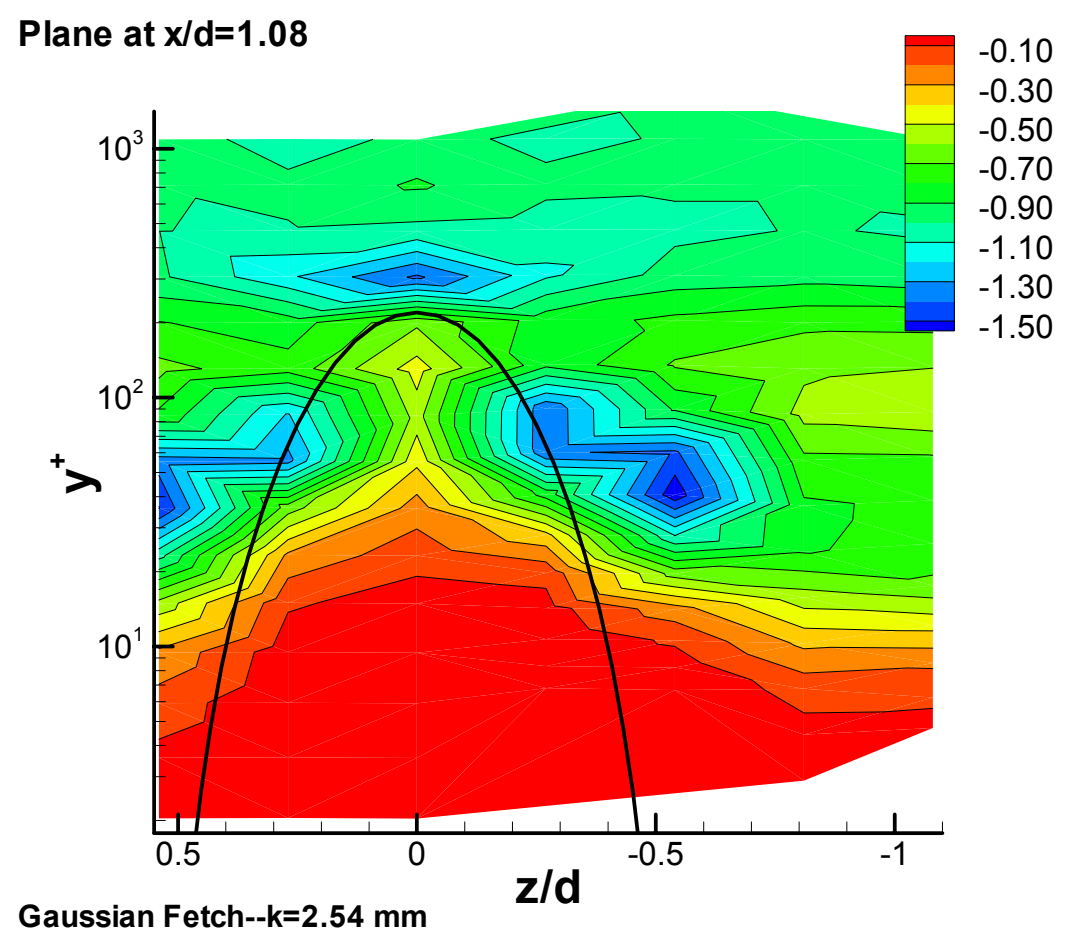

Figure D.8: Contours of $\overline{u v} / U_{\tau}^{2}$ in the $\mathbf{y}-z$ plane at a location of $\mathbf{x} / \mathbf{d}=\mathbf{1 . 0 8}$

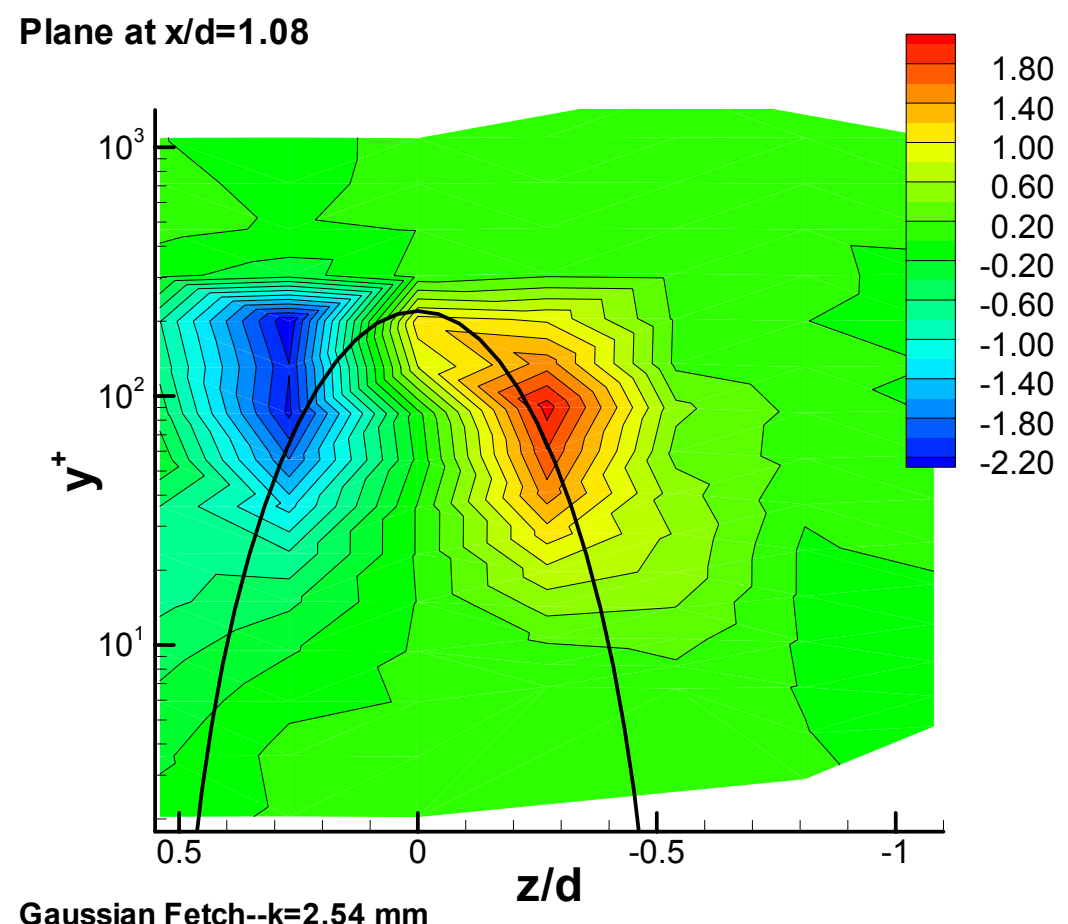

Figure D.9: Contours of $\overline{u w} / U_{\tau}^{2}$ in the $\mathbf{y}-\mathbf{z}$ plane at a location of $\mathbf{x} / \mathbf{d}=1.08$ 


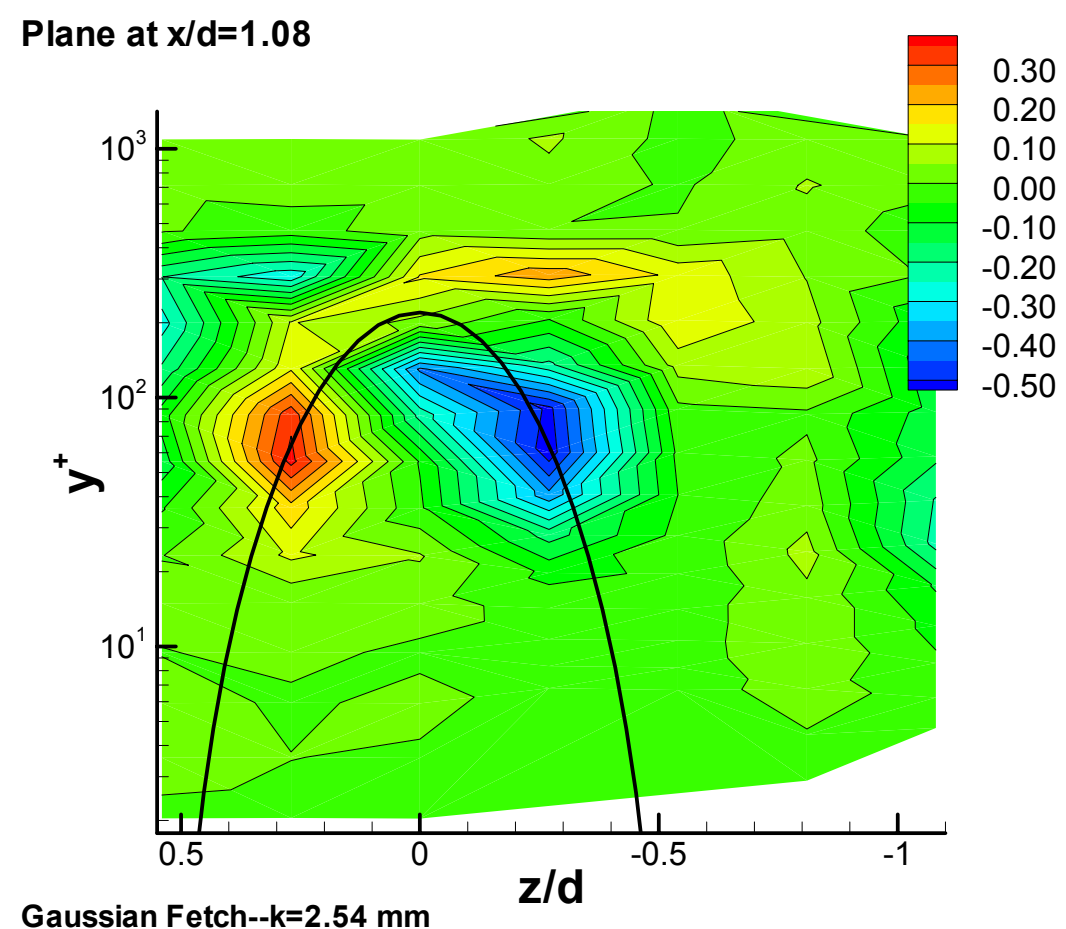

Figure D.10: Contours of $\overline{v w} / U_{\tau}^{2}$ in the $\mathbf{y}-\mathrm{z}$ plane at a location of $\mathbf{x} / \mathbf{d}=\mathbf{1 . 0 8}$

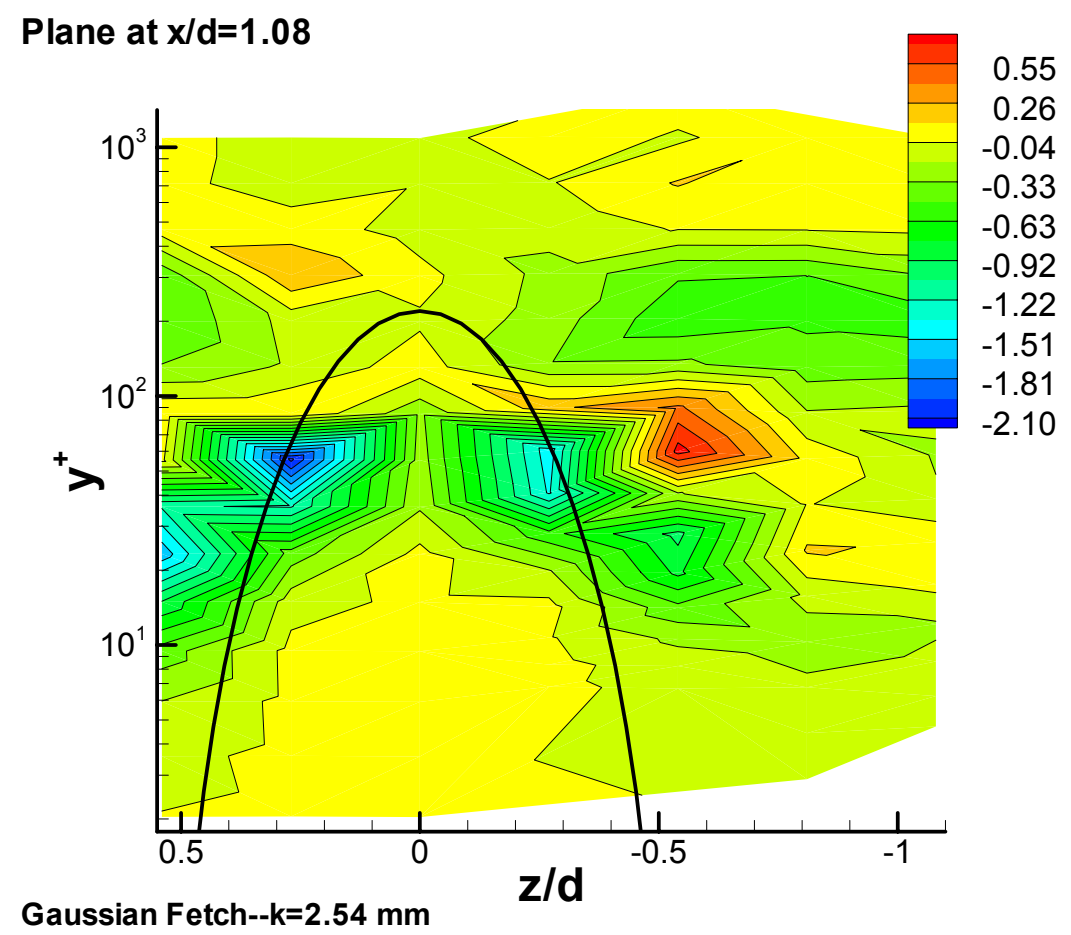

Figure D.11: Contours of $\overline{u^{2} v} / U_{\tau}^{3}$ in the $\mathbf{y - z}$ plane at a location of $\mathbf{x} / \mathbf{d}=1.08$ 


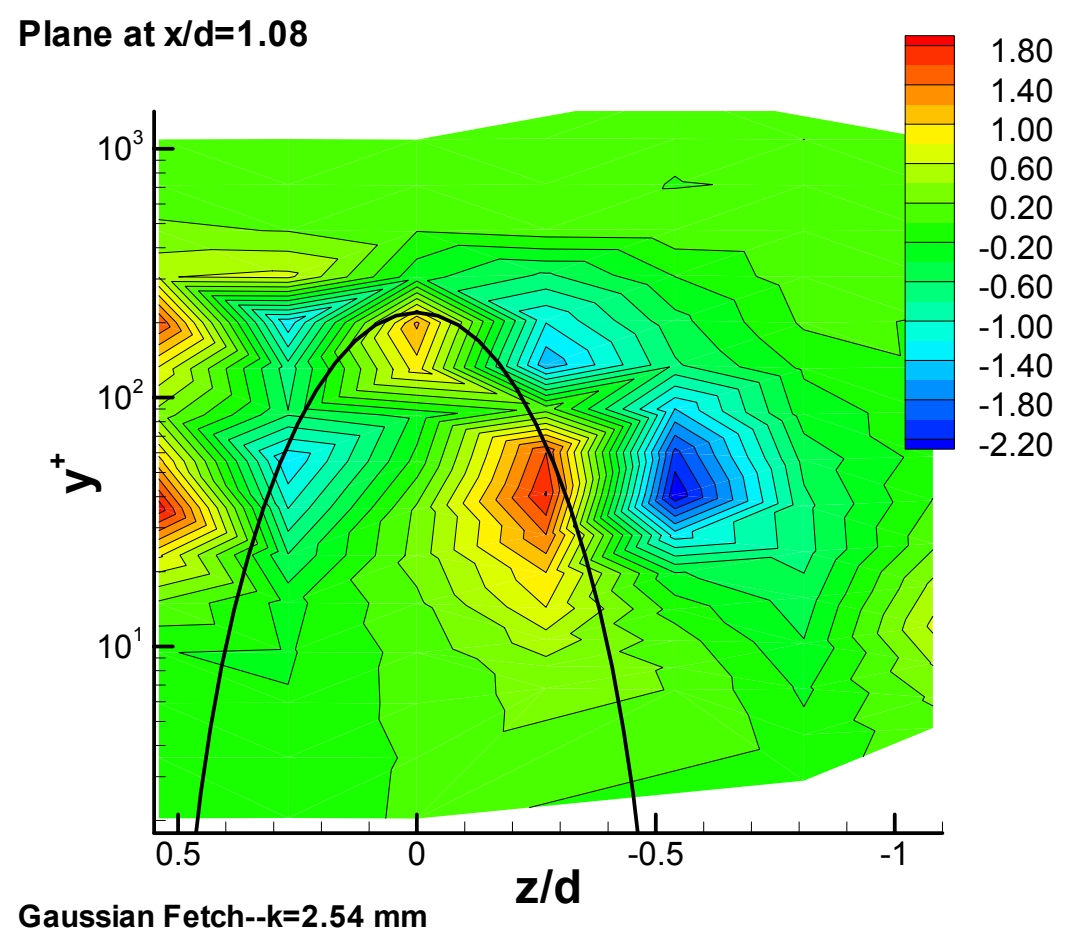

Figure D.12: Contours of $\overline{u^{2} w} / U_{\tau}^{3}$ in the $\mathbf{y}-z$ plane at a location of $\mathbf{x} / \mathbf{d}=1.08$

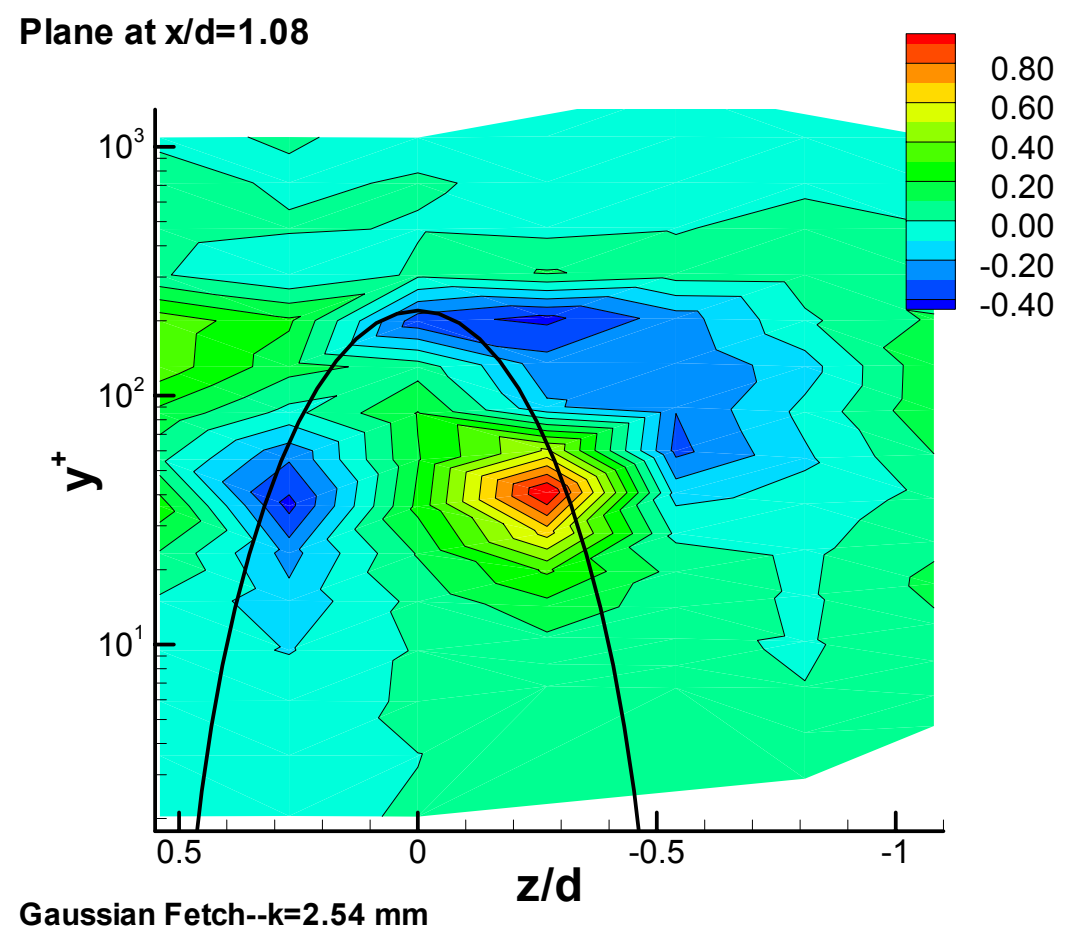

Figure D.13: Contours of $\overline{v^{2} w} / U_{\tau}^{3}$ in the $\mathbf{y}-z$ plane at a location of $\mathbf{x} / \mathbf{d}=1.08$ 


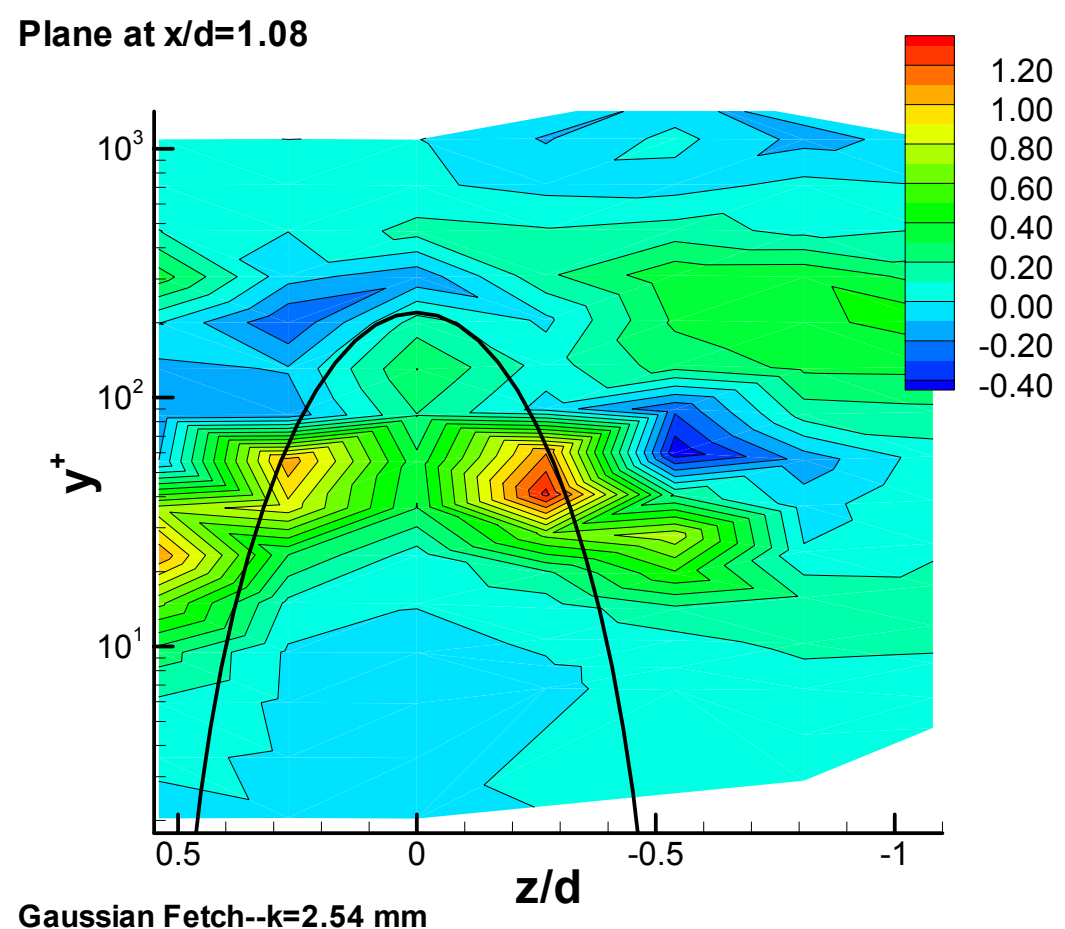

Figure D.14: Contours of $\overline{u v^{2}} / U_{\tau}^{3}$ in the $y-z$ plane at a location of $\mathbf{x} / \mathbf{d}=1.08$

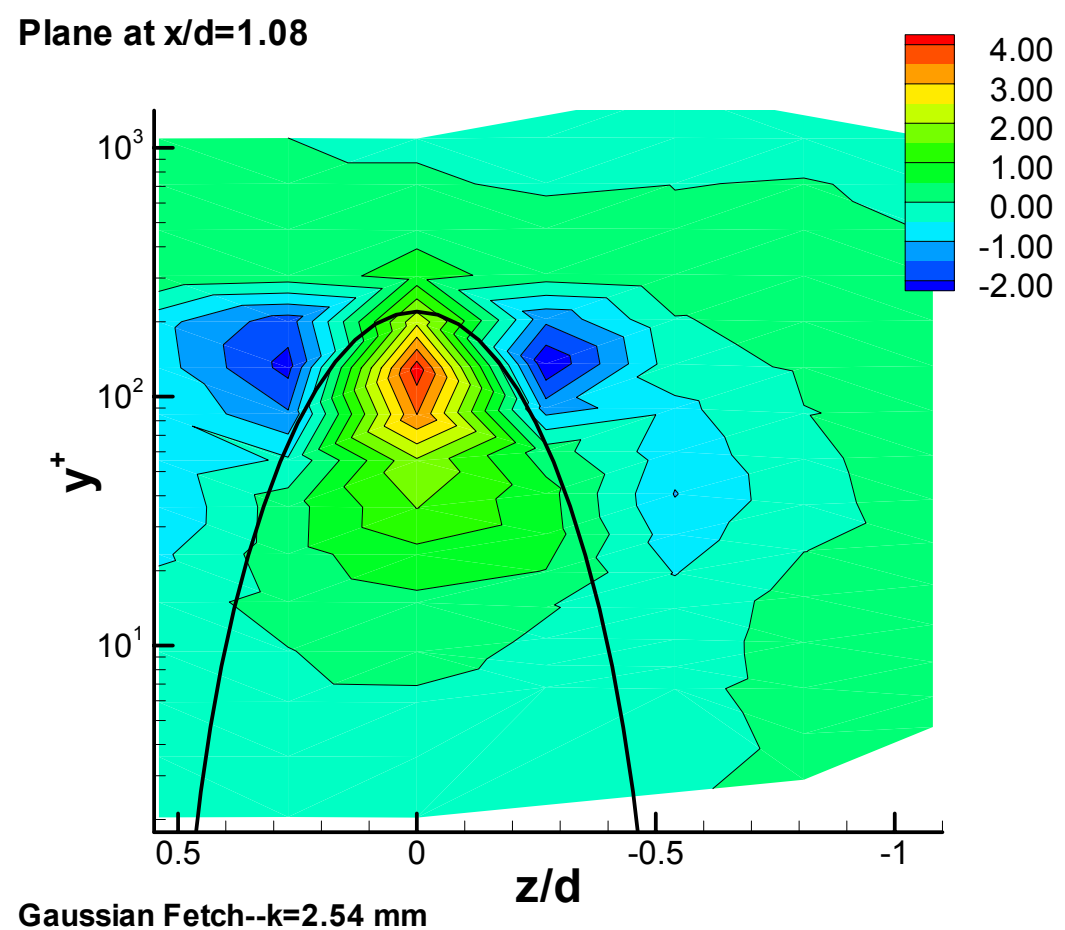

Figure D.15: Contours of $\overline{u w^{2}} / U_{\tau}^{3}$ in the $\mathbf{y}-\mathrm{z}$ plane at a location of $\mathbf{x} / \mathbf{d}=1.08$ 


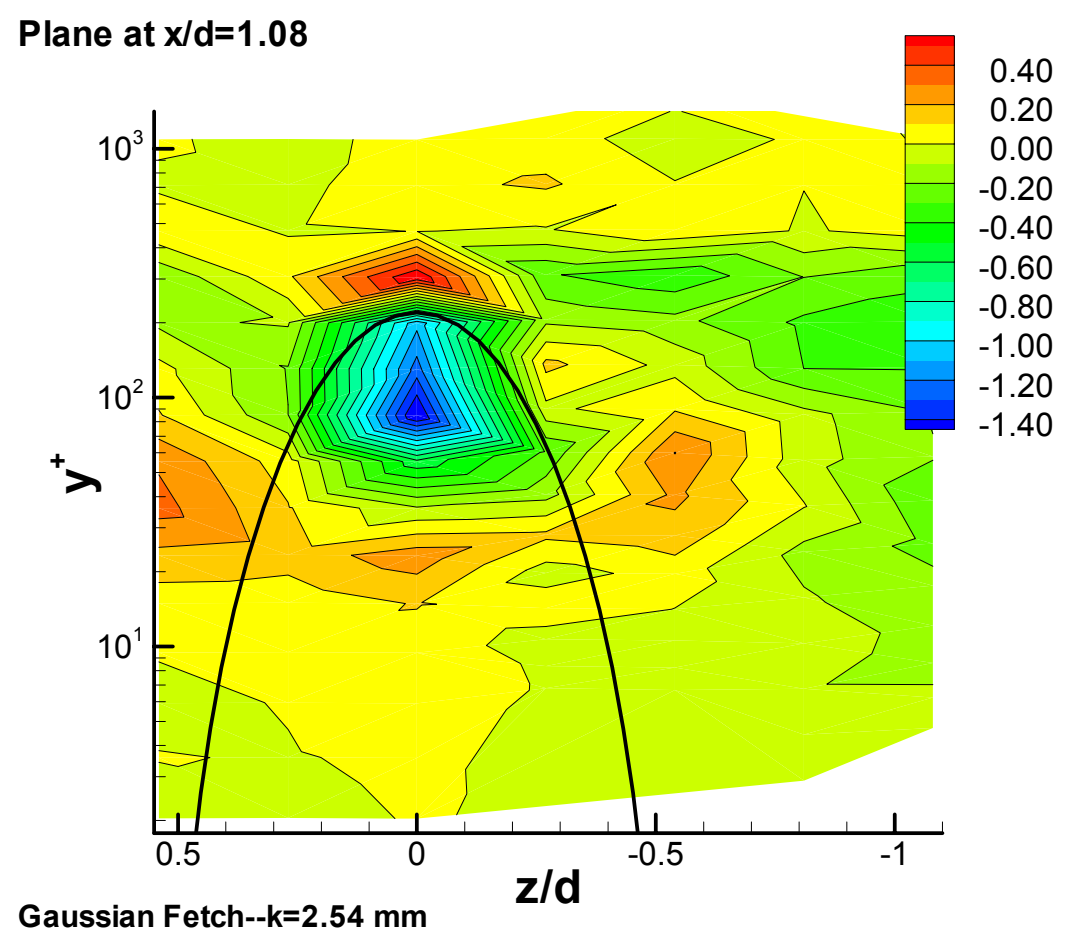

Figure D.16: Contours of $\overline{v w^{2}} / U_{\tau}^{3}$ in the $\mathbf{y}-\mathrm{z}$ plane at a location of $\mathbf{x} / \mathbf{d}=\mathbf{1 . 0 8}$

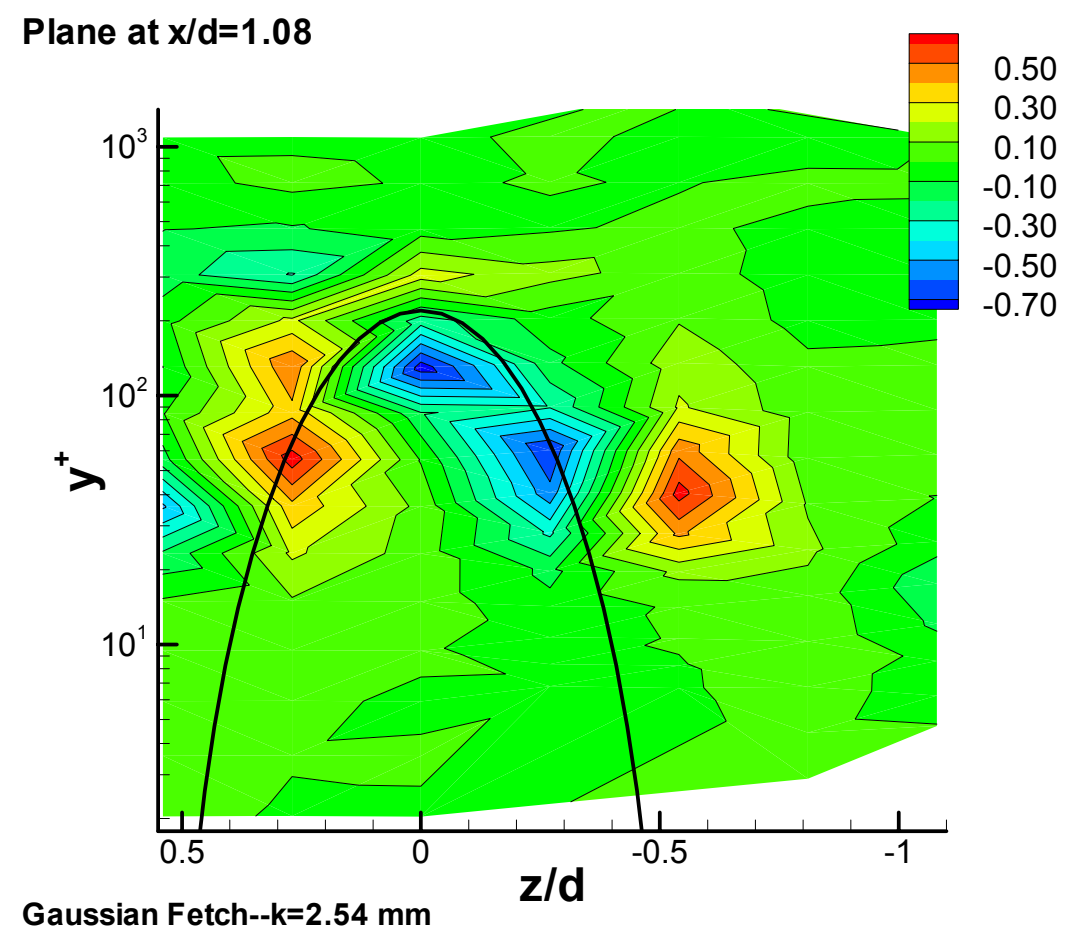

Figure D.17: Contours of $\overline{u v w} / U_{\tau}^{3}$ in the $\mathbf{y}-z$ plane at a location of $\mathbf{x} / \mathbf{d}=\mathbf{1 . 0 8}$ 


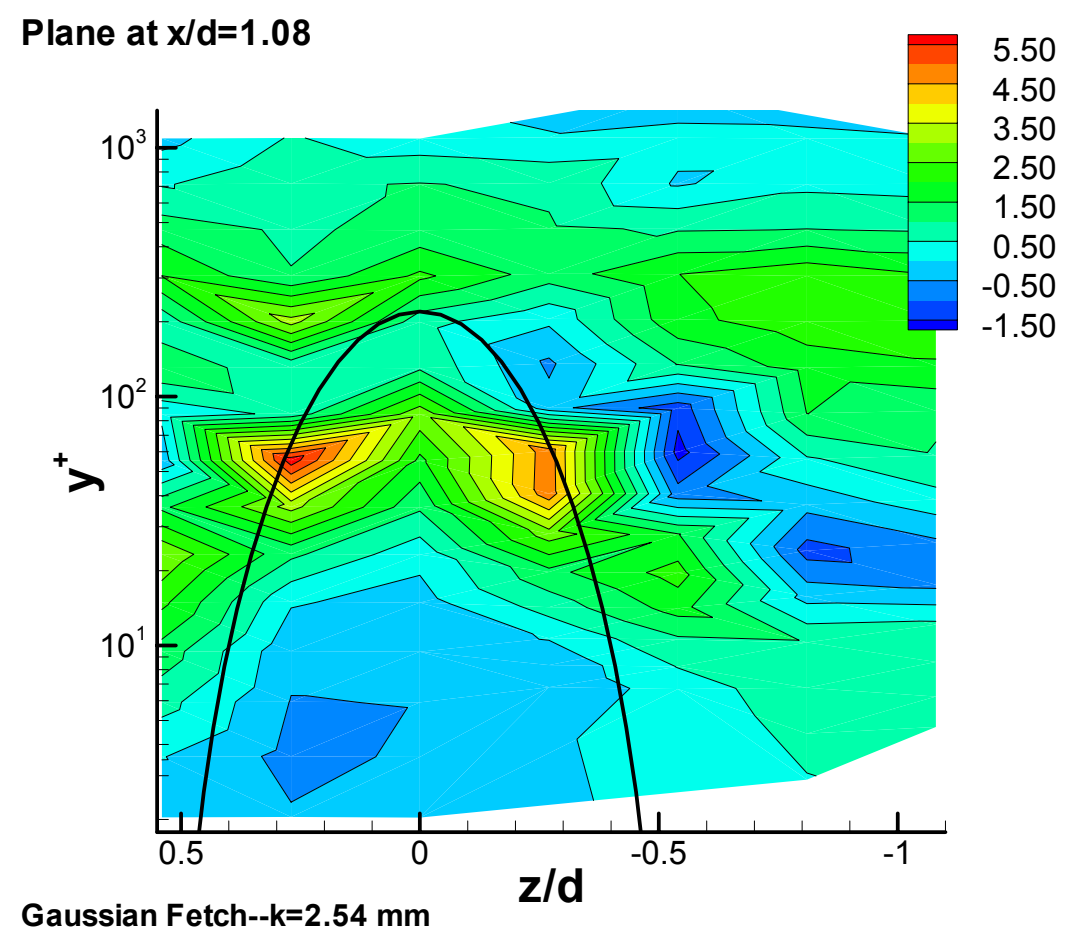

Figure D.18: Contours of $\overline{u^{3}} / U_{\tau}^{3}$ in the $y-z$ plane at a location of $\mathbf{x} / \mathbf{d}=1.08$

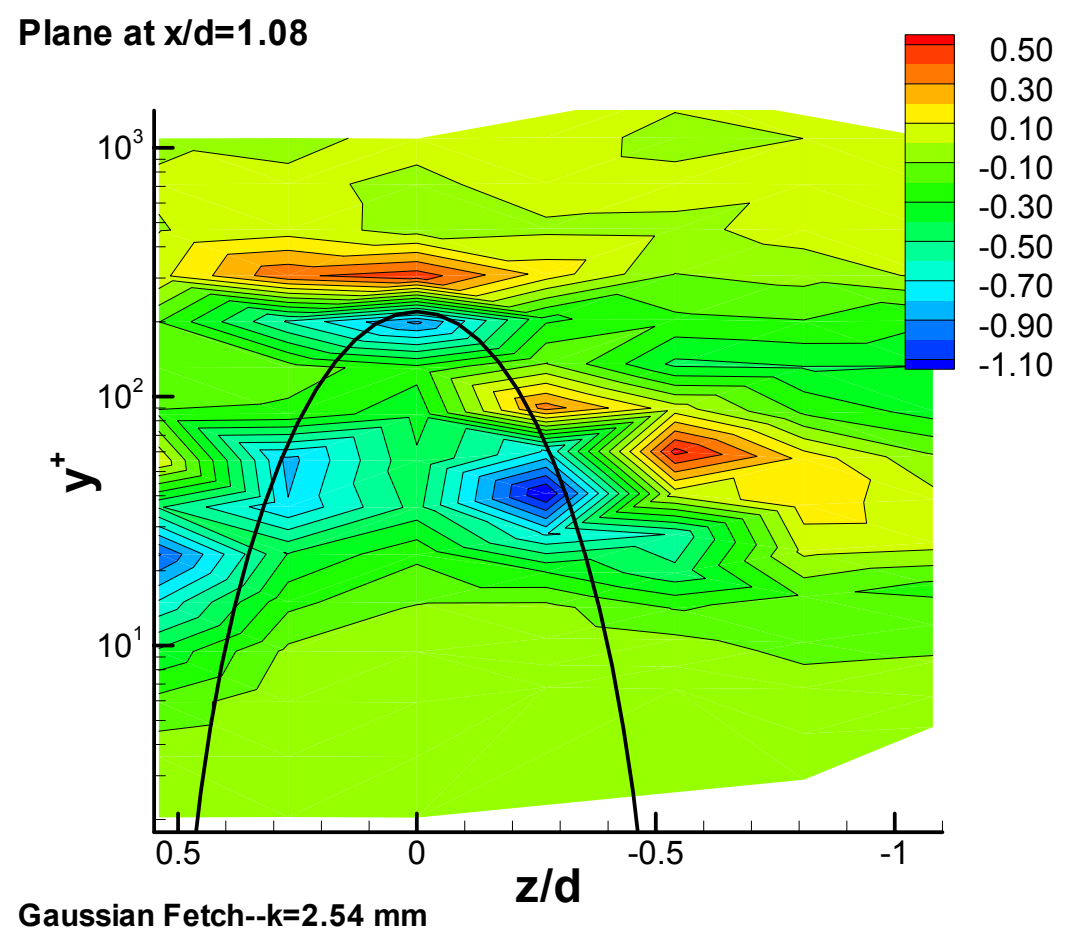

Figure D.19: Contours of $\overline{v^{3}} / U_{\tau}^{3}$ in the $y-z$ plane at a location of $x / d=1.08$ 


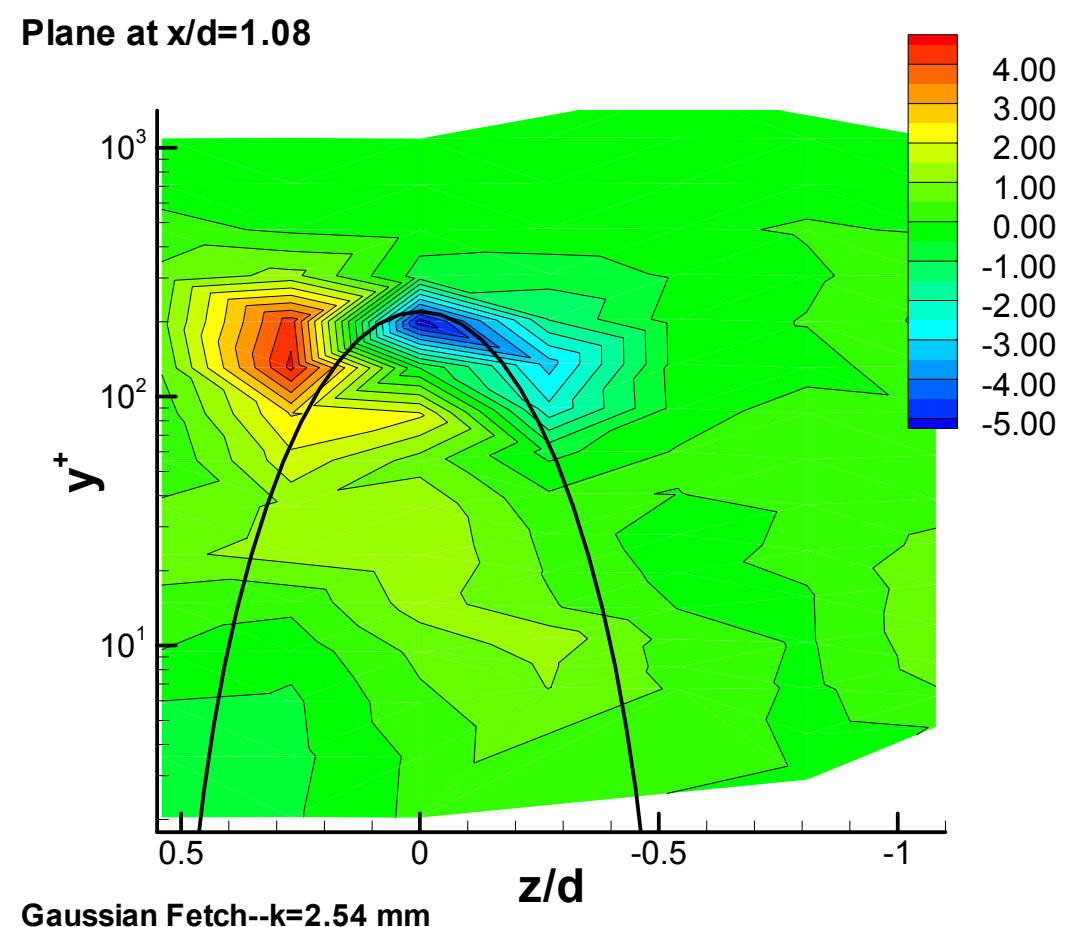

Figure D.20: Contours of $\overline{w^{3}} / U_{\tau}^{3}$ in the $\mathbf{y}-z$ plane at a location of $x / d=1.08$

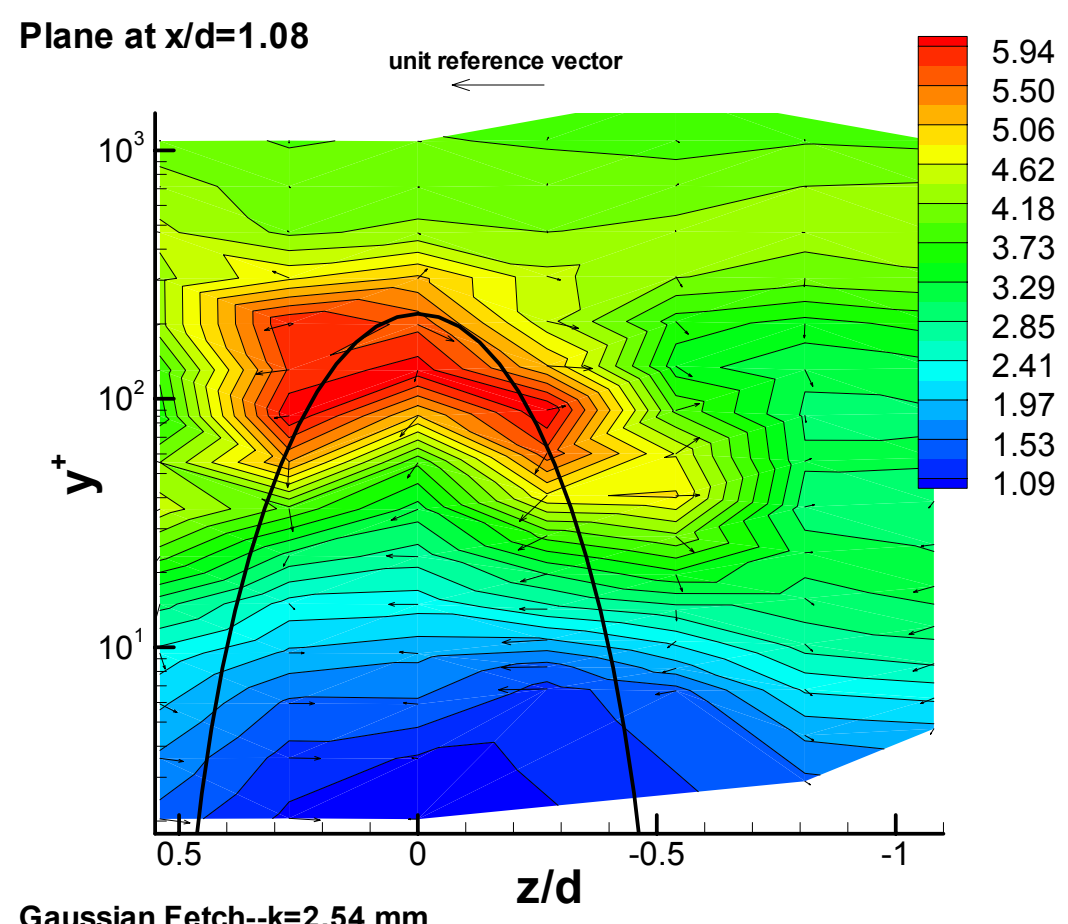

Figure D.21: Contours of TKE $\left[\left(\overline{q^{2}} / 2\right) / U_{\tau}^{2}\right]$ with diffusion velocity vectors, $V_{q} / U_{\tau}=\left(\overline{v q^{2}} / \overline{q^{2}}\right) / U_{\tau}$ and $W_{q} / U_{\tau}=\left(\overline{w q^{2}} / \overline{q^{2}}\right) / U_{\tau}$, in the $\mathbf{y}-z$ plane at $\mathbf{x} / \mathbf{d}=\mathbf{1 . 0 8}$ 


\section{Appendix E Flow Angle Data}

\section{E.1 Mean Flow Angle}

The mean flow angle (FA) is calculated via Equation E.1 below, where $\mathrm{W}$ is the mean spanwise velocity and $U$ is the mean streamwise velocity.

$$
F A=\tan ^{-1}\left(\frac{W}{U}\right)
$$

The flow angle enables one to determine the degree of cross-flow (in the spanwise direction) due to three-dimensional effects. If the flow angle is larger than $45^{\circ}$ at any point within the flow, the cross-flow is deemed to be locally larger than the flow in the streamwise direction. The calculated uncertainty in the flow angle is $\delta \mathrm{FA} \approx \pm 0.7^{\circ}$; see Appendix C, Section C.6. Figure E.1 shows the mean flow angle variation along the centerline of the elements. The mean flow angle is expected to be zero along the centerline due to symmetry reasons within the flow. Within the bounds of the uncertainty, this is generally the case at all streamwise centerline measurement locations except for the first location at $\mathrm{x} / \mathrm{d}=1.36$. Due to the proximity of this location with respect to the element and the highly turbulent flow that is present; even the slightest error in probe alignment will produce results that deviate from the expected zero flow angle value. As is shown at the subsequent downstream measurement locations, these small alignment errors do not propagate very far downstream. Figures E.2 and E.3 show contour plots of the flow angles at the first measurement plane downstream of the elements. All axisymmetric elements show FA values that are very comparable with respect to each other. At the measurement location, $x / d=2.75$, mean $U$ is positive throughout all boundary layer profiles. Consequently, negative flow angles indicate that the flow is being directed in the (-)z-direction, (-)spanwise direction, where the positive flow angle values indicate the opposite flow behavior. The flow angles are related to the strength of the secondary flow and thus directly related to the strength of the horseshoe vortex structures produced by each element. Therefore, as is discussed in the previous chapters, the cube at $45^{\circ}$ has the largest magnitude in FA followed by the cube at $90^{\circ}$ and the axisymmetric elements. The flow angle created by the cube at $45^{\circ}$ indicates that the 
local cross-flow is nearly $50 \%$ of the local streamwise velocity value. The range of the flow angles across the measurement plane is a good indicator of the three-dimensionality of the flow. Thus, there is highly three-dimensional flow near the wall $\left(\mathrm{y}^{+}<10\right)$ in the vicinity of the horseshoe vortex structures. This three-dimensionality in the flow tends to dissipate with distance from the vortex structure.

Contours of the FA at a downstream measurement location of $\mathrm{x} / \mathrm{d}=10.0$ can be seen in Figures E.4 and E.5. As the flow progresses downstream the strength of the horseshoe vortex structure dissipates and causes a decrease in flow angle magnitude. The cube elements still produce the most significant cross-flow at this location with the cube at $45^{\circ}$ being the largest. The axisymmetric elements are still very comparable to one another but the large grooved element and the hemisphere tend to have the lowest values of crossflow at this downstream location.

\section{E.2 Flow Gradient Angle}

The flow gradient angle (FGA) indicates the flow strain change associated with the flow and is calculated using Equation E.2 below.

$$
F G A=\tan ^{-1}\left[\frac{\partial W / \partial y}{\partial U / \partial y}\right]
$$

A magnitude in the FGA larger than $45^{\circ}$ indicates that the flow in the spanwise direction changes faster than the flow in the streamwise direction. Contour plots of the flow gradient angle at the $\mathrm{x} / \mathrm{d}=2.75$ plane $(\mathrm{x} / \mathrm{d}=1.36$ for fine grooved element) can be seen in Figures E.6 and E.7. The fine grooved element at $\mathrm{x} / \mathrm{d}=1.36$ shows very large values of FGA near the peak of the element which indicate that the spanwise flow changes significantly faster than the streamwise flow. Again, the cube elements have the most effect on the FGA followed by the axisymmetric elements which have comparable magnitudes. In these contour plots it is possible to see the regions where there is the fastest change in flow direction. As the distance from the wall increases, a region of large FGA is seen around $15<\mathrm{y}^{+}<40$. In this region $\partial W / \partial y$ decreases rapidly as the distance to the core of the vortex gets less and less. Likewise, $\partial W / \partial y$ increases rapidly 
as you move away from the center of the vortex in the positive y-direction. It is in this region that negative FGA's exist, in the neighborhood of $100<\mathrm{y}^{+}<300$. Contour plots of the FGA in the $y-z$ plane at a location of $x / d=10.0$ can be seen in Figures E. 8 and E.9. As is expected the magnitudes of the FGA's have decreased to values close to $50 \%$ of the upstream, $\mathrm{x} / \mathrm{d}=2.75$, magnitudes. The higher magnitude regions have moved farther away from the wall and centerline as compared to the upstream measurement plane. This result is quite obvious due to the fact that the FGA's are directly related to the horseshoe vortex structures. These vortices have previously been shown to move away from the wall and centerline as they propagate downstream.

\section{E.3 Shear Stress Angle}

The shear stress angle (SSA) is used to describe the direction of the effective turbulent stress in the flow field (Tang, 2004). The definition of the SSA can be found in Equation E.3.

$$
S S A=\tan ^{-1}\left(\frac{-\overline{v w}}{-\overline{u v}}\right)
$$

The calculated uncertainty in the shear stress angle is $\delta \mathrm{SSA} \approx \pm 2.4^{\circ}$; see Appendix $\mathrm{C}$, Section C.6. For similar reasons expressed in Section E.1, the SSA should be zero along the centerline. Likewise, due to the same proximity issues previously discussed the SSA is not exactly zero at the near element locations as is evidenced by Figure E.10. At the downstream locations the SSA is seen to collapse to zero except at very near-wall locations. Contour plots of the SSA in the $y-z$ plane at $x / d=2.75(x / d=1.36$ for fine grooved) and $\mathrm{x} / \mathrm{d}=10.0$ for all elements can be found in Figures E.11 through E.14. There are higher values of SSA located near the peaks and edges of each element indicating regions of large directional changes in the turbulent stresses produced by the roughness elements' presence in the boundary layer. These areas of significant direction change in the effective turbulent stress have diminished by the $\mathrm{x} / \mathrm{d}=10.0$ measurement plane. 


\section{E.4 Appendix E Figures}

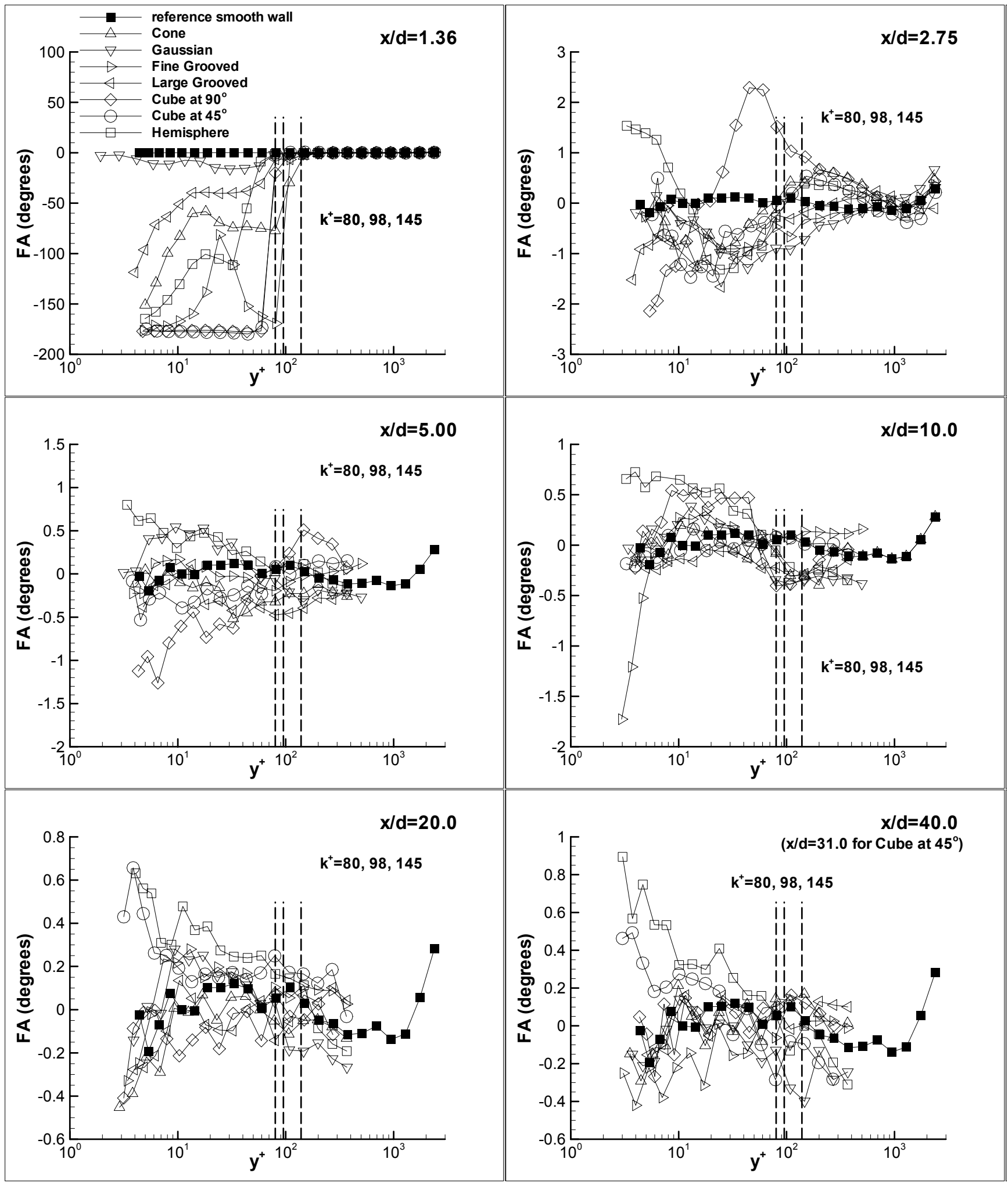

Figure E.1: Mean flow angle variation along the centerline, where $F A=\tan ^{-1}(W / U)$ 

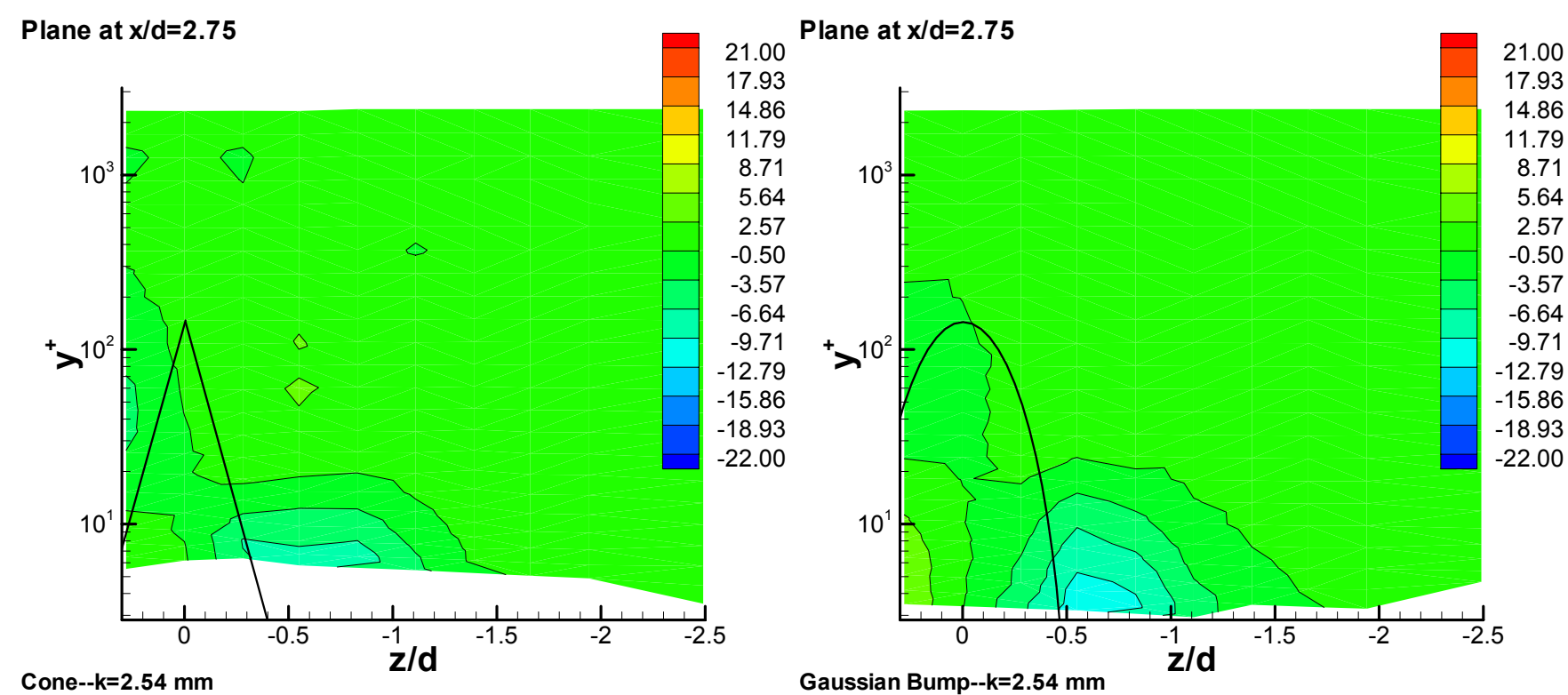

Plane at $\mathrm{x} / \mathrm{d}=\mathbf{2 . 7 5}$

Gaussian Bump--k=2.54 mm

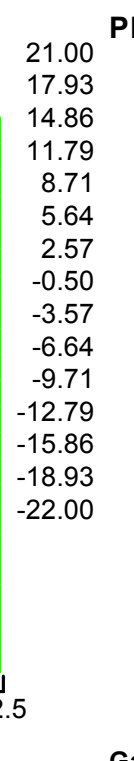

Plane at $x / d=1.36$
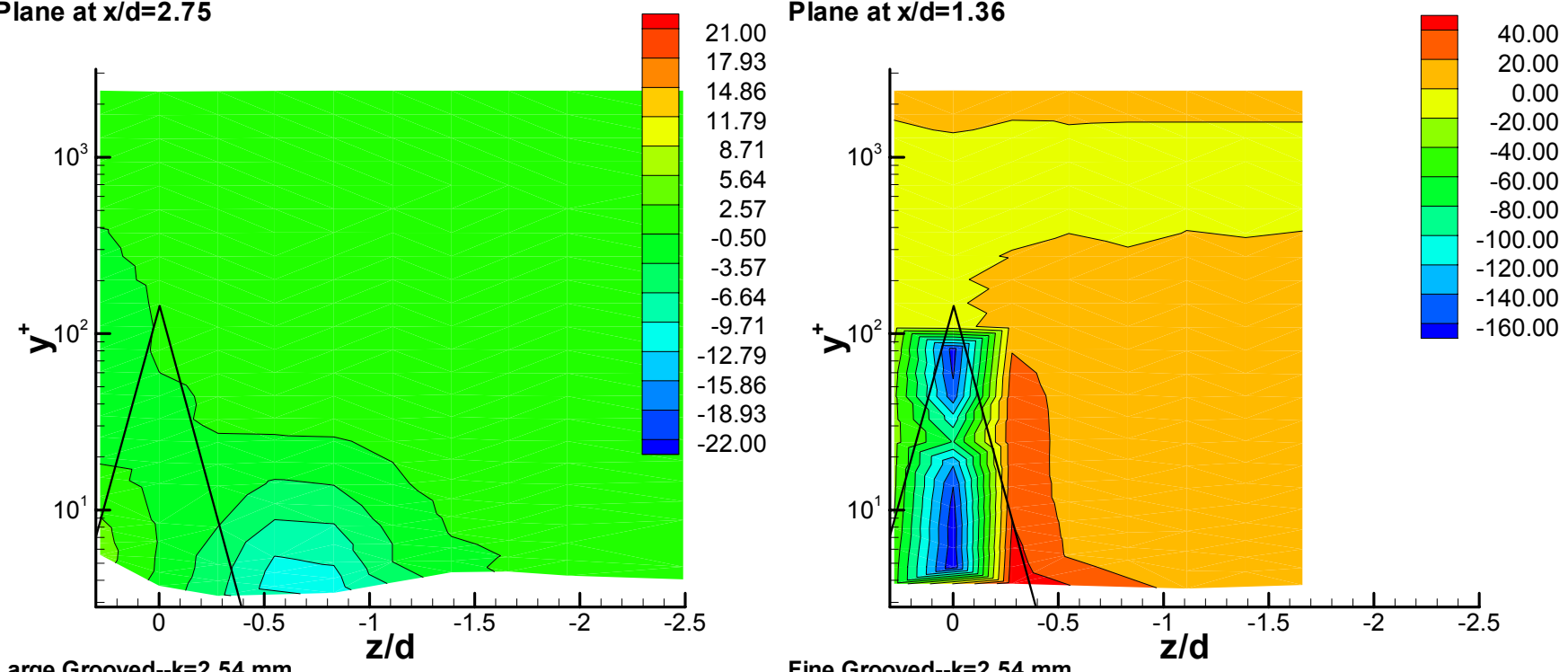

Large Grooved--k=2.54 mm

Fine Grooved--k=2.54 mm

Figure E.2: Contours of mean flow angle (FA) in the $y-z$ plane at $x / d=2.75$ (Fine Grooved $x / d=1.36)$ 


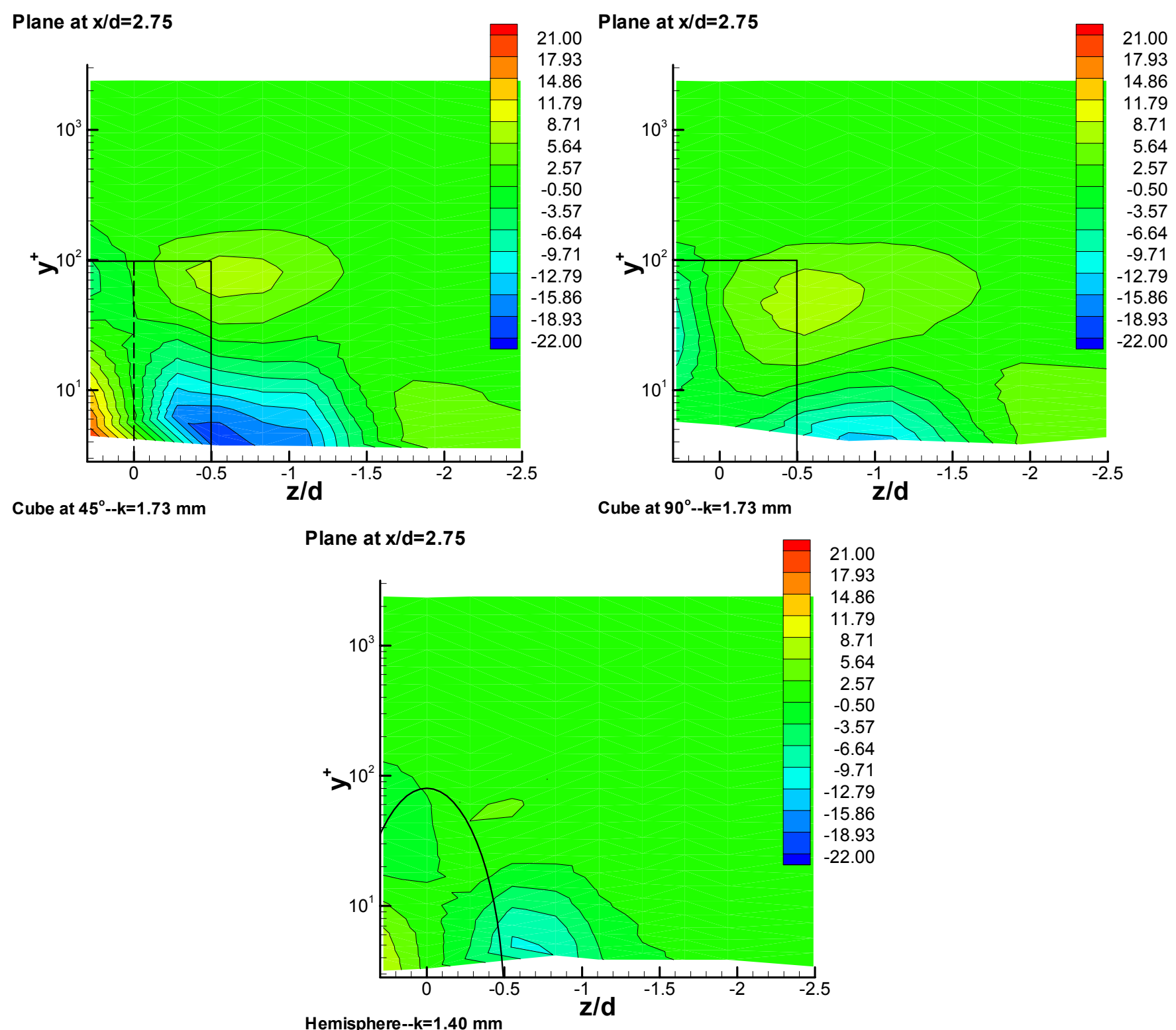

Figure E.3: Contours of mean flow angle (FA) in the $y-z$ plane at $x / d=2.75$ 

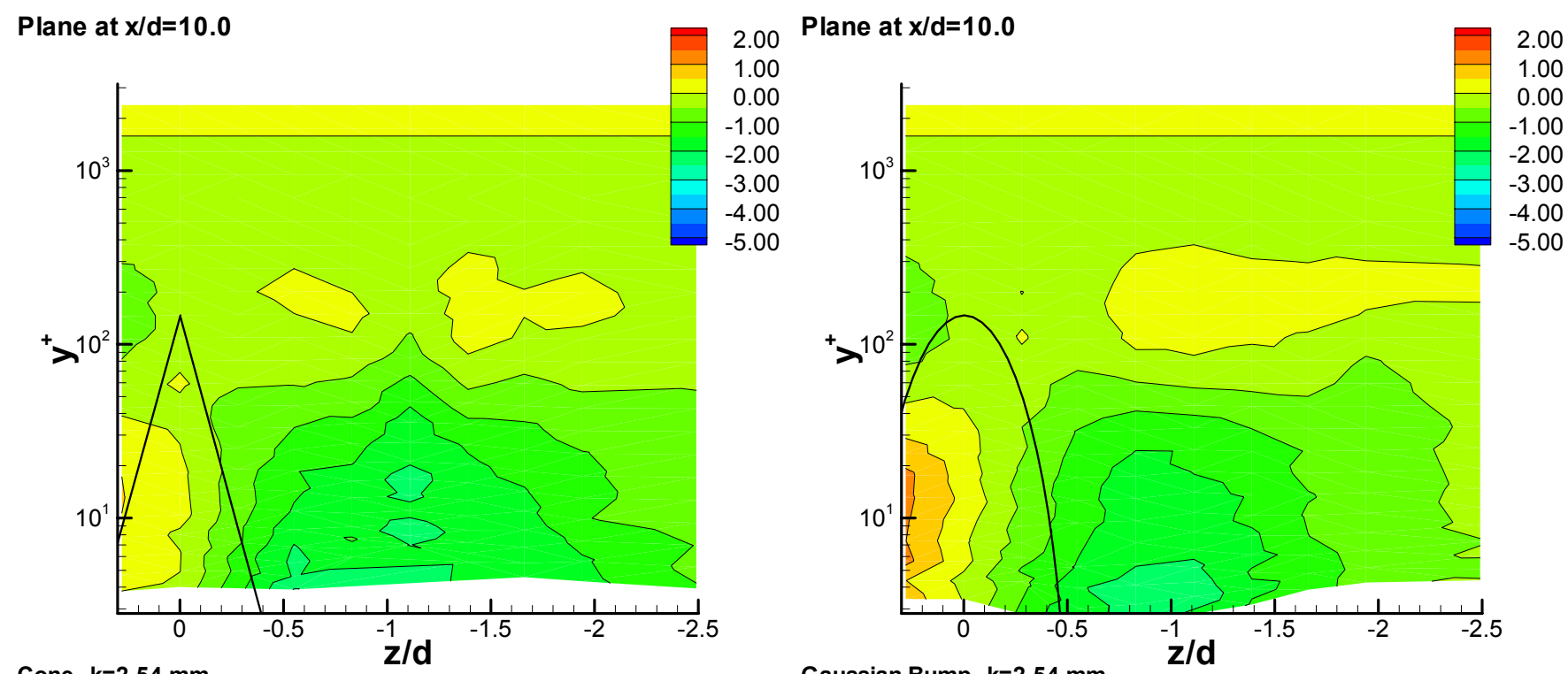

Gaussian Bump--k=2.54 mm

Plane at $x / d=10.0$

Plane at $x / d=10.0$
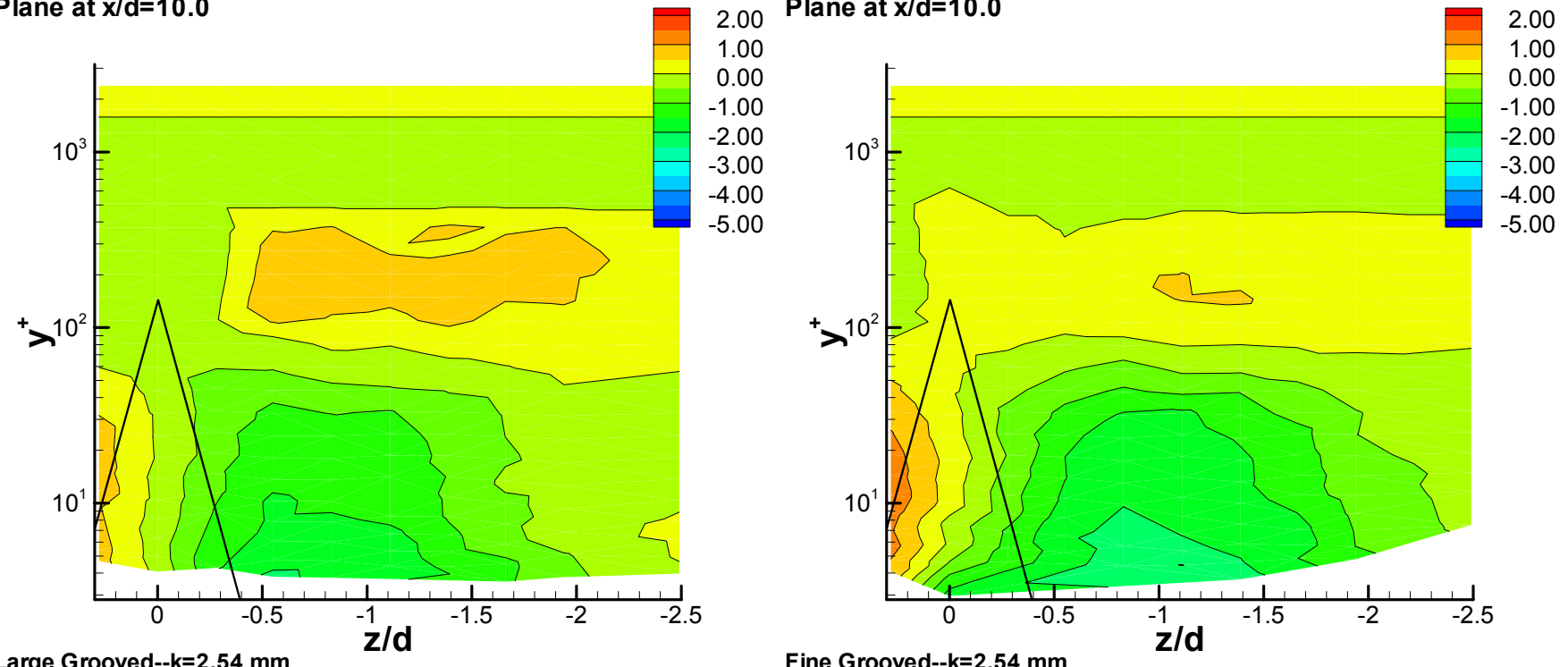

Figure E.4: Contours of mean flow angle (FA) in the $y-z$ plane at $x / d=10.0$ 


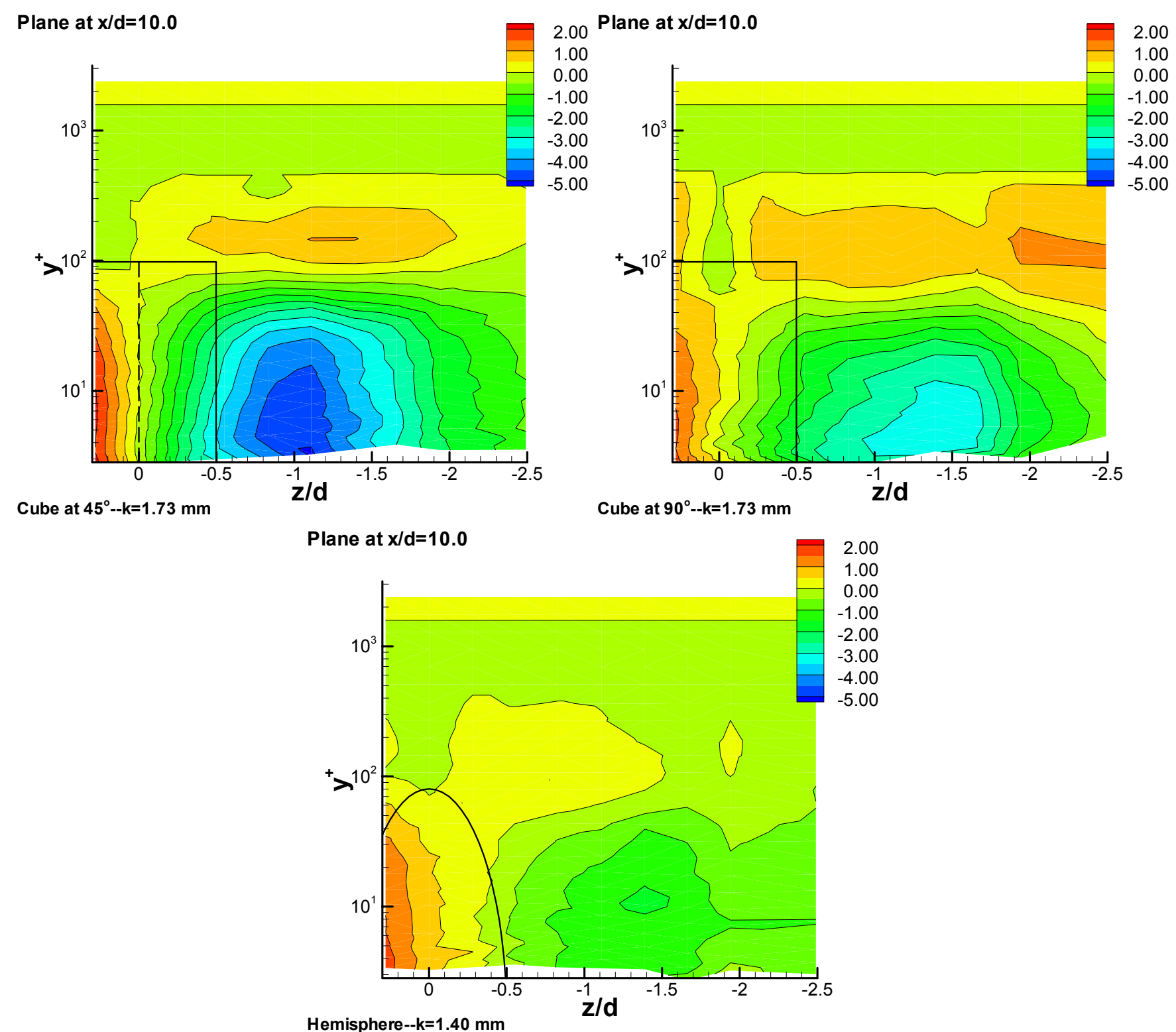

Figure E.5: Contours of mean flow angle (FA) in the $y-z$ plane at $x / d=10.0$ 

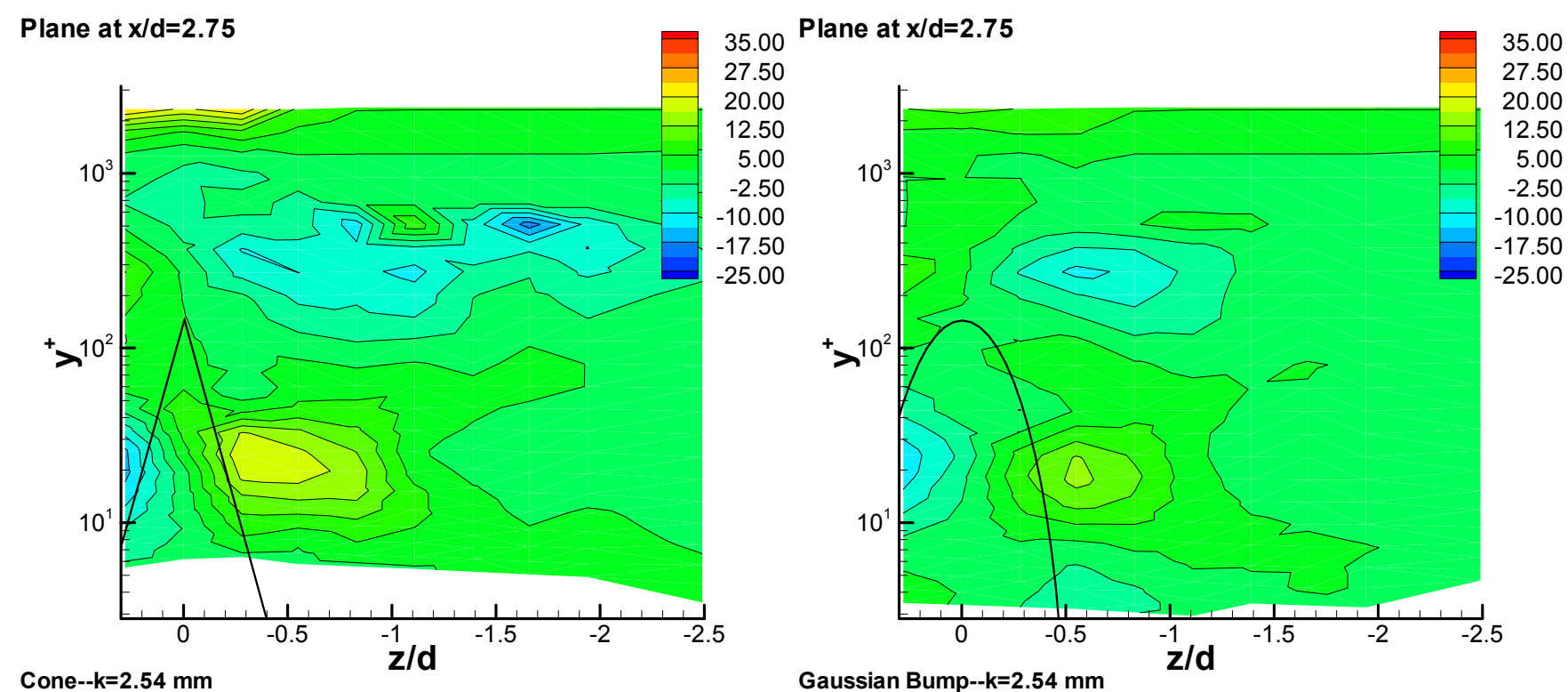

Plane at $\mathrm{x} / \mathrm{d}=\mathbf{2} . \mathbf{7 5}$

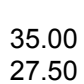

Gaussian Bump--k=2.54 mm

Plane at $x / d=1.36$

160.00 27.50
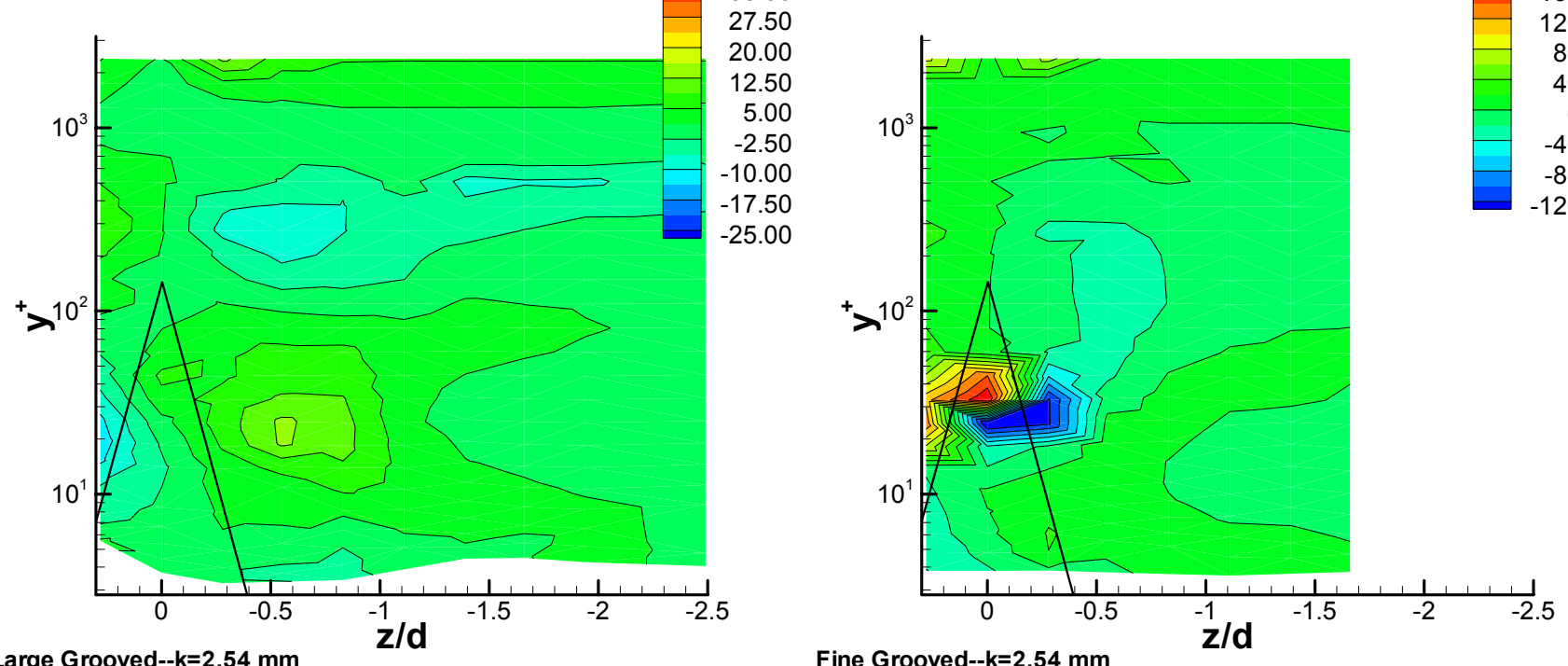

Figure E.6: Contours of flow gradient angle (FGA) in the $y-z$ plane at $x / d=2.75$ (Fine Grooved $x / d=1.36)$ 


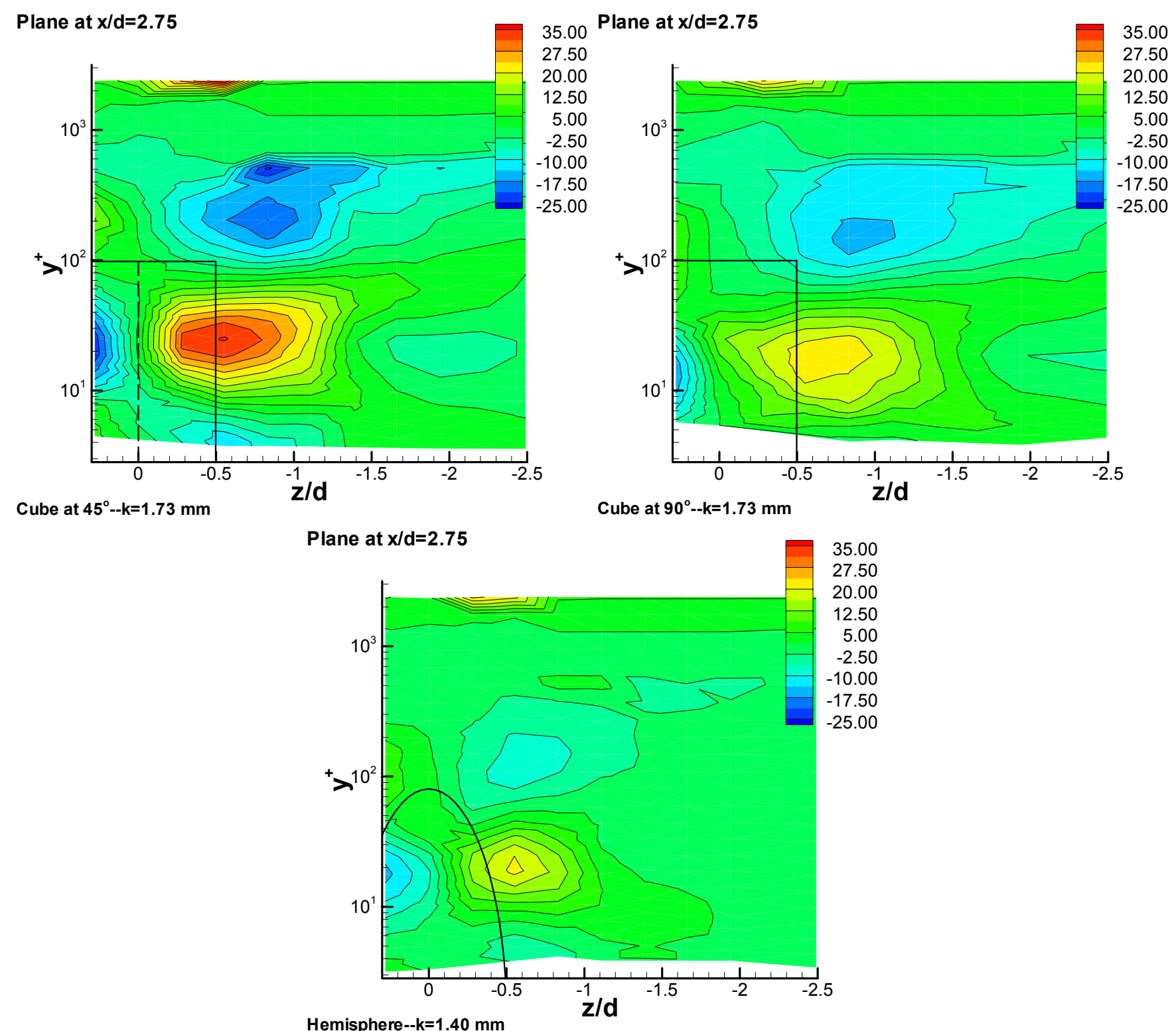

Figure E.7: Contours of flow gradient angle (FGA) in the $y-z$ plane at $x / d=2.75$ 

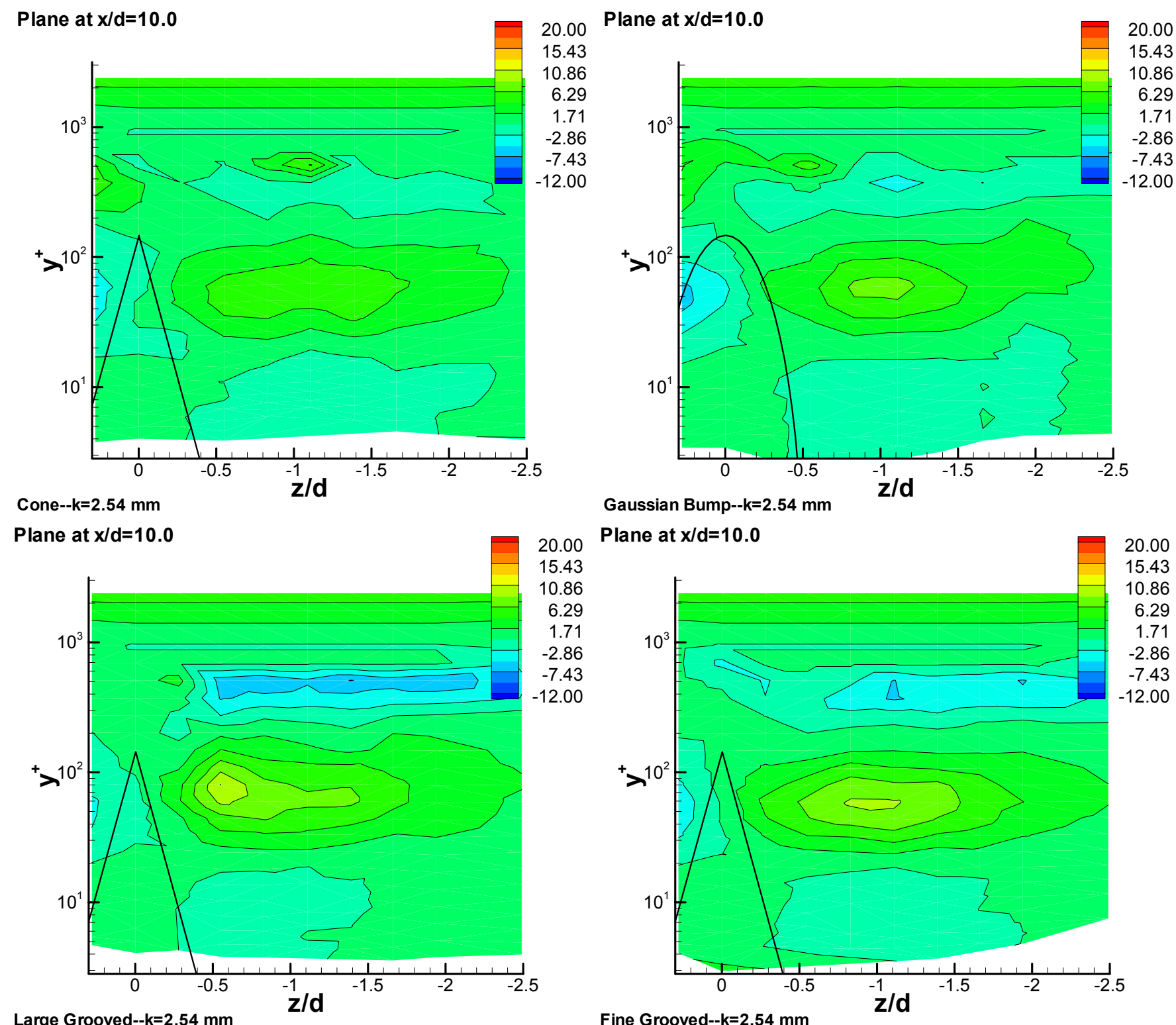

Figure E.8: Contours of flow gradient angle (FGA) in the $y-z$ plane at $x / d=10.0$ 


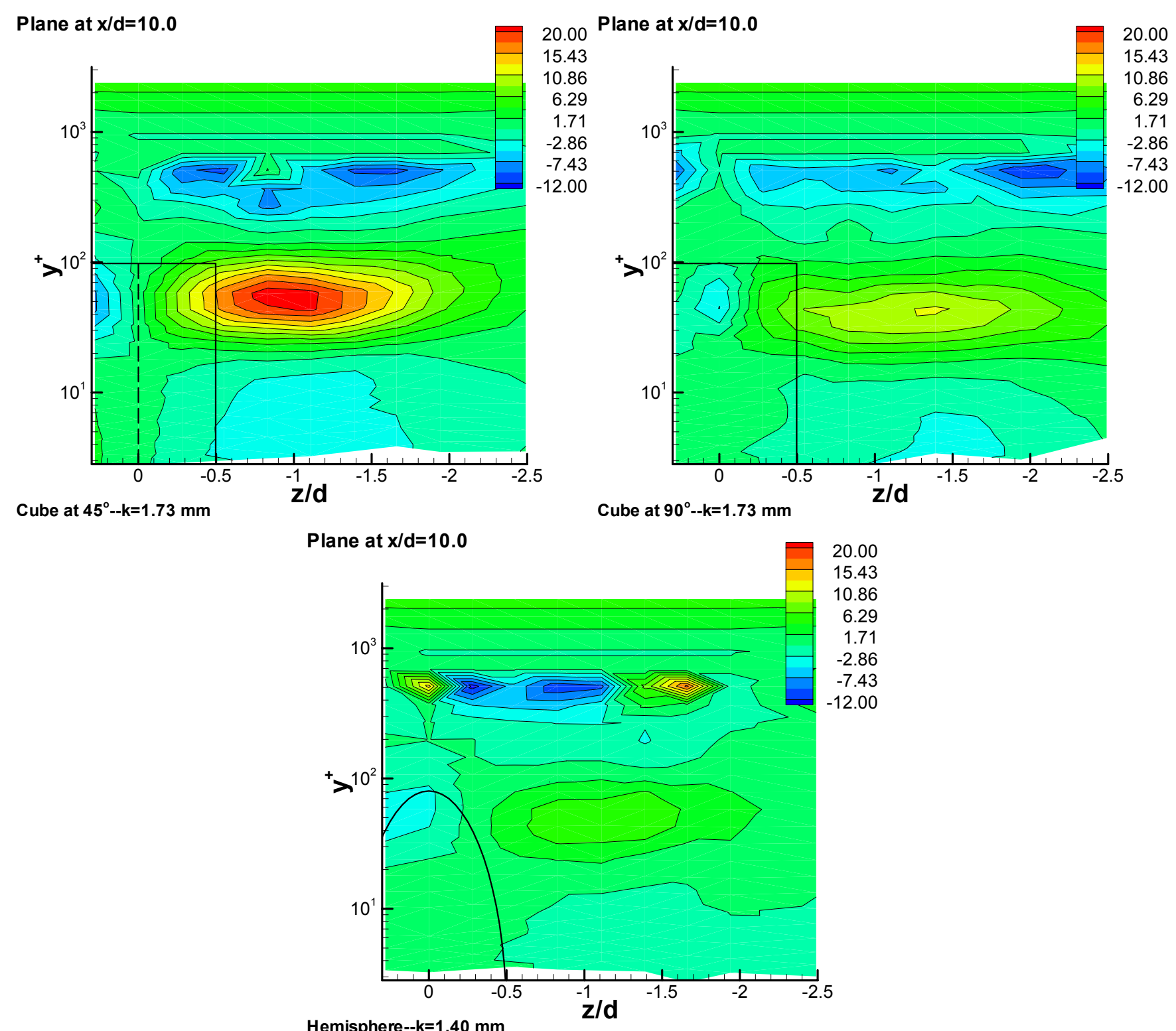

Figure E.9: Contours of flow gradient angle (FGA) in the $y-z$ plane at $x / d=10.0$ 


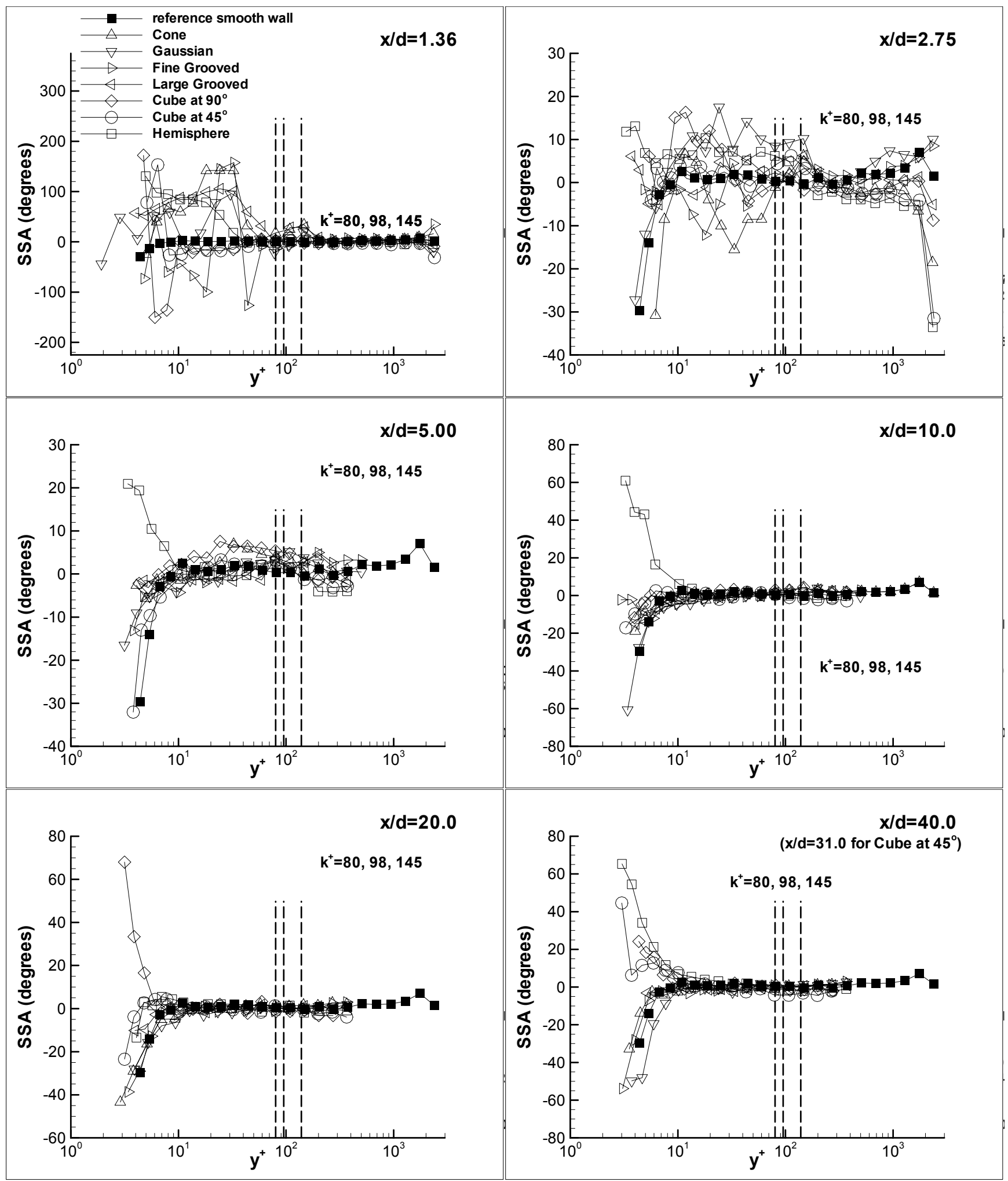

Figure E.10: Shear stress angle (SSA) variation along the centerline, where

$$
S S A=\tan ^{-1}(-\overline{v w} /-\overline{u v})
$$



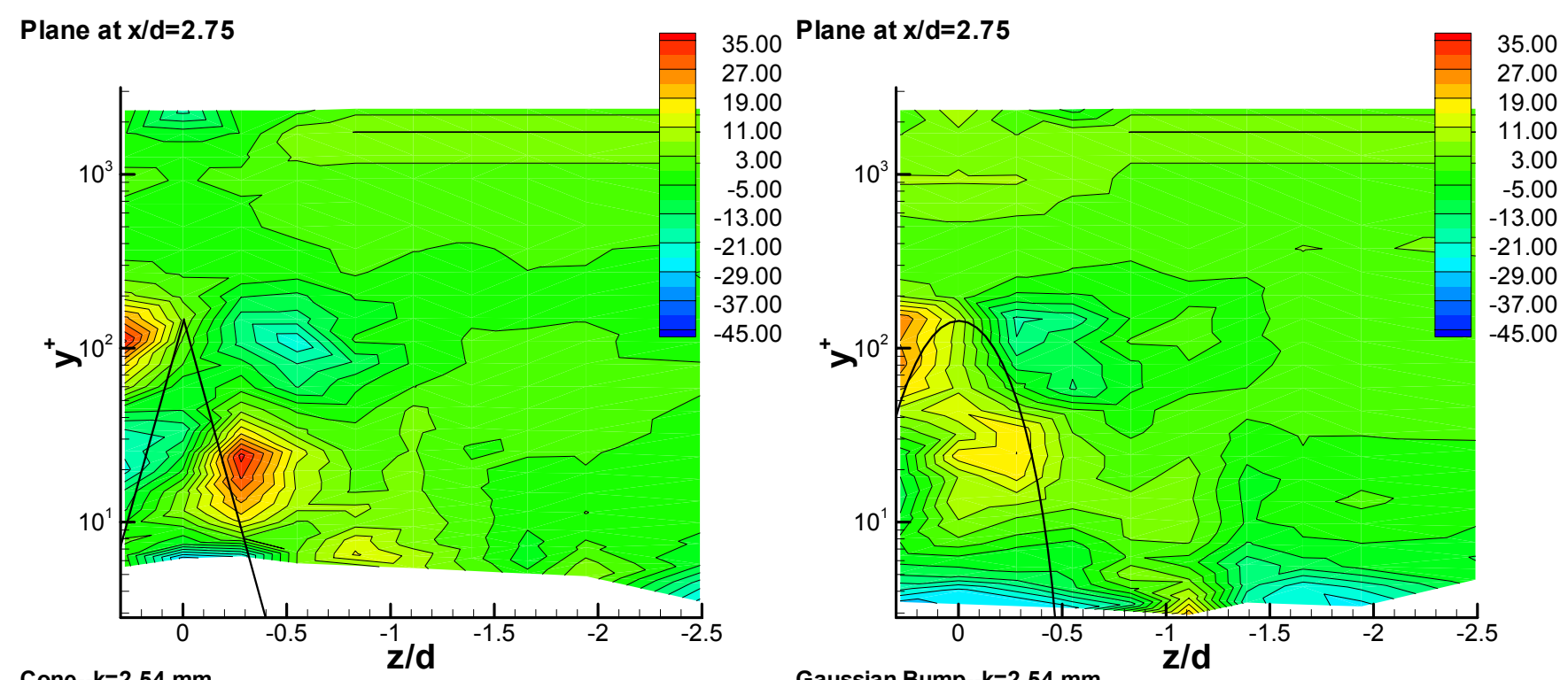

Plane at $x / d=2.75$

Gaussian Bump--k=2.54 mm
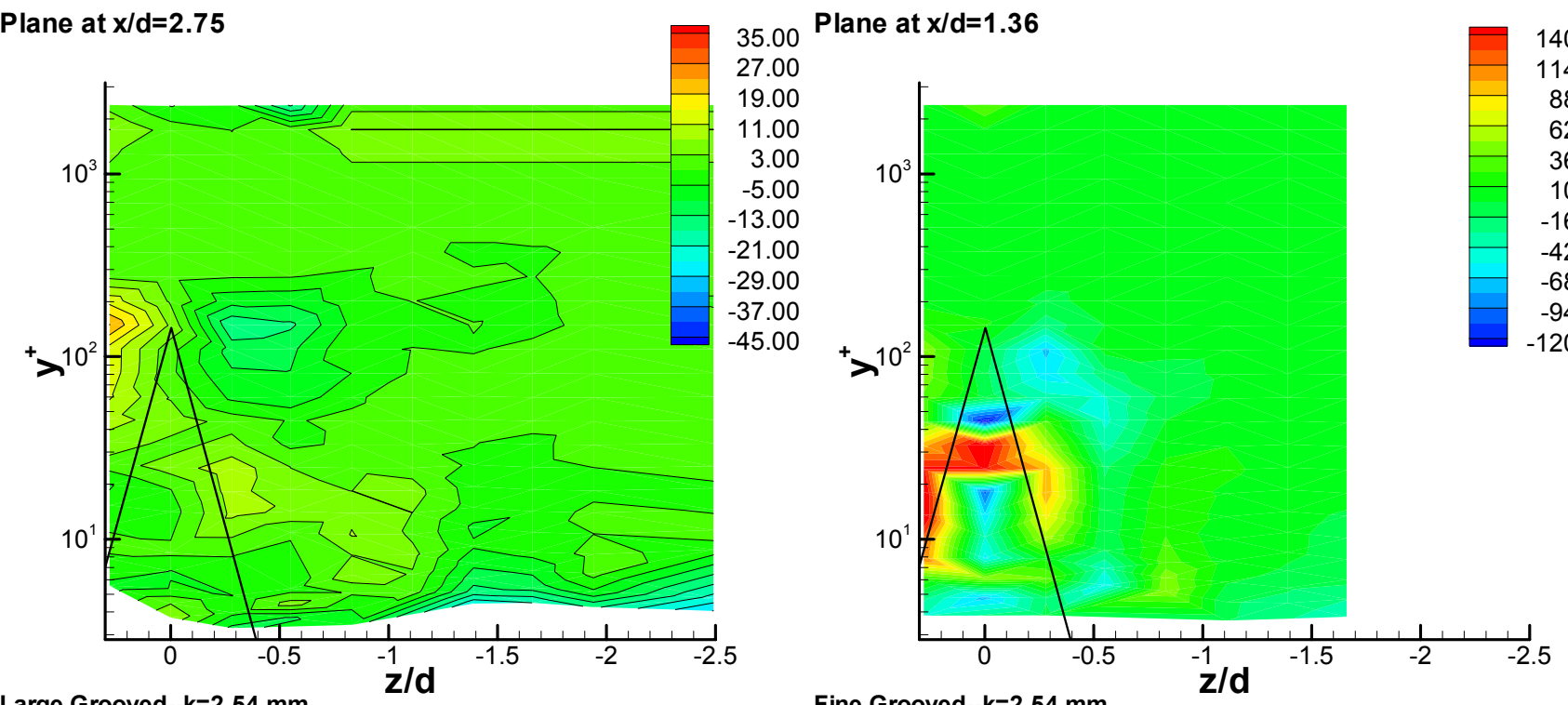

Large Grooved--k=2.54 mm

Fine Grooved--k=2.54 mm

Figure E.11: Contours of shear stress angle (SSA) in the $y-z$ plane at $x / d=2.75$ (Fine Grooved $x / d=1.36)$ 


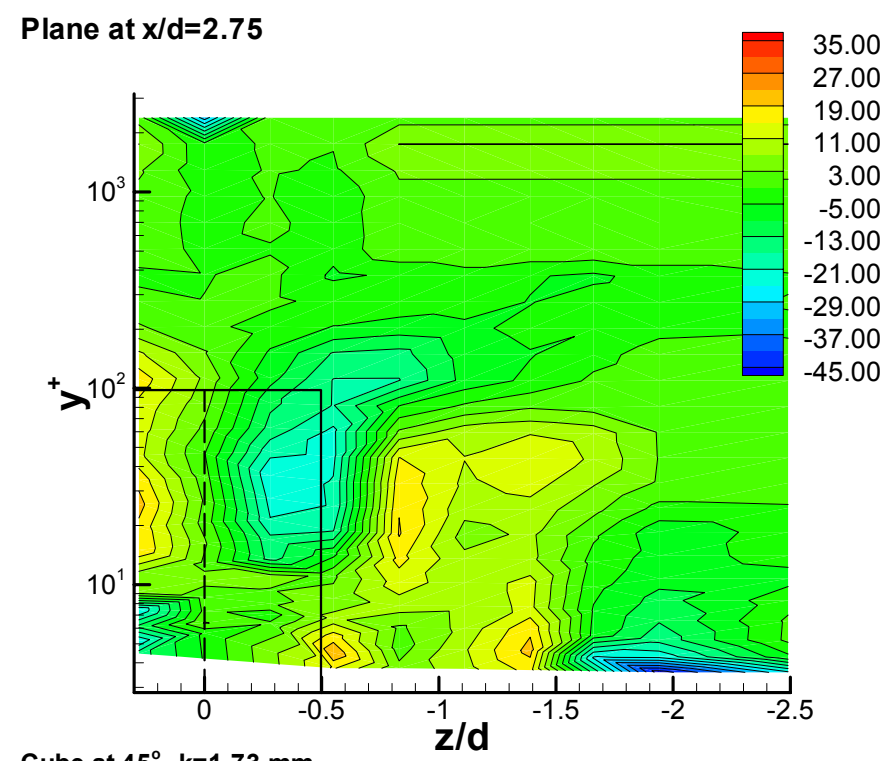

Cube at $45^{\circ}--\mathrm{k}=1.73 \mathrm{~mm}$

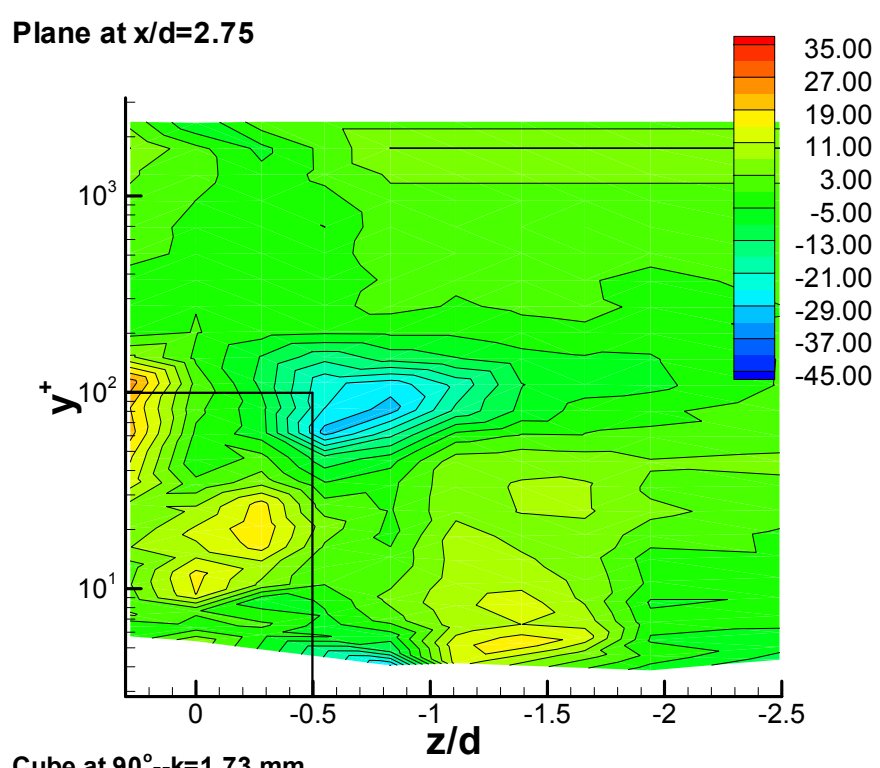

Plane at $x / d=2.75$

Cube at $90^{\circ}--\mathrm{k}=1.73 \mathrm{~mm}$

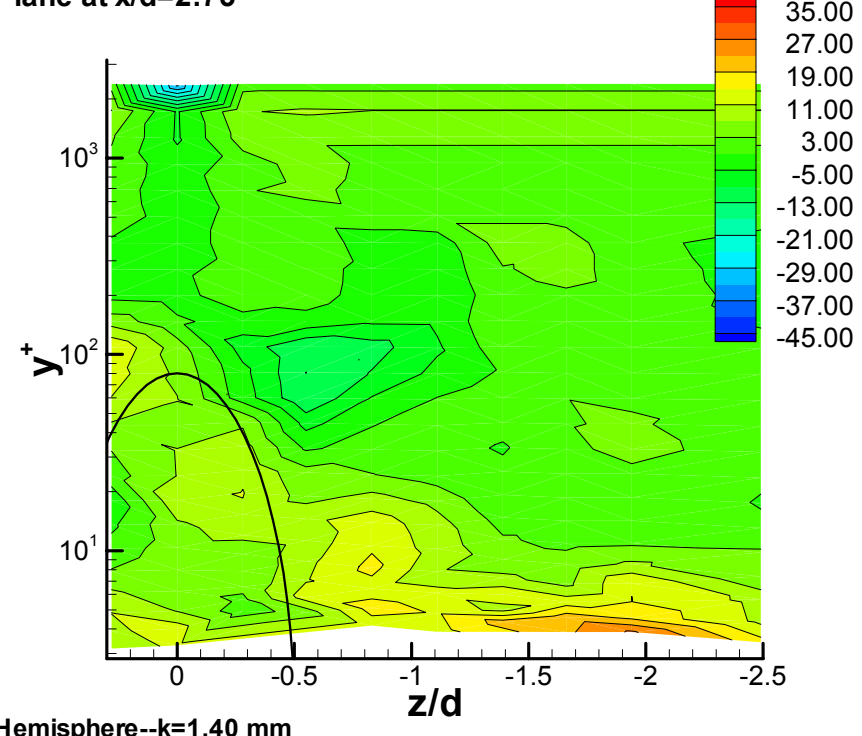

Figure E.12: Contours of shear stress angle (SSA) in the $y-z$ plane at $x / d=2.75$ 

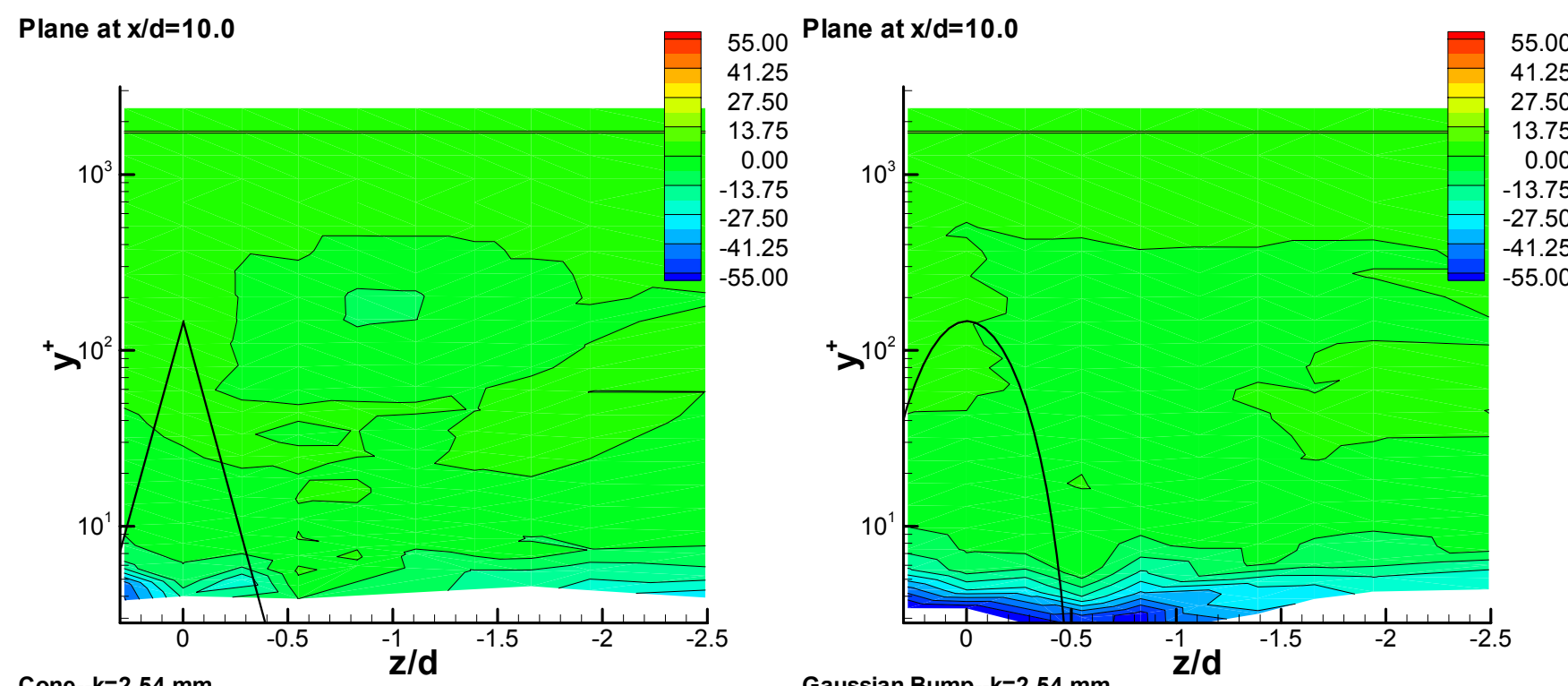

Gaussian Bump--k=2.54 mm

Plane at $x / d=10.0$

55.00

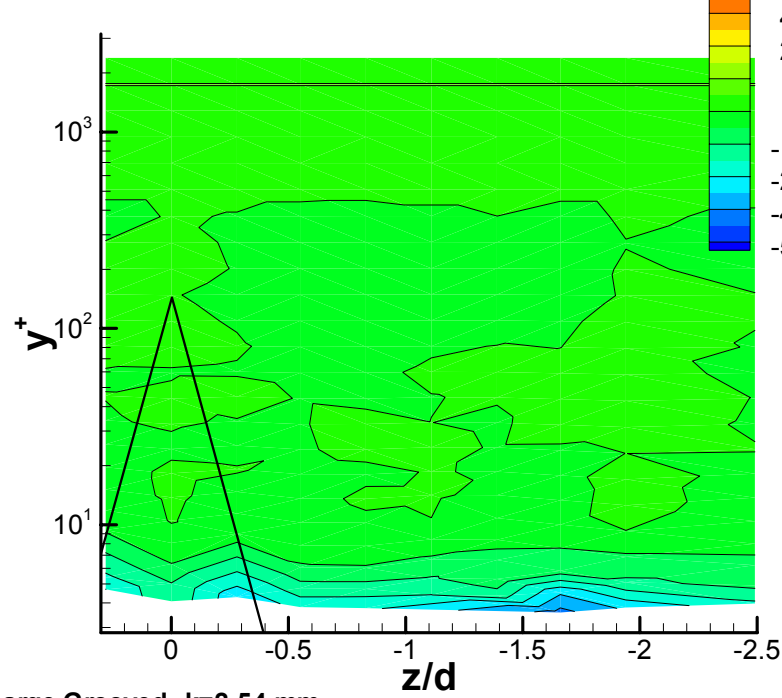

Plane at $x / d=10.0$

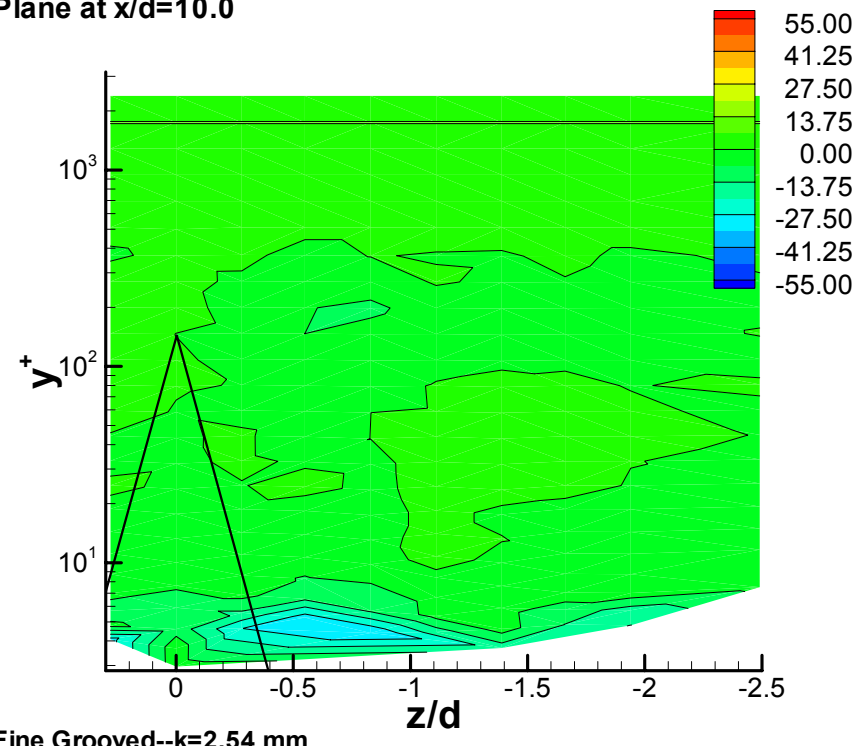

Large Grooved--k=2.54 mm

Fine Grooved--k=2.54 mm

Figure E.13: Contours of shear stress angle (SSA) in the $y-z$ plane at $x / d=10.0$ 


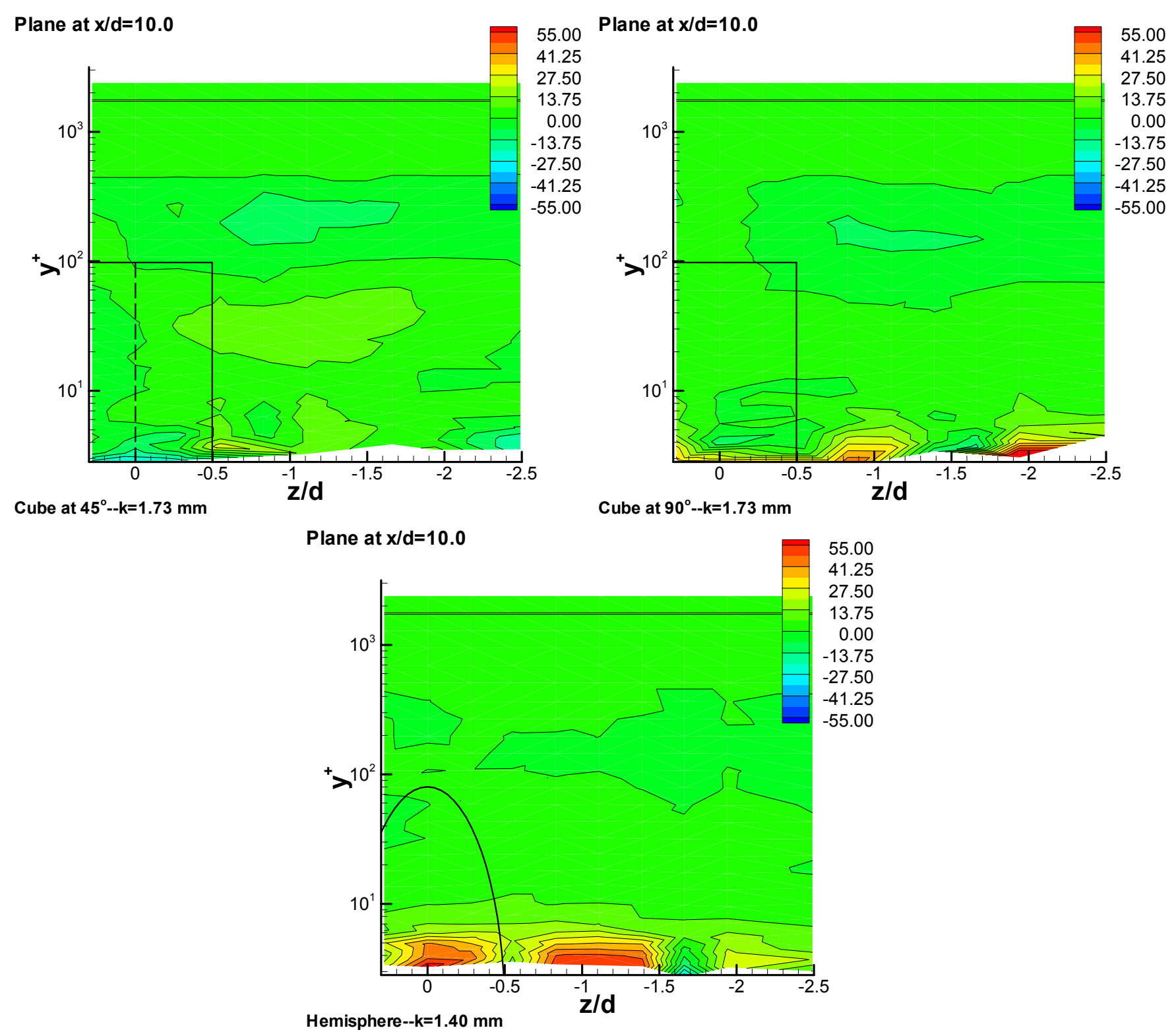

Figure E.14: Contours of shear stress angle (SSA) in the $y-z$ plane at $x / d=10.0$ 


\section{Appendix F Element Profile Shape Data}

Table F.1 gives the $y-z$ coordinates that define the shape of both the large and fine grooved elements. The shape of the profiles for all other roughness elements can be defined via their respective equations.

Table F.1: Coordinate locations for profile shapes of grooved elements

\begin{tabular}{|c|c|}
\hline \multicolumn{2}{|c|}{ Fine Grooved Element } \\
\hline $\mathrm{y}(\mathrm{m})$ & $\mathrm{z}(\mathrm{m})$ \\
\hline 0 & 0.0013462 \\
\hline 0.00002032 & 0.00133604 \\
\hline 0.00004064 & 0.00132588 \\
\hline 0.00006096 & 0.00131572 \\
\hline 0.00008128 & 0.00130556 \\
\hline 0.0001016 & 0.0012954 \\
\hline 0.00012192 & 0.00128524 \\
\hline 0.00014224 & 0.00127508 \\
\hline 0.00016256 & 0.00126492 \\
\hline 0.00018288 & 0.00125476 \\
\hline 0.0002032 & 0.0012446 \\
\hline 0.0002032 & 0.00102616 \\
\hline 0.00022352 & 0.001037844 \\
\hline 0.00024384 & 0.001049528 \\
\hline 0.00026416 & 0.001061212 \\
\hline 0.00028448 & 0.001072896 \\
\hline 0.0003048 & 0.00108458 \\
\hline 0.00032512 & 0.001096265 \\
\hline 0.00034544 & 0.001107949 \\
\hline 0.00036576 & 0.001119633 \\
\hline 0.00038608 & 0.001131317 \\
\hline 0.0004064 & 0.001143 \\
\hline 0.0004064 & 0.000924561 \\
\hline 0.00042672 & 0.000936245 \\
\hline 0.00044704 & 0.000947929 \\
\hline 0.00046736 & 0.000959613 \\
\hline 0.00048768 & 0.000971297 \\
\hline 0.000508 & 0.000982981 \\
\hline 0.00052832 & 0.000994665 \\
\hline 0.00054864 & 0.001006349 \\
\hline 0.00056896 & 0.001018033 \\
\hline 0.00058928 & 0.001029717 \\
\hline 0.0006096 & 0.001041401 \\
\hline 0.0006096 & 0.000822961 \\
\hline 0.00062992 & 0.000834645 \\
\hline
\end{tabular}

\begin{tabular}{|c|c|}
\hline \multicolumn{2}{|c|}{ Large Grooved Element } \\
\hline $\mathrm{y}(\mathrm{m})$ & $\mathrm{z}(\mathrm{m})$ \\
\hline 0 & 0.0009398 \\
\hline 0.0000508 & 0.00097028 \\
\hline 0.0001016 & 0.00100076 \\
\hline 0.0001524 & 0.00103124 \\
\hline 0.0002032 & 0.00106172 \\
\hline 0.000254 & 0.0010922 \\
\hline 0.0003048 & 0.00112268 \\
\hline 0.0003556 & 0.00115316 \\
\hline 0.0004064 & 0.00118364 \\
\hline 0.0004572 & 0.001214121 \\
\hline 0.000508 & 0.001244601 \\
\hline 0.000508 & 0.000685801 \\
\hline 0.0005588 & 0.000716281 \\
\hline 0.0006096 & 0.000746761 \\
\hline 0.0006604 & 0.000777241 \\
\hline 0.0007112 & 0.000807721 \\
\hline 0.000762 & 0.000838201 \\
\hline 0.0008128 & 0.000868681 \\
\hline 0.0008636 & 0.000899161 \\
\hline 0.0009144 & 0.000929641 \\
\hline 0.0009652 & 0.000960121 \\
\hline 0.001016 & 0.000990601 \\
\hline 0.001016 & 0.000431801 \\
\hline 0.0010668 & 0.000462281 \\
\hline 0.0011176 & 0.000492761 \\
\hline 0.0011684 & 0.000523241 \\
\hline 0.0012192 & 0.000553722 \\
\hline 0.00127 & 0.000584202 \\
\hline 0.0013208 & 0.000614682 \\
\hline 0.0013716 & 0.000645162 \\
\hline 0.0014224 & 0.000675642 \\
\hline 0.0014732 & 0.000706122 \\
\hline 0.001524 & 0.000736602 \\
\hline 0.001524 & 0.000177802 \\
\hline 0.0015748 & 0.000208282 \\
\hline
\end{tabular}




\begin{tabular}{|c|c|}
\hline 0.00065024 & 0.000846329 \\
\hline 0.00067056 & 0.000858013 \\
\hline 0.00069088 & 0.000869697 \\
\hline 0.0007112 & 0.000881381 \\
\hline 0.00073152 & 0.000893065 \\
\hline 0.00075184 & 0.000904749 \\
\hline 0.00077216 & 0.000916433 \\
\hline 0.00079248 & 0.000928117 \\
\hline 0.0008128 & 0.000939801 \\
\hline 0.0008128 & 0.000721361 \\
\hline 0.00083312 & 0.000733045 \\
\hline 0.00085344 & 0.000744729 \\
\hline 0.00087376 & 0.000756413 \\
\hline 0.00089408 & 0.000768097 \\
\hline 0.0009144 & 0.000779781 \\
\hline 0.00093472 & 0.000791465 \\
\hline 0.00095504 & 0.000803149 \\
\hline 0.00097536 & 0.000814833 \\
\hline 0.00099568 & 0.000826517 \\
\hline 0.001016 & 0.000838201 \\
\hline 0.001016003 & 0.000619763 \\
\hline 0.001036323 & 0.000631447 \\
\hline 0.001056643 & 0.000643131 \\
\hline 0.001076963 & 0.000654815 \\
\hline 0.001097283 & 0.000666499 \\
\hline 0.001117603 & 0.000678183 \\
\hline 0.001137923 & 0.000689867 \\
\hline 0.001158243 & 0.000701551 \\
\hline 0.001178563 & 0.000713235 \\
\hline 0.001198883 & 0.000724919 \\
\hline 0.0012192 & 0.000736601 \\
\hline 0.0012192 & 0.000518162 \\
\hline 0.00123952 & 0.000529846 \\
\hline 0.00125984 & 0.00054153 \\
\hline 0.00128016 & 0.000553214 \\
\hline 0.00130048 & 0.000564898 \\
\hline 0.0013208 & 0.000576582 \\
\hline 0.00134112 & 0.000588266 \\
\hline 0.00136144 & 0.00059995 \\
\hline 0.00138176 & 0.000611634 \\
\hline 0.00140208 & 0.000623318 \\
\hline 0.0014224 & 0.000635002 \\
\hline 0.0014224 & 0.000416562 \\
\hline 0.00144272 & 0.000428246 \\
\hline 0.00146304 & 0.00043993 \\
\hline 0.00148336 & 0.000451614 \\
\hline 0.00150368 & 0.000463298 \\
\hline 0.001524 & 0.000474982 \\
\hline & \\
\hline
\end{tabular}

\begin{tabular}{|c|c|}
\hline 0.0016256 & 0.000238762 \\
\hline 0.0016764 & 0.000269242 \\
\hline 0.0017272 & 0.000299722 \\
\hline 0.001778 & 0.000330202 \\
\hline 0.0018288 & 0.000360682 \\
\hline 0.0018796 & 0.000391162 \\
\hline 0.0019304 & 0.000421642 \\
\hline 0.0019812 & 0.000452122 \\
\hline 0.002032 & 0.000482602 \\
\hline 0.0020828 & 0.000457202 \\
\hline 0.0021336 & 0.000431802 \\
\hline 0.0021844 & 0.000406402 \\
\hline 0.0022352 & 0.000381002 \\
\hline 0.002286 & 0.000355602 \\
\hline 0.0023368 & 0.000330202 \\
\hline 0.00235712 & 0.000320042 \\
\hline 0.00235966 & 0.000315277 \\
\hline 0.00238506 & 0.00029889 \\
\hline 0.00241046 & 0.00027925 \\
\hline 0.00243586 & 0.000255608 \\
\hline 0.00246126 & 0.000226715 \\
\hline 0.00248666 & 0.000190195 \\
\hline 0.00251206 & 0.000140207 \\
\hline 0.00253746 & $4.30303 \mathrm{E}-05$ \\
\hline 0.00254 & 0 \\
\hline & \\
\hline
\end{tabular}




\begin{tabular}{|c|c|}
\hline 0.00154432 & 0.000486666 \\
\hline 0.00156464 & 0.00049835 \\
\hline 0.00158496 & 0.000510034 \\
\hline 0.00160528 & 0.000521718 \\
\hline 0.0016256 & 0.000533402 \\
\hline 0.0016256 & 0.000314962 \\
\hline 0.00164592 & 0.000326646 \\
\hline 0.00166624 & 0.00033833 \\
\hline 0.00168656 & 0.000350014 \\
\hline 0.00170688 & 0.000361698 \\
\hline 0.0017272 & 0.000373382 \\
\hline 0.00174752 & 0.000385066 \\
\hline 0.00176784 & 0.00039675 \\
\hline 0.00178816 & 0.000408434 \\
\hline 0.00180848 & 0.000420118 \\
\hline 0.0018288 & 0.000431802 \\
\hline 0.0018288 & 0.000213362 \\
\hline 0.00184912 & 0.000225046 \\
\hline 0.00186944 & 0.00023673 \\
\hline 0.00188976 & 0.000248414 \\
\hline 0.00191008 & 0.000260098 \\
\hline 0.0019304 & 0.000271782 \\
\hline 0.00195072 & 0.000283466 \\
\hline 0.00197104 & 0.00029515 \\
\hline 0.00199136 & 0.000306834 \\
\hline 0.00201168 & 0.000318518 \\
\hline 0.002032 & 0.000330202 \\
\hline 0.002032003 & 0.000111764 \\
\hline 0.002052323 & 0.000123448 \\
\hline 0.002072643 & 0.000135132 \\
\hline 0.002092963 & 0.000146816 \\
\hline 0.002113283 & 0.0001585 \\
\hline 0.002133603 & 0.000170184 \\
\hline 0.002153923 & 0.000181868 \\
\hline 0.002174243 & 0.000193552 \\
\hline 0.002194563 & 0.000205236 \\
\hline 0.002214883 & 0.00021692 \\
\hline 0.0022352 & 0.000228602 \\
\hline 0.00225552 & 0.000218442 \\
\hline 0.00227584 & 0.000208282 \\
\hline 0.00229616 & 0.000198122 \\
\hline 0.00231648 & 0.000187962 \\
\hline 0.0023368 & 0.000177802 \\
\hline 0.00235712 & 0.000167642 \\
\hline 0.00237744 & 0.000157482 \\
\hline 0.00239776 & 0.000147322 \\
\hline 0.00241808 & 0.000137162 \\
\hline 0.0024384 & 0.000127002 \\
\hline
\end{tabular}




\begin{tabular}{|c|c|}
\hline 0.00245872 & 0.000116842 \\
\hline 0.00247904 & 0.000106682 \\
\hline 0.00249936 & $9.65225 \mathrm{E}-05$ \\
\hline 0.0025019 & $9.68869 \mathrm{E}-05$ \\
\hline 0.00250825 & $8.95778 \mathrm{E}-05$ \\
\hline 0.0025146 & $8.11211 \mathrm{E}-05$ \\
\hline 0.00252095 & $7.11087 \mathrm{E}-05$ \\
\hline 0.0025273 & $5.87504 \mathrm{E}-05$ \\
\hline 0.00253365 & $4.20253 \mathrm{E}-05$ \\
\hline 0.00254 & 0 \\
\hline
\end{tabular}




\section{Vita}

Jeremy Bennington started his undergraduate career at the University of Northern Colorado (UNC) in Greeley, CO the fall of 1997. While at UNC he studied PreEngineering and played baseball at the varsity and semi-professional levels. In the fall of 1999 he transferred to Kansas State University in Manhattan, KS to pursue a degree in Mechanical Engineering. In the spring of 2000 he transferred to the University of Wyoming located in Laramie, WY. He graduated cum laude in the spring of 2002 with a BS in Mechanical Engineering while majoring in Mechanical Engineering and Mathematics. In pursuit of higher education, he entered the graduate program in the Aerospace and Ocean Engineering Department at Virginia Polytechnic Institute and State University in Blacksburg, VA. He completed the requirements for a Master of Science degree in Aerospace Engineering in the fall of 2004. 